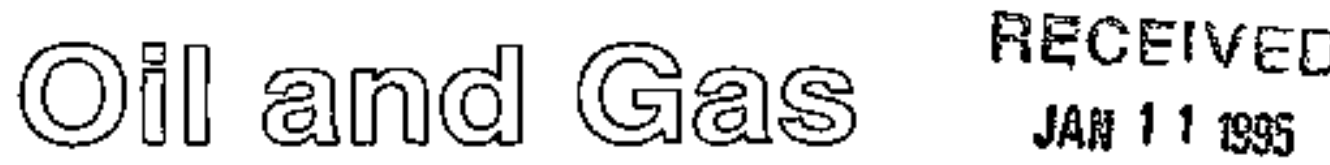 [F[eld Code Master "y 1995
}

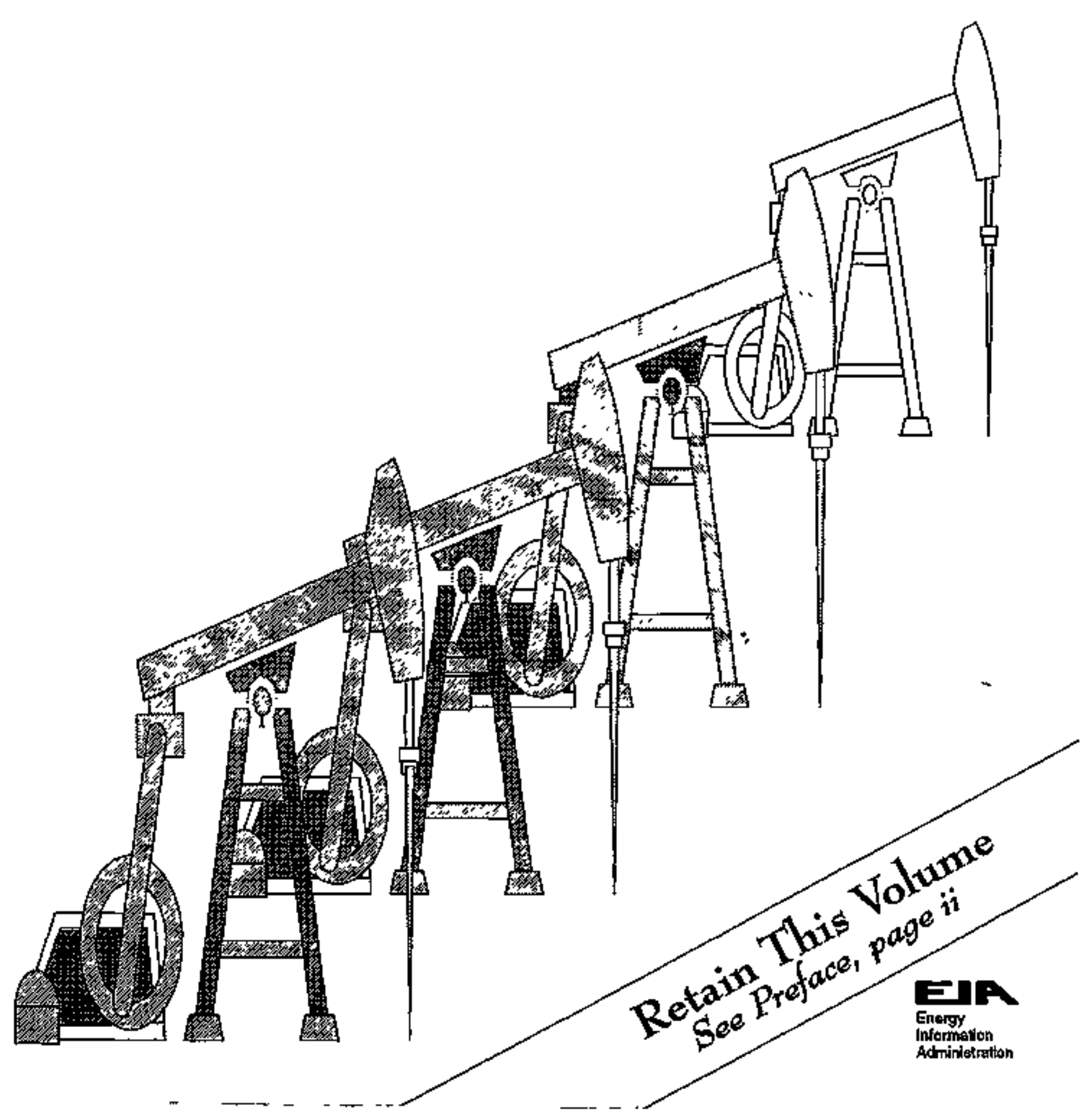


This publication and other Energy Information Administration (ElA) publications may be purchased from the Superintendent of Documents, U.S. Government Printing Office.

Telephone orders may be directed to:

Superintendent of Documents

U.S. Government Printing Office

Main Order Desk

(202) $512-1800$

FAX: (202) $\$ 12-2250$

8 a.m. 10 4:30 p.m., eastem time, $M-F$

Alt mail orders should be directed to:

U.S. Government Printing Office

P.O. Box 371954

Pittsburgli, PA 15250-7954

Compltmentary subscriptions and single issues are available to certain groups of subscribers, such as public and academic libraries. Federal, State, local and foreign governments, ElA survey respondents, and the media. For furthet information and for answers to questions on energy statistics, please contact EIA's National Energy Information Center. Address, telephone ulumbers, and hours are as follows:

National Energy Information Center, EI-23।

Energy Information Administration

Forrestal Building, Room IF-048

Washington, DC 20585

(202) $586-8800$

(202) 586-0727 (FAX)

TTY: For people who are deaf or hard

of hearing: (202) 586-1181

9 a.Im. to 5 p.m., eastem time, M-F

Internet Addresses:

E-mail: infoctr@eia.doe.gov

World Wide Web Site: http:/www,eia.doe.gov

Gopher Site: gopher//gopher.eia.doe.gov

FTP Site: ftp:/ftp.eia.doe.gov

Released tor Printing December 13, 1995

GPO Stock No.: 061-003-00944-1 


\section{Oil and Gas \\ Field Code Master List}

\section{5}

December 1995

\section{Energy Information Administration \\ Office of Oil and Gas \\ U.S. Department of Energy \\ Washington, D.C. 20505}

Thla report was prepared by the Eneray Intormatlon Admintatrellon, the Independent siatlatical and analyleal

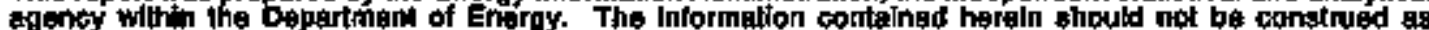
edwecting or restecting any polloy poshlon of the Depariment of Enerdy or any oiber organdzation.

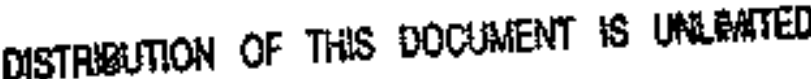
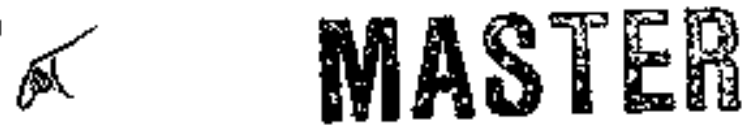


\section{Preface}

The Oil and Gas Fleld Code Master List 1995 is the fourteenth annual listing of all identiffled ofl and gas fields in the United States. It is updated with field information collected through October 1995. The purpose of this publication is to provide tuique, standardized codes for identification of domestic fields. Uise of these field codes fosters consistency of field identification by government and industry.

The use of freld nanses and codes listed in this publicstion is required on survey forms and other reports regarding field-specific data collected by the Energy Information Administration (EIA). The surveys currently using these field codes are the following: Form EIA-23, "Annual Survey of Domestic Oll and Gas Reserves" and Form EIA-191, entitled "Underground Gas Storage Report".
A glossary of key terms is provided to assist readers in more fully understanding the information in this Field Code Master List.

EIA gratefully acknowledges the assistance provided by the various State organjzations and trade associations in verifying the existence of fields and confirming their officially recognized names.

General information regarding this publitation may be obtained from Craig H. Cranston (202) 586-6083, Chief, Reserves and Production Branch, Reserves and Nature] Gas Division, in the Energy Information Administration's Office of Oil and Gas. Detailed information regarding the preparation of this document may be obtatined from Robert F. King (202) 386-4787, Reserves and Production Branch (rking (9eia.doe.gov).

The Field Code Index, a listing of all freld names and the States in which they occur, ordered by field code, has been removed from this year's publication to reduce printing and postage costs. Complete copies (incinding the Field Code Index) will be available on the EIA CD-ROM and the EIA World-Wide Web Site. Future editions of the complete Master List will be available on CD-ROM and other efectronic media. Respondents to EIA Forms 23 and 191 will receive only printed updates to this volume in future years. It should therefore be retafned. 


\section{Contents}

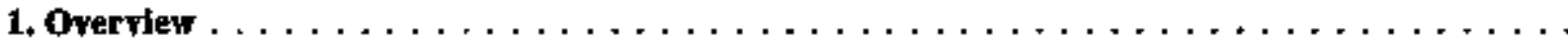

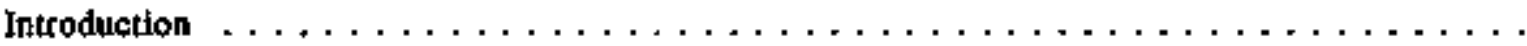

Summery Statistics $\ldots \ldots+\ldots+\ldots+\ldots \ldots+\ldots+\ldots+\ldots+\ldots+\ldots+\ldots+\ldots+\ldots+\ldots+\ldots$

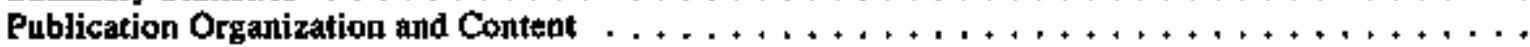

History

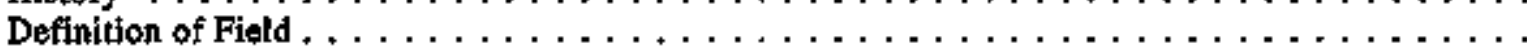

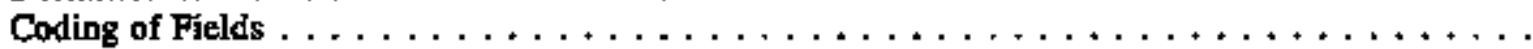

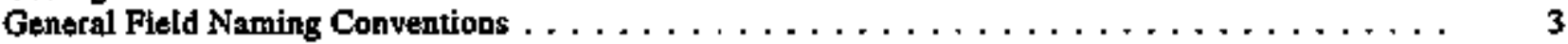

2. Methodology for Field Code Assignments $\ldots \ldots \ldots \ldots \ldots$

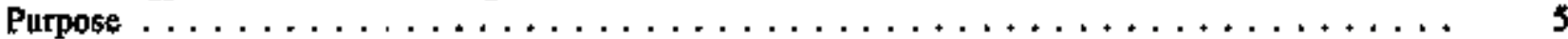

General System Overview . . . . . . . . . . . . . . . . . . . . . . 5

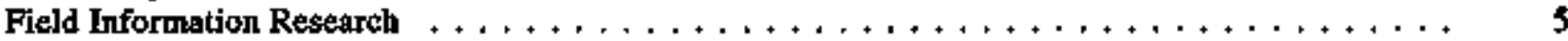

State Source Review Procedures $\ldots \ldots \ldots \ldots \ldots \ldots$

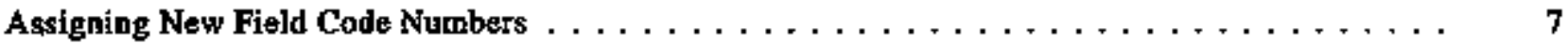

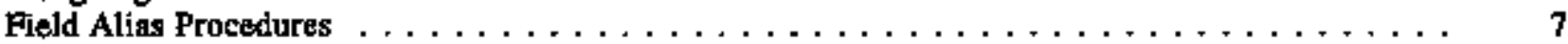

Offshore Code Assignments $\ldots \ldots \ldots+\ldots \ldots \ldots$

Field Naming Conventions . . . . . . . . . . . . . . . . . . . . . . . 8

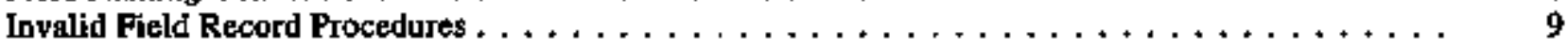

3. User's Guide $\ldots \ldots \ldots \ldots \ldots \ldots$

Field Code Master List $\ldots \ldots \ldots \ldots$

Coalbed Methane Field List . . . . . . . . . . . . . . . . . . . . . . . . . . . 14

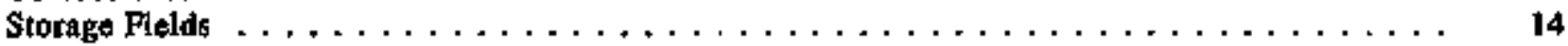

Fields Lacated in Multiple Juristictions $\ldots \ldots \ldots \ldots$

Outer Continental Sbelf Planntng Ateas $\ldots \ldots \ldots \ldots \ldots$

Glossinty $\ldots \ldots \ldots \ldots \ldots \ldots \ldots$

Oil and Gas Field Code Master List $\ldots \ldots \ldots \ldots \ldots \ldots$

Oll and Gas Invalid Fleld Record List $\ldots \ldots \ldots \ldots$

\section{Tables}

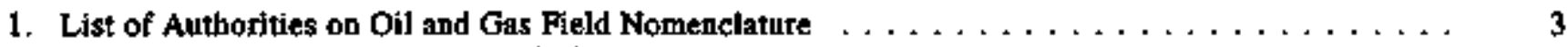

2. State Abbreviations and Geographic Subdivision Codes . . . . . . . . . . . . . . . . . 16

3. U.S. Geological Survey Alaska Quadtangles and Associated Codes . . . . . . . . . . . . . . . 17

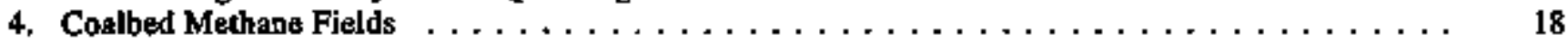

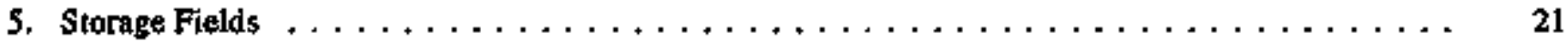

6. Fjelds Loctated in Multiple Jariadictions $\ldots \ldots \ldots \ldots$ 


\section{Figures}

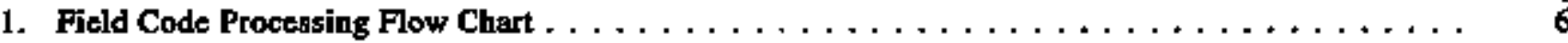

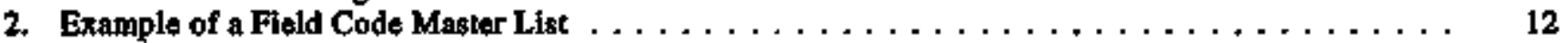

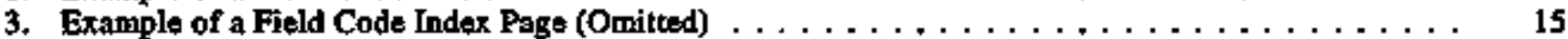

4. Subdivisions of Alaska and U.S. Geological Survey Quadrangles $\ldots \ldots \ldots \ldots \ldots \ldots \ldots \ldots \ldots$

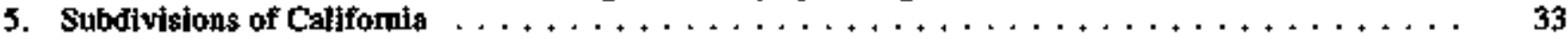

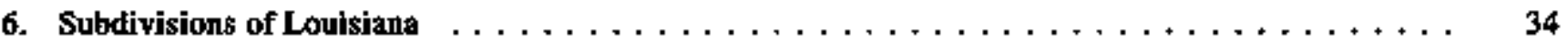

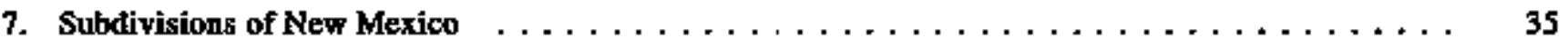

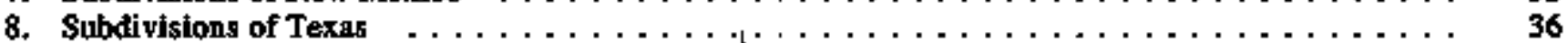

9. Western Planning Area, Gulf of Mexico Onter Continental Shelf Region . . . . . . . . . . . . . 37

10. Central Planning Ares, Gulf of Mexico Outer Continental Shelf Region . . . . . . . . . . 38

11. Eastern Planning Area, Gulf of Mexico Otiter Continental Shelf Region . . . . . . . . . . . . . . 39 


\section{Overview}

\section{Introduction}

This is the fourteenth anmual edition of the Energy Information Administration's (ETA) Oil and Gas Field Code Master List. It reflects data collected through October 1995 and provides standardized field name spellings and codes for all identified oil and/or gas fields in the United States. The Field Code Index; listing of all field names and the States in which they occur, ordered by field code, has been removed frota this year's publication to reduce printing and phstage costs. Complete copies (Inciudilng the Field Code Index) will be avalable on the RIA CD-ROM and the EIA World-Wide Web Stte. Futare editions of the complete Mastar List will be available on CD-ROM and ather electronic media. Respondents to EIA Forms 23 and 191 will receive only printed updates to this volume in fature years. If should therefore be retained, The master field nime spellings and codes are to be used by respondents when filing the following Department of Energy (DOE) forms:

- Form EIA-23, "Annual Survey of Domestíc Ot] and Gas Reserves", filed by oil and gas well operators (fteld codes are reguired from larger operators only).

- Form EIA-191, "Underground Gas Storage Report", flled by natural gas producers and distributors who operate underground naturat gas storage facilities.

Other Federal and State government agencies, as wel] as industry, use the ELA Oil and Gas Field Code Master List as the standard for field identification. A machinereadable version of the Oit and Gas Field Code Master List is avaitable from the National Tochnical Information Service, 5285 Port Royal Road, Springfield, Vis. ginia 22161, (703) 487.4650 .

In order for the Master List to be useful, it must be accurate and remain current. To accomplish this, EIA constantly reviews and revises this list. The EIA welcomes all comments, corrections, and additions to the Master List. All such information should be given to the BIA Field Code Coordinator at (202) 586-4787.

EIA gratefully acknowledges the sssistance provided by Dumerons State organizations and trade associations in verifying the existence of ftelds and their official nomenclature.

\section{Summary Statistics}

There are 57,400 fietd records in this year's otl and Gas Fleld Code Master List (FCML). This is 646 more than in last year's report. As it is maintained by EIA. the Master List includes the following:

- Field records for each State and county in which a field resides.

- Field records for each offebore area block in the Gulf of Mexico in which a neld resides.

- Field records for each alias field name (see definition of aljas below).

- Fields crossing State boundarles that may be assigned different names by the respective State naming authorities.

Taking into consideration the double-sounting of fields under such circumstances, EIA identifies 46,312 distinct fields in the United States as of October 1995. This count includes fields that no longer produce oil or gas, and 383 fields used in wbole or in part for oil or gas storage. The following is a summary count by hydrocarbon type of distinct fields in the United States:

\begin{tabular}{|c|c|}
\hline Fildi Type & Number \\
\hline U.S. oil and gas distiuct fiends. . .....+... & 46,312 \\
\hline Findos with oil,$\ldots++\ldots \ldots++++\ldots \ldots \ldots+++$ & 34,341 \\
\hline Fitelds with gas $\ldots, \ldots \ldots \ldots \ldots \ldots \ldots$, & 31,663 \\
\hline 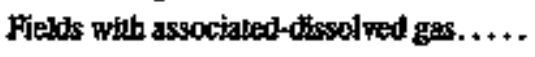 & 15,863 \\
\hline Fiethe wheh nomassociated ges .......... & 21,131 \\
\hline Fiesls with mespecified bydroctation ...+ & 145 \\
\hline
\end{tabular}

Note: The sum of these fields is areater than the lotal number of distinet fields, since many fieids have more than ont type of bydrocarton present.

EIA has assigned a unique six-digit field code to each field name. The field names recognized by the state Naming Authorities or by tradition total 52,054. Of these, 45,311 are currently considered official, while 6,743 have been declared unoficial by the State Naming Authorities and are designated as altas vames by BIA. 
This report also contains an Invalid Field Record List of 40 records that have been semoved from the FCML since last year's report. These records were found to be either technically incorrect or to represent field names which were never recognized by State naming authorities.

\section{Publication Organization and Content}

Chapter 2 provides details on the methodology nsed to assign field codes and the procedures followed to standardize field aames. Chapter 3, the User's Guide, and the Glossary provide users with the definitions and explanations needed to utilize this publication. The FCML follows the Glossary. It is organized by state, with fields sorted alphabetically by name witbin each individual State. Fields in the Federal Offshore Outer Continental Shelf are listed separately at the end after Wyoning. Each field aame entry contains the field code, field name, geographical information, and other related information. The Invalid Field Record List identifies those field records which were determined to be invalid since the previous repart.

\section{History}

The EIA FCML evolved from the Federal Power Commission's Field/Plant Code List (FPC Field Code List). The FPC Field Code List, originally developed over $\mathbf{2 0}$ years ago, bad a unique code assigned to each field on the list. That is, two fields having identical games in separate States bas separate six-digit field codes. However, some respondents to Form FPC 15, "Interstate Pipeline's Annual Report of Gas Supply", began using the first code given in the list for a field agme, regardless of the State involved. With few respondents utllizing computerized edits on their submisstons at that time, miscoding of fields became a problem. The solution applied to this problem was to recode the fields on the list so that any fieids with identical names were assigned the same sjx-digit code (a fleld name code) but were differentiated by the State and county codes incorporated in the foll field code. For example, $145385 \mathrm{KS} 101$ is the field code for the CLARK field in Kansas and 145385TX285 is the field code for the CLARK field in Texas, as 145385 is the field name code for CLARK.

The FPC Field Code List, otiginally designed to handts data relating to interstate gas fields, was expanded over the years, as resources permitted, 10 include the names of oil fields and intrastate gas fields. Six-digit codes were assigned in ascending order to alphabetically sorted field names. Codes from the FPC Field Code List were used in flling Form FPC 15 and Form FPC \&. "Underground Gas Storage Repott".

After the establishment of the DOE in 1977, the requitement to gather annual, verifiable oil and gas re- serves estimates led to the development of Form BLA23. "Annual Survey of Domestic Oil and Gas Reserves". Form EIA-23 collects certain data by field. and the ase of the FPC Field Code List aided the reporting and processing of these data. As use of the Master List expanded by way of the Form ELA-23 program, additional work to verify and update the code list was necessary to keep the list current. In 1981, the correlation between the code number secuence and the alphabetical field name sequence was dropped. This change precluded the necesstty of periodically teassigning field codes in order to maintain the list in parallel numeric and alphabetic order.

A procedure was developed for the 1988 Field Code Master List pubtication which determined the actual count of distinct oil and gas fields in the United States. A part of this procedure was to aggregate the separate county portions of existing fields into their recognized entirety, even tf the field crossed a State boundary. This protedure is time-consuming and does entail manual intervention, so that it is accomplished on a yearly cycle.

\section{Definition of "Field"}

\section{A. field is defined as:}

\begin{abstract}
"An area consisting of a single reservoir or multiple reservoits all grouped on, or telated to, the same individual geological structural feature and/or strat:graphise conditon. There may be two or mapre reservoirs in a field which are separated vertically by intervening impervious strata, or laterally by local geologic bariers, or by both."
\end{abstract}

Thts definition is not used by all States in their designation of fields; consequently, areas classified as individual frelds by some States may be found combined in this Master Eist.

\section{Coding Of Flelds}

As noted above, the six-digit field name code is common to a specific field name, regardless of whether one or several fields exist with that particular name. In order to uniquely jdentify a particular field, the field name code must be coupled with the cotresponding State abbrevfation and county code.

Most codes on the Master List remain in a numerically ascendins order by alphabetized fietd name. However, field names added since 1981 have been assigned the first available (numerically lowest) nnused code. Fields Jocated in the Federal Offshore area and large State offshore blocks of the Gulf of Mexico will continue to be represented by codes above 800000 , according to their erea, subarea, and block number. 
Records that appear on the list with field name codes in light jtalics are alias records. The correct field name and field name code appear as part of the alias field name record aftor the label "Alias For:". Alias field names are not currently recogoized by State naming authorities, and alias field names and their codes should not be used in DOE fillings.

Records that were on the Field Code Master List last year bot which were sabsequently foutad to be invalid are entered on a separate Invalid Field Record List. These records were incorrectly placed on the FCML of when complete information was not available. Field names and codes on the Invalid Field Record List should not be used in DOE filings witb the indicated State and county, Note, bowever, that the identified Gield name and field name code may still be valid for a different State/connty combination.

\section{General Field Naming Conventions}

Field name speltings in the Master List reflect a number of convertions and conditions. In most instances, the 26-character name reflects the conventions imposed by the data block length in the DOE forms and by the State naming authority, nsually the oil and gas regulatory agency. Fteld identification for a well, lease, block, unit, or section is the responsibility of the State naming authority. A listing of these agencies appears in Table 1 . In the absence of a \$tate authority, field aames which bave come into general acceptance in an arta may be listed. In the Appalachlan Region, ffeld area names are often used. Detailed informatton is provided in Chapter 2, Methodology for Field Code Assignments, concerning the field naming conventions followed.

Table 1. Lfat of Authorities on Oil and Gas Field Nomenclature

\begin{tabular}{|c|c|c|c|}
\hline State & Naming Authority & State & Neming Authorily \\
\hline Alobeme & Slate On and Gas Boend of Alabana & Nabracke & $\begin{array}{l}\text { Nabraska of and Get Consorvalton } \\
\text { Coramiasion }\end{array}$ \\
\hline $\begin{array}{l}\text { Alater } \\
\text { Atrond }\end{array}$ & 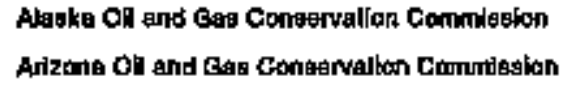 & Navada & 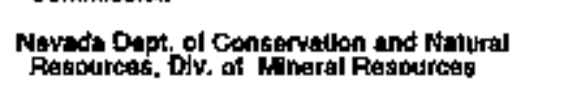 \\
\hline Aklangag, & 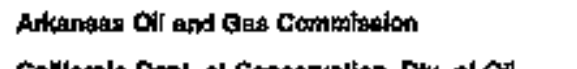 & New Mexdiso & 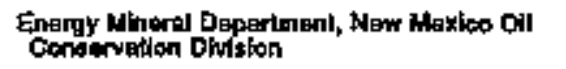 \\
\hline Gevelinomia & $\begin{array}{l}\text { Calloornle Depl, of Conservalion, DFy, of OAl } \\
\text { and Geg }\end{array}$ & Now York & 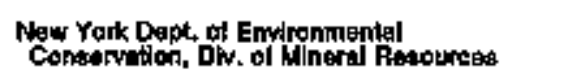 \\
\hline Coniaradia & 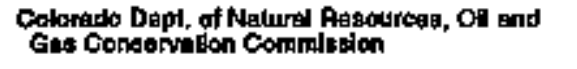 & North Dakota & North Dakele Geolopionl Sunvy \\
\hline Florida & 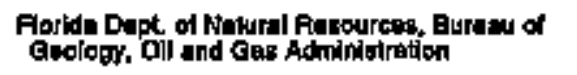 & Onlo & $\begin{array}{l}\text { Ohlo Dept. of Natural Resaurces, Dlv. of On } \\
\text { and Gas }\end{array}$ \\
\hline Wilnoln & 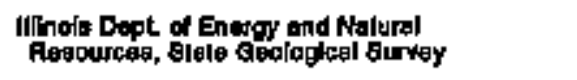 & Okfehomina & 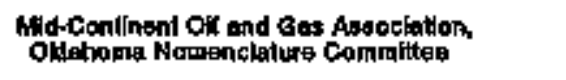 \\
\hline Indland & 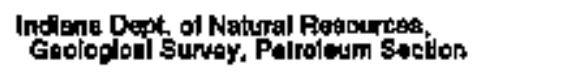 & Orepont & 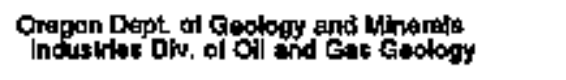 \\
\hline $\begin{array}{l}\text { Ionia } \\
\text { Kansass }\end{array}$ & $\begin{array}{l}\text { Lowh Guologle Survey } \\
\text { Kmates Geological Survay }\end{array}$ & Pennsylvanit: & 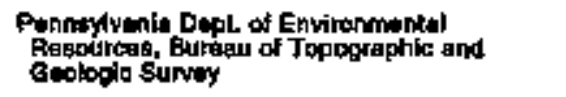 \\
\hline Kantwaky & Kenitucky Geologtoed Eurvey & South Dakaila & Dept. of Emviranment and Maturnl Recourroes \\
\hline Louletana & $\begin{array}{l}\text { Louldune Dept. of Hetural Fesources, Otiles } \\
\text { of Contirvition }\end{array}$ & Tonnusgen & 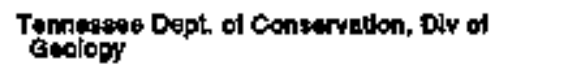 \\
\hline Maryland & Manjiand Georglical Surwey & Texas & 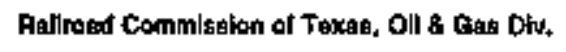 \\
\hline Mketaban & 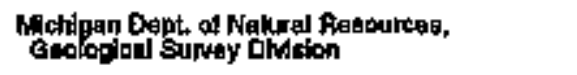 & Utah & 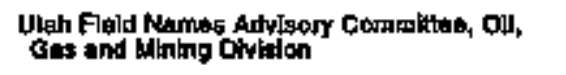 \\
\hline Mlinnanola & 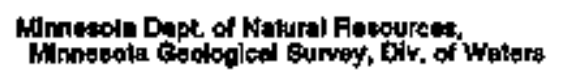 & Virginia & $\begin{array}{l}\text { Oapartment of Mines, Minarats and Enargy. } \\
\text { Of and Gas DFvitlen }\end{array}$ \\
\hline Malsesiselpp[ & 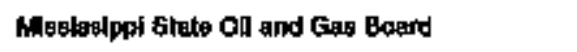 & Waghington & 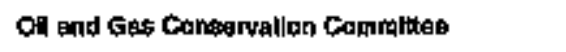 \\
\hline Mlowoun & 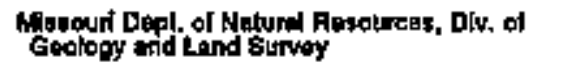 & Wost Virginiz & 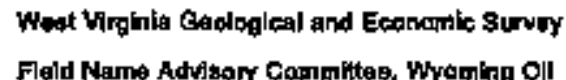 \\
\hline \multirow[t]{2}{*}{ Mantane } & \multirow{2}{*}{ 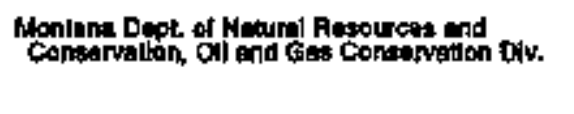 } & & and Gao Conservalion Commlalon \\
\hline & & Foderel Offohora & 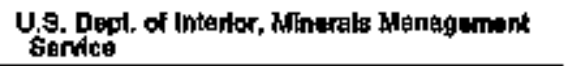 \\
\hline
\end{tabular}

Sousce: Energy Informallon Adinintstratlon, Office of Oil and Gas 


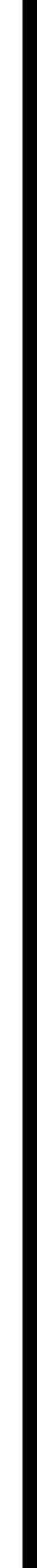




\section{Methodology for Field Code Assignments}

\section{Purpose}

The purpose of the Field Code Master List effort is to provide the Energy Information Administration (EIA) and others with standardized field name spellings and associated codes for all crode oil and datural gas fields thronghout the United States. The codes are compiled on the computerized Field Code Master Fle (FCMF), which is updated on a quarterly cycle. The Oil and Gas Field Code Master List, derived from the FCMP, is published annually. The first edition was released by EIA in December 1982. Computer tapes containing the latest field code information are delivered to the $\mathrm{Na}$ tional Technical Information Service in January of each year,

\section{General System Overview}

Figure 1 presents a flow chart of the activities necessary to process new fleld information. All new field information recelved by EIA goes through this cycle, which is designed to process fietd information received from respondents filing Forms EIA-23 and EIA-191, as wel] as from other sources such as State publications.

The system incorporates both a Field Code Working File (FCWF) and the FCMF. The FCWP contains att new, or unverifled, field information. It exists for two reasons:

- The large size of the FCMF makes it relatively difficult to manipulate.

- It is useful in tracking the progress made toward verifying the large number of in process fields, while at the same time assuring that reduedant activities are not taking place.

The FCWF is updated as information about the field is obtained. Quarterly, and more often when watranted by the number of completed transactions, all fully resolved records are extracted from the working file and used to update the FCMF. Once the master file update is contpleted, the resolved records are deleted from the FCWF.

\section{Field Information Research}

Geologists and petroleum engineers are responsible for supervising research and final resolution of field information. Listings of the FCWF are produced by region for review. There are several possible explanations why field Information under review is not already con- tained on the FCMF. That information could reflect the following:

- A relatively recene field discovary.

- A recently discovered exteasion of an old field into a new county or State.

- An alias used for the official name.

- An etror exists in the reported information (e,g., a reporting error on the Form EIA-23).

The official recognision of a new field discovery by a State field noming authority is a prerequisite for the assignment of an official EIA field code. Table 1 on page 3 lists these naming antborities. Information segarding State recognition is obteined through official State publications and computer tapes, or through other contact with the State agencies.

If the field name in question has not been officially recoguized, several sources of information exist for further investigation into the third and fonrth possibilities listed above. These include:

- Analysis of State data files (the Texas and New Mexico files, for example, contain detailed taformation for each field and operator.)

- Review of otber oil and gas publications.

- Telephone contact with the source of the ioformation (e.g., a respondent to Form EIA-191 or Form EIA-23).

\section{State Source Review Procedures}

State sources provide most of the field names. Field information is processed on a routine basis from State sources so that EIA field codes will hopefully be assigned as this inforration is needed.

As State publications are released, they are routinely reviewed, and thejr information regarding new fields is compared to the information on the FCMF. If the FCMP does not contain the new field information, the working file is updated to reflect it. Prior to updating the working file, entries on it are reviewed in order to identify any research which might be in progress on the same field. Information added to the working file from State publications is added as tesolved information. 
Figure 1. Fleld Code Processing Ftow Chart

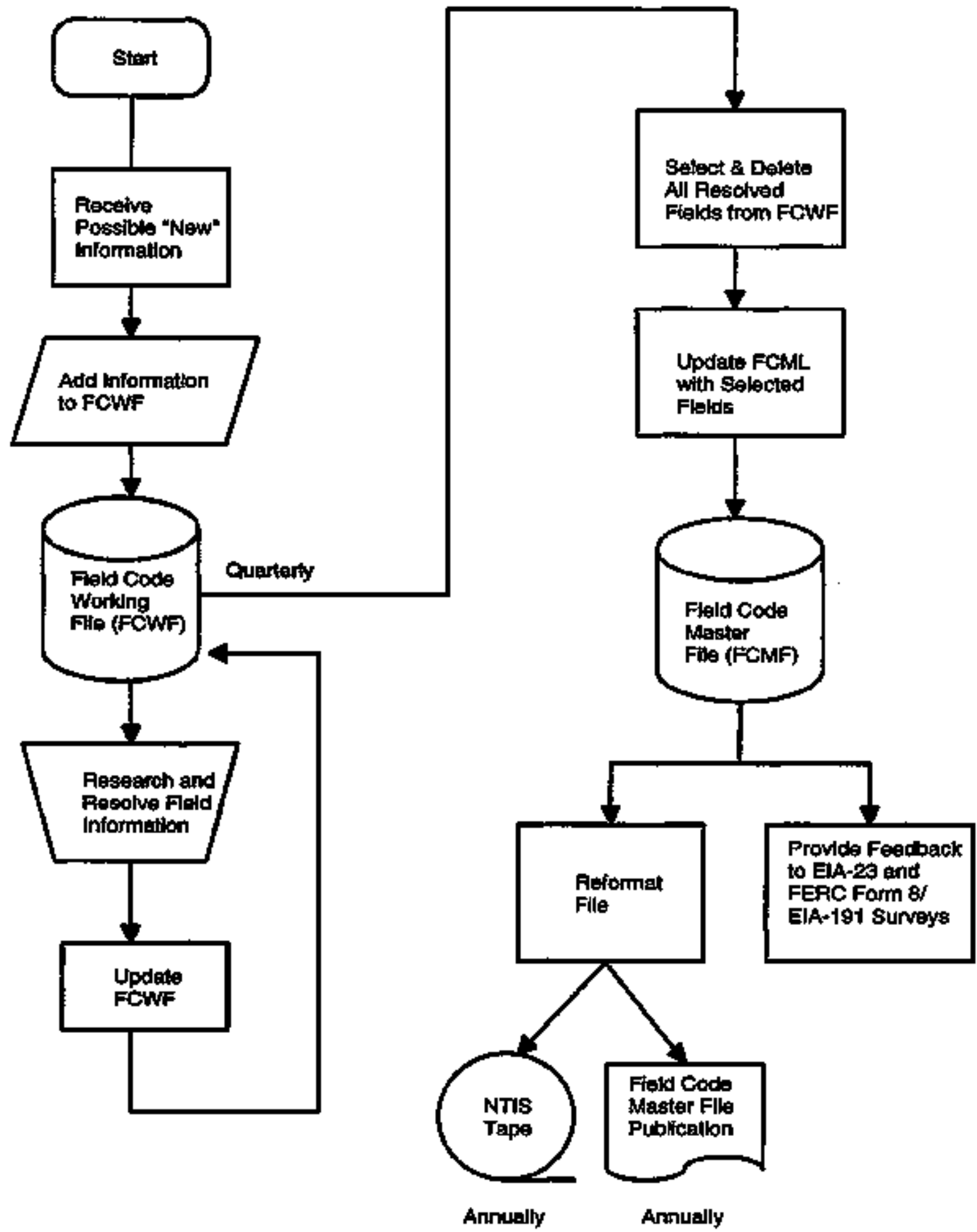

Bource: Energy Information Administration, Ofitice of Oll and Gas 
ready to be entered on the FCMF, since the States are the final authority for this information.

The quality and quantity of information available tbrough the State publjcations varies. Some States publisb new field informatton relatively frequently $(e+g$., monthly) in a format that is easily reviewed for incorporation into the FCMF, Routine processing of State sources begins with these publications. Additional State publications, such as State geological papers, are also included in these rontine reviews.

Periodically, all FCMF records for a State are compared to the most recent State pubjication, Some States do not publish field information, or the field information is not carried in the latest publication but in a provious one. In an overall review of the FCMF, when a particular fieid is not found in any available State publication, the source NISP (not in State publication) is initially coded into the source section of the FCMF. When an earlier State source teports "No Production", No Reports", or some similar remark for one or more years, and does not show the field in subsezurent sonrces, the field is stit] considered to be official, whether it is producing at this time or not. Whon a field is found in an older State source in some sort of combination or in subordination with another field name, the State is contacted and asked to clarify the status of the field. This research resolves most of the NISP records, allowing them to be finally catsorized as a master freld tecord with a State naming authorty source, as an alias field record, or as an tnvaltd field record. Certain field records represented field names assigned by non-State Naming Authority pubijcations which were coded prior to initiation of production of the fjeld, which subsequently was abandoned without any recorded production. Tbese records have been classified as invalid.

\section{Assigning New Fleld Code Numbers}

A sequential listing of available field code numbers starting with 000101 , has been produced for use by the Field Code Coordinator when new fteld codes are assigned. The first available code (numerically lowest) is used for a now field, regerdless of tame spelltng, with the following exception: codes $800000-999998$ are ex. cloded from sequential code assiguments, as they are reserved for offshore Gulf of Mexico fielde.

\section{Field Alias Procedures}

A fteld alias is an unofficital name for at officiatly recogaized field. Each name designated as an alias is cross-referenced to the official name. An official name Is the current, State-sanctioned name. An unofficial name is one which no longer js, or newet was, a Statesanctioned name.
A field alias may reflect the following possibilities:

- An alternate name for the official name.

- A field that has been renamed to something else.

- A field that has been combined, consolidated, or metged into another field.

- A field that has split into two or more new fietdis and the old name is no longer used.

For the combined or renamed types of aliases, both the official and alias names are on the FCMF.

For alternate name aliases, the official name must be on the FCMF in all cases. The alias name will be on the FCMF in the following cases:

- A representative from the State agency is aware of the aljas name as an alternative for the oftlcial name.

- The alias name was submitted by a Form FERC IS 'Jnterstate Pipeline's Annual Report of Gas Supply" cespondeot (formerly collected by the Federal Energy Regulatory Commission).

- Two or more operators report the stame alias.

- The alias is aiready on the FCMF for some reason.

An alternate name alias which does not meet one of these four criteria normally would not be added to the FCMP.

\section{Field Allas Records That Have Several Official Nemes}

Certain alias field records result from the splitting of a fleld into two or more fjelds, with none of the resulting fields using the original field name. For example, Field $A$ was split into three fields: Field B, Field C, and Field D. Fields B, C, and D would be listed as field records on the Master List. Field A would be listed as a field alias record referenciug Field B, but not Fields $C$ and $D$ and would show SPLIT as its reason for alias. Because of space limitations, the appropriate State naming Authority or the Field Code Coordinator must be contacted to determine names of the other flelds created out of Field $A$ when it was split, as well as bow many chers were created.

\section{Fields With More Than One Name Change}

The curront offlcial name may be the result of several ntme changes. For instance, in Oklahoma, RINGWOOD NE was combined with RINGWOOD NORTH 
in 1965, which wes subsequently combined into RINGWOOD in 1966. The listing for RINGWOOD NE would show the correct field name as RINOWOOD. If multiple names were involved, the alias field name recotd would list only the current (correct) tield naine. (For example, in Oklahoma, HENNESSEY SE is an alias for HENNESSEY EAST whtch has stuce been changed ftrst to DOVER-HENNESSEY and finally to SOONER TREND. The correct name for the alias HENNESSEY SE, HENNESSEY EAST and DOVER-HENNESSEY is SOONER TREND.) Information on the chain of successive field names may be obtained from the appropriate State raming authority or the Field Code Coordinator.

\section{Offshore Code Assignments}

Offshore fields on the Federal Gulf of Mexico Onter Contibental Shelf receive a field code that is determined by the lease block (or blocks) for whtch they ate named by the U.S. Minerals Management Service (MMS). The last three digits of the code are the block number. For instance, East Cameron (offshore area prefix code 824) Block 071 , receives the fietd code of 824071 . If several blocks are included in one field, the field code reflects the number of the block for which the ffeld is named. The other blocks included in the field are aliases to the officiaI name. When die MMS has named two fields with the same basic block number t.e., Ship Shoal Blk 113 field and \$bip Shoal Btk 113A field, for the second designated field the basic block number is combined with the prefix code of an adjacent area within which this block number wonld not occur.

There are cases where a partictular OCS lease block cobtains portions of two or more fields. If one of the fields is named for the block concerned, there will be an entry in the REMARKS section indicating PBL, or Part of Block In, followed by the field code of the other included field. If the block concerned is only an aljas to all the fields involved, it will be designated in MULTI to highlight that more than one field is included within the lease block.

Fields in large offshote State blocks in the Gulf of Mexico are assigned cotes in the manoer of offshore Federal fjelds. Special prefixes, generally unassigned prefixes for area surveys, are used for the small state block fields. A special prefix has been set aside for High Island-State. This prefix is used only with the small block fields. For State blocks tbot exceed 999, an unused three-digit profix ending in 1 is used, such as SOUTH PADRE IS BLK 1068 with code 951068.

Federal offshore fields in areas other than the Oulf of Mexico are given names in the same manter as for onshore fields, and are coded with the next sequential code.

\section{Fleld Naming Conventions}

\section{Use of Compass Directions in Field Names}

As a general rule, a compass direction used as part of a field name is placed at the end of the name. For example, the field named West Davenport by a State source bears the name DAVENPORT WEST on the FCMF. A fiold named after a known landmark, such as the town of East Daveaport for example, bears the name EAST DAVENPORT on the FCMF.

If the field DAVENPORT WEST is then combined with other wells or fields and the word District, of some sintilar word, is added to the name, the name will ap. pear on the FCMF as DAVENPORT WEST DISTRICT. This enables users to distinguish between two fields, one aamed as a result of forming a distrlct with the DAVENPORT WEST field as its nuclens, e.g., DAV. ENPORT WEST DISTRICT, and the other which is a new district to the west of DAVENPORT DISTRICT, e.g., DAVENPORT DISTRICT WEST.

Abbreviations are not used in connection with onshore field names except for noncardinal compass points, such as NW for Northwest or SE for Southeast, and for names of combined fields in certain States which use all the individual names of the former fields to torm the new field name. Cardinal compass points are spelled out in onshore field names.

Ofishore tield nemes in the Gutf of Merico usually consist of an offshore area name and block number specified by the U.S. Minerais Management Service (MMS). Example: EAST CAMERON BLOCK 299. The Field Code Master List has retained the subarea identifiers such as EAST CAMERON SOUTH ADDITION BLOCK 299 field which yields a 37-character field name. Snch lengtby offshore area agmes must be abbrsviated to flt within the 26 characters avatiable. When thłs is aecessary, the following standard abbre. viations are used:

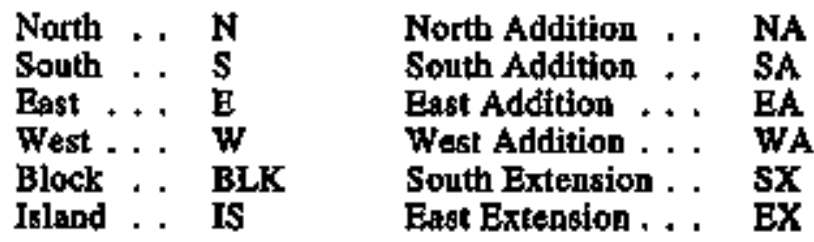

For example, High Igland East Addition South Extension Block A376 is abbrevisted HIGH IS EA SX BLK A376. 


\section{Special Naming Conventions}

Some States regard reservoirs as fields and keep their records on that basis (e.g. Texas and New Mexico). The Master List does not follow the State conventions in these instances. For example, in Texas, PARKER (PENNSYLVANIAN) and PARKER (WOLFCAMP) are considered to be separate fields by the State. PARKER is actually the name of the field and PENNSYLVANLAN and WOLFCAMP are the names of reservoirs in the field. The Texas Railroad Commission requites that the reservoir name appear in parentheses after the field name in its publications. In the Master List, you will only find PARKER listed as the field.

The MMS quarterly publication, OCS Operations Fteld Names Master List, Gulf of Mexico OCS Region. (FNML) is the primary source for the Federal offshore Fields in the Gutf of Mexico. This publication, and the MMS computer file on which it is based, does not use the subarea identifiers, such as the SOUTH ADDITON, although maps of the region still carry these subareas. That is, where the MMS FNML carries the name wEST CAMERON BLOCK 617, most maps and the Fjelit Code Master List call the field WEST CAMERON SA BLK 617 with SA the abbrevjation for SOUTH ADDITION.

\section{Reused Field Names}

Some States occasionally rense field names for areas other than the original freld location. This problem is bandled on the FCMF by Indicaling (OLD) and (NEW) after the field name, with each being assigned a differ- ont fjeld code. This procedure facilitates computerized usage of the database.

\section{Invalid Field Record Procedures}

Field records are removed from the FCML when they are found to be incerrect for one of the following reasons:

- The field name as it appears was never approved by the State naming authority for that State and county; i.e, it could have been a misspelled field name or the name of a producing unit, well, lease or offshore platform.

- County or State location data are incorrect.

- Two separate field codes were assigned to the stame field name.

When research on a NISP field record determines that one of those reasons applies to a field of field alias record, the record type is changed to INVALID. Invalid field records appear in the Invalid Field Record List section of the FCML publication for the year in which they have been designated INVALID. They will not appear in subsequent years. A cumulative list of INVA. LII records may be obteined by contacting the Field Code Coordinator. Tite Nationel Technical Informetion Service machine-readable version of the EIA Oit and Gas Field Code Master List inclodes a separate and cumulative Invalid Field Record List. 



\section{User's Guide}

\section{Fleld Code Master List}

Fields in the Field Code Master List (FCML) are sorted alphabetically by State and alphabetically by field name within a State. Frelds in the Federal Offshore are listed separately. Fields that occur in multiple States are listed in each and are further sublisted by county.

The layout of the FCML is shown in Figure 2. Each field record is one line long except for records with a "REMARK", which is on a second line. Each aljas field record is two lines long. A brief description of each data item follows.

\section{Master Field Record}

Iten I, FIELD CODE. The six-digit field code assigned to this fleld name.

Item 2, PIELD NAME. This is the field name (26-character límit).

Item 3, STATE POSTAL ABBREVIATION AND STATE SUBDIVISION CODE, This is a four-chreracter code Indicating the State and State subdivisloa. The first two positions ars the State postal abbreviation. The last two positions are the two-dtgit subdivjsion code, used only In Alaska, California, Lonisiana, New Mexico, and Texas, and in offshore areas to differentiate between State and Federal waters. Table 2 on page 16 is a listing of these cades. Figtres 4 through 8 , at the end of the Users' Guide, are subdivision maps fot the aforementioned States.

Item 4, COUNTY CODE. This is a three-charecter code for the county or parish. For all States except Alaska this is the Federal Information Processing Standards (FIPS) county code, as presented in FIPS publication 6-3 dated December 15, 1979 and its amendments. As Alaska bas no counties, the FCML nses the U.S. Geo. logical Survey $1 \times 3^{6}$ quadrangles for Alaska and the three-digit psendo-county codes assigned to them by the American Petroleum Institute. Codes used for fields loceted in State or Federal offshore areas are: Offshore-State, 990; Offshore-Federal, 995; OfflivuteCleneral, 999.

Inem 5, coumT NAME. This is the county of parish name (23-character limit) as deffined in FIPS publication 6-3 for all State onshore areas except Alaskg, For Alaskg, the nambe associated with the USGS $1 \times 3^{\circ}$ guadrangle is used. Table 3 on page 17 is a listing of the pseudo-county codes and names for Alaska. If the field is in an offshore area, see the list to Item 4 above.

hem 6, FIELD DISCOVERY YEAR. This is the fousdigit year of first tiscovery of oil or gas in this field, if it is kmown. In the case of combined sields, this is the earliest date antong the formerly separate fields.

Item 7, FIELD TYPE. This is a three-character block giving the type of hydrocarben found in the field using the symbols defioed below.

\begin{tabular}{|c|c|}
\hline Symbol & Manintog of Symbol \\
\hline ONA & 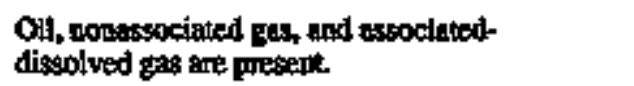 \\
\hline ON & $\begin{array}{l}\text { Oil and nomassociated gas present: } \\
\text { associgien-dissolved gas absent. }\end{array}$ \\
\hline $\mathbf{N}$ & $\begin{array}{l}\text { Nowassockased gas present; } \\
\text { oil and associated-dissolved gas alsent }\end{array}$ \\
\hline 0 & $\begin{array}{l}\text { Oil presenti nonassocianed gas and assoclated-dis- } \\
\text { oolved pers ahoent. }\end{array}$ \\
\hline $\mathrm{OA}$ & $\begin{array}{l}\text { On mad associsted-dissolved gas present; } \\
\text { nonassociated gas dbseal. }\end{array}$ \\
\hline Etank & Type of bydrocarbon is unhown. \\
\hline
\end{tabular}

Item 8, REMARKS. This is a 23-character entry specifying an atcribute of the field. 
Figure 2. Example of a Fleld Code Master Llet

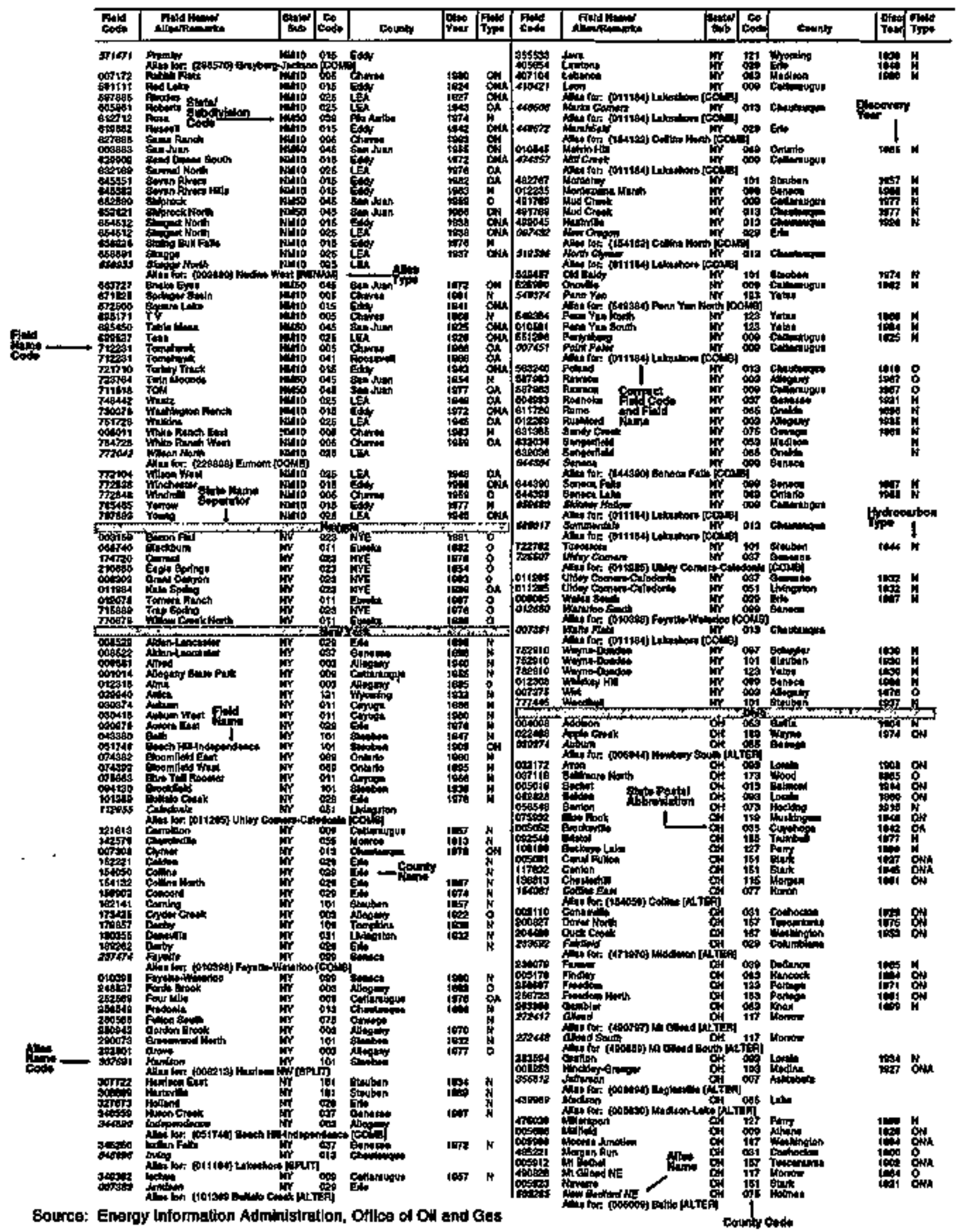


Common remarks are described below:

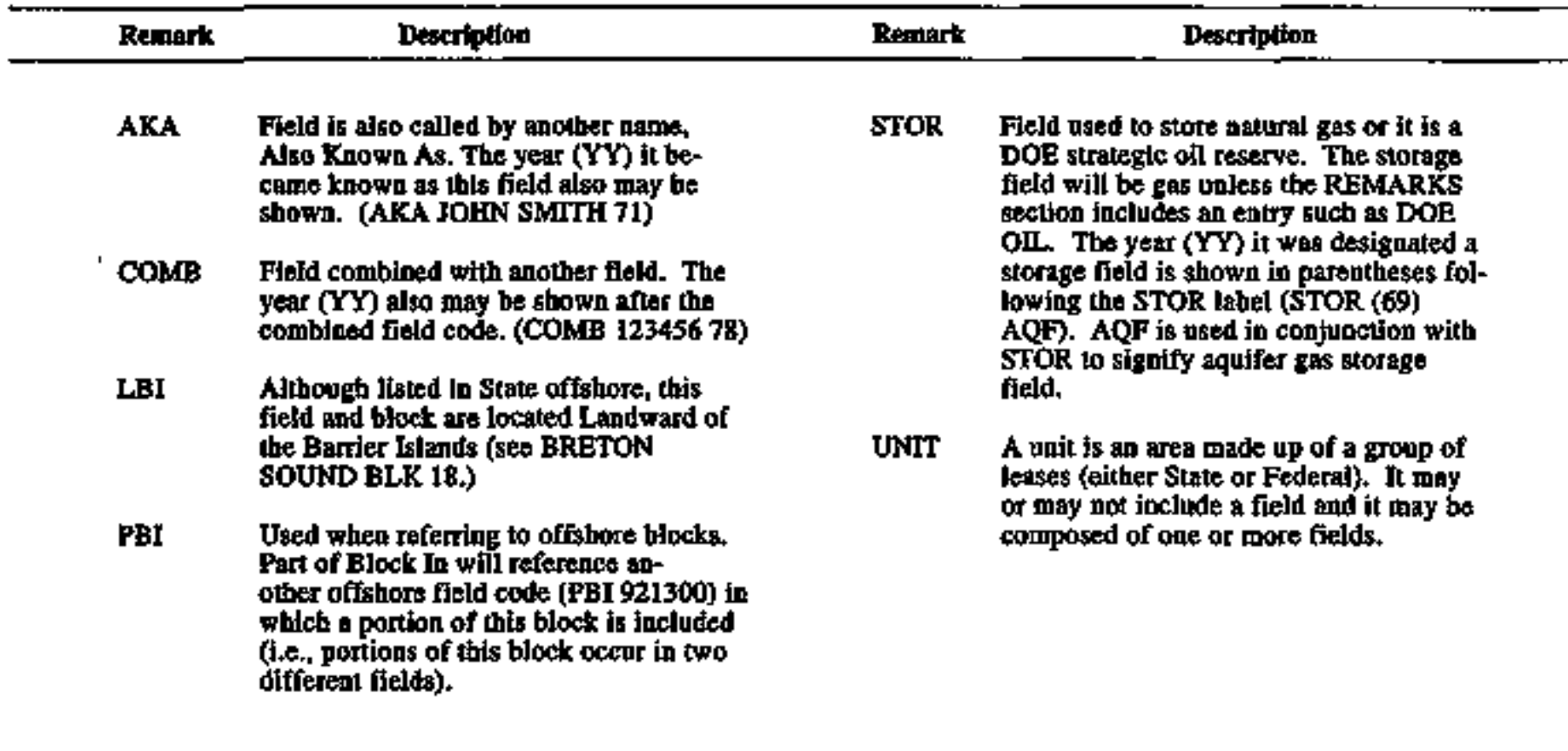

\section{Allas Field Record}

Ihem 1, ALIAS FIELD CODE. The six-digit field name onde assigned to thits altas freld name, printed in light italics.

IJem 2, ALAS FIELD NAME. This is the alias field name (26-cbaracter limit), printed in italics.

Itam 3, STATE POSTAL ABBREVIATION and STATE SUBDMVSION CODE. Same as Item 3 under Master Field Record.

Ilem 4, COUNTY CODE, Same as Item 4 under Master Fiteld Record.

Ihen 5, COUNTY NAME Same as ftem 5 moder Marter Frekd Record.

Iten 6, CROSS-REFERENCE. This is the identification of the master neld coule and fleld name which should be osed in place of the alias name listed in Iten 2 of this record. This item consists of the lagel "Allas For:" followed by the correct six-digit field name code and the correct (the, current) field name.

Item 7, ALIAS TYPE. This code (five-character limit) indicating type of aljas is present only for alias fietds. The types of allas codes are as follows:
ALTFR Thts filed name is an alternative for the official fleld mame and should not be nsed in DOE forms. The cotrect narrie and code are llated niter "Alhas Far:".

BLOCK This offshme feld extends into mone than one offihome black. This offshore bilock name ts not the ote for which the fiekd is named. The black for which the field is nouned is given after "Alizs For".

COMB This Gitk has been corrbined with another field which is Ested after "Alins Ror:".

MULTI This offshors lease block has portions of two oc mare fields in It. In the Fteld Code Master List, the record is itsted once for each field to thich it is aliasect so that aptupurigte cross reference is mende.

RENAM This fitld has been renamed. The new name is lisied after "Altas For:".

SPLIT This field has been cplit into two or more fields, bone of which retalns this name. Cne of the new fields is listed after Alias Fort". Conact the Field Code Coordinator for the atditionat ftelds and inconmation rebating to this alias fietd.

Included on the following page is a listing of the State names and the page of the Oil and Gas Field Code Master List on which the first fteld in those States occurs. 


\begin{tabular}{|c|c|c|c|c|c|c|c|}
\hline $\begin{array}{l}\text { First Fleld } \\
\text { In State }\end{array}$ & $\begin{array}{c}\text { On } \\
\text { Paga }\end{array}$ & $\begin{array}{l}\text { First Field } \\
\text { In State }\end{array}$ & $\begin{array}{l}\text { On } \\
\text { Page }\end{array}$ & $\begin{array}{l}\text { First Field } \\
\text { In State }\end{array}$ & $\begin{array}{r}\text { On } \\
\text { Page }\end{array}$ & $\begin{array}{l}\text { First Field } \\
\text { In State }\end{array}$ & $\begin{array}{r}\text { On } \\
\text { Page }\end{array}$ \\
\hline Alabams & 42 & Konses & $\$ 6$ & Nevada & 145 & Texes & 203 \\
\hline Alastan & 43 & Rentucisy & 104 & New Hexteo & 145 & Utah & 301 \\
\hline Arizona & 44 & Louisions & 315 & New York & 151 & Virgintas & 300 \\
\hline Aftomsas & 44 & Mraylead & 125 & North Daknte & 153 & Whstington & 303 \\
\hline Callifornla & 46 & Mchligan & 125 & Ohlos & 156 & Wesl Vrrghta & 303 \\
\hline Colorido & 49 & Mhenescita & 134 & Ortahoma & 163 & Wyoming & 30 \\
\hline Forda & 56 & MLostsstppl & 134 & Oregon & 197 & F.O.Collfomba & 315 \\
\hline Illionols & 56 & Mitsourd & 138 & Pencsylvariq & 197 & F.O.-Loutstana & 315 \\
\hline Iafilana & 62 & Madisa & 138 & Sooth Dakota & 202 & F.O-OUher Galf & 329 \\
\hline Lowa & $\infty$ & Neturska & 141 & Tennessed & 202 & F.O.-Тexas & 329 \\
\hline
\end{tabular}

\section{Coalbed Methane Field List,}

A dramatic ise in coalbed methane's share of natural gas production has beightened interest in those fields with coalbed methane potential. In Table 4, p. 18, Coalbed Methane Fields, the field name, field code, county code and name and state are given for those fitds currently productive or with drilling activity.

\section{Storage Fields}

Storage fields have been developed in many States. Some were developed in existing, official oil and/or gas fields. All storage fields are listed in Table 5 . In certain States, the storage portion of a field or pool bas been given a different name. In this chse, the storage field name is camied on the FCML as an alias to the official field name. The storage field pames which are altases are listed in both Table 5 and the FCML in italics. These alias field names and codes in itolics should only be used in filing Form EIA-191, "Underground Gas Storage Report".

\section{Flelds Located in Multiple Jurisdictions}

Table 6, Fields Located in Multiple Jurisdictions, is compiled to reflect those oil andfor gas fields which cross State boumdaries. In developing the stummary statistics on page 1 , a field is only counted once, no matter how many counties or States it is in.

\section{Outer Continental Shelf Planning Areas}

Gulf of Mexico Outer Contipental Shelf statistics published by the Minerals Management Service (MMS) folIow the boundary lines of their Western, Central and Eastern Planning areas, shown respectively in Figures 9, 10 , and 11 . In order that reports developed from the U.S. Crude Oll, Natural Gas, and Notural Gas Liquids Reserves track the reporting by MMS, fields currently found in the Garden Bonks leasing area (GB), and those which may be found in GB or in the Keathley Canyon leasing area, will henceforth be tisted in the FCML as Texas Federal Offshore rather than Louisiana-South Federal Offshore. 
This figure has been omitted dne to removal of the Fiteld Code Index. As this figure is included in all electronic versions of the Field Code Master List, the figures in this publication have not been renumbered. 


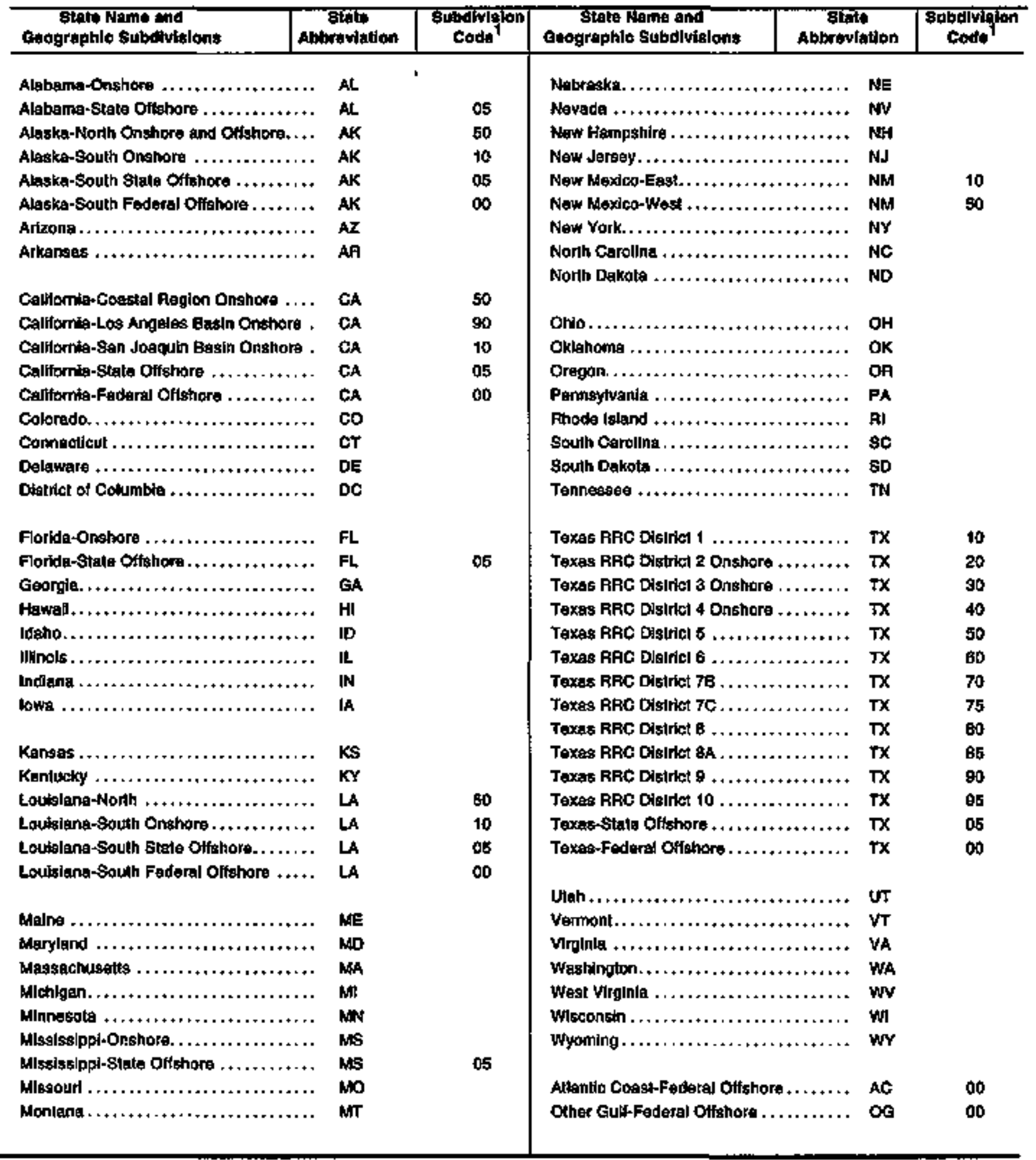

1 if no subditision code ls specilied, there is no subtilusion.

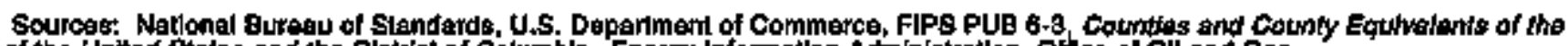
States of the Unifed states and the District of Columbia. Eneryy Informatlon Adninistrailon, Ohice of Oll and Gas. 
Table 3. U.S. Geologic Survey Alaska Quadrangles and Assoclated Codes

\begin{tabular}{|c|c|c|c|c|c|c|c|}
\hline Oupuranglis & $\begin{array}{l}\text { aused } \\
\text { Code }\end{array}$ & Quraditang & $\begin{array}{l}\text { Cuad } \\
\text { Cods }\end{array}$ & Ouatrangle & $\begin{array}{l}\text { Quadd } \\
\text { Code } \\
\end{array}$ & Ouadrangle & $\begin{array}{l}\text { Dued } \\
\text { Cods }\end{array}$ \\
\hline Adak & 001 & Dillingham & 077 & Marshell. & 157 & Sowerd. & 203 \\
\hline Afognak $\ldots+\ldots+\ldots \ldots$ & 009 & Dixcen Entiranoe. & 079 & Accorthy ...... & 169 & Shlshmarof & 235 \\
\hline Ambter Flver ......... & oos & Eagle ......... & 081 & MoGrath ......+...... & 181 & Shungnak. & 237 \\
\hline Amukta .............. & 007 & Falrtatiks ..., & o8g & Megade Fiver ........ & 163 & \$trieonof Istand ..... & 299 \\
\hline Anchorage .......... & 009 & Fatso Pass ... & 097 & Medtra............ & 165 & $\sin _{\mathrm{n}} \mathrm{a}_{+\ldots+\cdots}$ & 241 \\
\hline Arctic............... & 011 & Flexman Isiand...... & 099 & Malozbne ........... & 167 & Skegway .. & 243 \\
\hline Alks.................. & 013 & Forl Yukon .......... & 091 & Middleton Istand ..... & 169 & Sleotmule. & 245 \\
\hline Ailln $\ldots++\ldots, \ldots$ & 015 & cokd Bay ...... & 093 & Mishaguk Mth .. & 171 & Solarmon . & 247 \\
\hline Altu ...... & 017 & Geretoi lstand. & 095 & Mt Falrweather & 085 & si Lewrence & 25 \\
\hline Ealrd InIed+. . . & 019 & Goodnaws... & ogs & Mil Hayes. & 173 & S1 Matthew & 249 \\
\hline Gaird bits ...... & OEI & Gulksna ${ }_{+} \ldots+$, & 098 & Mt toamai... & 175 & S1 Minchapl & 255 \\
\hline Barrow ........ & 023 & Hexemaister Island + . & tor & Mil Mokinley. + & 177 & Slepovak Be & 251 \\
\hline Barter Island ... . & 925 & Harrieon Bay........ & 103 & Mil Michalson... & 179 & Sumchum ... & 257 \\
\hline Benver $\ldots+\ldots+\ldots$ & 027 & Healy ........." & 105 & Mt st Eligs ..... & $16 \mathrm{O}$ & Survey Pass, & 259 \\
\hline Beochey Polnt , ....... & 029 & Holy Cross .......... & 107 & Nabsina...... & 189 & Sutwlk Istand ....... & 281 \\
\hline Bendetogen .......... & ost & Hooper Bay......... & 109 & Naknek....... & 188 & Tebila Min ........... & 269 \\
\hline Bering Clacter. . & $\mathrm{QBS3}$ & Howard Pass .. & 111 & Noatak ..... & 167 & Taku Riwer. . & 265 \\
\hline Belhel .... & 035 & Hughes .... & 113 & Nome,..... & 169 & Talkeeina . & 267 \\
\hline Betiles $+\ldots . .+$. & 037 & ky Bay.... & 115 & Narton Bay.. & 191 & Talkgeing Mils........ & 269 \\
\hline Big Oend . . . & 009 & tdllarod ... & 117 & Nulato... & 199 & Tanacross . & 271 \\
\hline Blatk...... & 041 & 1kplkpuk Rwer........ & 119 & Nurivak bylgnd. & 195 & Tenana... & 273 \\
\hline Black Rlver... & 049 & It:armna........ & 121 & Nushagak Bay. & 197 & Taylor Mts. & 275 \\
\hline Btylng sound... & 048 & Juneas...... & 123 & Ophlr........ & 189 & Teller ...... & 277 \\
\hline Bradifeld Canal. & 047 & Kagigualk,... & 125 & Pettersburrg... & 201 & Teshappuk. . & 279 \\
\hline Btlatal Bay +......... & 049 & Kantshne Piver $+\ldots+$. & 127 & Phillp smilh Mts . . .... & 200 & Trnity Istands........ & 281 \\
\hline Candle. & 051 & Karluk............... & 129 & Patnt Hopd.......... & 205 & Tyonak,............ & 263 \\
\hline Cape Nendenhall . .... & 059 & Kateel River . ........ & 131 & Polm Lay ........... & 207 & Woshik ...+....... & 286 \\
\hline Chandalar....... & 055 & Kenal $\ldots \ldots+\ldots \ldots, \ldots$ & 133 & Pon Alexander , ...... & 209 & Unlat ........ & 287 \\
\hline Chandler Lake. . & 067 & Ketchikan .... & 138 & Pont Maller.......... & 211 & Umnak,... & 289 \\
\hline Charley Fuver... & 059 & Kallk Rher.... & 137 & Prlbllof Isiands. & 213 & Uhalakleat.+ & 291 \\
\hline Chignlk .. & 081 & Kkskg ... & 139 & Prince Furgert .. & 215 & Uhalaskg. . & 290 \\
\hline chrristian.. & 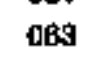 & Kodlak ..... & 141 & Pat Iskands.... & 217 & Unimak.... & 295 \\
\hline Circla..... & 065 & Kotzebue........... & 148 & Ruby ... & 219 & ytukok Rwer... & 297 \\
\hline Cold Bay... & 093 & Kutskokwim Bay ...... & 145 & Putsstan Mtisstion .... & 221 & Valdez ... & 299 \\
\hline Colien....... & 067 & Kwiguk.... & 147 & Segevanitktok $+\ldots \ldots$. & 223 & Wainwitght...$\ldots \ldots$ & 301 \\
\hline Cordove ....... & $A B g$ & Lake Cark.......... & 140 & Samalga letand ..... & 228 & Whseman .... & 309 \\
\hline Cralg................ & 071 & Lima Hills . ., , , ...... & 151 & Seguarn $\ldots \ldots \ldots \ldots$ & 227 & Yakutat...$\ldots \ldots \ldots$ & 305 \\
\hline Do Long Hils ......... & 073 & Lwengood ............ & 158 & Selewks..... & 229 & & \\
\hline Demareailon Point ... & 075 & Lookoun Fidga ....... & 155 & Soldovta...$\ldots \ldots \ldots$ & 231 & & \\
\hline
\end{tabular}

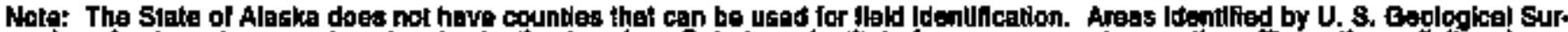

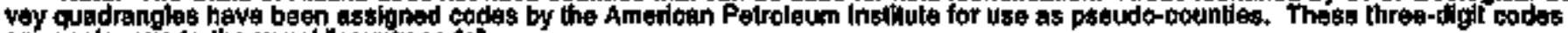
are andioghlus to the ugual "county coute".

Sourcet The API Well Number and Standard slate and Counly Numerle Codes Including Ofishore Waters. API Bulletin D12A, Jantiany 1679 . American Peiroleum Instihute, Washingion, DC. 


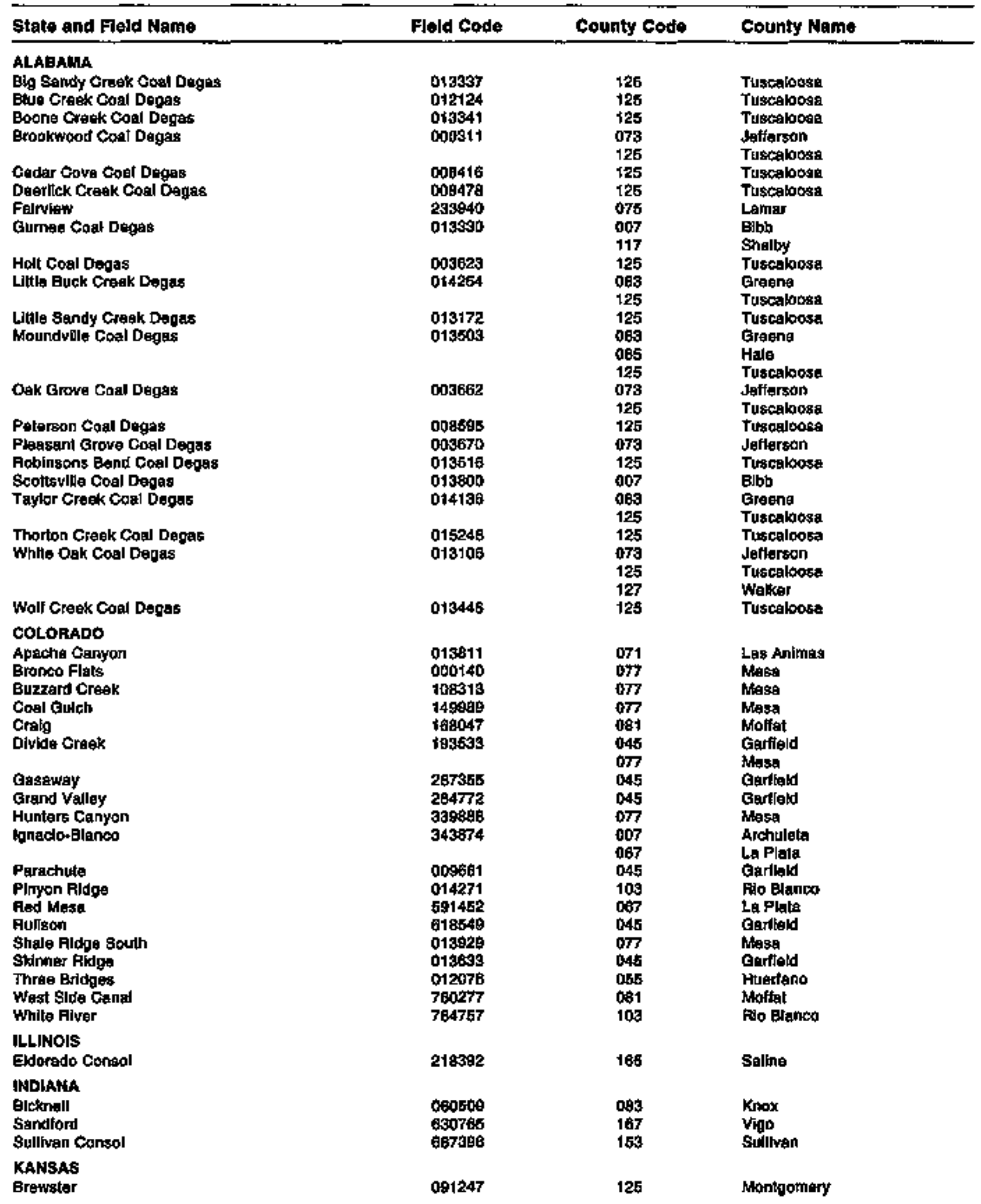


Table 4. Coalbed Methane Fields (continued)

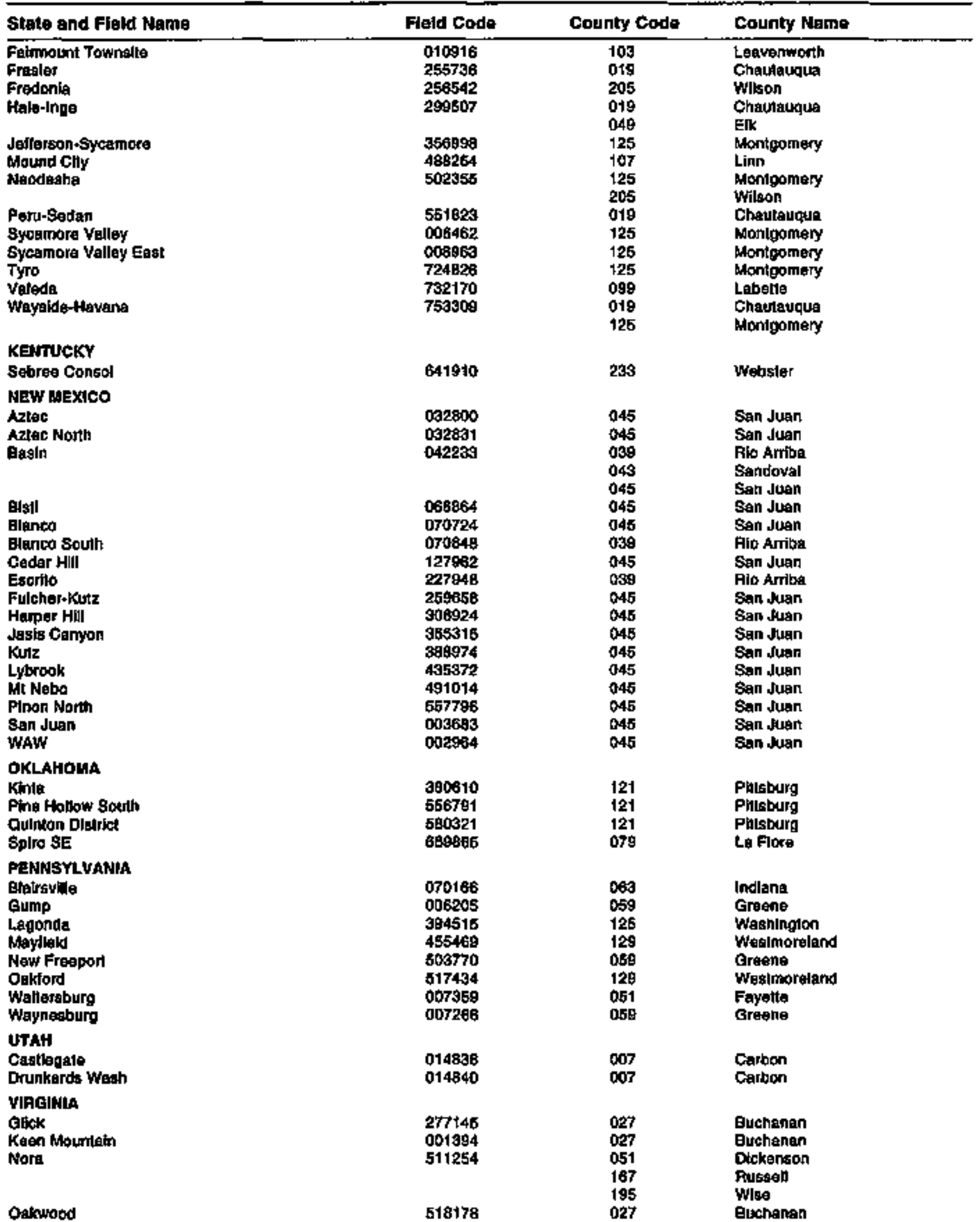




\begin{tabular}{|c|c|c|c|}
\hline State and Fleid Hame & Field Code & County Cods & County Name \\
\hline $\begin{array}{l}\text { WEST VIRCINEA } \\
\text { Btg Run-B|rchlleld }\end{array}$ & 063381 & 103 & Wetzel \\
\hline
\end{tabular}

Source; Eneroy Intarmailan Adminlstration, Offioe of Oll and Gas 
Table 5. Storage Flelds*

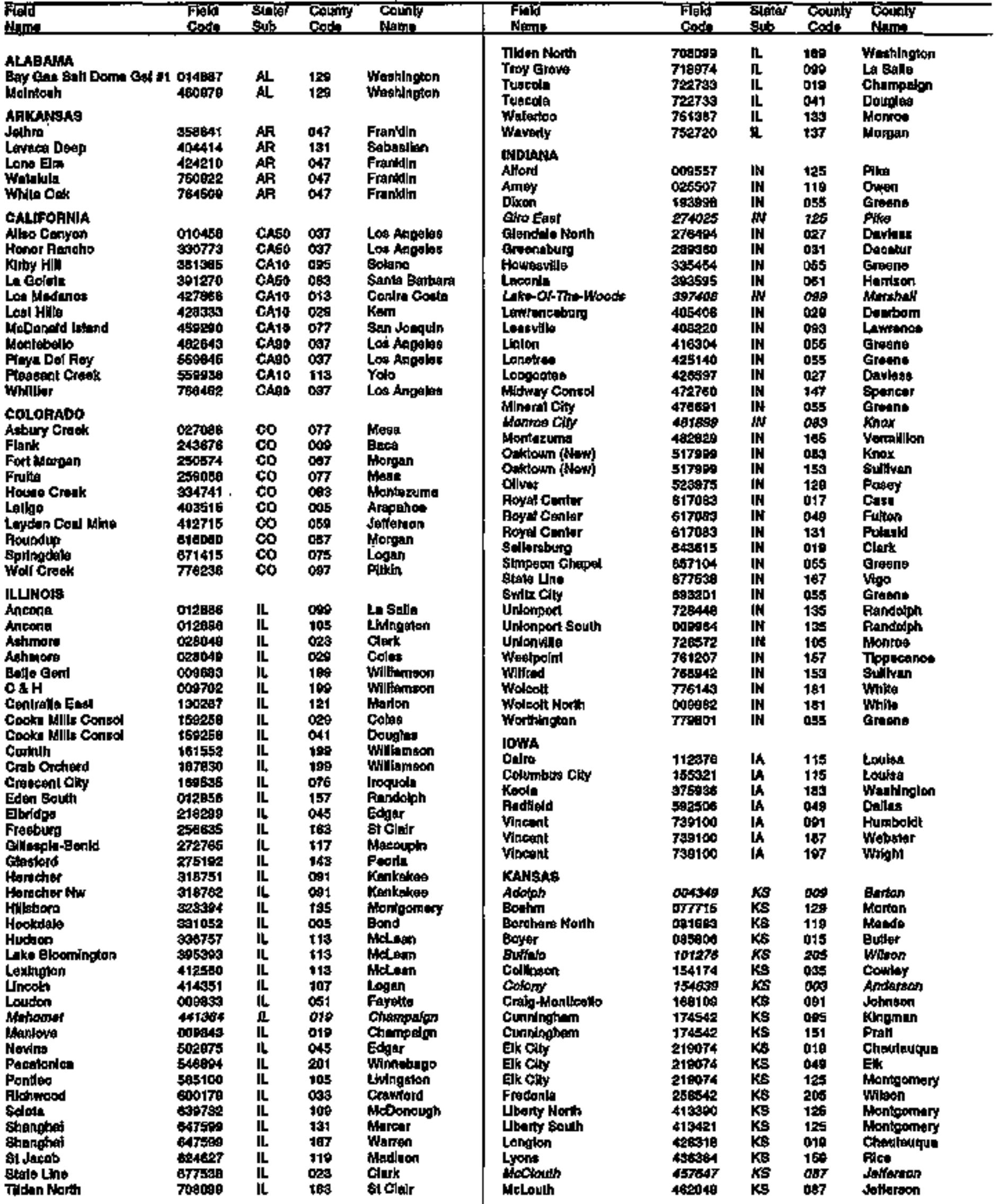

*Alias field names are in itolics. 
Table 5. Storage Fletds" (Continued)

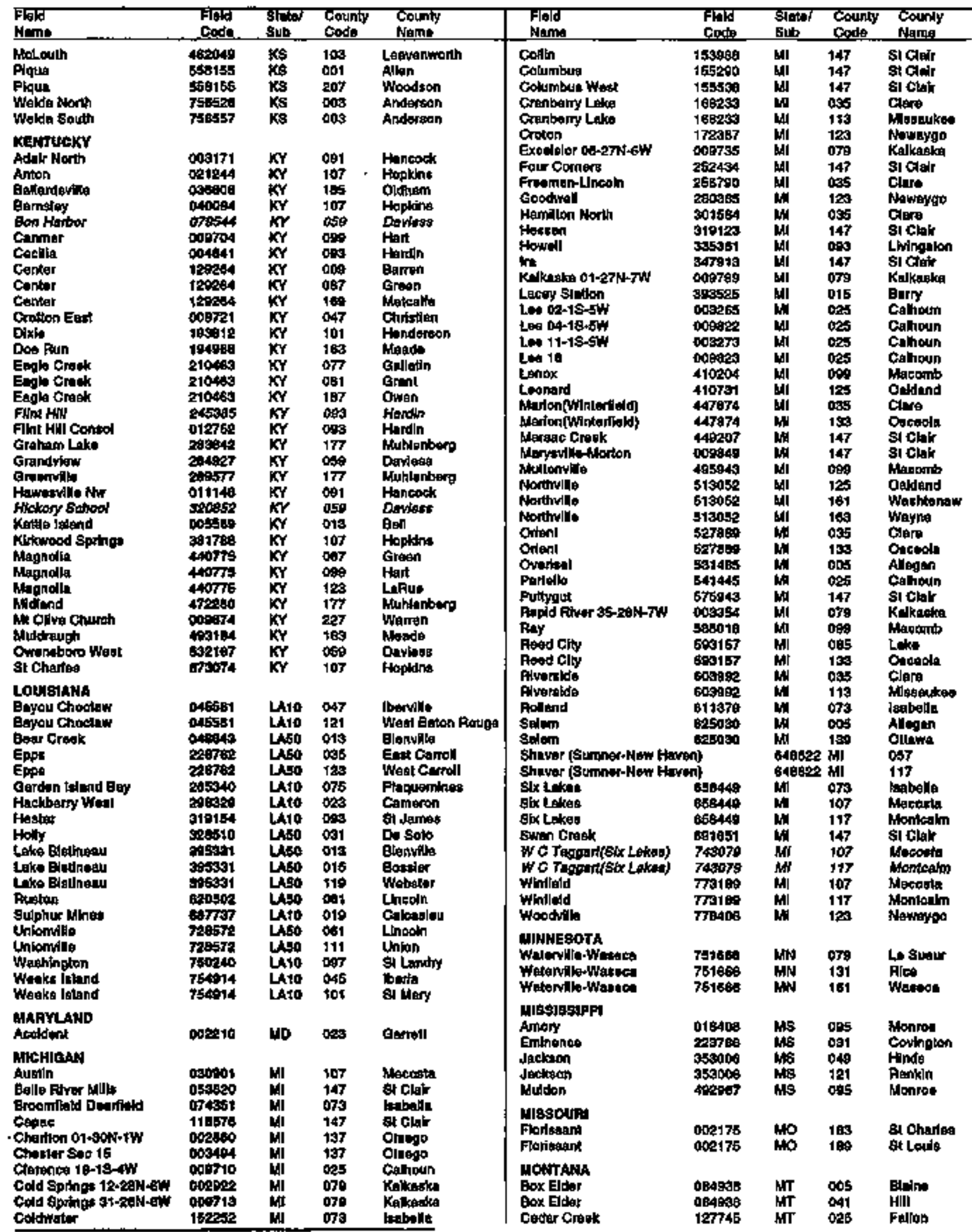

*Alias field admes are in italies. 
Table 5. Storage Flelds* (Continued)

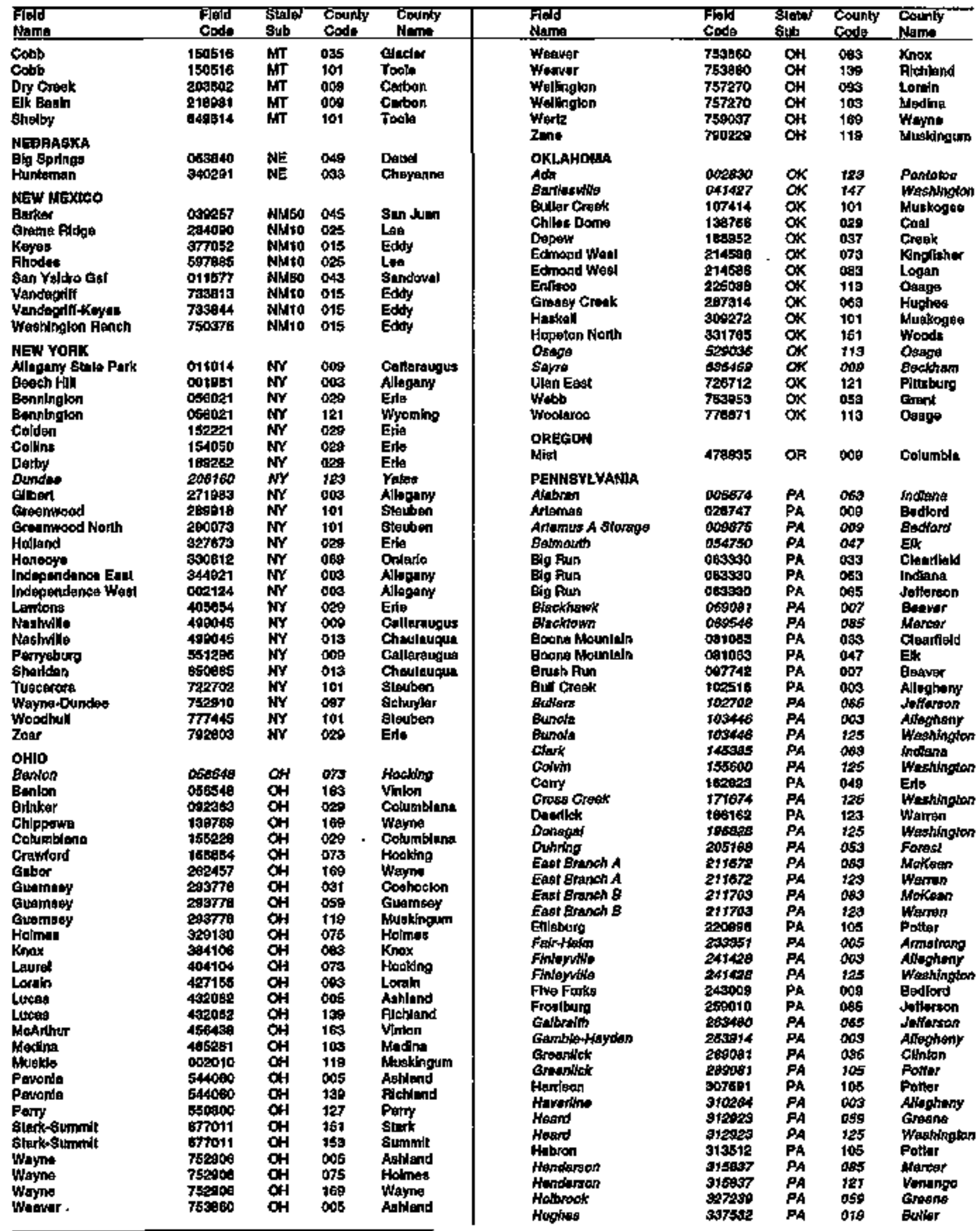

*Aljas field names are in italics. 
Table 5. Storage Fielde' (Continued)

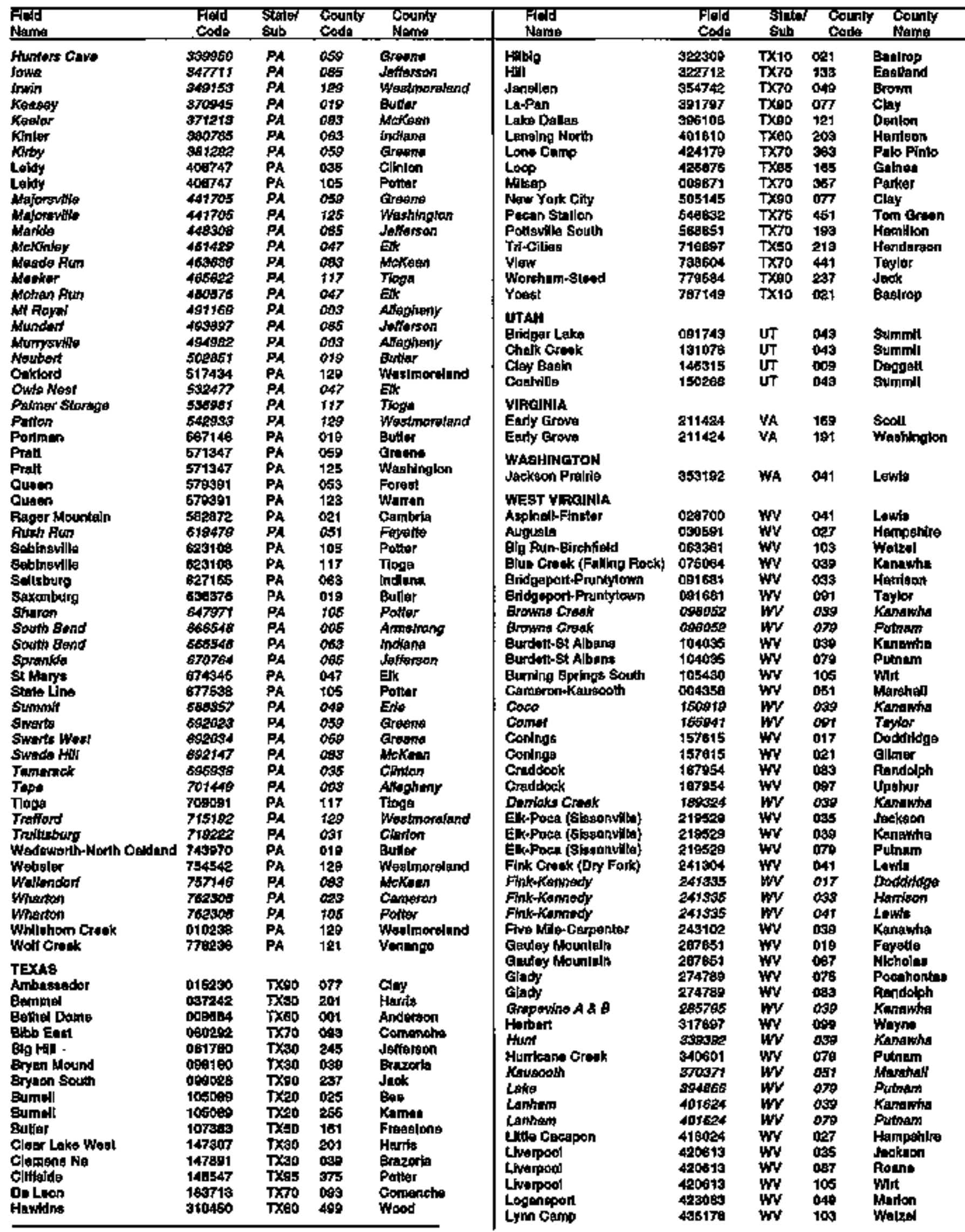

*Alias field rames are in italics. 
Table 5. Storage Flelds* (Contlnued)

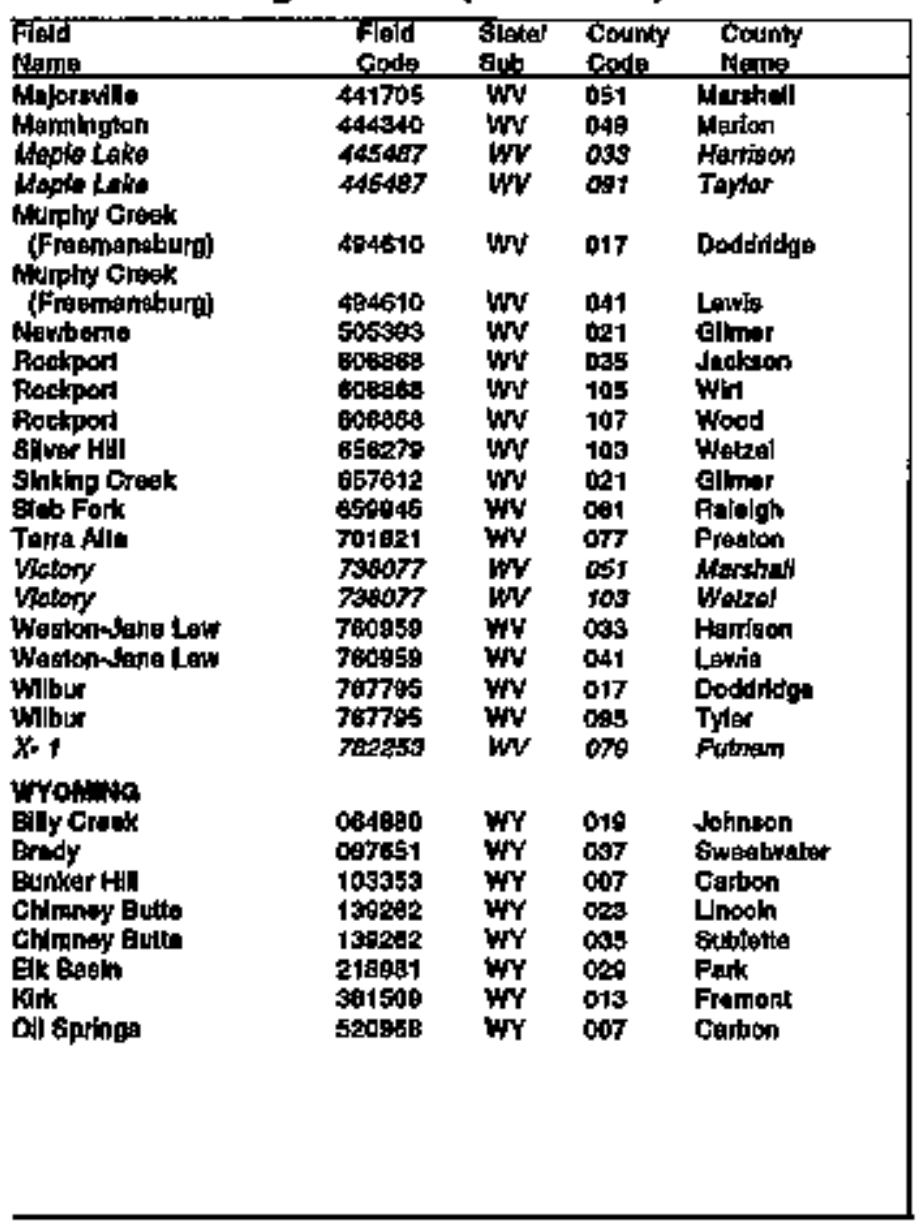

*Alias field names are in italics. 
Table 6. Flelds Loested in Multiple Jurisdictions

\begin{tabular}{|c|c|c|c|c|c|c|c|}
\hline $\begin{array}{l}\text { Find } \\
\text { Codo }\end{array}$ & $\begin{array}{l}\text { Find } \\
\text { Kent: }\end{array}$ & $\begin{array}{l}\text { sis. } \\
\text { sith }\end{array}$ & $\begin{array}{l}\text { County } \\
\text { Cods. }\end{array}$ & $\begin{array}{l}\text { Filid } \\
\text { Cond }\end{array}$ & $\begin{array}{l}\text { Fing } \\
\text { Neme }\end{array}$ & $\begin{array}{l}\text { State } \\
\text { sub }\end{array}$ & $\begin{array}{l}\text { Cotitity } \\
\text { cands }\end{array}$ \\
\hline \multicolumn{4}{|c|}{ 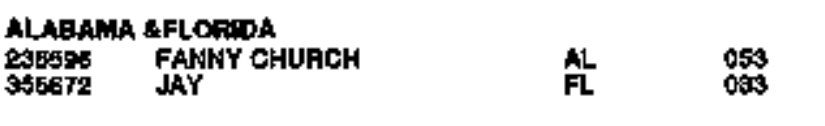 } & \multirow{2}{*}{$\begin{array}{l}013490 \\
019500 \\
581392 \\
\text { Ba1476 }\end{array}$} & JACE & co & 061 \\
\hline $\begin{array}{l}416506 \\
35672\end{array}$ & UTMLE EBCAMGAA GREEK & AL & 05 & & $\begin{array}{l}\text { sTockHoLn } \\
\text { sTockHOLN sw }\end{array}$ & co & $\begin{array}{l}017 \\
071\end{array}$ \\
\hline $\begin{array}{l}410505 \\
355072\end{array}$ & JTTLE EBCANABA GREEK & ALL & $\operatorname{sos}$ & $\begin{array}{l}\text { CDLOR } \\
\text { 640553 } \\
544553\end{array}$ & $\begin{array}{l}\text { D BNEARASKA } \\
\text { FEaBLE } \\
\text { PEBgLE }\end{array}$ & CO & $\begin{array}{l}075 \\
039\end{array}$ \\
\hline $\begin{array}{l}\text { ALABAn } \\
\text { ogsas1 } \\
090311\end{array}$ & $\begin{array}{l}\text { MISSIBSIPPI } \\
\text { EUCATUNAA CF:EEX } \\
\text { BUCATUNAA CFEEX }\end{array}$ & ALs & 023 & 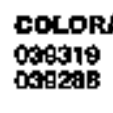 & 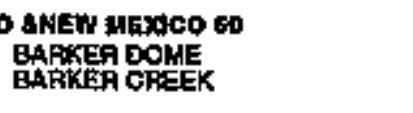 & co & $\begin{array}{l}067 \\
045\end{array}$ \\
\hline $\begin{array}{l}401314 \\
401314\end{array}$ & $\begin{array}{l}\text { GANGSDALE } \\
\text { LANGSDAIE }\end{array}$ & At & 020 & $\begin{array}{l}343974 \\
070724\end{array}$ & $\begin{array}{l}\text { KANACLO-BLANCO } \\
\text { BLANICO }\end{array}$ & CO & 007 \\
\hline \multicolumn{2}{|c|}{ 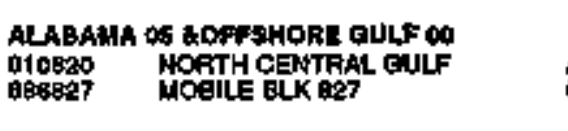 } & ALO5 & $\begin{array}{l}900 \\
905\end{array}$ & $\begin{array}{l}348974 \\
070724\end{array}$ & $\begin{array}{l}\text { IENACIO-BLANTCO } \\
\text { BLANCO }\end{array}$ & co & $\begin{array}{l}\text { CB7 } \\
\text { OA5 }\end{array}$ \\
\hline $\begin{array}{l}010021 \\
\operatorname{6os} 2023\end{array}$ & $\begin{array}{l}\text { MORTHWEST GULF } \\
\text { WOEILE EUK G2B }\end{array}$ & $\underset{O Q 00}{A L O S}$ & 电 & \multicolumn{2}{|c|}{$\begin{array}{l}\text { COLORADO SUTAH } \\
\text { 246474 FLODNE PAFK } \\
346911\end{array}$} & cơ & ogs \\
\hline a1089s & 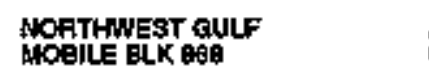 & Alos & 690 & $\begin{array}{l}\text { Bes6441 } \\
098420\end{array}$ & $\begin{array}{l}\text { SOUTH GANYOW } \\
\text { BAR X }\end{array}$ & UT & $\begin{array}{l}\text { O45 } \\
019\end{array}$ \\
\hline $\begin{array}{l}0142992 \\
0186521\end{array}$ & $\begin{array}{l}\text { STATE } 109 \\
\text { WOEILE ELK BZ1 }\end{array}$ & $\frac{\operatorname{Alos}}{0000}$ & og & $\begin{array}{l}\text { B8E841 } \\
\text { O9B420 }\end{array}$ & $\begin{array}{l}\text { SOUTH GAHYON } \\
\text { BAP } X\end{array}$ & Co & or7 \\
\hline \multicolumn{2}{|c|}{$\begin{array}{l}\text { ARKAN8AS \&LOUISIAHA } 60 \\
\text { O2499\% ARKAWA } \\
\text { O24391 ARACANA }\end{array}$} & AP & $\begin{array}{l}073 \\
015\end{array}$ & \multirow{2}{*}{$\begin{array}{l}606041 \\
626058 \\
606541 \\
677652\end{array}$} & $\begin{array}{l}\text { SOU'T GANYOW } \\
\text { SAN ARAOYO }\end{array}$ & co & 045 \\
\hline $\begin{array}{l}311380 \\
311300\end{array}$ & $\begin{array}{l}\text { HAYNESVILE } \\
\text { HAYNESVILE }\end{array}$ & AR & 027 & & SOUTH CANYON & co & or \\
\hline $\begin{array}{l}3+1300 \\
351300\end{array}$ & $\begin{array}{l}\text { HAYNESVLLE } \\
\text { HAYNESVILE }\end{array}$ & AF & $\begin{array}{l}097 \\
119\end{array}$ & \multicolumn{2}{|c|}{ 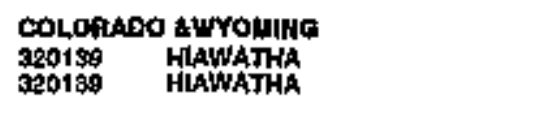 } & co & 001 \\
\hline $\begin{array}{l}\text { 50185s } \\
\text { 653551 }\end{array}$ & \multicolumn{2}{|c|}{ 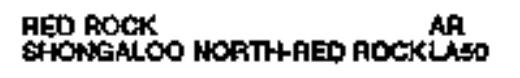 } & 027 & $\begin{array}{l}410621 \\
410521\end{array}$ & $\begin{array}{l}\text { LITTRE SNAKE } \\
\text { LITTLE SNAKE }\end{array}$ & co & 061 \\
\hline $\begin{array}{l}\operatorname{sog} \theta \sin 0 \\
\cos \theta \sin 0\end{array}$ & $\begin{array}{l}\text { RODESSA } \\
\text { RODESSA }\end{array}$ & AP & $\begin{array}{l}\text { oot } \\
017\end{array}$ & $\begin{array}{l}\text { Seg181 } \\
\text { Seg130 }\end{array}$ & $\begin{array}{l}\text { POWDEA WASH } \\
\text { POWDEA BPAINGS }\end{array}$ & $\underset{w r}{c o}$ & 091 \\
\hline $\begin{array}{l}\text { 65ssego } \\
653551\end{array}$ & $\begin{array}{l}\text { SHOHGALOG NORTH } \\
\text { SHOHGALOO NORTH-REO ROCK }\end{array}$ & KLAEO & 0027 & $\begin{array}{l}615812 \\
615812\end{array}$ & $\begin{array}{l}\text { RGUND TAGLE } \\
\text { ROUND TAGLE }\end{array}$ & co & $\begin{array}{l}001 \\
087\end{array}$ \\
\hline 677795 & $\begin{array}{l}\text { SPRINGHIL } \\
\text { SPR|MGHILL }\end{array}$ & AR & 007 & $\begin{array}{l}6776002 \\
6776018\end{array}$ & $\begin{array}{l}\text { STATELLNE } \\
\text { STATE UNE }\end{array}$ & wr & 001 \\
\hline \multicolumn{2}{|c|}{ 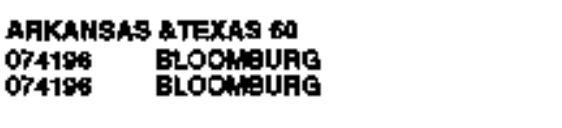 } & Aft & 001 & \multirow{2}{*}{$\begin{array}{l}700277 \\
034079 \\
700277 \\
700277\end{array}$} & $\begin{array}{l}\text { WEST SDE CANAL. } \\
\text { BACES SOUTH }\end{array}$ & co & on1 \\
\hline $\begin{array}{l}608050 \\
600980\end{array}$ & $\begin{array}{l}\text { RODESSA } \\
\text { RODESSA }\end{array}$ & $\stackrel{\text { AR: }}{\text { DX60 }}$ & 001 & & $\begin{array}{l}\text { WEST SDE CANAL } \\
\text { WEST SIDE GANAL } \\
\text { LWHANA }\end{array}$ & op & $\begin{array}{l}001 \\
007\end{array}$ \\
\hline \multirow{2}{*}{\multicolumn{2}{|c|}{ 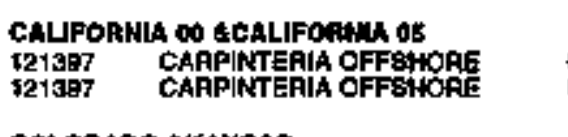 }} & $\begin{array}{l}\text { capos } \\
\text { CAOS }\end{array}$ & $\begin{array}{l}995 \\
980\end{array}$ & $\begin{array}{l}011572 \\
145740\end{array}$ & $\begin{array}{l}\text { ALENDALE } \\
\text { CLAYPOLE HILLS CONSOL }\end{array}$ & 比 & 165 \\
\hline & & & & $\begin{array}{l}055122 \\
049498\end{array}$ & $\begin{array}{l}\text { EGMAN EAST } \\
\text { BEAMAN EKST CONSOL }\end{array}$ & 虽 & to1 \\
\hline $\begin{array}{l}012459 \\
011646\end{array}$ & $\begin{array}{l}\text { APAPAHOE EAST } \\
\text { ARAPAKOEKAN }\end{array}$ & KS & $\begin{array}{l}017 \\
190\end{array}$ & 0.835es & $\begin{array}{l}\text { QLACK RAEA } \\
\text { ELACK RHEA CONSOL }\end{array}$ & in & $\begin{array}{l}199 \\
128\end{array}$ \\
\hline $\begin{array}{l}012159 \\
601382\end{array}$ & $\begin{array}{l}\text { FFONTERA } \\
\text { STOCKHOLM }\end{array}$ & co & $0 \$ 7$ & 34698 & MWAN EAST CONSOL & W & 证。 \\
\hline $\begin{array}{l}012189 \\
\text { 011est }\end{array}$ & $\begin{array}{l}\text { FFONTERA } \\
\text { STOCKHOLH SOUTH }\end{array}$ & $\begin{array}{c}\mathrm{CO} \\
\mathrm{KB}\end{array}$ & 017 & $\begin{array}{l}371627 \\
291313\end{array}$ & $\begin{array}{l}\text { SEENSQURG 80UTH } \\
\text { OFIFFIN CON\$OL }\end{array}$ & 触 & $\begin{array}{l}186 \\
051\end{array}$ \\
\hline 290919 & 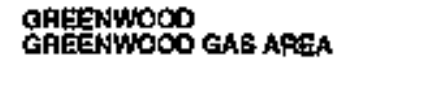 & 而 & 000 & $\begin{array}{l}40626 x \\
673384\end{array}$ & $\begin{array}{l}\text { LAWAENCE COUNTY DIVISION } \\
\text { ST FRAHCISVILE CONBOL }\end{array}$ & 林 & $\begin{array}{l}101 \\
063\end{array}$ \\
\hline
\end{tabular}


Table 6. Flelds Leogted in Multiple Jurlsdletlons (Contlnued)

\begin{tabular}{|c|c|c|c|c|c|c|c|}
\hline $\begin{array}{l}\text { Fivid } \\
\text { Cada }\end{array}$ & $\begin{array}{l}\text { FiEd } \\
\text { Neme }\end{array}$ & $\begin{array}{l}\text { Sixtor } \\
\text { sub. }\end{array}$ & $\begin{array}{l}\text { Gounty } \\
\text { Coda }\end{array}$ & $\begin{array}{l}\text { Find } \\
\text { Coda }\end{array}$ & $\begin{array}{l}\text { Fient } \\
\text { Yema }\end{array}$ & 9 & $\begin{array}{l}\text { Coupky } \\
\text { Code }\end{array}$ \\
\hline 453702 & MAUNE EAST & 叫 & 199 & $\begin{array}{l}265805 \\
002598\end{array}$ & $\begin{array}{l}\text { GAFAND } \\
\text { SWART-CAMLAMD }\end{array}$ & $\mathbf{K s}$ & $\begin{array}{l}011 \\
217\end{array}$ \\
\hline $\begin{array}{l}490332 \\
490369\end{array}$ & $\begin{array}{l}\text { WT CABHEL } \\
\text { WT CABHEL CONBOL }\end{array}$ & 占 & $\begin{array}{l}185 \\
0 \mathrm{a} 1\end{array}$ & $\begin{array}{l}429108 \\
002651\end{array}$ & LOMSBUting & Ks & $\begin{array}{l}121 \\
\text { ost }\end{array}$ \\
\hline 400032 & $\begin{array}{l}\text { MT CARAML } \\
\text { HT CAFHEL NOATH }\end{array}$ & in & 186 & apatos & $\begin{array}{l}\text { LOMSEUAB } \\
\text { Uuptis }\end{array}$ & KS & $\log _{12}^{121}$ \\
\hline $\begin{array}{l}509874 \\
201313\end{array}$ & $\begin{array}{l}\text { NEW HAFACNY CONSOL } \\
\text { CAFFIN CONSOL }\end{array}$ & 步 & $\begin{array}{l}199 \\
129\end{array}$ & $\begin{array}{l}429108 \\
002503\end{array}$ & $\begin{array}{l}\text { LOUREAnO } \\
\text { USLE }\end{array}$ & KB & 19: \\
\hline $\begin{array}{l}500374 \\
201313\end{array}$ & $\begin{array}{l}\text { NEW HAPMONY CONSOLL } \\
\text { GRIFFIN CONEOL }\end{array}$ & 齿 & 193 & $\begin{array}{l}\text { 881041 } \\
747512\end{array}$ & BTILLWEM & $\mathbf{k g}$ & $\begin{array}{l}091 \\
0 \$ 7\end{array}$ \\
\hline $\begin{array}{l}50 \times 1967 \\
756200\end{array}$ & $\begin{array}{l}\text { NEW HAPINONY SOUTH } \\
\text { WELOOFN HOATH CONBOL }\end{array}$ & in & $\begin{array}{l}198 \\
129\end{array}$ & $\begin{array}{l}\text { KansAE } \\
\text { O71458 } \\
\text { S5T643 }\end{array}$ & $\begin{array}{l}\text { YcaRAsk } \\
\text { 9LEES } \\
\text { SNAK }\end{array}$ & 總 & 145 \\
\hline 504029 & $\begin{array}{l}\text { NEW HAVEN CONSOL } \\
\text { HOWEY WEST }\end{array}$ & 怰 & 109 & $\begin{array}{l}330487 \\
30487\end{array}$ & $\begin{array}{l}\text { HOFinNEK } \\
\text { HOFiNEK }\end{array}$ & K8 & $\begin{array}{l}143 \\
0.57\end{array}$ \\
\hline 504029 & $\begin{array}{l}\text { NEN HAYEN CONSOL } \\
\text { WELBOAN CONSOL }\end{array}$ & 林 & 189 & $\begin{array}{l}30797 \\
012684\end{array}$ & $\begin{array}{l}\text { HUFFSTUTTEA } \\
\text { ALHA SOUTH }\end{array}$ & KE & 147 \\
\hline $\begin{array}{l}\text { 510721 } \\
\text { 516721 }\end{array}$ & $\begin{array}{l}\text { OAK GAOVE } \\
\text { OAK BROVE }\end{array}$ & 紧 & $\begin{array}{l}059 \\
128\end{array}$ & $\begin{array}{l}\text { 00155y } \\
\text { Tr2tas }\end{array}$ & 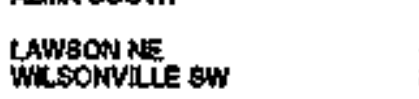 & NE & 039 \\
\hline $\begin{array}{l}907411 \\
607411\end{array}$ & $\begin{array}{l}\text { ROOHESTEA } \\
\text { ROCHESTER }\end{array}$ & IL & $\begin{array}{l}185 \\
051\end{array}$ & \multicolumn{4}{|c|}{ 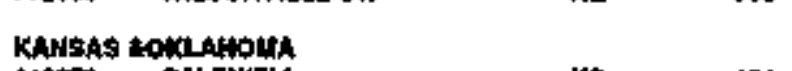 } \\
\hline $\begin{array}{l}679415 \\
679344\end{array}$ & $\begin{array}{l}\text { ST FAANCISVILAE EAST } \\
\text { ST FAANCISNILLE CONBOL }\end{array}$ & 歫 & 101 & +12672 & CALDWEL & $\%$ & 053 \\
\hline $\begin{array}{l}\text { grasas } \\
677589\end{array}$ & $\begin{array}{l}\text { STATE UNE } \\
\text { STATE UNE }\end{array}$ & 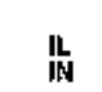 & 187 & $\begin{array}{l}t 16084 \\
\text { t16623 }\end{array}$ & GANEY & ks & $\begin{array}{l}125 \\
147\end{array}$ \\
\hline ILnials & KENTUCKY & & & $\begin{array}{l}145497 \\
145497\end{array}$ & $\begin{array}{l}\text { CLARK CAEEK } \\
\text { GLARK GAEEK }\end{array}$ & Ks & 005 \\
\hline 049050 & $\begin{array}{l}\text { SHAWWEETOWN EAST } \\
\text { SHAWWEETOWN EAST }\end{array}$ & IL & 959 & $\begin{array}{l}218702 \\
218730\end{array}$ & Elein & ks & $\begin{array}{l}016 \\
113\end{array}$ \\
\hline 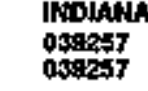 & $\begin{array}{l}\text { KENTUCKY } \\
\text { BARKEA } \\
\text { BAAKER }\end{array}$ & IN & $\begin{array}{l}108 \\
101\end{array}$ & $\begin{array}{l}215693 \\
129006\end{array}$ & $\begin{array}{l}\text { GLKHAFT } \\
\text { CAPTHAEE OLISTECT NE }\end{array}$ & kik & 1289 \\
\hline 909595 & $\begin{array}{l}\text { LACONIA } \\
\text { DOERUN }\end{array}$ & $\begin{array}{l}\text { IN } \\
\mathbf{K Y}\end{array}$ & $\begin{array}{l}\text { osi } \\
1 \in 3\end{array}$ & $\begin{array}{l}241115 \\
480070\end{array}$ & FWCHAM & Ko & 119 \\
\hline $\begin{array}{l}568002 \\
580471\end{array}$ & $\begin{array}{l}\text { POWELS LAKE CONSOL } \\
\text { POWHELS LAKE }\end{array}$ & IN & 129 & 200011 & $\begin{array}{l}\text { CGEENMOOD GAS AAEA } \\
\text { CARTHAEE BAS ARE }\end{array}$ & Ke & 129 \\
\hline $\begin{array}{l}602584 \\
6625354\end{array}$ & $\begin{array}{l}\text { SHTH 44LS NOFTH } \\
\text { SHTH HL CONBOL }\end{array}$ & IN & 19a & $\begin{array}{l}397790 \\
295545\end{array}$ & $\begin{array}{l}\text { HUCOTON GAB AGEA } \\
\text { BUMUON HUSOTON OAS AREA }\end{array}$ & Ks & $\begin{array}{l}175 \\
130\end{array}$ \\
\hline $\begin{array}{l}\text { NoIAHA } \\
219894 \\
2 \text { tE496 }\end{array}$ & 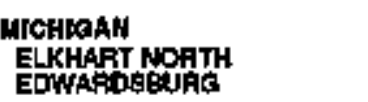 & 岿 & $\frac{090}{\cos 7}$ & $\begin{array}{l}353790 \\
295345\end{array}$ & $\begin{array}{l}\text { HUEOTON GAS AAEA } \\
\text { GUYWOA+HUGOTOW GAB AAEA }\end{array}$ & Ko & $\begin{array}{l}189 \\
130\end{array}$ \\
\hline INDIANA & OHIO & & & $\begin{array}{l}413048 \\
480070\end{array}$ & 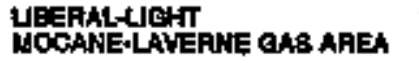 & ok & $\begin{array}{l}175 \\
007\end{array}$ \\
\hline 716880 & $\begin{array}{l}\text { TRENTON } \\
\text { CHATTANDOCA }\end{array}$ & is & 901 & $\begin{array}{l}46+150 \\
139064\end{array}$ & MckKaY & Ke & ast \\
\hline $\begin{array}{l}716880 \\
005094\end{array}$ & $\begin{array}{l}\text { TRENTON } \\
\text { CHATTANOOFA }\end{array}$ & 唯 & $\begin{array}{l}975 \\
207\end{array}$ & $\begin{array}{l}464081 \\
490050\end{array}$ & $\begin{array}{l}\text { MCKANEY } \\
\text { MOCANELAVEAHE RAS AAEA }\end{array}$ & Ko & 007 \\
\hline $\begin{array}{l}719600 \\
005777\end{array}$ & $\begin{array}{l}\text { TPENTON } \\
\text { WILLSHIRE }\end{array}$ & ON & 001 & $\begin{array}{l}481481 \\
400070\end{array}$ & $\begin{array}{l}\text { WCKWNNEY } \\
\text { WOCANE-LAVERWE CAAG AREA }\end{array}$ & kp & $\begin{array}{l}119 \\
007\end{array}$ \\
\hline $\begin{array}{l}\text { KANSAt } \\
178946 \\
411537\end{array}$ & $\begin{array}{l}\text { Postual } \\
\text { DALCAS } \\
\text { LESTEA }\end{array}$ & $\mathbf{k}$ & 991 & $\begin{array}{l}404679 \\
102479\end{array}$ & $\begin{array}{l}\text { MUFPHY } \\
\text { DILWOFTH DISTAICT }\end{array}$ & KS & $\begin{array}{l}101 \\
071\end{array}$ \\
\hline
\end{tabular}


Table 6. Fields Lecated In Multiple Jurisdietions (Continued)

\begin{tabular}{|c|c|c|c|c|c|c|c|}
\hline $\begin{array}{l}\text { Frold } \\
\text { Codio }\end{array}$ & $\begin{array}{l}\text { Figo } \\
\text { Namis }\end{array}$ & $\begin{array}{l}\text { siatar } \\
\text { sub }\end{array}$ & $\begin{array}{l}\text { Gounty } \\
\text { copip }\end{array}$ & $\begin{array}{l}\text { Find } \\
\text { conds }\end{array}$ & $\begin{array}{l}\text { Frend } \\
\text { timens }\end{array}$ & $\begin{array}{l}\text { Sitale } \\
\text { Bylb }\end{array}$ & $\begin{array}{l}\text { County } \\
\text { Cods }\end{array}$ \\
\hline $\begin{array}{l}551629 \\
160519\end{array}$ & $\begin{array}{l}\text { PEPUN+BEOAN } \\
\text { OOLESPOND CAEEK }\end{array}$ & Ks & $\begin{array}{l}019 \\
119\end{array}$ & 950060 & $\begin{array}{l}\text { MNN PASS BLK } 6 \\
\text { MAN PASS BUK BO }\end{array}$ & $\begin{array}{l}\text { LAOD } \\
\text { LAOS }\end{array}$ & 998 \\
\hline$\frac{5510 p 9}{721307}$ & $\begin{array}{l}\text { PEAt.BEDNN } \\
\text { TURKEYCAEEK }\end{array}$ & Ks & $\begin{array}{l}019 \\
113\end{array}$ & $\begin{array}{l}966075 \\
0.9074\end{array}$ & 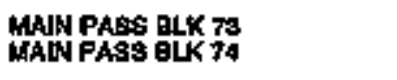 & $\begin{array}{l}\text { LAOD } \\
\text { LAOS }\end{array}$ & $\infty_{0 \rightarrow 0}^{\infty}$ \\
\hline $\begin{array}{l}60850 \\
244402\end{array}$ & $\begin{array}{l}\text { TALOSA } \\
\text { FLATS NW }\end{array}$ & ks & 120 & 000415 & $\begin{array}{l}\text { SHF SHOAL EUK } 15 \\
\text { SHIF BHOAL ELK } 15\end{array}$ & LAOP & 905 \\
\hline $\begin{array}{l}723065 \\
420790\end{array}$ & $\begin{array}{l}\text { TUTTES SE } \\
\text { LOVEDALE NW }\end{array}$ & $\begin{array}{l}\mathbf{K S} \\
\mathbf{D K}\end{array}$ & $\underset{060}{03}$ & $\begin{array}{l}09400 \% \\
60006\end{array}$ & 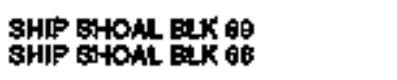 & $\begin{array}{l}\text { Lano } \\
\text { LAOS }\end{array}$ & 905 \\
\hline $\begin{array}{l}724800 \\
116825\end{array}$ & TYAO & $\underset{C K}{K \&}$ & 125 & $\begin{array}{l}\text { axporz } \\
\text { Estort }\end{array}$ & $\begin{array}{l}\text { SHIP SHOAL BUK 7z } \\
\text { \&tip SHOAL BƯ72 }\end{array}$ & LAOD & $\log _{000}$ \\
\hline 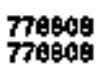 & $\begin{array}{l}\text { wolesmort } \\
\text { worshort }\end{array}$ & $\mathbf{K S}$ & 1507 & poopor & $\begin{array}{l}\text { SOUTH PASS BLK } 1 \\
\text { \$OUTH PASB BLK } 1\end{array}$ & LAOD & 905 \\
\hline $\begin{array}{l}780539 \\
760502\end{array}$ & $\begin{array}{l}\text { YELONNTONE NORTH } \\
\text { YELIONSTOHE }\end{array}$ & Ks & 039 & 900020 & $\begin{array}{l}\text { SOUTH PASS 最 } 20 \\
\text { SOUTH PASS BEK } 20\end{array}$ & LA0D & 905 \\
\hline $\begin{array}{l}\text { KE:muc } \\
\text { 004s72 } \\
\text { or7755 }\end{array}$ & $\begin{array}{l}\text { RTENHESSEE } \\
\text { BEATY } \\
\text { STATIC }\end{array}$ & TH & $\begin{array}{l}053 \\
137\end{array}$ & $\begin{array}{c}900021 \\
\cos 021\end{array}$ & $\begin{array}{l}\text { SOUTH PASS ELK } 21 \\
\text { SOUTH PASS BLK } 21\end{array}$ & $\begin{array}{l}\operatorname{LaO0} \\
\mathrm{LAOS}\end{array}$ & gis \\
\hline nermuc & armednia & & & copose & $\begin{array}{l}\text { SOUTH PASS BLK } 28 \\
\text { \$OUTH PASS BLK } 26\end{array}$ & LAt00 & $\cos$ \\
\hline $\begin{array}{l}\text { ox1711 } \\
\text { oxto15 }\end{array}$ & $\begin{array}{l}\text { EKKHOANCATY BS EAST } \\
\text { BREAKSHAVSI }\end{array}$ & $\underset{\text { Wh }}{\mathrm{K}}$ & $\begin{array}{l}195 \\
027\end{array}$ & $\begin{array}{l}900027 \\
900047\end{array}$ & $\begin{array}{l}\text { SOUTH PASS } \text { QLK } 27 \\
\text { SOUTH PASS BLK } 27\end{array}$ & LAOD & $\underset{905}{900}$ \\
\hline 000578 & WOODAMAN EAST & KY & 196 & $\underset{\operatorname{cog} 0000000}{\cos 0}$ & $\begin{array}{l}\text { SOUTH PASS 朂 } 30 \\
\text { SOUTTH PASB BLK } 30\end{array}$ & LAOD & 905 \\
\hline 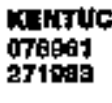 & $\begin{array}{l}\text { AVIST YIRCINIAA } \\
\text { BOART TREE } \\
\text { GILESAT }\end{array}$ & Wr & $\begin{array}{l}195 \\
059\end{array}$ & $\begin{array}{l}009049 \\
000042\end{array}$ & $\begin{array}{l}\text { SOUTH PASS BLK } 42 \\
\text { 8OUTH PASS GUK } 42\end{array}$ & LAOS & 900 \\
\hline $\begin{array}{l}010087 \\
440 \pi / 5\end{array}$ & $\begin{array}{l}\text { BUSKIRK NOFTH } \\
\text { MAGNOLA }\end{array}$ & $\mathbf{K Y}$ & $\begin{array}{l}198 \\
059\end{array}$ & $\begin{array}{l}900061 \\
\operatorname{sog} 000\end{array}$ & $\begin{array}{l}\text { SOUTH PASS 朂E1 } \\
\text { SOUTH PASS BLK }\end{array}$ & EAOD & 905 \\
\hline $\begin{array}{l}014004 \\
420048\end{array}$ & $\begin{array}{l}\text { FALLBBUAC CONSOL } \\
\text { LOUISA }\end{array}$ & KY & 127 & $\begin{array}{l}\text { gajors: } \\
\text { o1sore }\end{array}$ & $\begin{array}{l}\text { BOUTH PASS SA EUK 76 } \\
\text { BOUTH PASS BA BLK } 78\end{array}$ & LAop & $\operatorname{sic}$ \\
\hline $\begin{array}{l}429045 \\
429045\end{array}$ & $\begin{array}{l}\text { LOUISA } \\
\text { LOUISA }\end{array}$ & Wr & 127 & 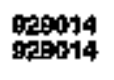 & VEAHLLON BYK 14 & LAONOS & 900 \\
\hline oos7es & $\begin{array}{l}\text { WOODMAN EAST } \\
\text { GLBEAT }\end{array}$ & Wr & $\begin{array}{l}185 \\
059\end{array}$ & $\begin{array}{l}929014 \\
920029\end{array}$ & 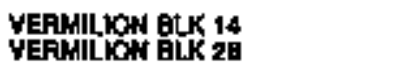 & $\begin{array}{l}\text { LAOOS } \\
\text { CaOS }\end{array}$ & 995 \\
\hline $\begin{array}{l}\text { ofosa? } \\
250140\end{array}$ & ZEEDA & WY & 127 & 935017 & $\begin{array}{l}\text { WEST CANERON BLK } 17 \\
\text { WEST CAMEFON ELK } 17\end{array}$ & $\begin{array}{l}\text { LAOD } \\
\text { LAOS }\end{array}$ & 89 \\
\hline $\begin{array}{l}\text { Loulepte } \\
003002 \\
003002\end{array}$ & 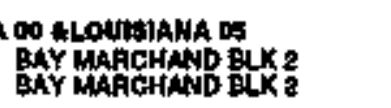 & Lapo & $\underset{690}{690}$ & 9350apas & $\begin{array}{l}\text { WEST CANEAON BLK } 26 \\
\text { WEST CANEFON BUK } 26\end{array}$ & LAOOS & $\stackrel{905}{900}$ \\
\hline $\begin{array}{l}\text { 910059 } \\
\text { 910005 }\end{array}$ & $\begin{array}{l}\text { BFETON SOLND ELK } 53 \\
\text { BFETON \$OWND BLK } 58\end{array}$ & Lios & 90 & 935005 & 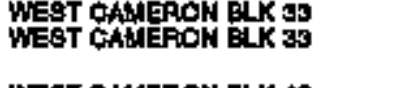 & $\begin{array}{l}\text { LAOOS } \\
\text { SAOS }\end{array}$ & 996 \\
\hline $\begin{array}{l}\text { 15:tot } \\
\text { 16tsot }\end{array}$ & $\begin{array}{l}\operatorname{cogN} \text { Polnt } \\
\text { coon PONTT }\end{array}$ & CAto & 89 & 25s5045 & $\begin{array}{l}\text { WEST CAMEFON ELK } 45 \\
\text { WES CAHEFON ELK } 45\end{array}$ & LAOD & ops \\
\hline $\begin{array}{l}842016 \\
6+2016\end{array}$ & 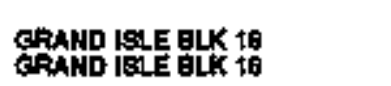 & $\begin{array}{l}\text { LAOS } \\
\text { LAOS }\end{array}$ & 690 & $\begin{array}{l}945049 \\
935049\end{array}$ & $\begin{array}{l}\text { WEST CAWEFON EUK } 49 \\
\text { WEST CAHERON BLK } 49\end{array}$ & $\begin{array}{l}\text { LAOS } \\
\text { LAOS }\end{array}$ & 90 \\
\hline $\begin{array}{l}\text { 94ap18 } \\
\text { 942018 }\end{array}$ & 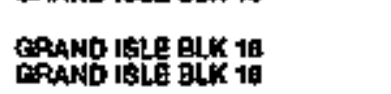 & Ledos & 905 & 949097 & WEST DELTA BLYK & LAOPS & $\stackrel{905}{900}$ \\
\hline $\begin{array}{l}\text { E42025 } \\
B 42026\end{array}$ & $\begin{array}{l}\text { GAAND ISAE ELK } 29 \\
\text { GFAND ISLE BLK } 26\end{array}$ & colos & 695 & 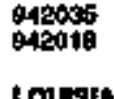 & $\begin{array}{l}\text { WEST DELTA 最 35 } \\
\text { WEAT DETA BL } 10\end{array}$ & $\begin{array}{l}\text { LAON } \\
\text { LAOS }\end{array}$ & $\underset{000}{905}$ \\
\hline 413924 & 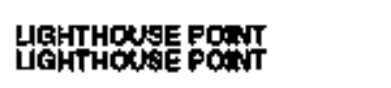 & LAOS & 696 & gespaces & $\begin{array}{l}\text { WOSOA KHOLL BLK } 22 \\
\text { WOONLE BLK OOO }\end{array}$ & 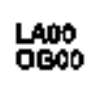 & $\cos$ \\
\hline $\begin{array}{l}\text { 0015155 } \\
\text { 0.6074 }\end{array}$ & MAN PASS 最 151 & Lion & 995 & $\begin{array}{l}\text { Loullotit } \\
\text { asasso } \\
\text { esesso }\end{array}$ & 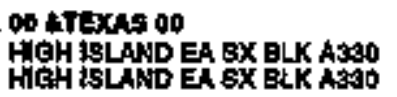 & $\frac{400}{1 \times 00}$ & toss \\
\hline $\begin{array}{l}\text { Bebonos } \\
\text { Begenos }\end{array}$ & $\begin{array}{l}\text { MANN PASS GLK } \\
\text { MAN PASS GLK }\end{array}$ & LAOD & 6995 & & & & \\
\hline
\end{tabular}


Table 6. Fields Located in Muttiple Juriadletions (Continued)

\begin{tabular}{|c|c|c|c|c|c|c|c|}
\hline $\begin{array}{l}\text { Frad } \\
\text { code }\end{array}$ & $\begin{array}{l}\text { flatd } \\
\text { lame }\end{array}$ & $\begin{array}{l}\text { slato } \\
\text { sub }\end{array}$ & $\begin{array}{l}\text { Cootnhy } \\
\text { Conth }\end{array}$ & $\begin{array}{l}\text { Fadd } \\
\text { Cote }\end{array}$ & $\begin{array}{l}\text { Alad } \\
\text { Nama } \\
\end{array}$ & $\begin{array}{l}\text { Enाल } \\
\text { Evl }\end{array}$ & $\begin{array}{l}\operatorname{cosin} f y \\
\cos d x\end{array}$ \\
\hline $\operatorname{sega70}$ & HGH ISLAND EA SX最 & LAco & $\operatorname{mos}$ & \multicolumn{2}{|c|}{ 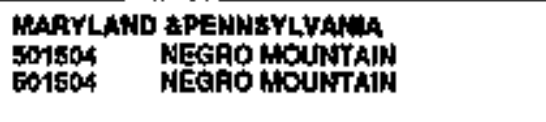 } & PA & oes \\
\hline $\begin{array}{l}800010 \\
800010\end{array}$ & $\begin{array}{l}\text { BABINE PAS\$ ELK 10 } \\
\text { SABINE PASB EUK 10 }\end{array}$ & $\begin{array}{l}\text { La09 } \\
\text { TX00 }\end{array}$ & \%5 & \multirow{2}{*}{\multicolumn{2}{|c|}{ 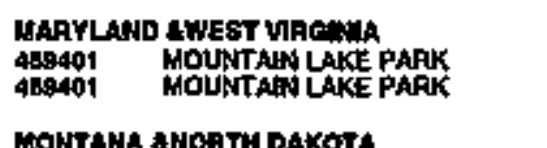 }} & & \\
\hline $\begin{array}{l}890017 \\
890017\end{array}$ & $\begin{array}{l}\text { SABINE PA\$B BUK } 17 \\
\text { SABINE PAES BUK } 17\end{array}$ & $\begin{array}{l}1400 \\
x_{014}\end{array}$ & 9:5 & & & w & og \\
\hline Qa7bos & WEST OANAFON \$A QLK & LAOD & $\cos$ & $\begin{array}{l}\text { month } \\
127746 \\
127745\end{array}$ & $\begin{array}{l}\text { ANOATH DAKOTA } \\
\text { CEDAA CAEEK } \\
\text { CEDAR CAEEK }\end{array}$ & Mo & ovs \\
\hline $\begin{array}{l}\text { LovisianA } \\
\text { o117g日 } \\
\text { o117as }\end{array}$ & $\begin{array}{l}\text { 10 ATEXAS 30 } \\
\text { ALTATOR LAKE } \\
\text { ALTATOR LAKE }\end{array}$ & $\operatorname{lis}_{T \times 0}^{410}$ & $\begin{array}{l}011 \\
011\end{array}$ & 206741 & GWYFARA & MD & 091 \\
\hline $\begin{array}{l}169094 \\
169094\end{array}$ & $\begin{array}{l}\text { MEAFYME1E } \\
\text { MEAFYVILE }\end{array}$ & $\frac{\operatorname{La10}}{T \times 30}$ & $\begin{array}{l}\text { 011 } \\
351\end{array}$ & $\begin{array}{l}243024 \\
243004\end{array}$ & $\begin{array}{l}\text { FLAT LAKE EAST } \\
\text { FLAT LAKE EAST }\end{array}$ & Nit & ogt \\
\hline $\begin{array}{l}554404 \\
654404\end{array}$ & $\begin{array}{l}\text { PHOENIX LAKE } \\
\text { PHOENIX LAKE }\end{array}$ & LX10 & $\begin{array}{l}\text { 018 } \\
\text { 3st }\end{array}$ & $\begin{array}{l}280729 \\
280720\end{array}$ & $\begin{array}{l}\text { GOOSE LAKE EAST } \\
\text { COOSE LAKE EAST }\end{array}$ & $\operatorname{Min}_{\text {No }}$ & $\begin{array}{l}\text { opi } \\
\text { ots }\end{array}$ \\
\hline$\frac{0.20029}{6202022}$ & $\begin{array}{l}\text { SABINE LAKE NOFTH } \\
\text { SABLNE LAKE NOFTH }\end{array}$ & UA10 & 023 & 481643 & MONDAK WEST & $\mathbb{N T}_{\mathbf{N D}}$ & $\begin{array}{l}\text { ogs } \\
\text { oss }\end{array}$ \\
\hline $\begin{array}{l}059728 \\
609720\end{array}$ & $\begin{array}{l}\text { SKWNEA LAKES } \\
\text { 8KMWEA LAKES }\end{array}$ & $\underset{T \times 30}{\operatorname{LA10}}$ & $\begin{array}{l}011 \\
351\end{array}$ & $\begin{array}{l}\operatorname{monta} \\
\text { op?150 } \\
\text { ofr150 }\end{array}$ & $\begin{array}{l}\text { WYOMING } \\
\text { ASH CAEEK } \\
\text { ASH CREEK }\end{array}$ & $\mathbf{M r}$ & 003 \\
\hline $\begin{array}{l}\text { LOULSIAHA } \\
\text { g6e415 } \\
00415\end{array}$ & 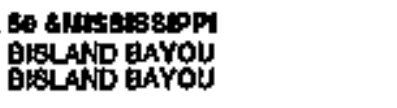 & 1450 & 000 & $\begin{array}{l}210001 \\
210051\end{array}$ & ELK EASIN & 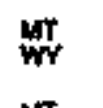 & $\cos _{009}$ \\
\hline $\begin{array}{l}224195 \\
278196\end{array}$ & $\begin{array}{l}\text { EAPERANCE POINT } \\
\text { ESPERANCE POINT }\end{array}$ & $\begin{array}{l}\text { LASO } \\
\text { HS }\end{array}$ & O20: & $\begin{array}{l}265550 \\
2505=0\end{array}$ & 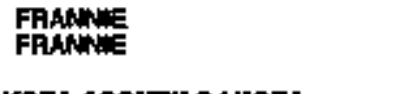 & $\underset{\mathbf{w r}}{\mathbf{4 T}}$ & $\begin{array}{l}099 \\
029\end{array}$ \\
\hline $\begin{array}{l}270479 \\
272479\end{array}$ & $\begin{array}{l}\text { CHLES BEND } \\
\text { GIIES BEKD }\end{array}$ & $\begin{array}{l}\text { LAso } \\
\text { Hs }\end{array}$ & nep & $\begin{array}{l}\text { MOATH } \\
330079 \\
465106\end{array}$ & $\begin{array}{l}\text { KOTA \&8OUTH OAKOTA } \\
\text { HORSE CAEE SOUTH } \\
\text { MEDIGINE POLE HIUS SOATH }\end{array}$ & so & $\begin{array}{l}011 \\
0.8\end{array}$ \\
\hline $\begin{array}{l}489169 \\
489168\end{array}$ & NATSHEZFEAR & LAso & 星 & 677589 & $\begin{array}{l}\text { STATE LINE } \\
\text { STATE LINE }\end{array}$ & $\begin{array}{l}\text { ND } \\
\text { So }\end{array}$ & cys \\
\hline $\begin{array}{l}\text { a10201 } \\
\text { ato201 } \\
\text { LounsianA }\end{array}$ & $\begin{array}{l}\text { ROPNEY ISLAN' } \\
\text { RODHEY ISLAW }\end{array}$ & LAso & 107 & $\begin{array}{l}\text { kow wi } \\
043760 \\
043762\end{array}$ & $\begin{array}{l}\text { OO 10 STIXAS Q⿱ } \\
\text { BATLEAXE } \\
\text { BATTLEAXE }\end{array}$ & $\underset{T X t 0}{\text { NMto }}$ & $\begin{array}{l}025 \\
301\end{array}$ \\
\hline 055572 & 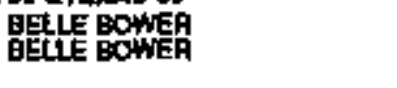 & Letop & $\begin{array}{l}031 \\
3 \times 5\end{array}$ & $\begin{array}{l}185809 \\
105809\end{array}$ & $\begin{array}{l}\text { DOUAFFADE } \\
\text { DOLAFHDE }\end{array}$ & TX10 & ods \\
\hline 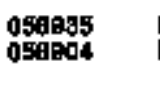 & $\begin{array}{l}\text { OETHANY.LONOSTREET } \\
\text { BETHANY }\end{array}$ & LAso & 017 & $\begin{array}{l}216085 \\
2169855\end{array}$ & 是 MAR & $\frac{\text { Nyiso }}{\text { TX80 }}$ & c25 \\
\hline $\begin{array}{l}111894 \\
447012\end{array}$ & $\begin{array}{l}\text { CADDO PINE WLAND } \\
\text { MARION COUNTY }\end{array}$ & $\begin{array}{l}\text { Lasp } \\
\text { TX00 }\end{array}$ & $\begin{array}{l}017 \\
316\end{array}$ & $\begin{array}{l}35 \times 3905 \\
3536800\end{array}$ & $\begin{array}{l}\text { JAE WAT } \\
\text { SCAARBOROUEH }\end{array}$ & $\begin{array}{l}\text { NW10 } \\
\text { TXEO }\end{array}$ & 1925 \\
\hline 200197 & $\begin{array}{l}\text { CAREENWOOO-WASKOH } \\
\text { WASKOH }\end{array}$ & Lxpo & 017 & 420117 & LITTMAN & $\frac{\text { Menco }}{\text { TXo }}$ & ogs \\
\hline 428003 & $\begin{array}{l}\text { LOAANAPOFT } \\
\text { LOBANSPOFT }\end{array}$ & $\begin{array}{l}\text { Latso } \\
\text { TXto }\end{array}$ & $\operatorname{los}_{305}^{001}$ & $\begin{array}{l}451470 \\
451470\end{array}$ & $\begin{array}{l}\text { MASON NOATH } \\
\text { MAON NOATH }\end{array}$ & $\frac{1}{T \times 10}$ & $\begin{array}{l}015 \\
301\end{array}$ \\
\hline 420504 & LONGWOOD & $\begin{array}{l}\cos 50 \\
\operatorname{T\times } 60\end{array}$ & 017 & $\begin{array}{l}451470 \\
461470\end{array}$ & MASON NOATH & Mxis & cos \\
\hline $\begin{array}{l}\operatorname{sog} a \operatorname{sen} \\
600900\end{array}$ & $\begin{array}{l}\text { RODESSA } \\
\text { RODESBA }\end{array}$ & $\underset{T \times 150}{\operatorname{Las}}$ & $\begin{array}{l}017 \\
037\end{array}$ & $\begin{array}{l}6000080 \\
600026\end{array}$ & $\begin{array}{l}\text { FED ELUFF } \\
\text { FEJ ELUFF }\end{array}$ & 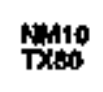 & $\begin{array}{c}\text { gis } \\
\text { soi }\end{array}$ \\
\hline 6009000 & $\begin{array}{l}\text { RODESSA } \\
\text { RODESBA }\end{array}$ & $\operatorname{Lxt}_{\operatorname{Tx} 0}$ & a17 & 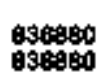 & $\begin{array}{l}\text { SCAFBOFOUSH } \\
\text { BCARBOFOUCH }\end{array}$ & 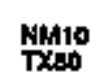 & 4025 \\
\hline & & & & $\begin{array}{l}\text { 6776652 } \\
\text { if76ta }\end{array}$ & $\begin{array}{l}\text { STATEUNE } \\
\text { STATIUNE }\end{array}$ & $\frac{N 410}{T \times 80}$ & tods \\
\hline
\end{tabular}


Table 6. Fields Located in Multiple Jurisdictions (Continued)

\begin{tabular}{|c|c|c|c|c|c|c|c|}
\hline $\begin{array}{l}\text { Fiakd } \\
\text { Gode } \\
\end{array}$ & $\begin{array}{l}\text { Fieto } \\
\text { Nen: }\end{array}$ & $\begin{array}{l}\text { sileter } \\
\text { sil: }\end{array}$ & $\begin{array}{l}\text { County } \\
\text { Code }\end{array}$ & $\begin{array}{l}\text { Fiad } \\
\text { Gods } \\
\end{array}$ & $\begin{array}{l}\text { Fitd } \\
\text { Niato } \\
\end{array}$ & $\begin{array}{l}\text { Sing } \\
\text { Sith }\end{array}$ & $\begin{array}{l}\text { Conty } \\
\text { cod!: }\end{array}$ \\
\hline $\begin{array}{l}093727 \\
093727\end{array}$ & $\begin{array}{l}\text { Co } 10 \text { irtexAs :5 } \\
\text { BRONGO } \\
\text { BRONAC }\end{array}$ & $\begin{array}{l}\text { NH10 } \\
\text { TXs }\end{array}$ & $\begin{array}{l}025 \\
501\end{array}$ & $\begin{array}{l}292554 \\
292564\end{array}$ & $\begin{array}{l}\text { GROUNDHOG CREEK } \\
\text { GROUNDHOO CAEEK }\end{array}$ & $\begin{array}{l}\mathrm{OH} \\
\mathrm{WW}\end{array}$ & $\begin{array}{l}105 \\
035\end{array}$ \\
\hline $\begin{array}{l}305720 \\
325720\end{array}$ & $\begin{array}{l}\text { HOABS EAST } \\
\text { HOBOBS EAST }\end{array}$ & NMtio & $\begin{array}{l}005 \\
165\end{array}$ & $\begin{array}{l}292504 \\
602758\end{array}$ & $\begin{array}{l}\text { EPOUNDHOG CREEK } \\
\text { FIPUEY NORTH }\end{array}$ & oH & $\begin{array}{l}105 \\
035\end{array}$ \\
\hline $\begin{array}{l}420117 \\
420+17\end{array}$ & $\begin{array}{l}\text { LITTHAN } \\
\text { LITTMAN }\end{array}$ & Nhiso & $\begin{array}{l}025 \\
165\end{array}$ & $\begin{array}{l}315775 \\
077094\end{array}$ & $\begin{array}{l}\text { HENDERSHOT } \\
\text { BOAZ }\end{array}$ & $\begin{array}{l}\text { OH } \\
\text { Wry }\end{array}$ & $\begin{array}{l}187 \\
107\end{array}$ \\
\hline \multicolumn{4}{|c|}{ NEW YORK \&PENWSYLVANIA } & $\begin{array}{l}407104 \\
500510\end{array}$ & $\begin{array}{l}\text { LEBANONA } \\
\text { NEPTUNE }\end{array}$ & OHH & 105 \\
\hline 086988 & $\begin{array}{l}\text { BRADFOAD } \\
\text { ERADFDAD }\end{array}$ & $\begin{array}{l}\text { NY } \\
\text { PA }\end{array}$ & $\begin{array}{l}000 \\
0 B 8\end{array}$ & $\begin{array}{l}420644 \\
135585\end{array}$ & $\begin{array}{l}\text { LVEAPOOL EAST } \\
\text { CHESTERHUGHOWN }\end{array}$ & 首 & o29 \\
\hline $\begin{array}{l}094130 \\
004130\end{array}$ & $\begin{array}{l}\text { BFOOKFIELD } \\
\text { BROOKFIELD }\end{array}$ & $\begin{array}{l}\text { NY } \\
\text { PA }\end{array}$ & $\begin{array}{l}101 \\
117\end{array}$ & $\begin{array}{l}430975 \\
077034\end{array}$ & $\begin{array}{l}\text { LOWER NEWPOAT } \\
\text { BOAZ }\end{array}$ & $\begin{array}{l}\text { OH } \\
\text { Wry }\end{array}$ & $\begin{array}{l}187 \\
107\end{array}$ \\
\hline $\begin{array}{l}107178 \\
696599\end{array}$ & $\begin{array}{l}\text { BUETI } \\
\text { SUGAR GROYE }\end{array}$ & $\begin{array}{l}\text { NY } \\
\text { PA }\end{array}$ & $\begin{array}{l}073 \\
123\end{array}$ & 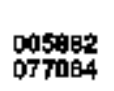 & $\begin{array}{l}\text { WNILE AUN } \\
\text { BOAZ }\end{array}$ & $\begin{array}{l}\text { OHH } \\
\text { wry }\end{array}$ & $\begin{array}{l}187 \\
107\end{array}$ \\
\hline $\begin{array}{l}307722 \\
307801\end{array}$ & $\begin{array}{l}\text { HARRISON EAST } \\
\text { HARPISON }\end{array}$ & $\begin{array}{l}\text { NY } \\
\text { PA }\end{array}$ & $\begin{array}{l}101 \\
105\end{array}$ & $\begin{array}{l}005906 \\
\text { orros4 }\end{array}$ & $\begin{array}{l}\text { MOOFES JUNCTION } \\
\text { BOAZ }\end{array}$ & QH & $\begin{array}{l}167 \\
107\end{array}$ \\
\hline $\begin{array}{l}442573 \\
759626\end{array}$ & $\begin{array}{l}\text { WALLOFY } \\
\text { WEST BAAREA }\end{array}$ & $\begin{array}{l}\text { NY } \\
\text { PA }\end{array}$ & $\begin{array}{l}000 \\
083\end{array}$ & $\begin{array}{l}005945 \\
\text { B广T4345 }\end{array}$ & $\begin{array}{l}\text { NEWWELL AUN } \\
\text { ST WAPYS }\end{array}$ & $\stackrel{o H}{W}$ & $\begin{array}{l}167 \\
073\end{array}$ \\
\hline $\begin{array}{l}590404 \\
552414\end{array}$ & $\begin{array}{l}\text { FICHPUAG } \\
\text { SHIHCLEHOUSE }\end{array}$ & $\begin{array}{l}\text { NY } \\
\text { PA }\end{array}$ & opa & $\begin{array}{l}\cos \theta 6 a \\
503889\end{array}$ & $\begin{array}{l}\text { CWNEWEIGS } \\
\text { NEW ENGLAND }\end{array}$ & QH & $\begin{array}{l}106 \\
107\end{array}$ \\
\hline $\begin{array}{l}690404 \\
652414\end{array}$ & 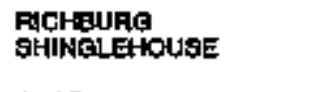 & $\begin{array}{l}\text { NY } \\
\text { PA }\end{array}$ & $\begin{array}{l}003 \\
106\end{array}$ & $\begin{array}{l}0059600 \\
276711\end{array}$ & $\begin{array}{l}\text { PATTOH RUN } \\
\text { CEENN RUN }\end{array}$ & OH & $\begin{array}{l}013 \\
460\end{array}$ \\
\hline $\begin{array}{l}947971 \\
948414\end{array}$ & $\begin{array}{l}\text { SHARON } \\
\text { SHINGLEHOUSE }\end{array}$ & $\begin{array}{l}\text { NY } \\
\text { PA }\end{array}$ & $\begin{array}{l}003 \\
\text { tos }\end{array}$ & 000090 & $\begin{array}{l}\text { PROCTOPYLLE } \\
\text { HUNTINGTOW }\end{array}$ & OH & $\begin{array}{l}067 \\
011\end{array}$ \\
\hline $\begin{array}{l}875523 \\
675523\end{array}$ & $\begin{array}{l}\text { STAGECOACH } \\
\text { STAGECOACH }\end{array}$ & $\begin{array}{l}\text { NY } \\
\mathbf{P A}\end{array}$ & $\begin{array}{l}\$ 07 \\
015\end{array}$ & $\begin{array}{l}006036 \\
340105\end{array}$ & $\begin{array}{l}\text { PAOCTOFuILLE } \\
\text { HutrTth'TON }\end{array}$ & OH & 097 \\
\hline $\begin{array}{l}677630 \\
677530\end{array}$ & $\begin{array}{l}\text { STATE UNE } \\
\text { STATE UARE }\end{array}$ & $\begin{array}{l}\text { MY } \\
\text { PA }\end{array}$ & $\begin{array}{l}003 \\
106\end{array}$ & $\begin{array}{l}\text { s7583a } \\
\text { 50658d }\end{array}$ & $\begin{array}{l}\text { PUTHANH } \\
\text { NEW ENGLAND }\end{array}$ & OH & 009 \\
\hline $\begin{array}{l}\text { ofto \&P } \\
\text { o15217 } \\
157082\end{array}$ & $\begin{array}{l}\text { WYYLVAHAR } \\
\text { GUSHNELL-CONHEAUT } \\
\text { CONHAAUT }\end{array}$ & $\stackrel{\phi_{H}}{\mathbf{P}}$ & $\begin{array}{l}007 \\
009\end{array}$ & $\begin{array}{l}003417 \\
050301\end{array}$ & $\begin{array}{l}\text { SHEETS RUN } \\
\text { EEHH RUN }\end{array}$ & OH & $\begin{array}{l}107 \\
1095\end{array}$ \\
\hline \multicolumn{4}{|c|}{ OtNO EMEST VIREIMA } & $\begin{array}{l}006417 \\
056301\end{array}$ & $\begin{array}{l}\text { SHEETS FUN } \\
\text { EEVHS RUN }\end{array}$ & oth & tor \\
\hline \multirow[t]{2}{*}{$\begin{array}{l}006001 \\
340105\end{array}$} & ATHALIA & OH & 09 & $\begin{array}{l}656139 \\
650139\end{array}$ & 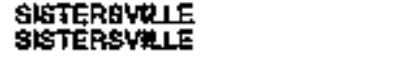 & $\underset{\text { QH }}{\text { or }}$ & $\begin{array}{l}111 \\
005\end{array}$ \\
\hline & & & & $\sin _{504}$ & S乡Tा\% & OH & 105 \\
\hline $\begin{array}{l}0 \times 4005 \\
540174\end{array}$ & $\begin{array}{l}\text { PAFILOW } \\
\text { PAFKERSAUAB }\end{array}$ & OH & $\begin{array}{l}\text { 167 } \\
\text { 10'? }\end{array}$ & \multicolumn{2}{|c|}{ DKLAHCWH ITEXAS OO } & Wh & $\mathbf{0 s}$ \\
\hline 054409 & $\begin{array}{l}\text { BELㅗ RUN } \\
\text { ST MARYS }\end{array}$ & ar & $\begin{array}{l}167 \\
073\end{array}$ & $\begin{array}{l}302024 \\
302824\end{array}$ & $\begin{array}{l}\text { HANDY SE } \\
\text { HANDY SE }\end{array}$ & $\frac{\text { Ox }}{\text { Txon }}$ & $\begin{array}{l}085 \\
1 \mathrm{t}\end{array}$ \\
\hline $\begin{array}{l}002030 \\
674345\end{array}$ & $\begin{array}{l}\text { BOgMOATH } \\
\text { ST MAAYS }\end{array}$ & GH & $\begin{array}{l}167 \\
073\end{array}$ & $\begin{array}{l}\text { a41879 } \\
480305\end{array}$ & $\begin{array}{l}\text { BEAY } \\
\text { MONTAGUE COUNTY RECULAA }\end{array}$ & $\begin{array}{l}\text { OK } \\
\text { TX90 }\end{array}$ & 307 \\
\hline $\begin{array}{l}005056 \\
606435\end{array}$ & $\begin{array}{l}\text { ERILUANI } \\
\text { \&UCKEAROD }\end{array}$ & oh & $\infty$ & $\begin{array}{l}\text { obases } \\
\text { 65ases }\end{array}$ & $\begin{array}{l}\text { \$NVEU\$ BENO } \\
\text { 8NVELS BEND }\end{array}$ & TXo & 005 \\
\hline $\begin{array}{l}104972 \\
276711\end{array}$ & $\begin{array}{l}\text { EUFIINGTON } \\
\text { GLENN FHN }\end{array}$ & QH & 013 & $\begin{array}{l}\text { OKI } 140 \\
036467 \\
011470\end{array}$ & $\begin{array}{l}\text { A ETEXAG } 95 \\
\text { EALKO SQUYTH } \\
\text { ALLEN-PAAKEA }\end{array}$ & Txos & 007 \\
\hline opsotio & $\begin{array}{l}\text { GHËIRE } \\
\text { POTI PUEASAN' }\end{array}$ & $\begin{array}{l}\text { OH } \\
\text { Why }\end{array}$ & $0 \%$ & $\begin{array}{l}116065 \\
\text { s513a9 }\end{array}$ & $\begin{array}{l}\text { GAWAICK OISTFICT } \\
\text { PERRYTOW WOATH }\end{array}$ & $\frac{9 x}{\text { Txis }}$ & 007 \\
\hline $\begin{array}{l}244927 \\
058331\end{array}$ & $\begin{array}{l}\text { GFANDWIEW } \\
\text { EENS RUN }\end{array}$ & oH & $\begin{array}{l}167 \\
073\end{array}$ & $\begin{array}{l}291498 \\
365493\end{array}$ & $\begin{array}{l}\text { GAIGGS SQUTH } \\
\text { JUDD }\end{array}$ & $\frac{\mathrm{WK}}{\mathrm{TXg}}$ & 025 \\
\hline $\begin{array}{l}284927 \\
058331\end{array}$ & $\begin{array}{l}\text { CFANDWIEW } \\
\text { EENS RNW }\end{array}$ & oH & $\begin{array}{l}187 \\
0056\end{array}$ & & & & \\
\hline
\end{tabular}


Table 6. Fields Located in Multiple Jurisdictions (Continued)

\begin{tabular}{|c|c|c|c|c|c|c|c|}
\hline $\begin{array}{l}\text { Fold } \\
\text { Cords }\end{array}$ & $\begin{array}{l}\text { Frold } \\
\text { Name }\end{array}$ & $\begin{array}{l}\text { Stald } \\
\text { sint }\end{array}$ & $\begin{array}{l}\text { Counhy } \\
\text { Codde }\end{array}$ & $\begin{array}{l}\text { Fakf } \\
\text { Cade }\end{array}$ & $\begin{array}{l}\text { Fink } \\
\text { Nemes }\end{array}$ & $\begin{array}{l}\text { glimar } \\
\text { suth }\end{array}$ & $\begin{array}{l}\text { County } \\
\text { Cods }\end{array}$ \\
\hline 3296496 & $\begin{array}{l}\text { GUYhON SOUTH } \\
\text { HTCHLAND WEST }\end{array}$ & OK & $\begin{array}{l}189 \\
189\end{array}$ & $\begin{array}{l}499029 \\
234157\end{array}$ & $\begin{array}{l}\text { MOUNT YOARIS } \\
\text { FAIAVIEW-STATLER RUN-WT ME }\end{array}$ & PA & ose \\
\hline 205545 & $\begin{array}{l}\text { GUYMONHWGOTON GAS AFEA } \\
\text { TEXAS HUEOTON }\end{array}$ & TXas & $\begin{array}{l}139 \\
195\end{array}$ & $\begin{array}{r}503779 \\
054502\end{array}$ & $\begin{array}{l}\text { NEW FAEEPOAT } \\
\text { BELTON }\end{array}$ & PA & 050 \\
\hline 295545 & $\begin{array}{l}\text { GUYMONHUGOTON BAS AREA } \\
\text { TEXAS HUEOTON }\end{array}$ & PXos & $\begin{array}{l}139 \\
421\end{array}$ & $\begin{array}{l}503770 \\
004858\end{array}$ & $\begin{array}{l}\text { TEW FAEEPOAT } \\
\text { GAMERON+KAUSOOTH }\end{array}$ & PA & 059 \\
\hline $\begin{array}{l}321545 \\
321545\end{array}$ & $\begin{array}{l}\text { Hgenns south } \\
\text { Hacmis south }\end{array}$ & oK & 2045 & $\begin{array}{l}503770 \\
507597\end{array}$ & $\begin{array}{l}\text { NEW FAEEPOHT } \\
\text { ROCK GAMP }\end{array}$ & PA & $\begin{array}{l}069 \\
103\end{array}$ \\
\hline 321565 & $\begin{array}{l}\text { HIGGINS sOUTH } \\
\text { HIGGINS SOUTH }\end{array}$ & $\underset{\mathrm{TX}}{\mathrm{OK}}$ & $\begin{array}{l}0.55 \\
2 \pm 1\end{array}$ & 907450 & $\begin{array}{l}\text { PODT MAPION } \\
\text { GASBVLLEOWLY }\end{array}$ & PA & 061 \\
\hline $\begin{array}{l}350269 \\
247557\end{array}$ & $\begin{array}{l}\text { IVANHOG } \\
\text { FOLETT }\end{array}$ & OKxas & $\underset{\cos }{\cos }$ & $\begin{array}{l}\text { 594846 } \\
\text { 141705 }\end{array}$ & RICH HAT, & PA & $\begin{array}{l}059 \\
051\end{array}$ \\
\hline $\begin{array}{l}423001 \\
247567\end{array}$ & $\begin{array}{l}\text { LOBAN SOUTH } \\
\text { FOLLETT }\end{array}$ & $\stackrel{\mathrm{OK}}{\mathrm{OX}}$ & $\frac{007}{285}$ & $\begin{array}{l}007351 \\
480030\end{array}$ & $\begin{array}{l}\text { SHANNON FUN } \\
\text { WOOAESWLLE }\end{array}$ & $\underset{W A}{P A}$ & 059 \\
\hline $\begin{array}{l}455582 \\
475752\end{array}$ & $\begin{array}{l}\text { MAYFIELD WEST } \\
\text { MS FANCHH }\end{array}$ & OKX & 480 & 682000 & $\begin{array}{l}\text { SHITHS FEARY } \\
\text { SHITHS FERAY }\end{array}$ & PA & $\cos$ \\
\hline $\begin{array}{l}190070 \\
247567\end{array}$ & $\begin{array}{l}\text { MOCANELLAVEFNE OAS AREA } \\
\text { FOLEET }\end{array}$ & $\begin{array}{l}\text { OK } \\
\text { TXas }\end{array}$ & $\underset{205}{007}$ & $\begin{array}{l}721514 \\
721808\end{array}$ & $\begin{array}{l}\text { TUAKEY FOOT } \\
\text { TUAKEYFOOT }\end{array}$ & PA & $\begin{array}{l}125 \\
029\end{array}$ \\
\hline $\begin{array}{l}\text { PENNST } \\
0072 B 1 \\
46558\end{array}$ & $\begin{array}{l}\text { VAHIA EWEST VRGWIA } \\
\text { ASHTREE } \\
\text { MAPLE-WADESTOWN }\end{array}$ & PA & obs & $\begin{array}{l}7 e 5853 \\
124404\end{array}$ & $\begin{array}{l}\text { WHITUY CFEEK } \\
\text { GASBYILE-QOWLEY }\end{array}$ & $P A$ & 058 \\
\hline $\begin{array}{l}\mathbf{1 0 1 0 8 2} \\
207324\end{array}$ & $\begin{array}{l}\text { CARSON } \\
\text { GAS VALLE }\end{array}$ & $\mathbf{P h}$ & $\underset{020}{\infty}$ & $\begin{array}{l}005437 \\
805438\end{array}$ & BAAZOS 最 437 & $\underset{T \times 00}{T \times 00}$ & $\log _{0}$ \\
\hline $\begin{array}{l}241424 \\
441705\end{array}$ & $\begin{array}{l}\text { FAHEY WEST } \\
\text { MANOASVILE }\end{array}$ & $\stackrel{P A}{W}$ & 959 & $\frac{\cos 479}{60479}$ & $\begin{array}{l}\text { BRAZOS BLK } 479 \\
\text { BAAZOS BLK } 479\end{array}$ & $\underset{T \times 00}{T \times 00}$ & 995 \\
\hline $\begin{array}{l}241424 \\
441706\end{array}$ & $\begin{array}{l}\text { FINEYY WEST } \\
\text { MANORSVILE }\end{array}$ & Ph & 125 & $\frac{848022}{000222}$ & 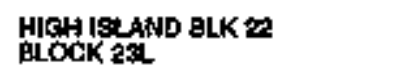 & $\underset{T \times a b}{T \times a 0}$ & 905 \\
\hline $\begin{array}{l}266510 \\
325102\end{array}$ & $\begin{array}{l}\text { GARRIBON } \\
\text { HIXENBWUSH (CAFPO RUN) }\end{array}$ & PA & $\begin{array}{l}0599 \\
109\end{array}$ & $\begin{array}{l}873519 \\
67 \$ 519\end{array}$ & $\begin{array}{l}\text { MATAGORDA ISLAND BLK } 510 \\
\text { MATMOORDA ISLAND BLK } 519\end{array}$ & $\begin{array}{l}\operatorname{Tx} 00 \\
7 \times 06\end{array}$ & $\begin{array}{l}995 \\
990\end{array}$ \\
\hline oorser & $\begin{array}{l}\text { HOOVERS RUN } \\
\text { EAAVE }\end{array}$ & PA & $\begin{array}{l}059 \\
051\end{array}$ & $\begin{array}{l}973527 \\
126721\end{array}$ & $\begin{array}{l}\text { MATAGOADA ISLAND BLK } 527 \\
\text { GAVALO }\end{array}$ & $\underset{T \times 00}{\operatorname{TX}}$ & 996 \\
\hline $\begin{array}{l}007361 \\
45565\end{array}$ & $\begin{array}{l}\text { MOOVEAS RUN } \\
\text { MAPLE-WADESTOWN }\end{array}$ & PA & 059 & 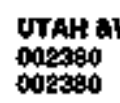 & $\begin{array}{l}\text { POUING } \\
\text { ANSCHUTZ RANCH EAST } \\
\text { ANSCHUTZ RANCH EAST }\end{array}$ & $\underset{W Y}{U T}$ & $\begin{array}{l}043 \\
041\end{array}$ \\
\hline $\begin{array}{l}374107 \\
721803\end{array}$ & KENDALL. & $\mathbf{P A}$ & $\begin{array}{l}007 \\
028\end{array}$ & \multicolumn{4}{|c|}{ VRGINIA \&WEST VIGKAA } \\
\hline $\begin{array}{l}458494 \\
004358\end{array}$ & $\begin{array}{l}\text { MCGAACKEN } \\
\text { CAMERON-KAUSOOTH }\end{array}$ & $\stackrel{P A}{W}$ & 059 & $\begin{array}{l}008087 \\
081408\end{array}$ & $\begin{array}{l}\text { BEAWIND } \\
\text { EAO CAEEK }\end{array}$ & WA & $\begin{array}{l}185 \\
047\end{array}$ \\
\hline 450484 & $\begin{array}{l}\text { MOCRACESEN } \\
\text { ROCKPOHT }\end{array}$ & $\underset{W N}{P A}$ & $\begin{array}{l}\text { 05g } \\
051\end{array}$ & 277145 & $\begin{array}{l}\text { COLKK } \\
\text { ROCKRIDEE }\end{array}$ & WA & 027 \\
\hline $\begin{array}{l}409099 \\
124004\end{array}$ & $\begin{array}{l}\text { MONNT MORREB } \\
\text { CASBVIIE-BOWLBY }\end{array}$ & $P_{A}$ & $\begin{array}{l}059 \\
061\end{array}$ & $\begin{array}{l}0064093 \\
271989\end{array}$ & $\begin{array}{l}\text { MUFLEY } \\
\text { GiLBEHT }\end{array}$ & $\stackrel{\text { WA }}{W}$ & 027 \\
\hline
\end{tabular}


Figure 4. Subdiviaions of Alaska and U.S. Geological Survey Quadrangles

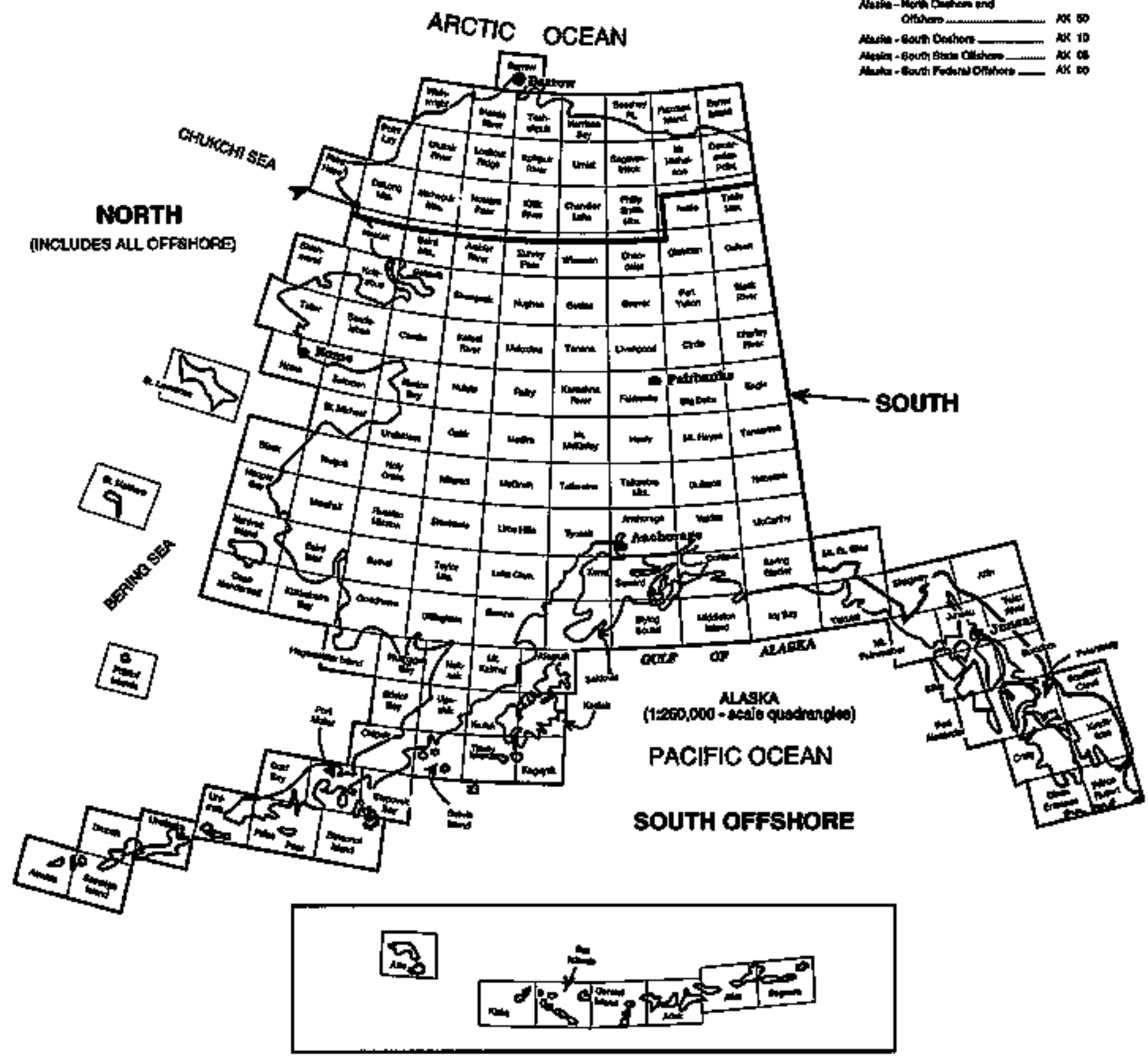

Saurce: Aner U.S. Geologlonl Survey 
Flgure 5. Subdivisions of California

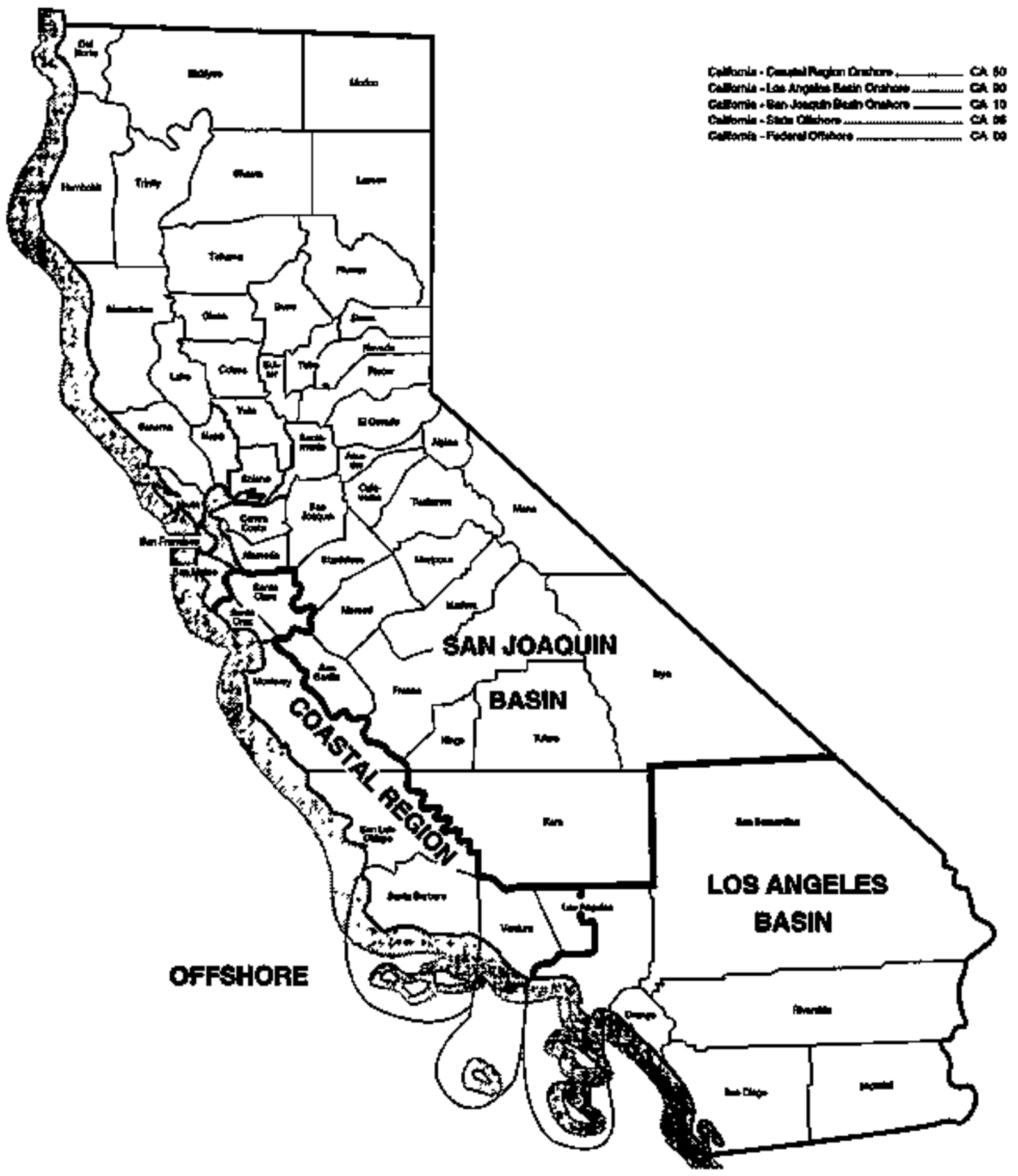

Source: Energy information Adrinisiration, Otfice of Oll and Bas 
Figure 6. Subdivisions of Loulslana

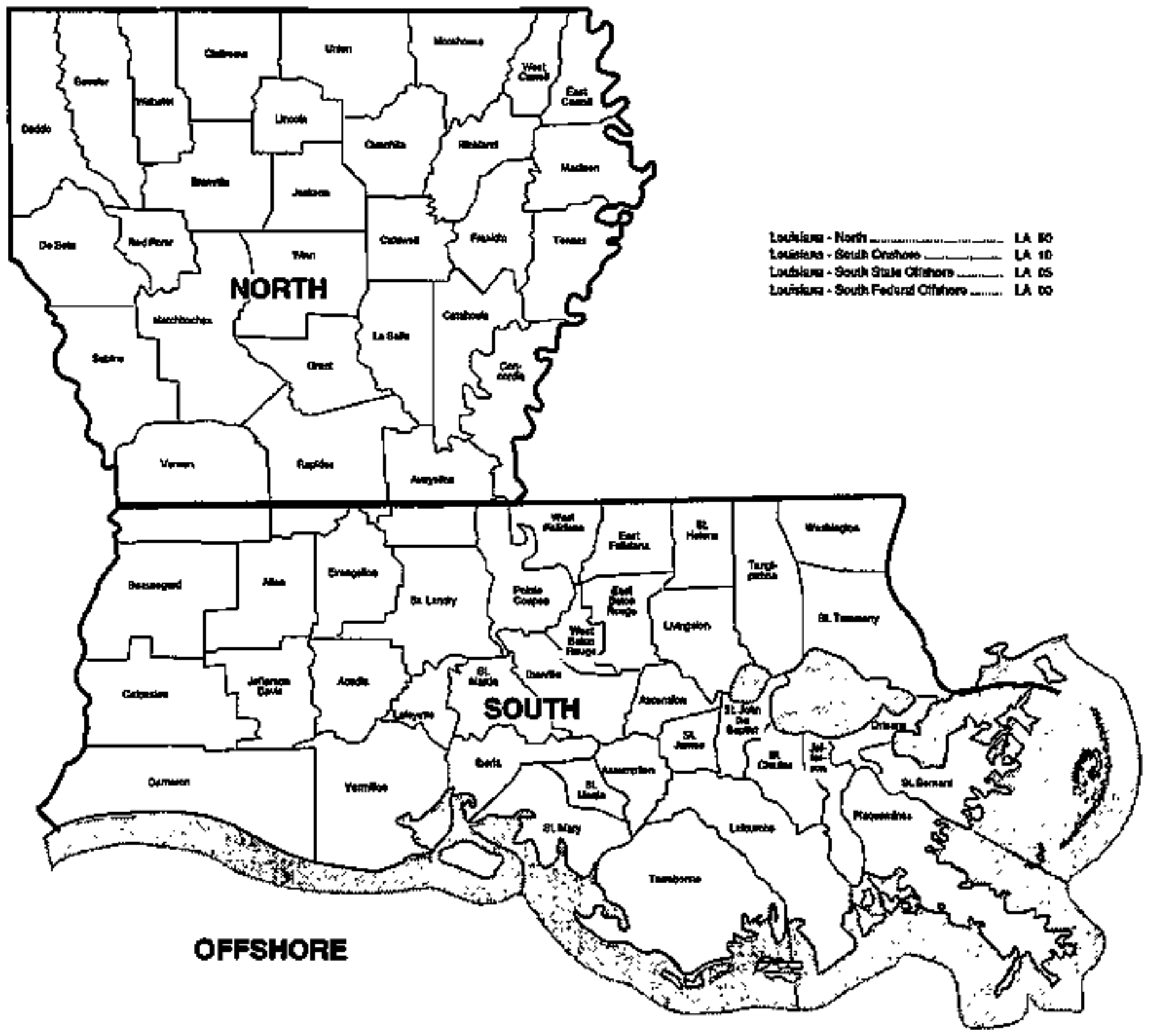

Source: Energy Information Adimtinlstratian, Offlea of Oil and Gas 
Figure 7. Subdivisions of New Mexlco

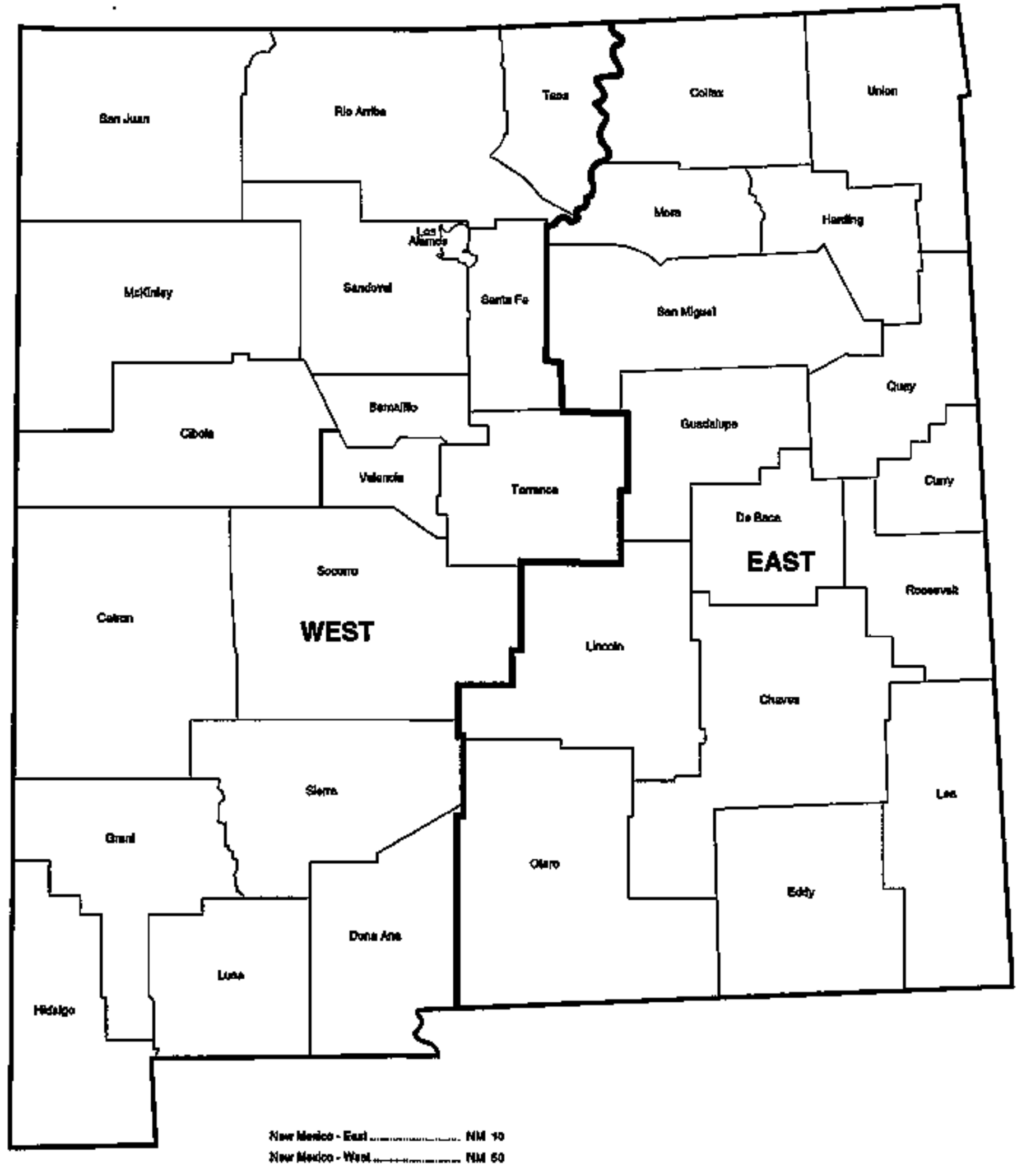

Source: Energy fniormation Adiminlstrallon, Office of Oll and Gas 
Figure 8. Subdiviatone of Texas

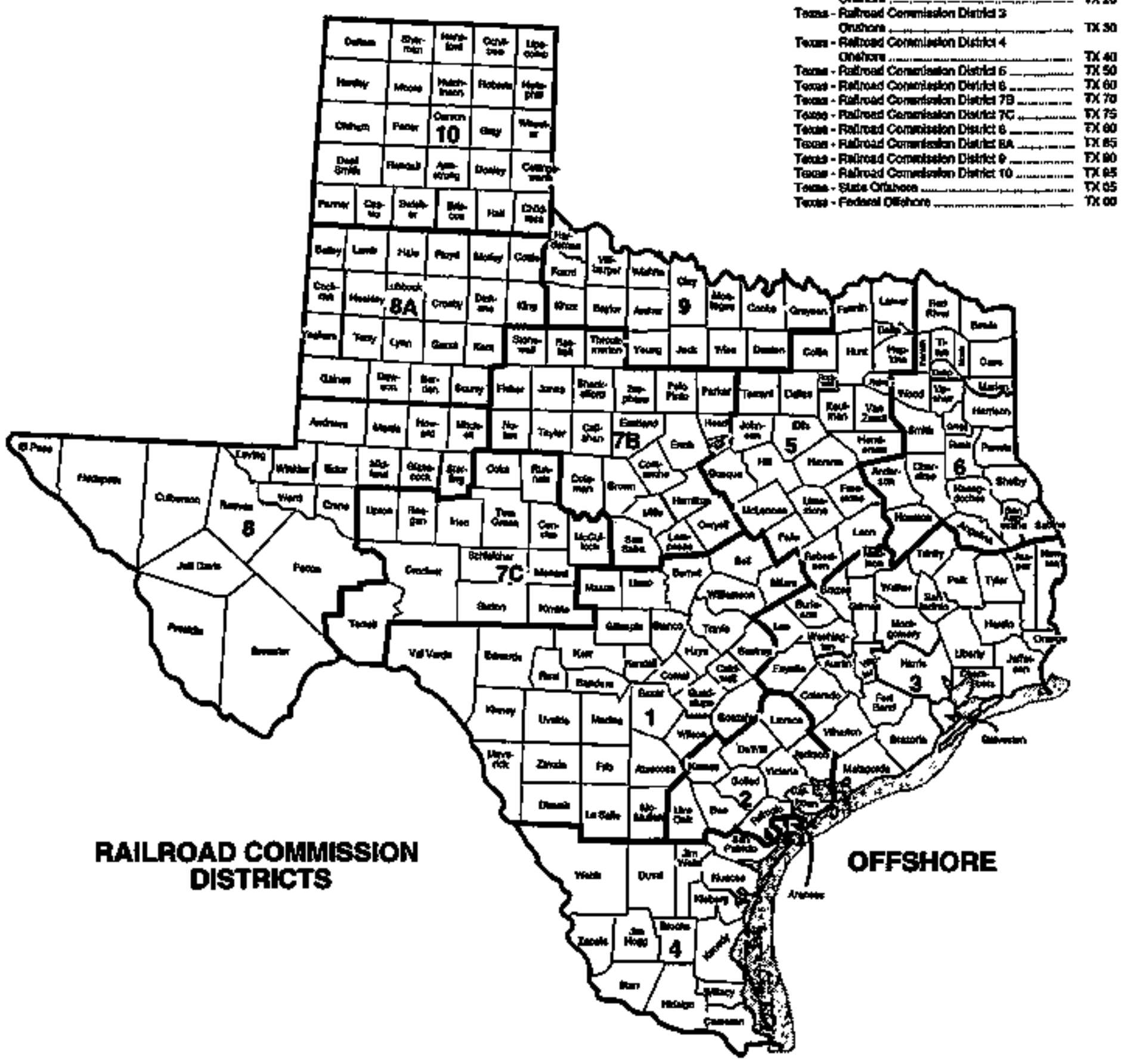

Source: Energy Intormallon Adminislration, Oftice of OAl and Ges 
Flgure 9. Western Planning Area, Guif of Moxlco Outer Continental Shelf Region

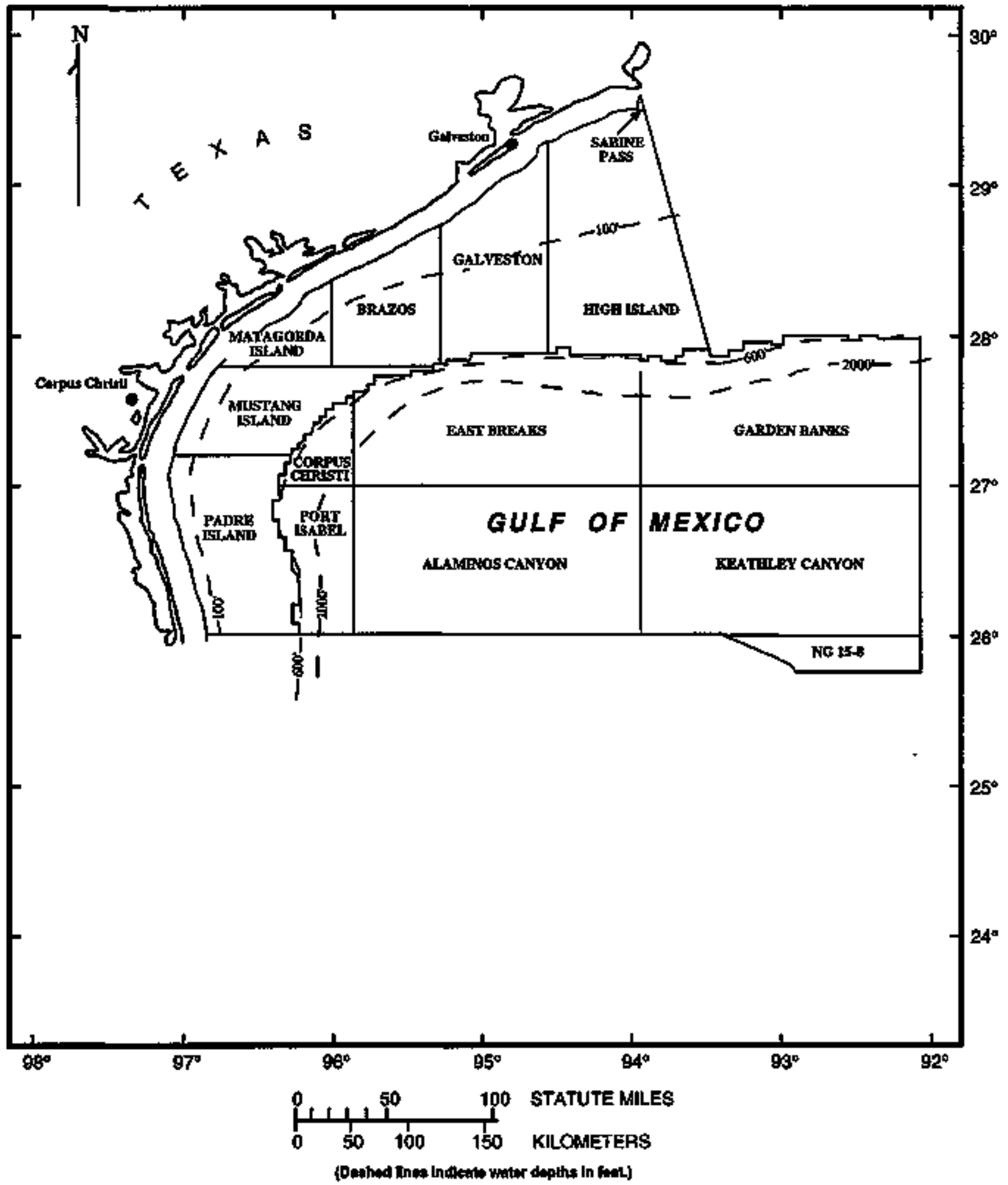

Source: Aflet Mlnerals Management Service, U.S. Department of the Iriterior 
Figure 10. Coniral Planning Aree, Culf of Mexico Outer Continental Sheif Region

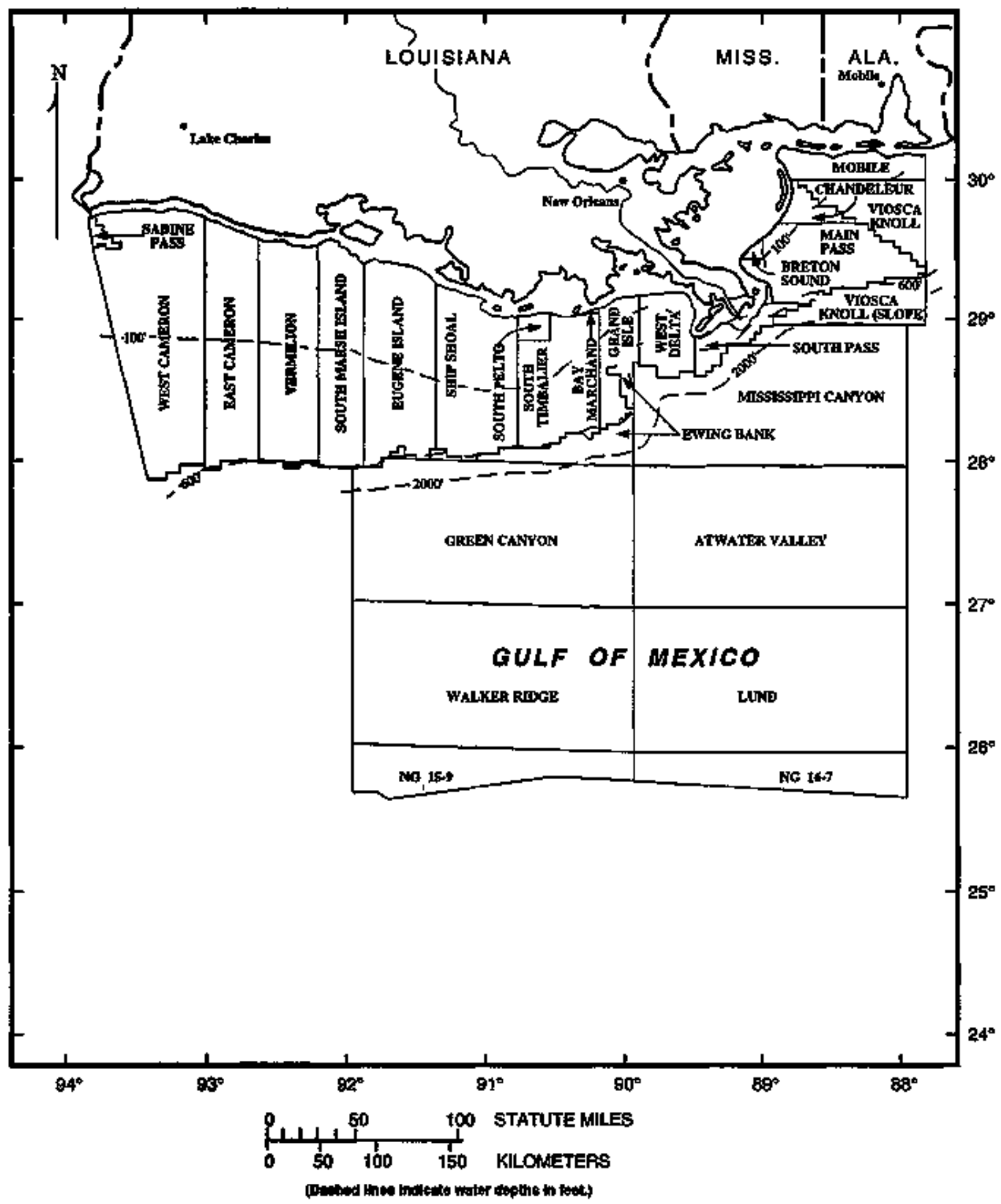

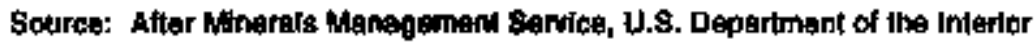


Figure 11. Eastern Planning Area, Gult of Mexico Outer Continental Shelf Region

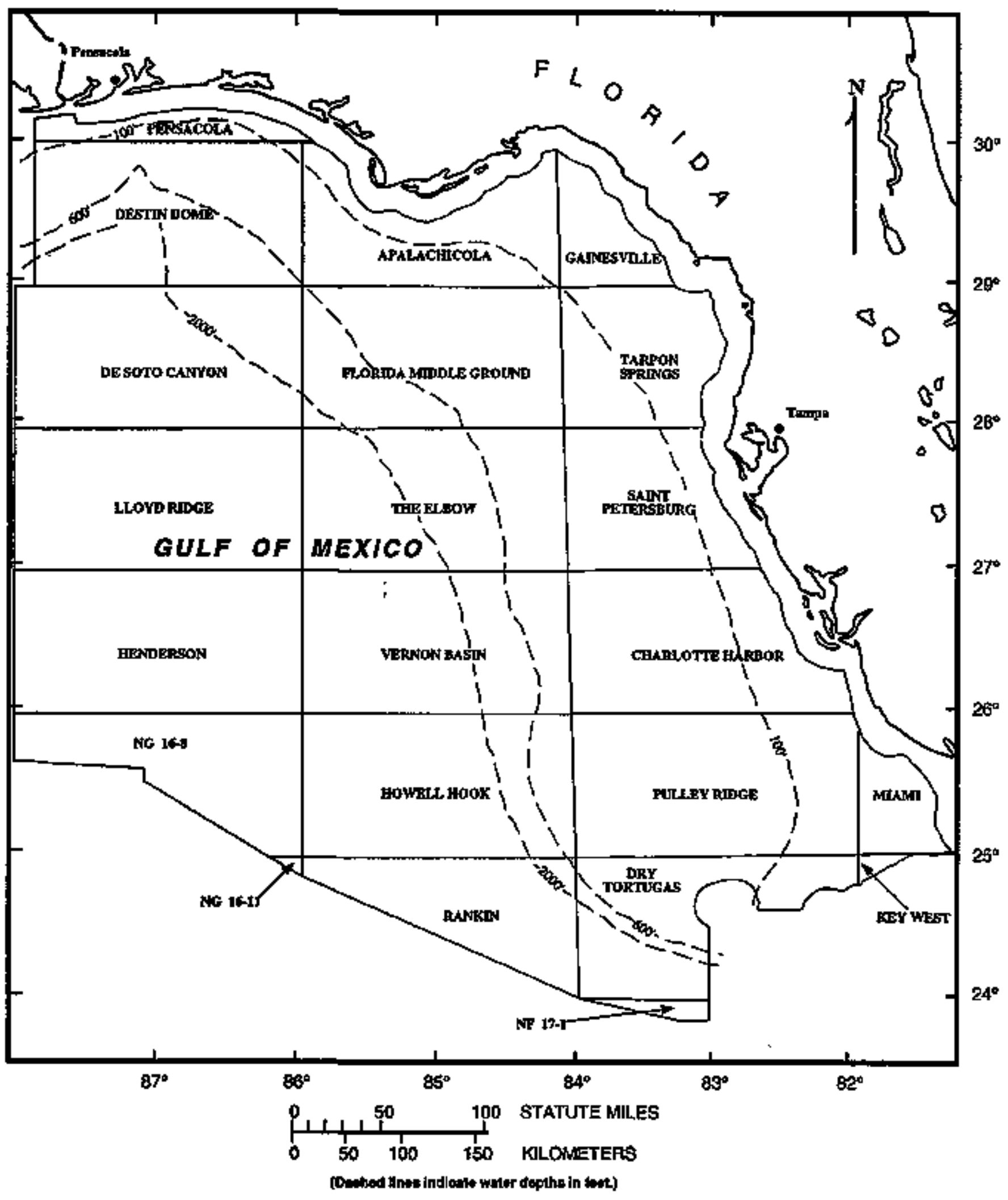

Source: Atler Mlnerals Manegernent Sarvice, U.S. Departreant of the Inlerior 


\section{Glossary}

This Glossary defines many of the technical terms used in this report.

Crude Oll: A mixture of hydrocarbons that exist in the liquid phase in aatural underground reservoirs and remain liquid at atmospheric pressure after passing through surface separating facilities. Crude oil may also inclode:

- Small amounts of bydrocarbons that exist in the gascous phase in natural underground reservoirs but are liquid at atmospheric pressure after being recovered from oil well (casinghead) gas in lease separators, and that subsequently are commingled with the crude stream without being separately weasured.

- Small amourts of nonhydrocarbons produced with the oil.

Field: An area consisting of a single reservoir or multiple reservoirs all grouped on or celated to the same individual geological stmetural feature and/or stratj. graphic condition. There may be two of more reservoirs in a field which are separated vertically by intervening impervious strata, or laterally by local geologic barriers, or by both. (Ses Reservoir.)

Field Area: A geograpbic area encompassing two or more pools that have a common gathering and metering system, the reserves of which are reported as a siogle unit. This concept applies primarily to the Appalachian region. (See Pool.)
Fleld Discovery Year: The calendar year in which a fleid was first recognized as containing economically recoverable accumulations of oil andor gas.

Natural Gas: A mixture of hydrocarbon compounds and suall quantities of various nonhydrocarbons existing in the gaseous phase or in solution with crude oil in natural undergiond reservoirs at reservolic conditions. The principal hydrocartons uspally contained in the mixture are methane, ethane, propane, butane, and pentane. Typical nonhydtocarbon gases which may be present in reservoir natural gas are carbon dioxide, helium. bydrogen sulfide, and nitrogen. Under reservoir conditions, attural gas and the liquefiable portions occur cither in a single gaseons phase in the reservoir or in solution with crode oil and are not distingnishable at the time as separate substances.

Pool: In general, a reservoir. In certain situations a pool may consist of more than one reservoir. (See Field Area.)

Reservolr: A porous and permeable undergtound formation contajning an indjuldual and separate natural accumulation of producible hydrocarbons (oil and/or gas) which is confined by impermeable rock or water barriers and is characterized by a single naturat pressure system.

Sabdiviston: A prescribed portion of a given State of other geographical region defied in this publication for statistical repotting pusposes. 


\section{Oil and Gas \\ Field Code Master List}


ALABA A A

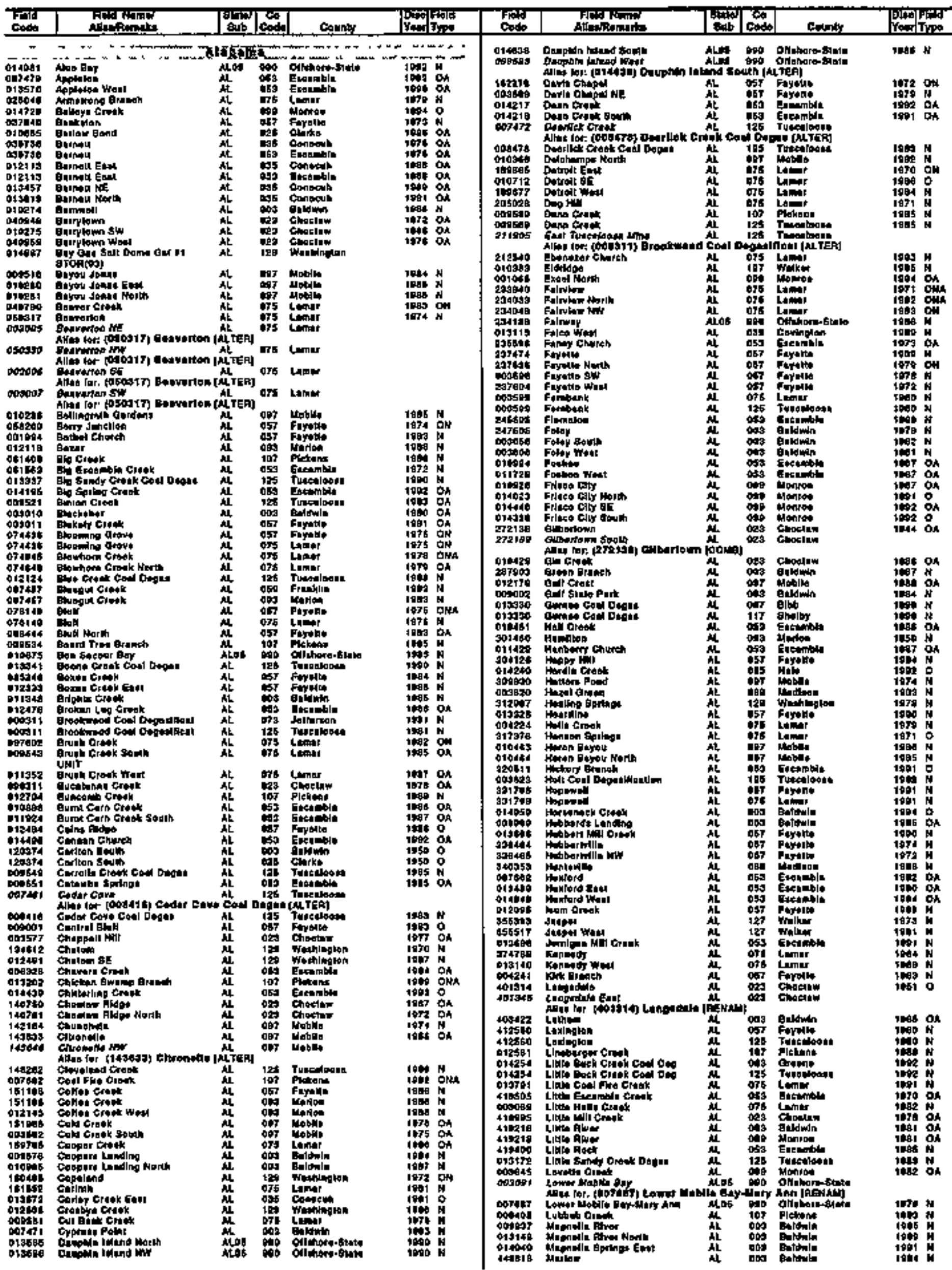


ALASKA

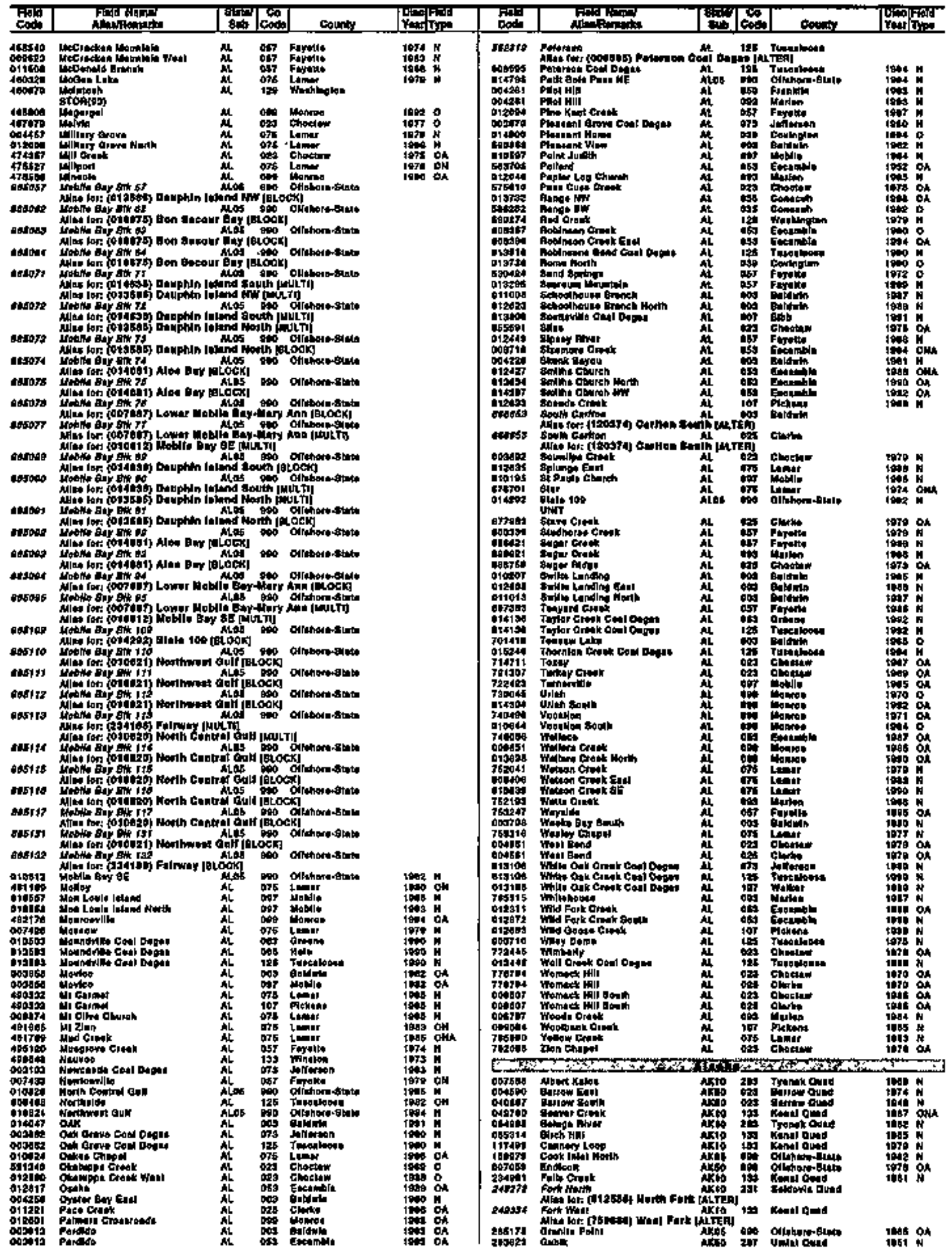


ALA BKA

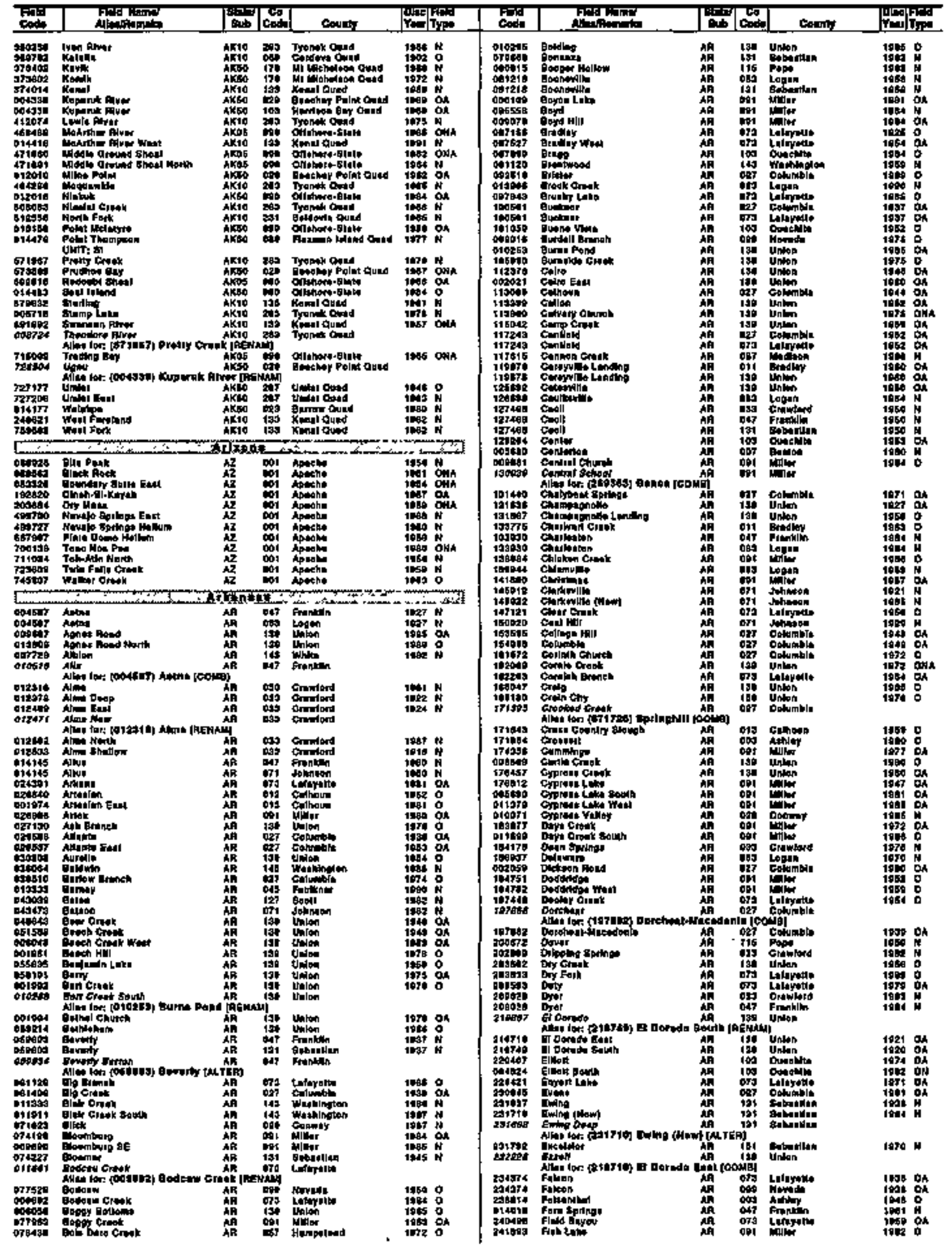


ARKANSAS

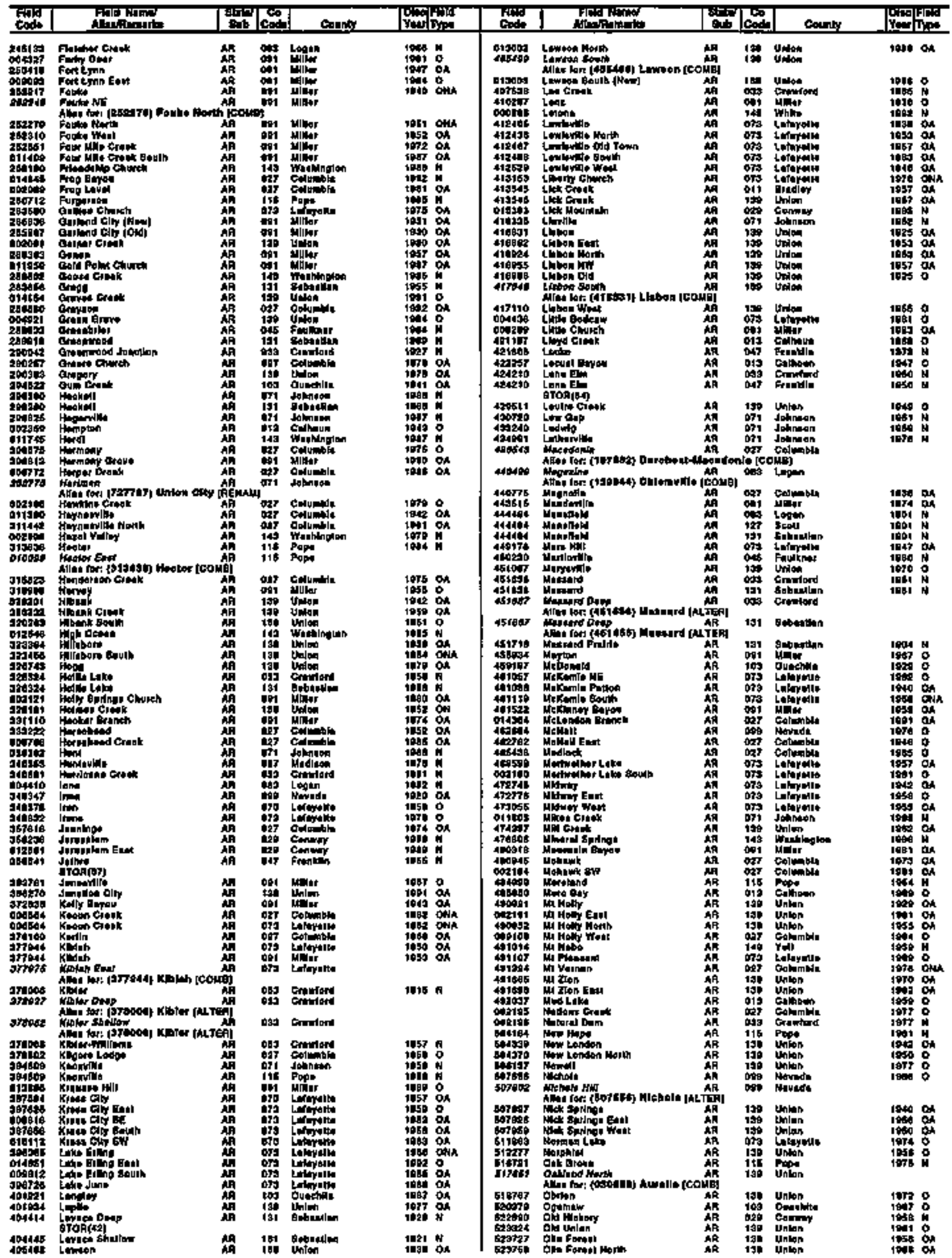




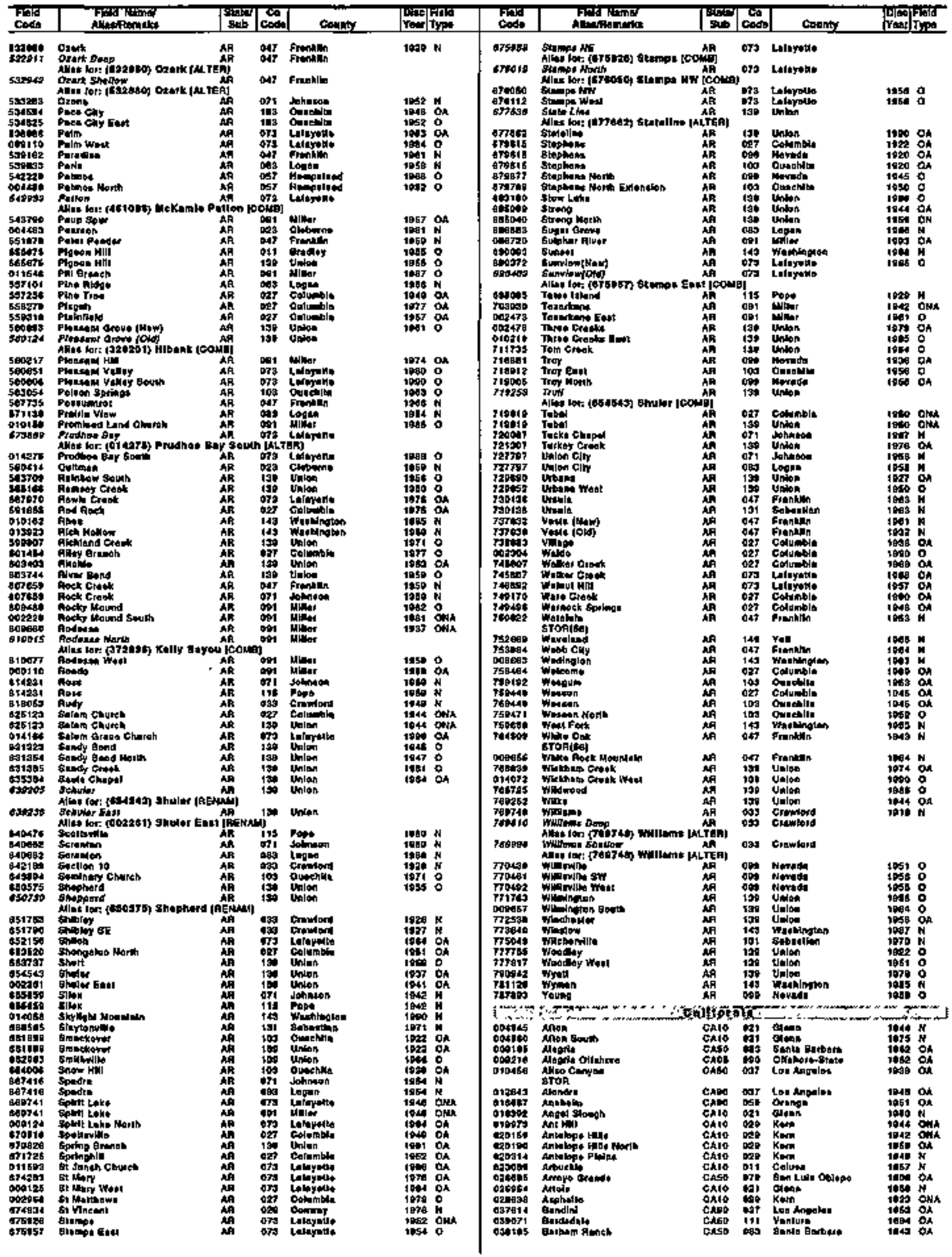


CALIFORNIA

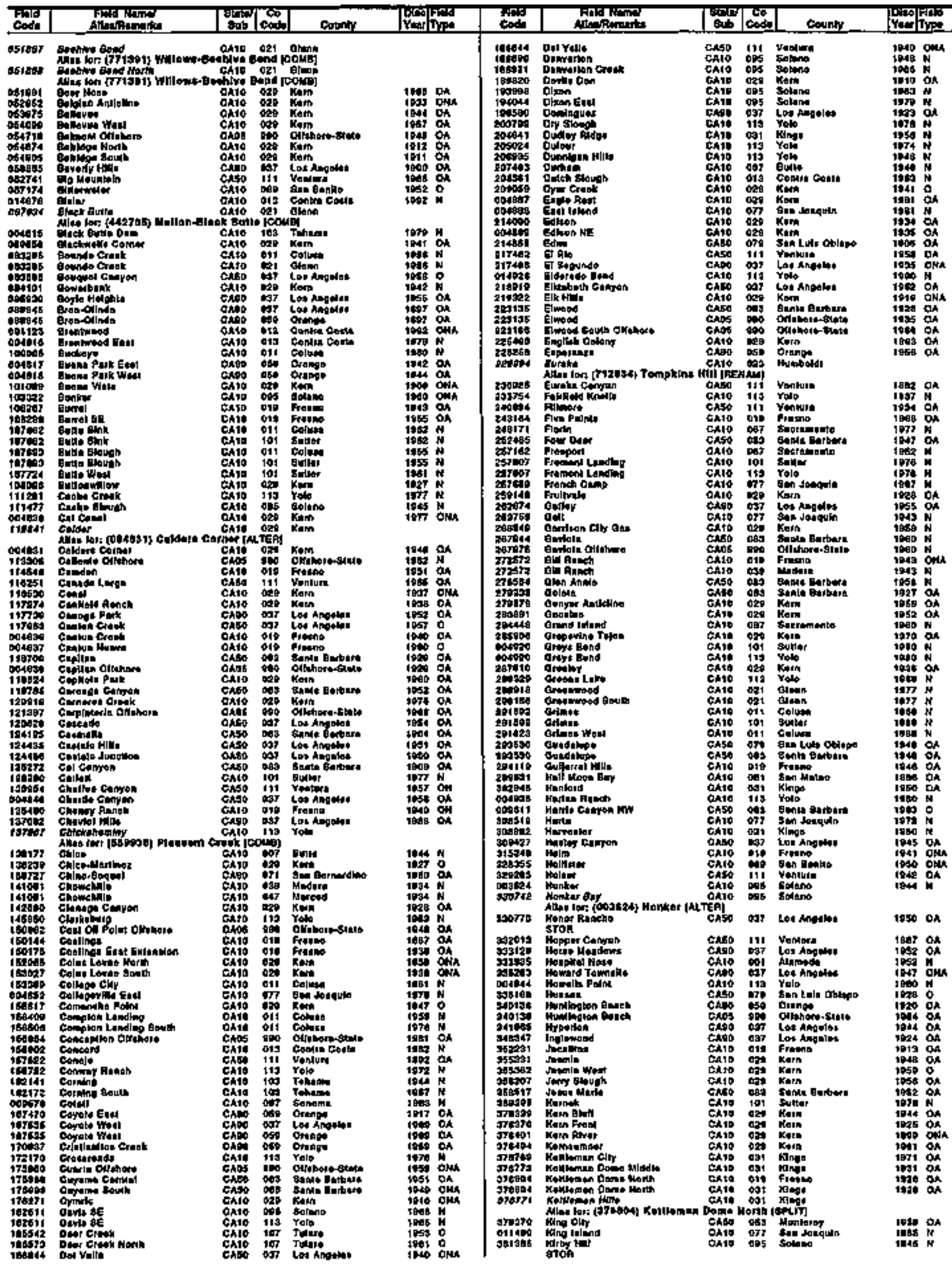


CALIFOANIA

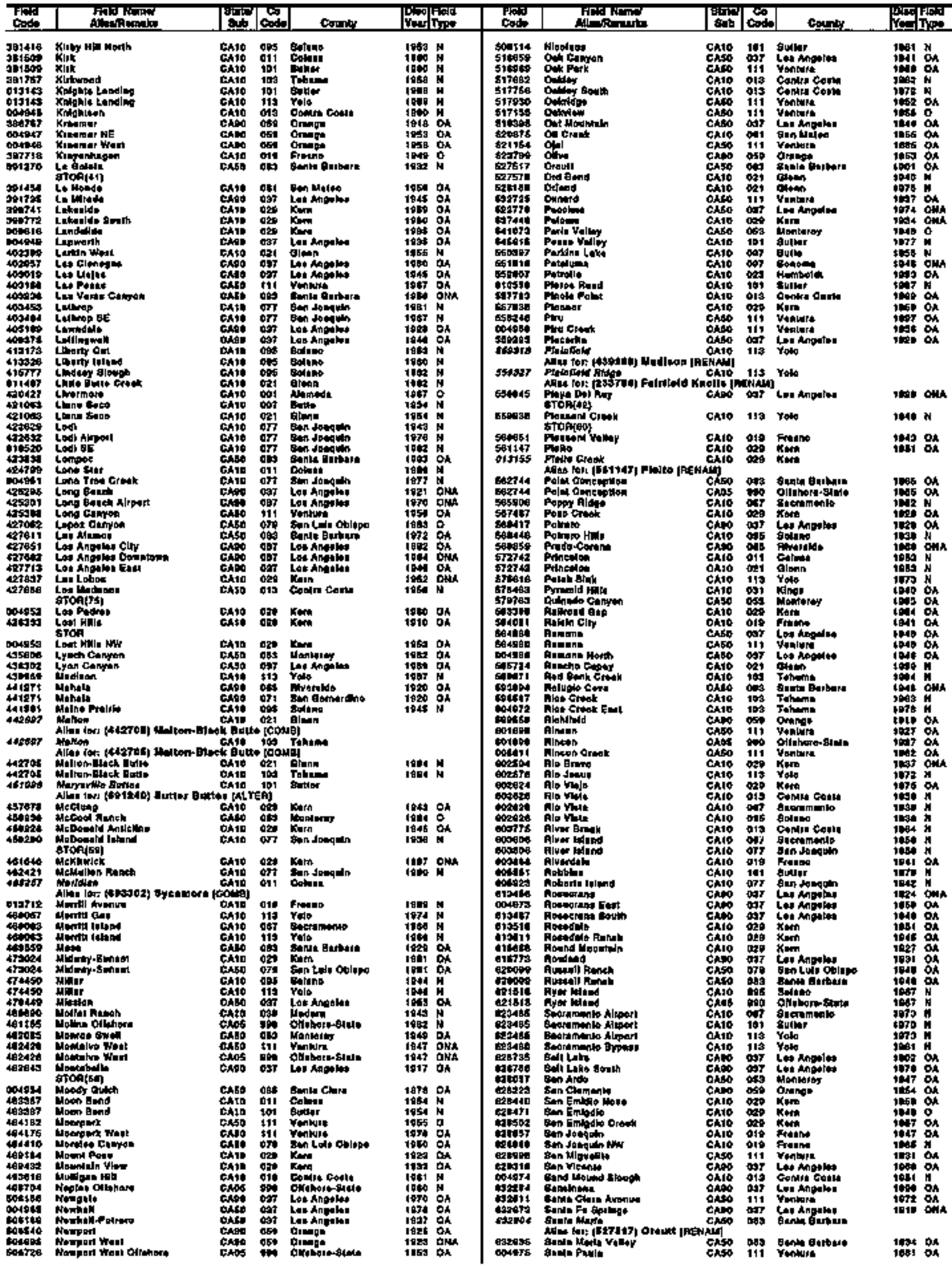


COLORADO

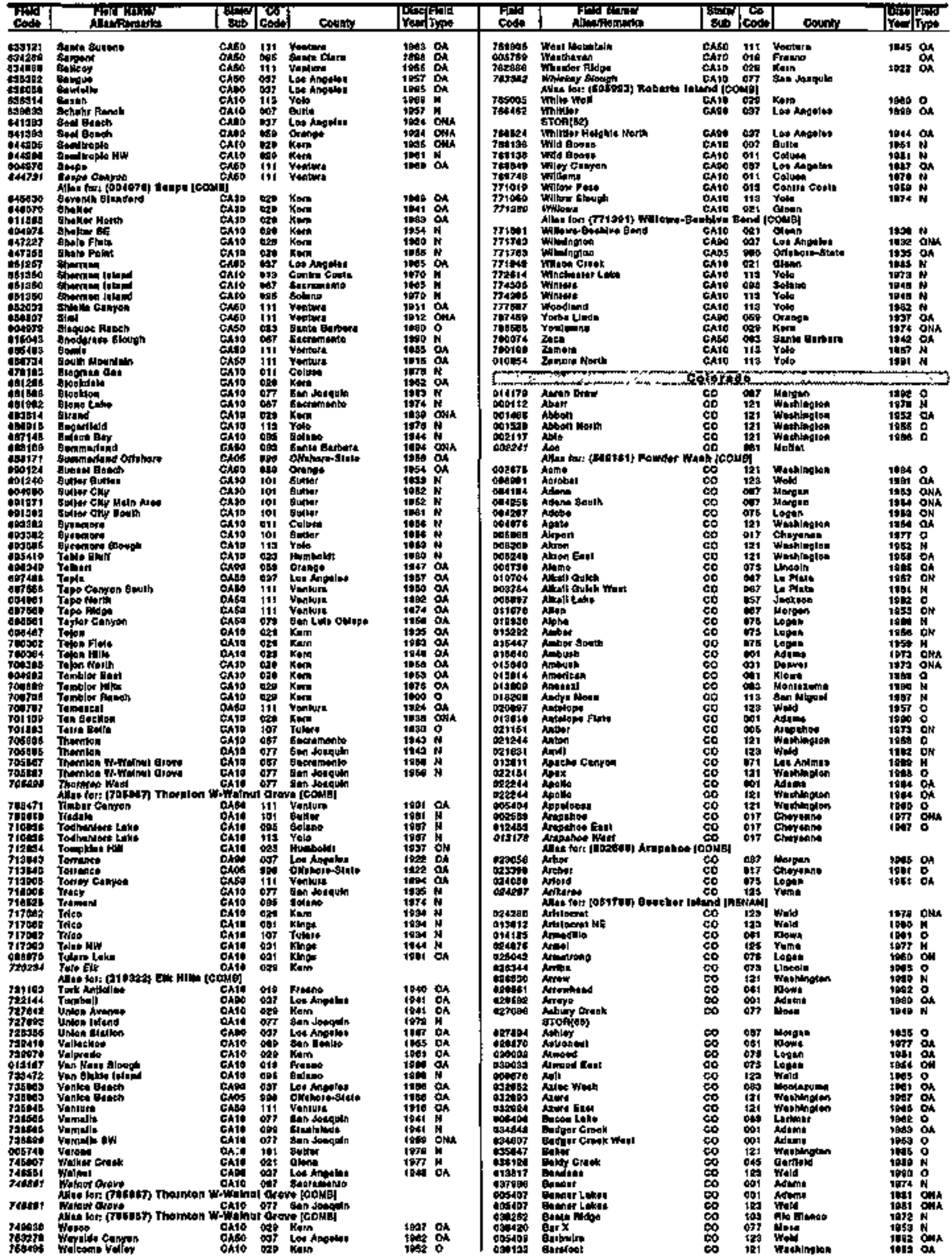




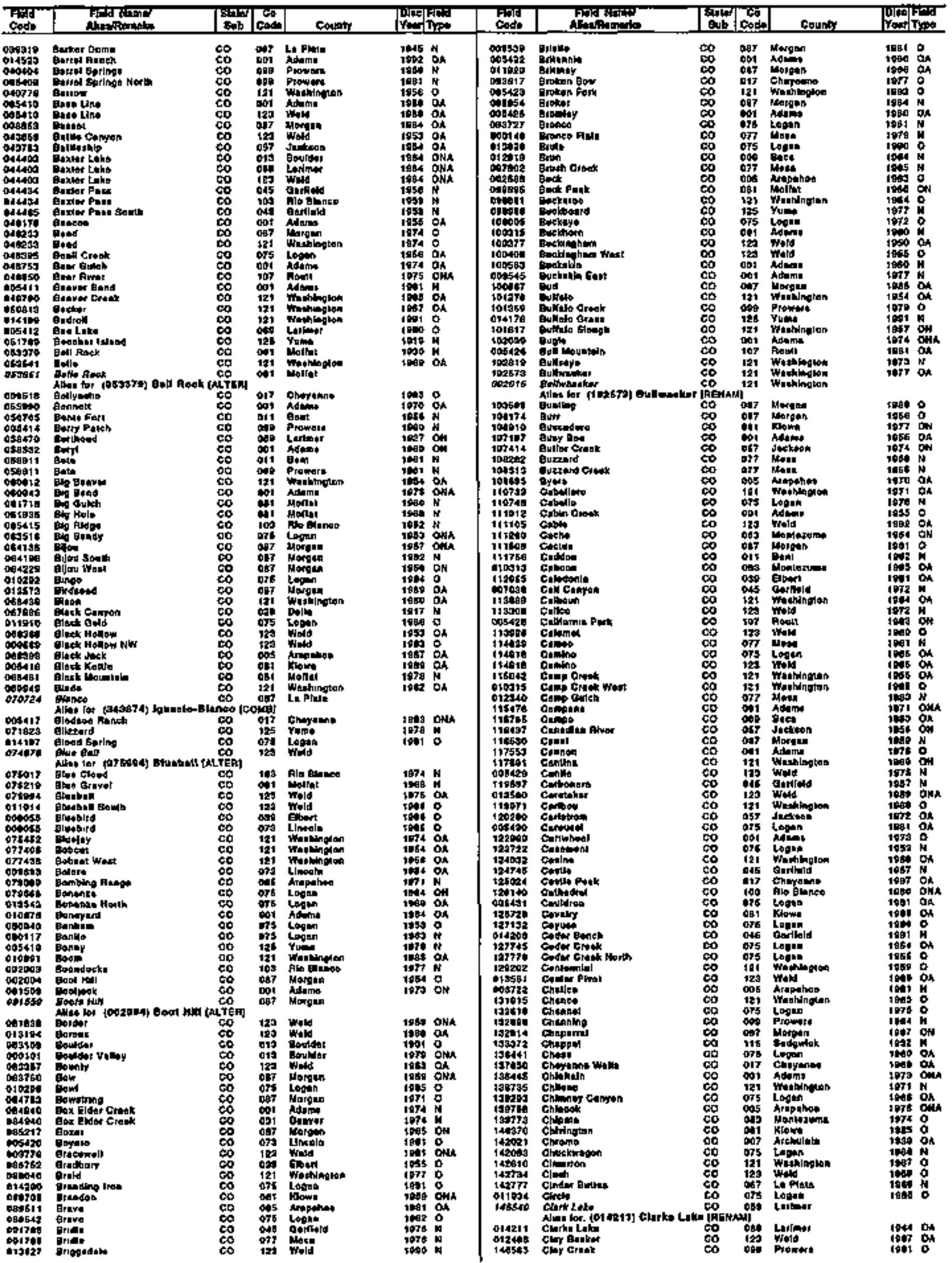


COLOAADO

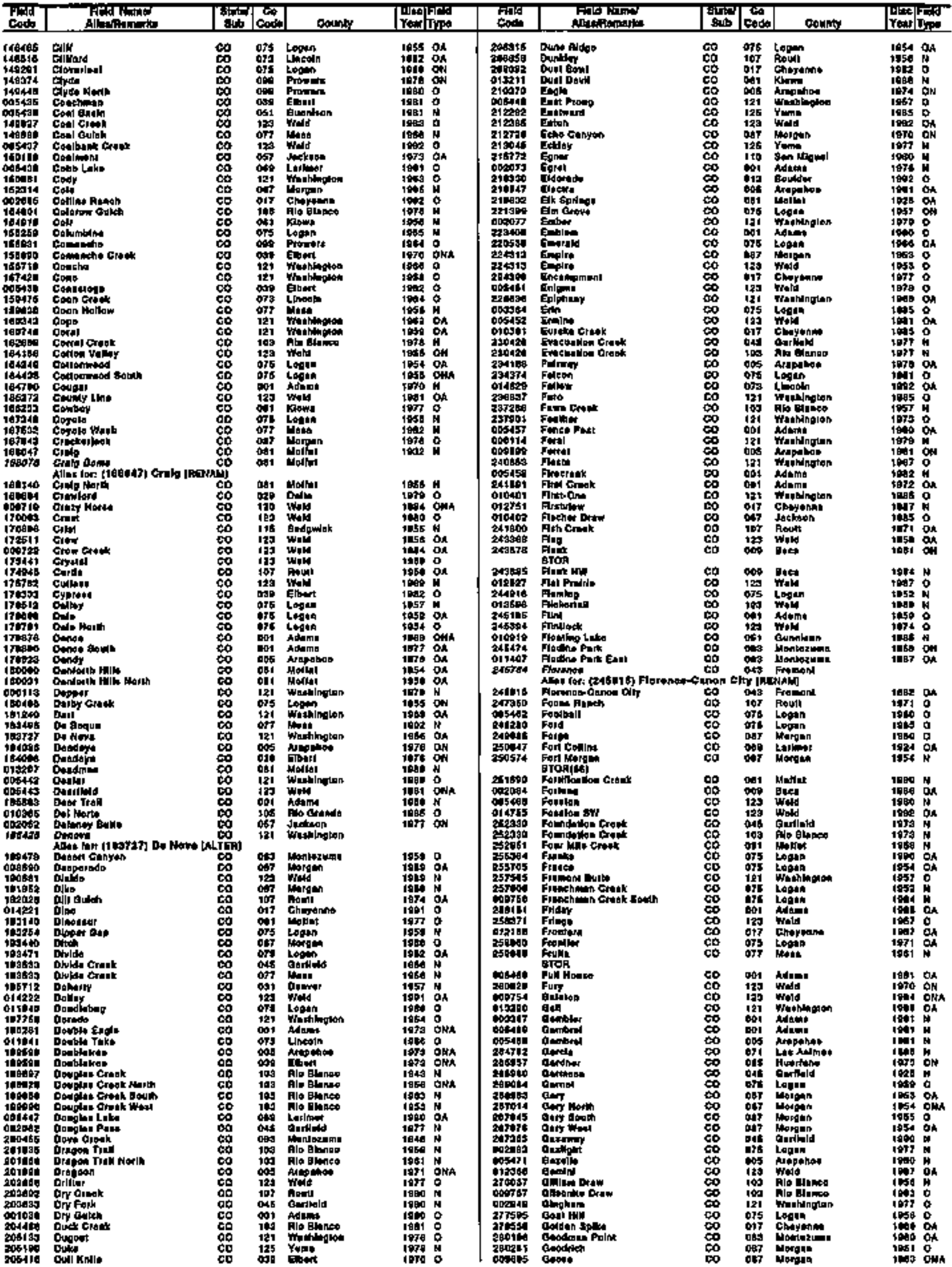

Energy tnformation Adminlstrekionioll and Gas Fleld Cade Master Llet 1995 


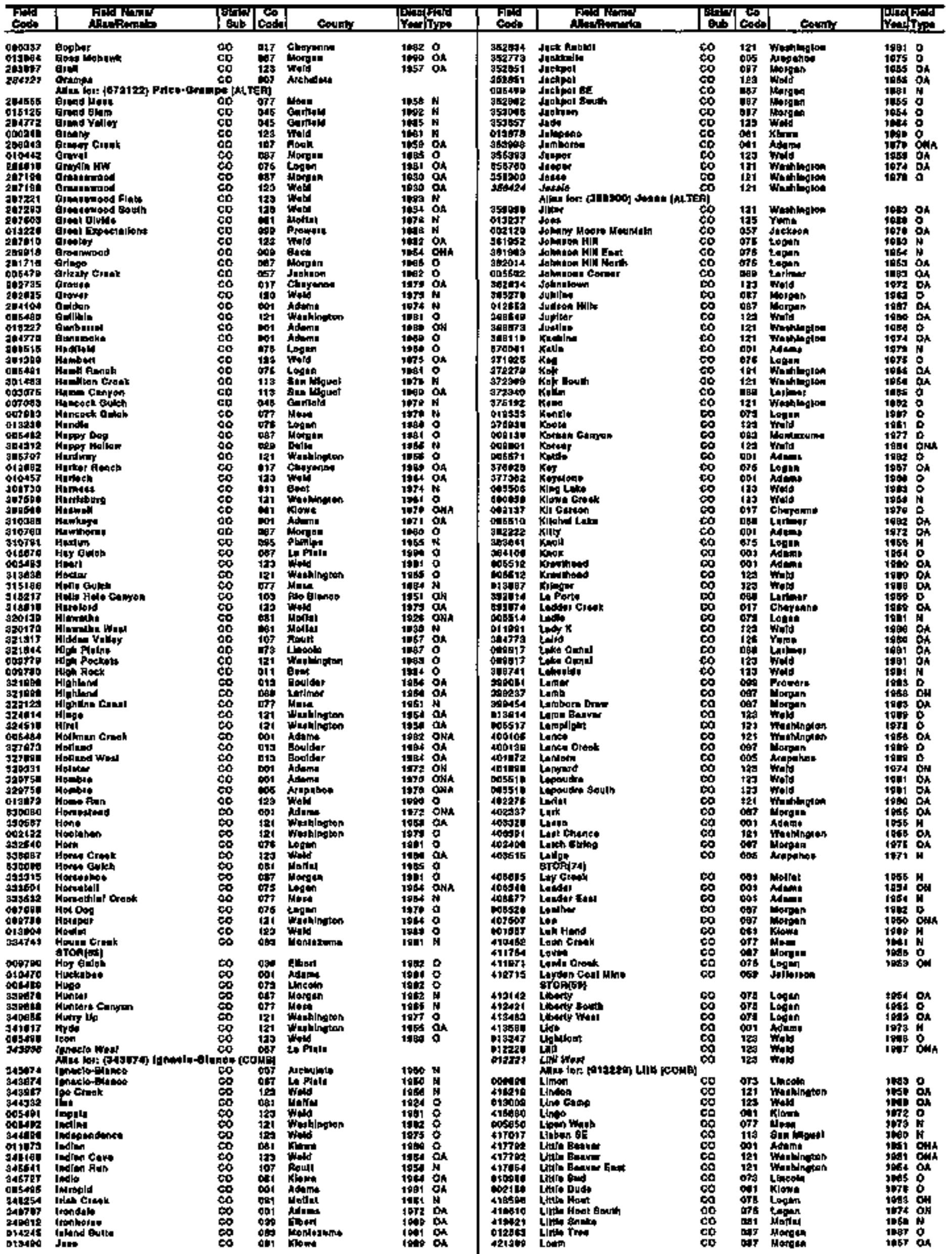


COLORADo

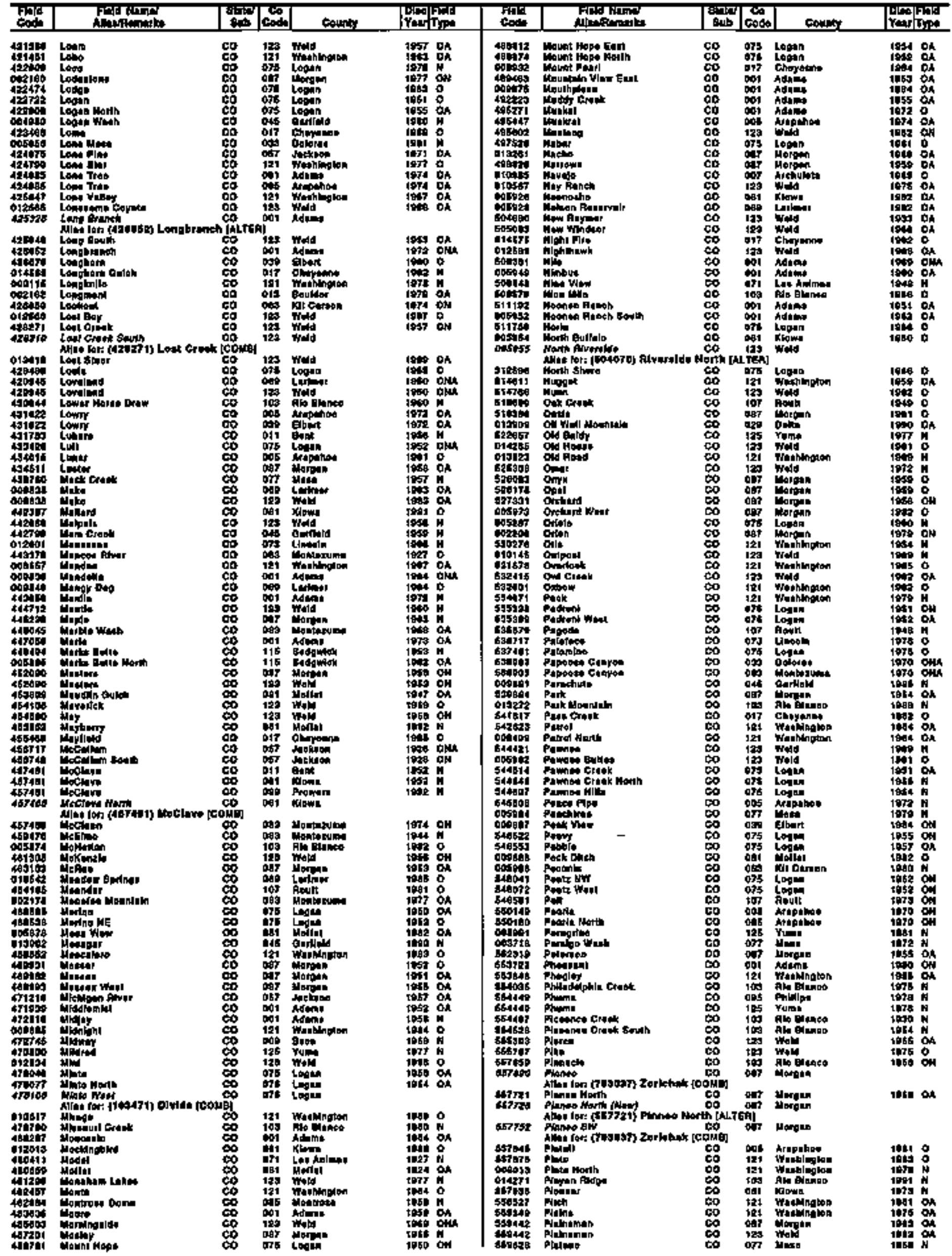




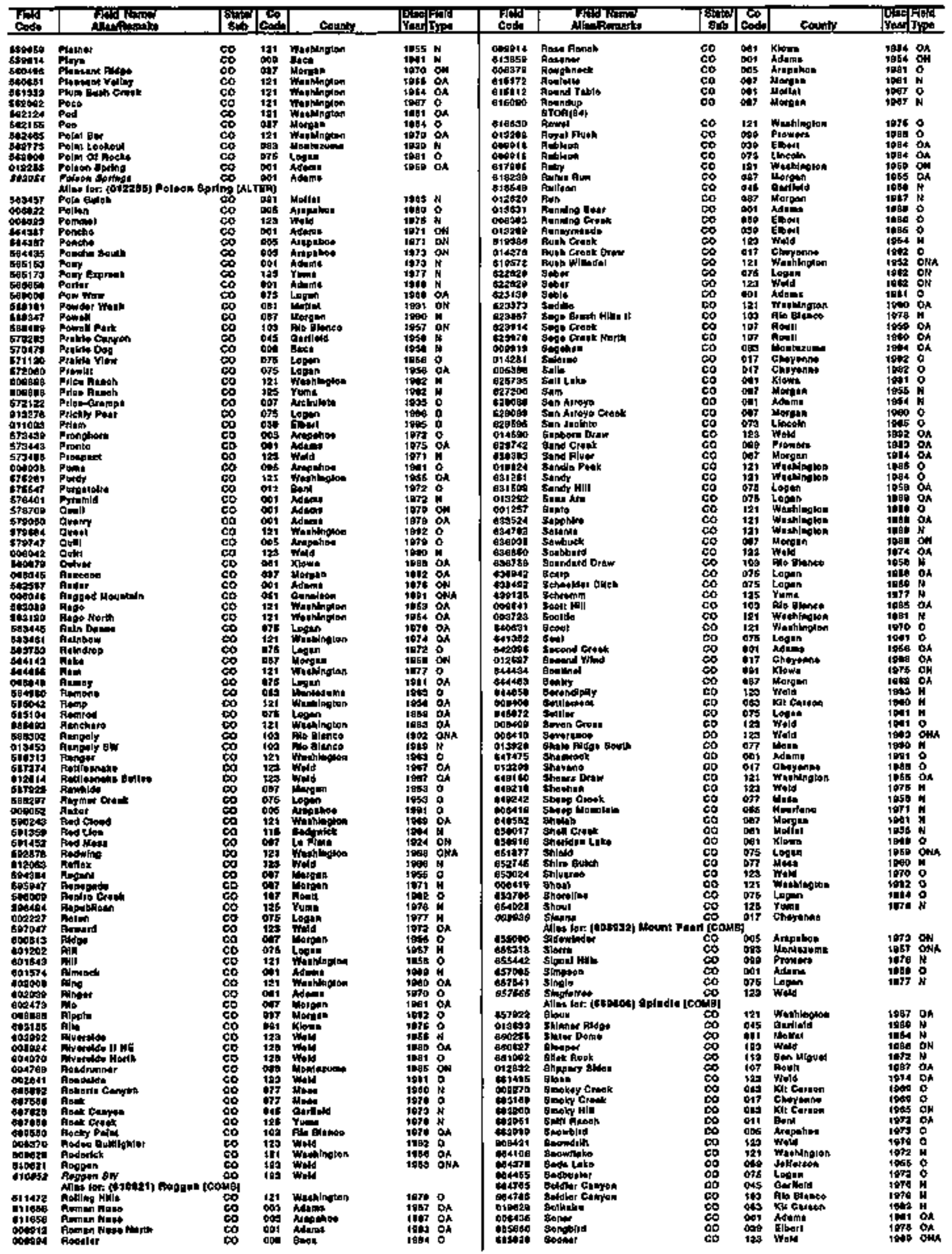


Colorado

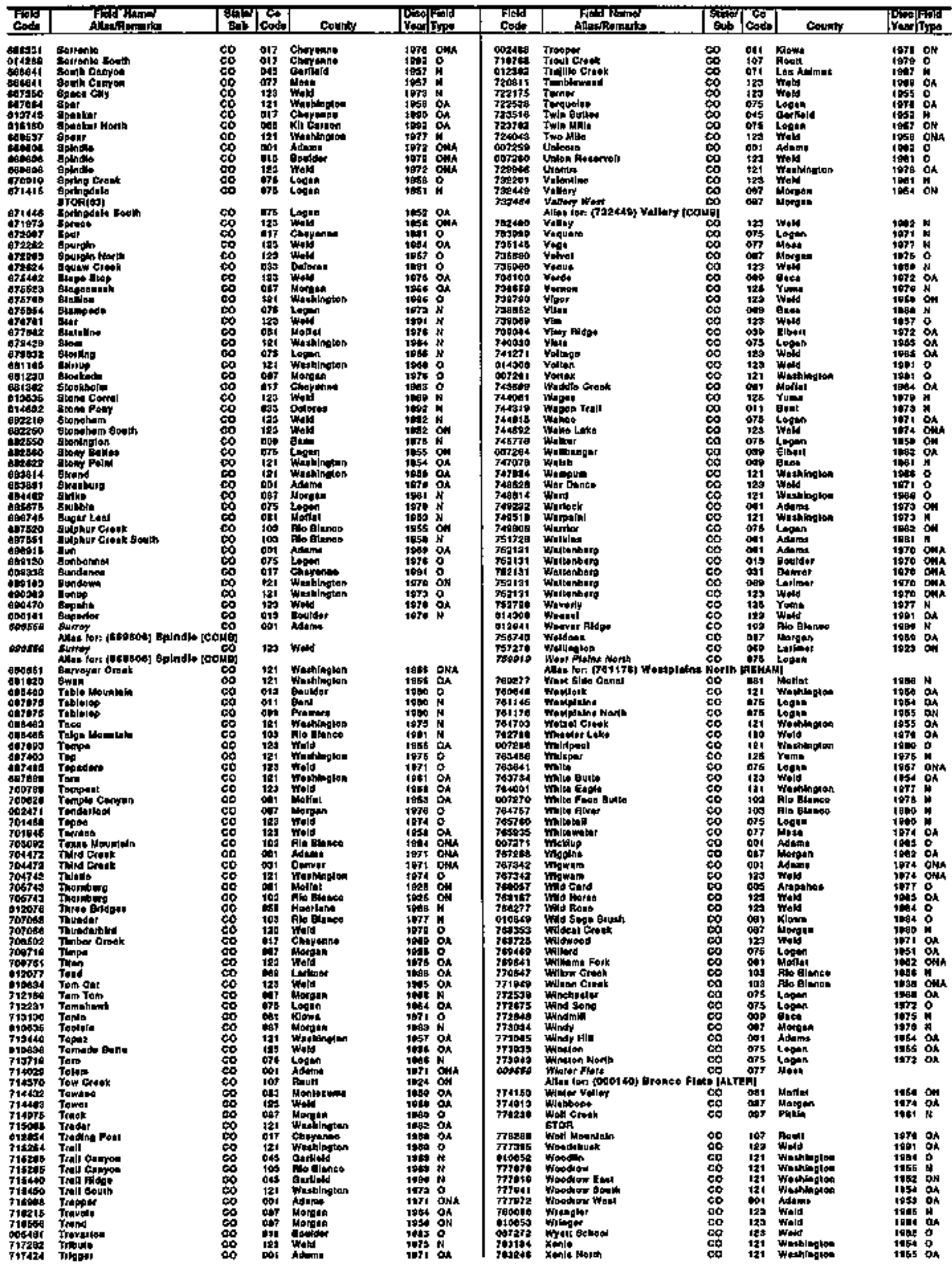




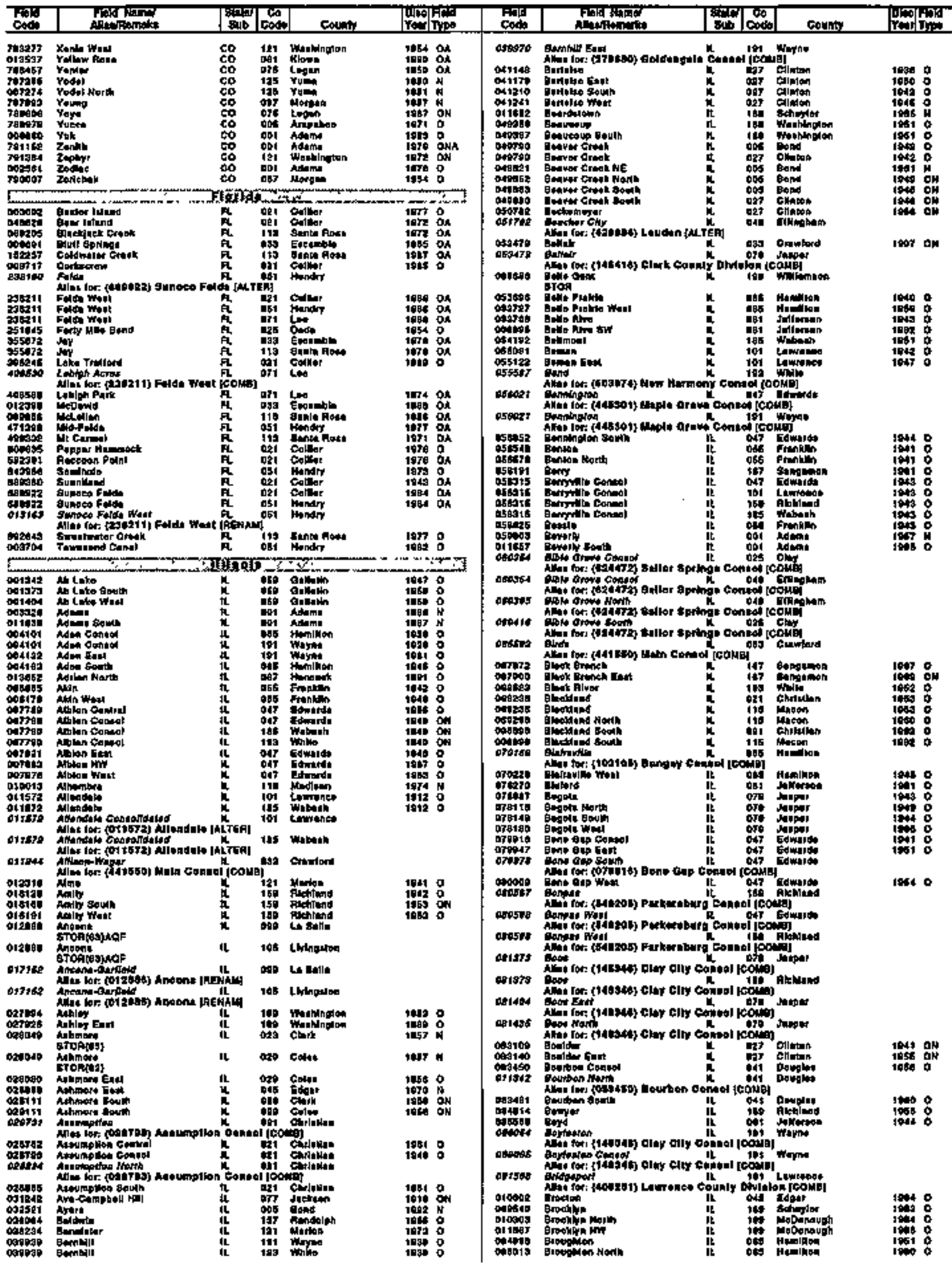




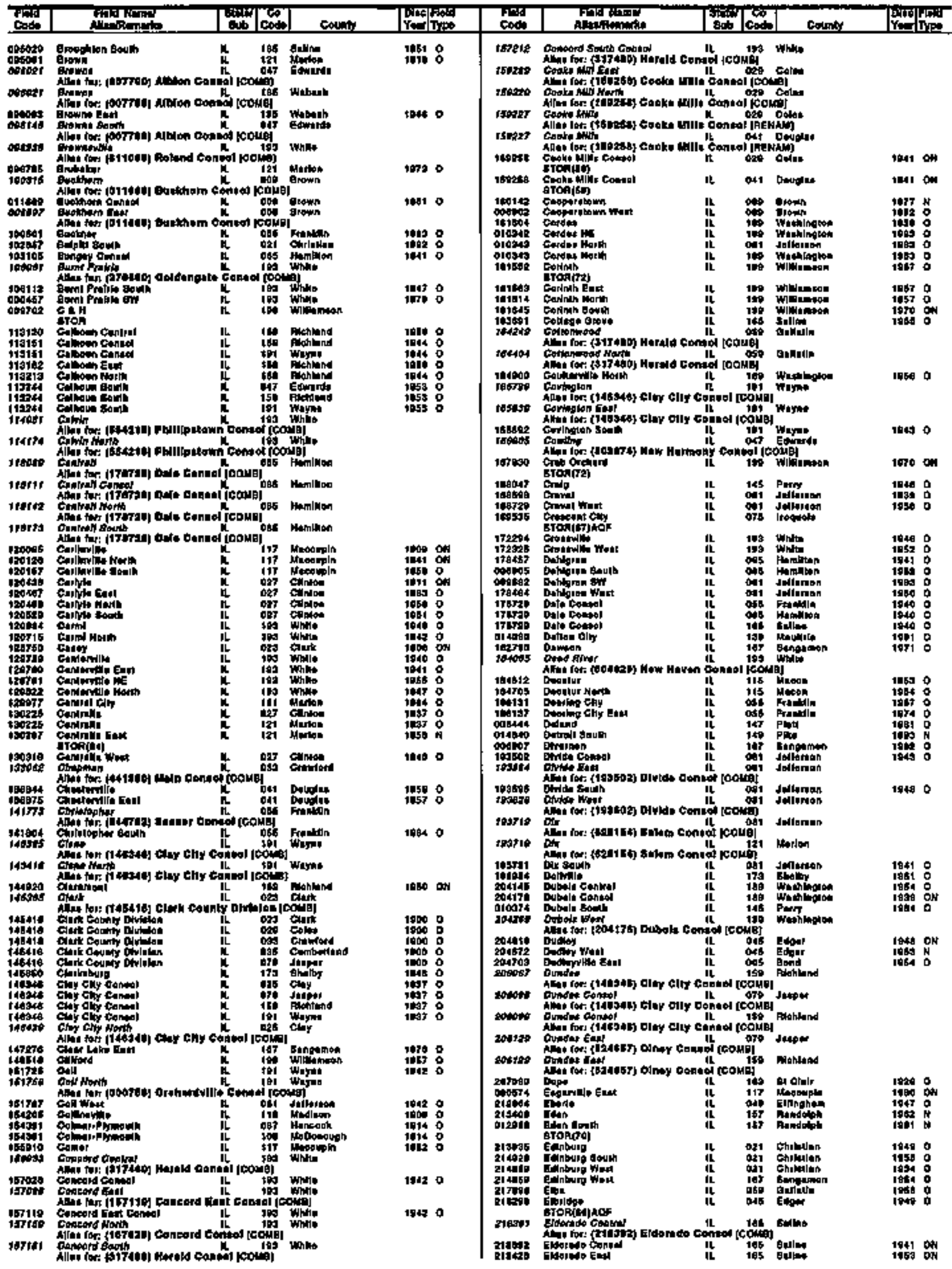




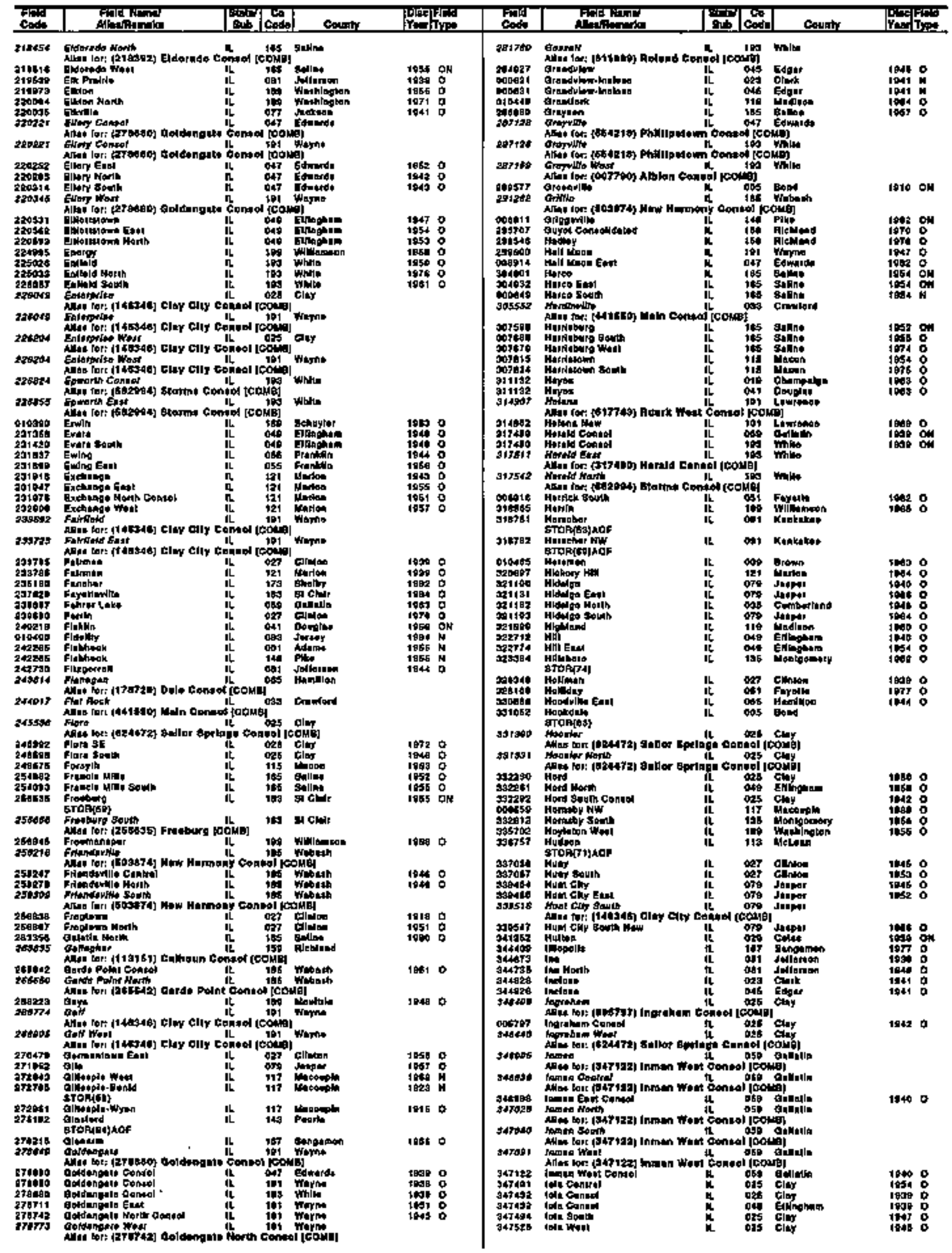


ILLINOIS

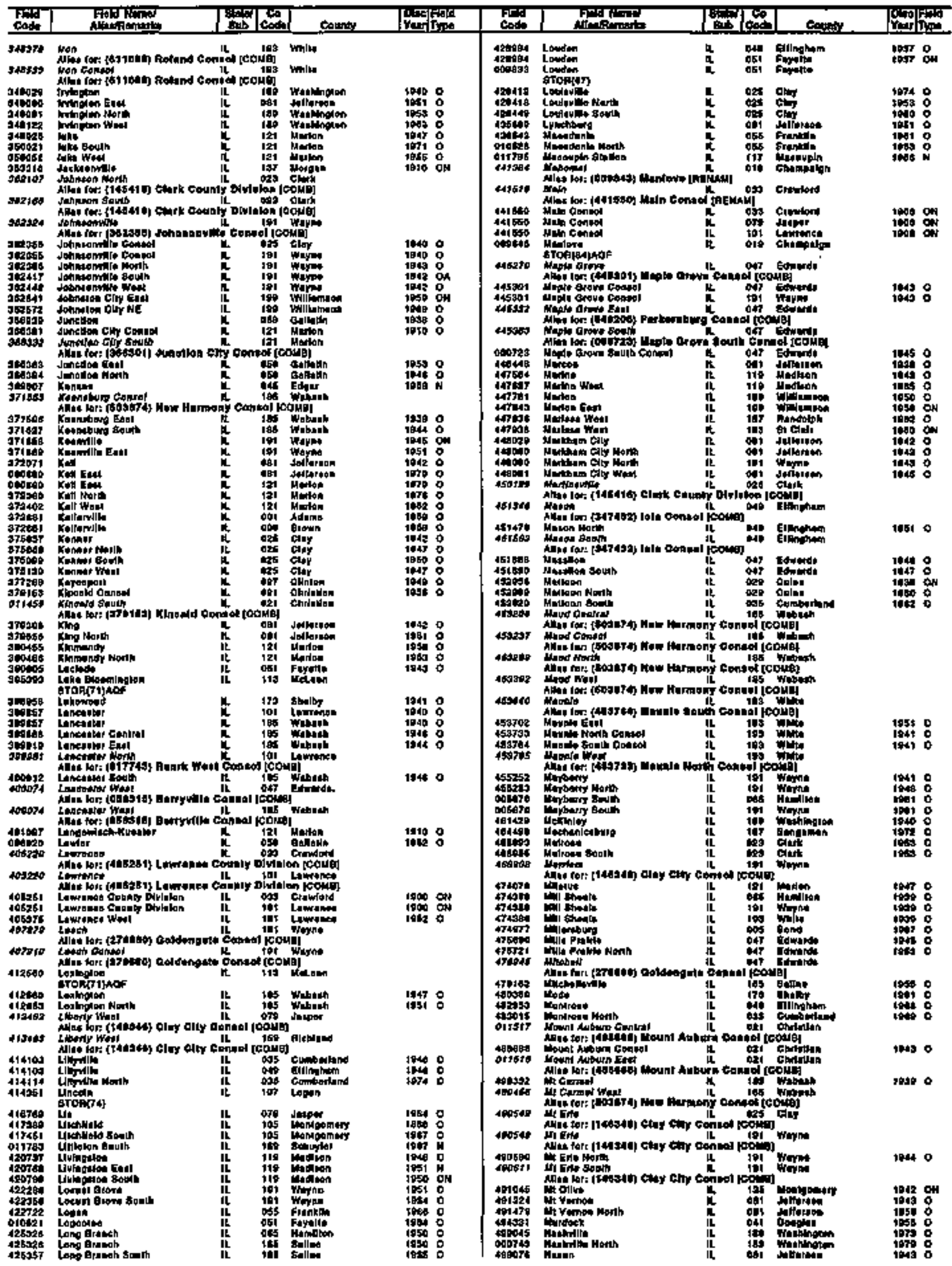




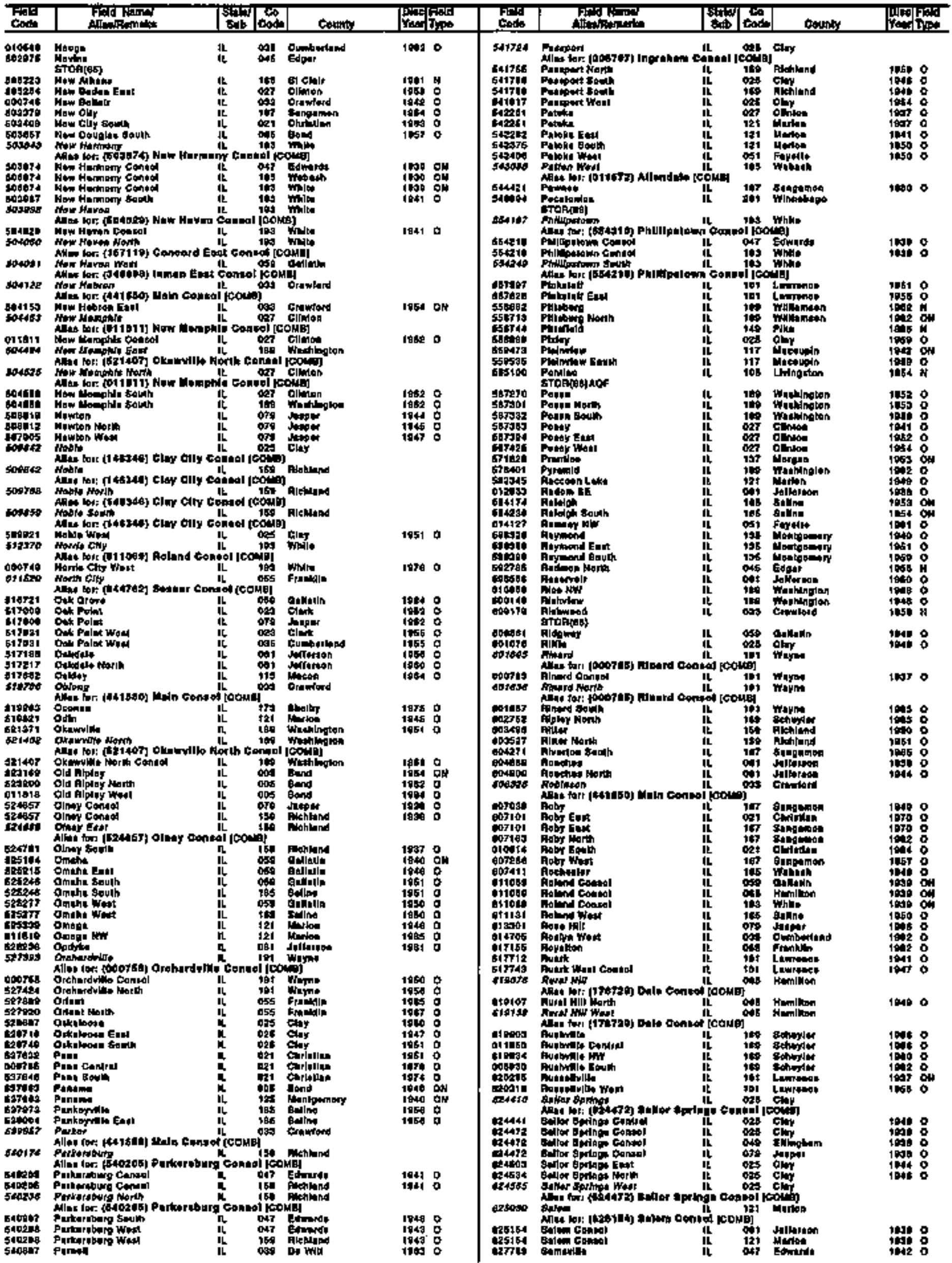


ILLINOIS

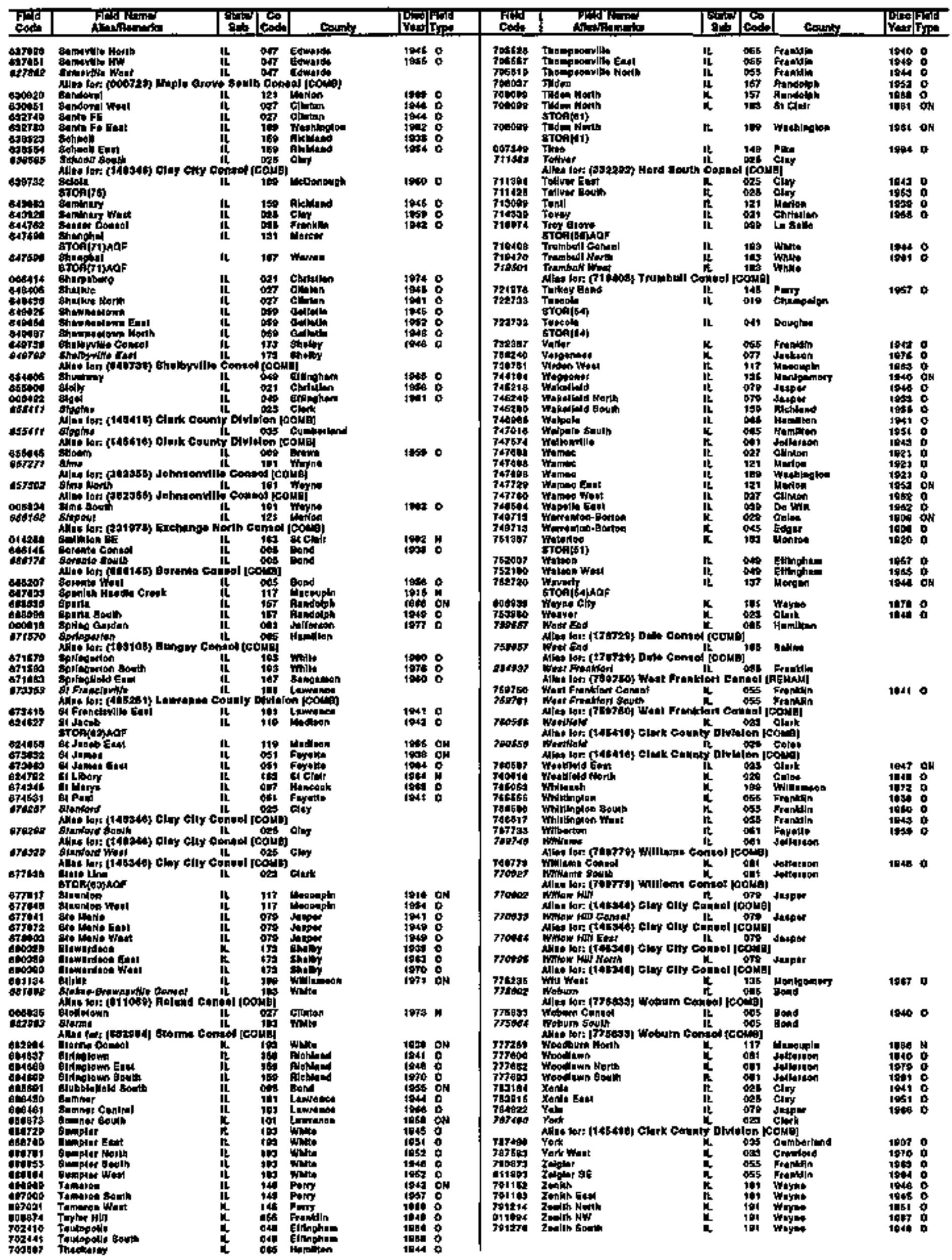


INDIANA

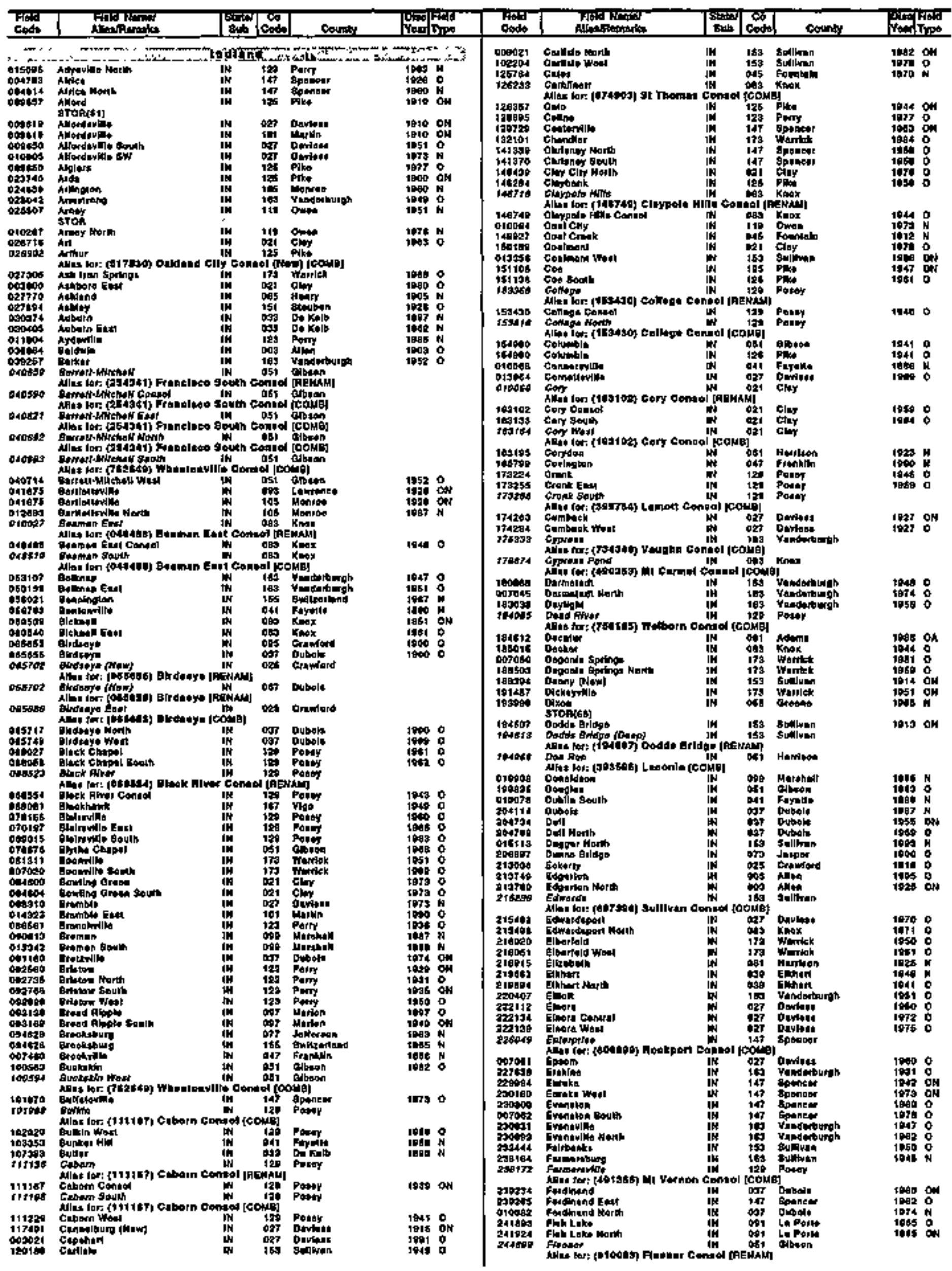


INDIANA

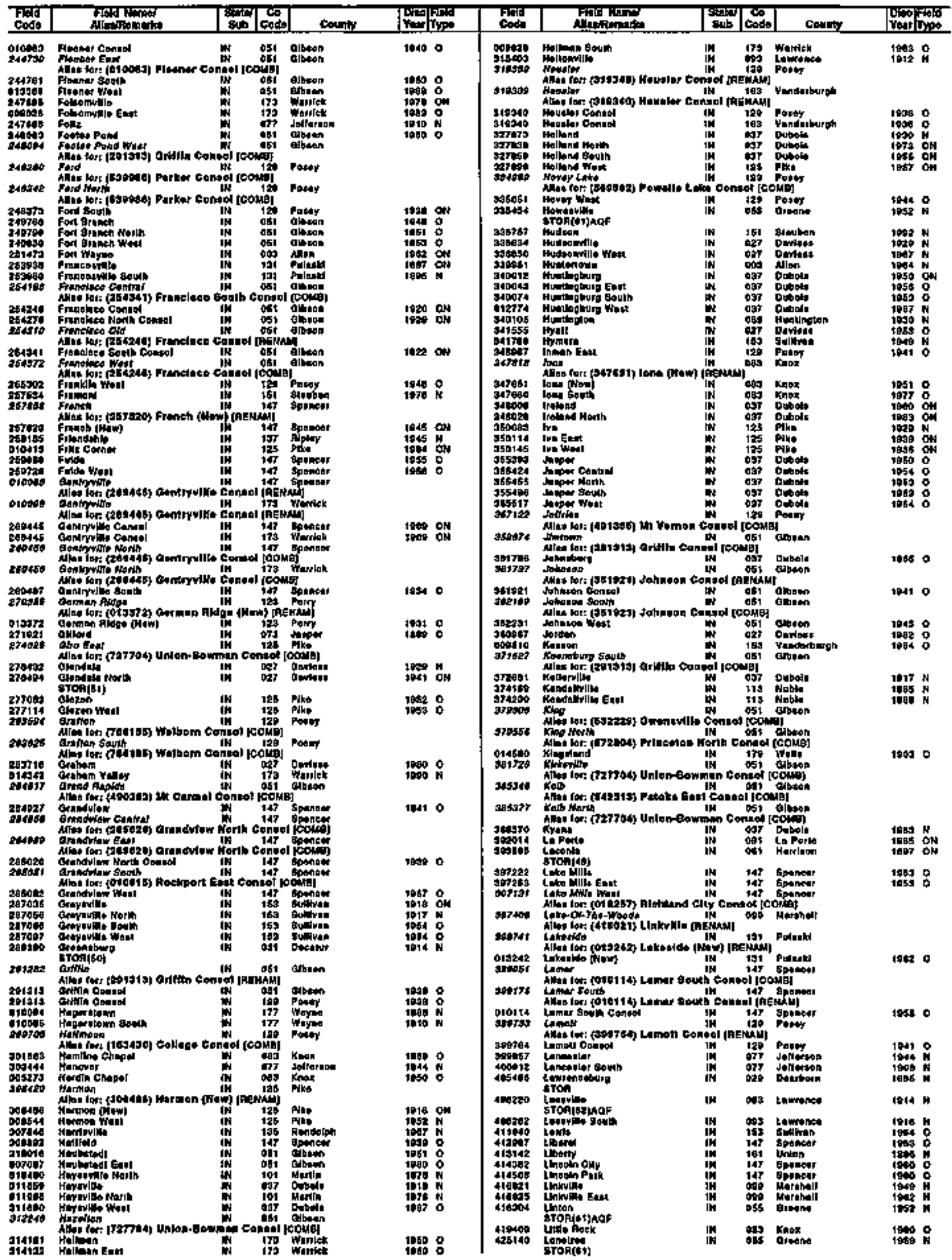


INDIANA

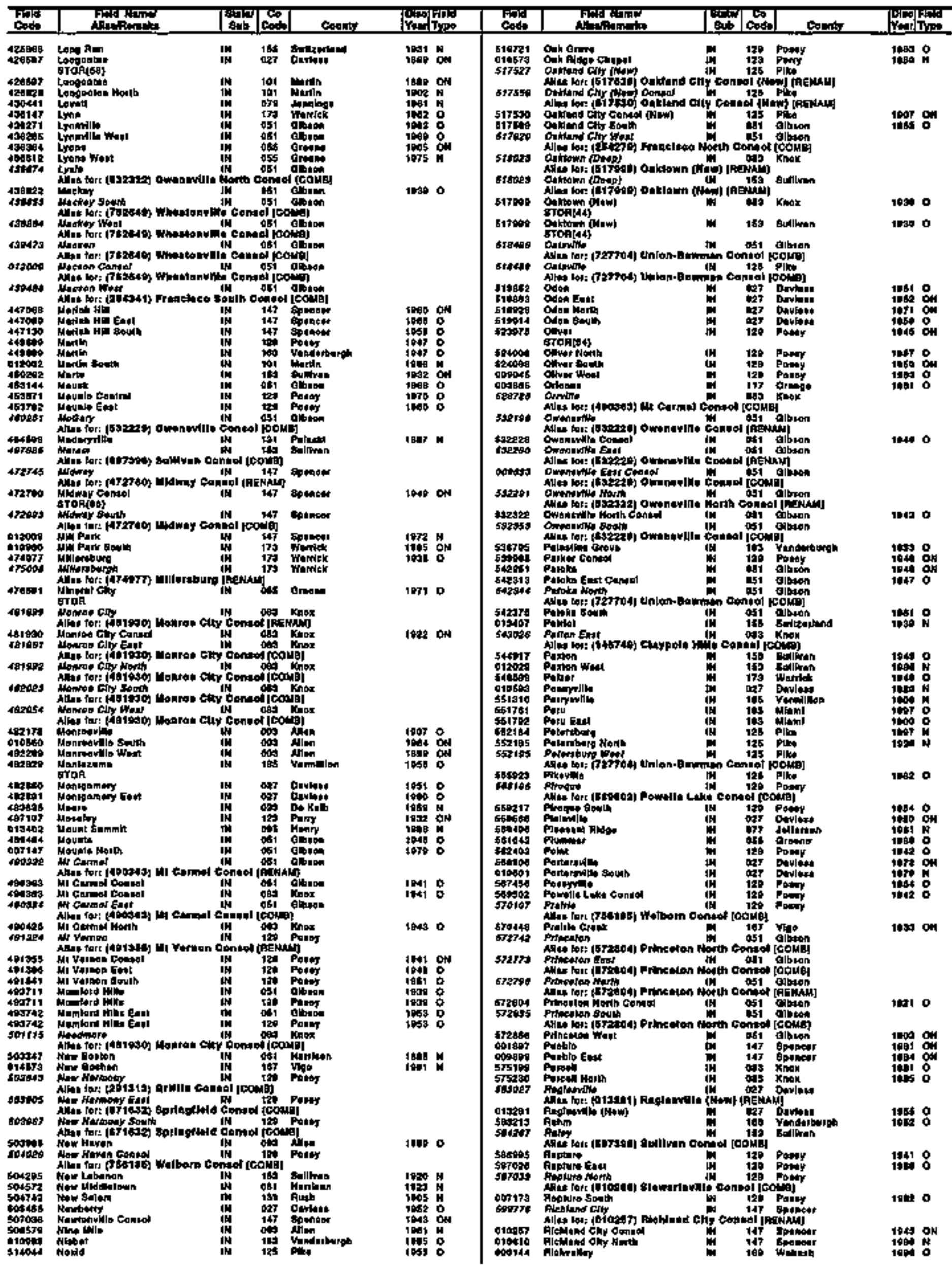




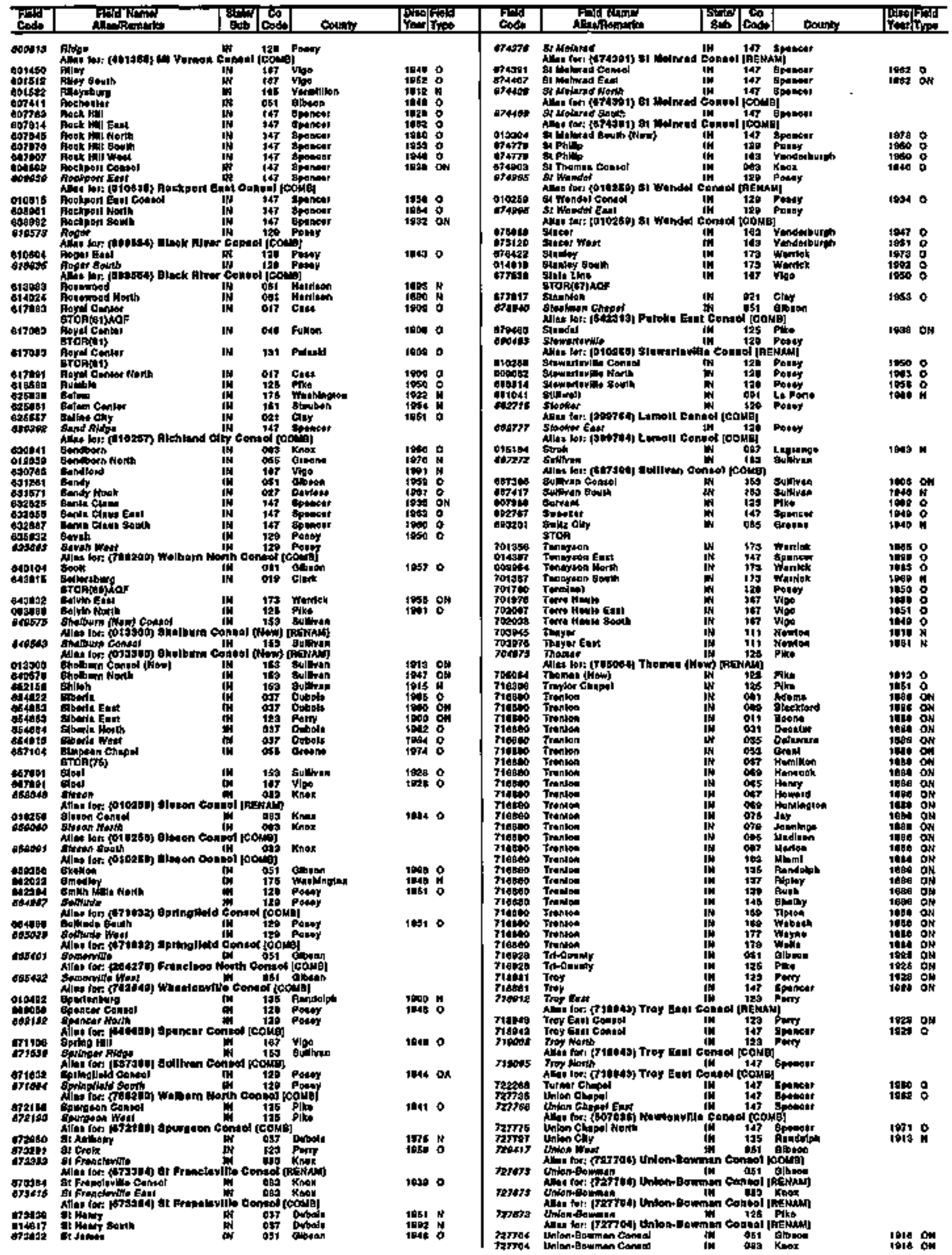


INDIANA

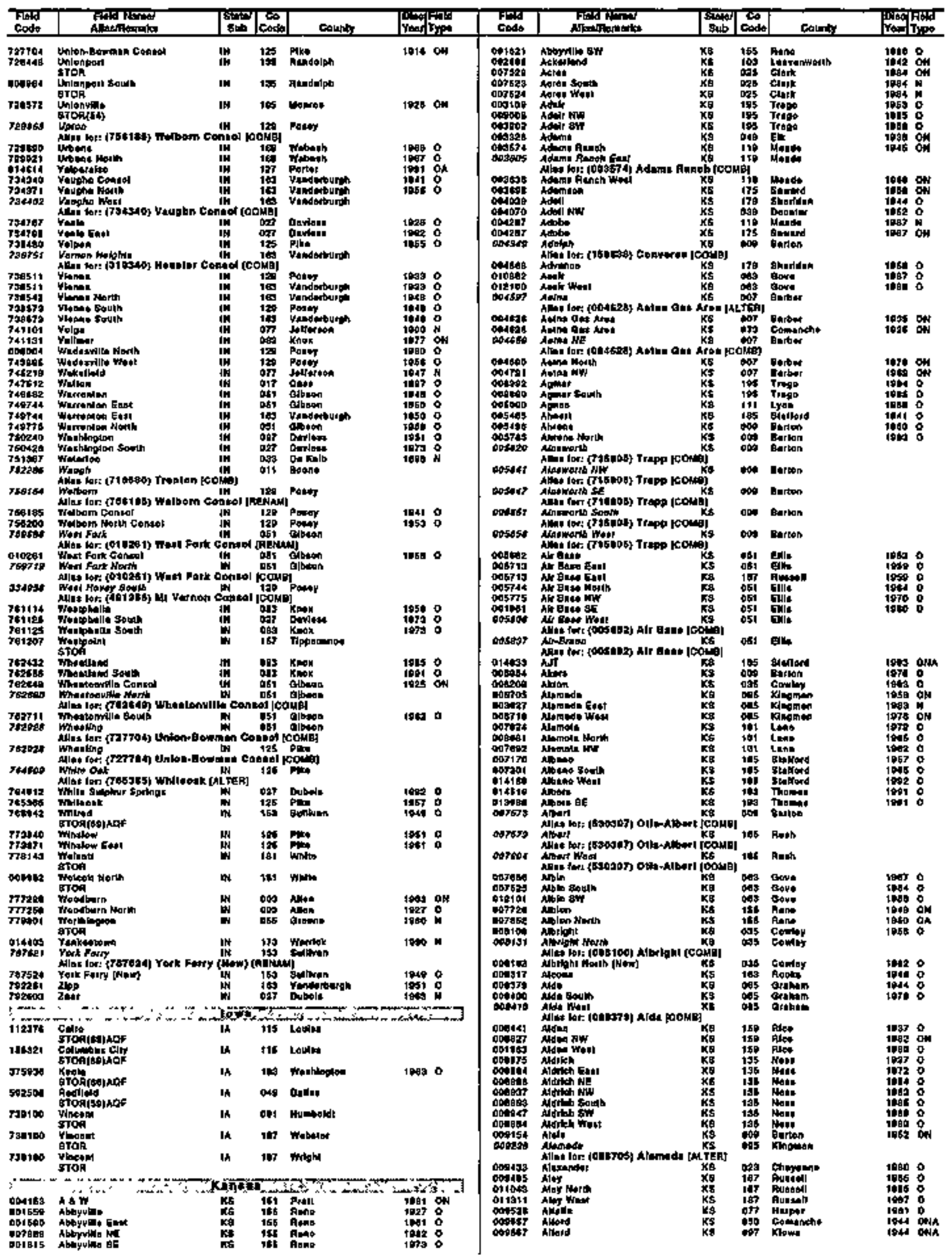


KANSAS

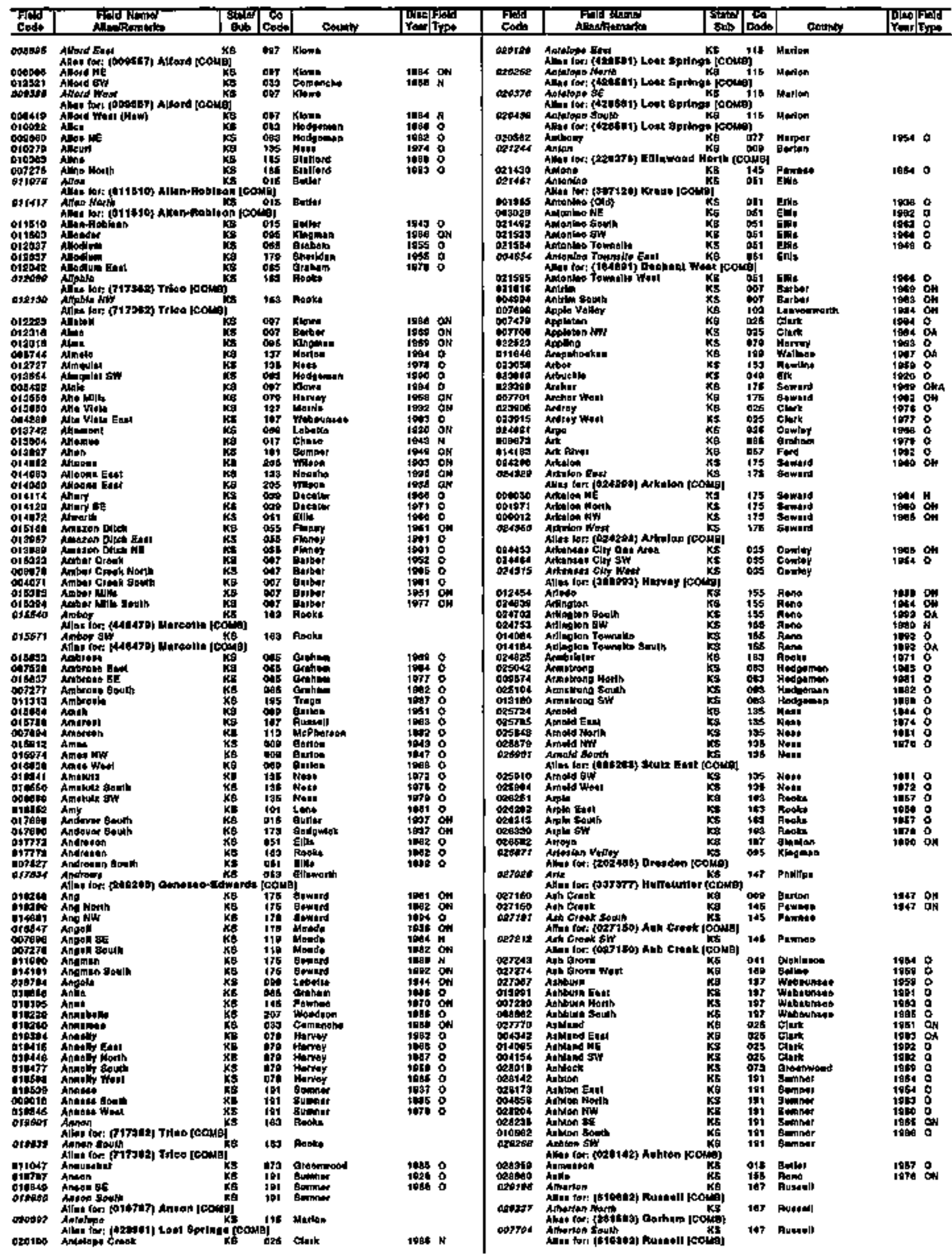




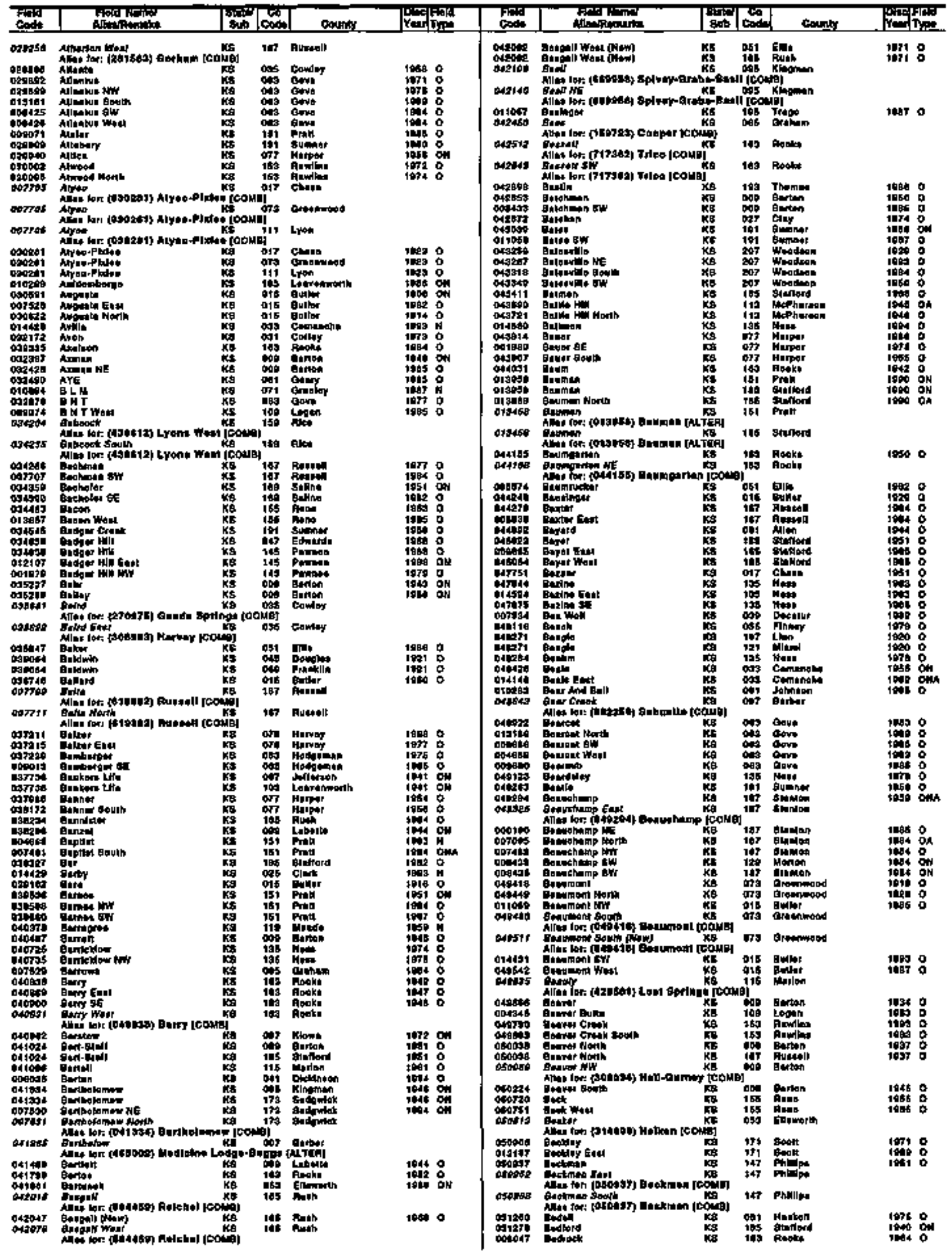


KANSAS

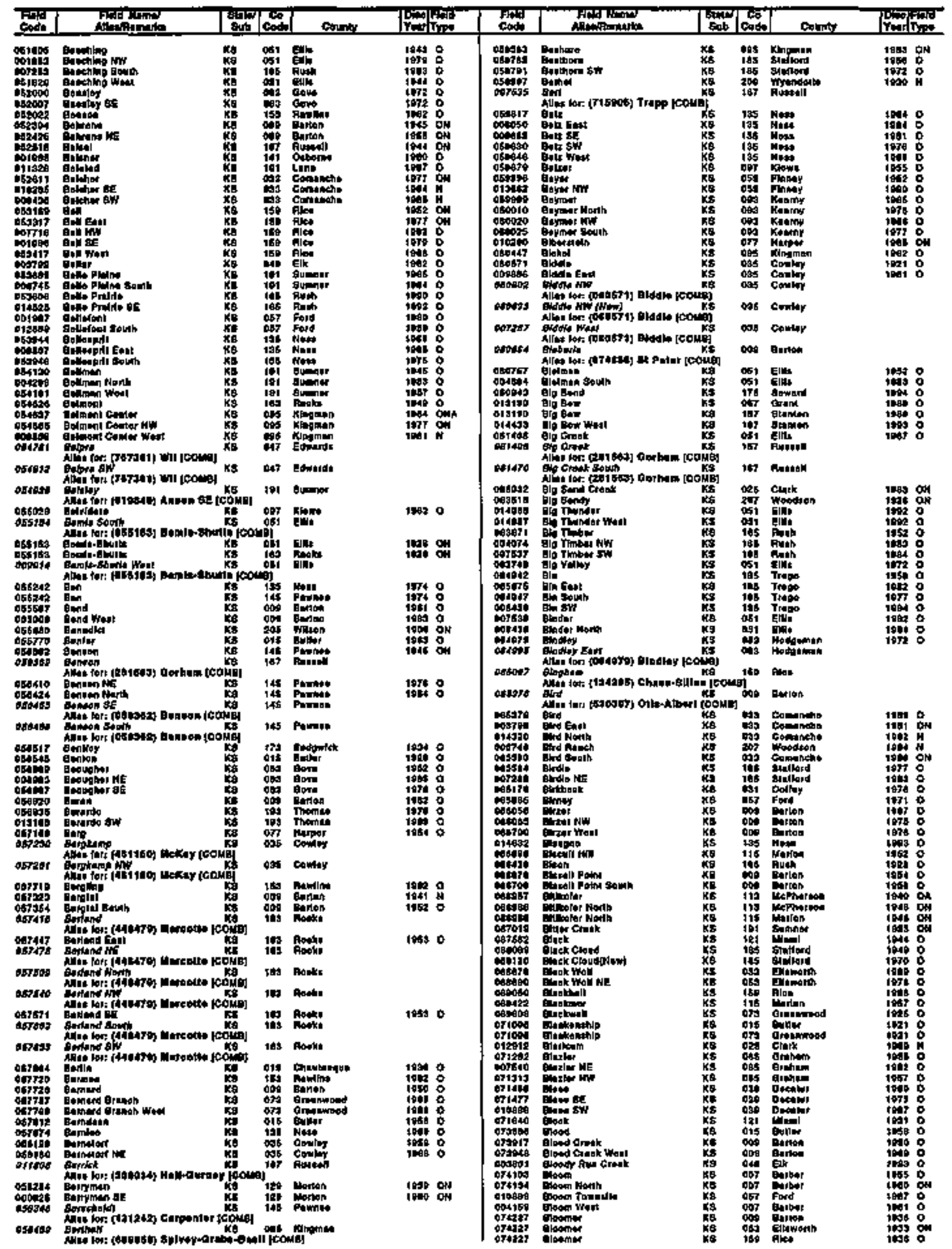




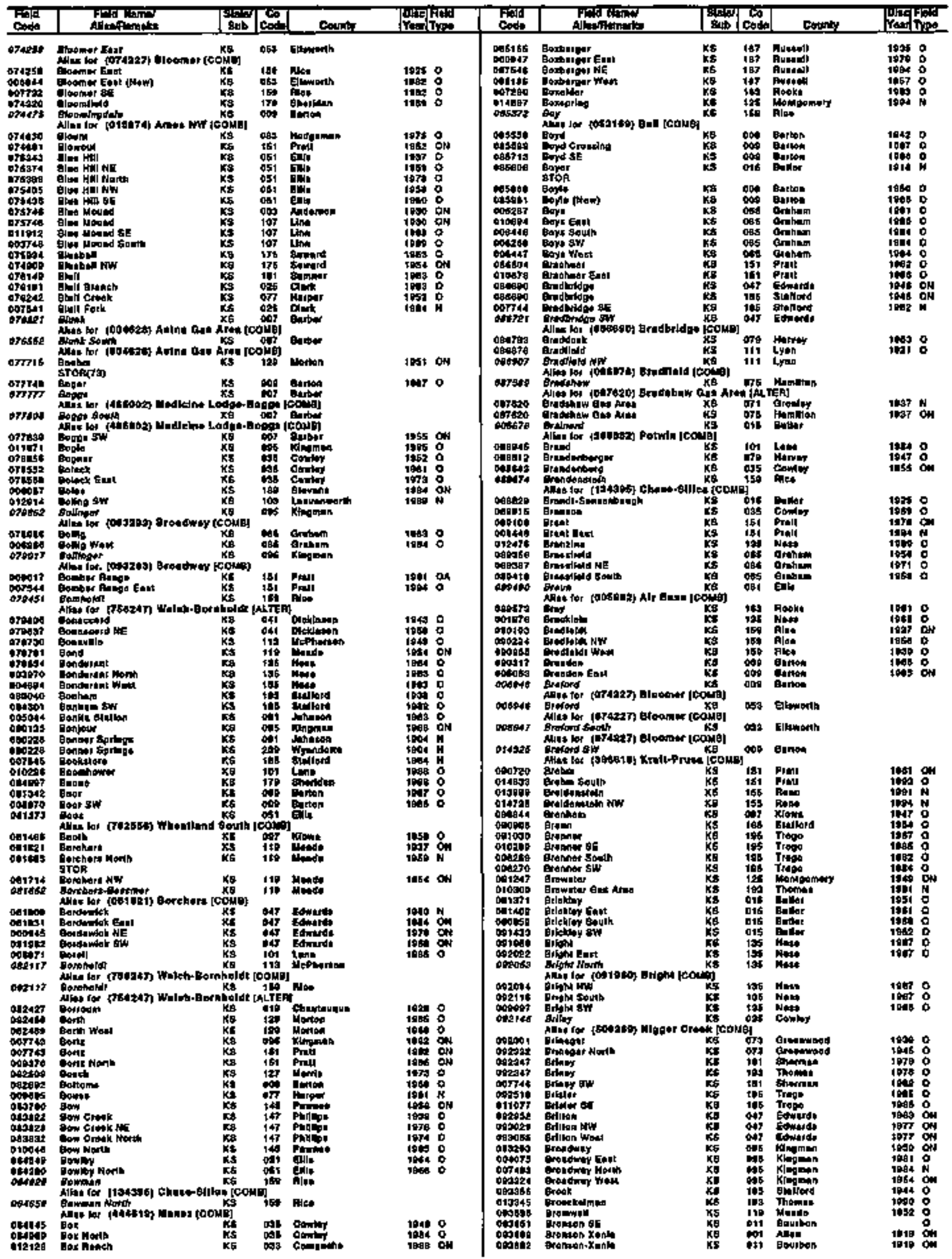


KANSAS

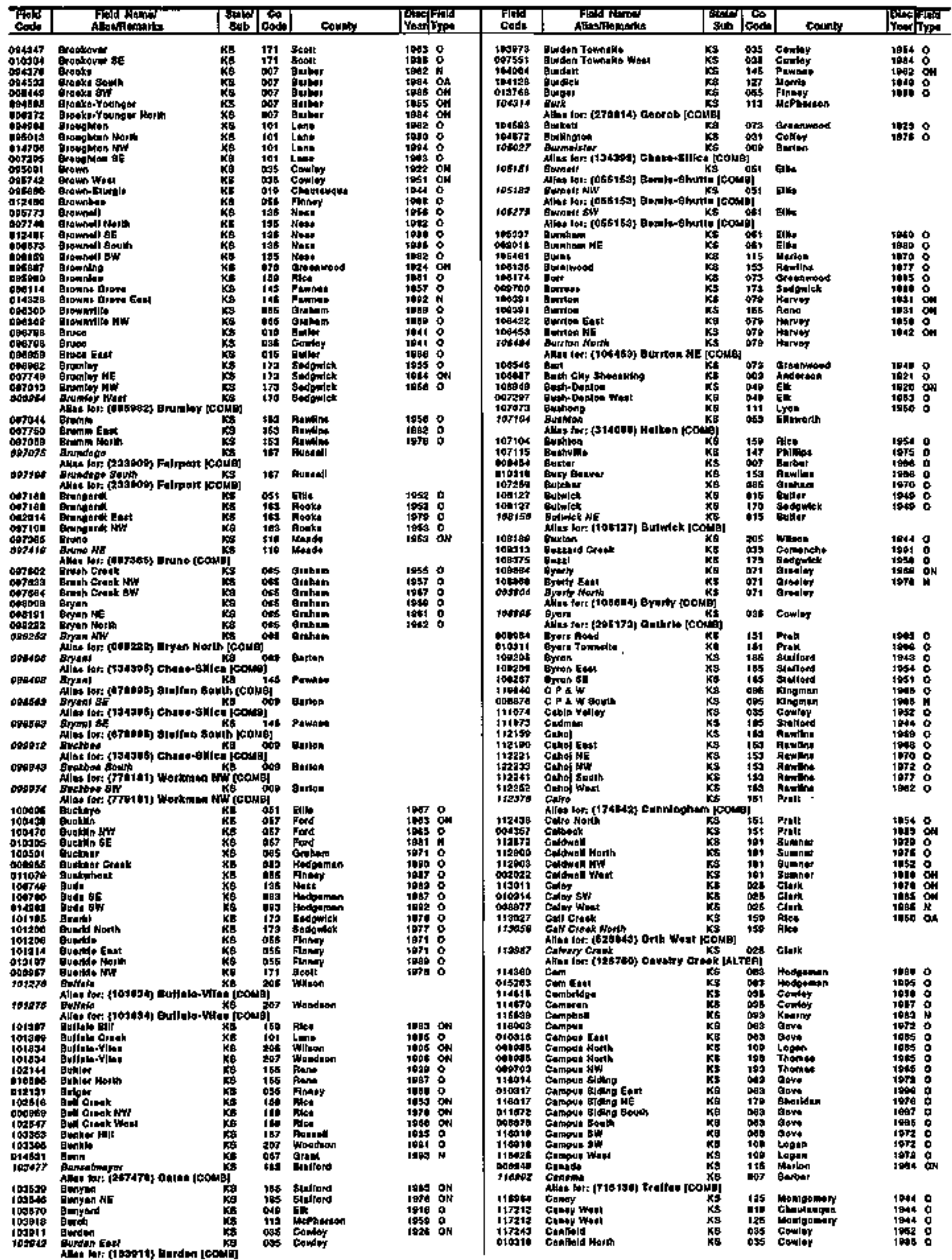




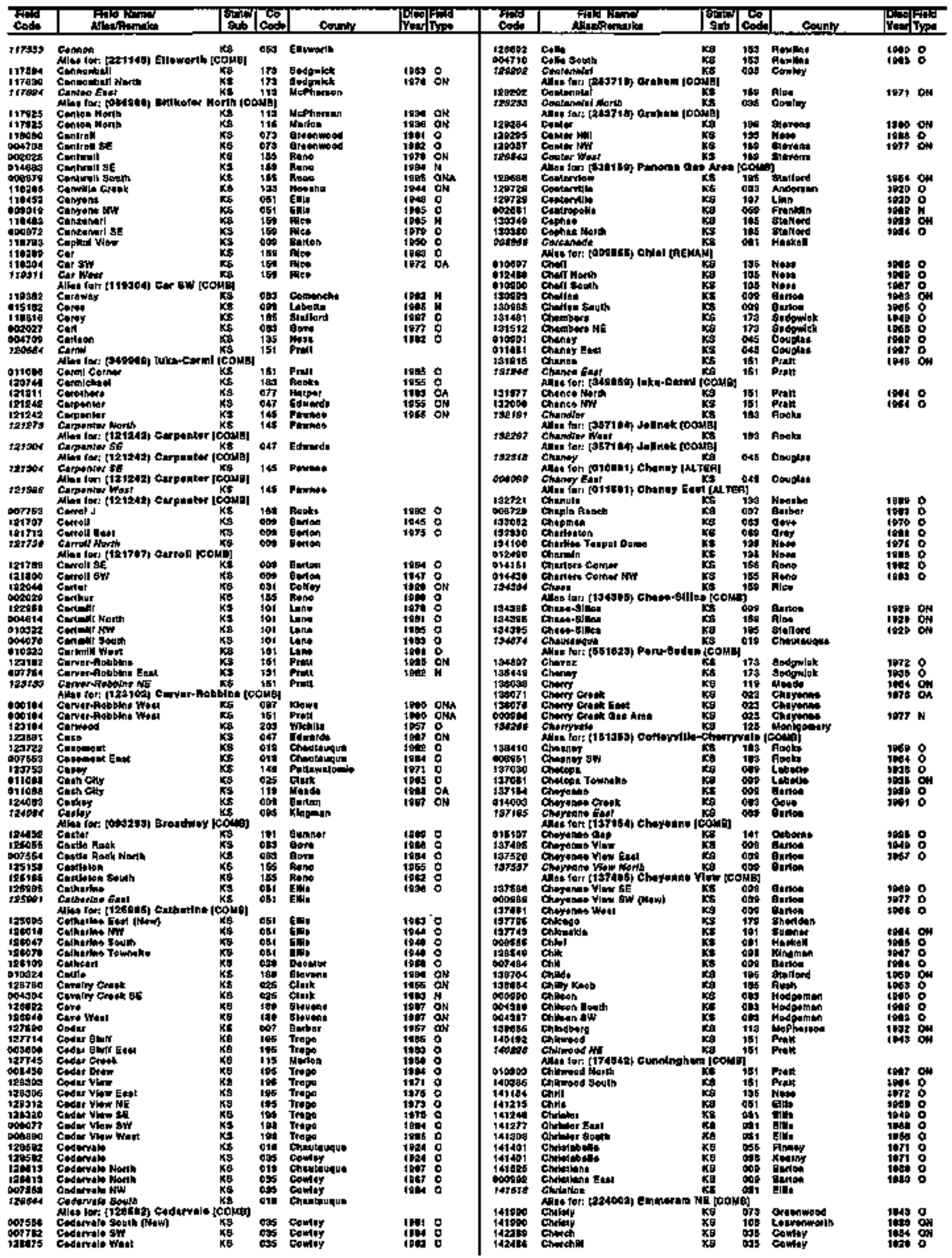


KANSAS

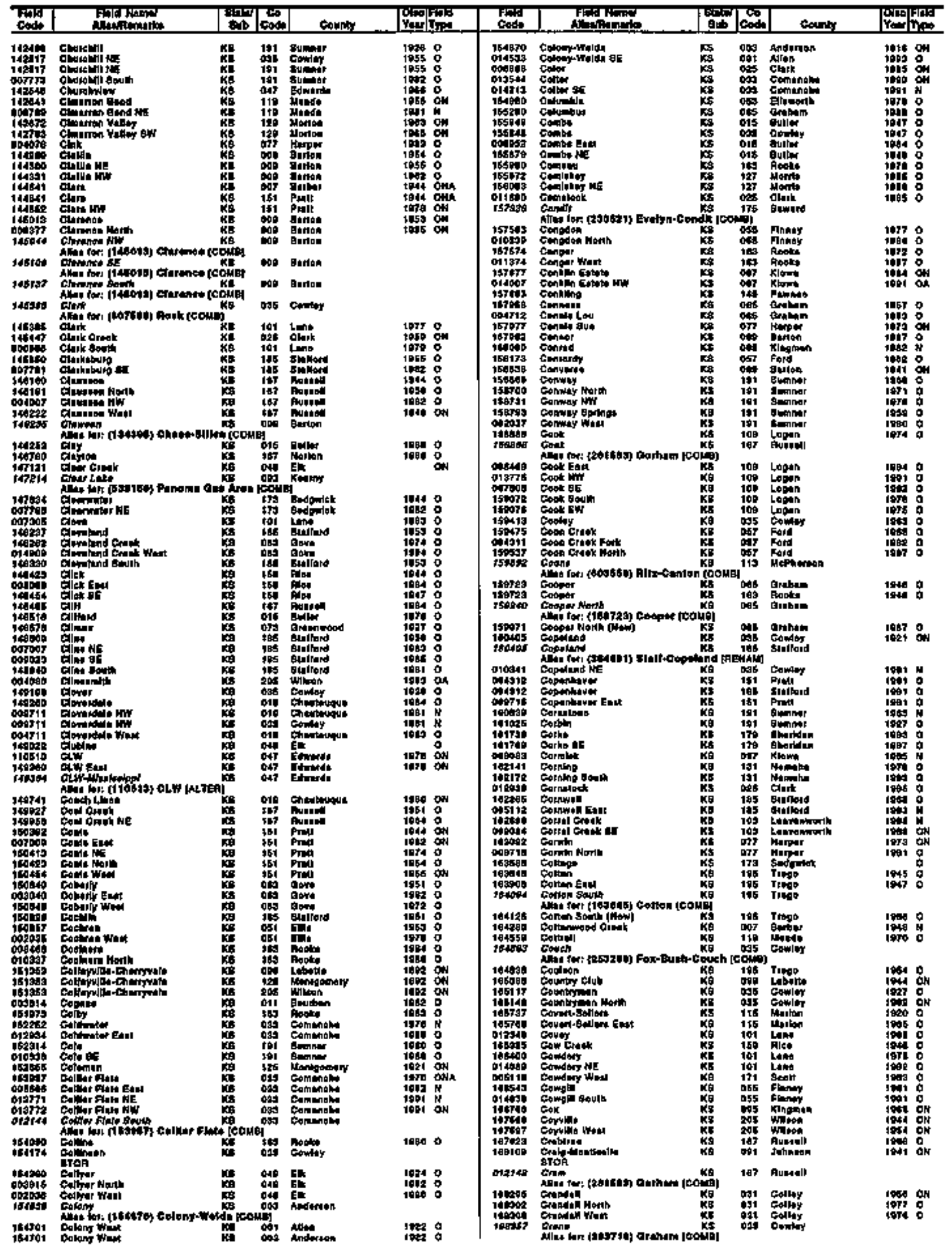


KANSA $\$$

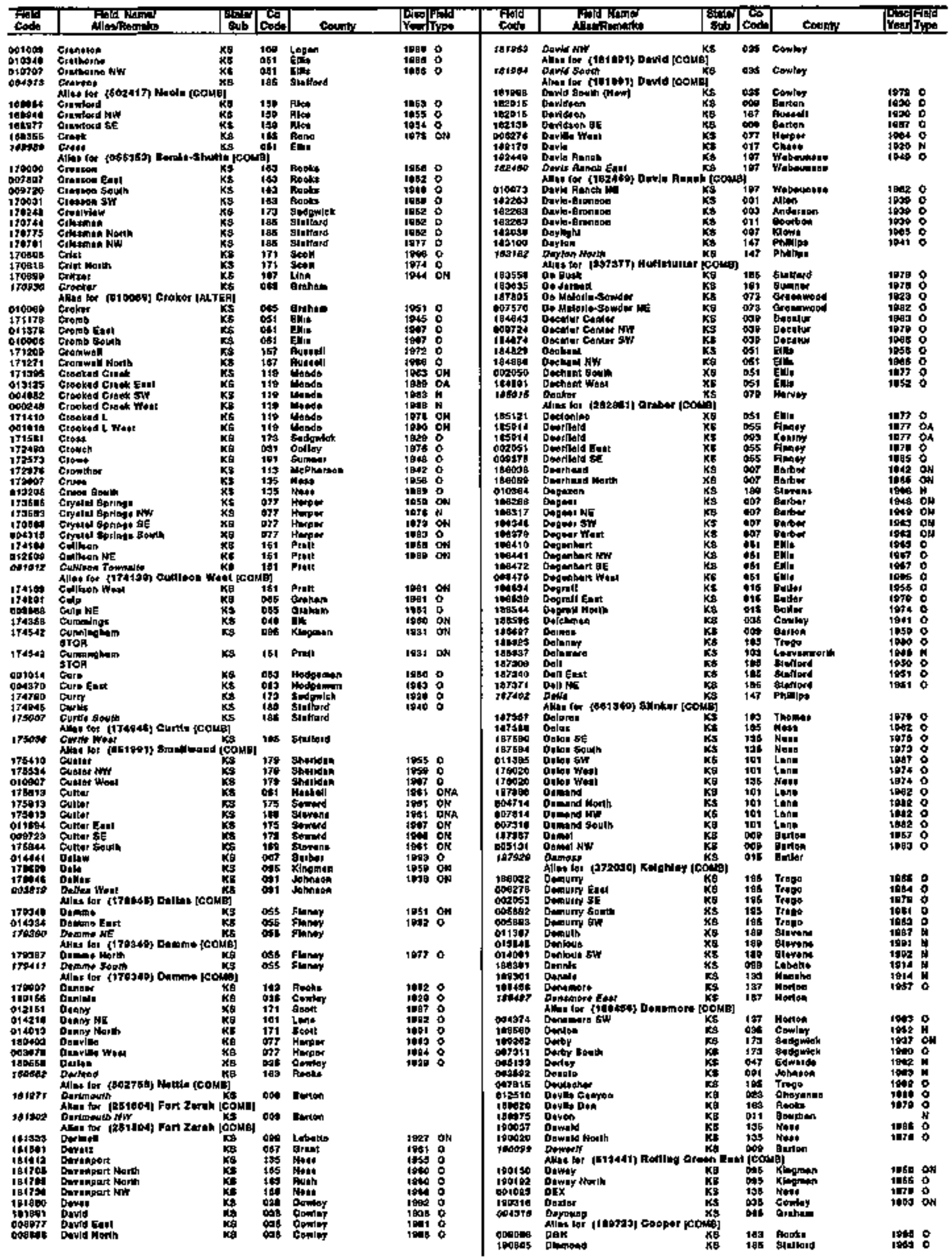


KANSAS

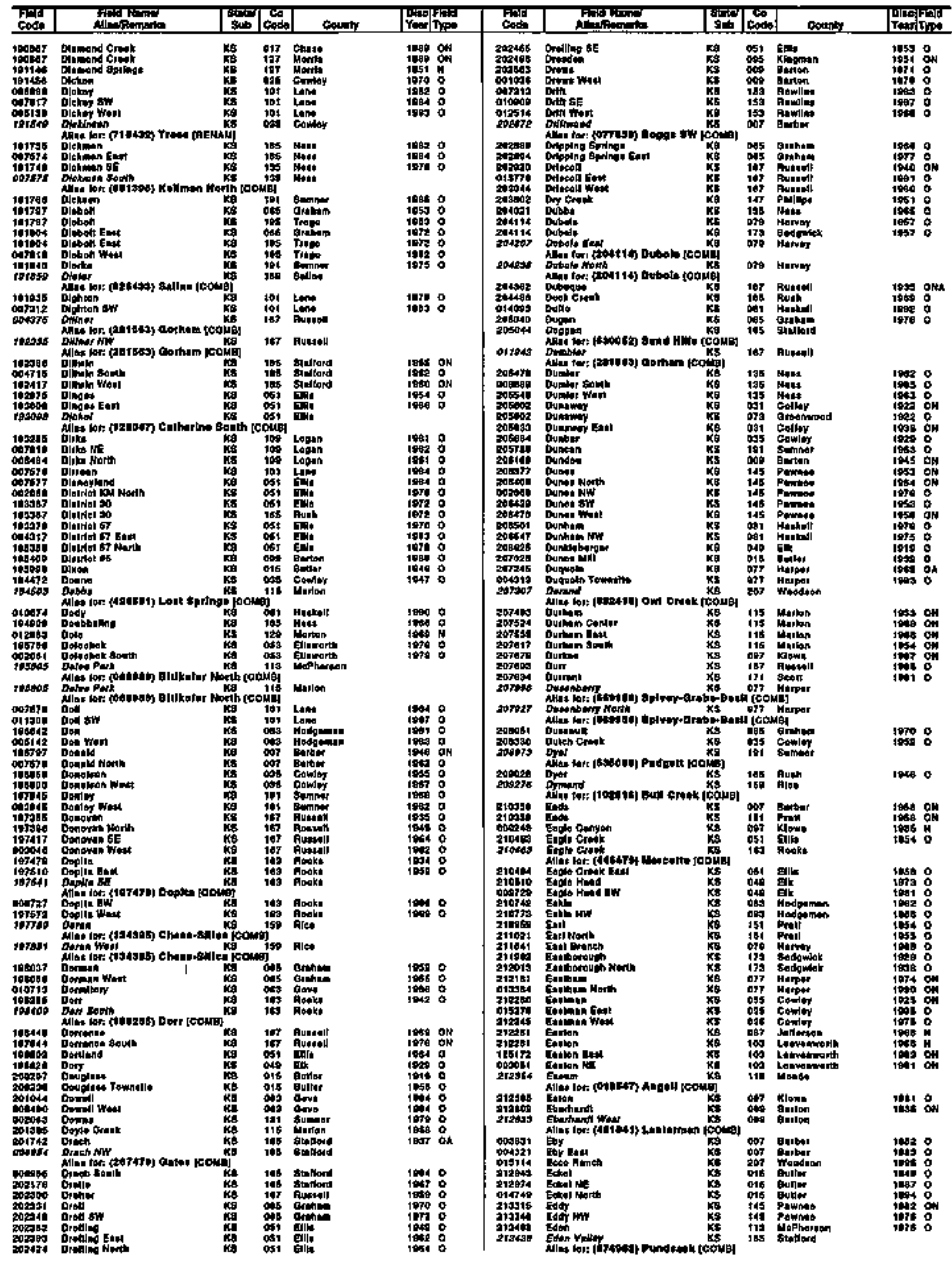




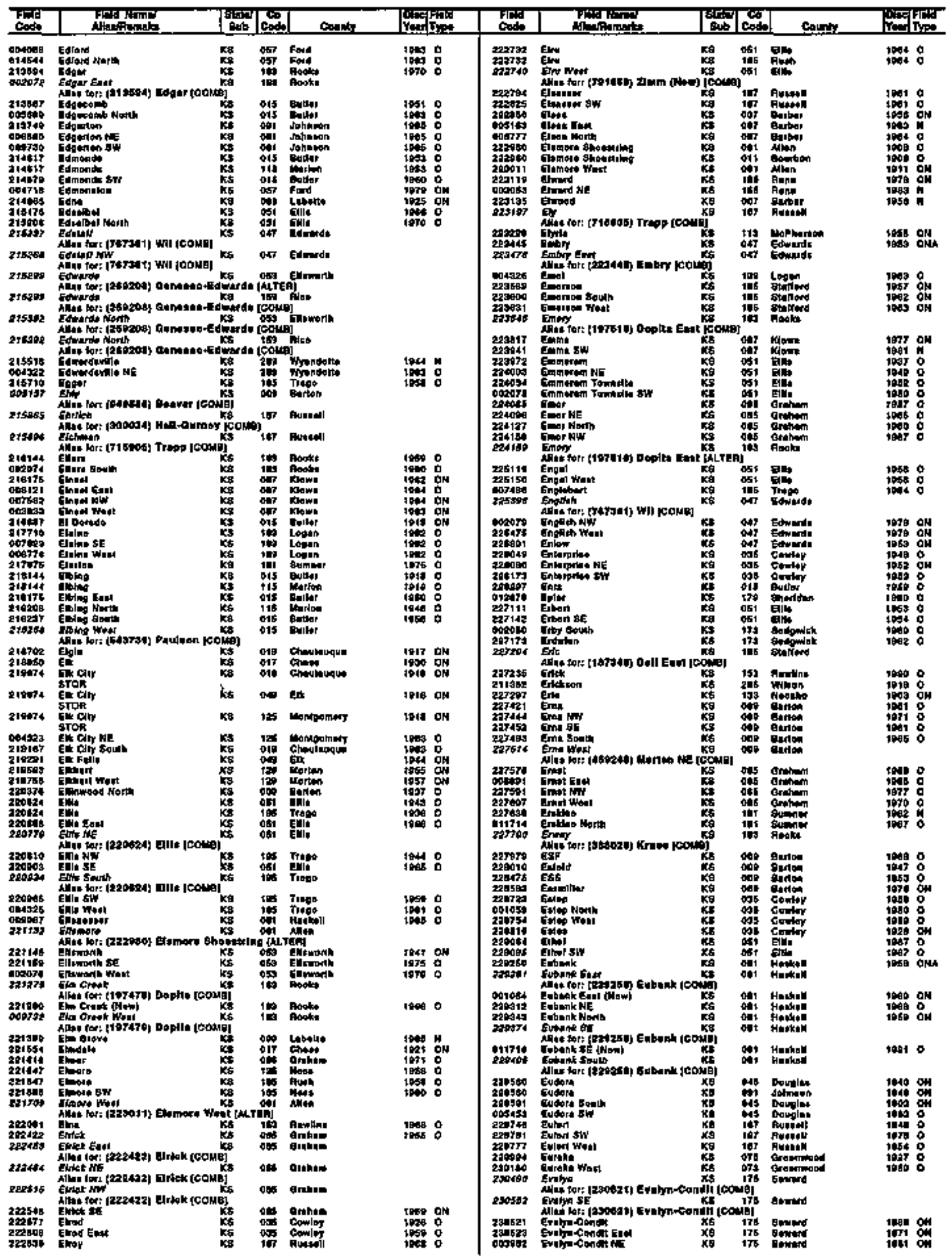




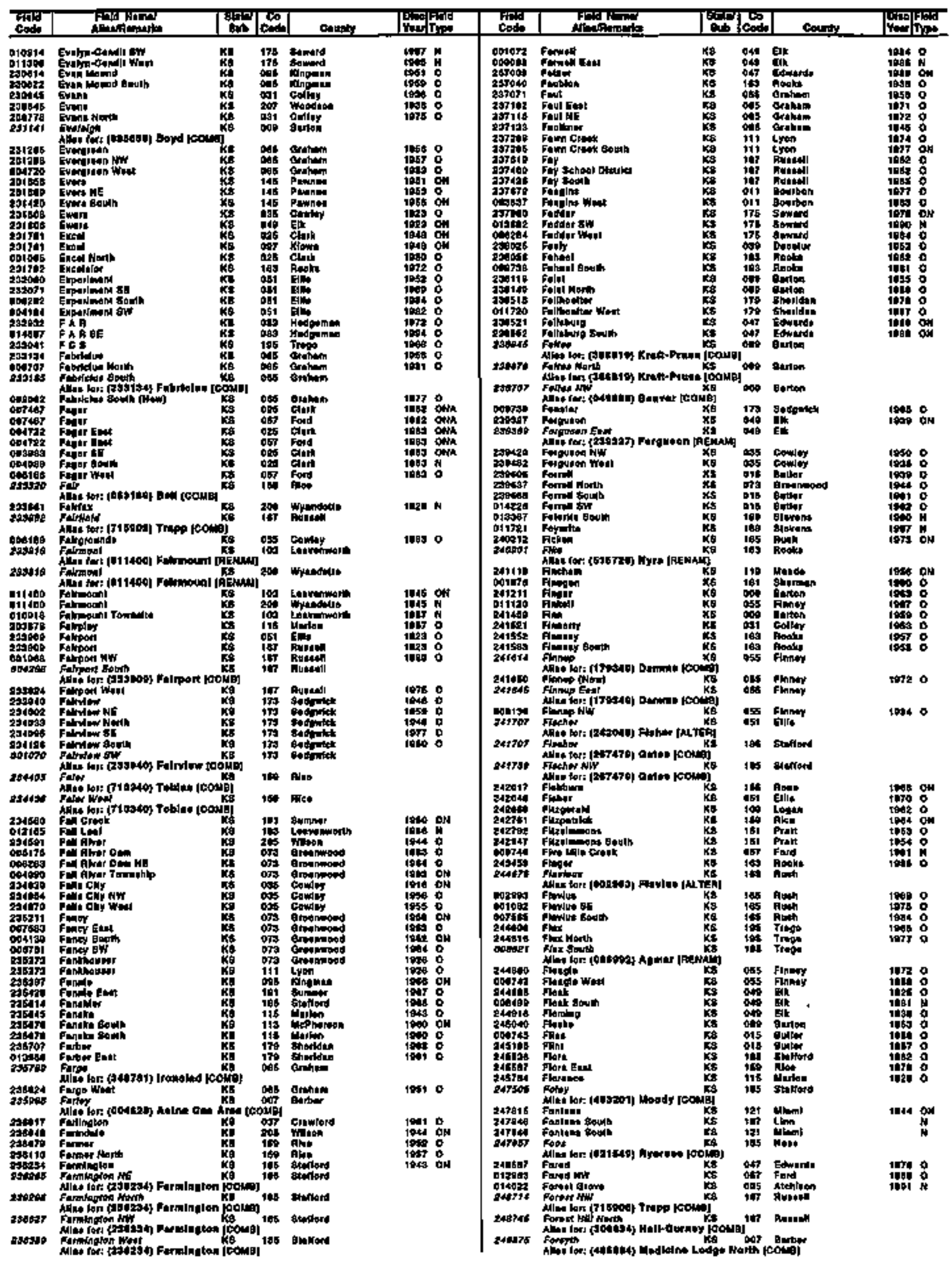


KANSAS

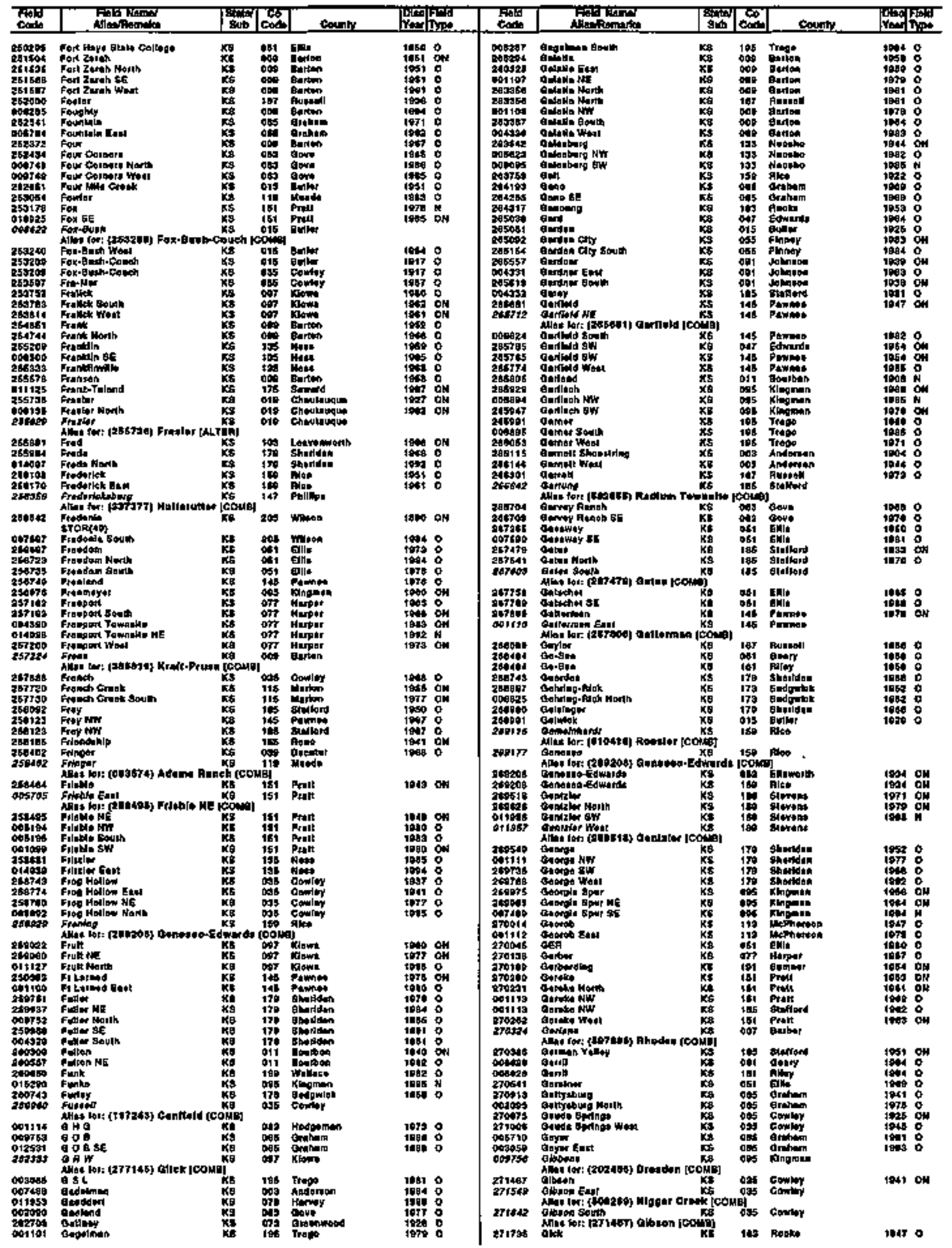


KANSAS

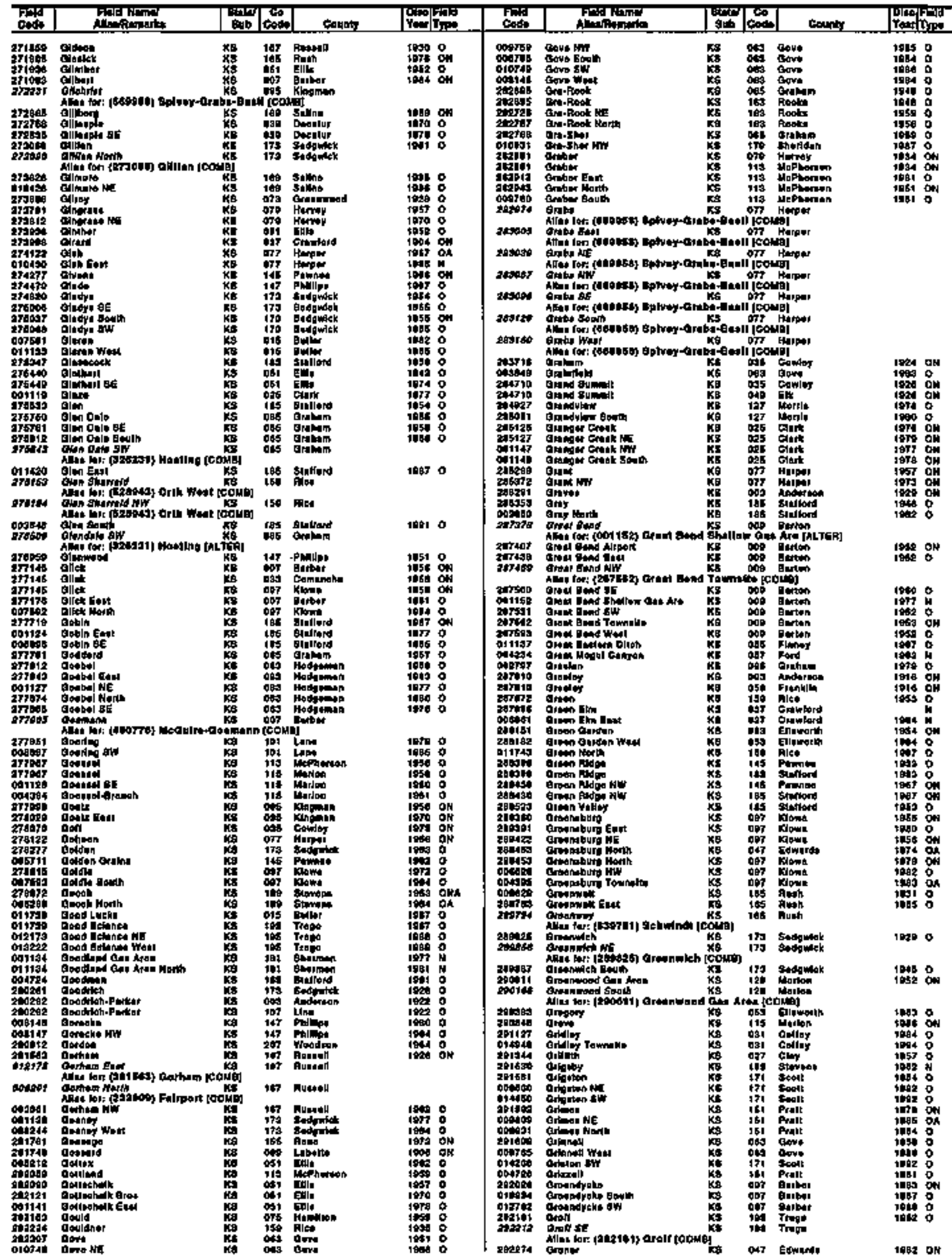




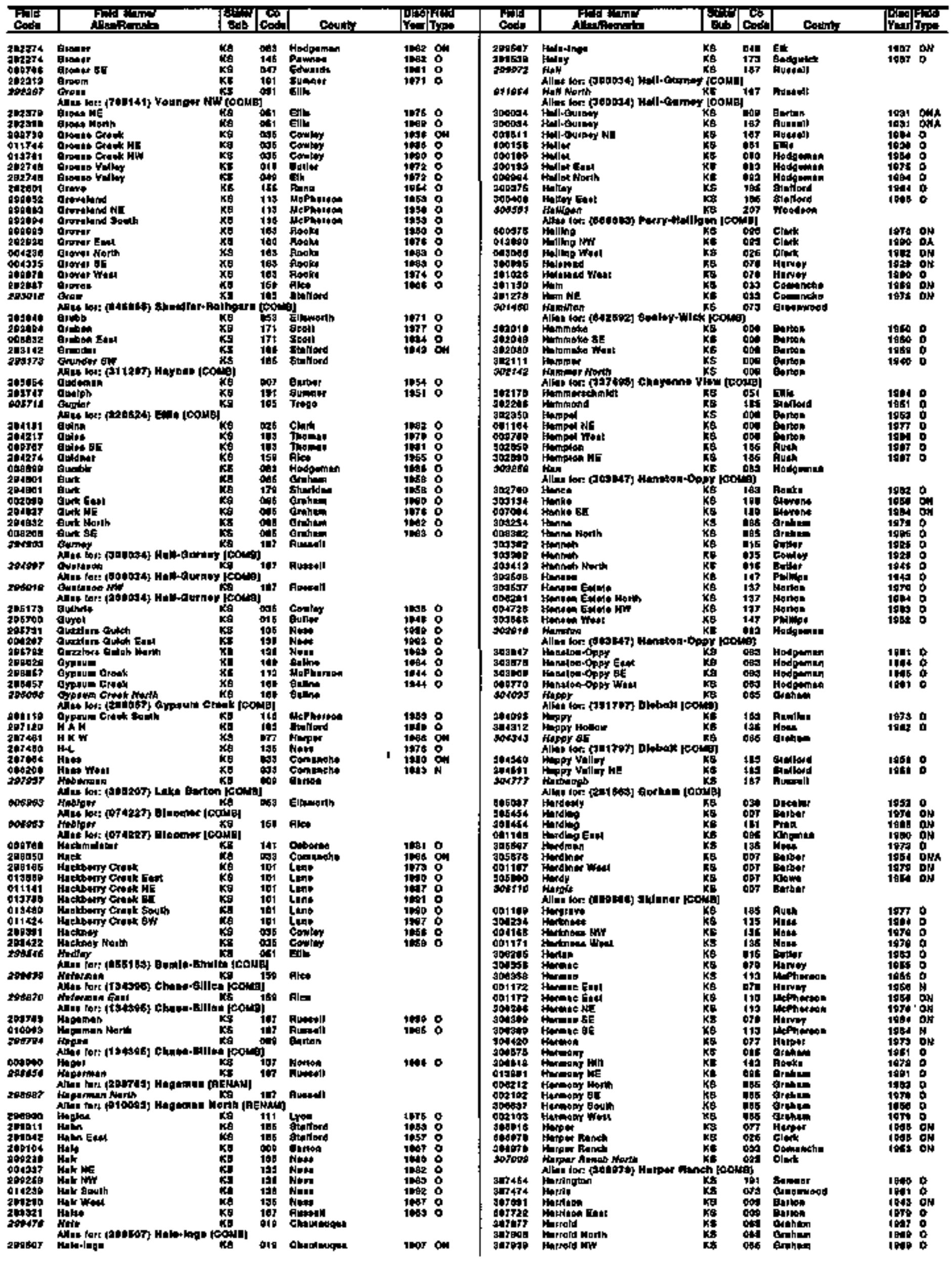


KANSAS

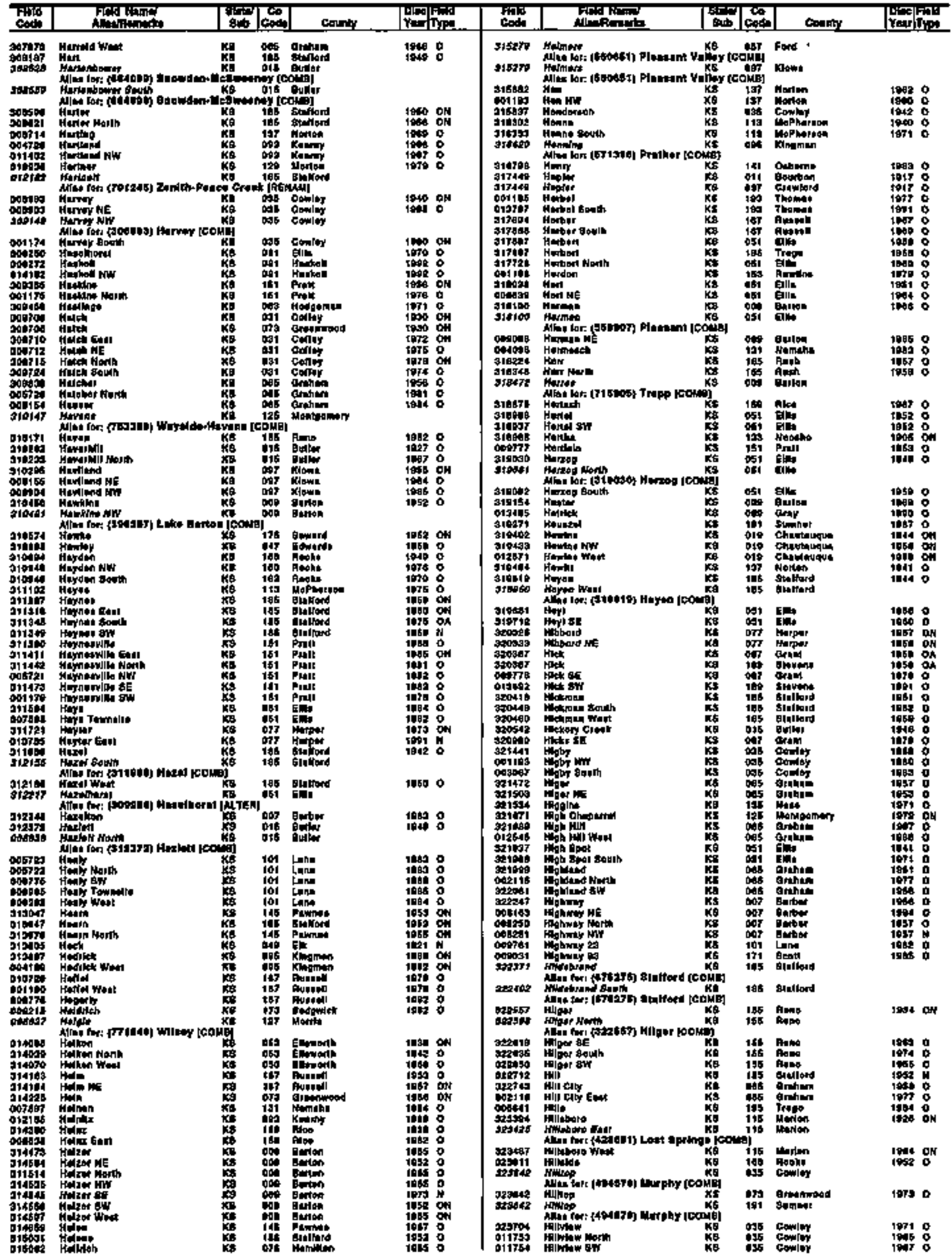


KANSAS

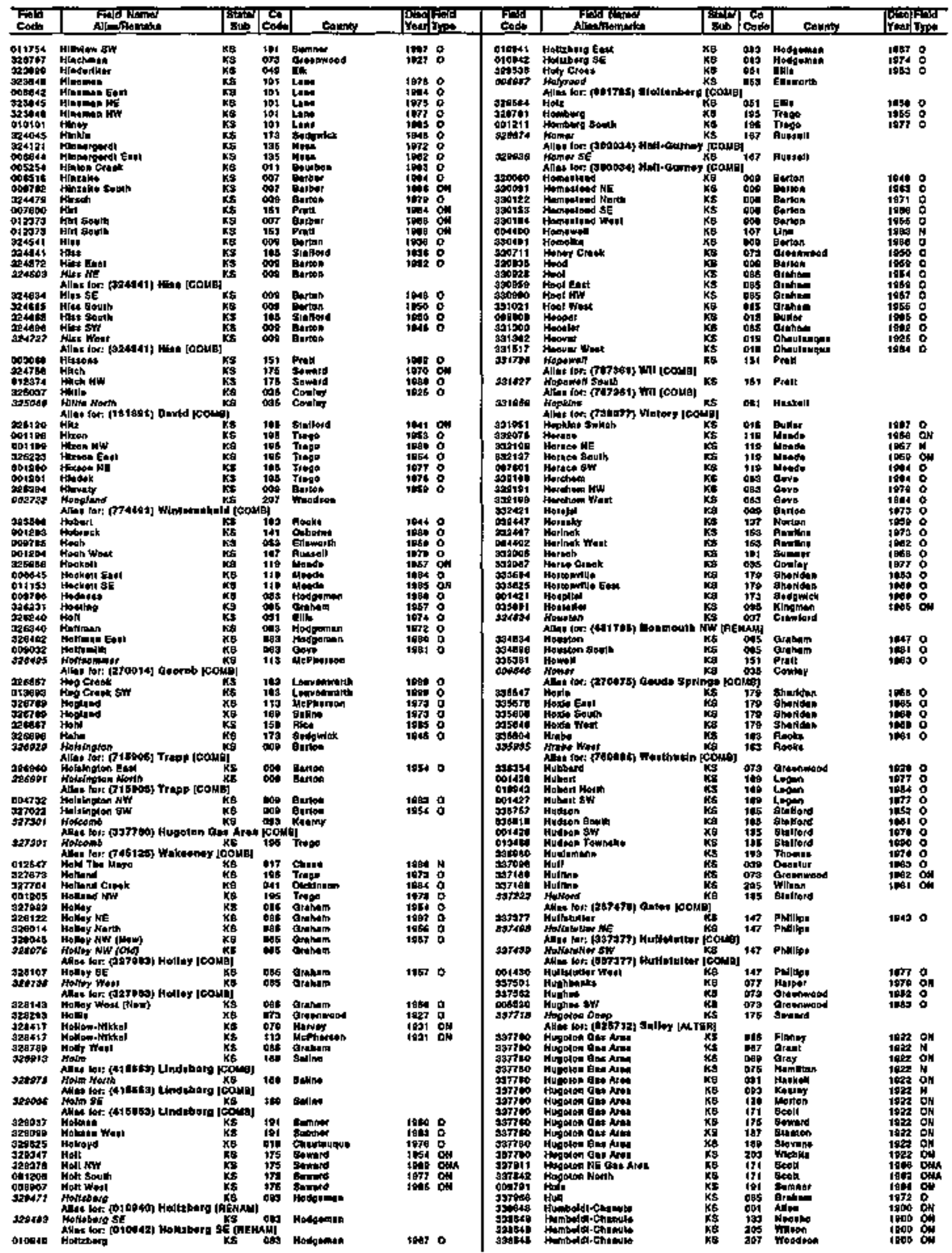


KANSAS

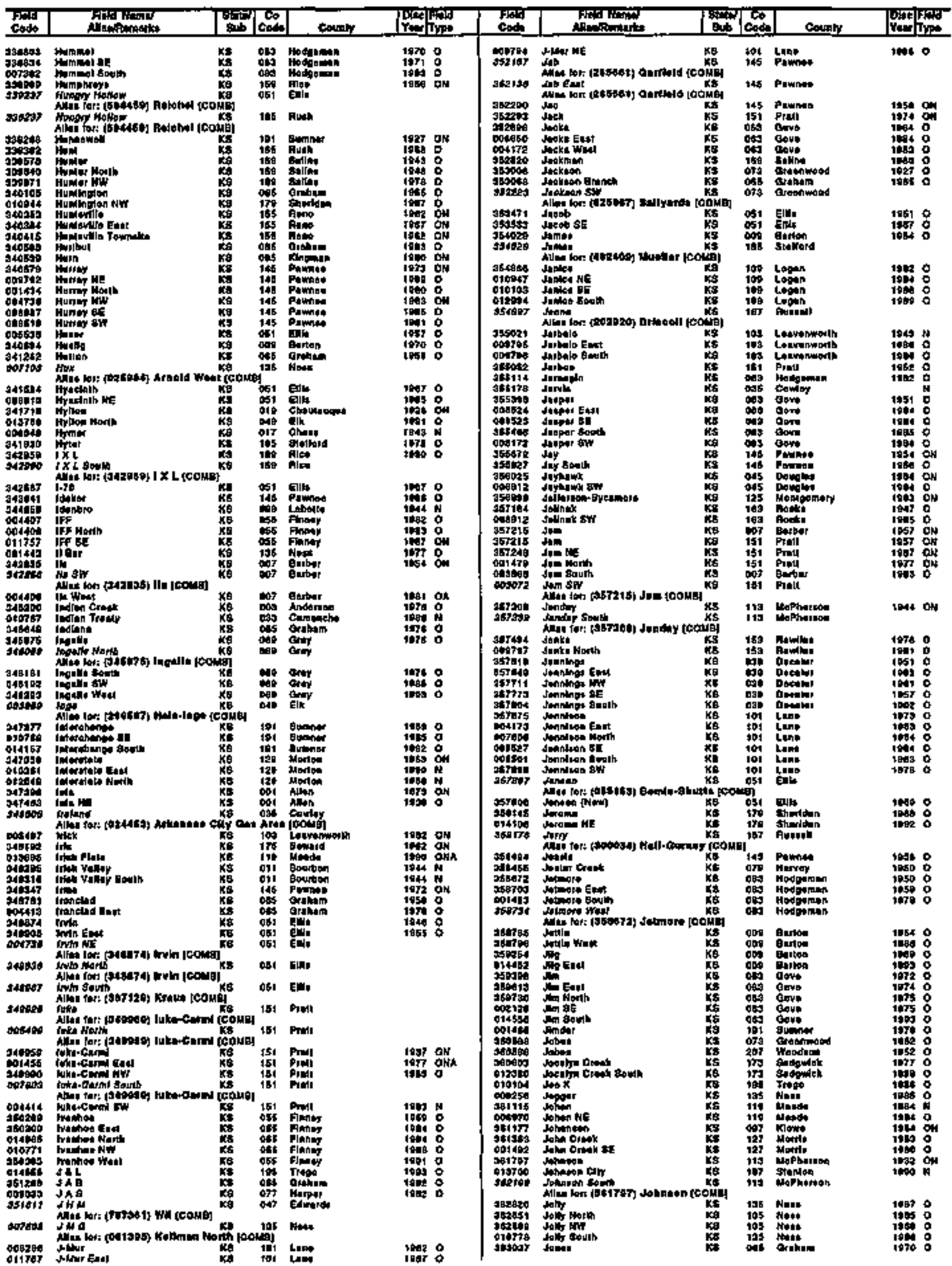




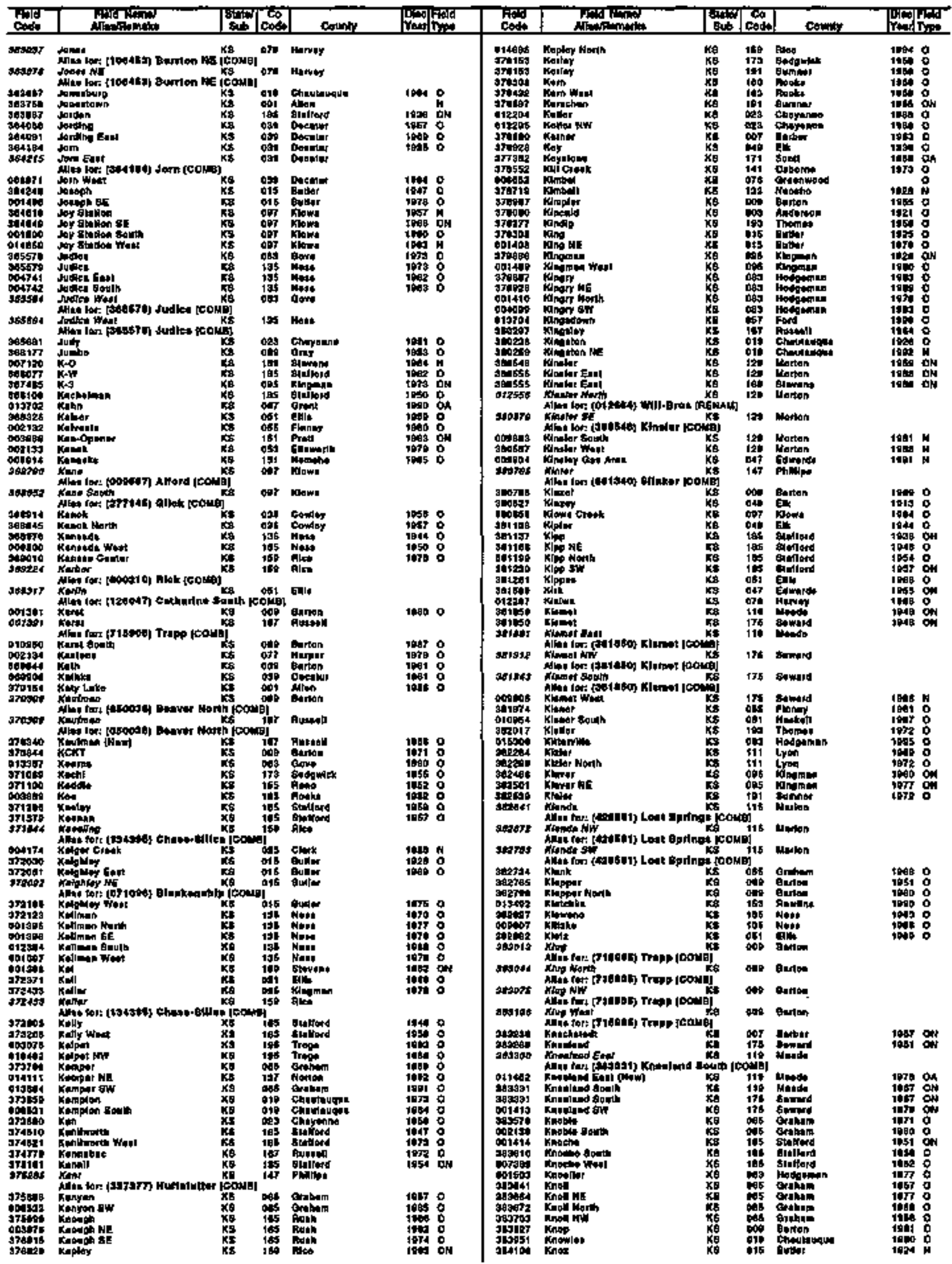


KANSAS

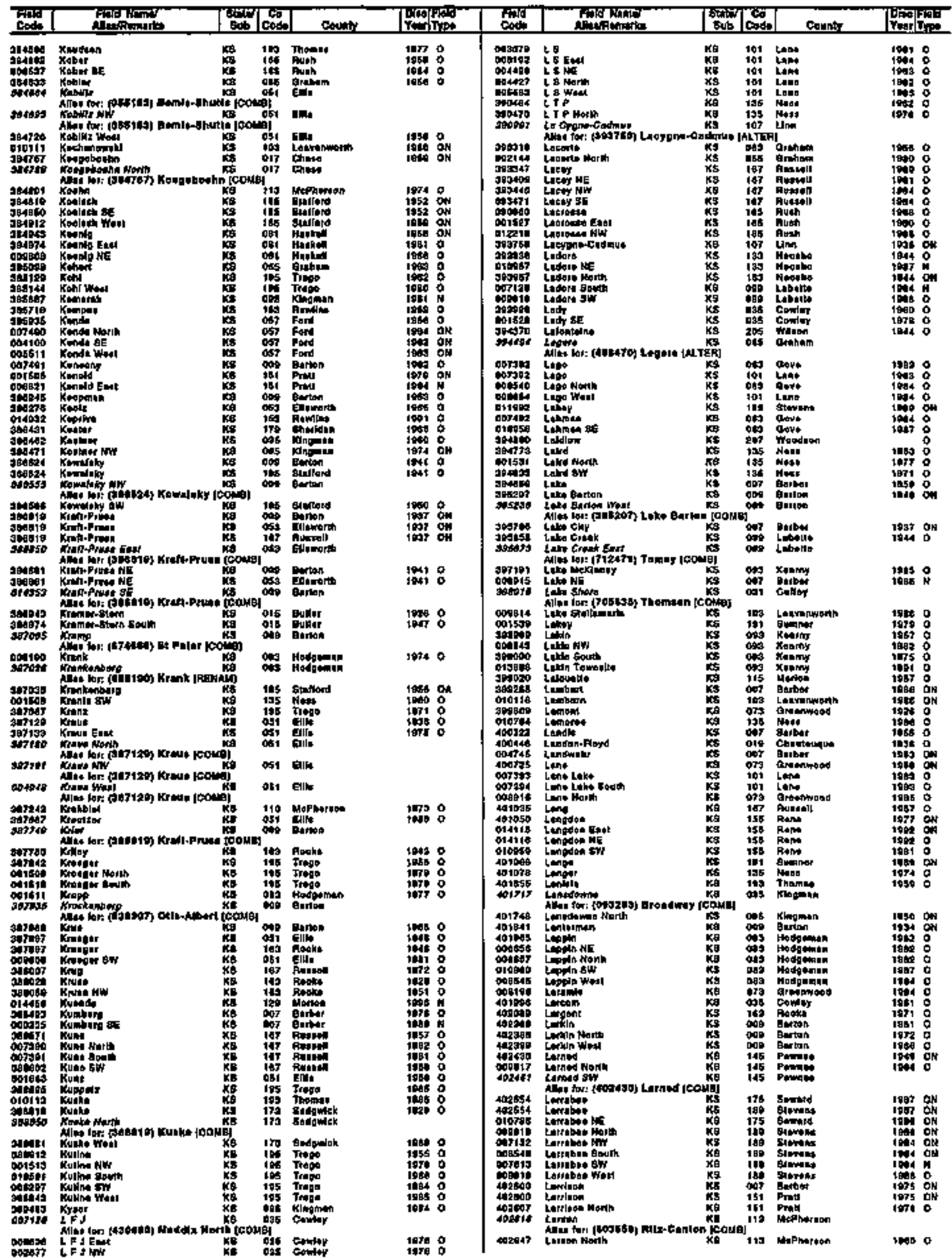


KANSAS

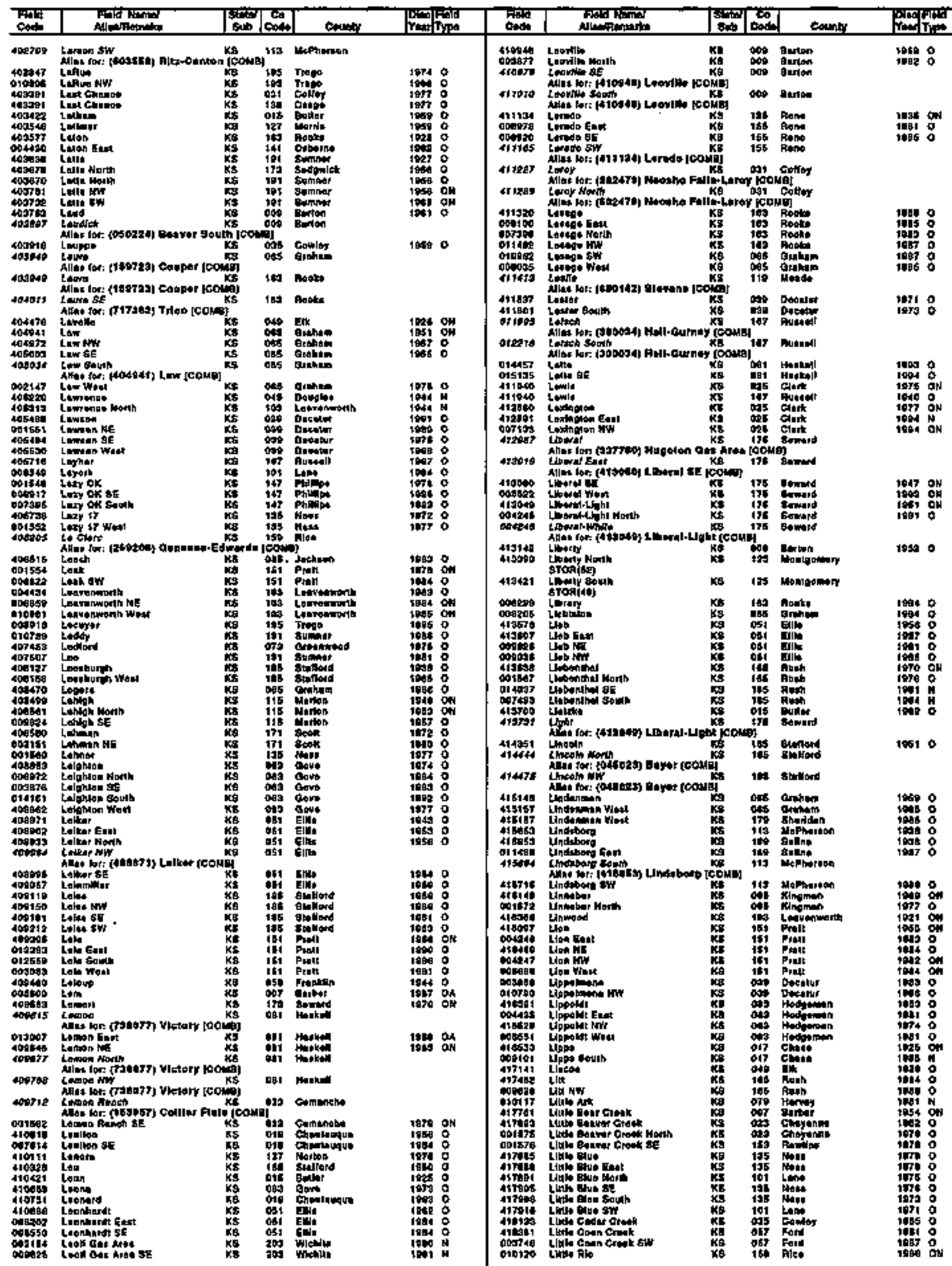


KANBAS

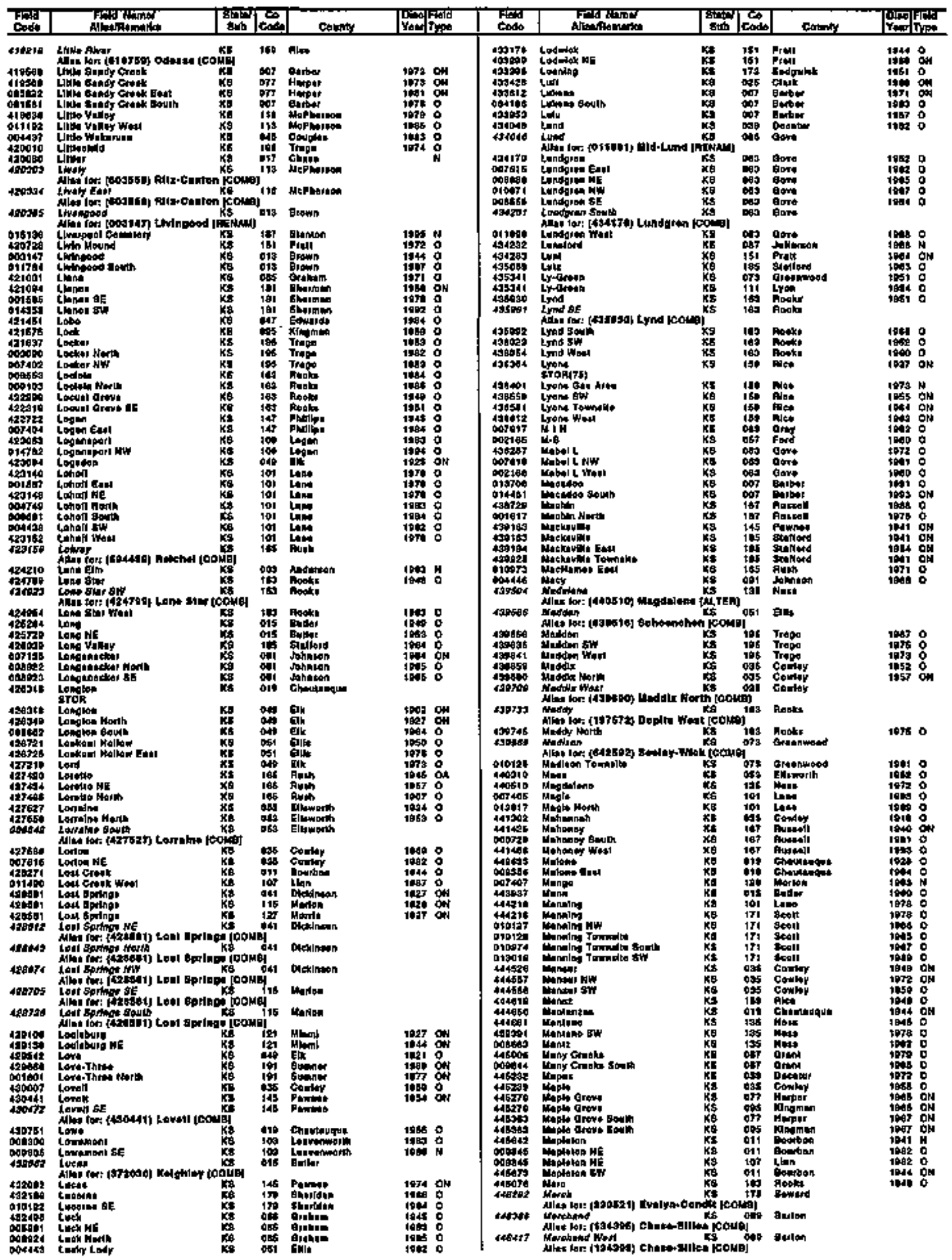




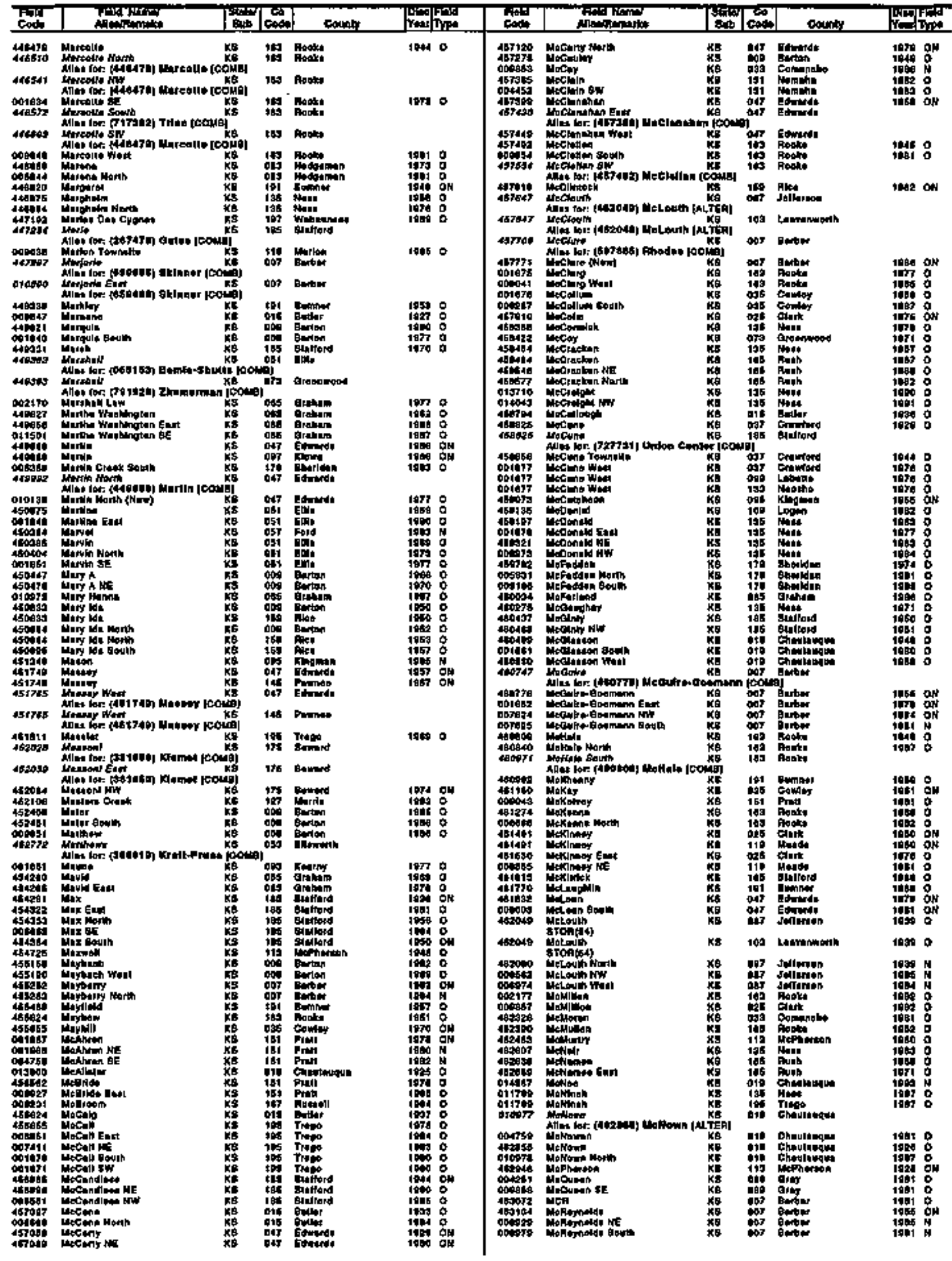


KANSAS

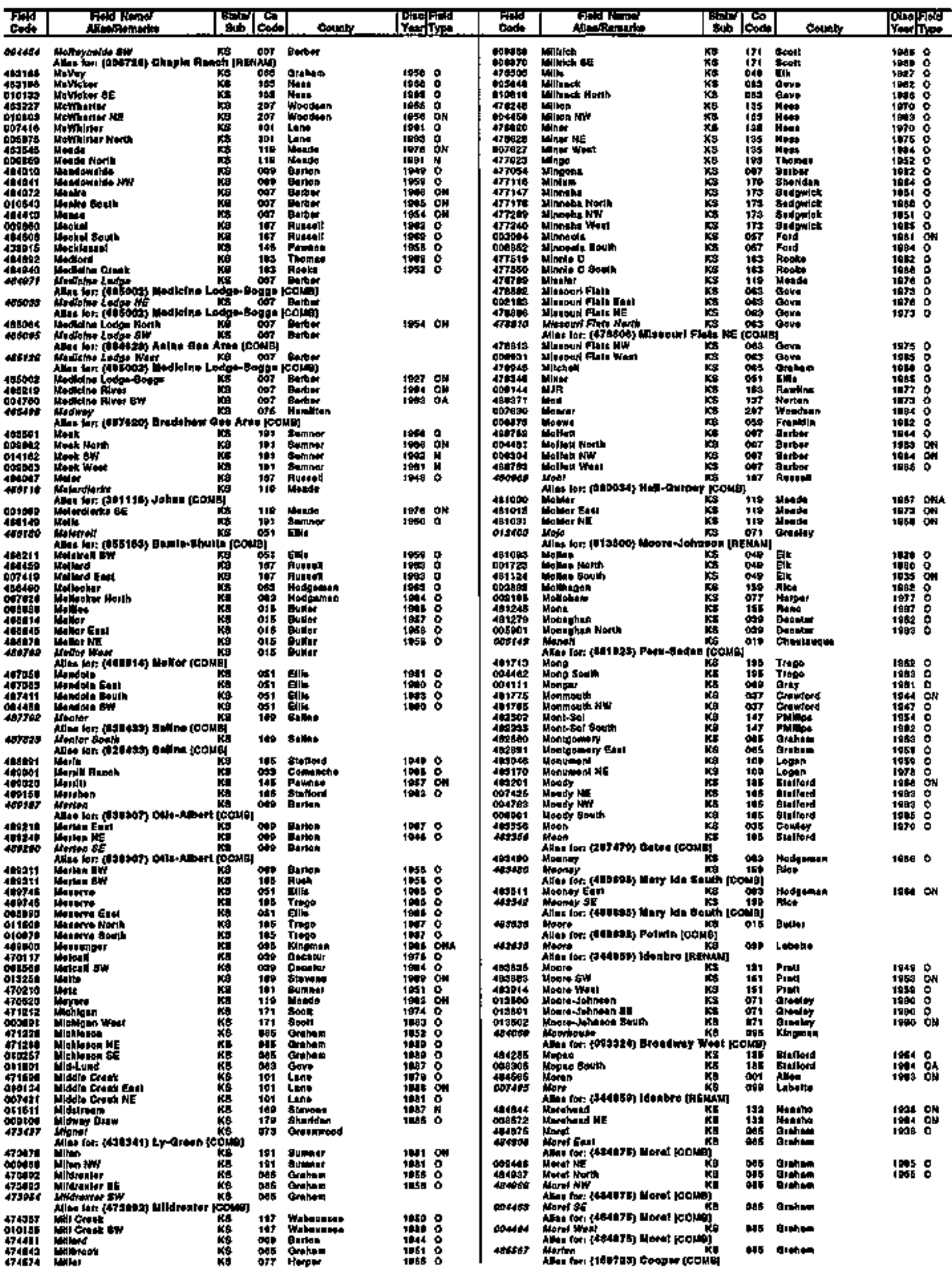




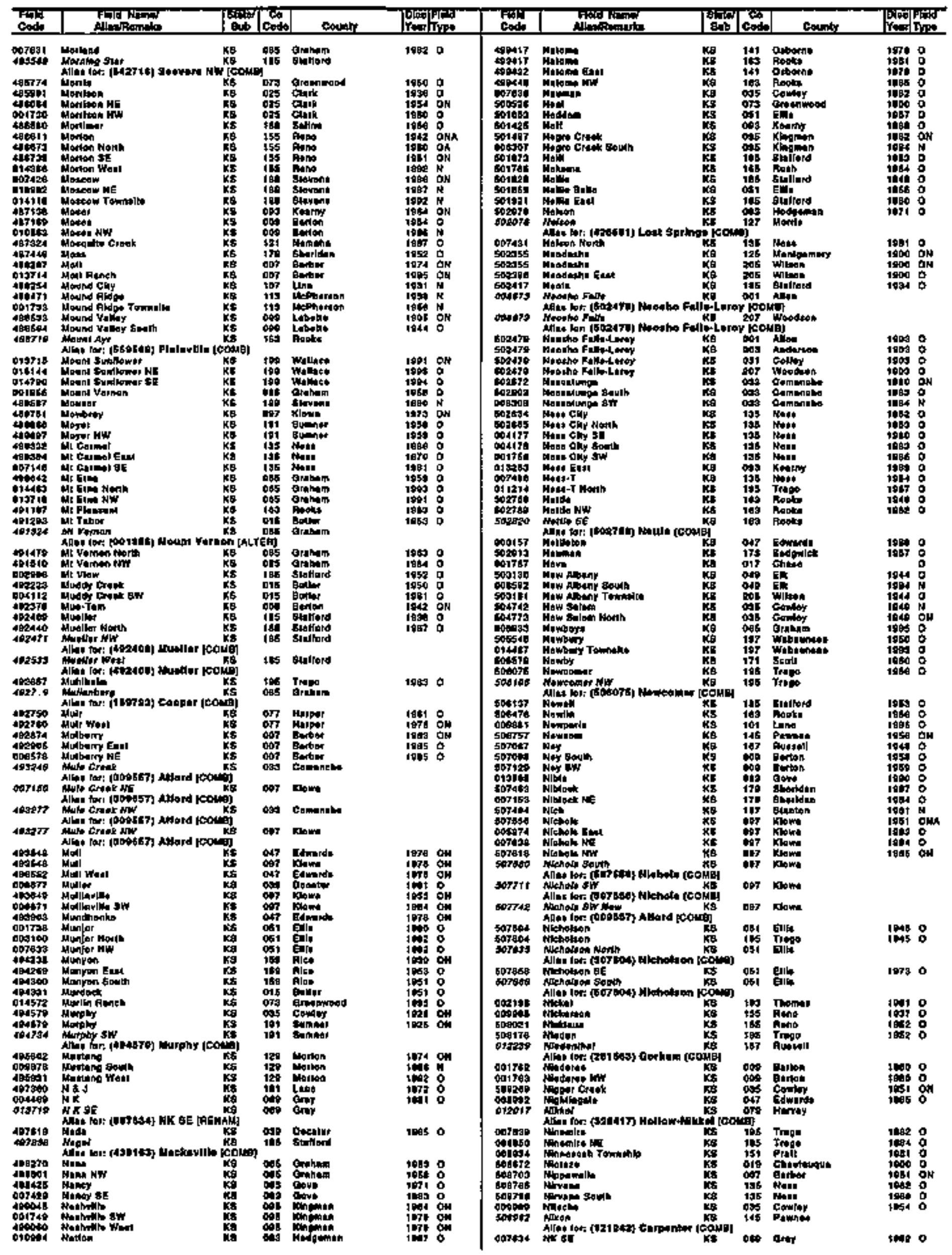


KANSAB

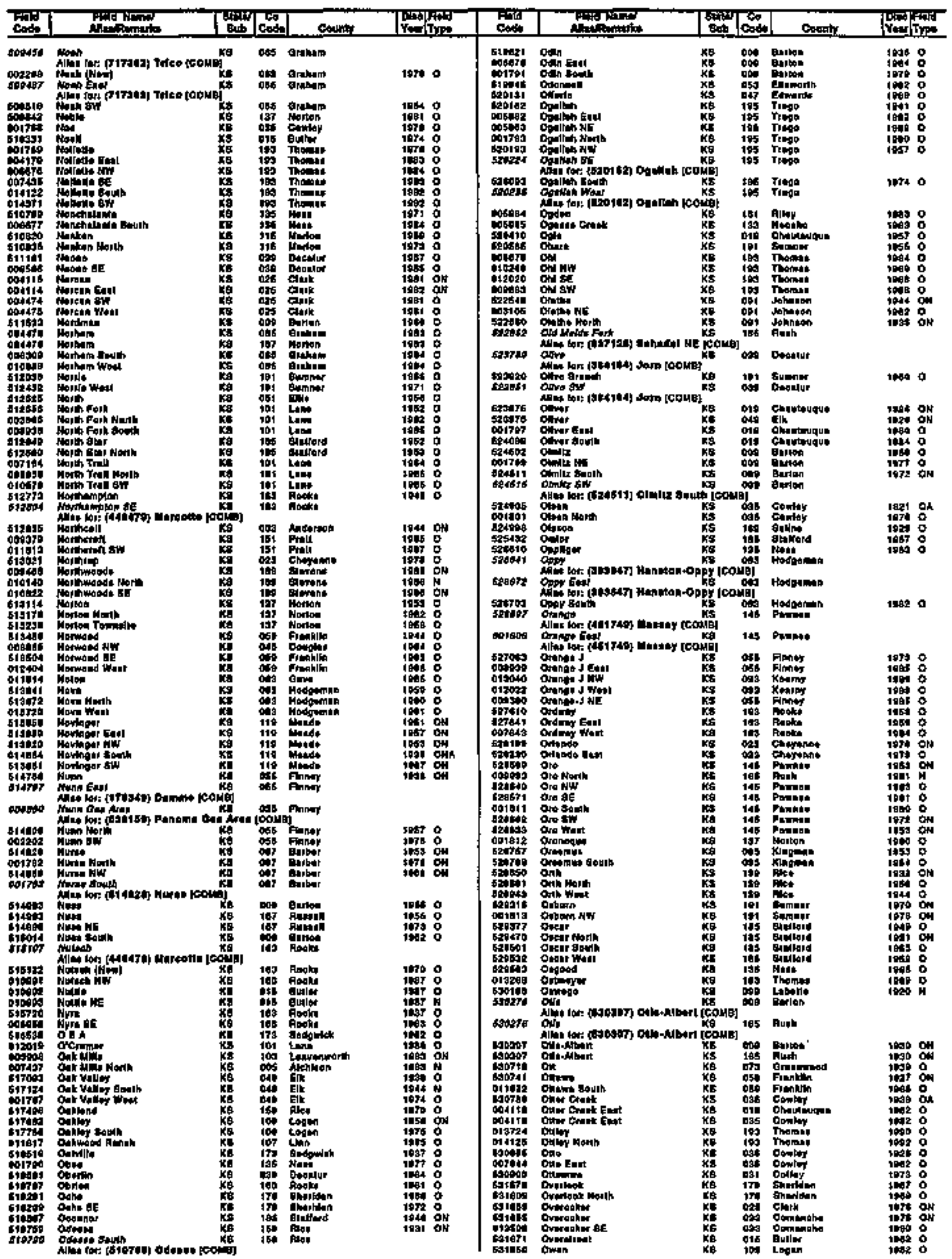




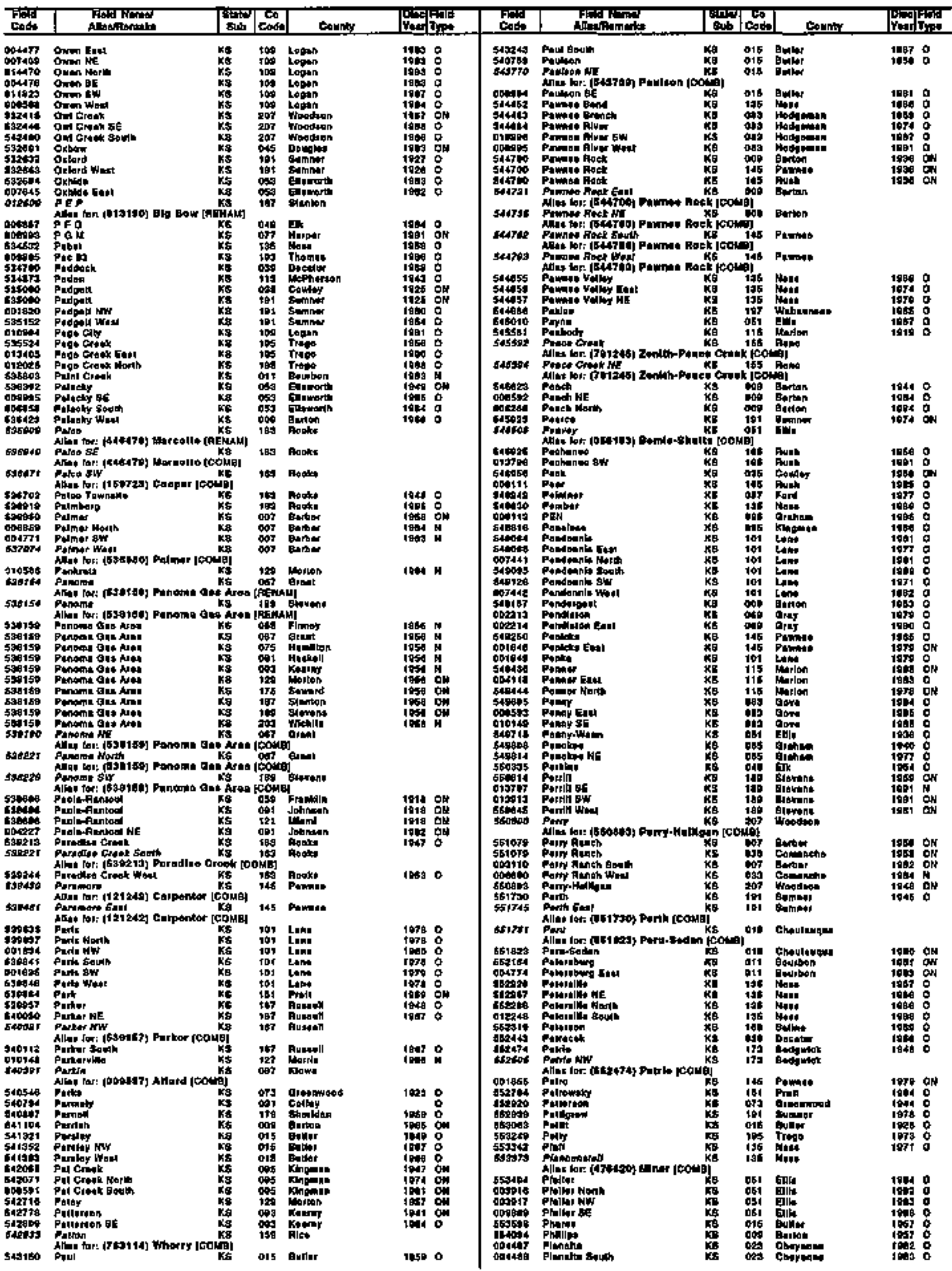


KANSAS

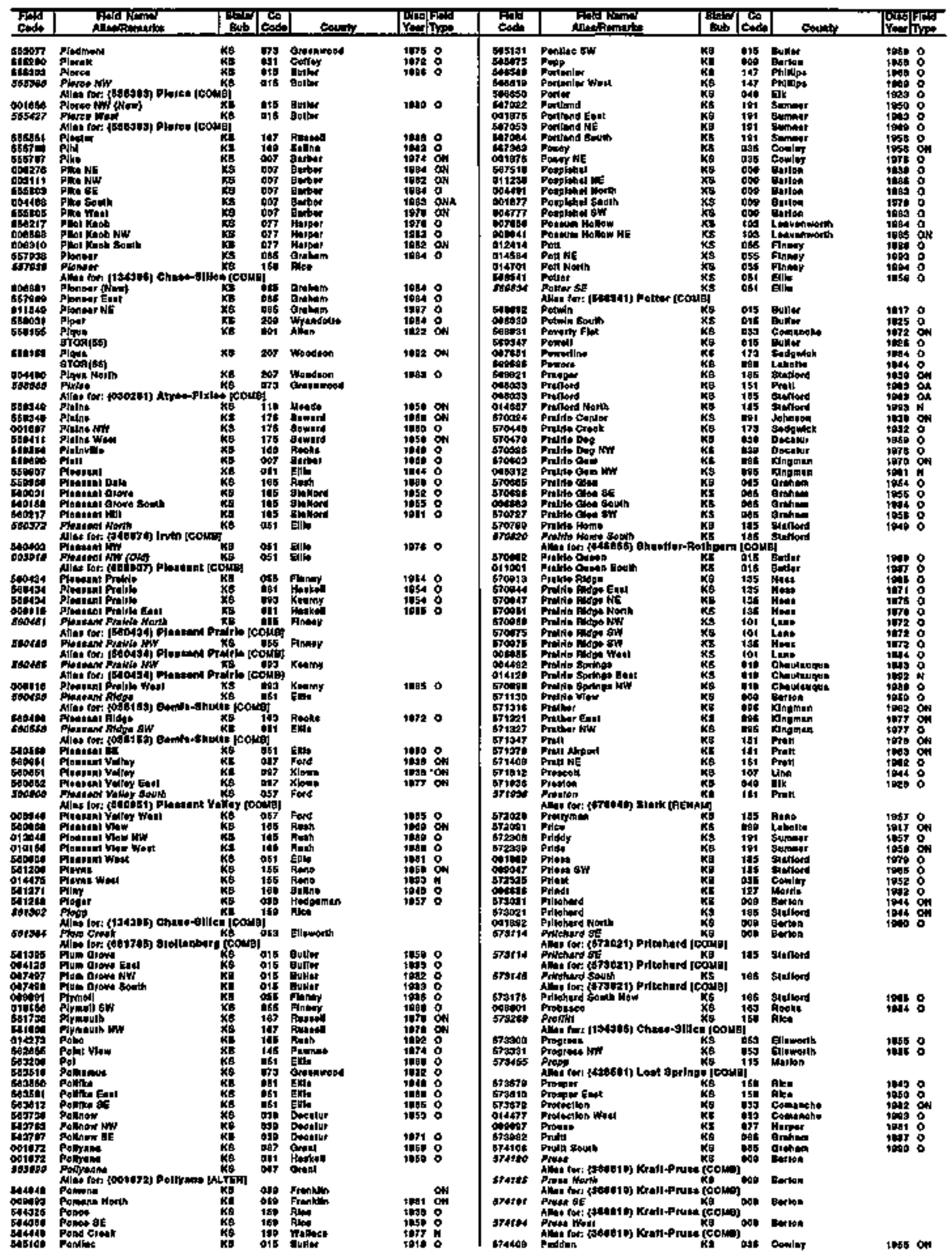


KANSAS

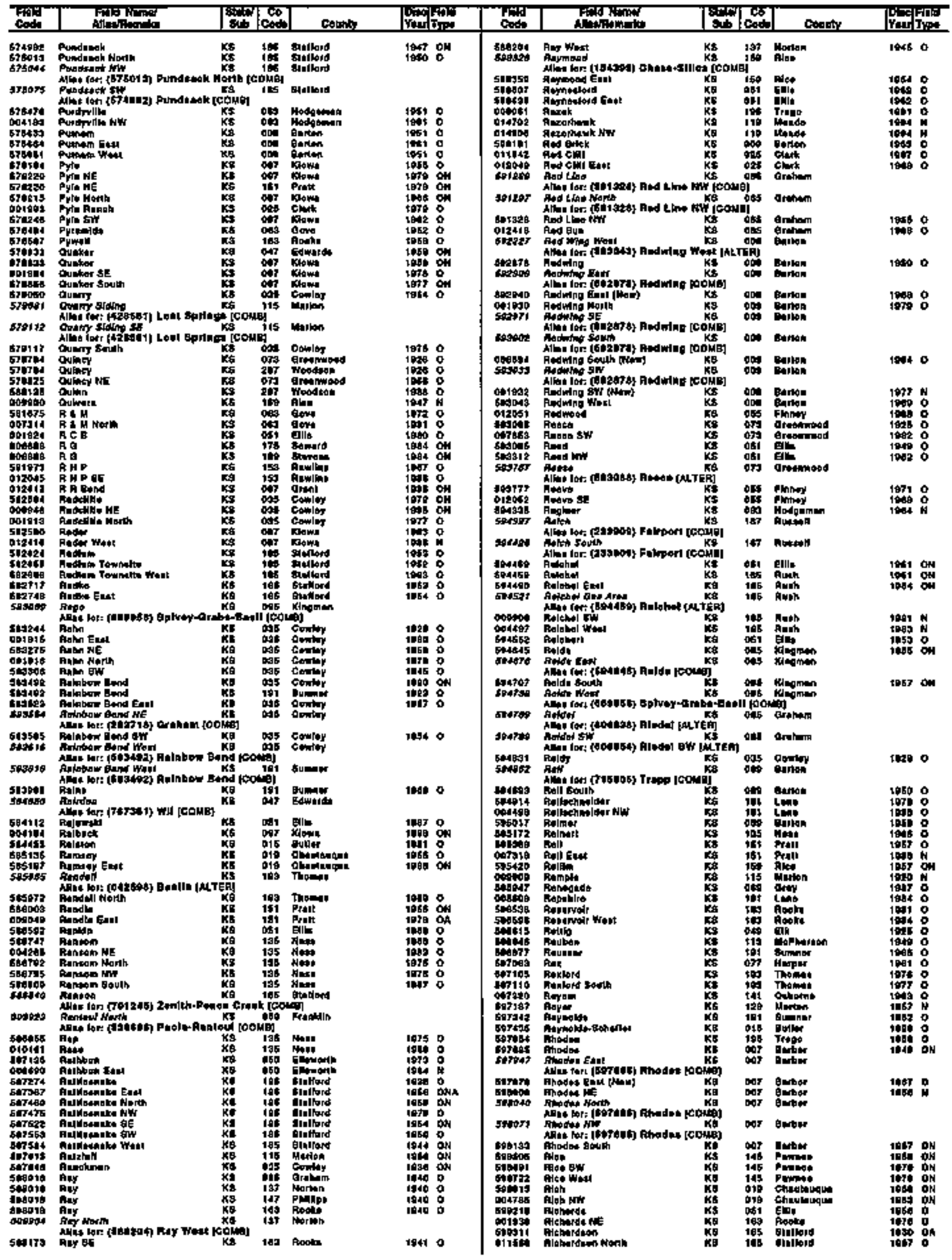


KAN\$AS

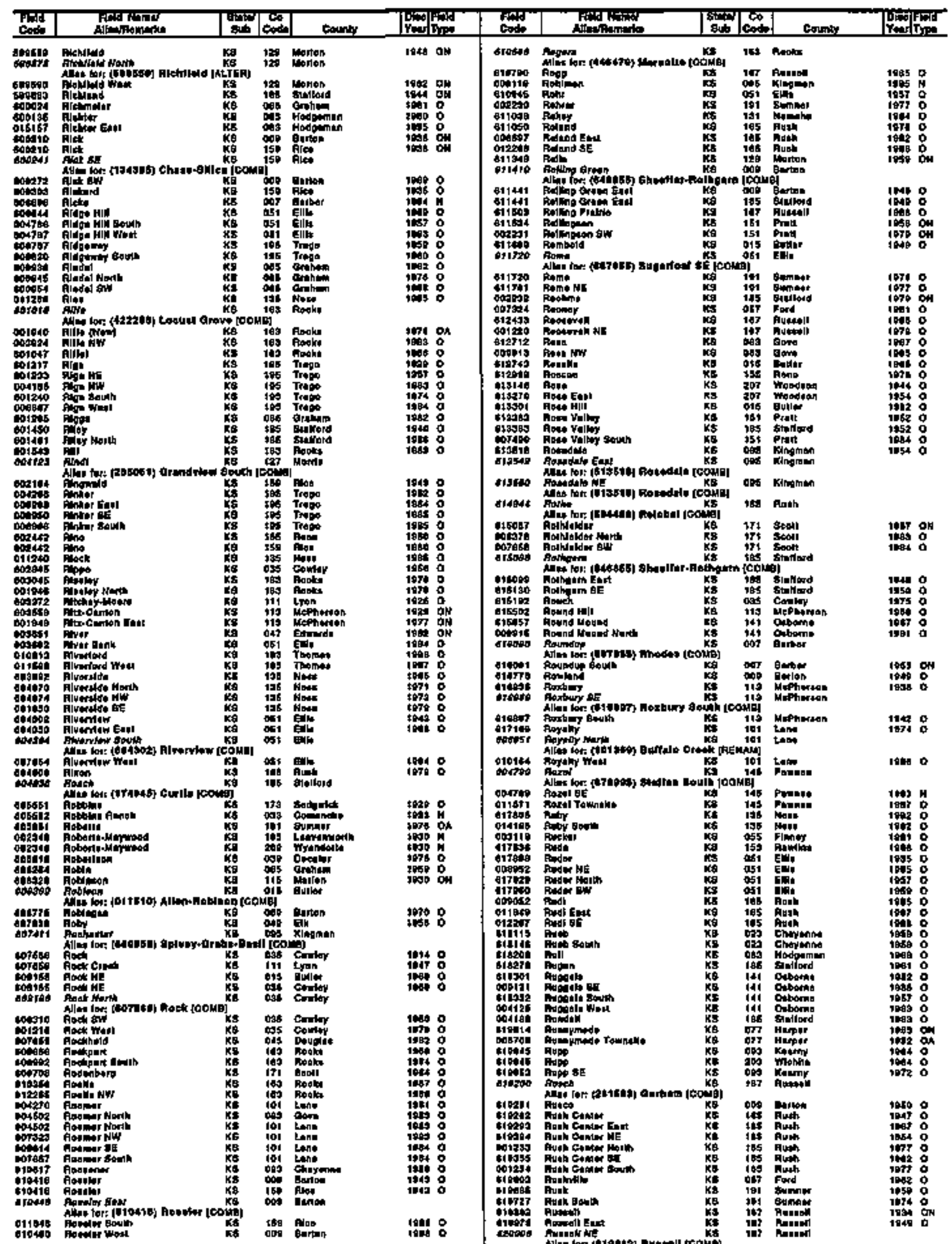




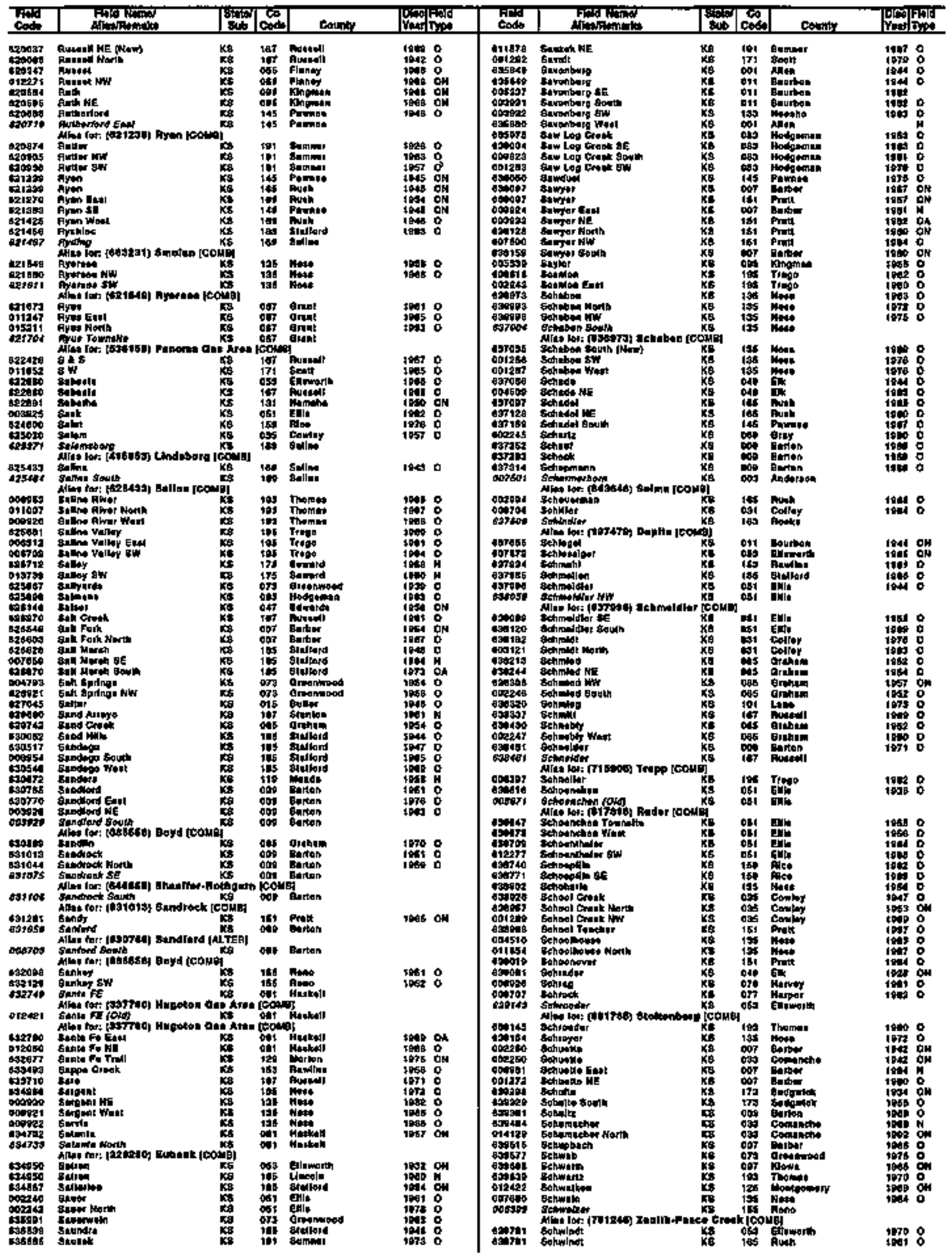


KANSAS

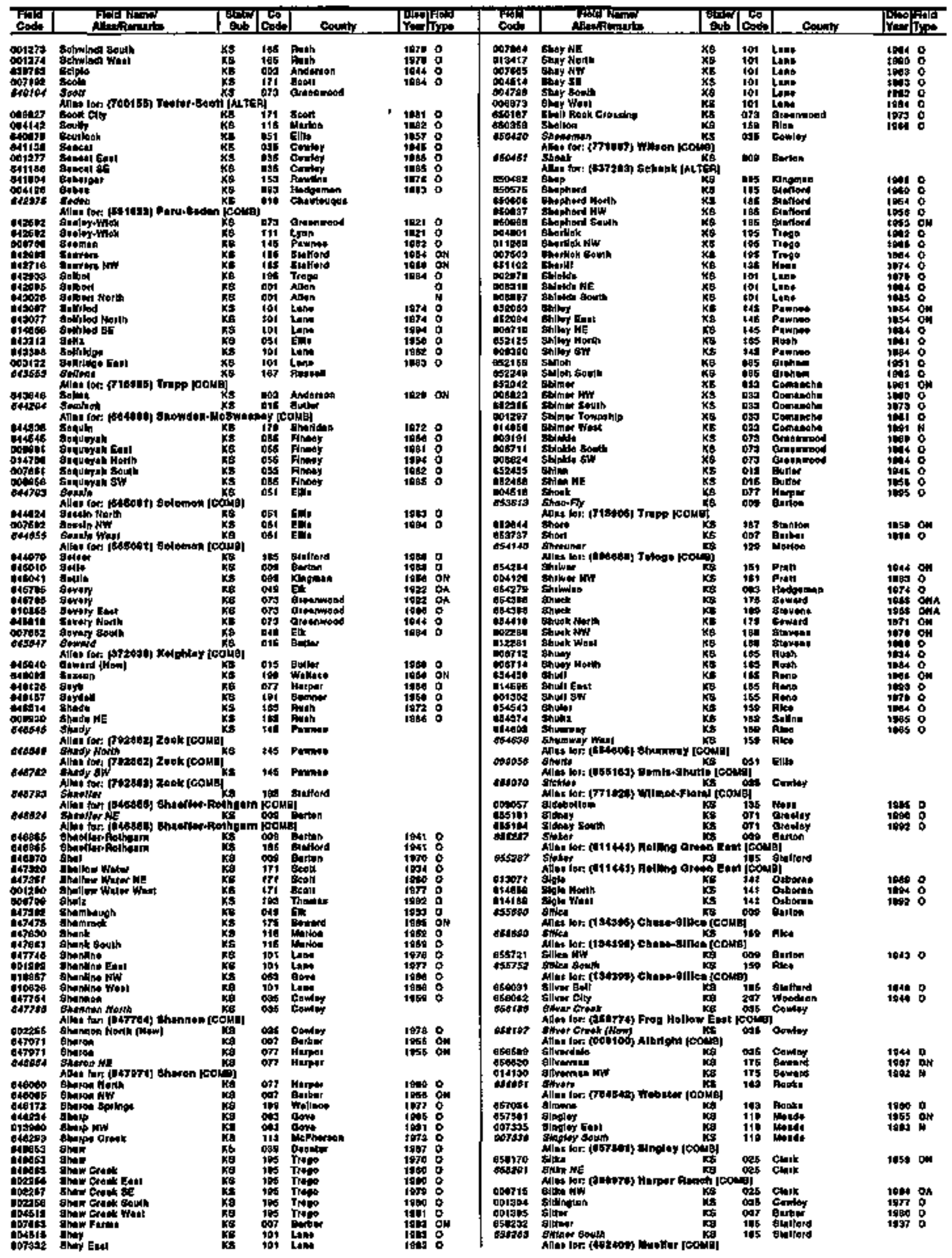


KANSAS

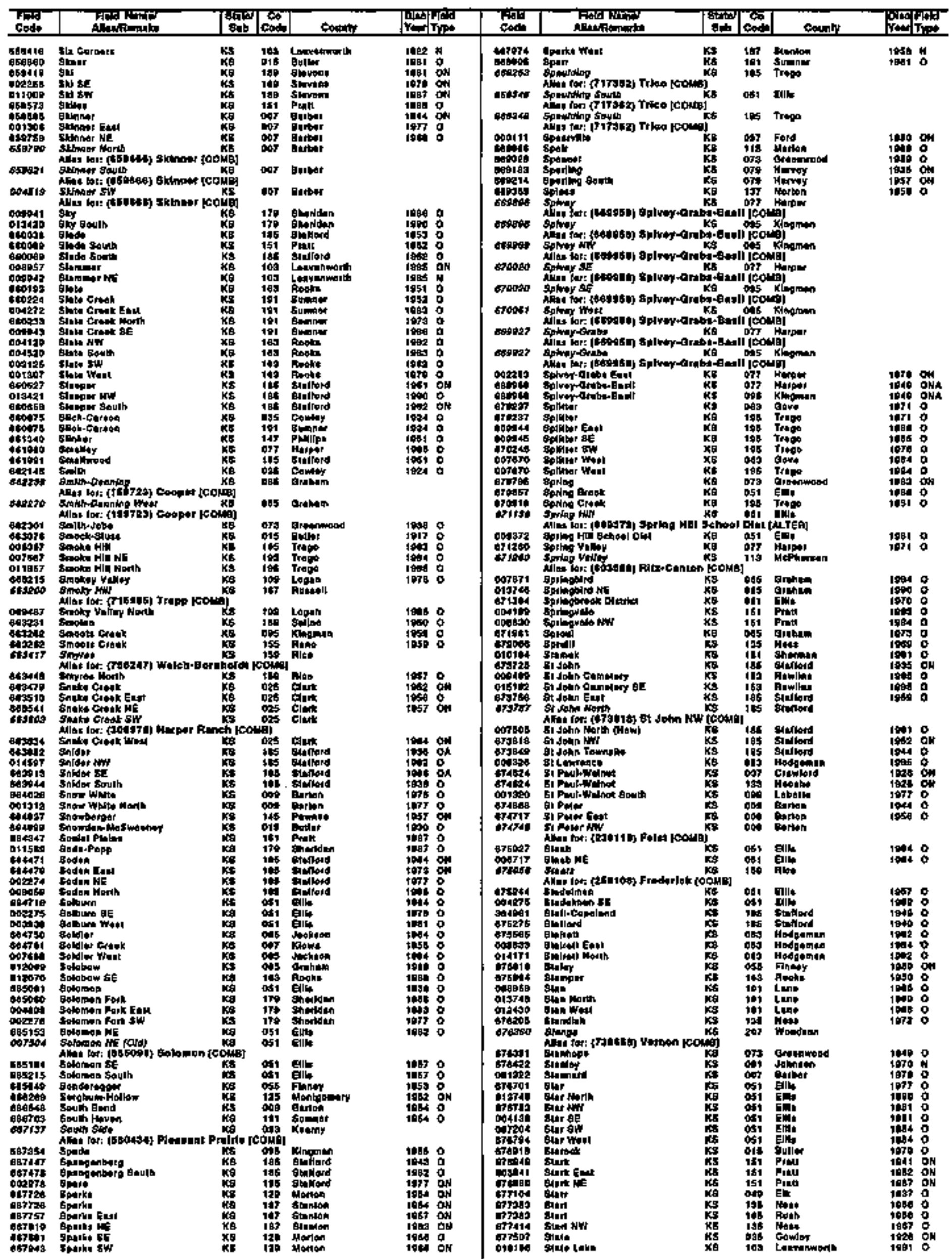


KANSAS

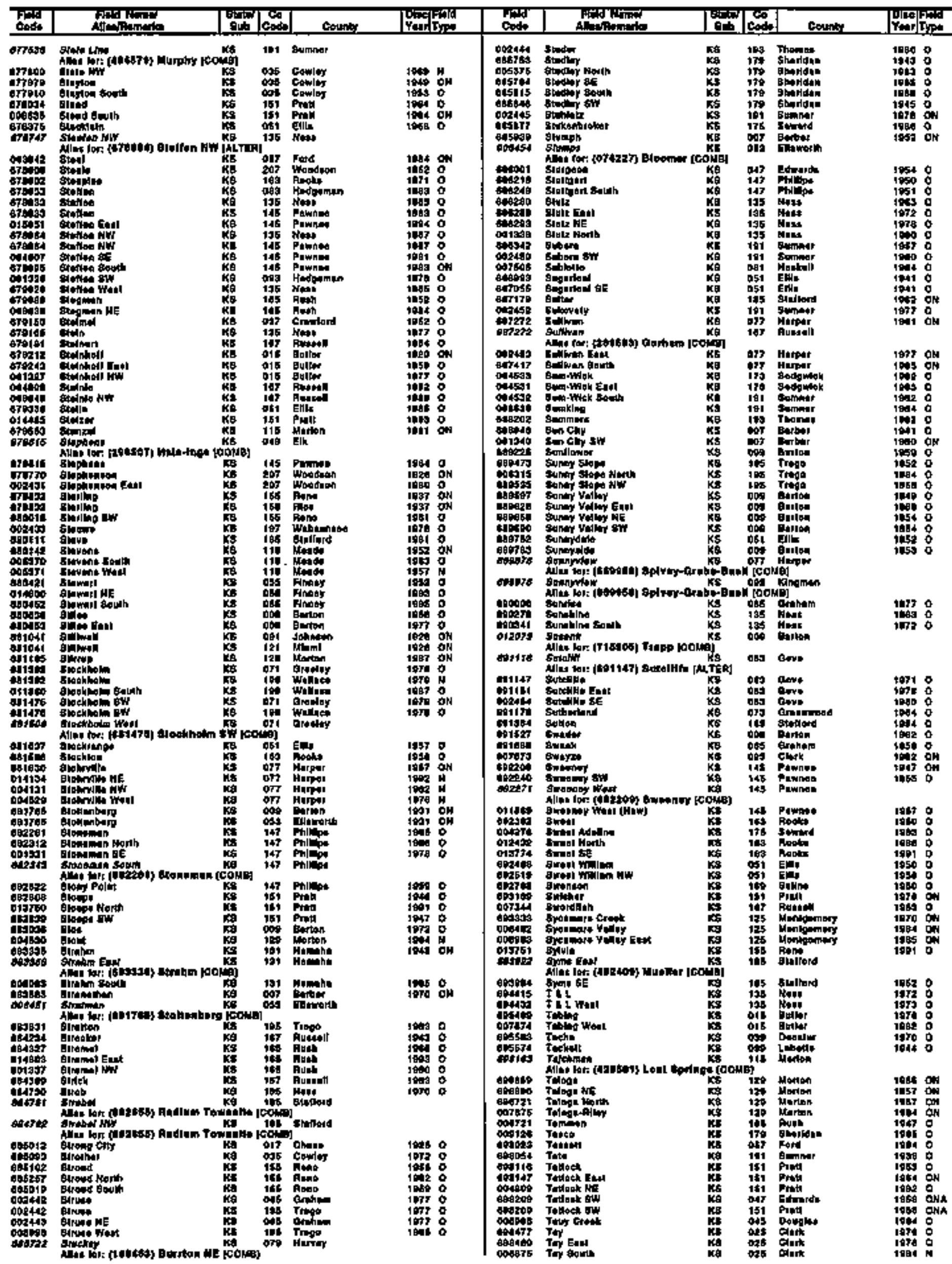




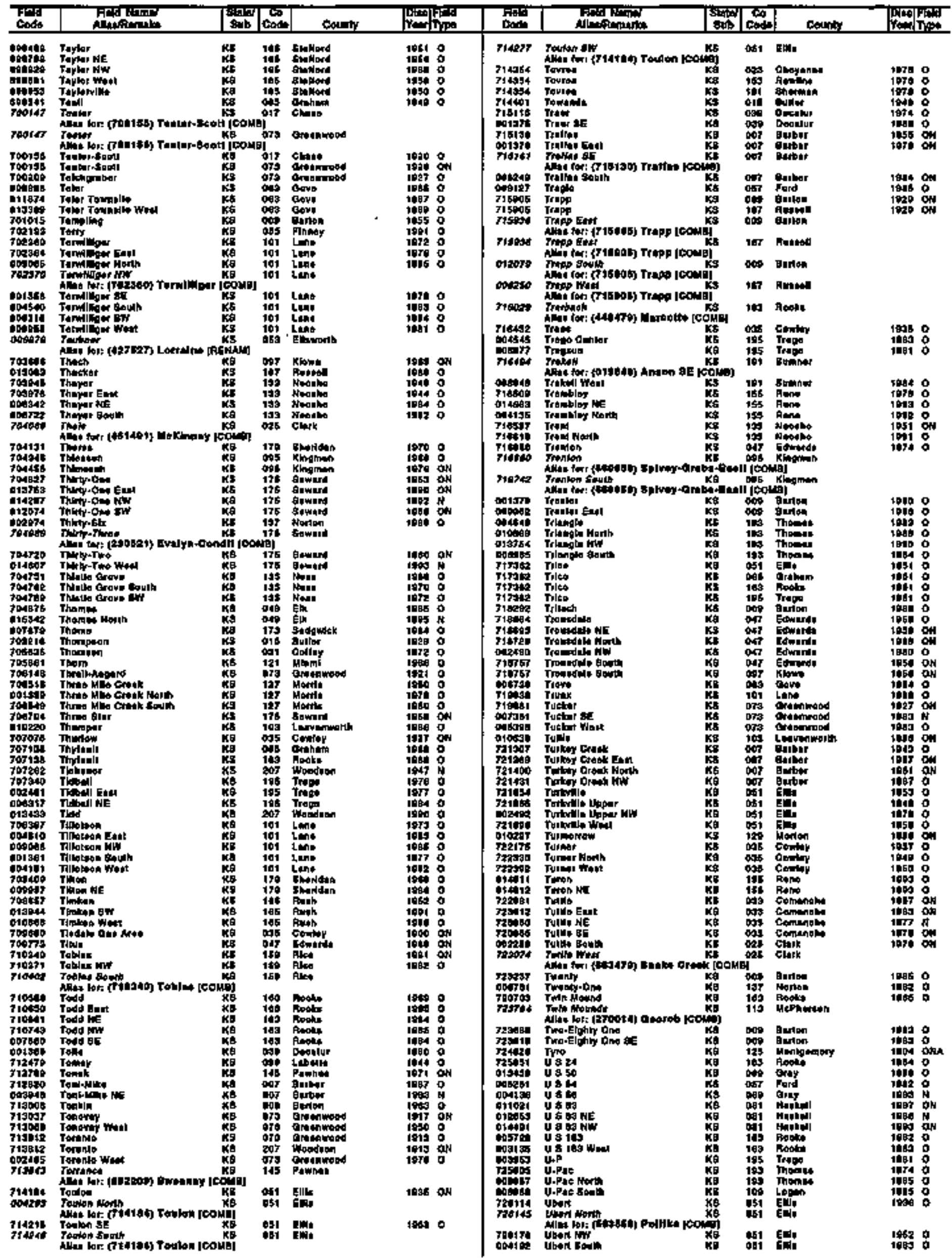


KANSAS

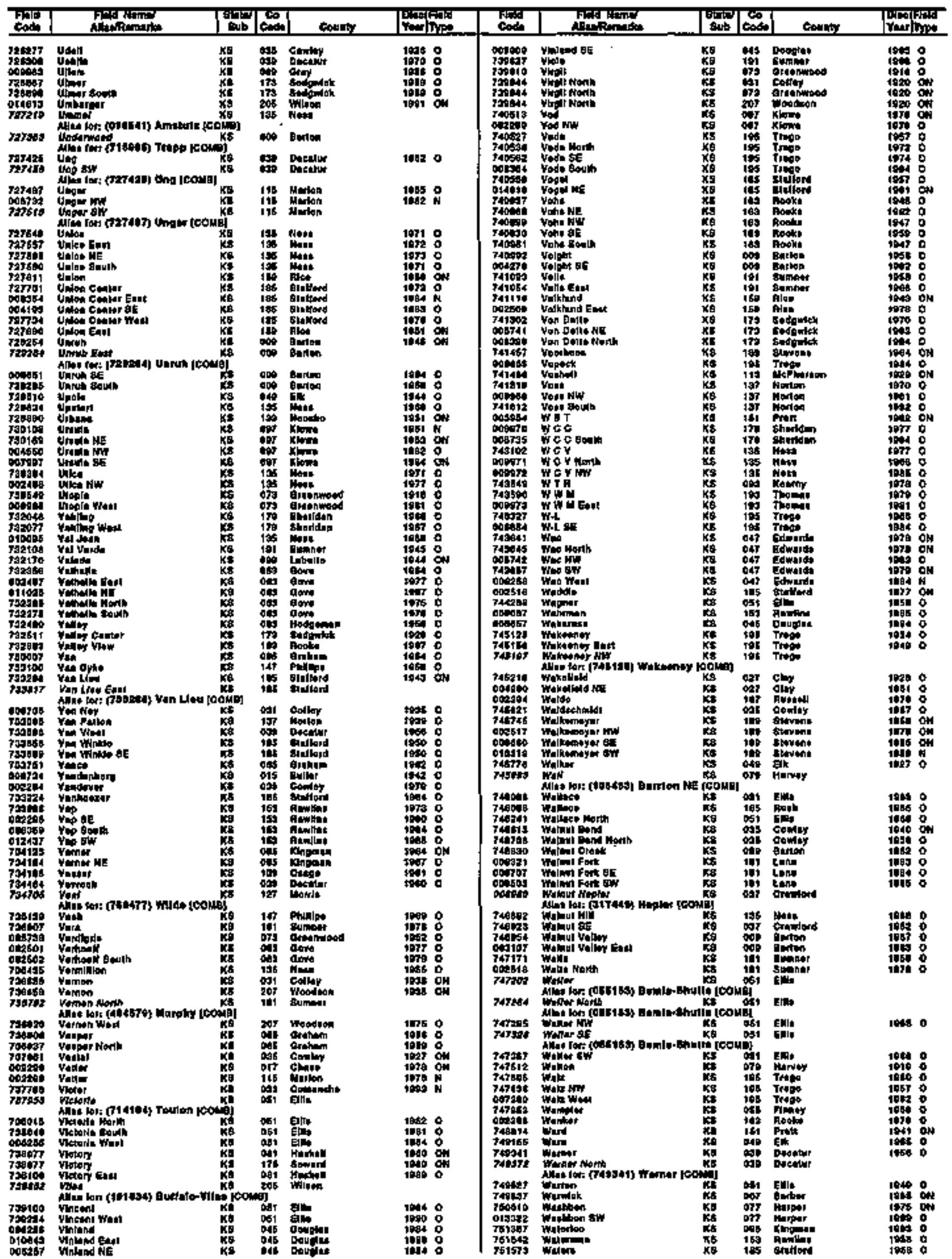




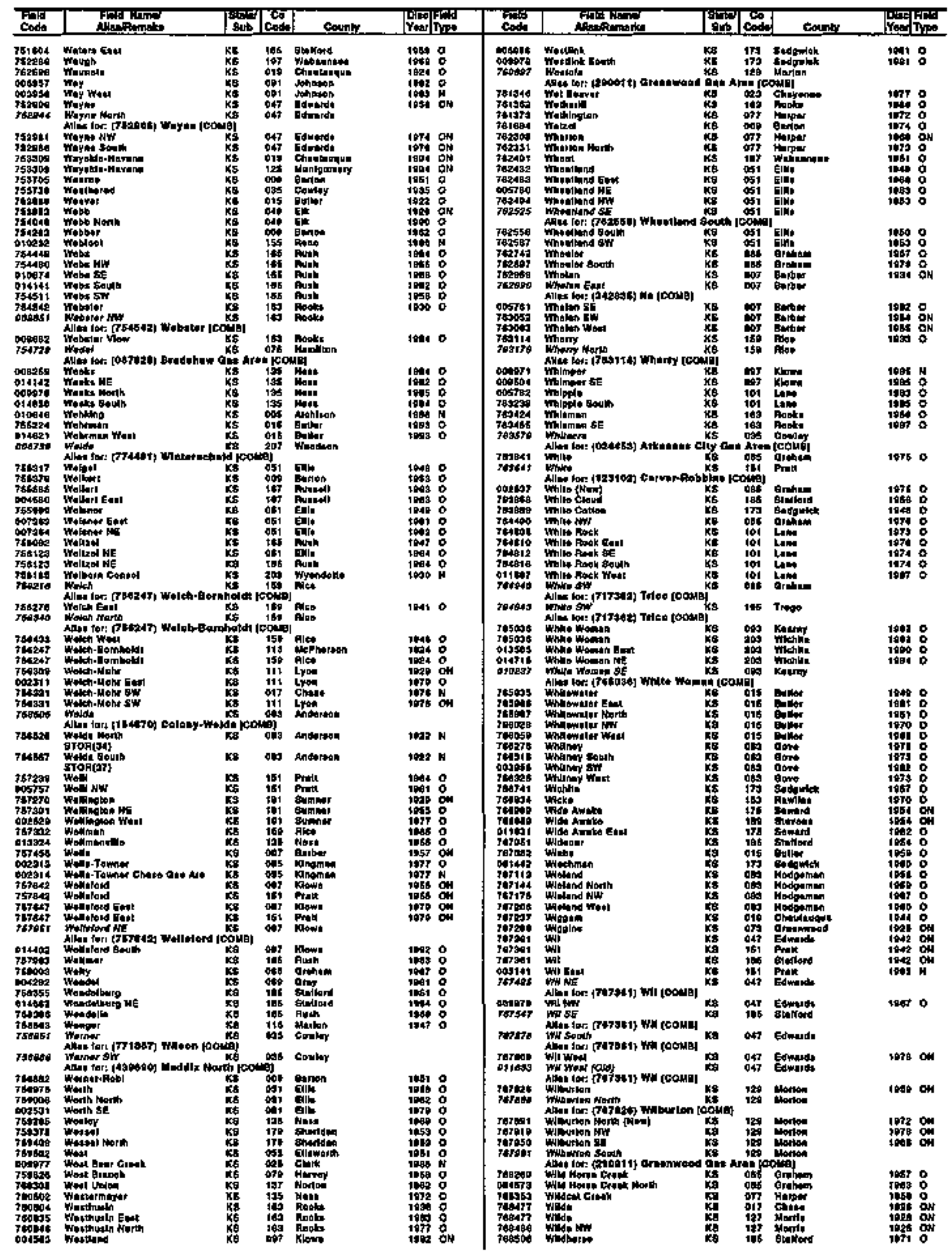


KANSAS

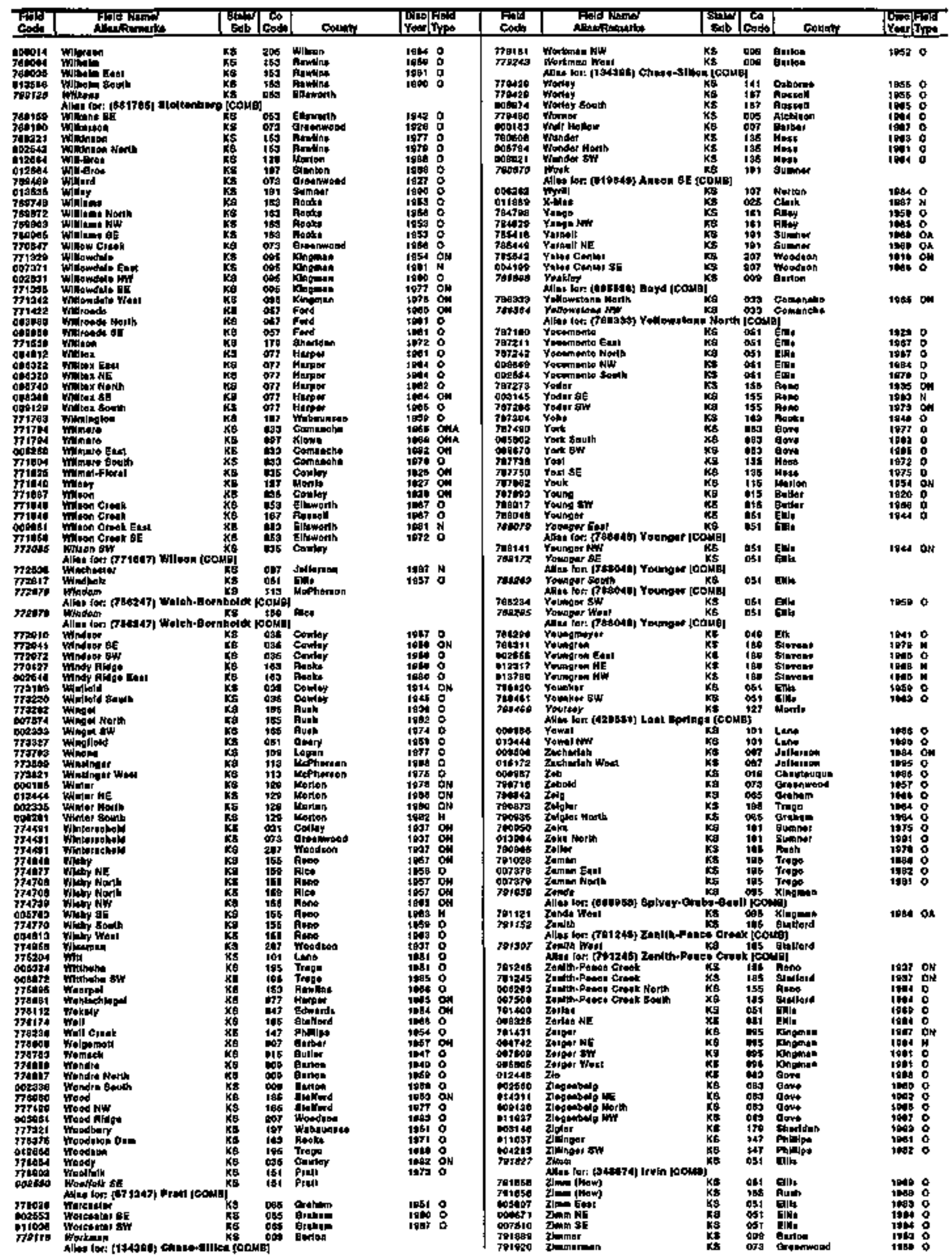




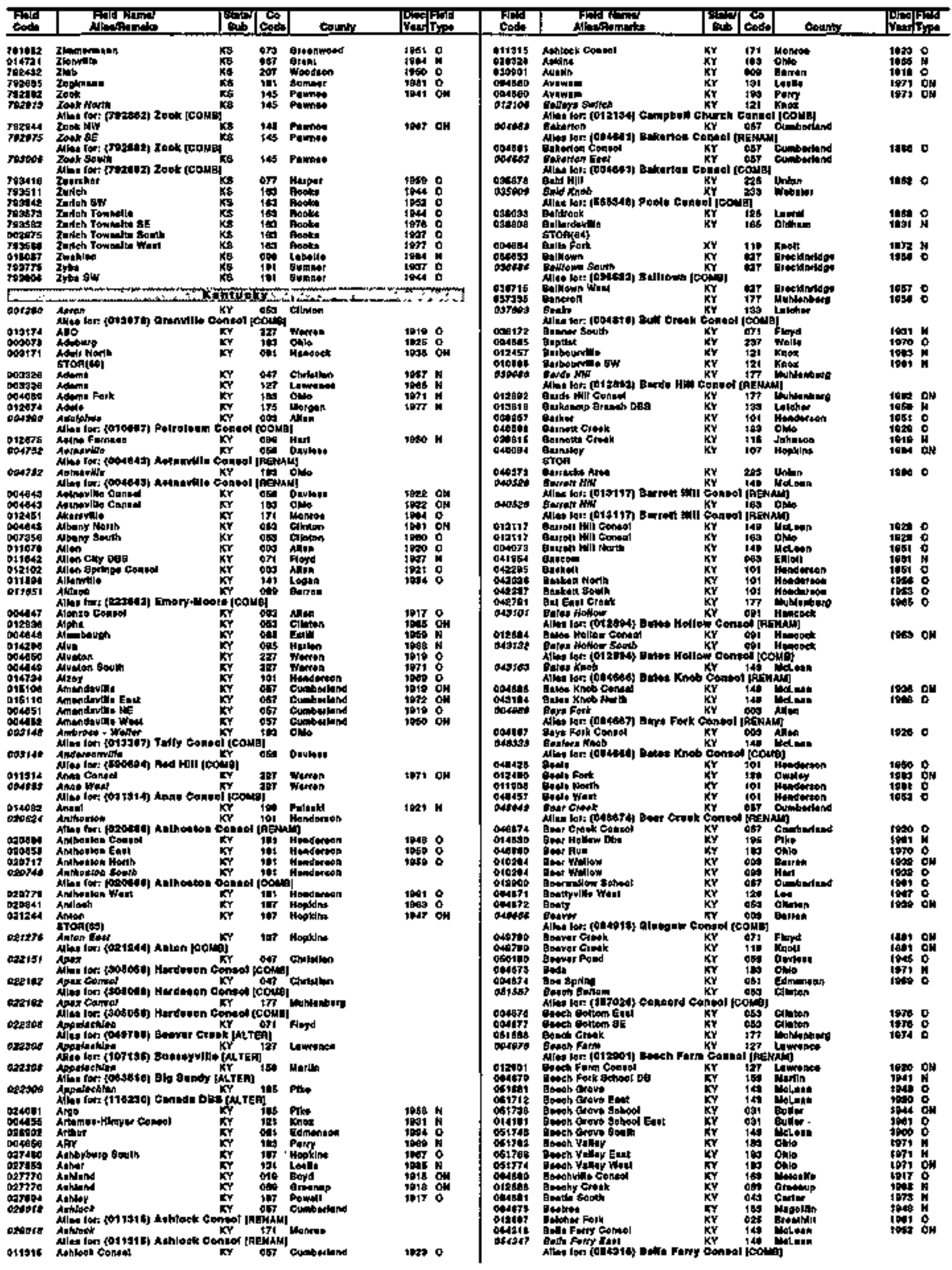


KENTUEKY

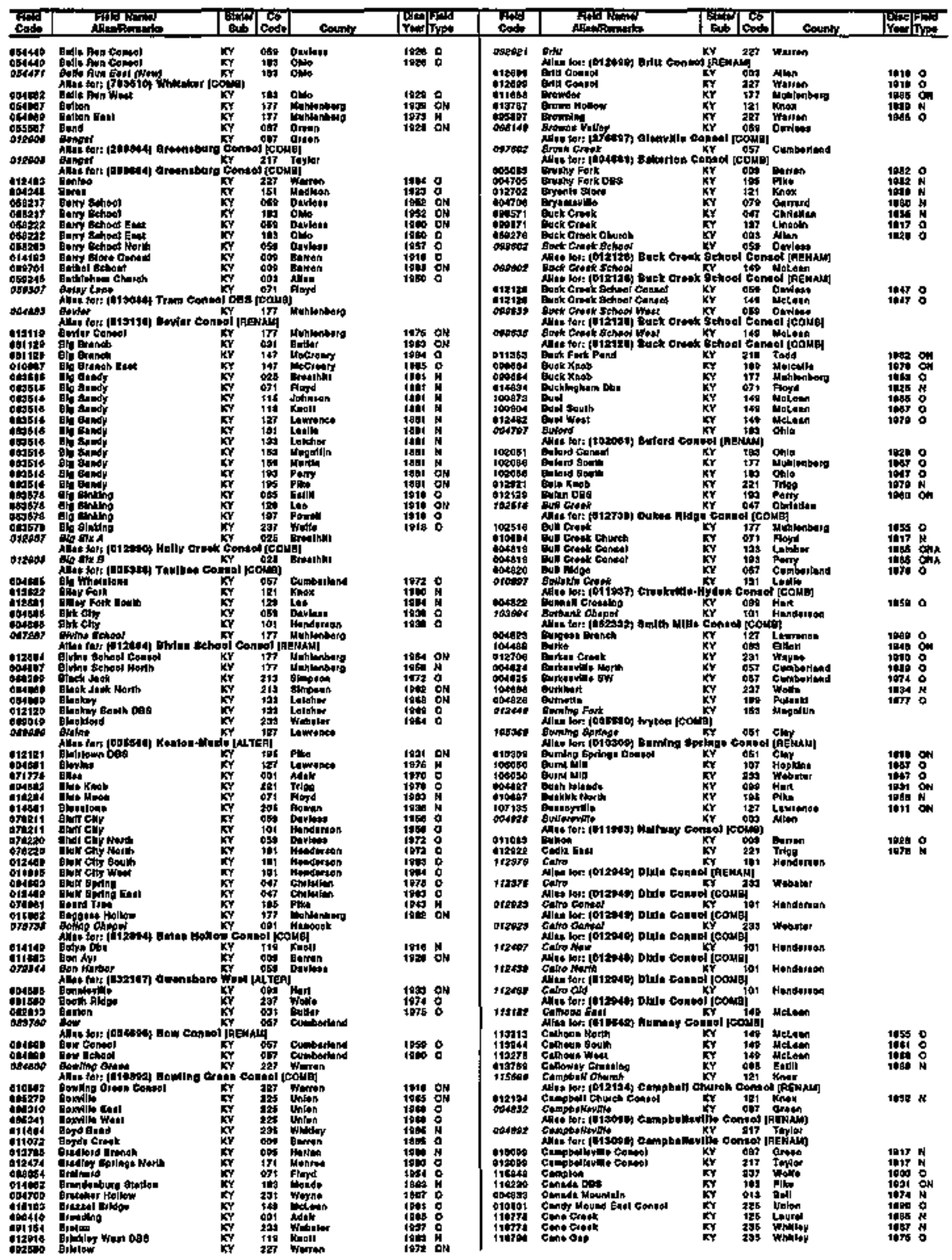




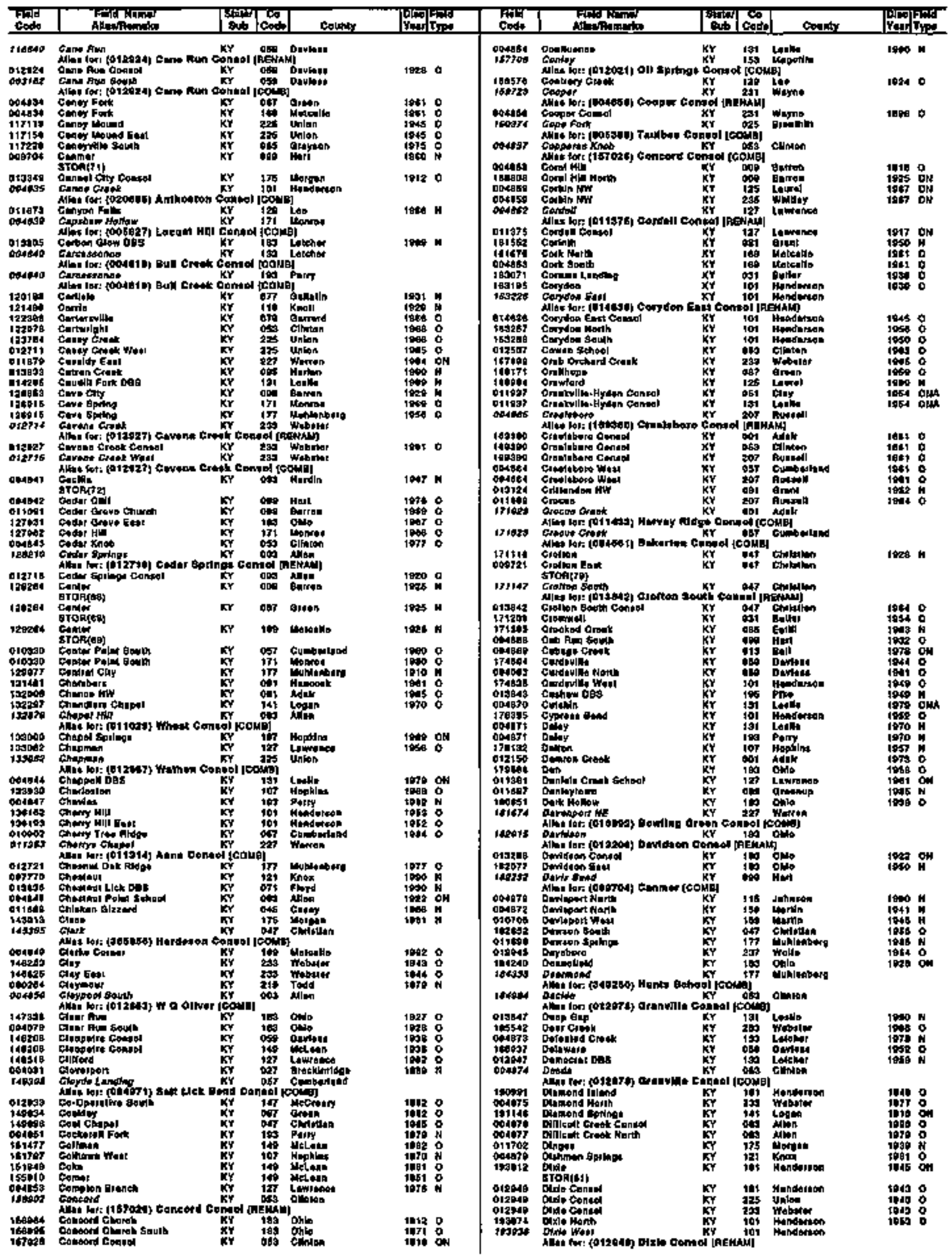


KENTUCKY

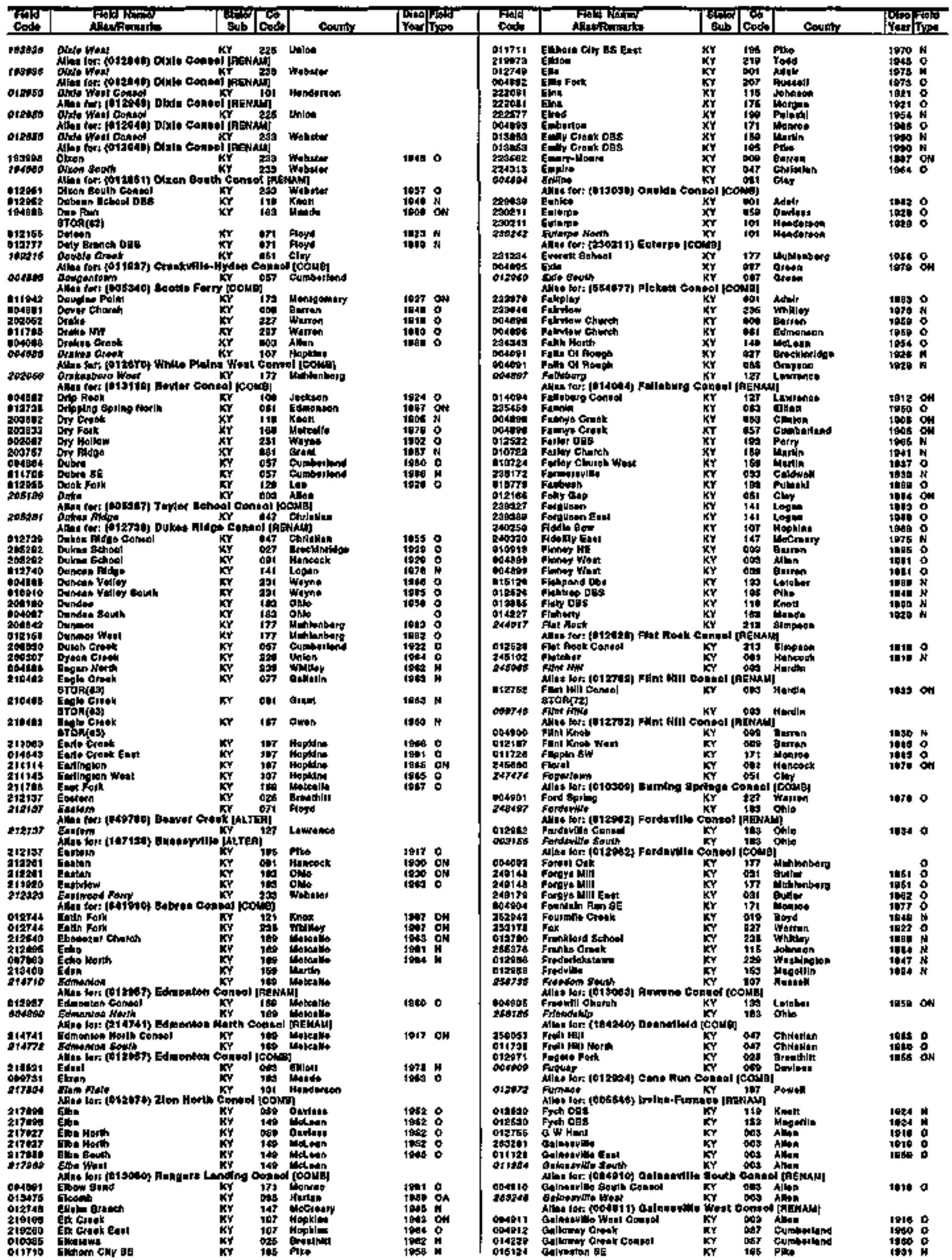




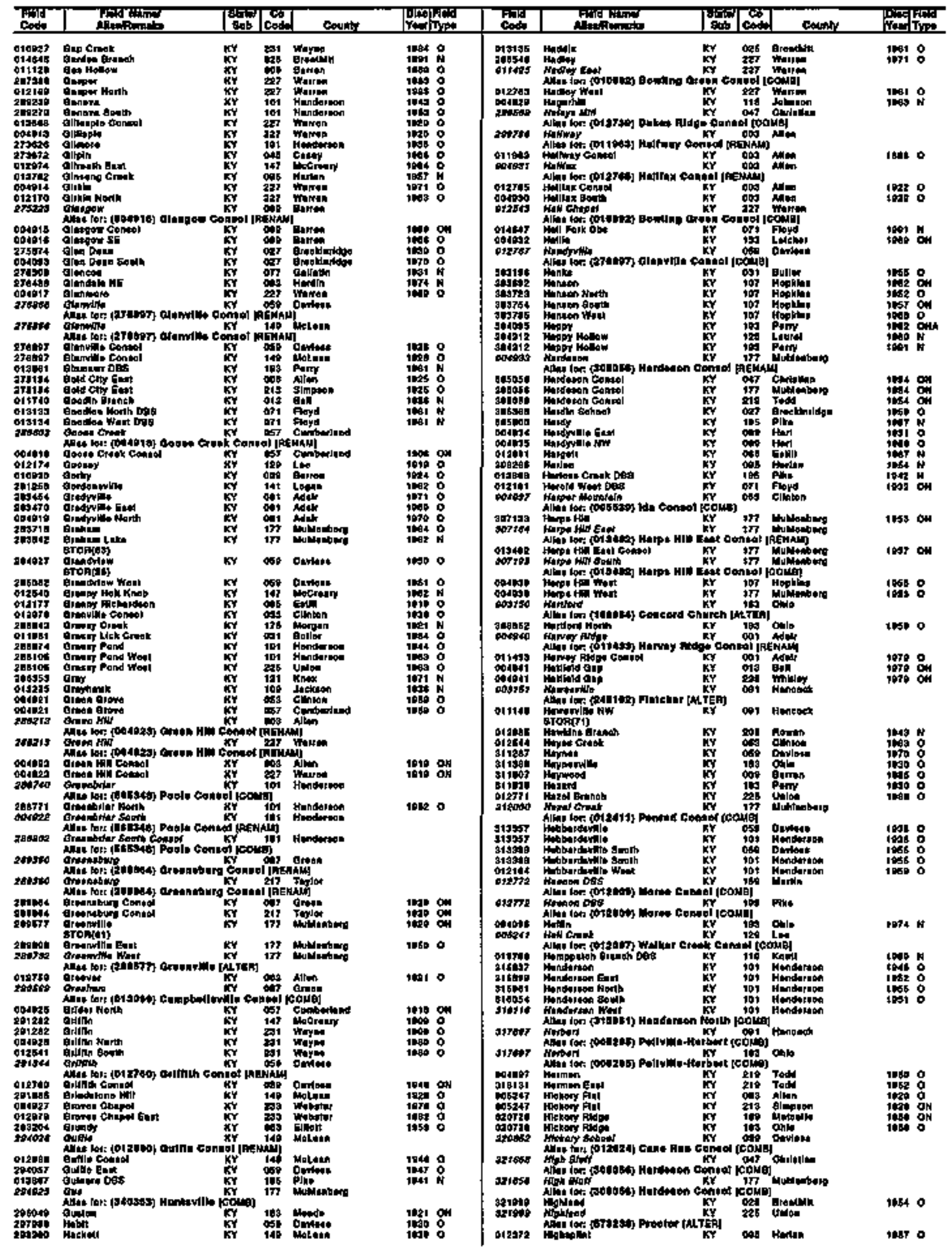




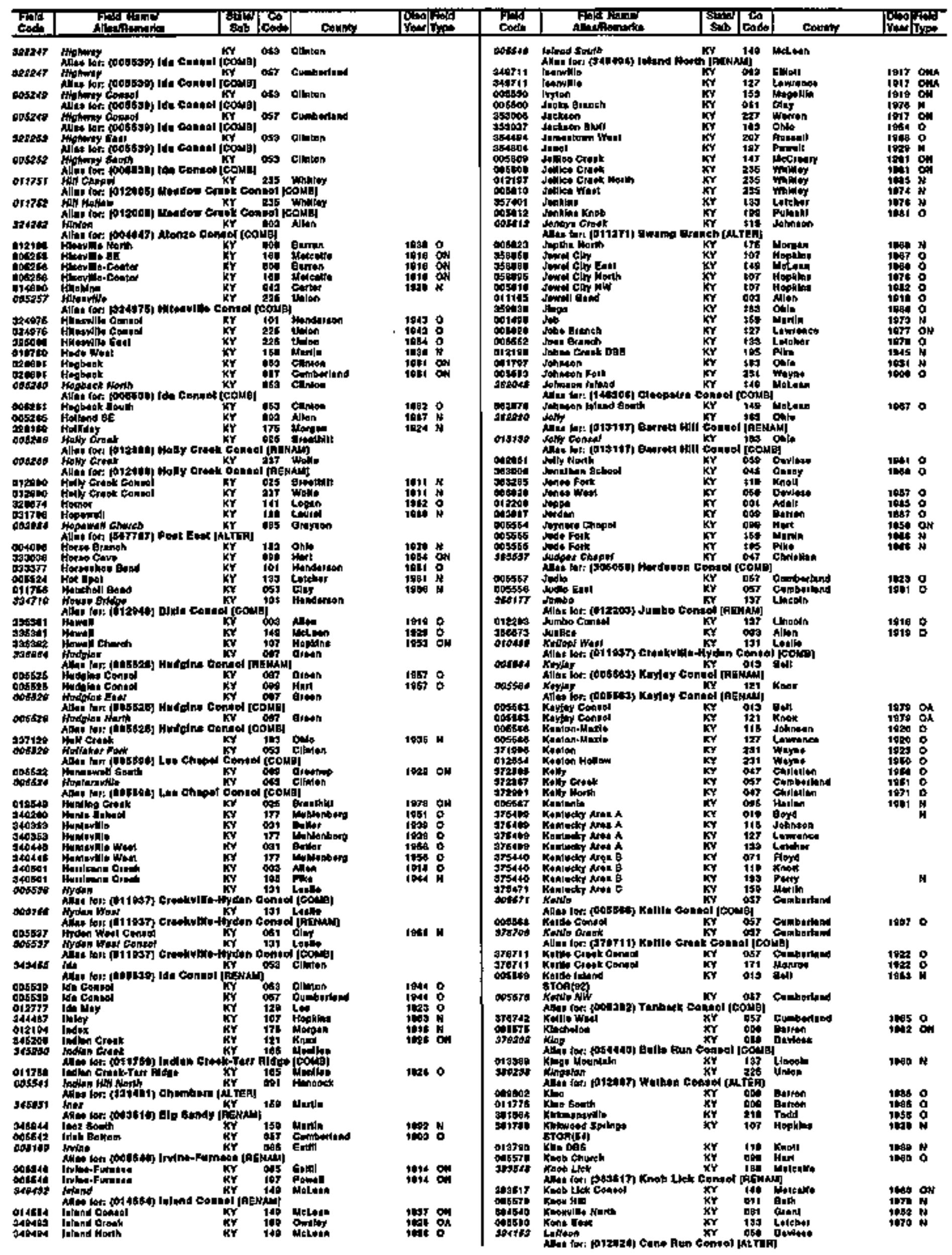




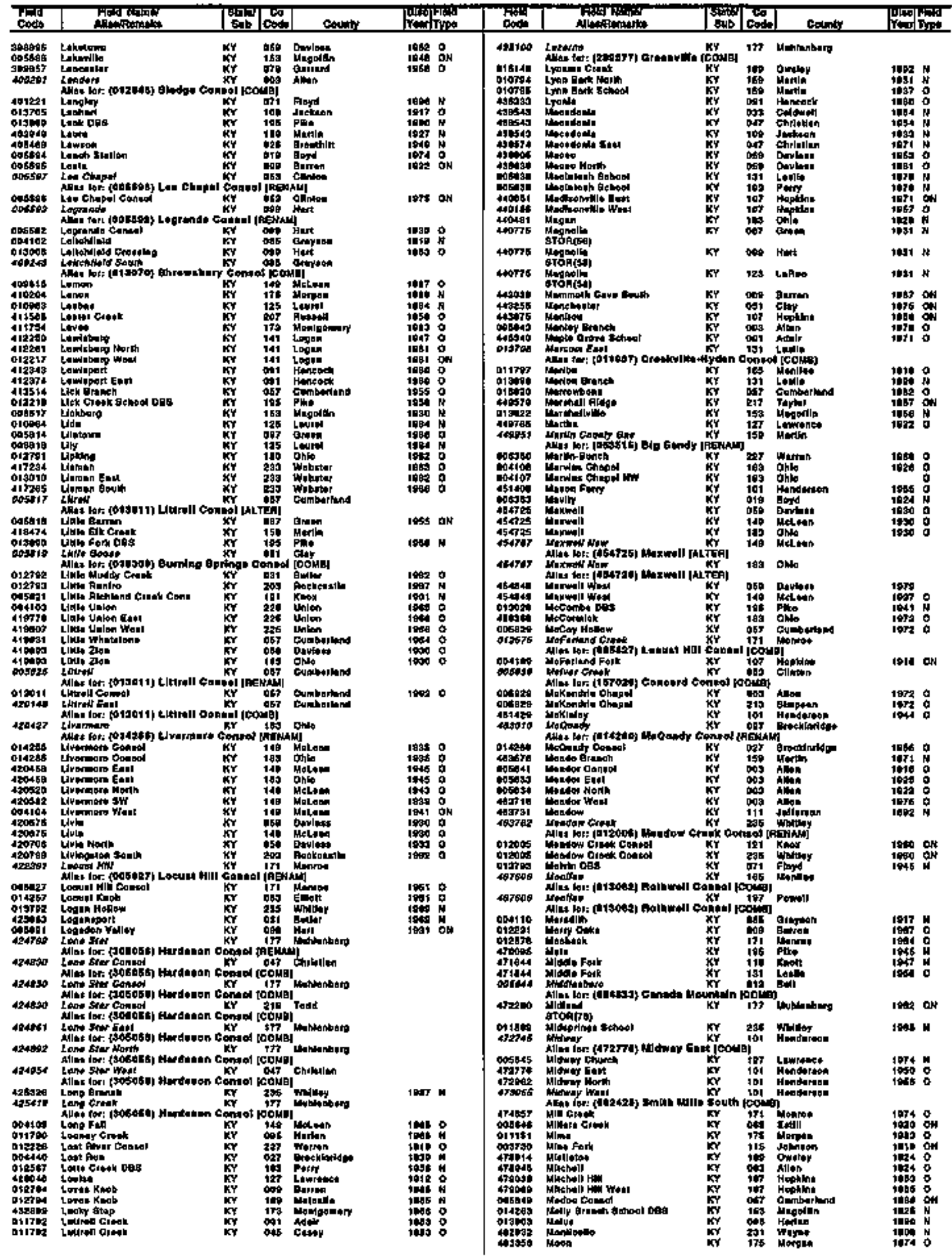


KENTUCKY

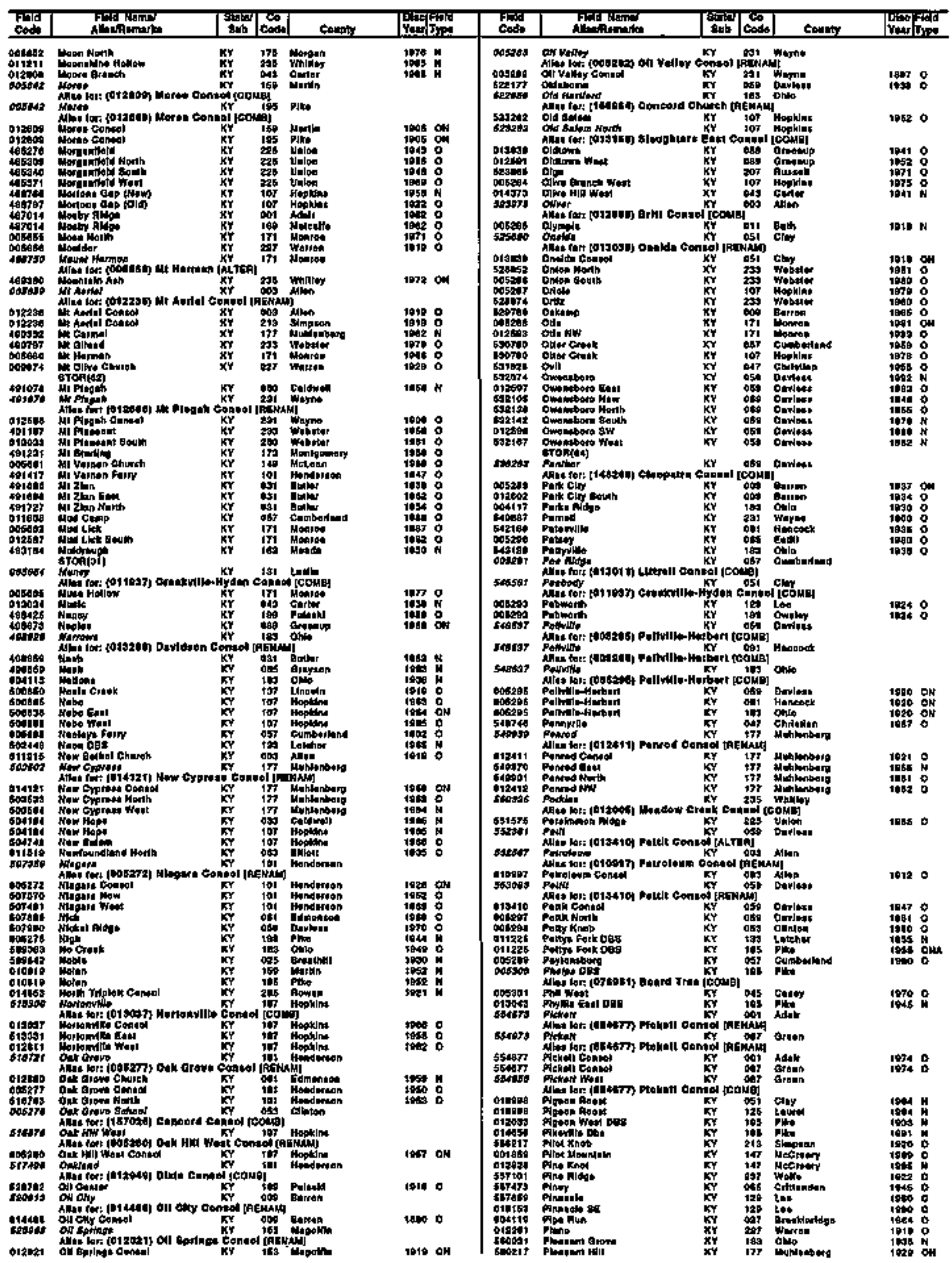




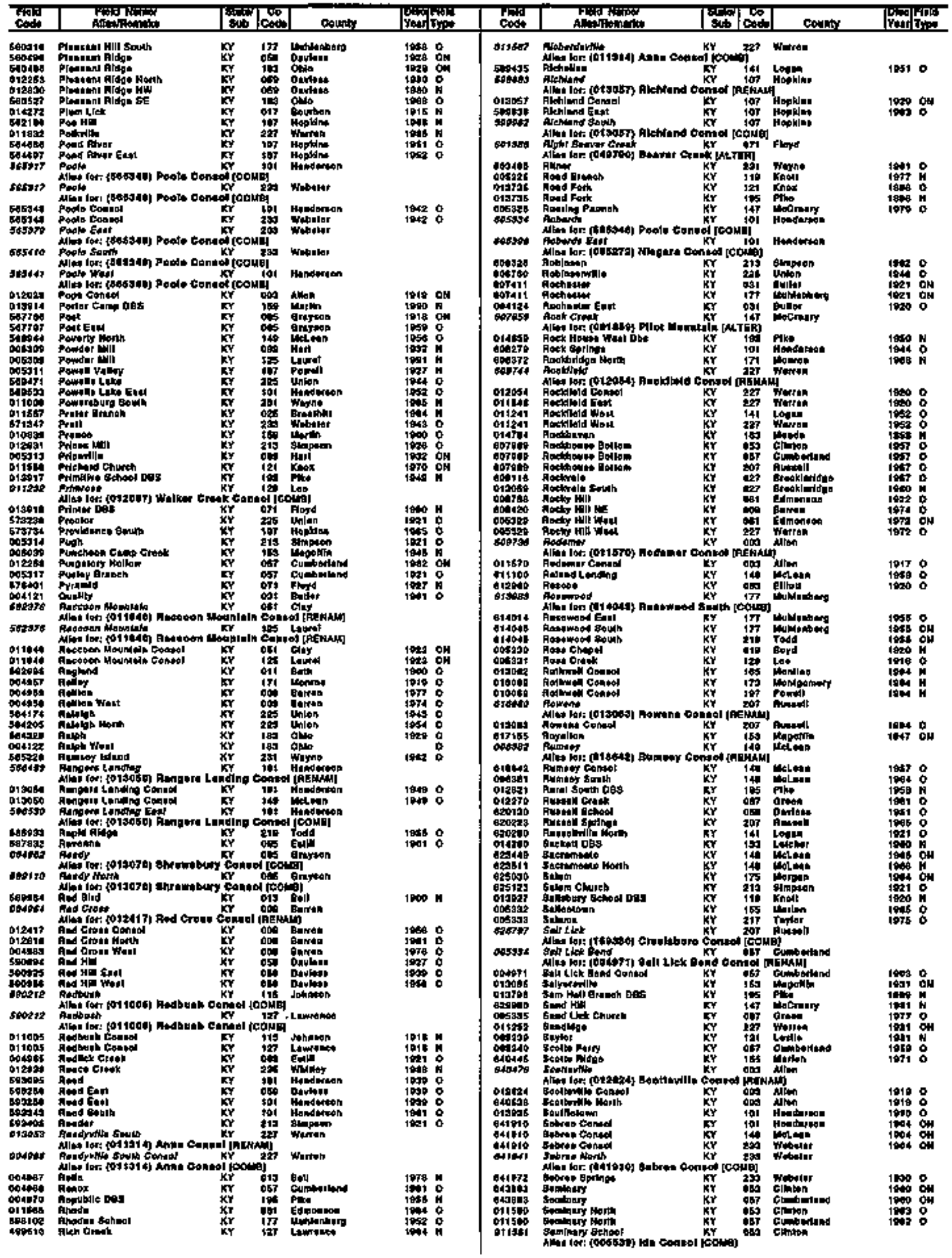


KENTUCKY

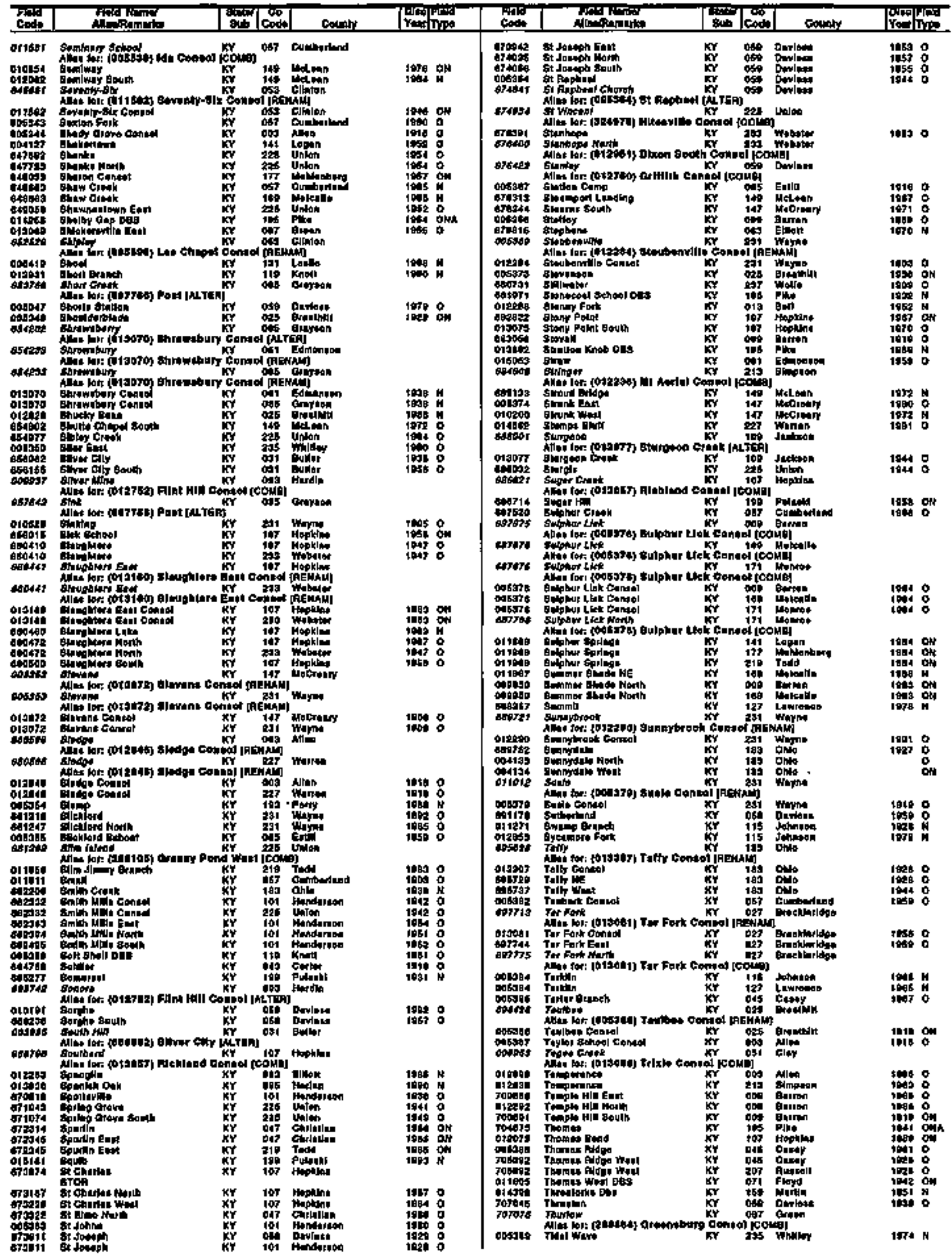




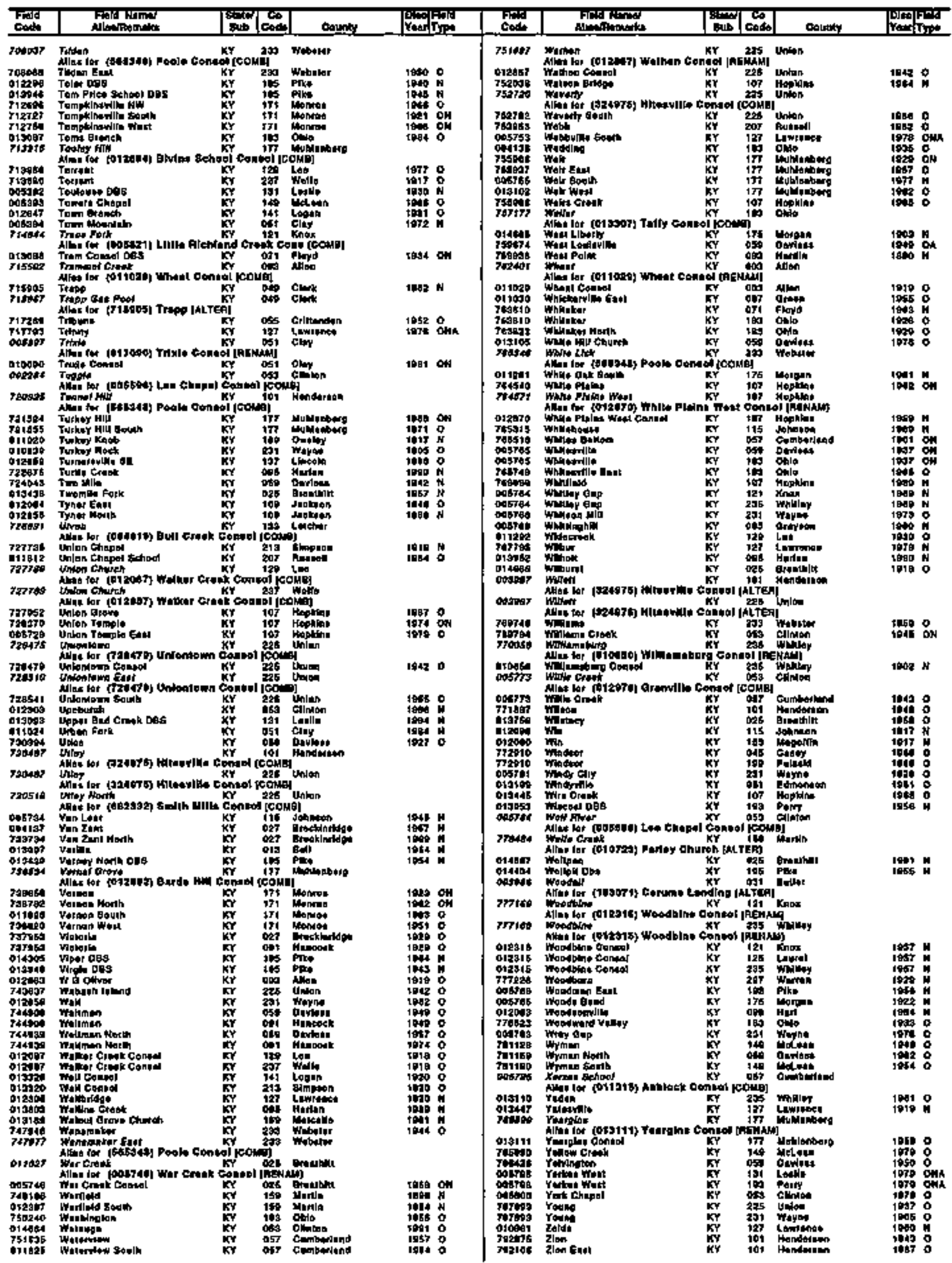


LOUISIANA

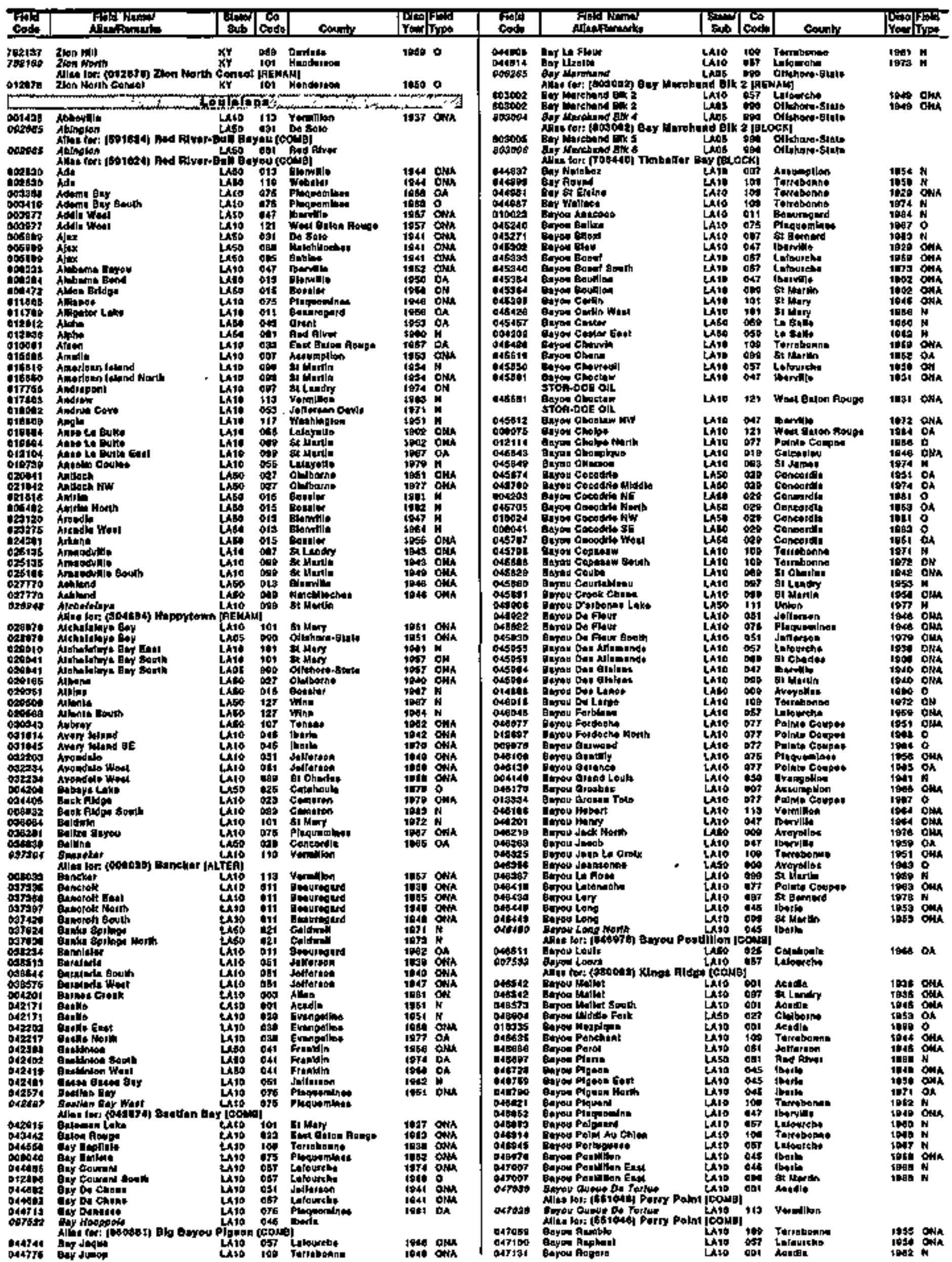




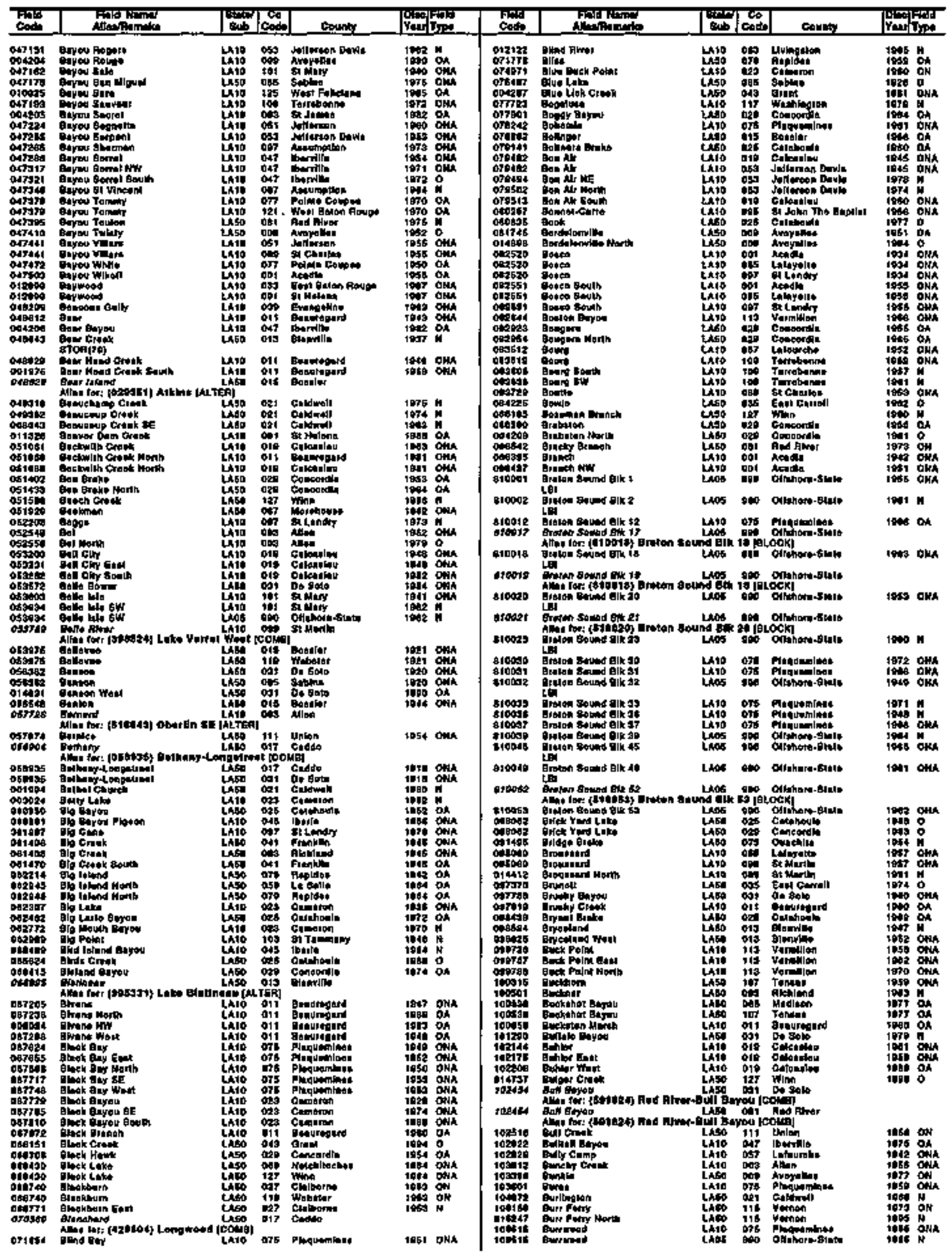


LOUISIANA

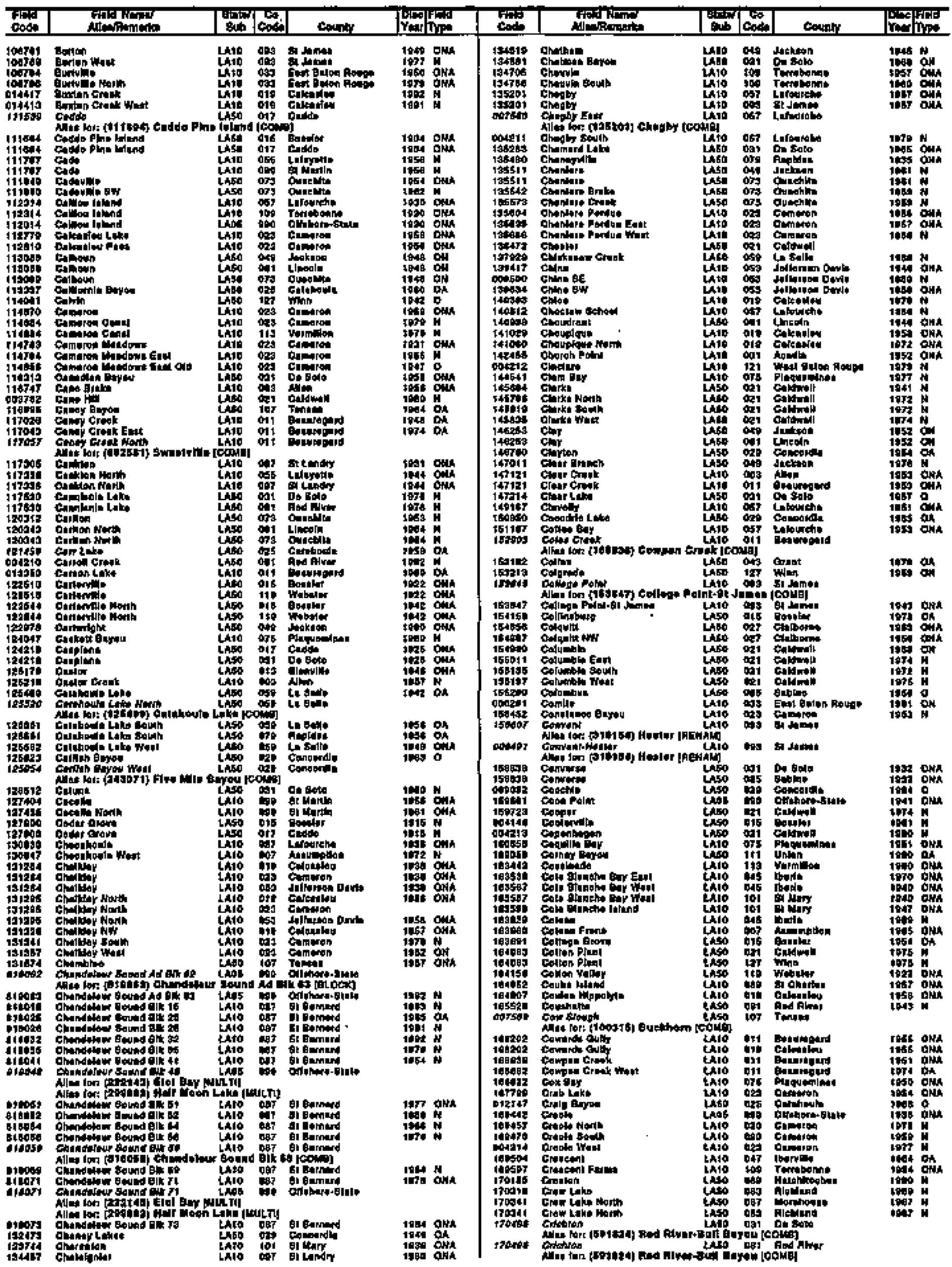




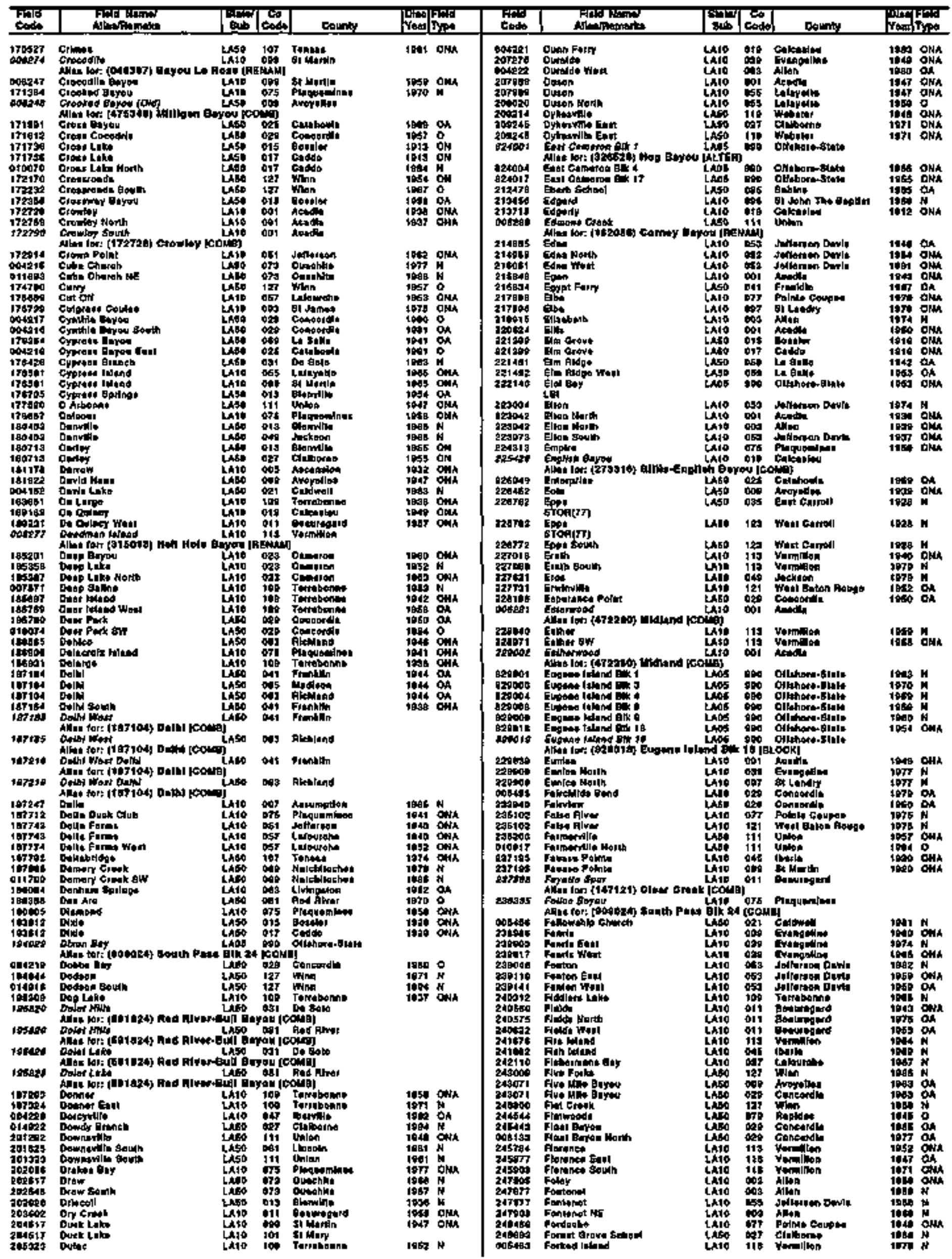


LOUISIANA

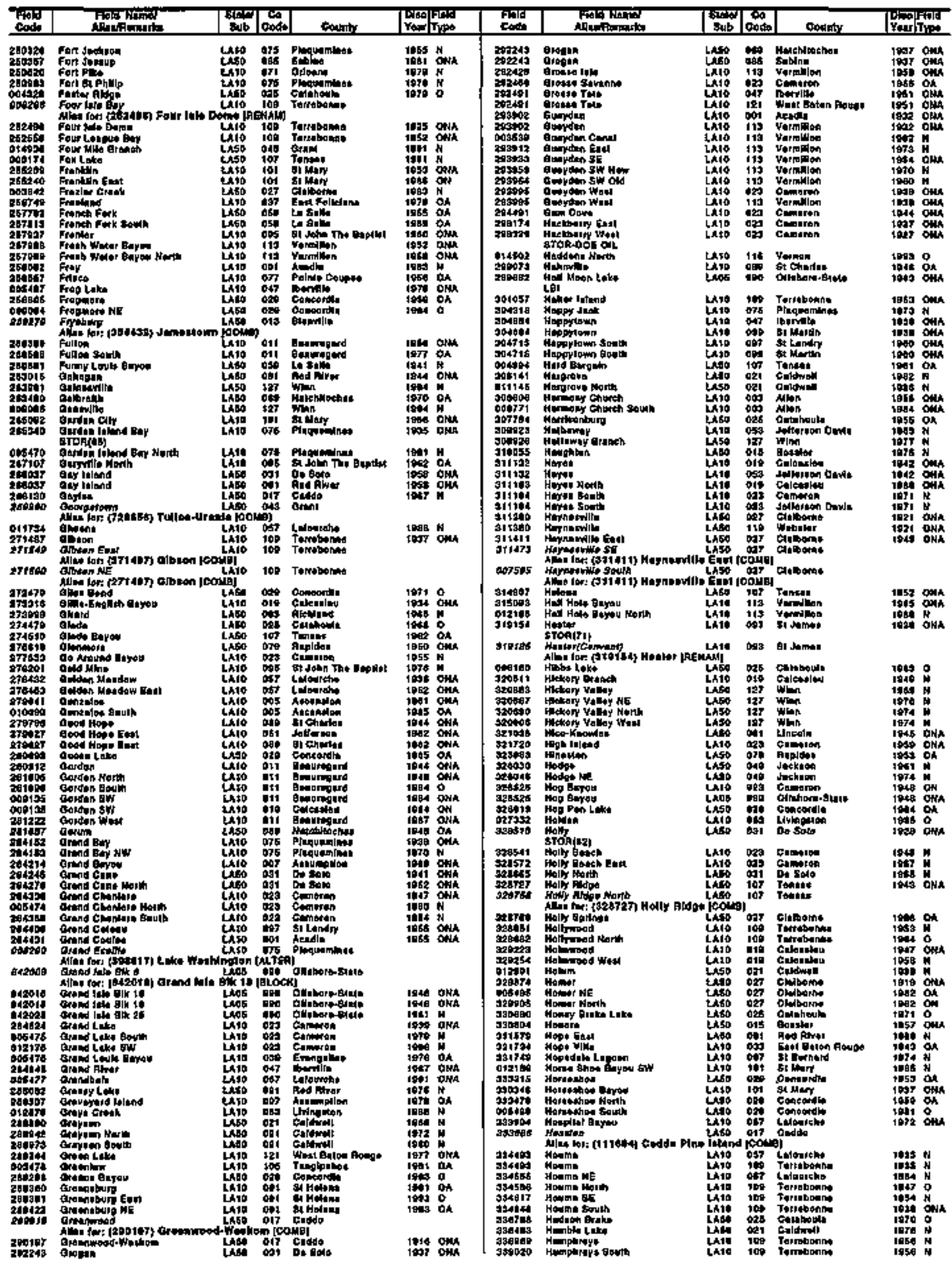




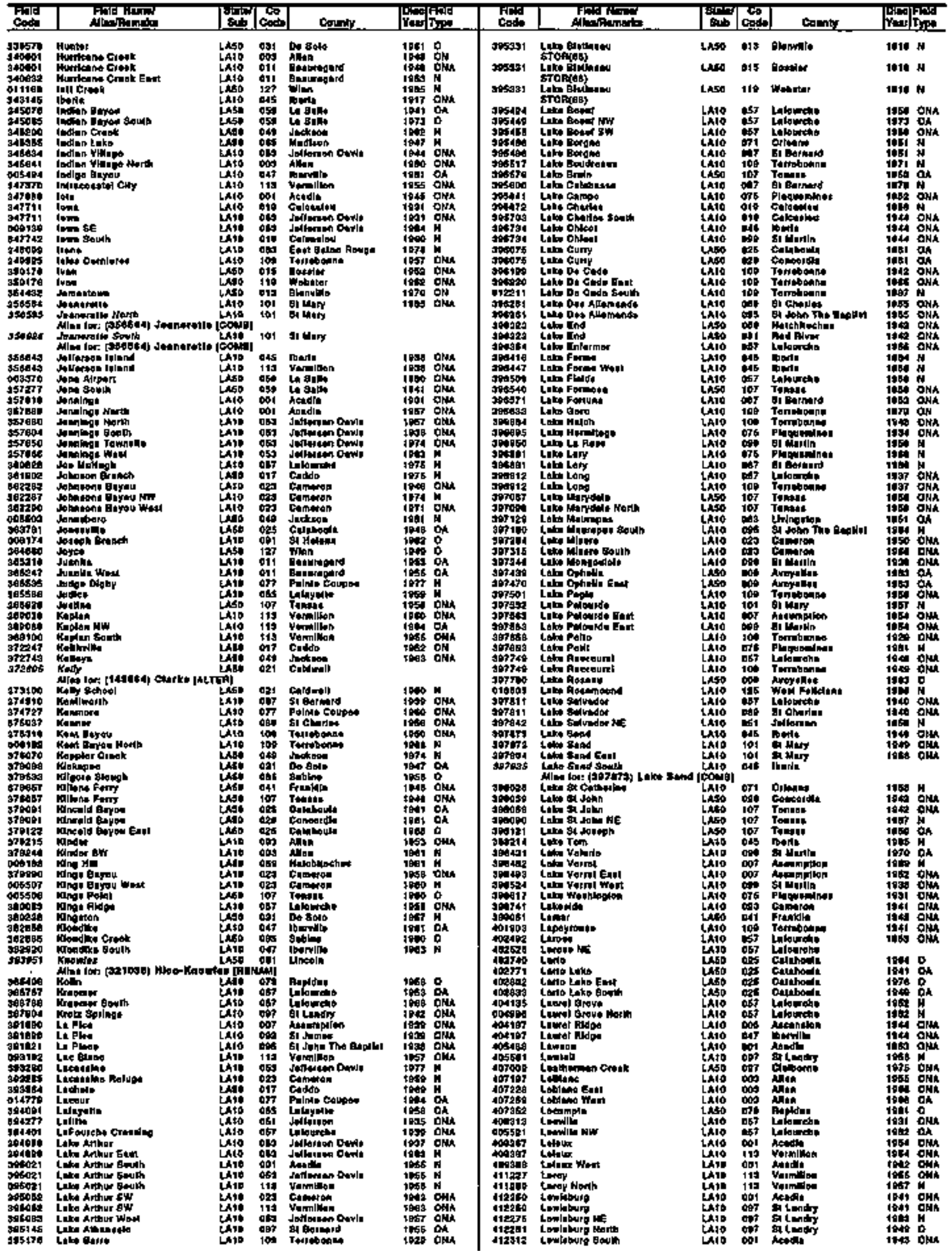


LOUISIANA

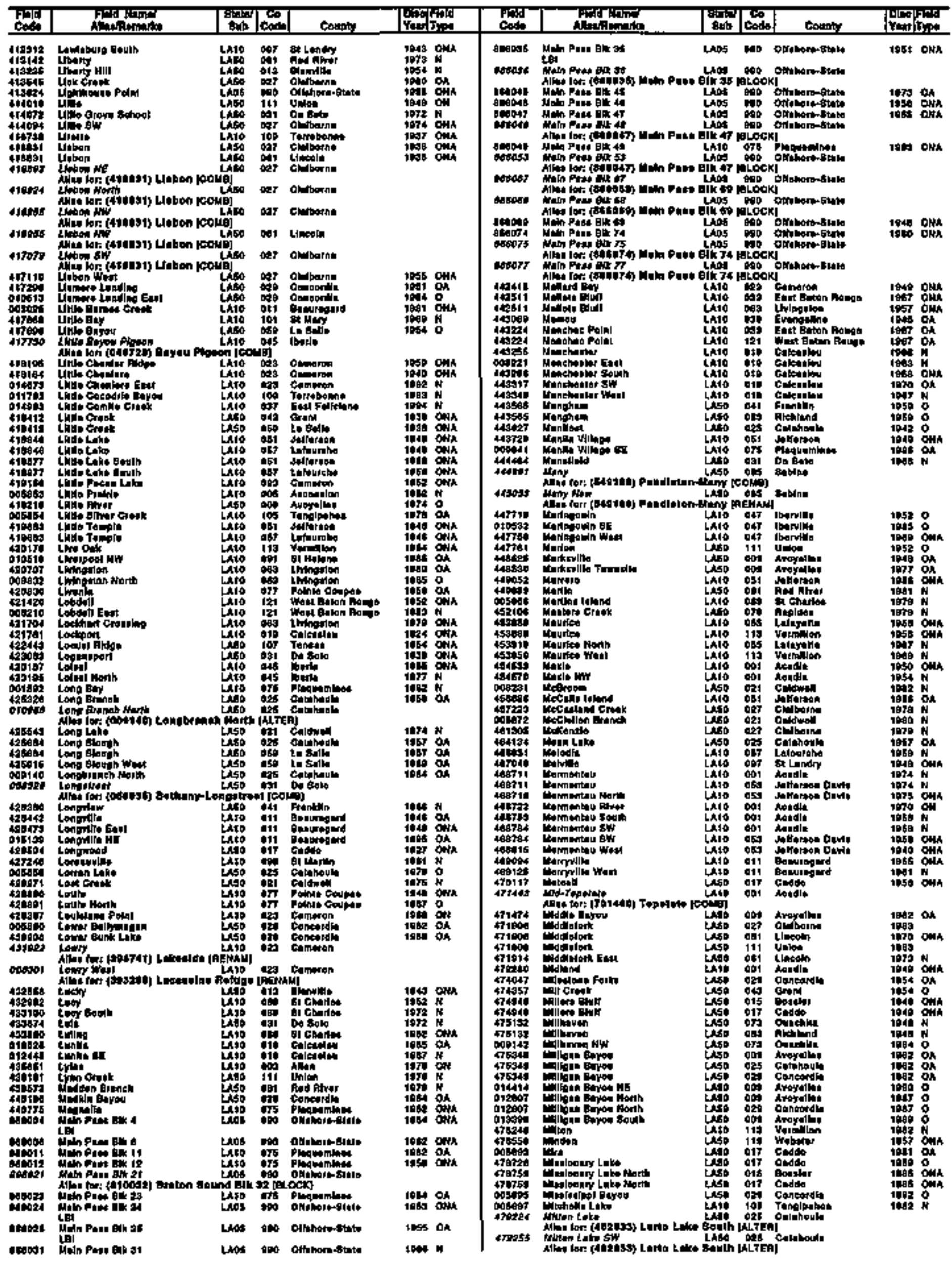


LOUISIANA

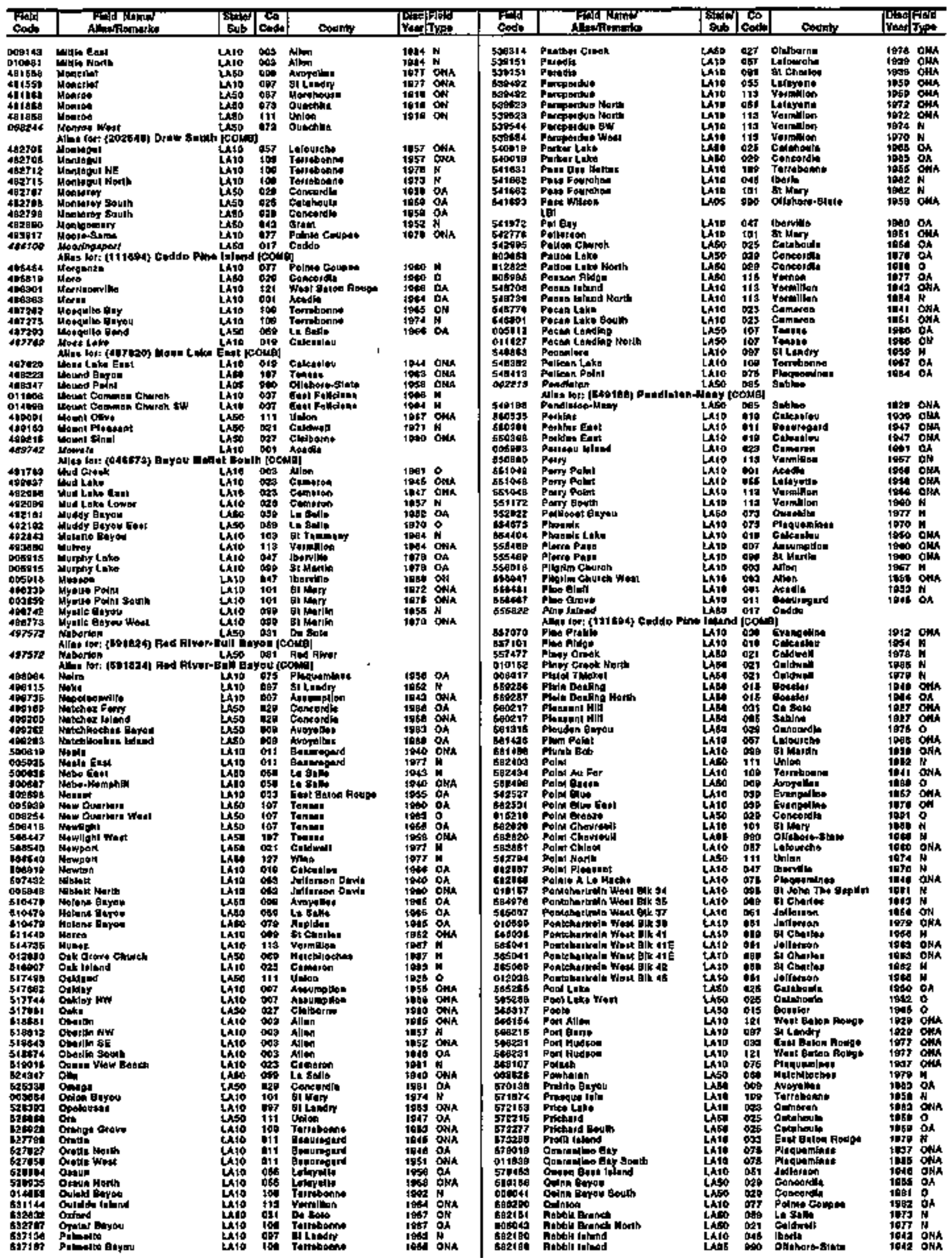


LOUISIANA

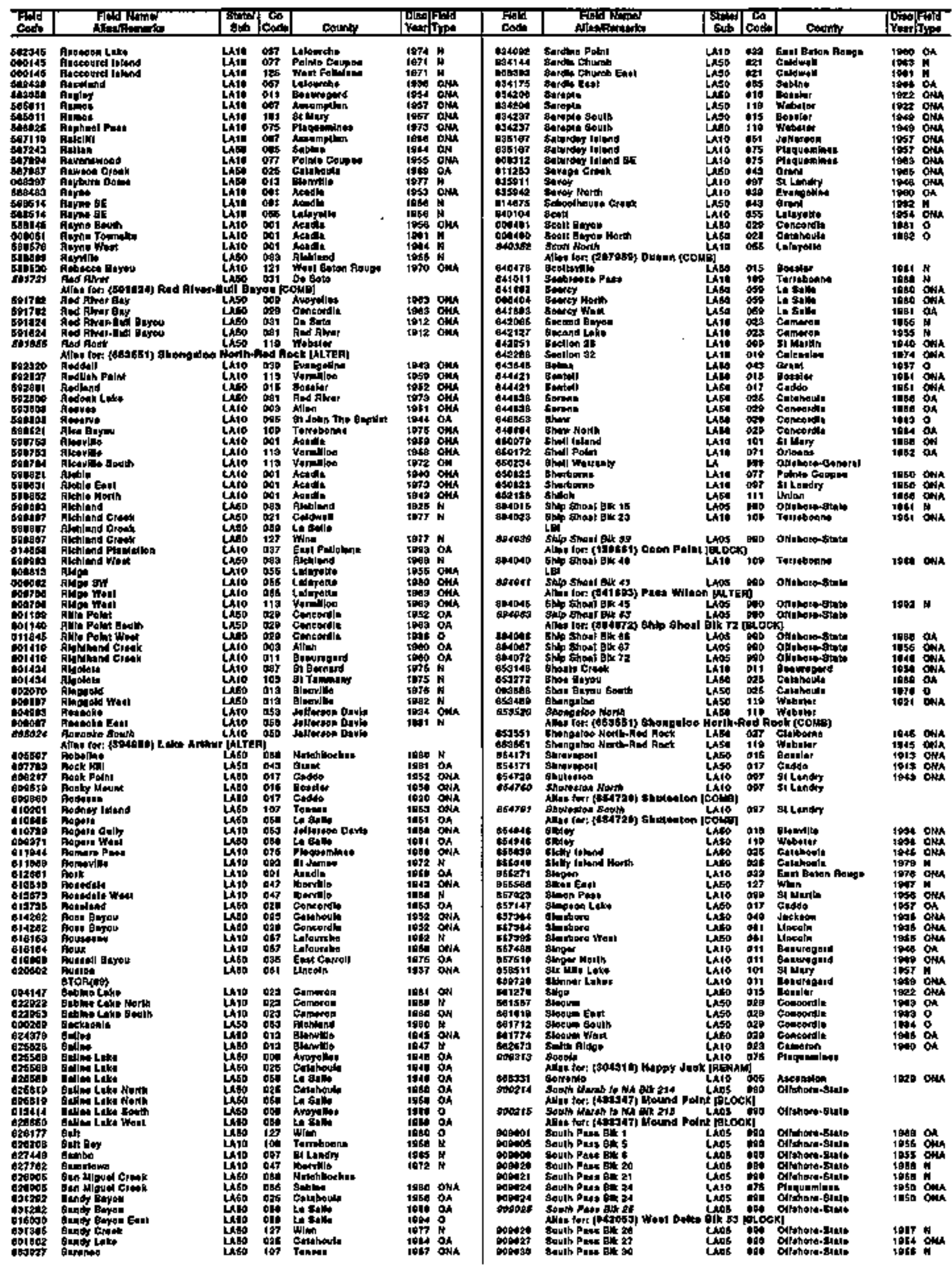




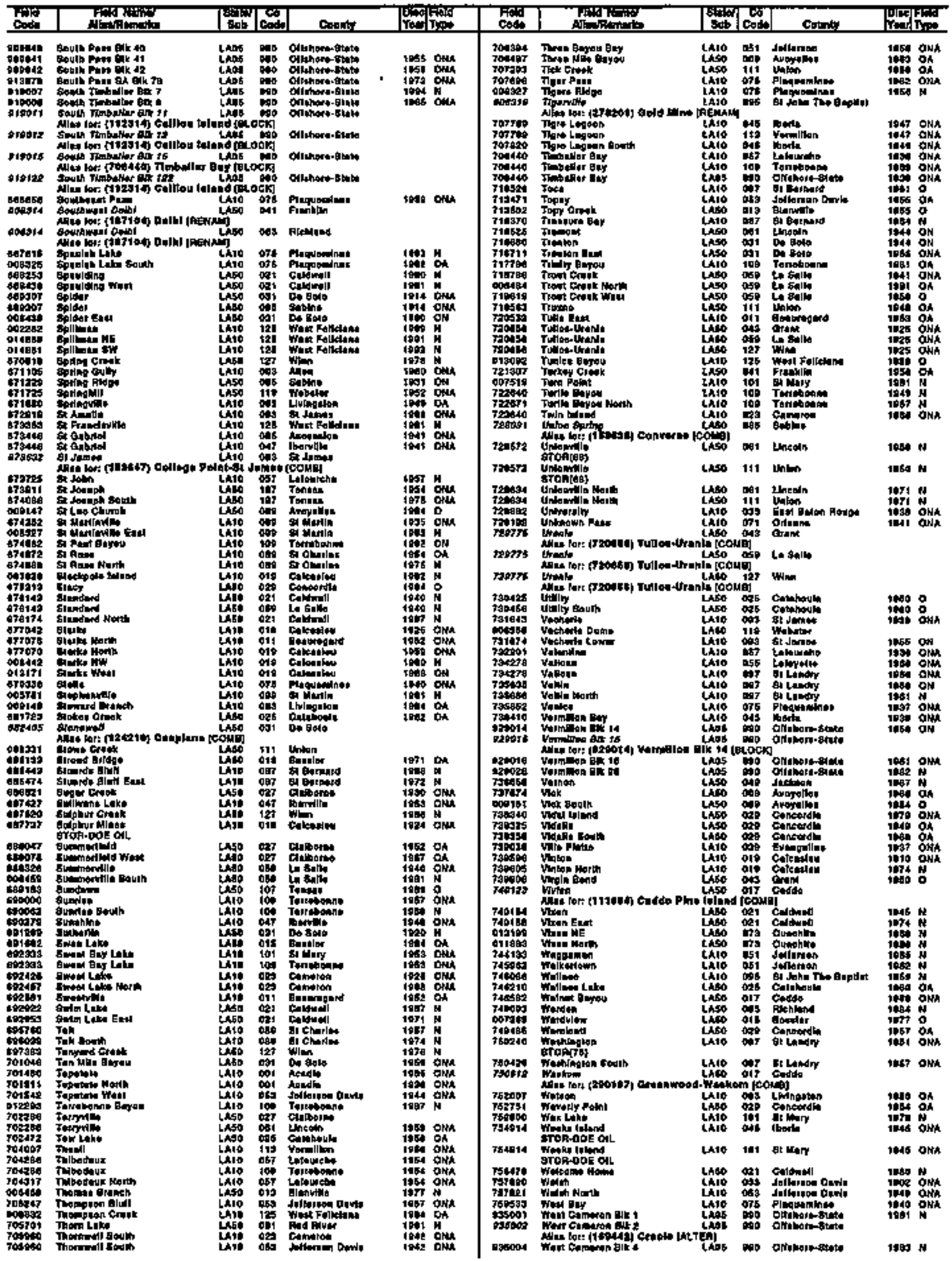


MICHIGAN

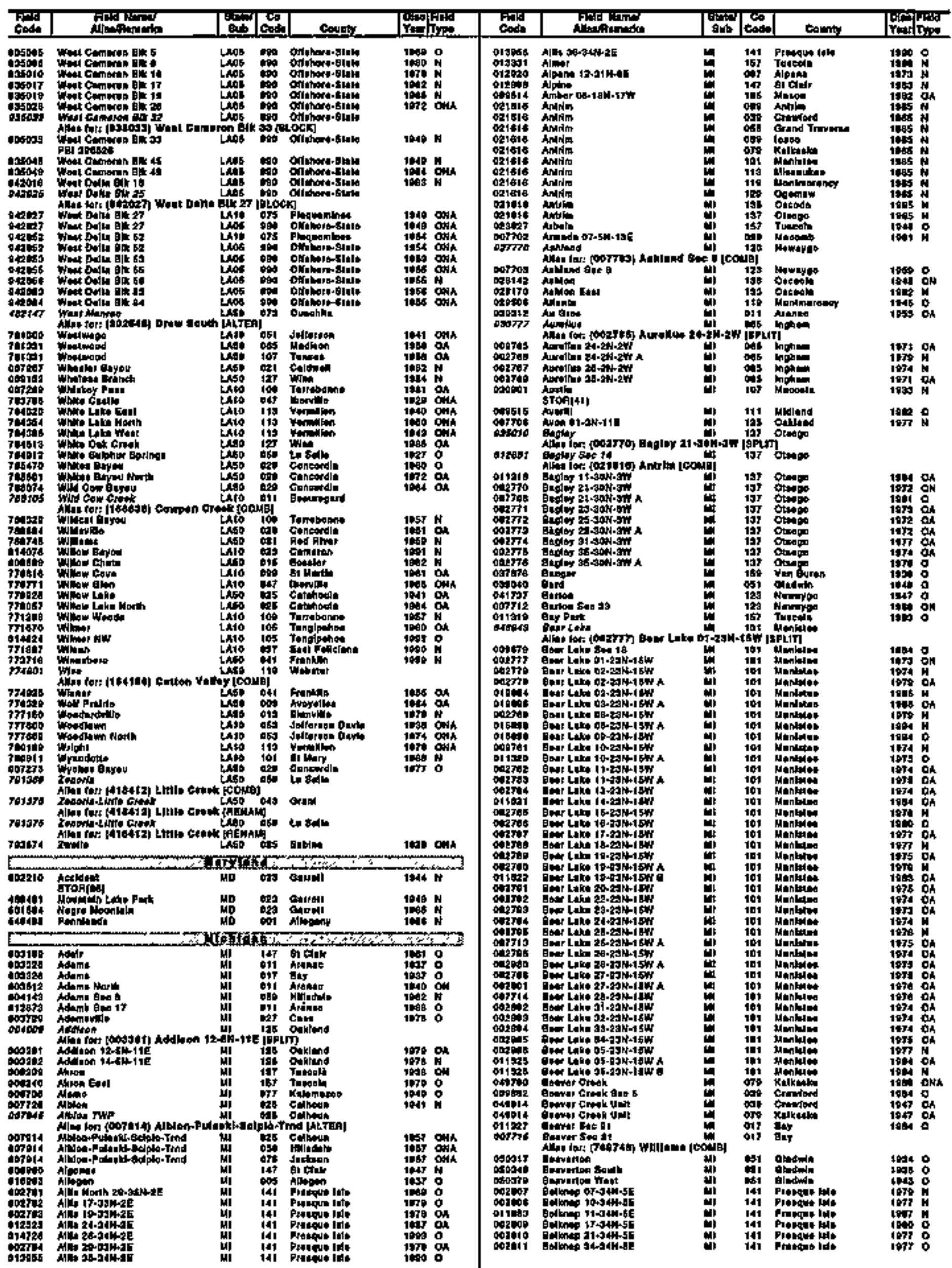


MICHIGAN

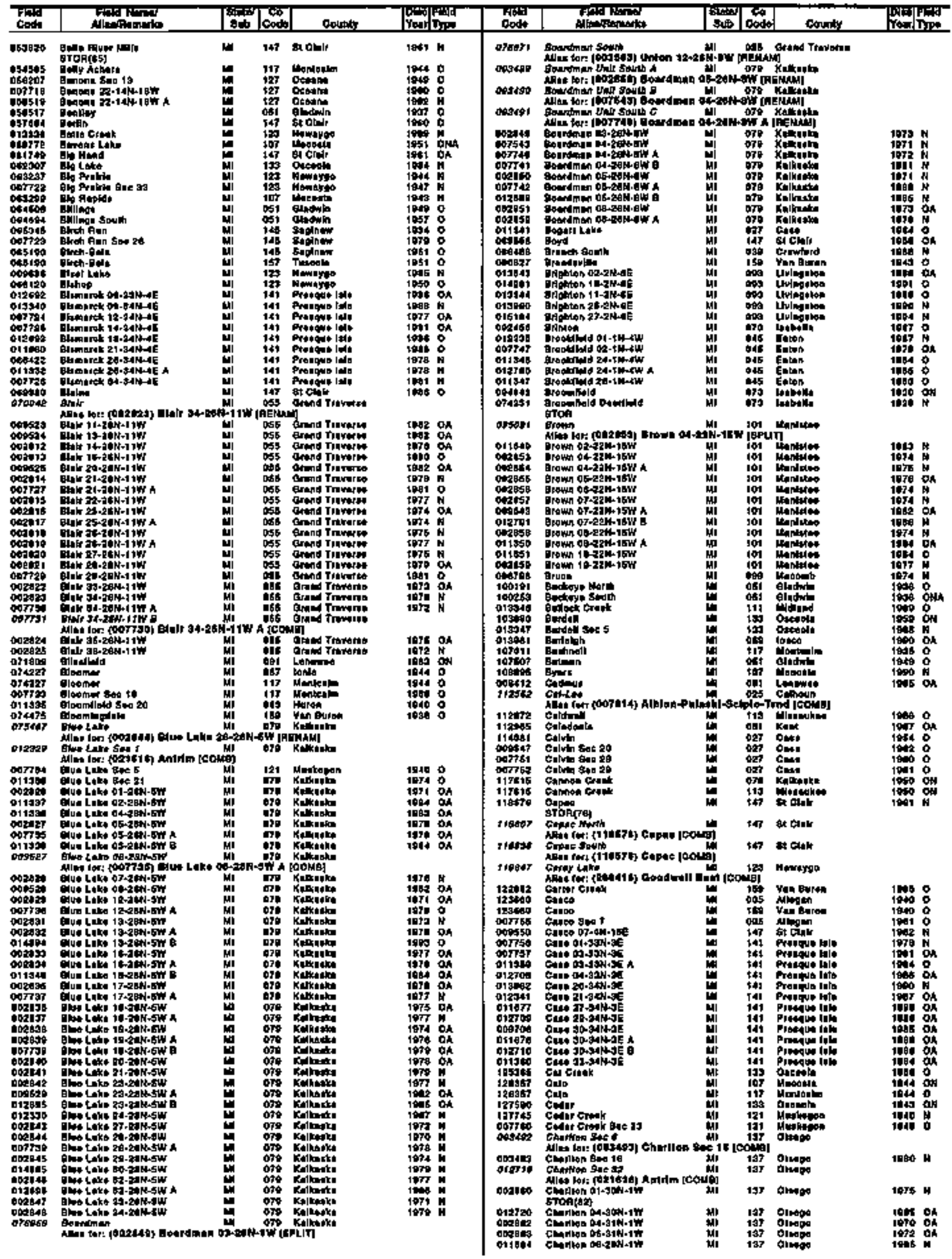


MICHIGAN

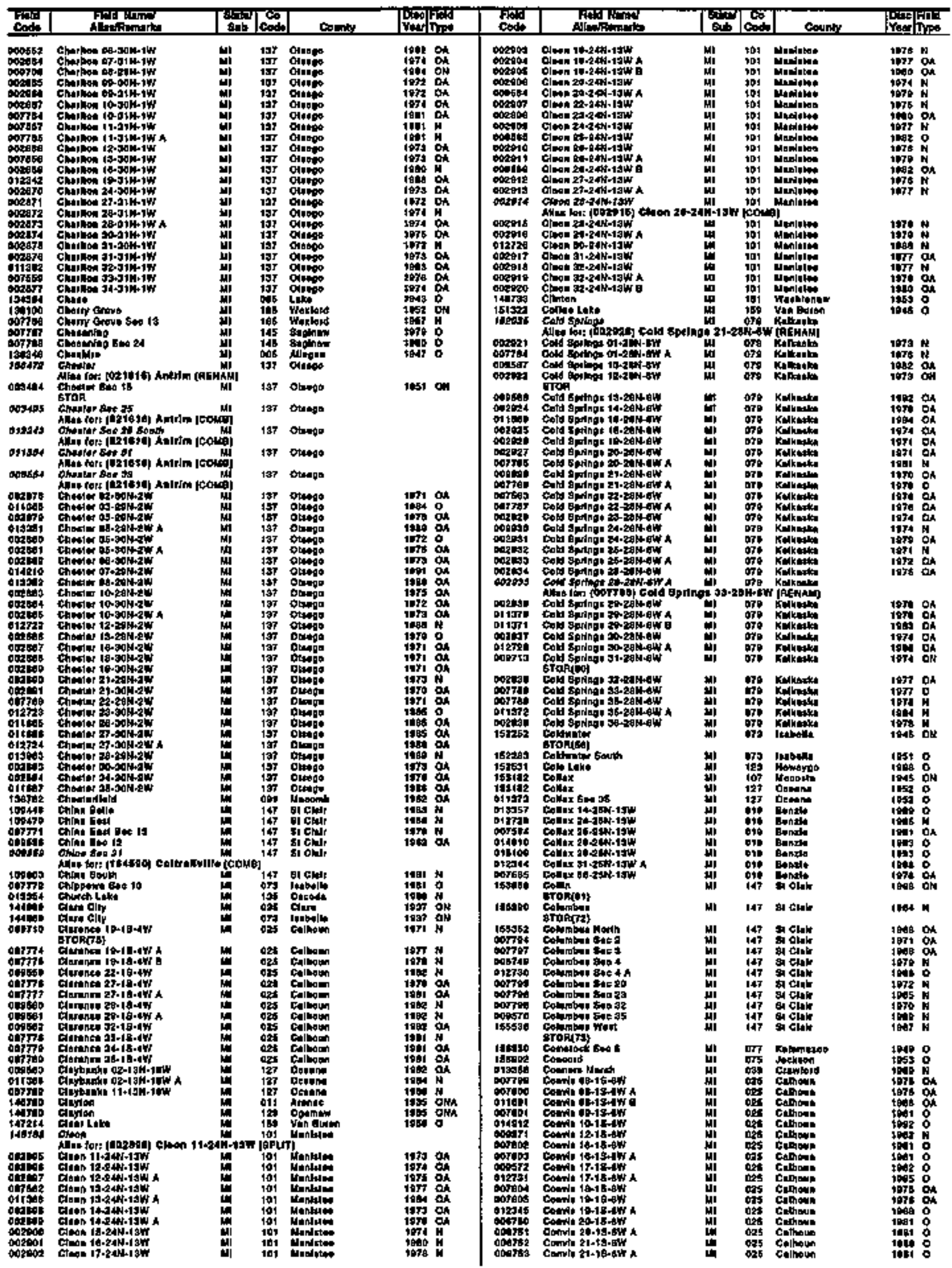




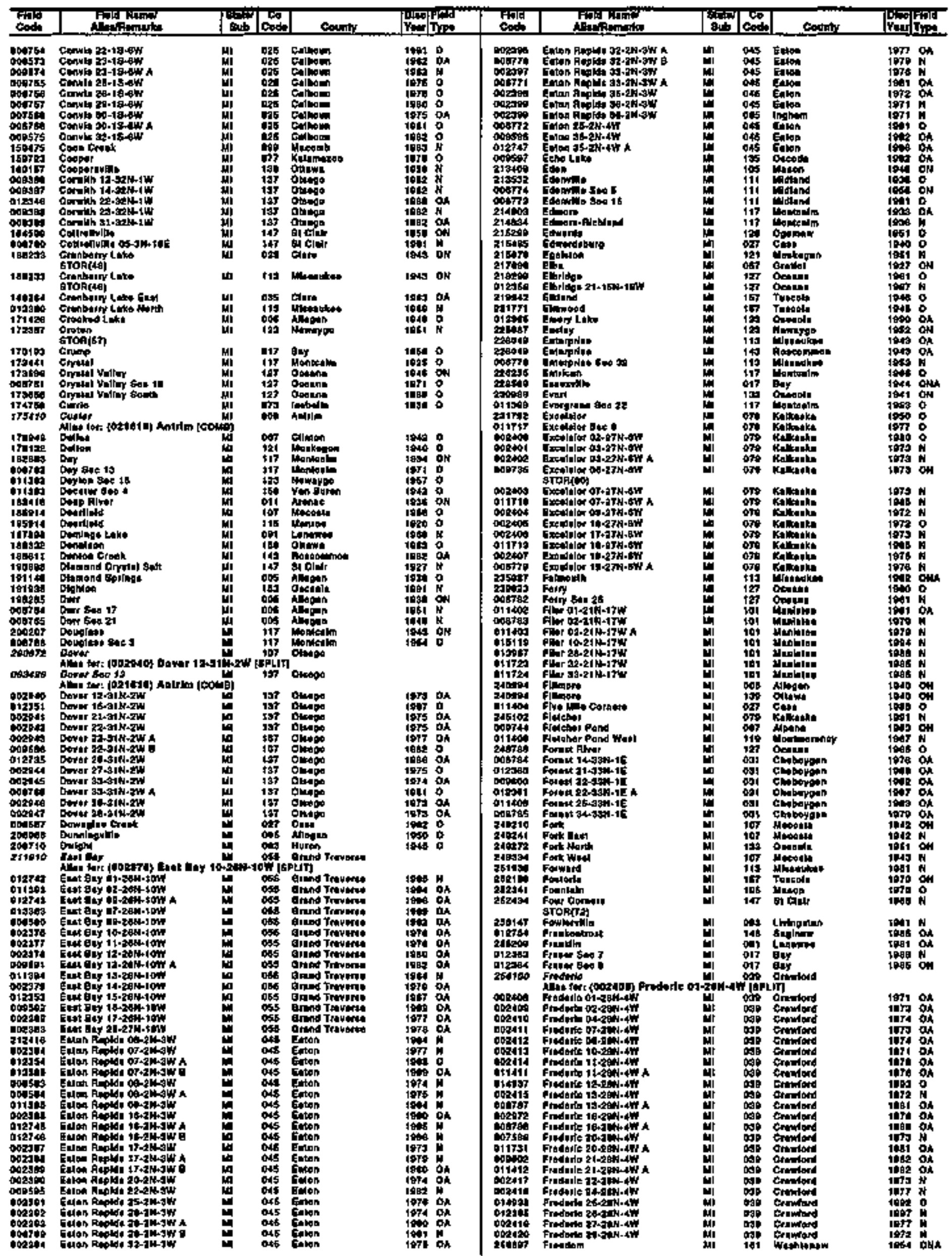


MICHIGAN

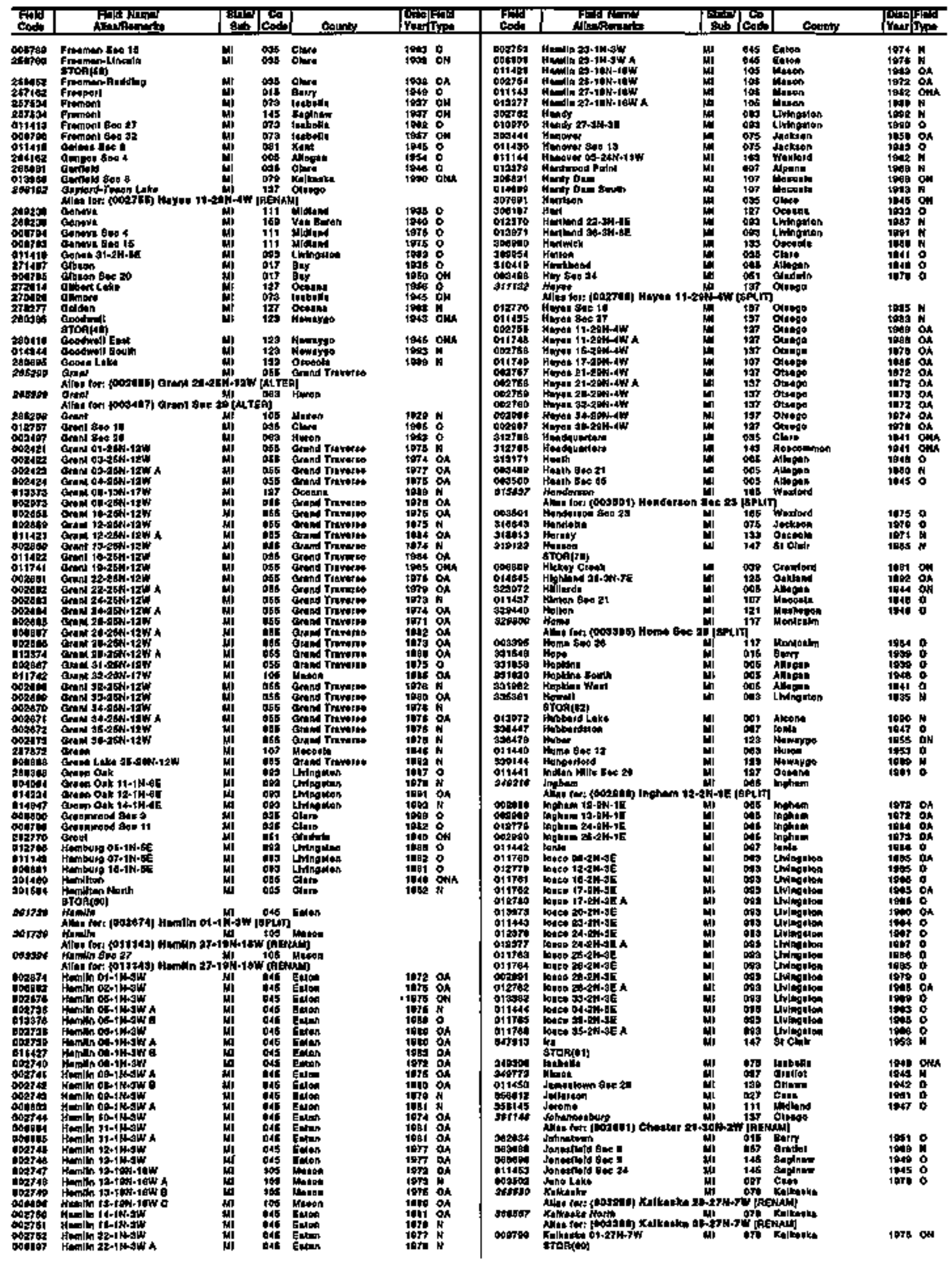




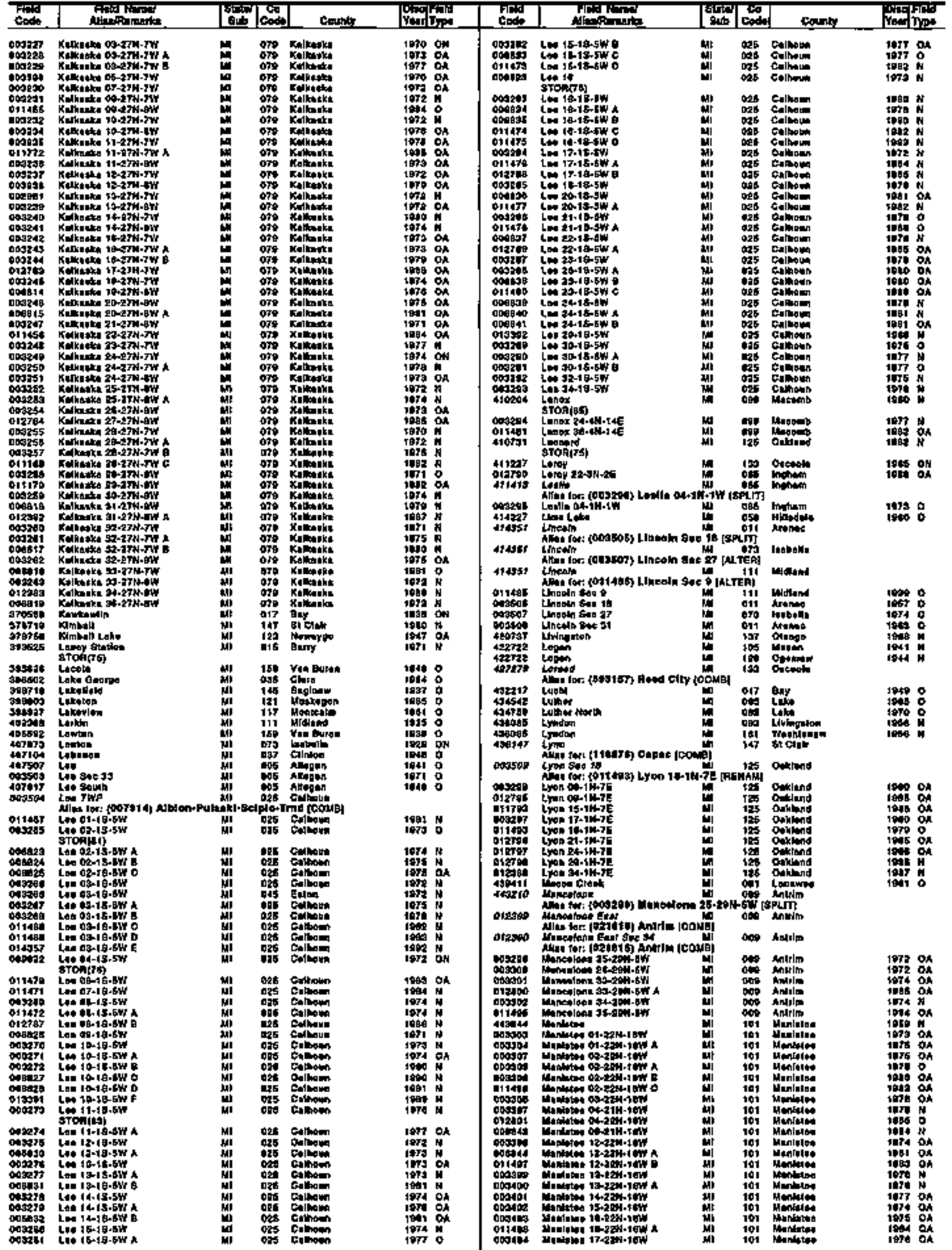


MICHIGAN

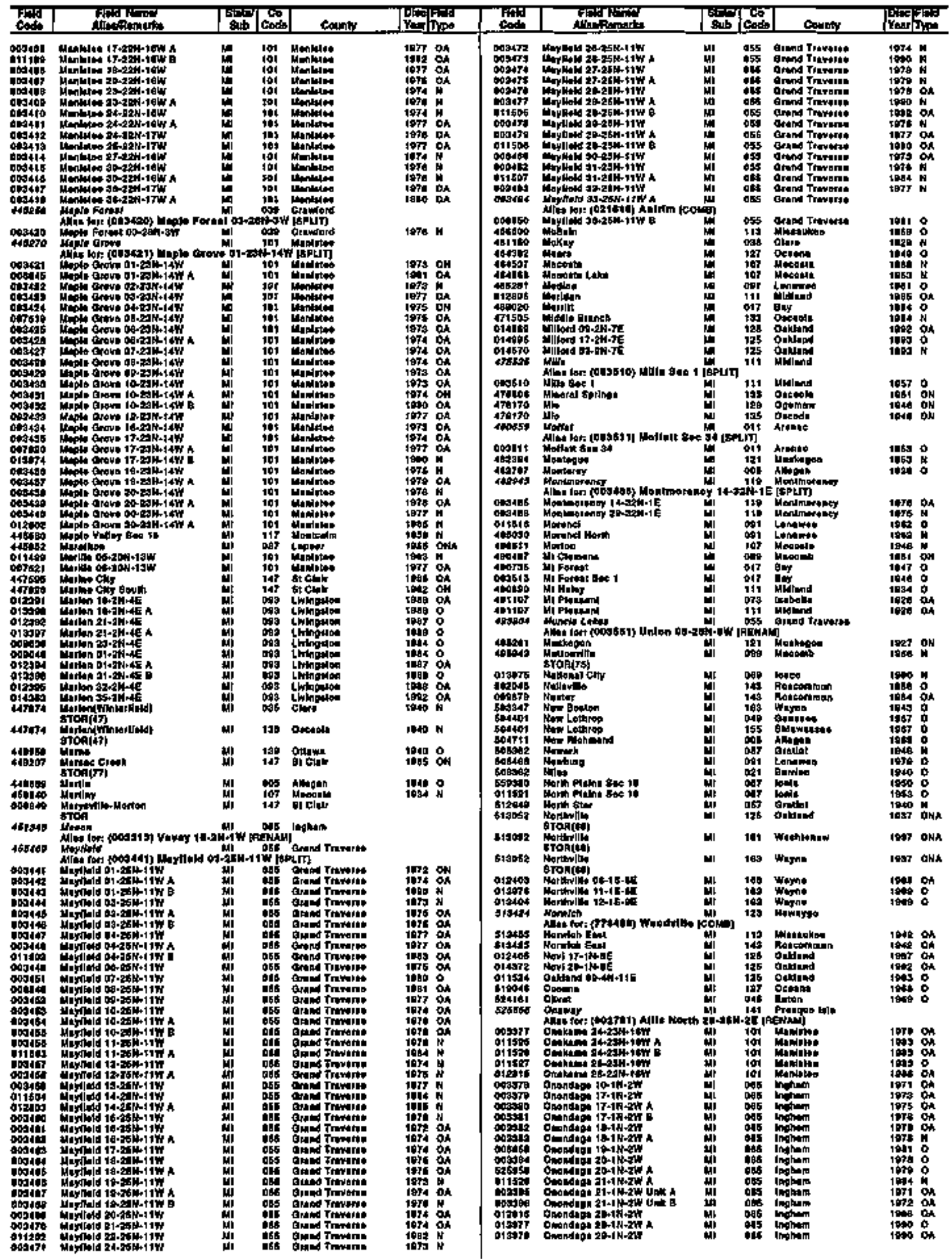




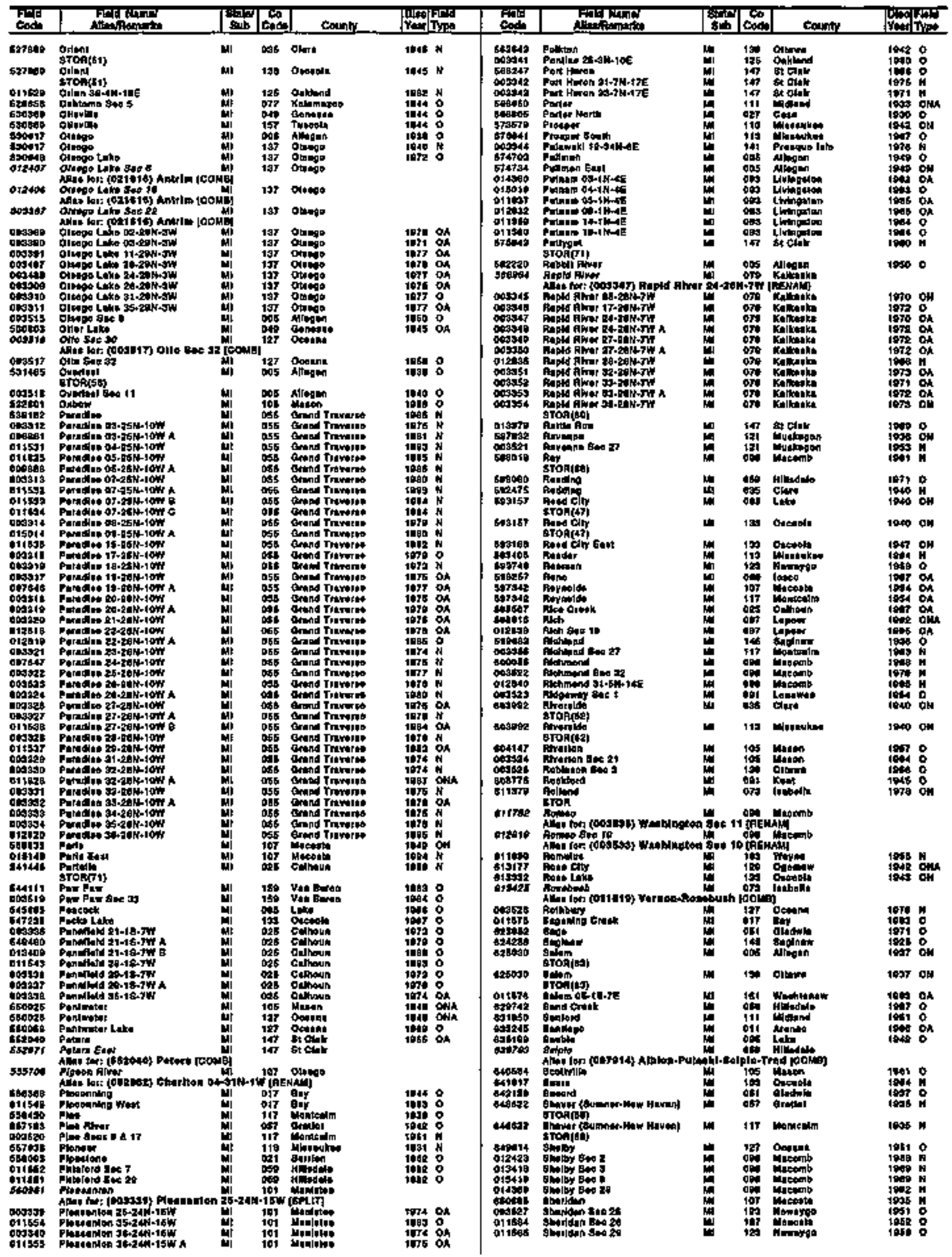




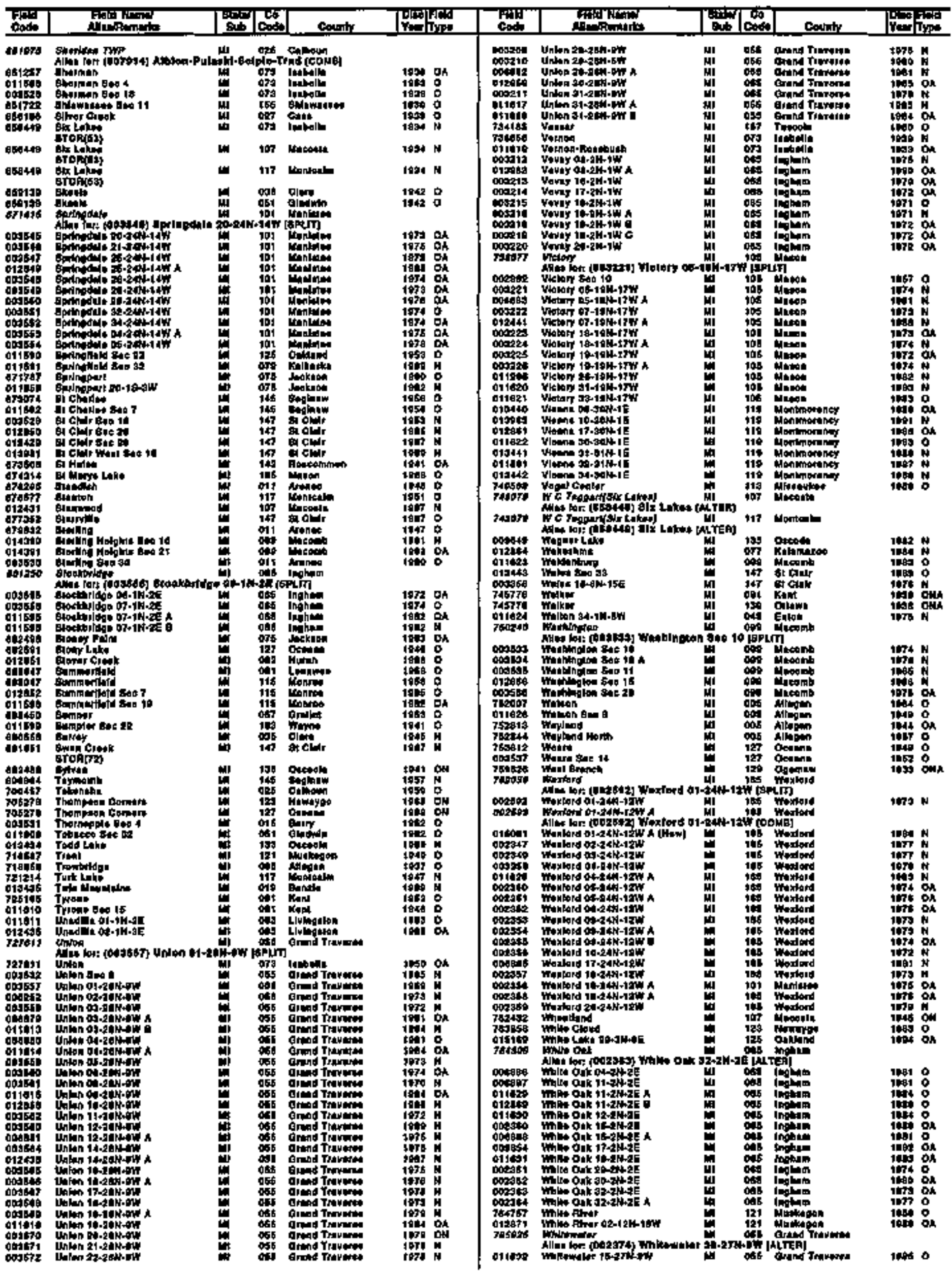




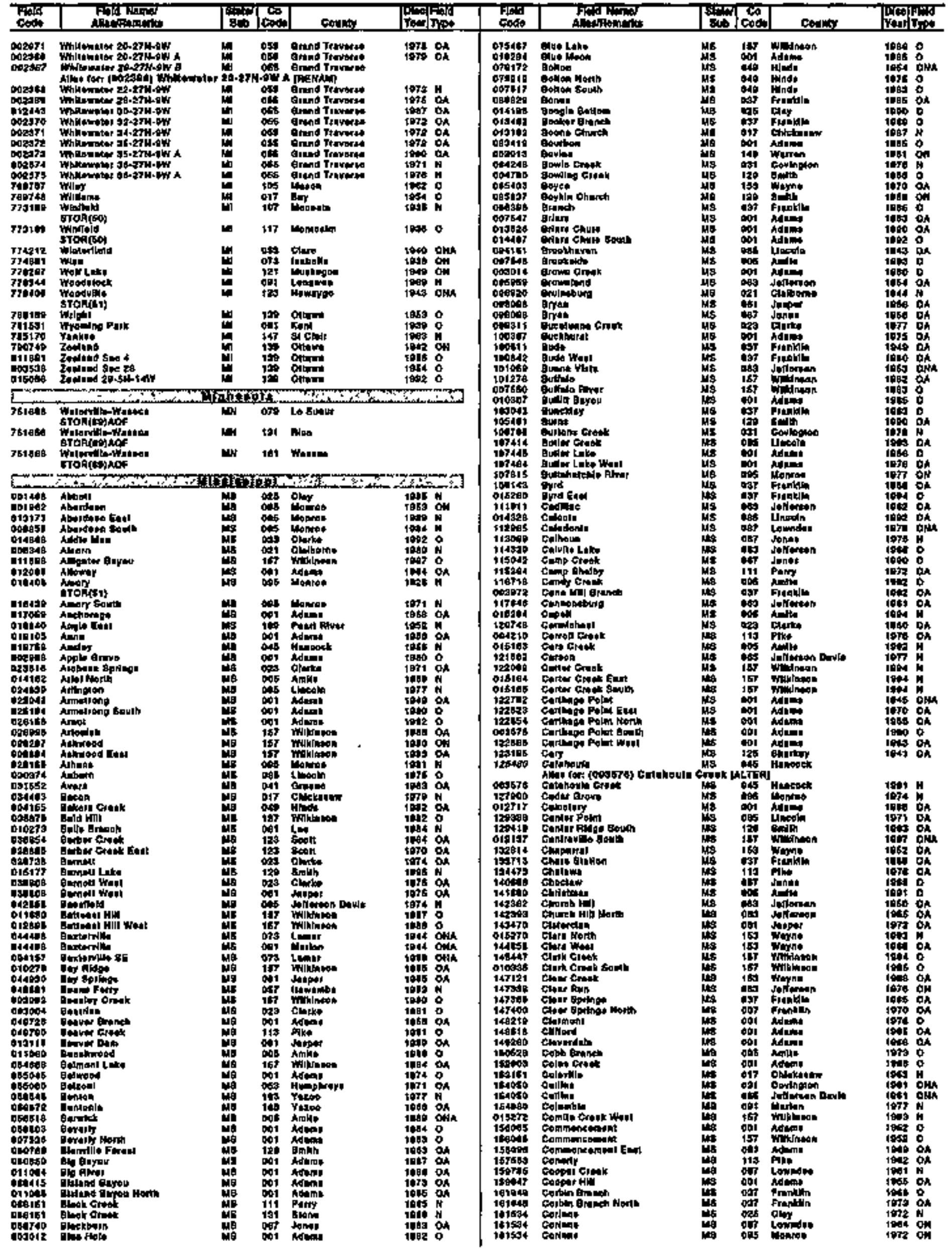


MISSISSIPPI

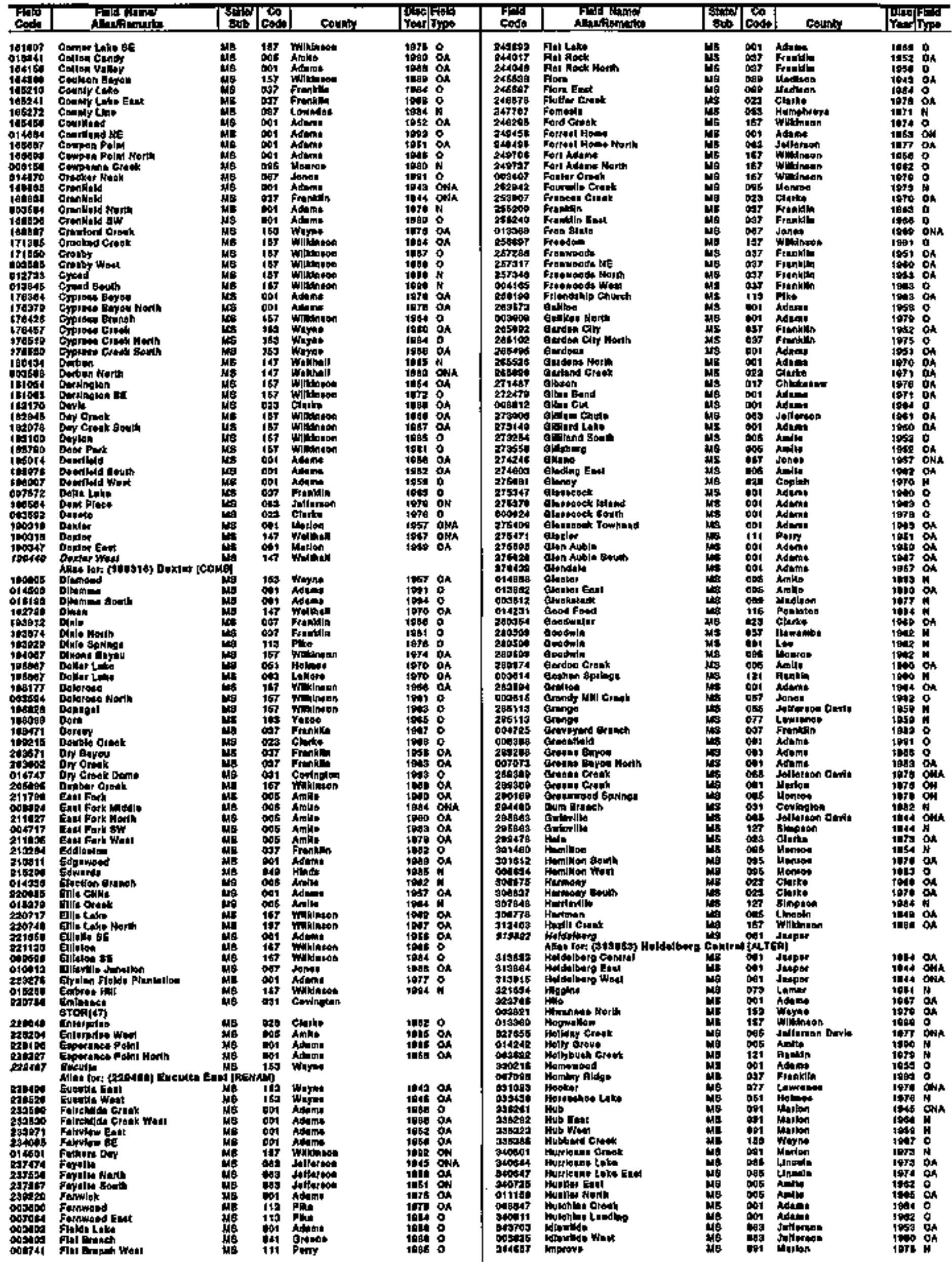




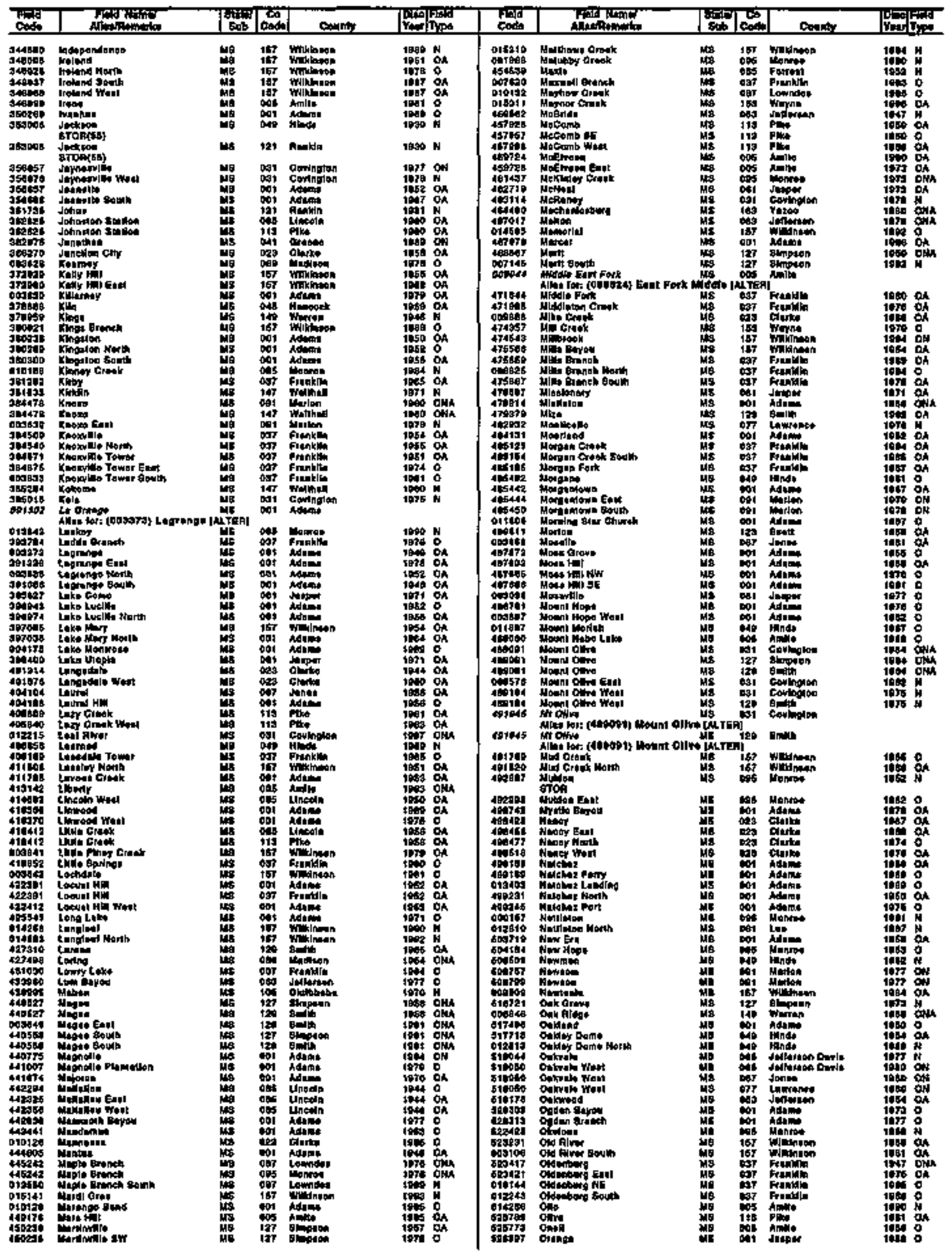


MISSISSIPPI

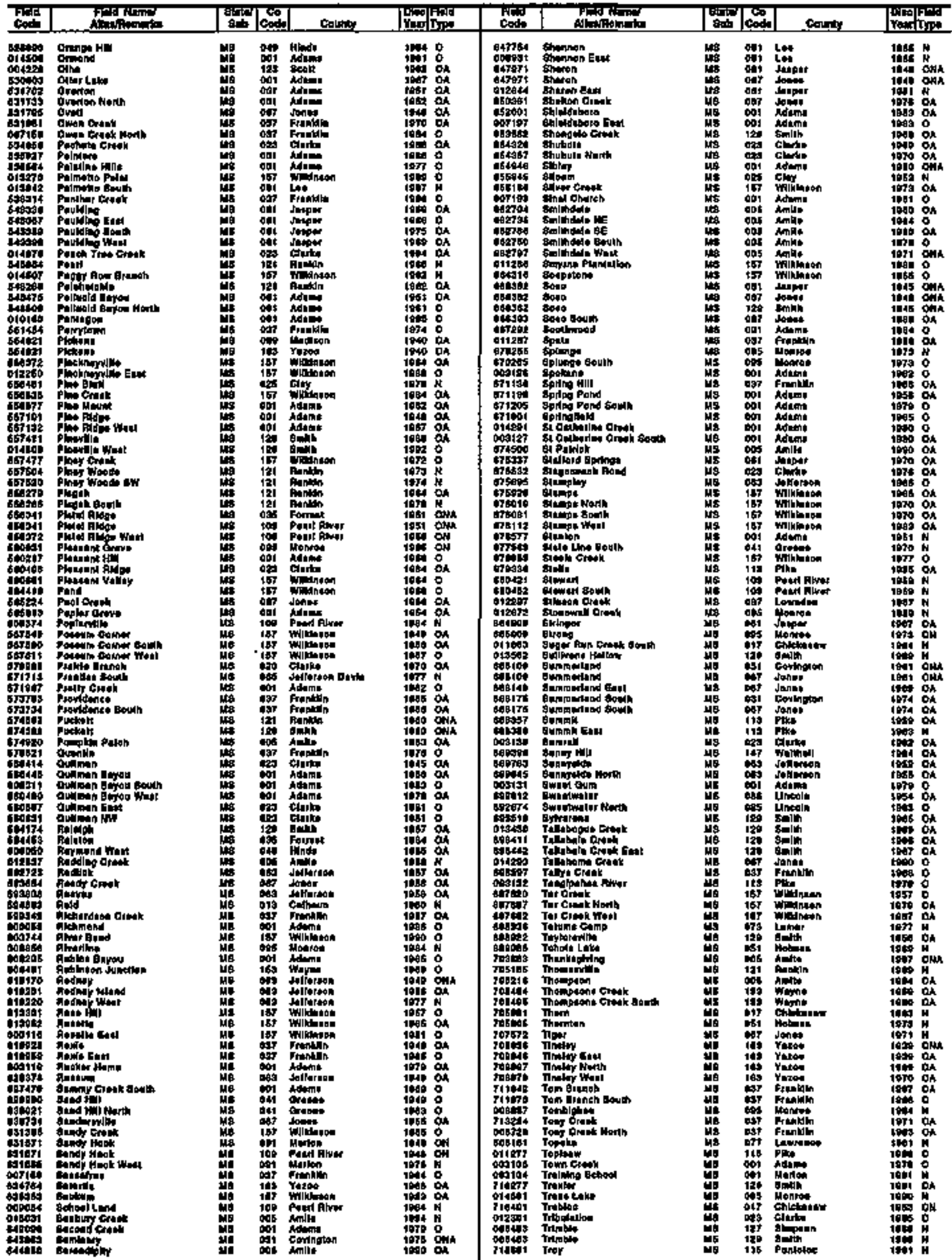


MISSISSIPPI

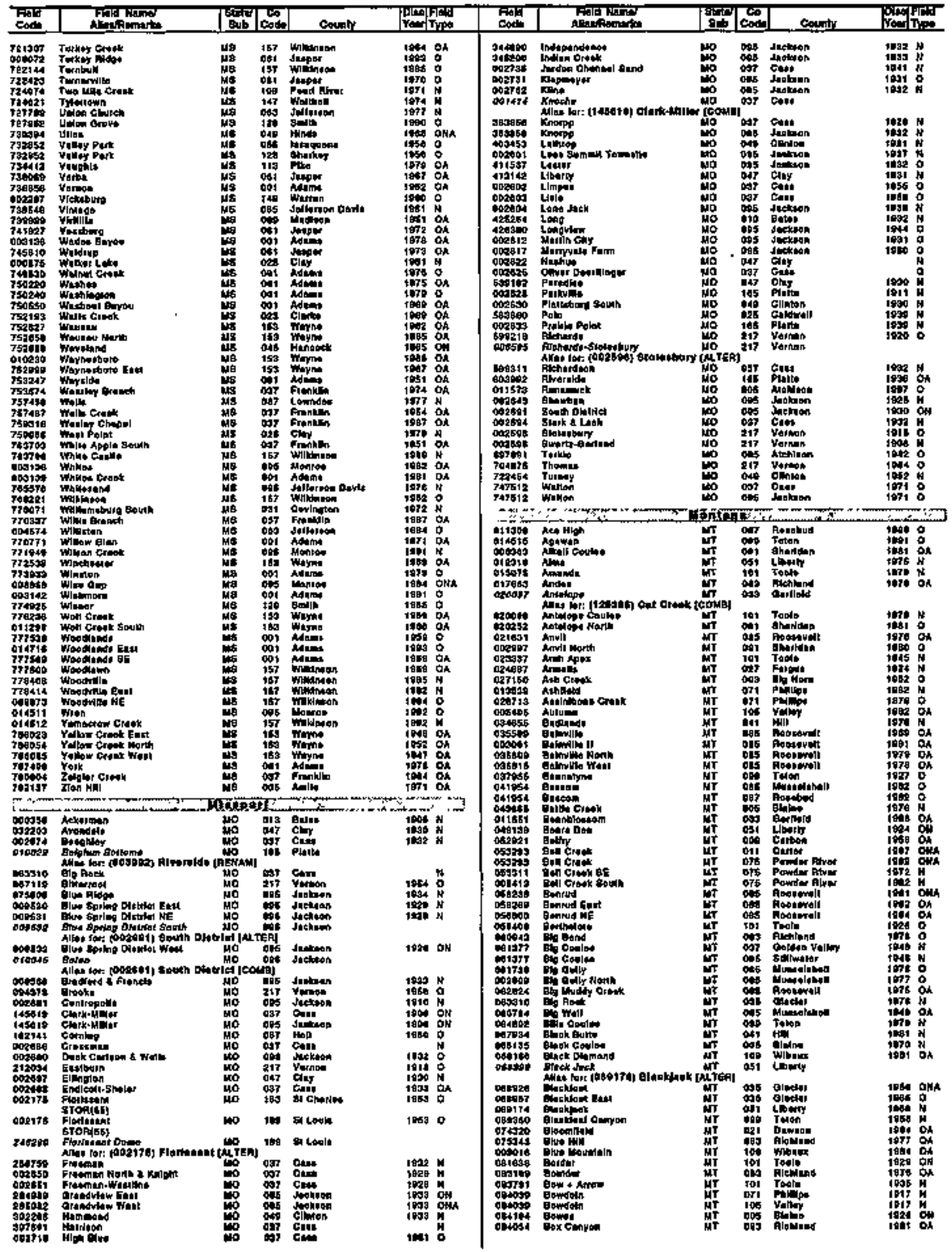


MONTANA

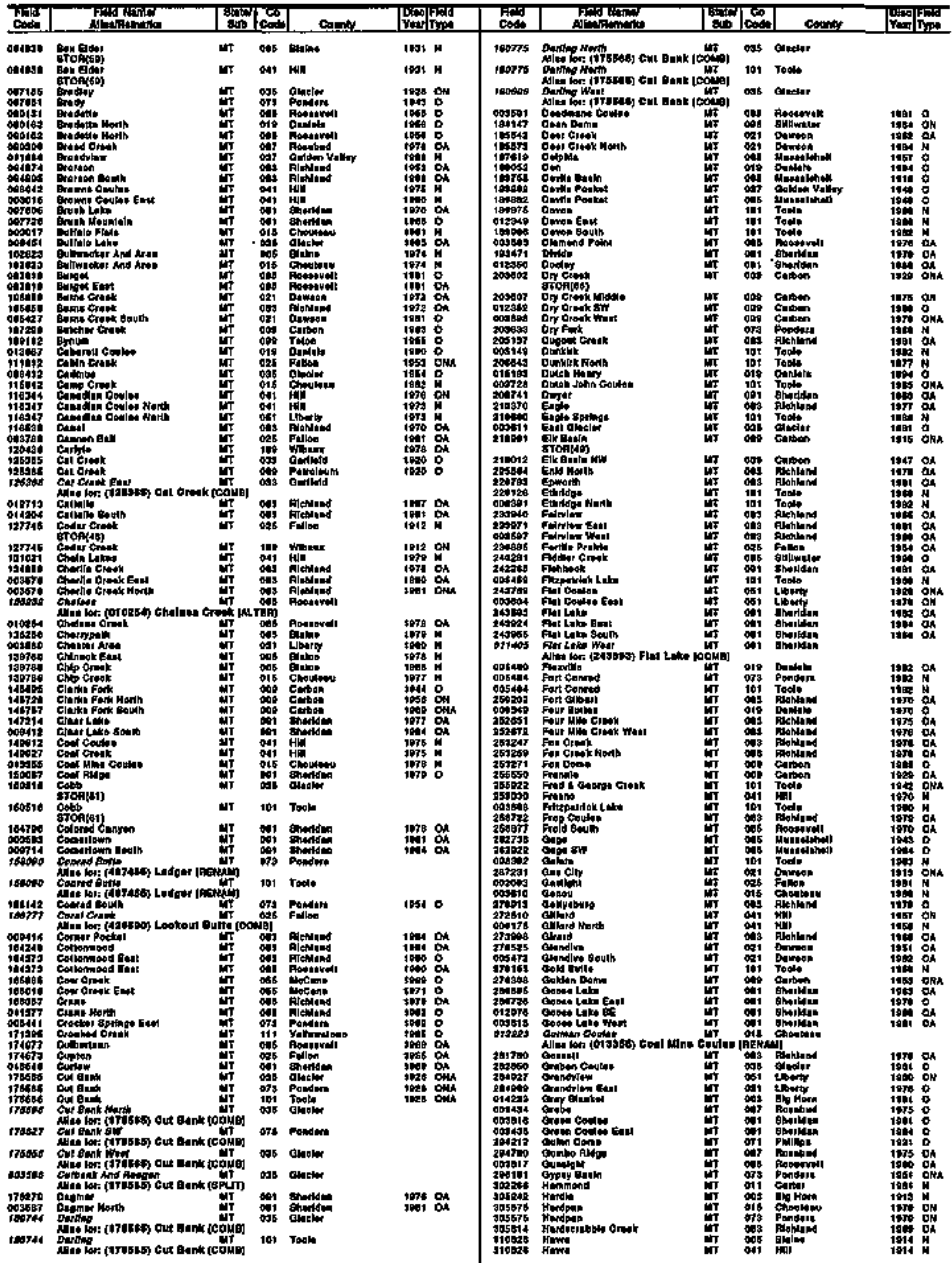


MONTANA

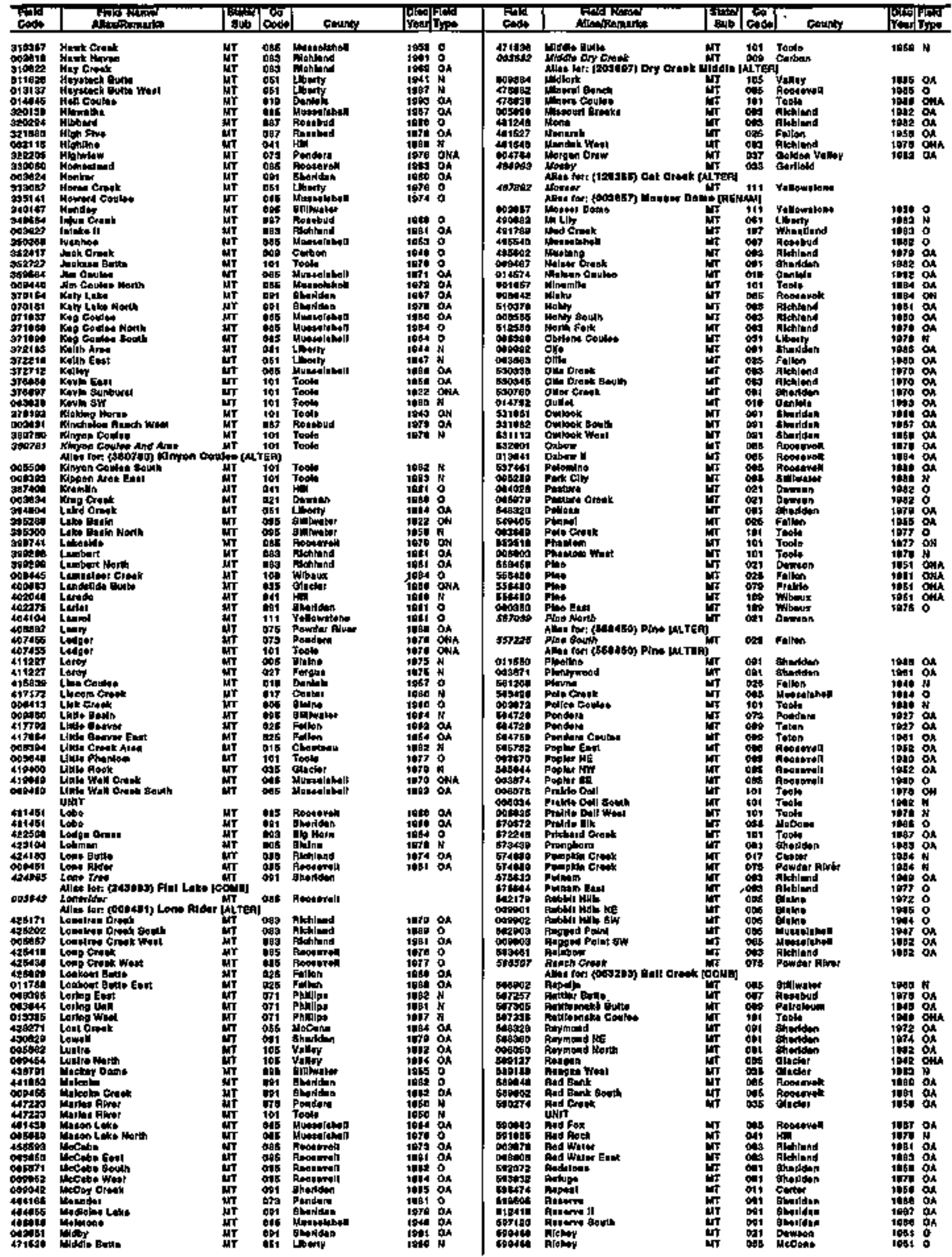


NEGRASKA

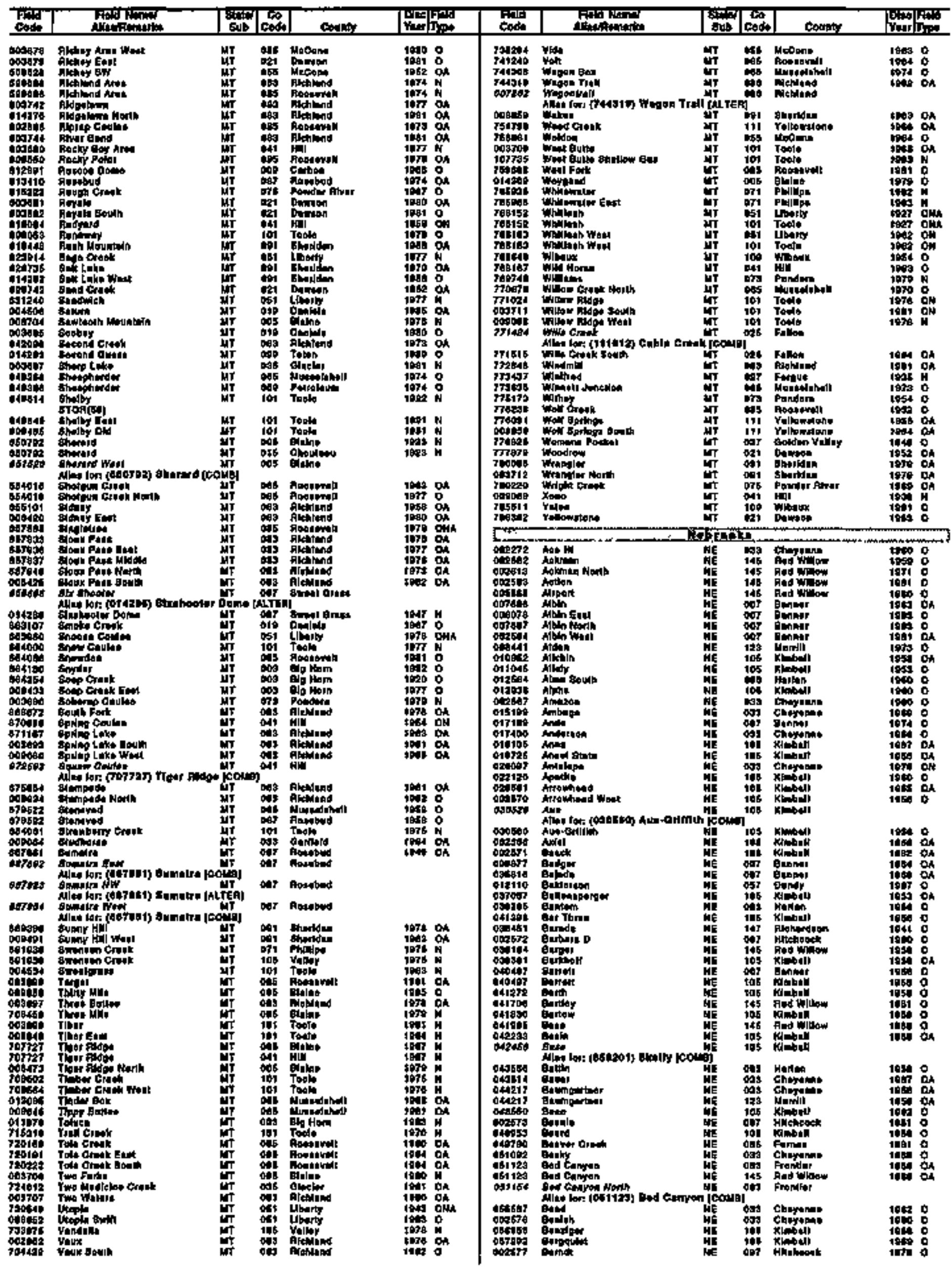




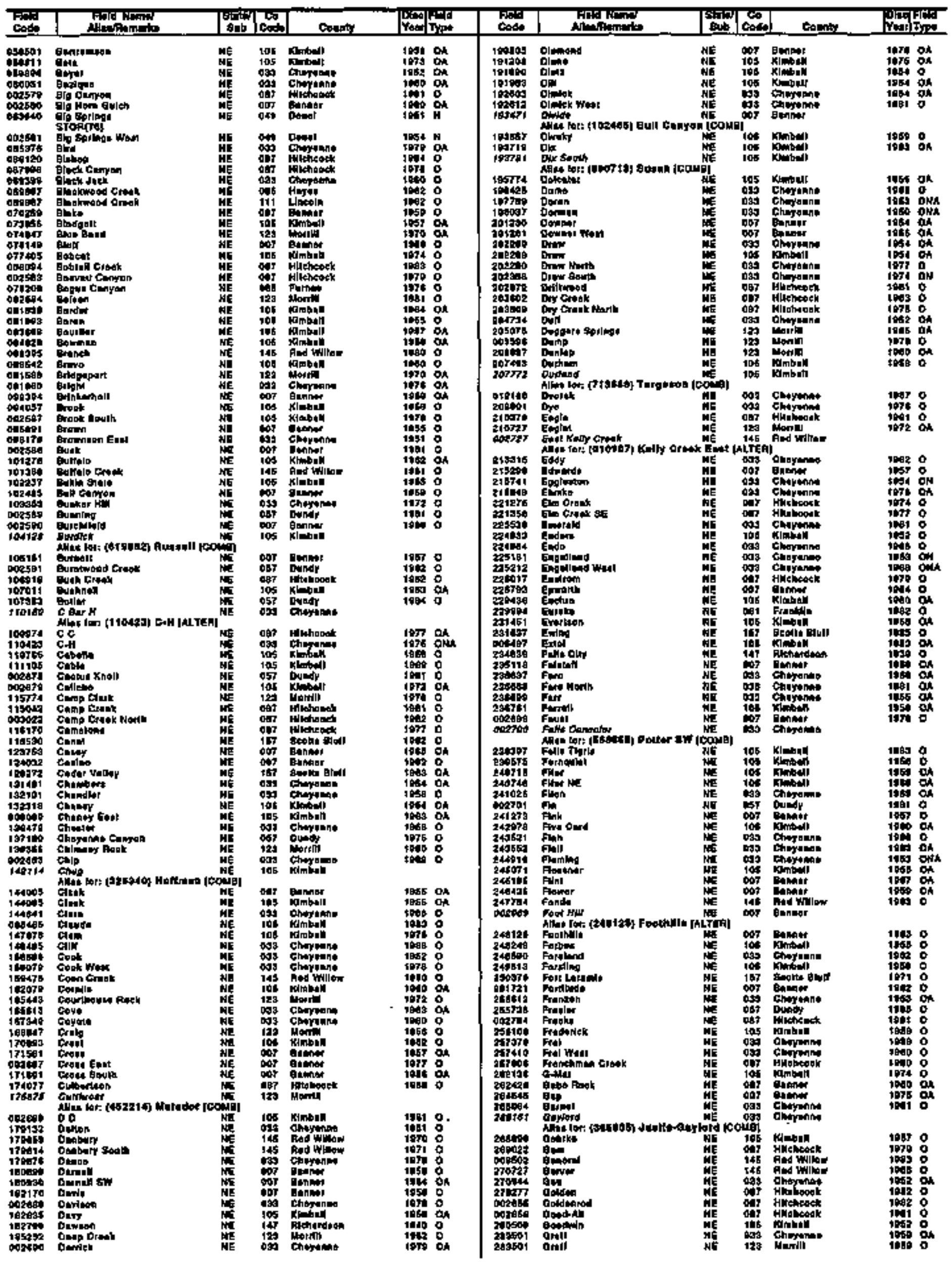


NEBRASKA

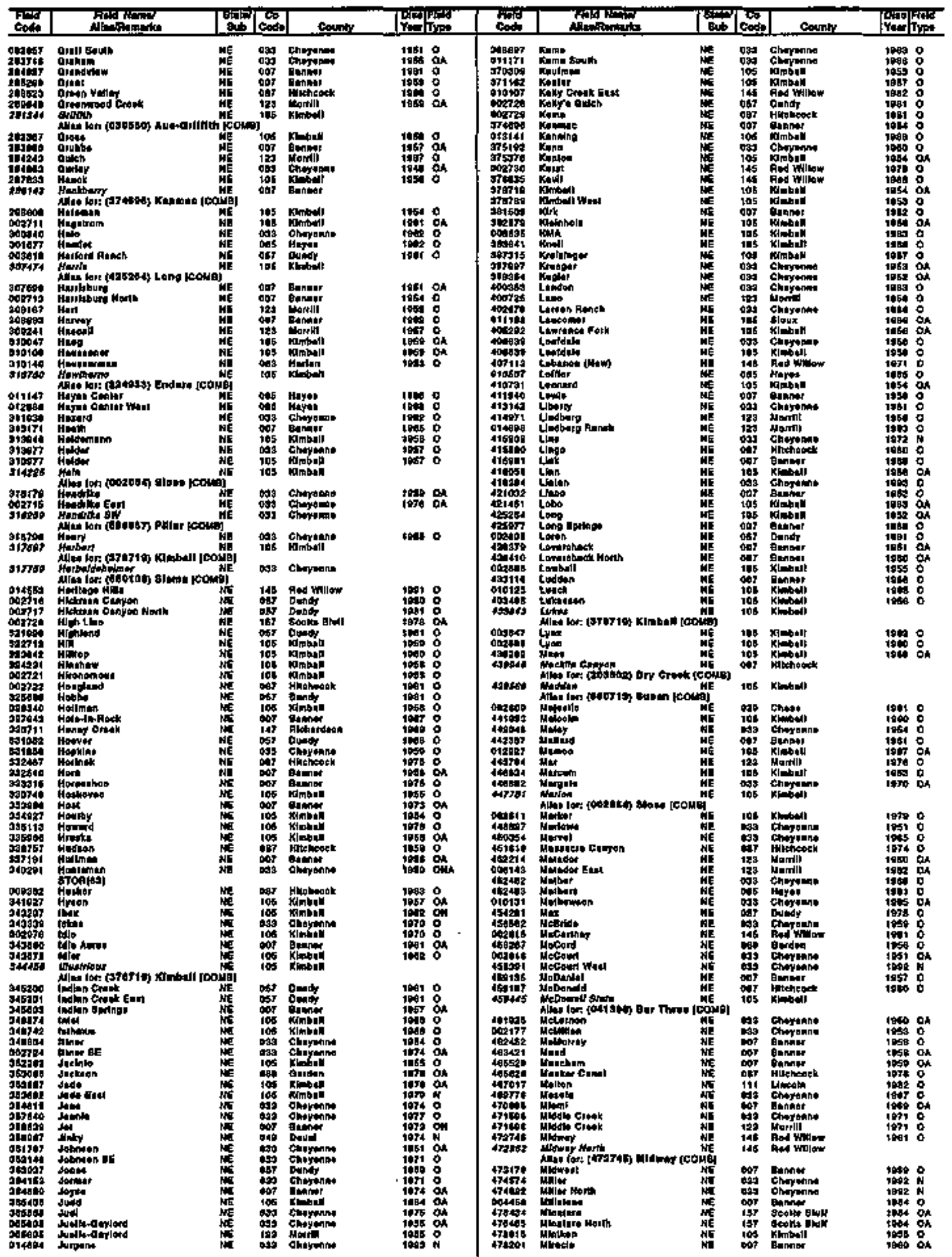


NEBRASKA

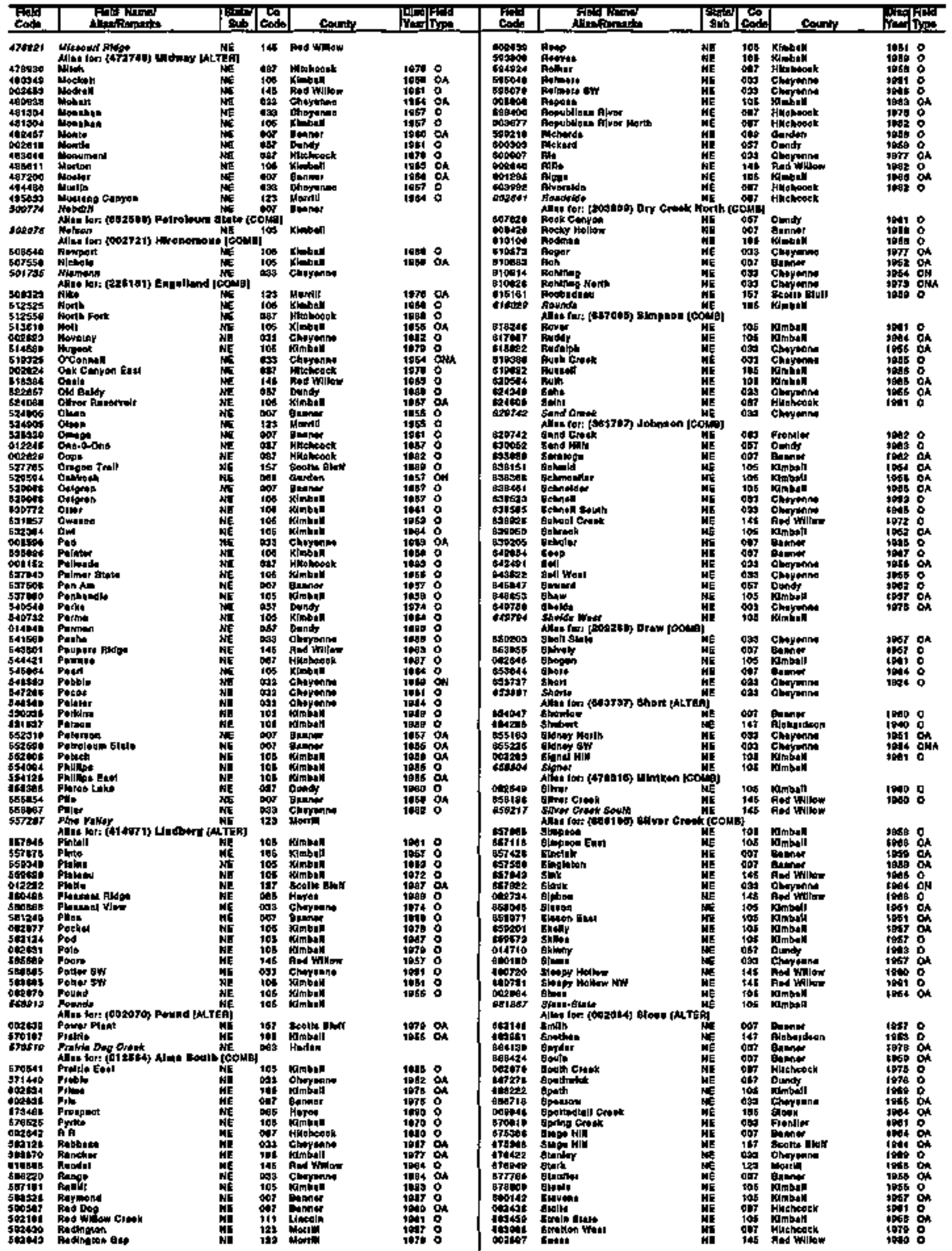


NEW MEXICO

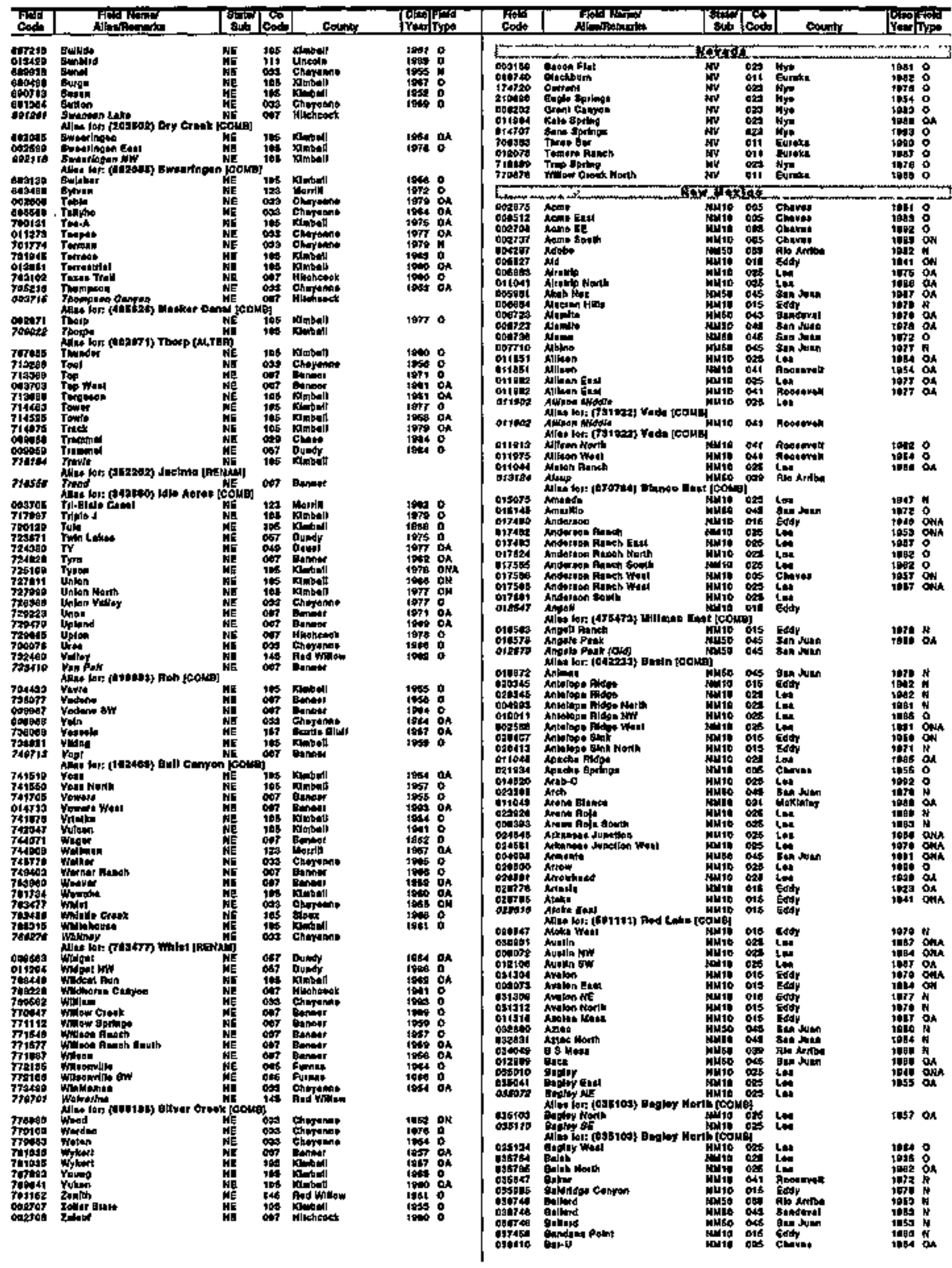


NEW MEXICO

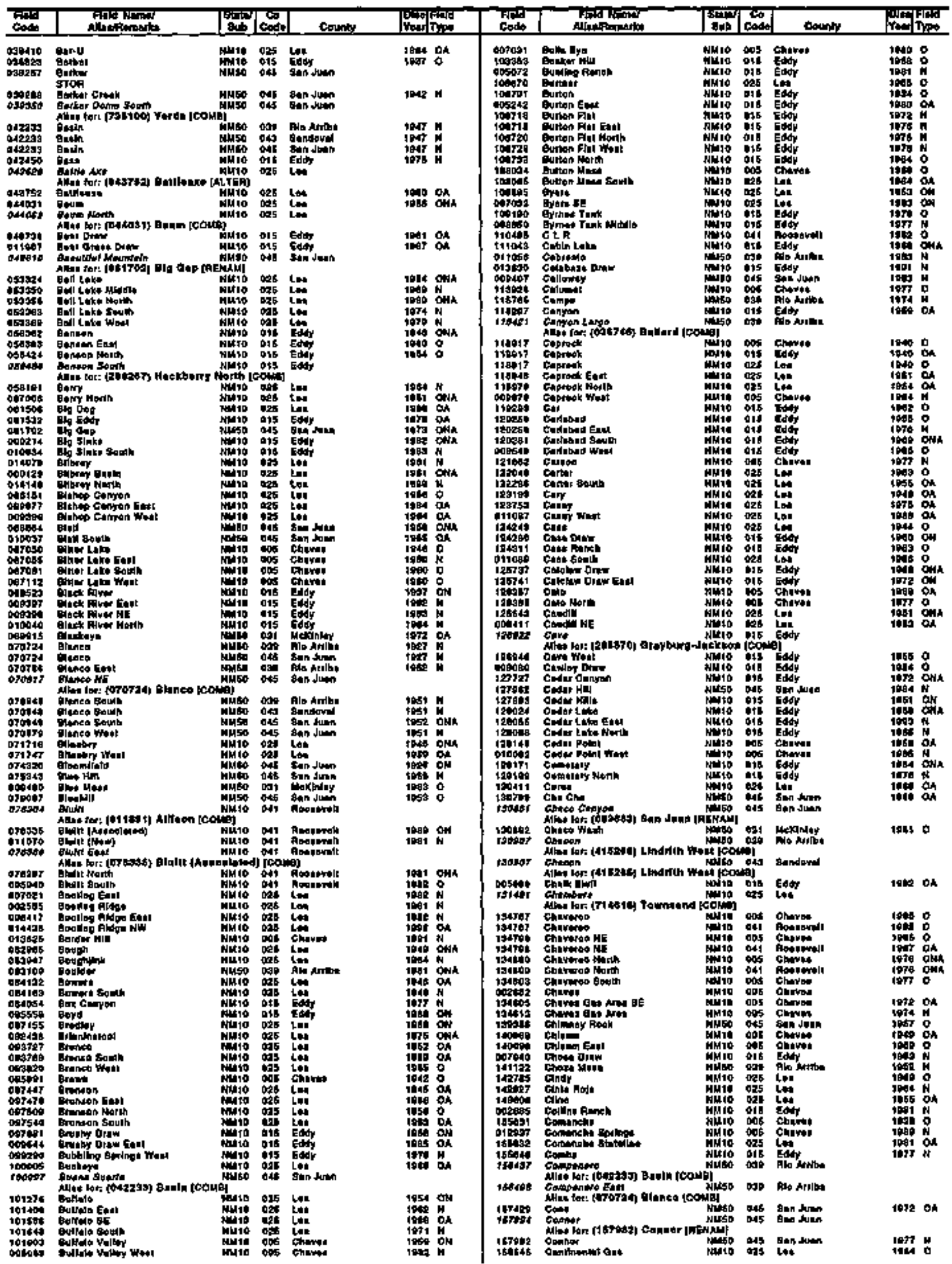


NEW MEXICO

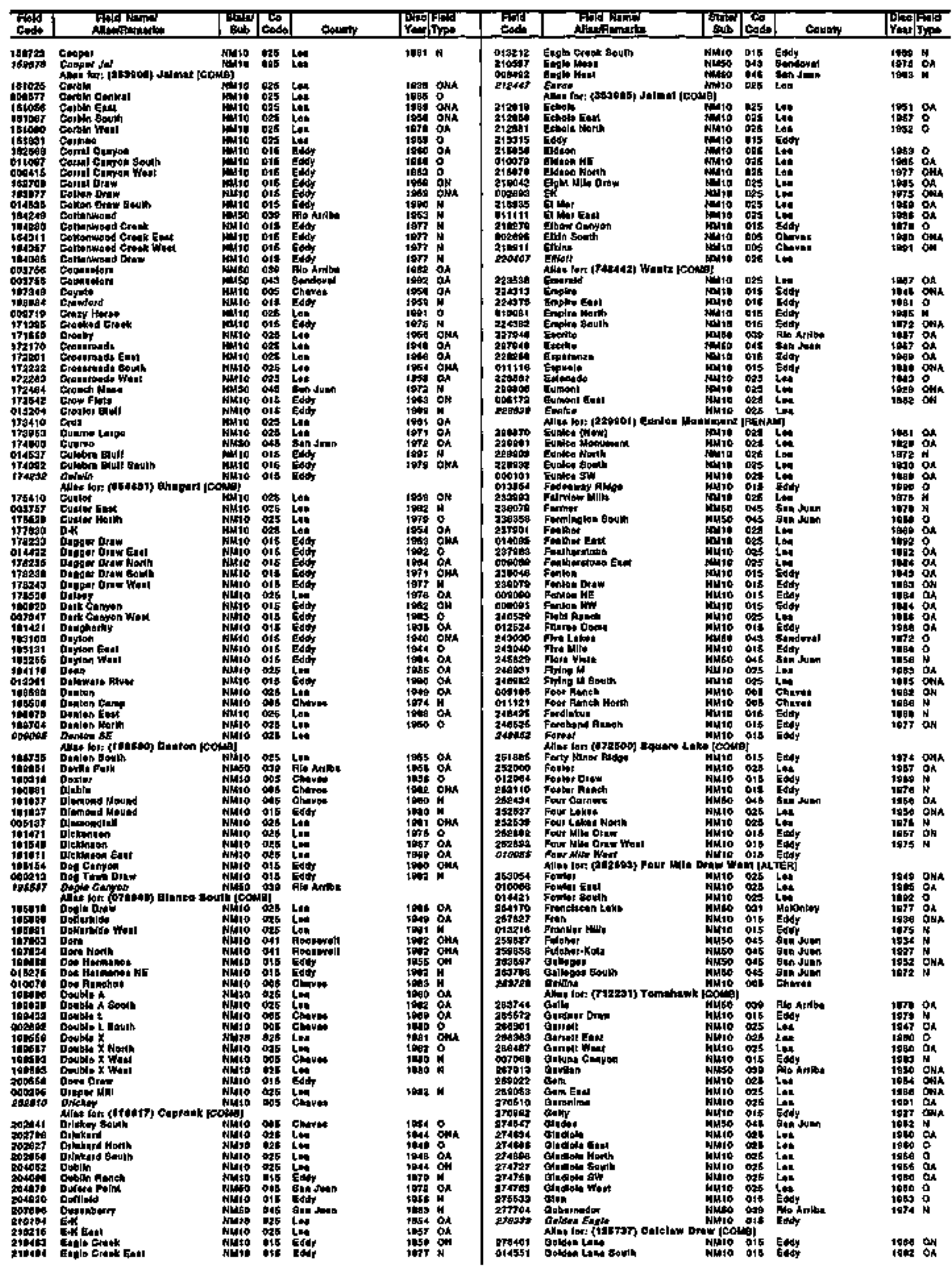


NEW MEXICO

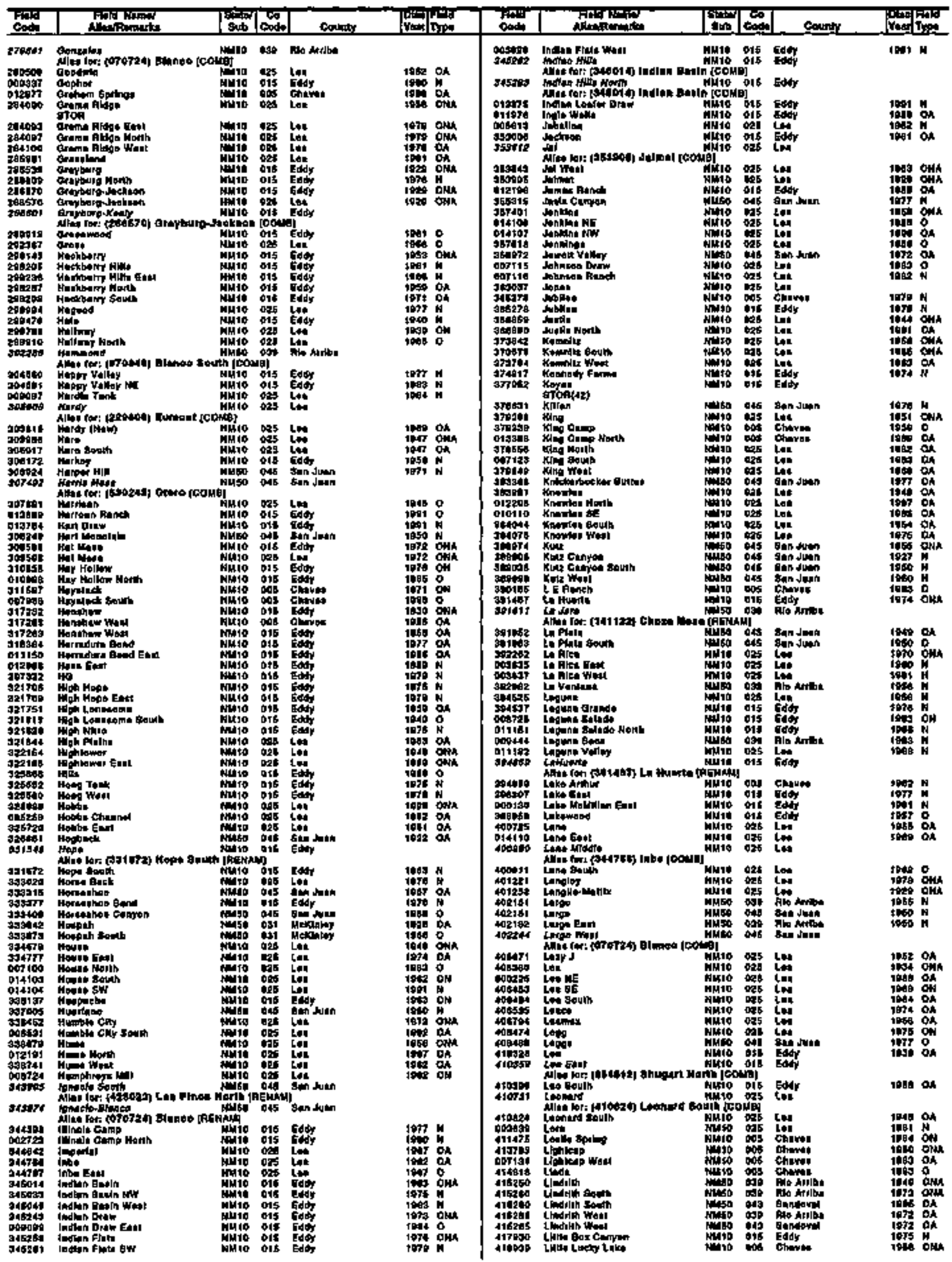


NEW MEXICO

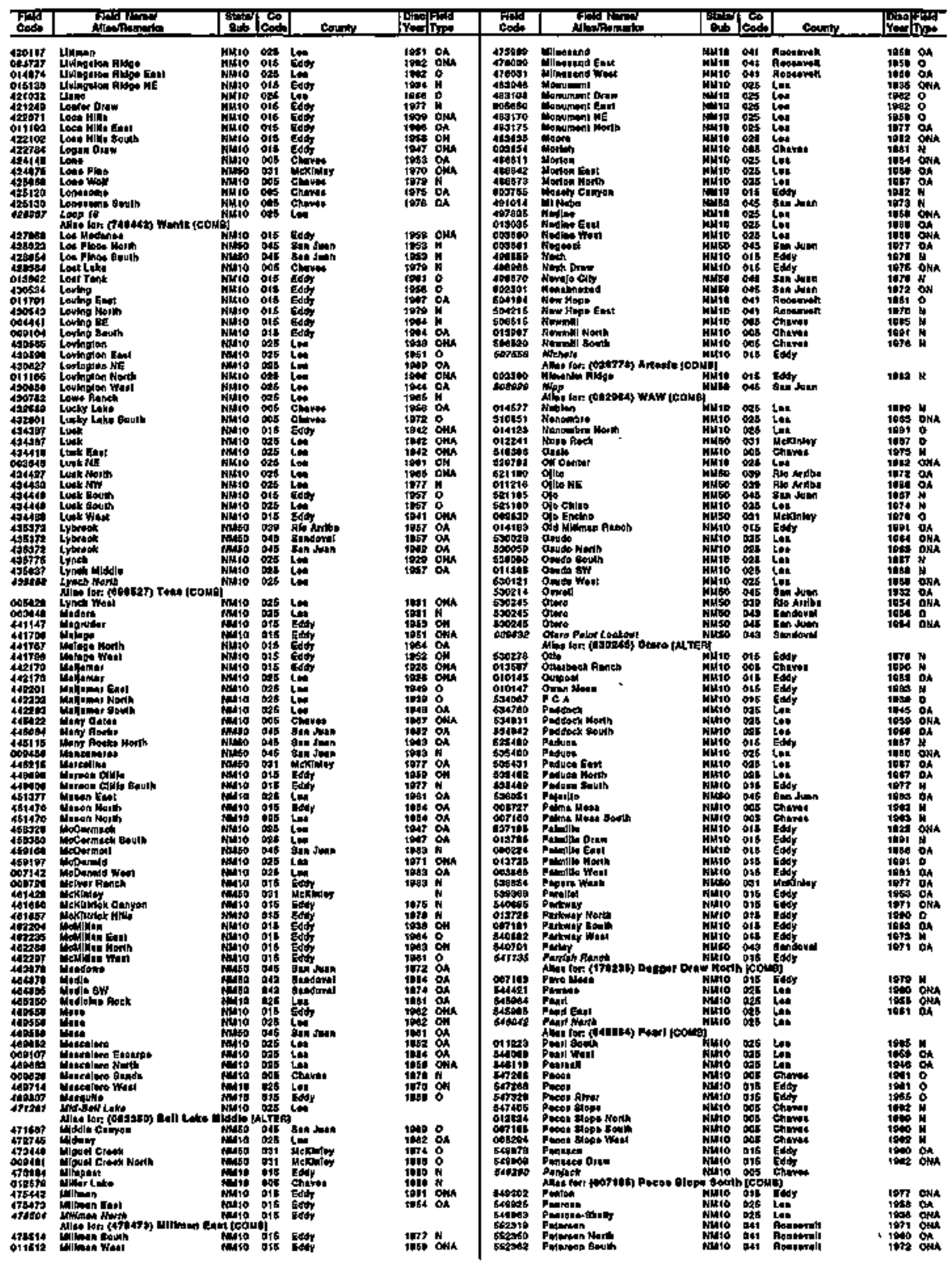




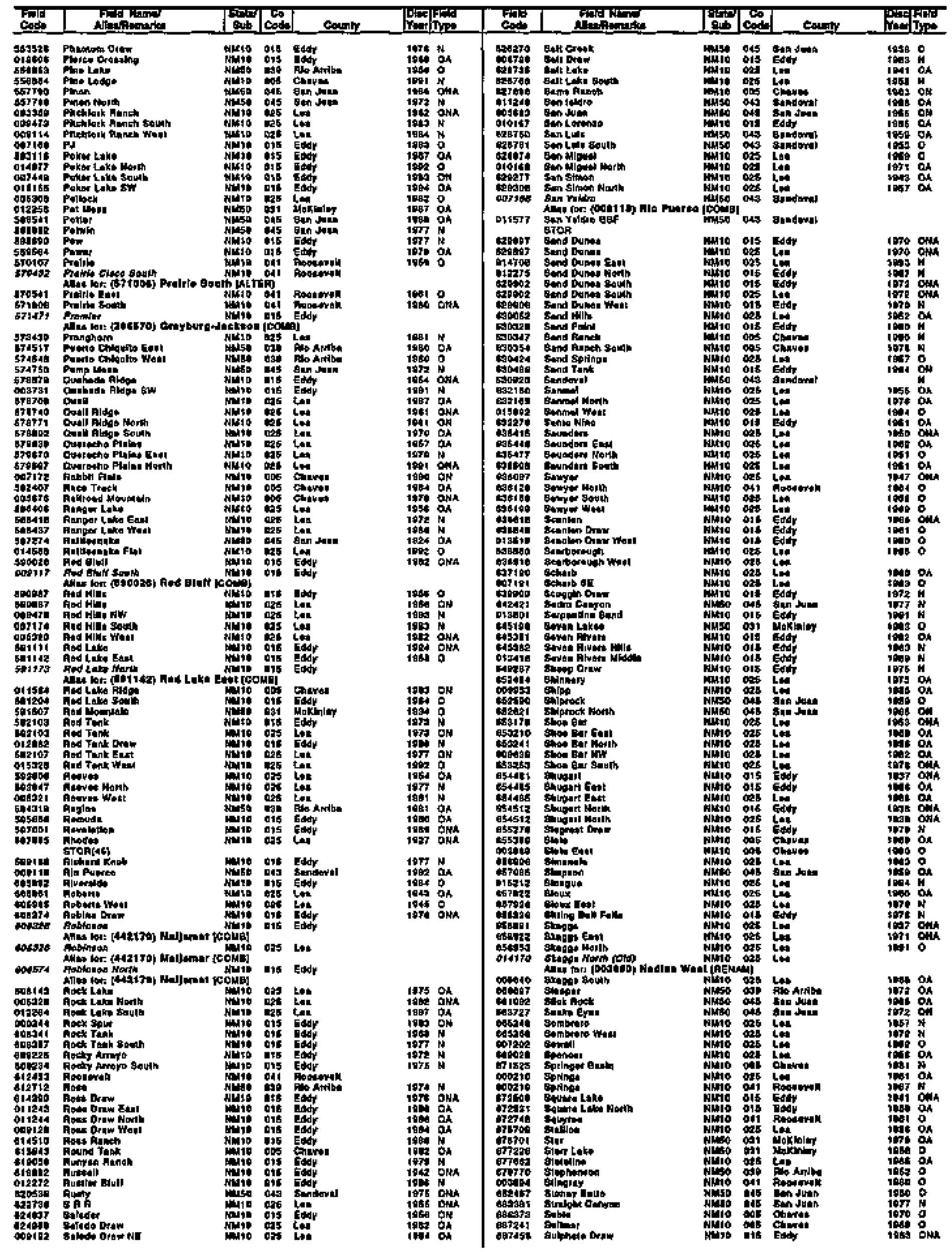


NEW YOAK

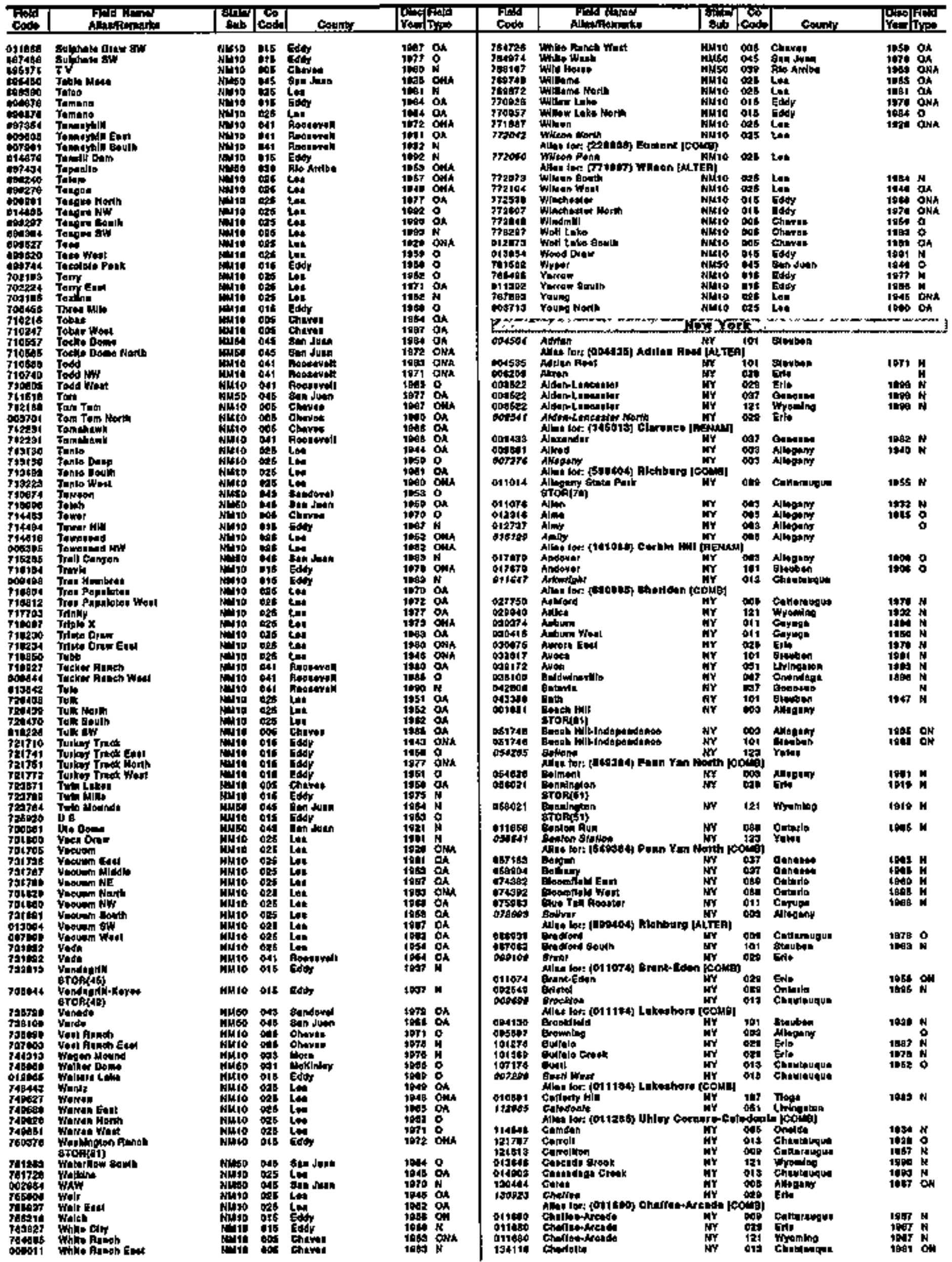




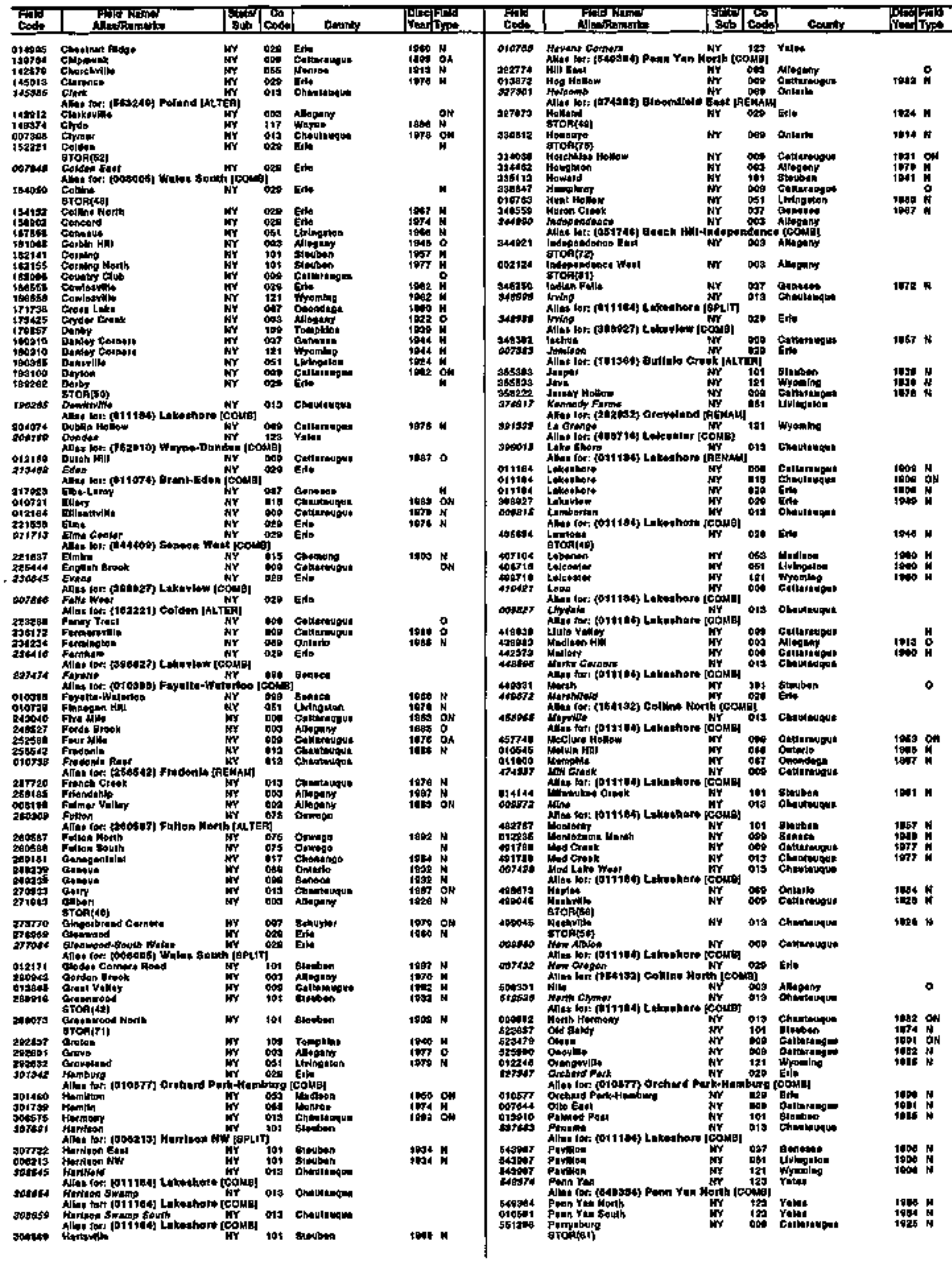


NORTH DAKOTA

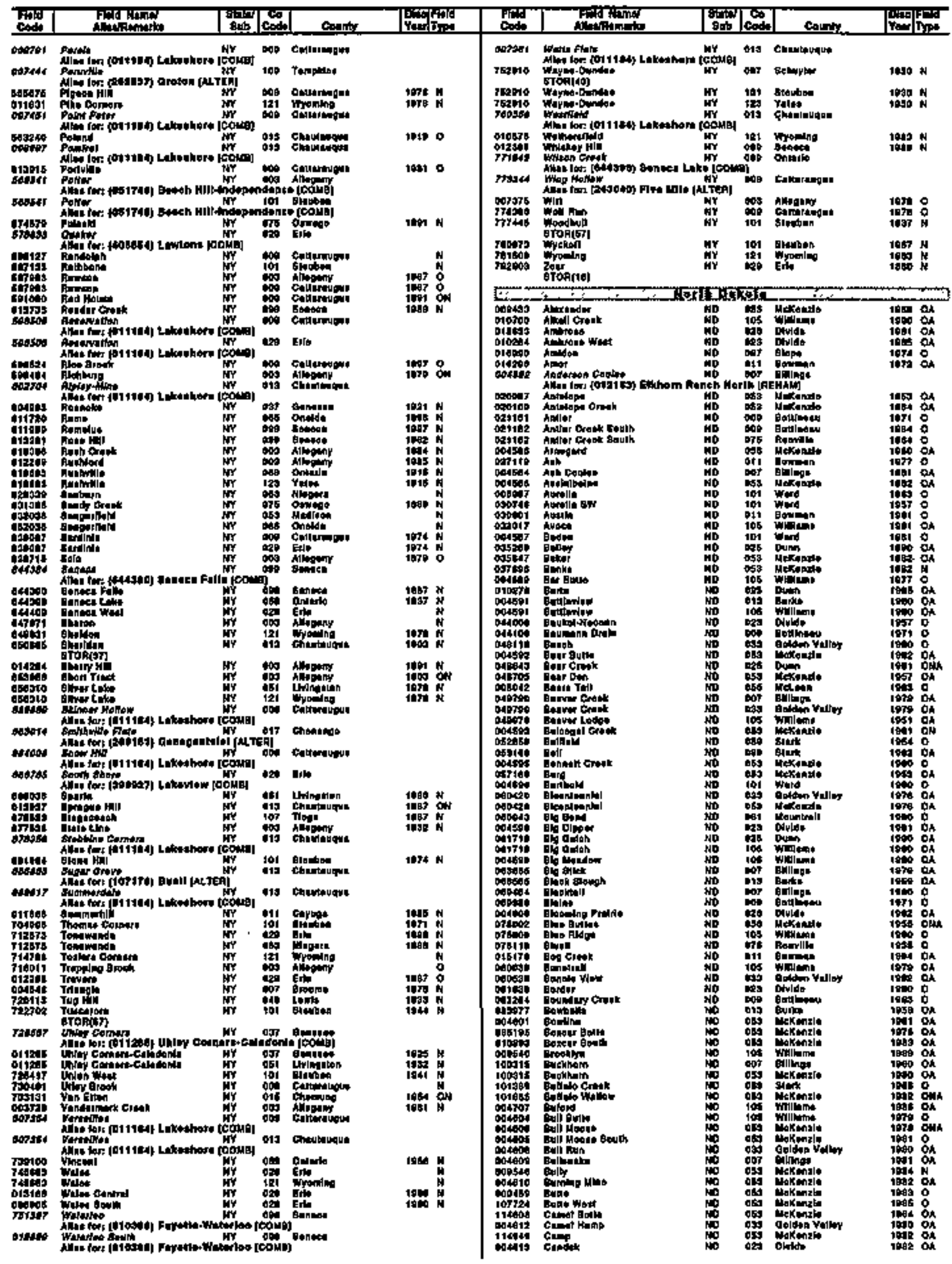


NORTH DAKOTA

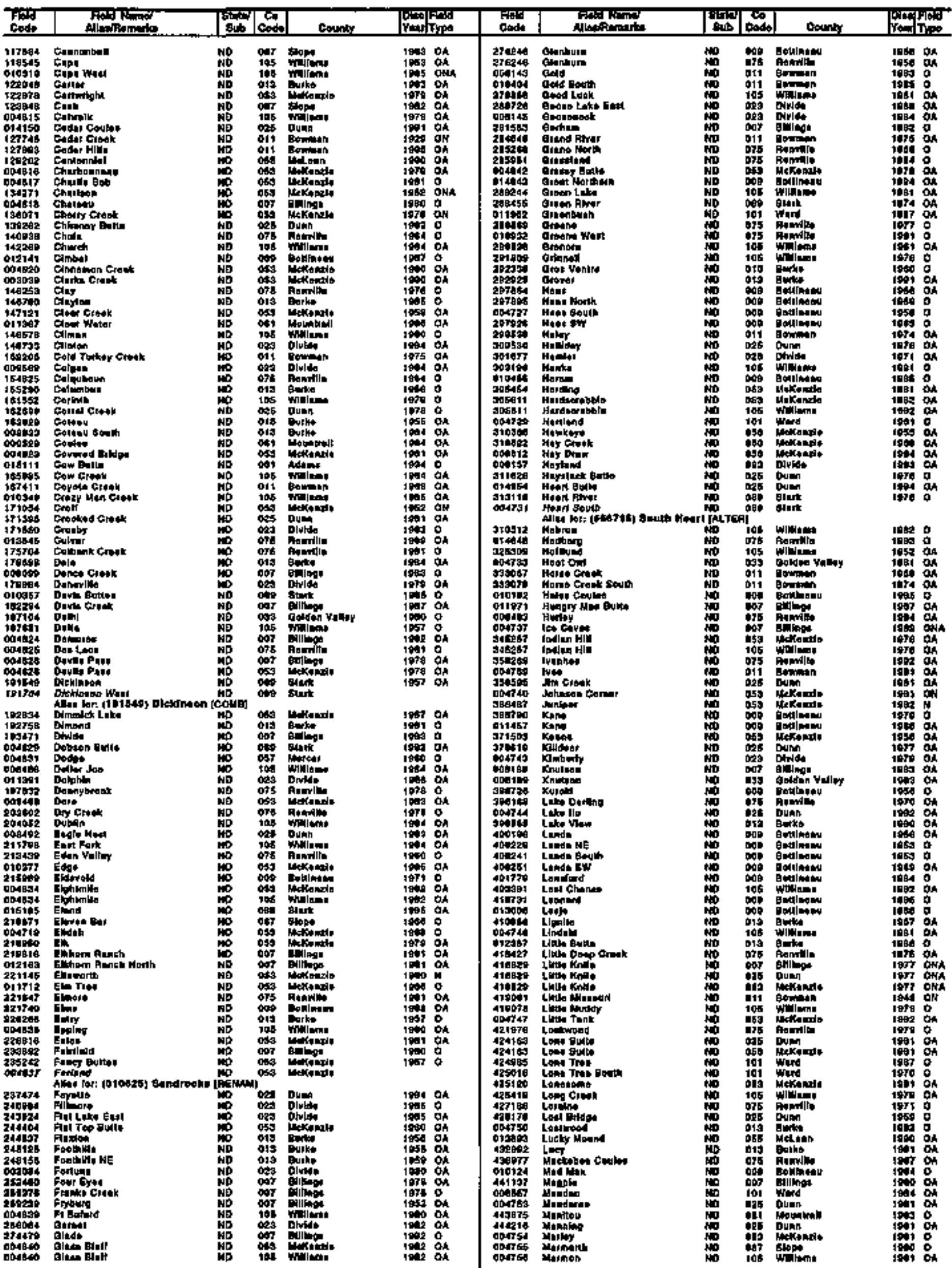


NORTH DAKOTA

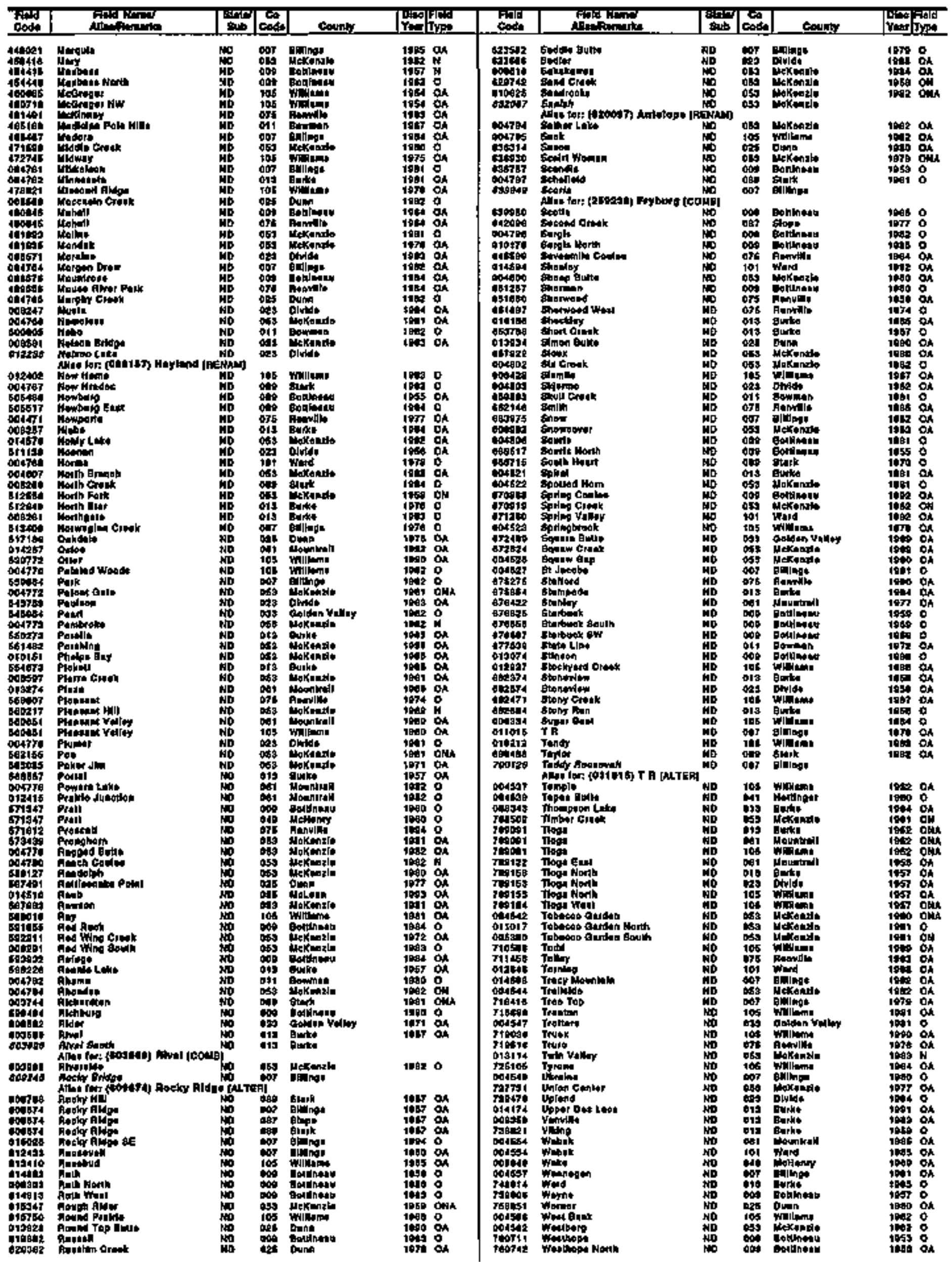




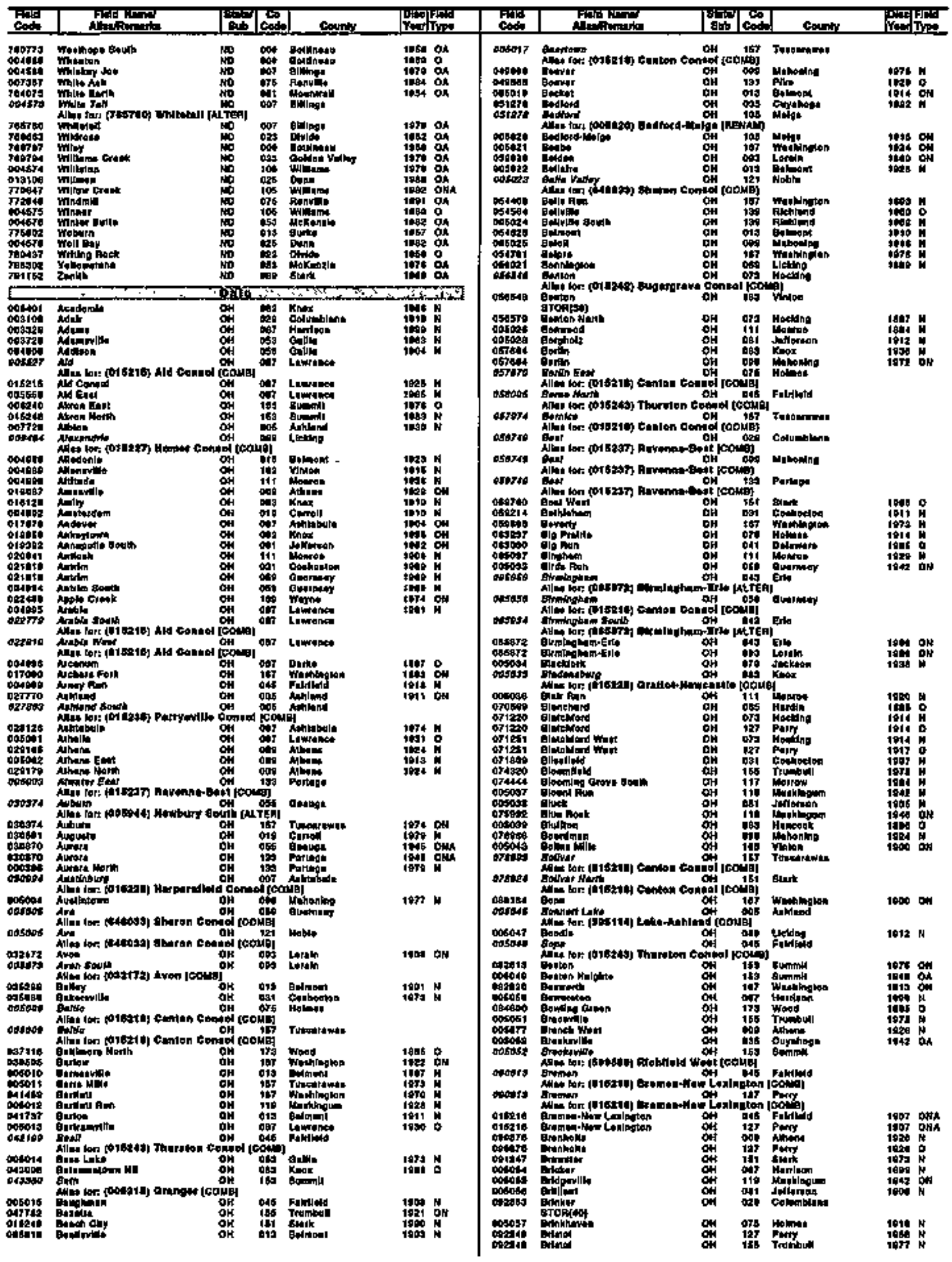




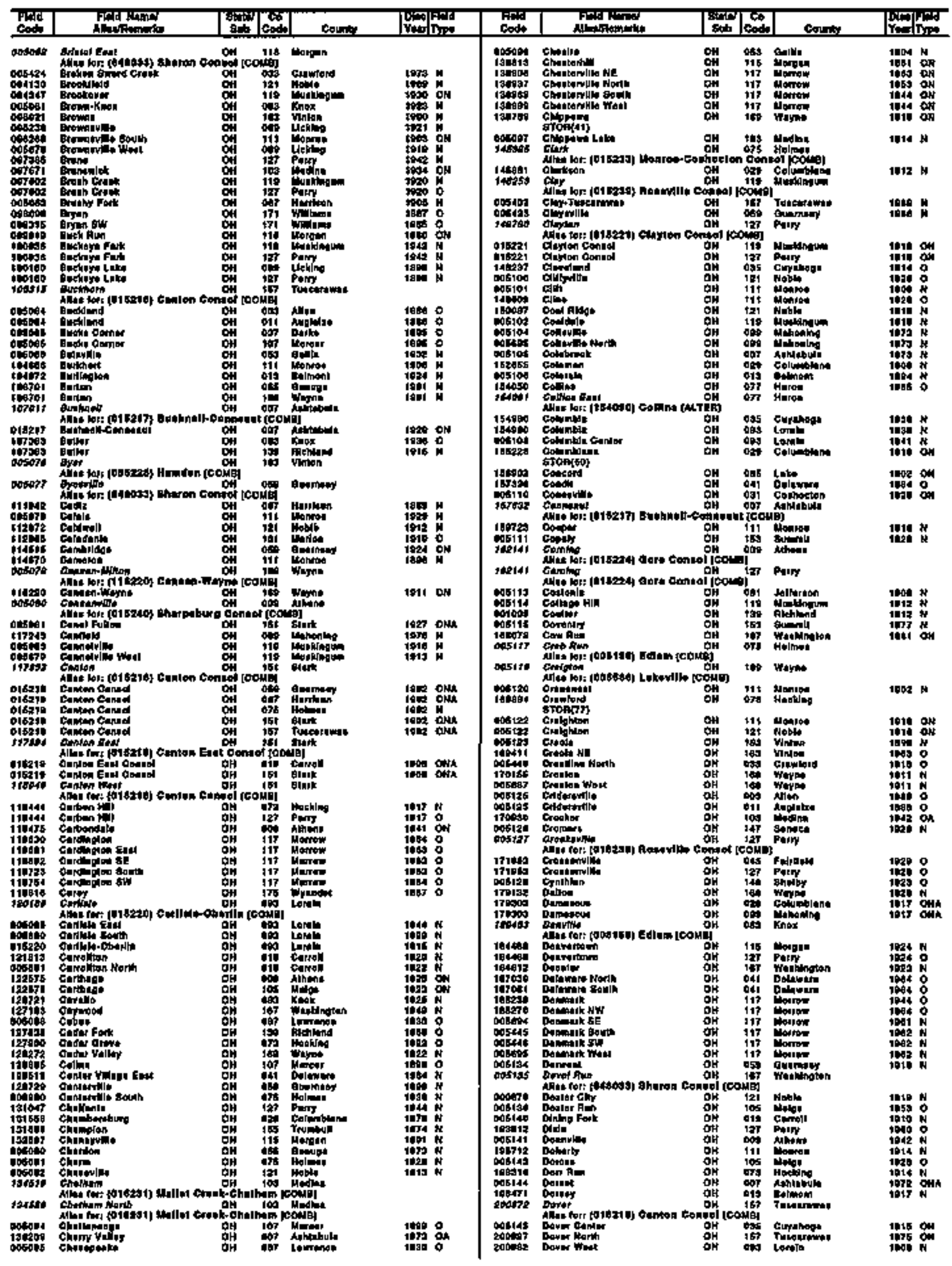




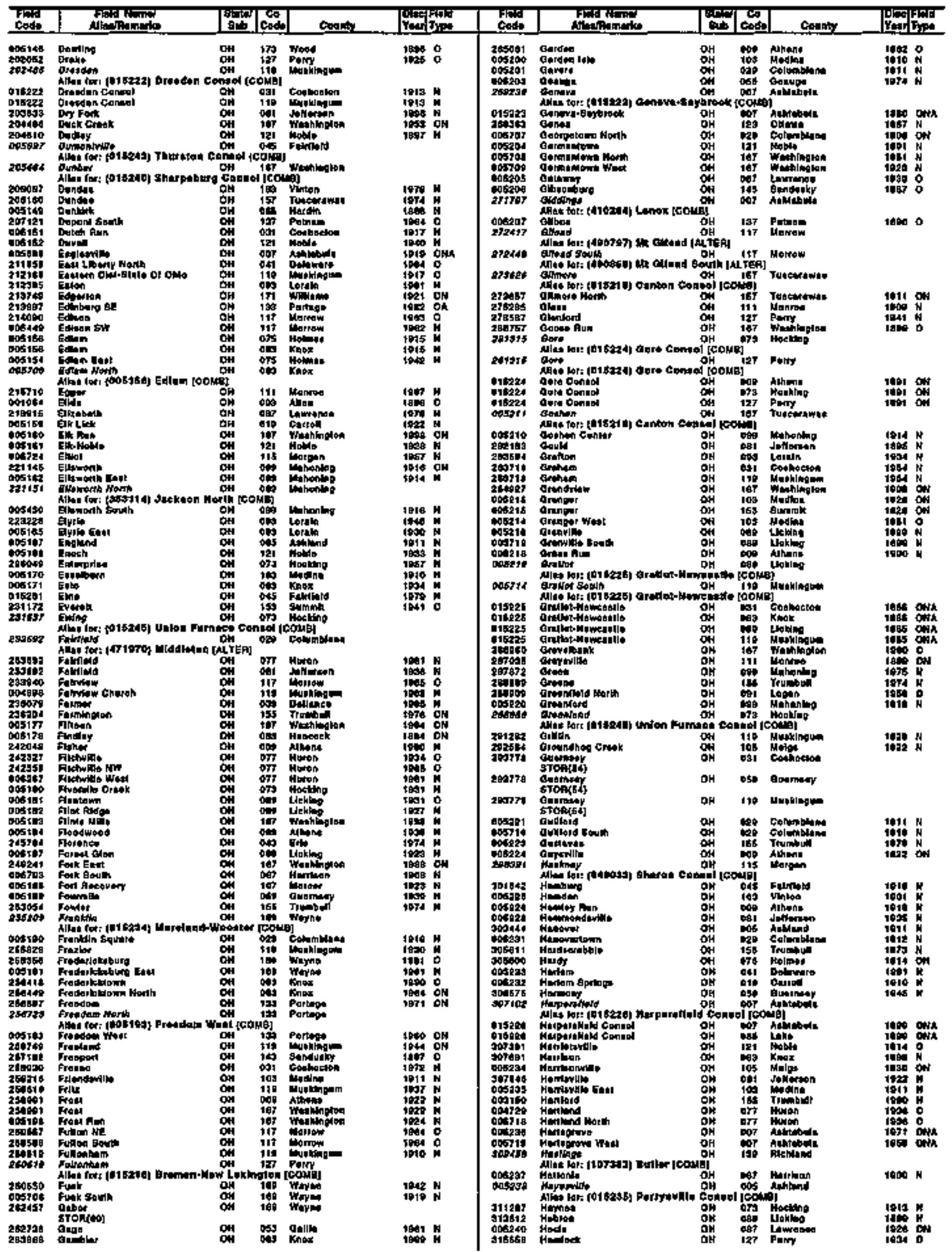


OHIO

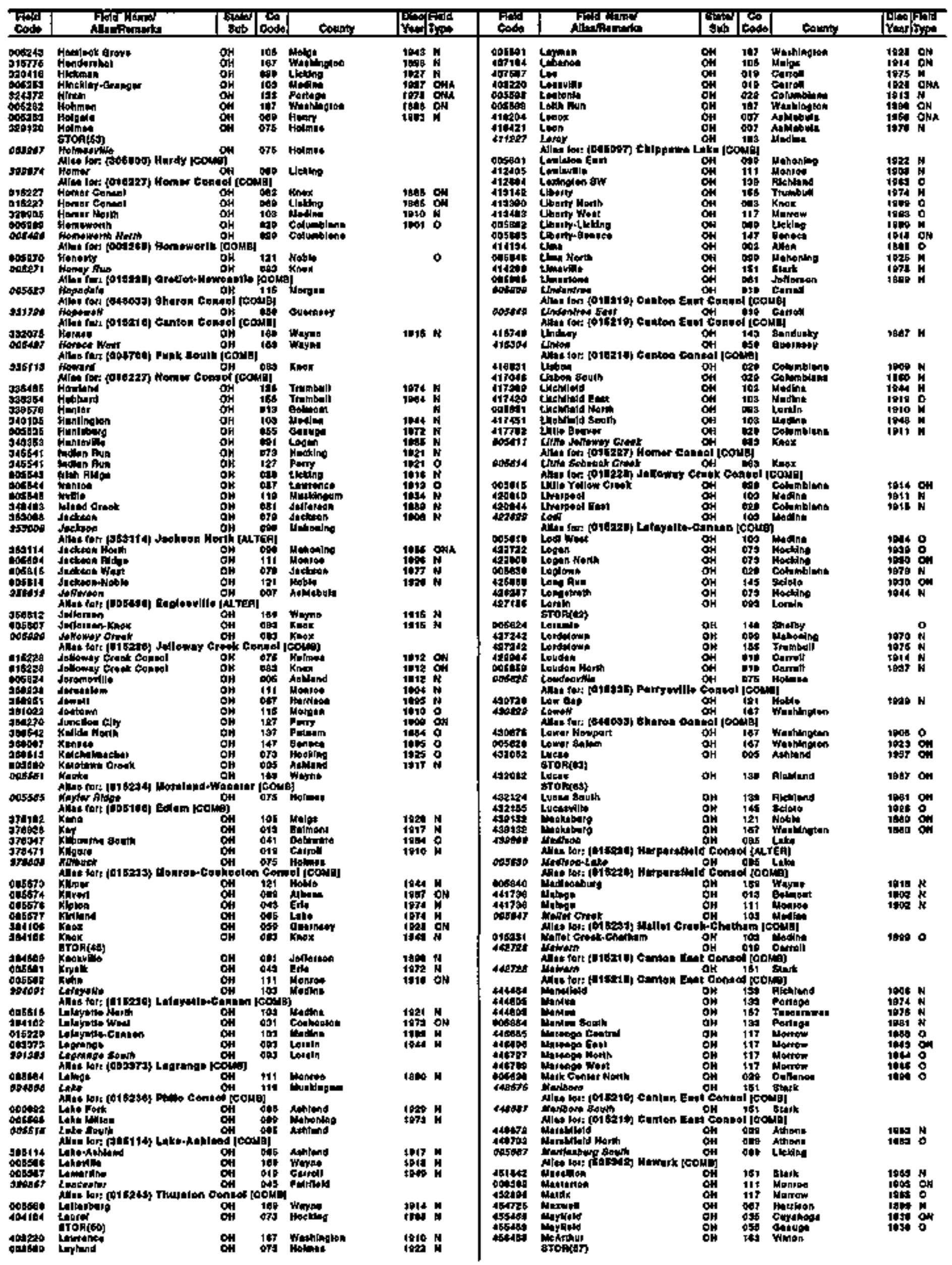




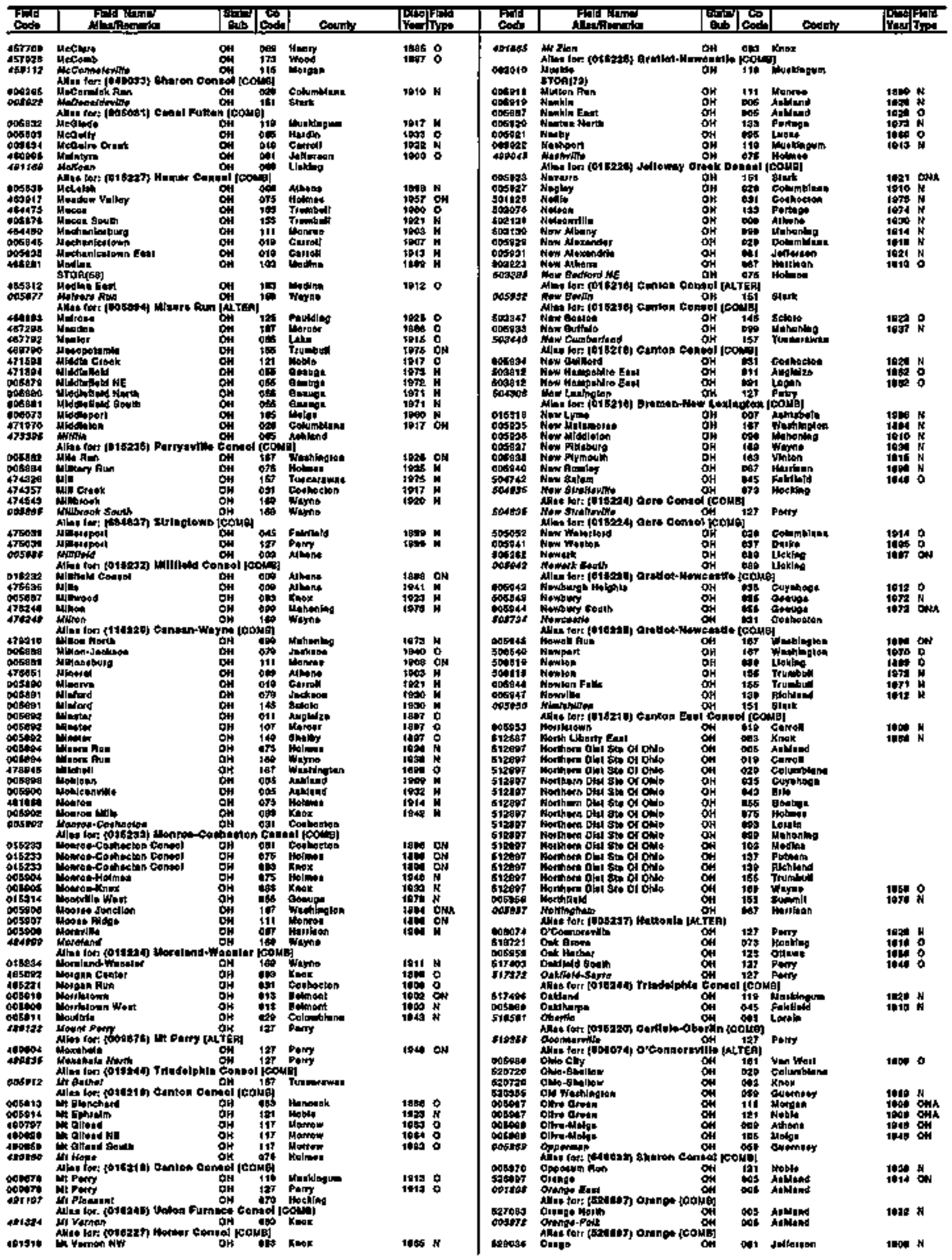




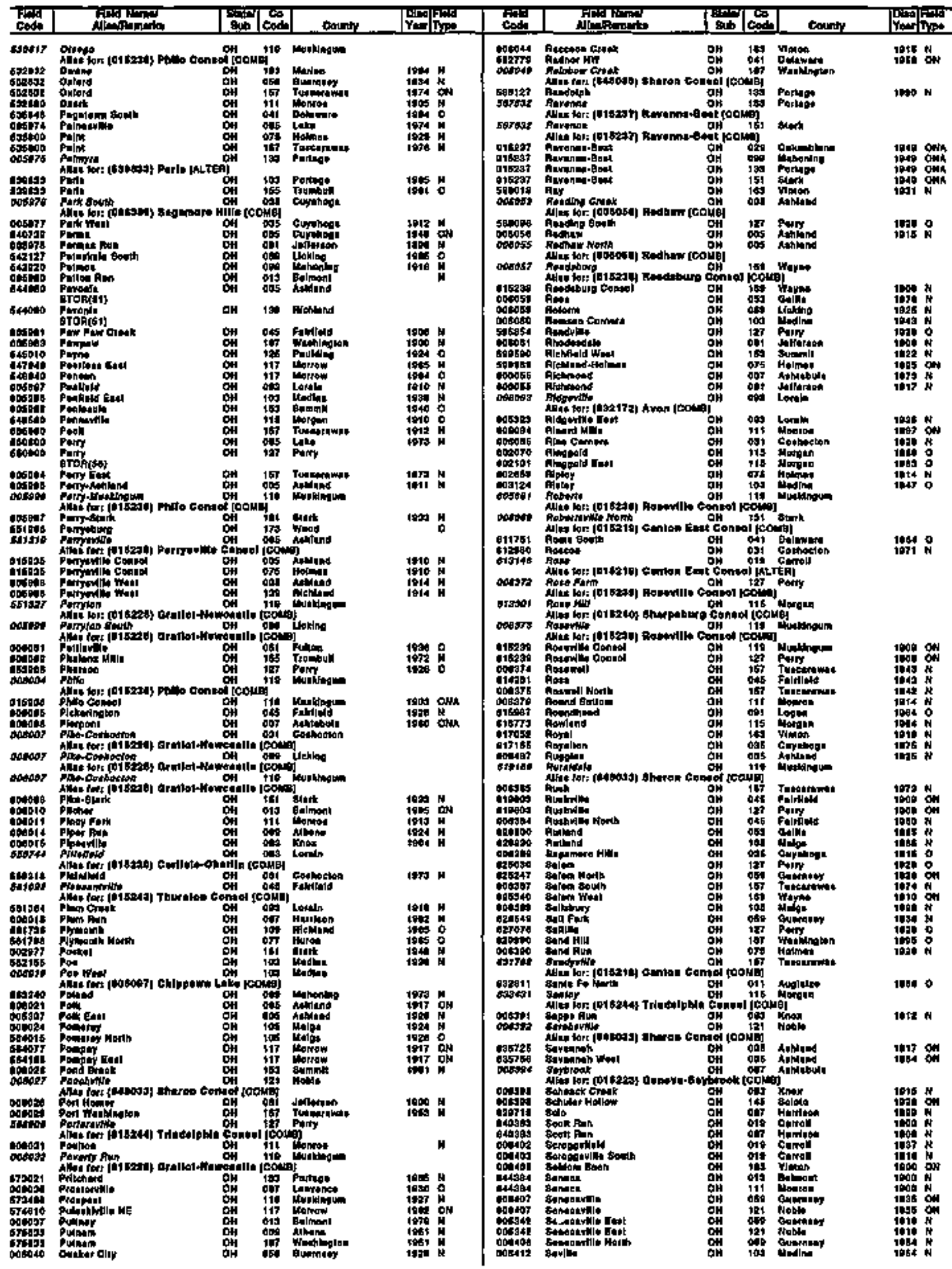




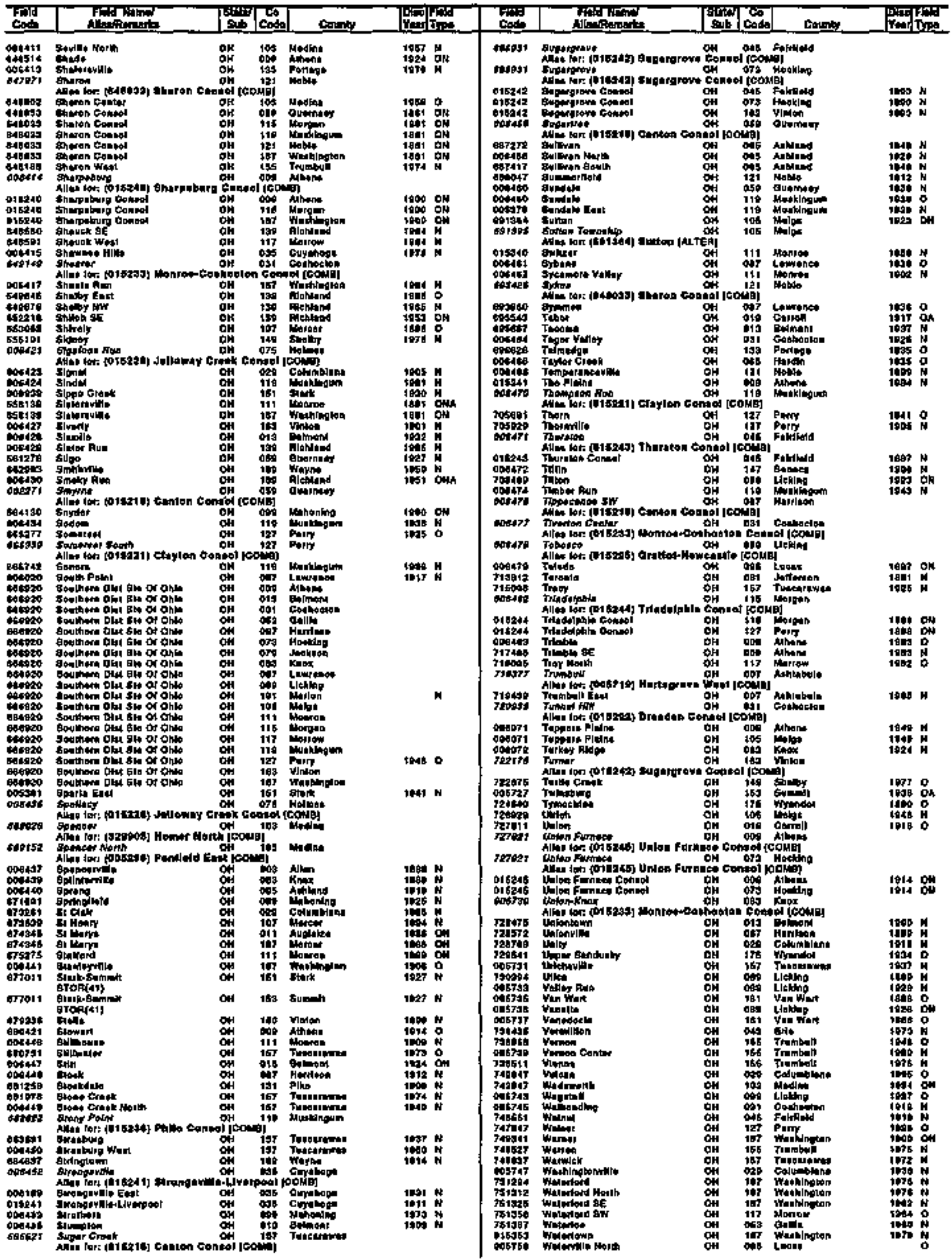


OKLAHOMA

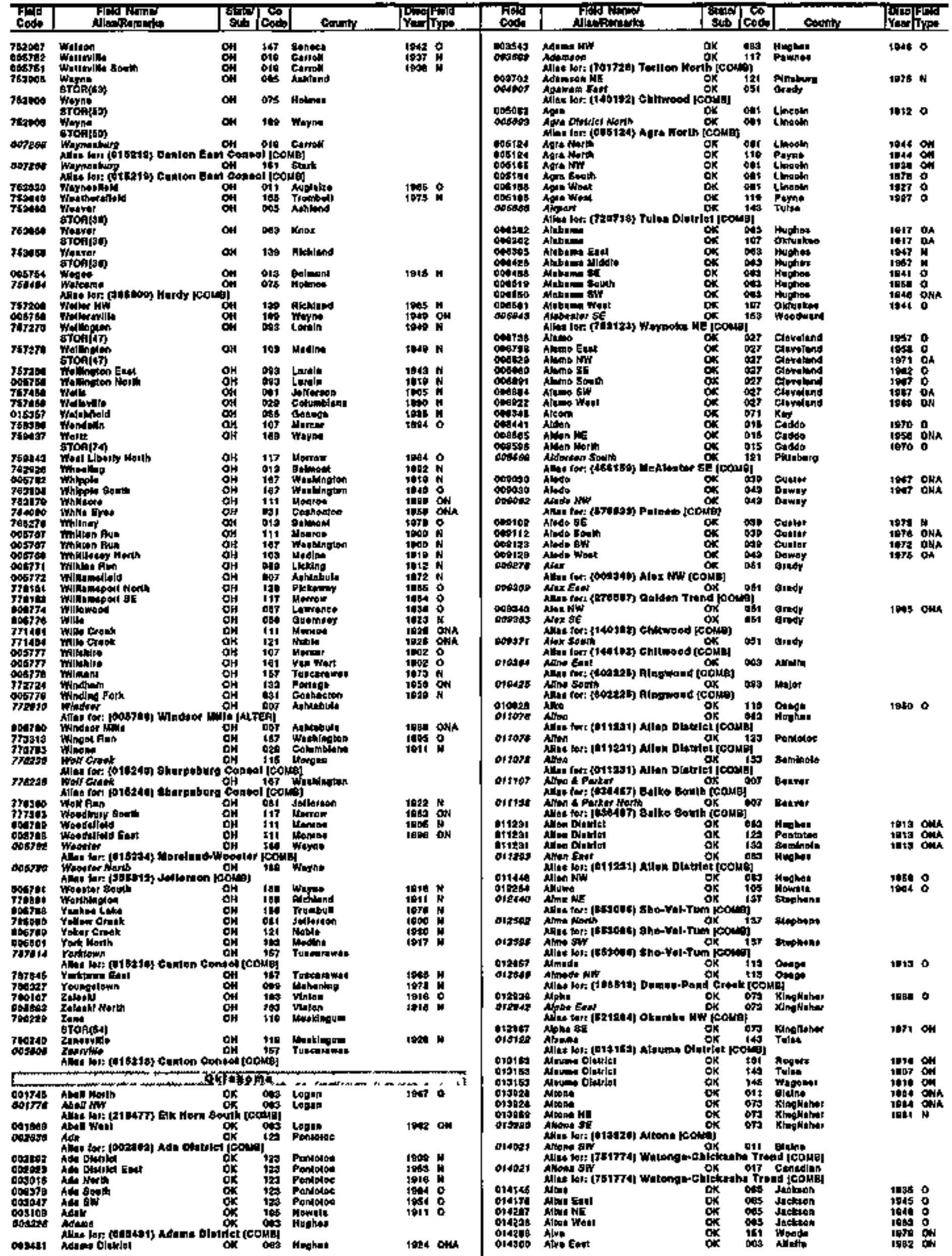


OKLAHOMA

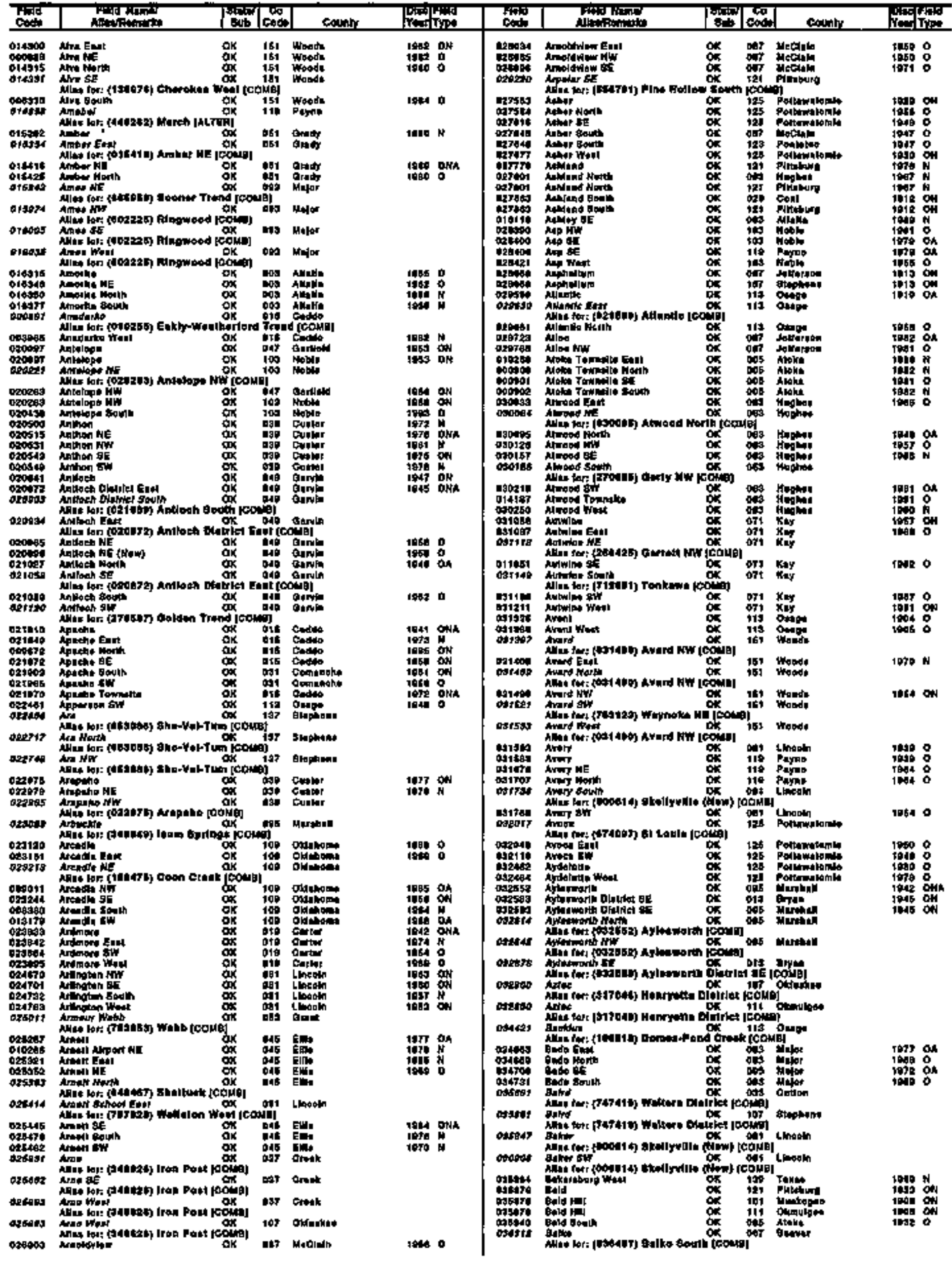


oklahoma

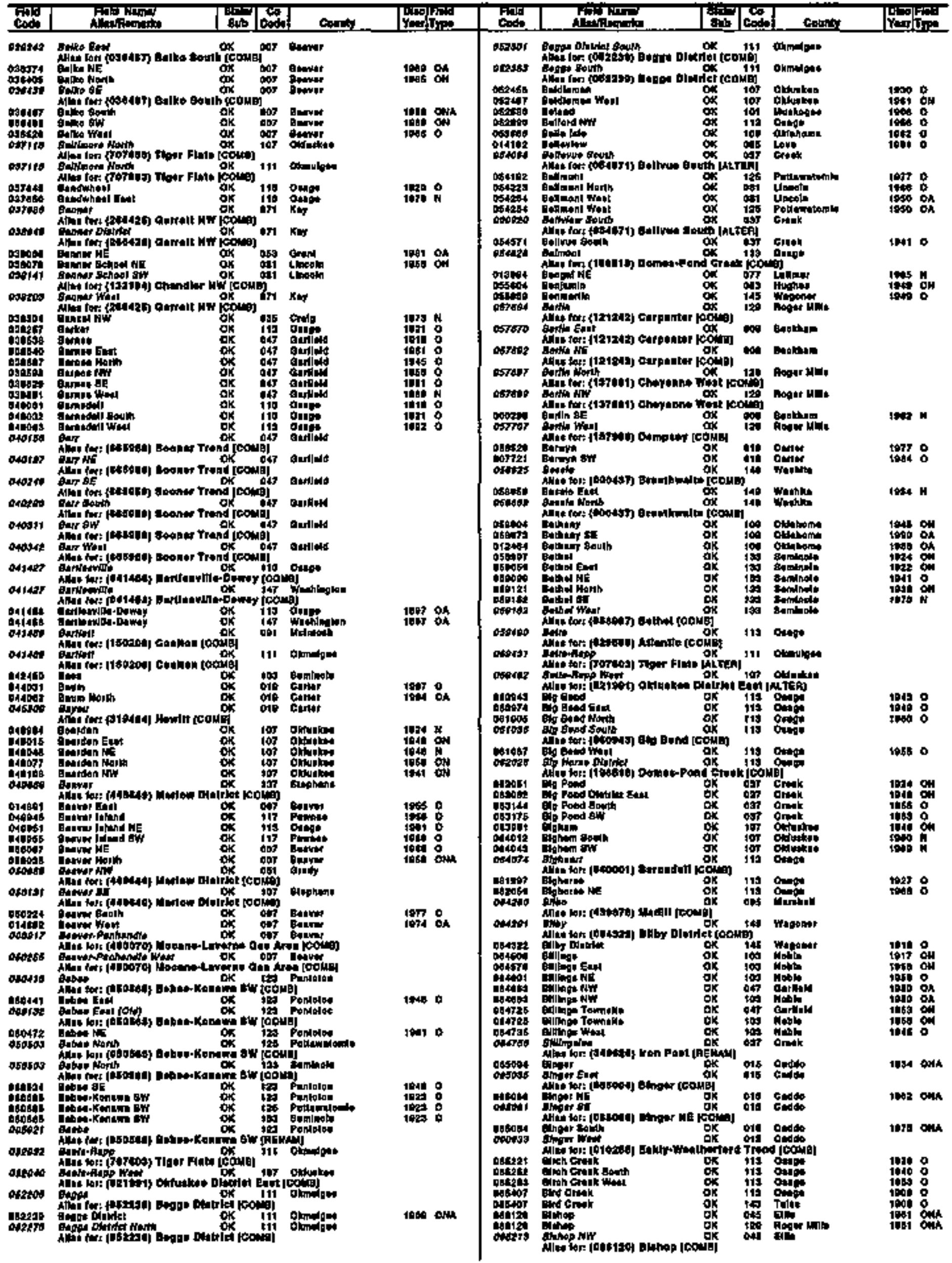


OKLAHOMA

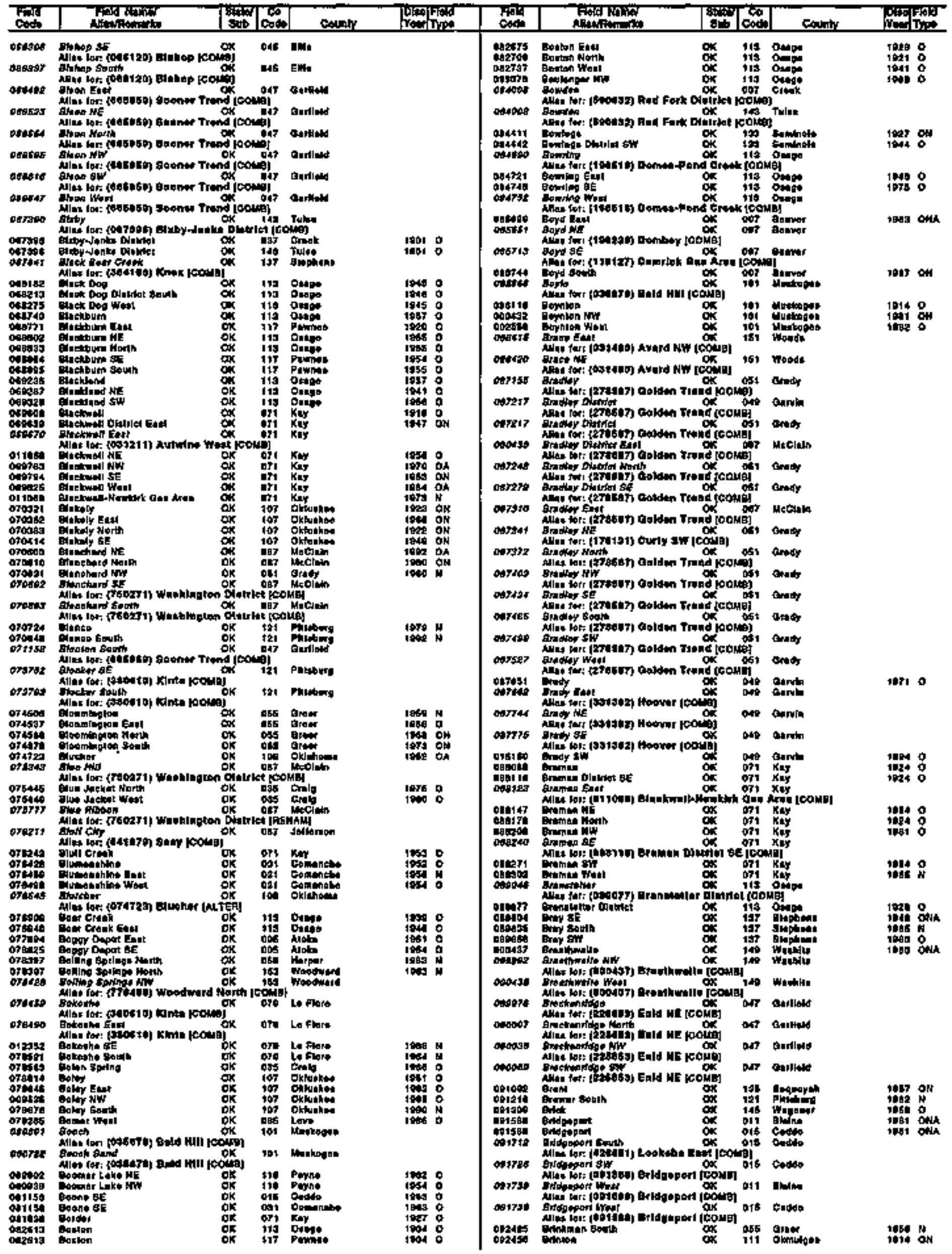


OKLAHOMA

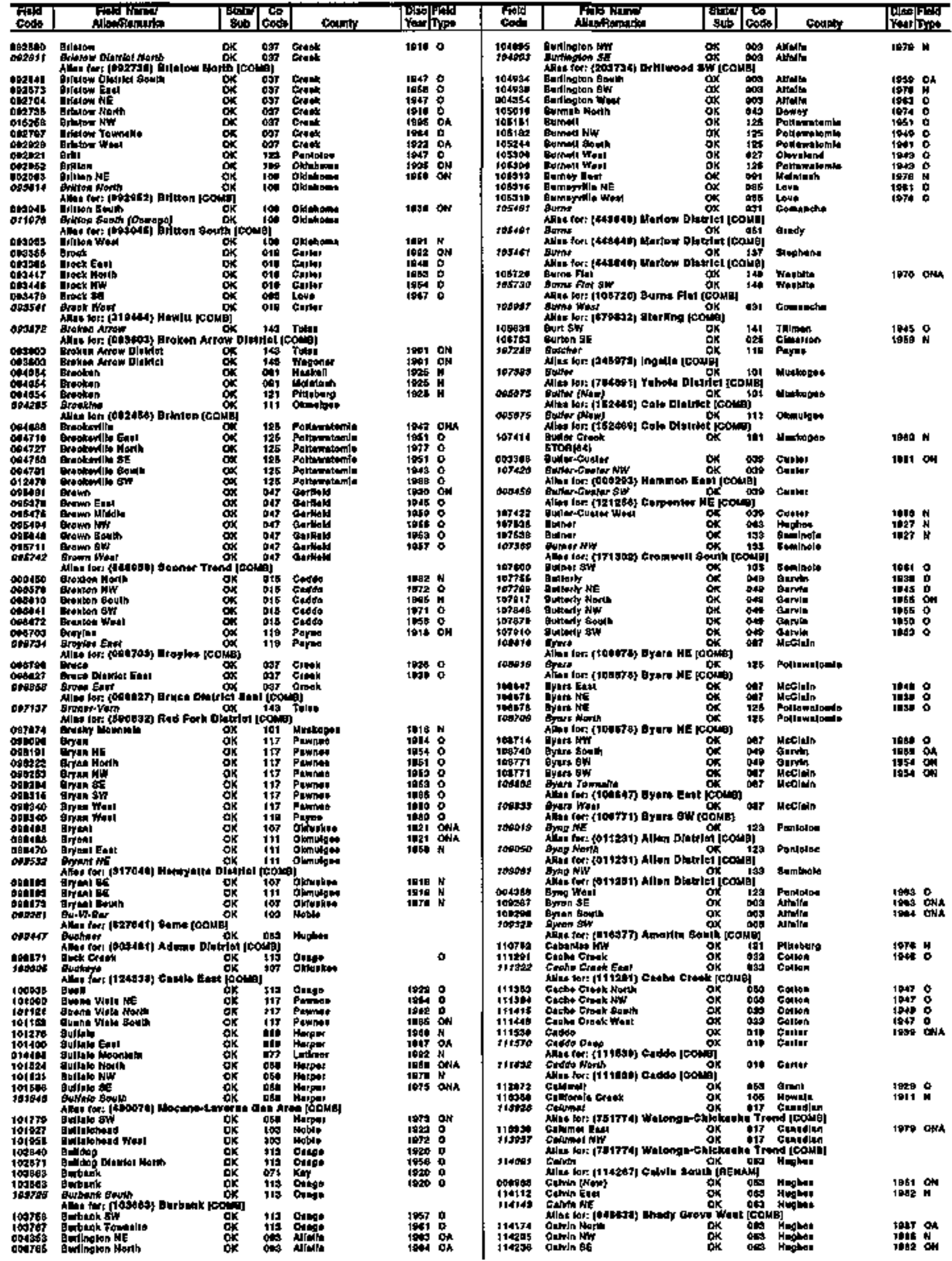


OKLAHOMA

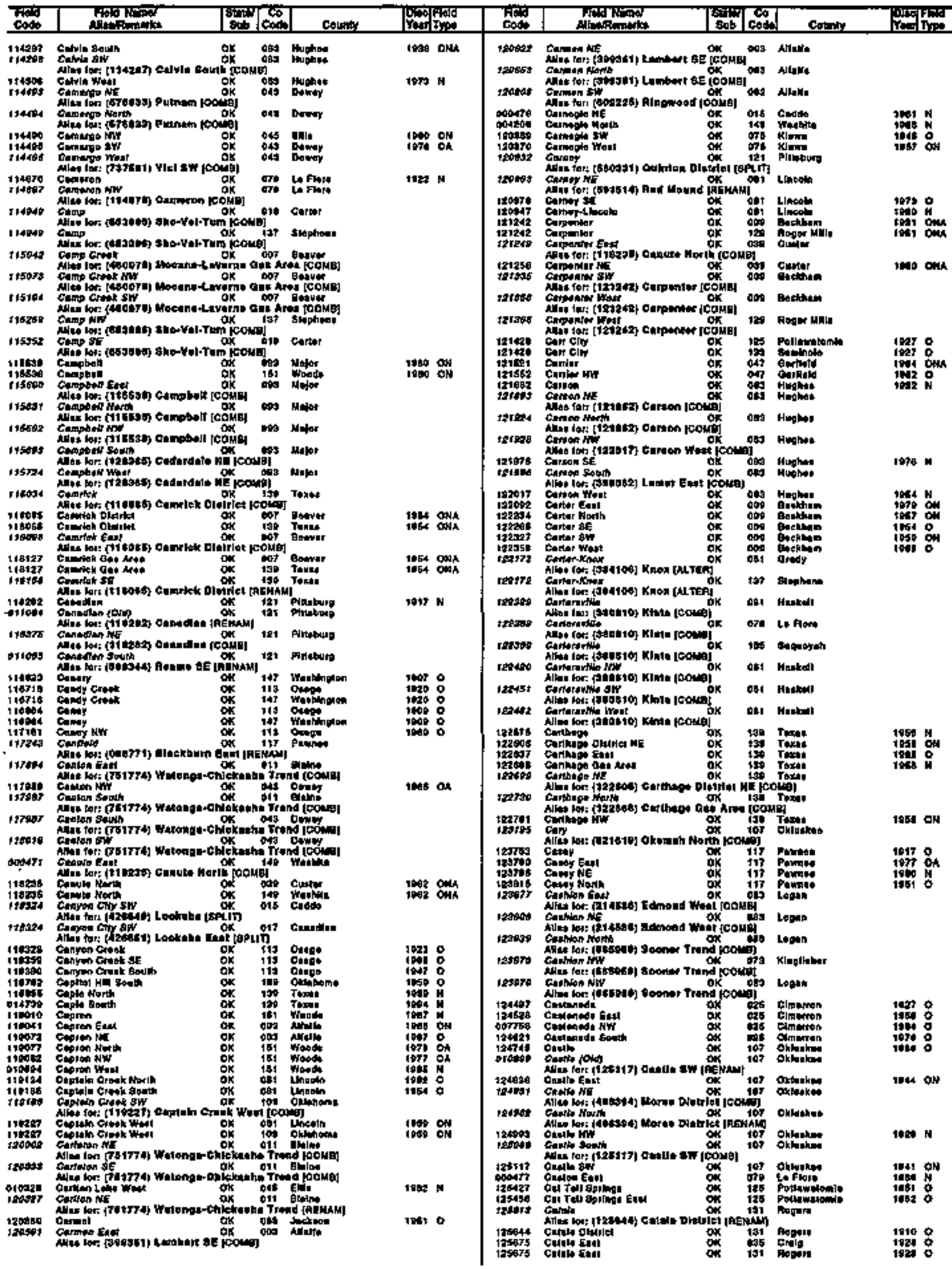


OKLAHOMA

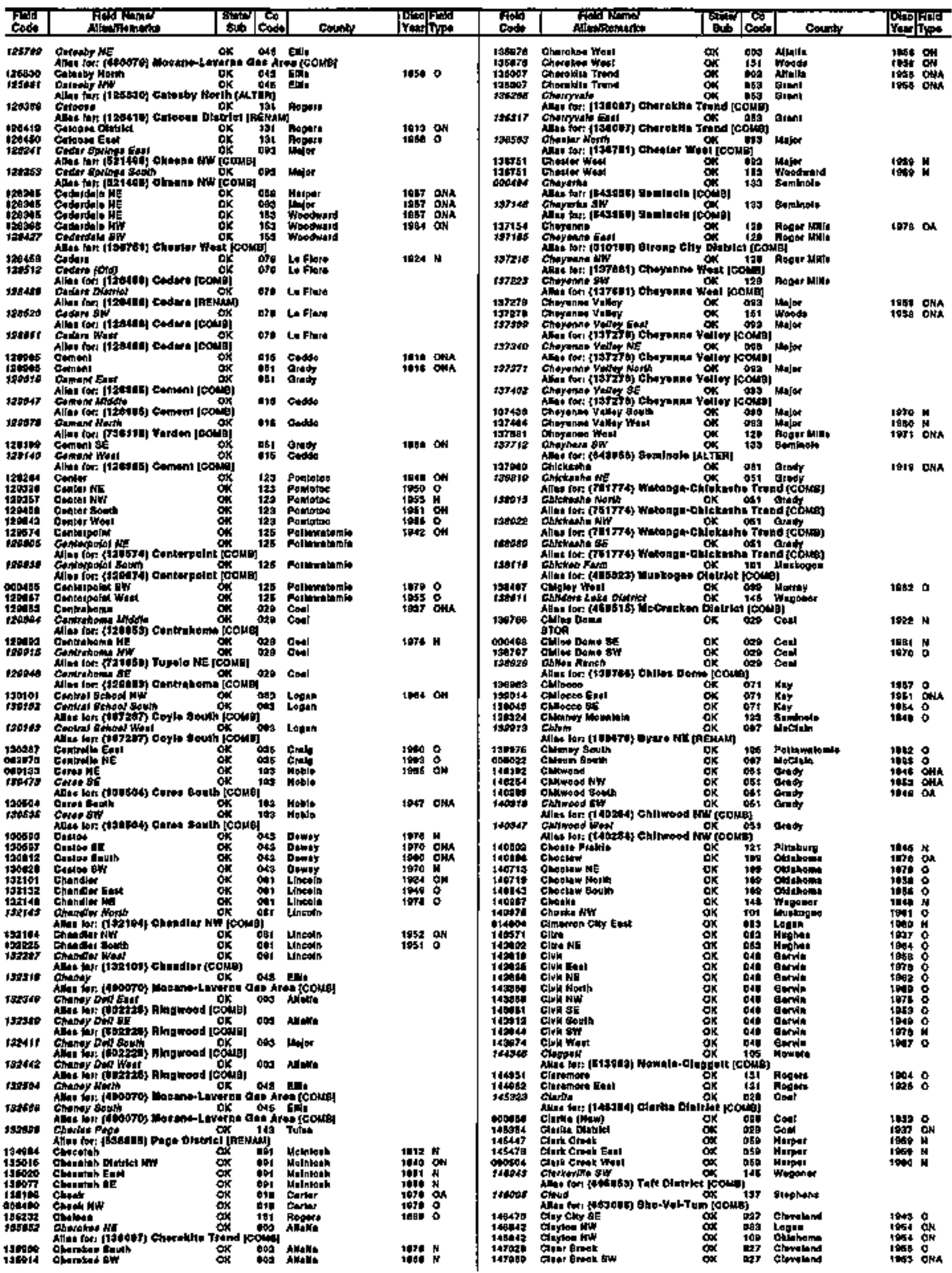




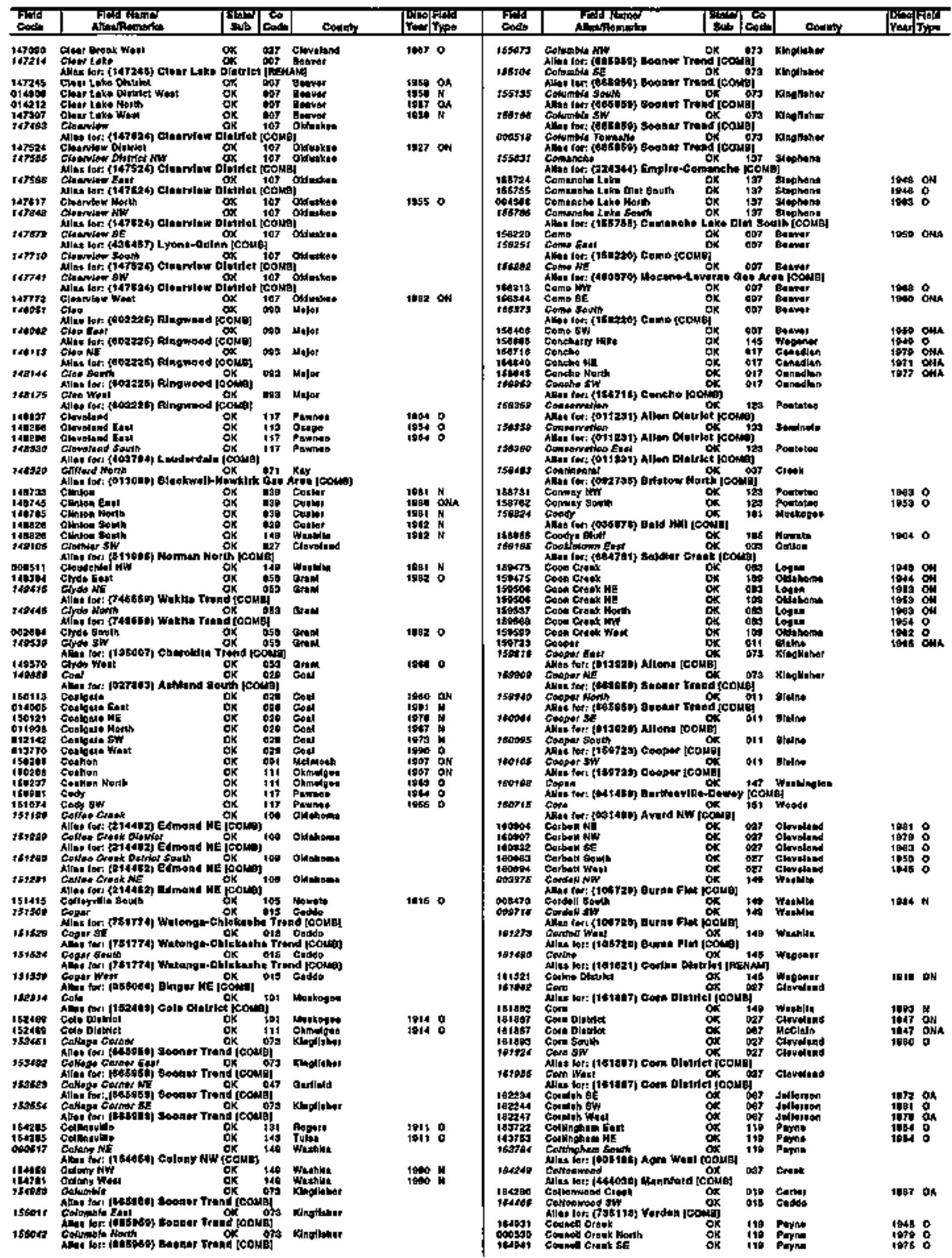


OKLAHOMA

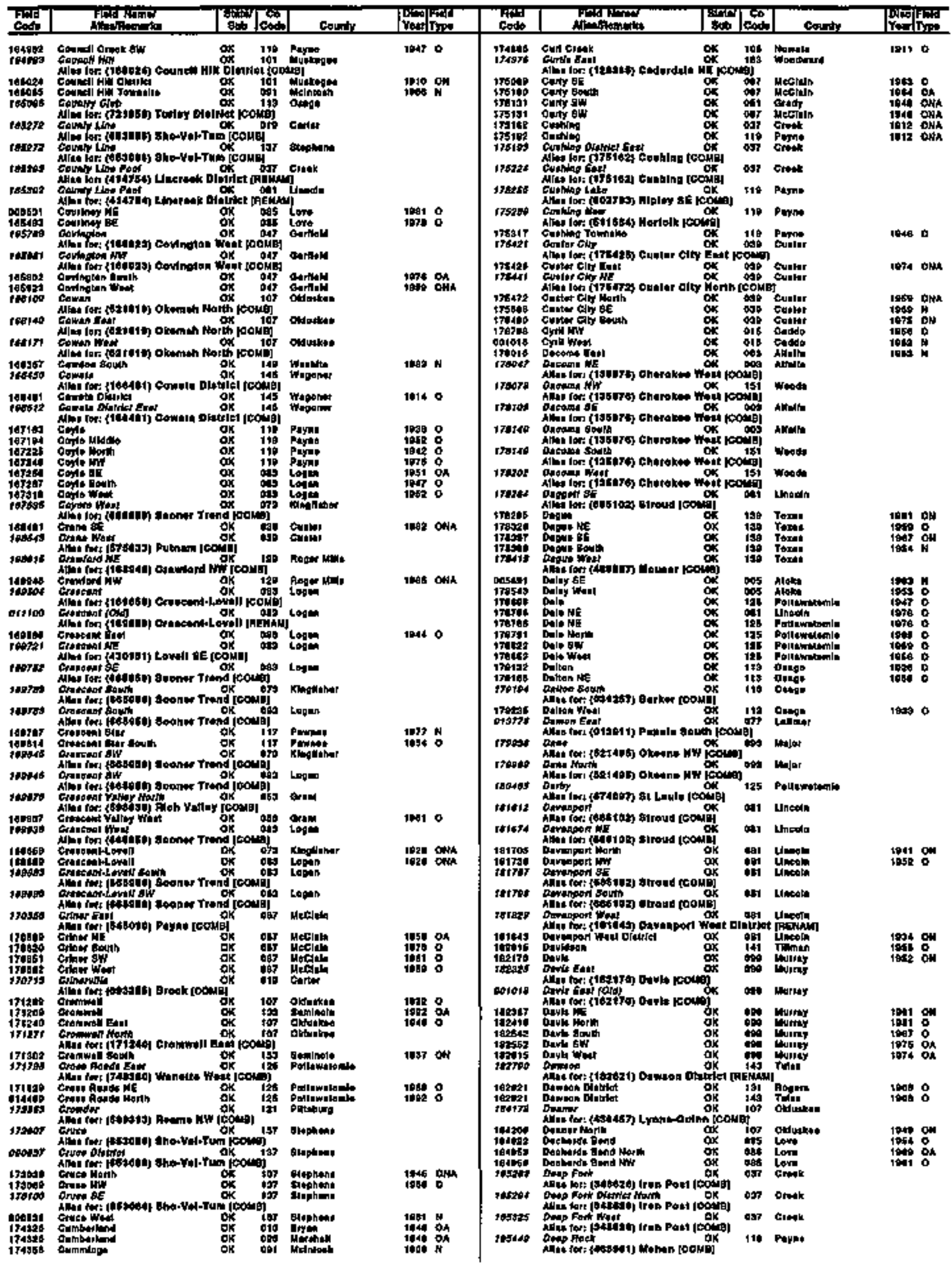




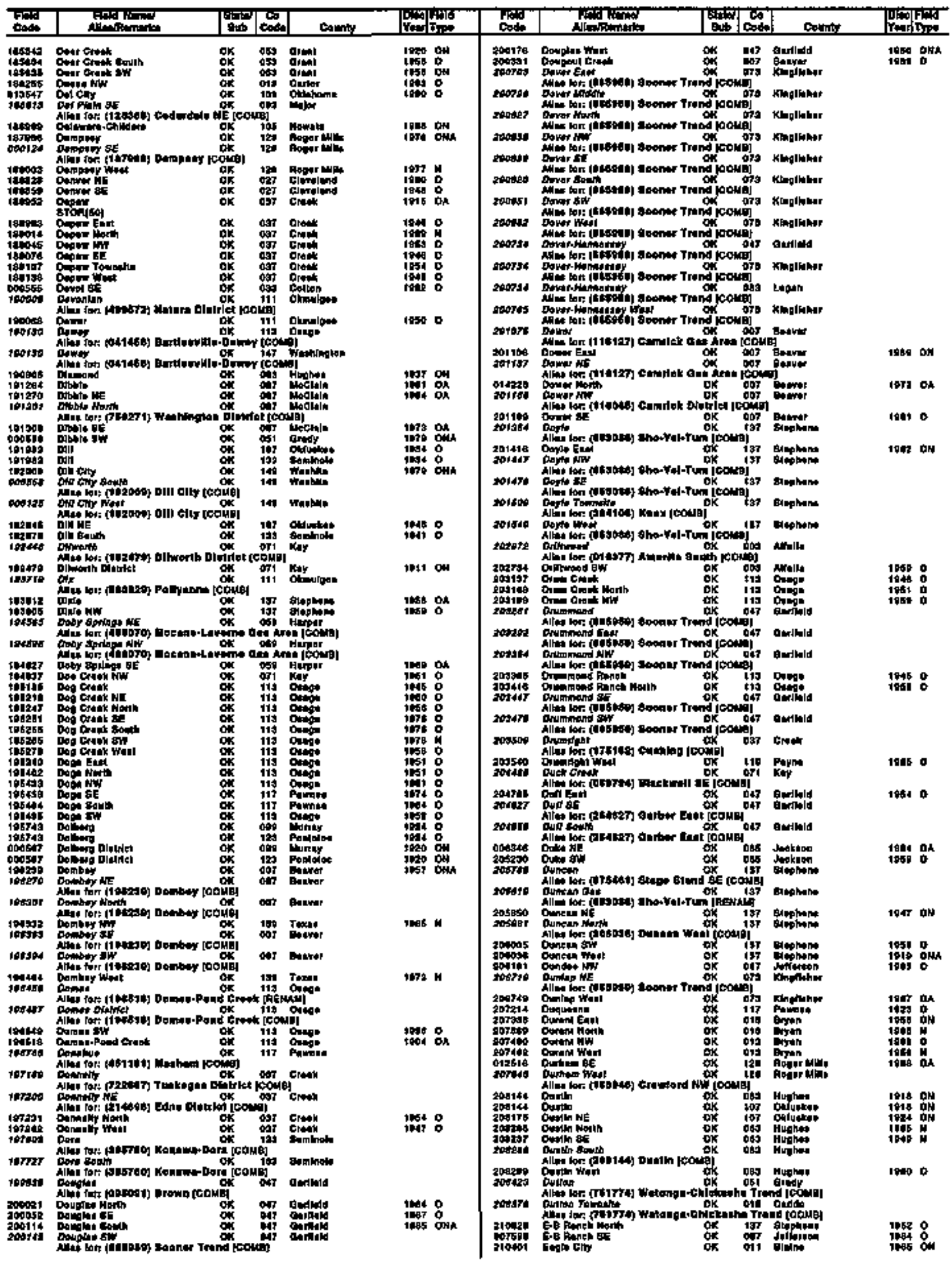


OKLAHOMA

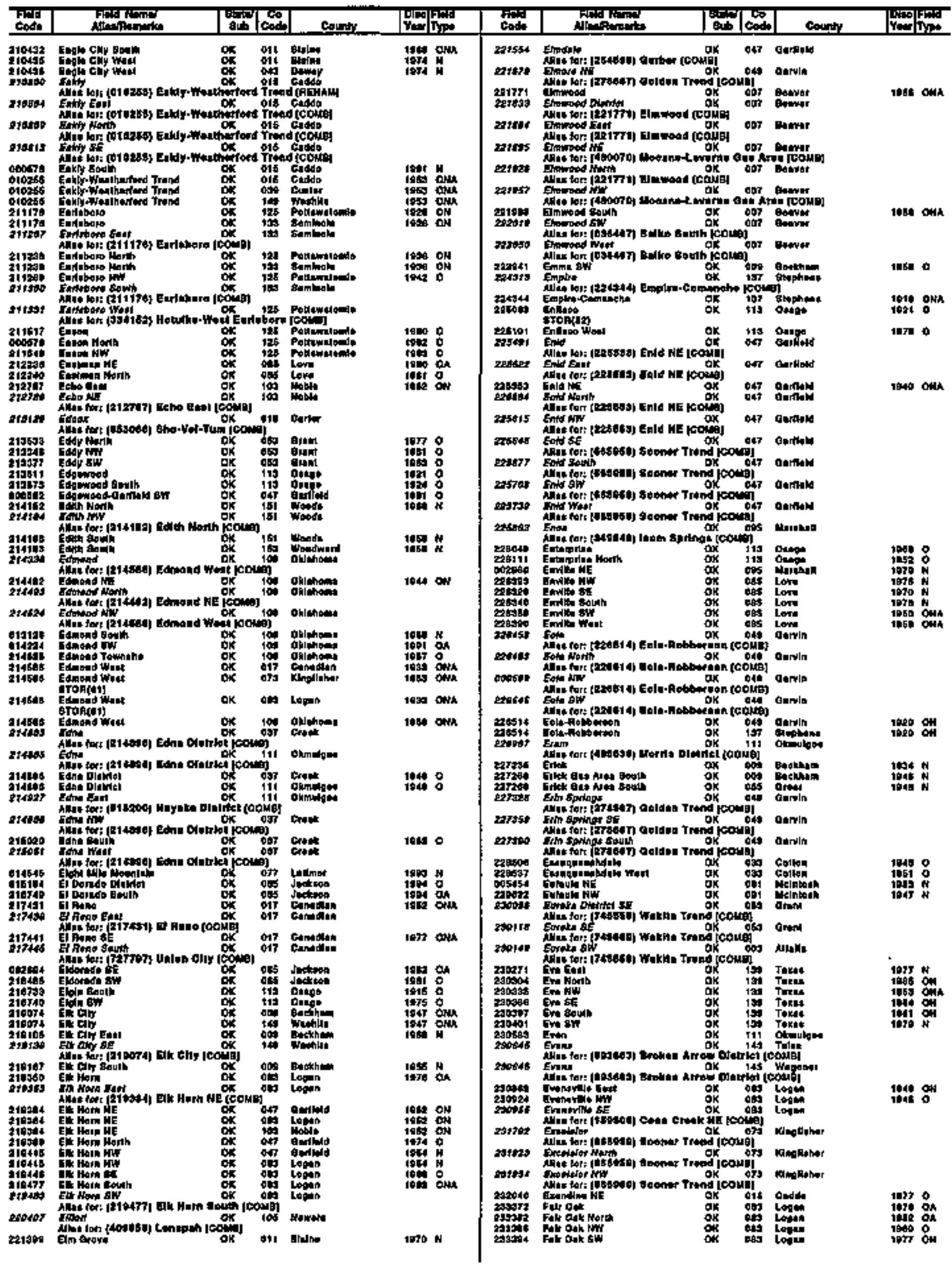




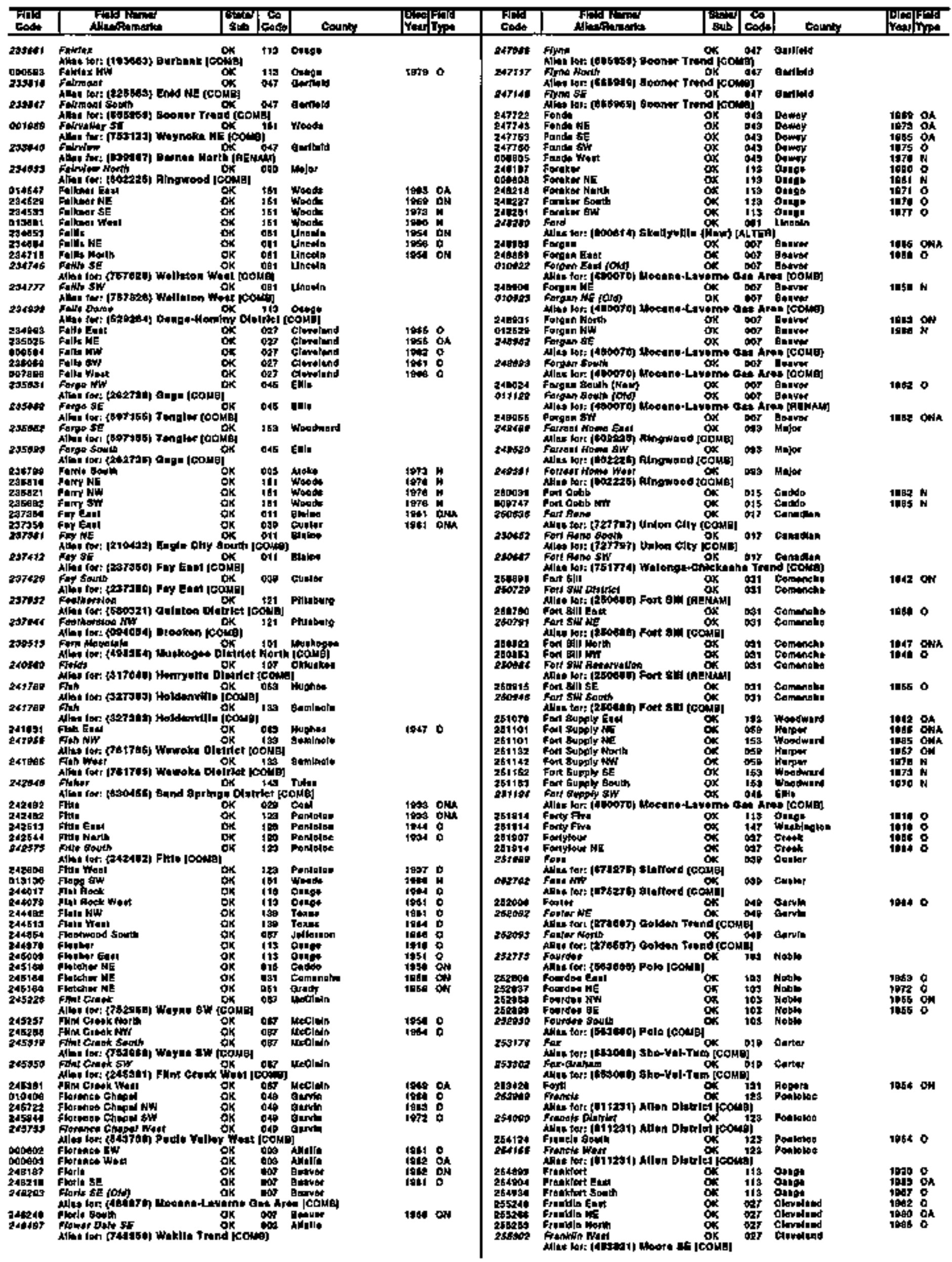


OKLAHOMA

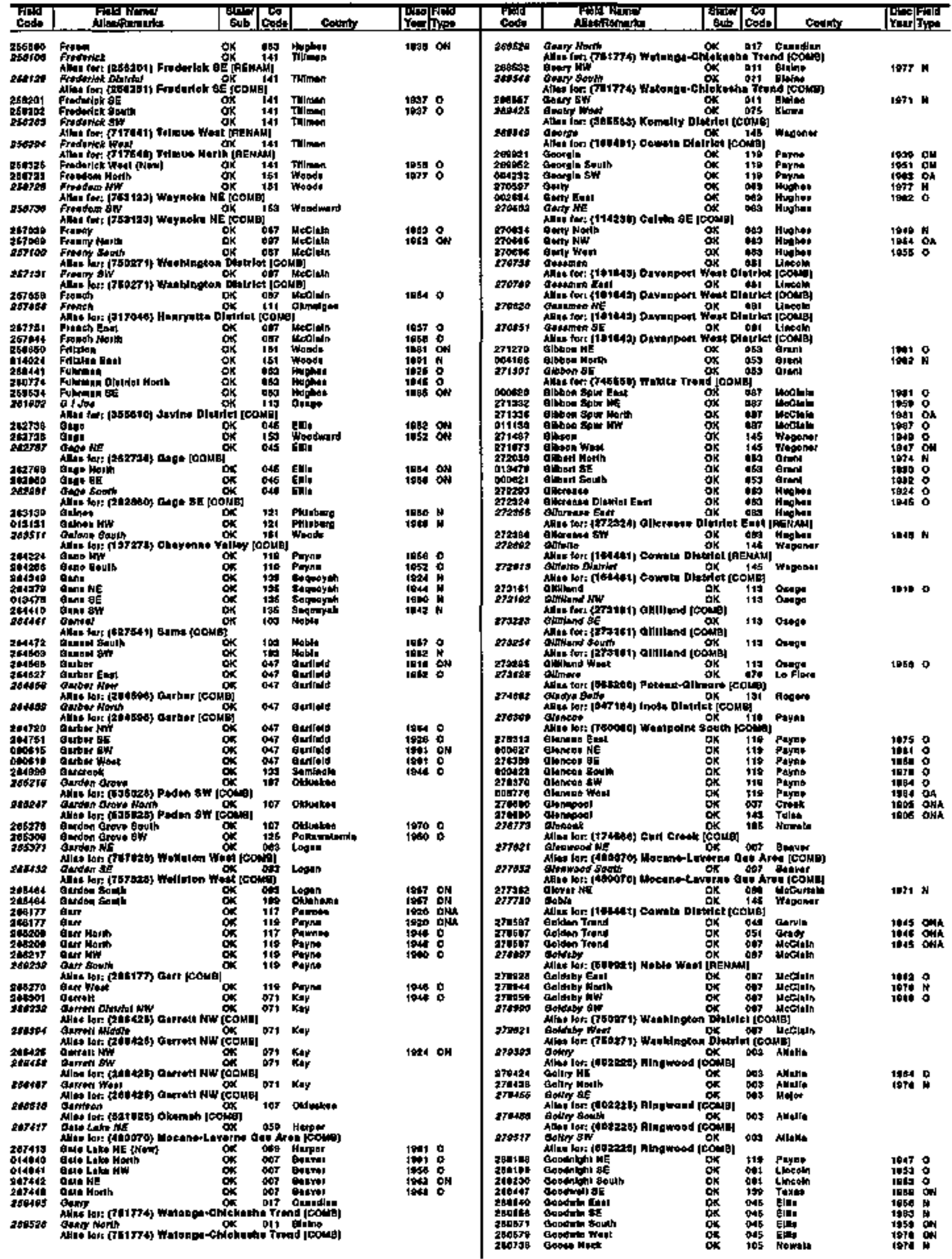




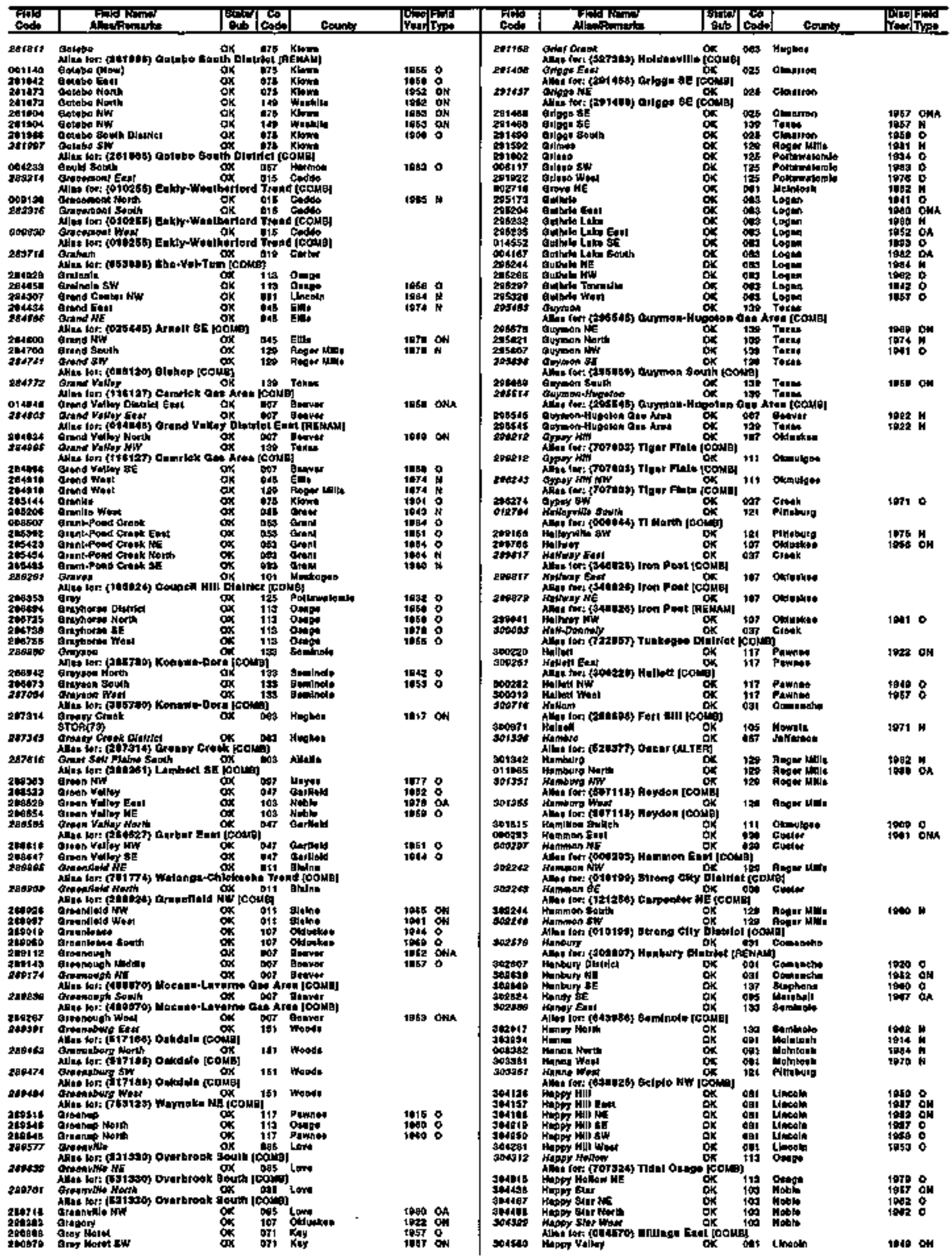


OKLAHOMA

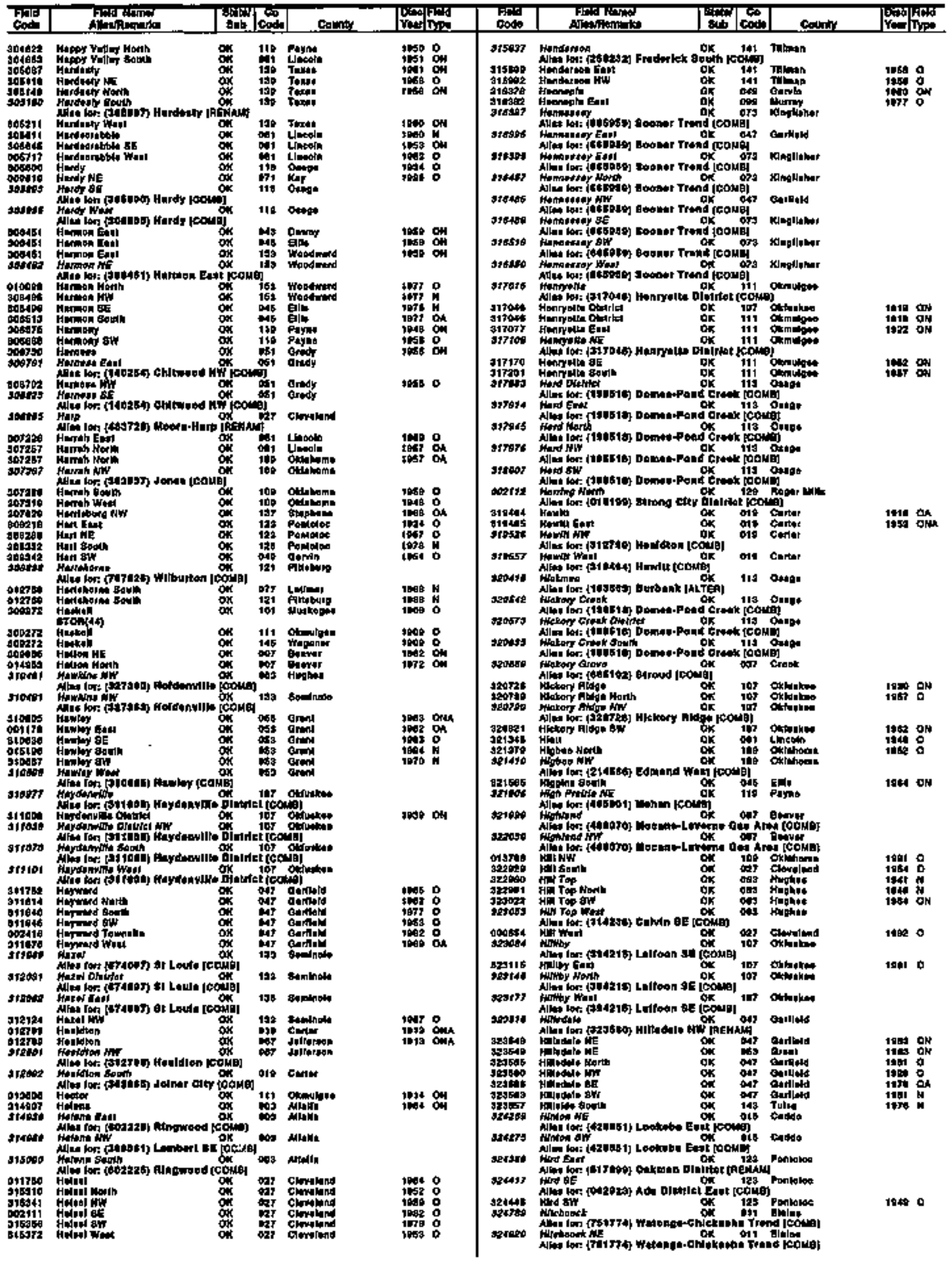


OKLAHOMA

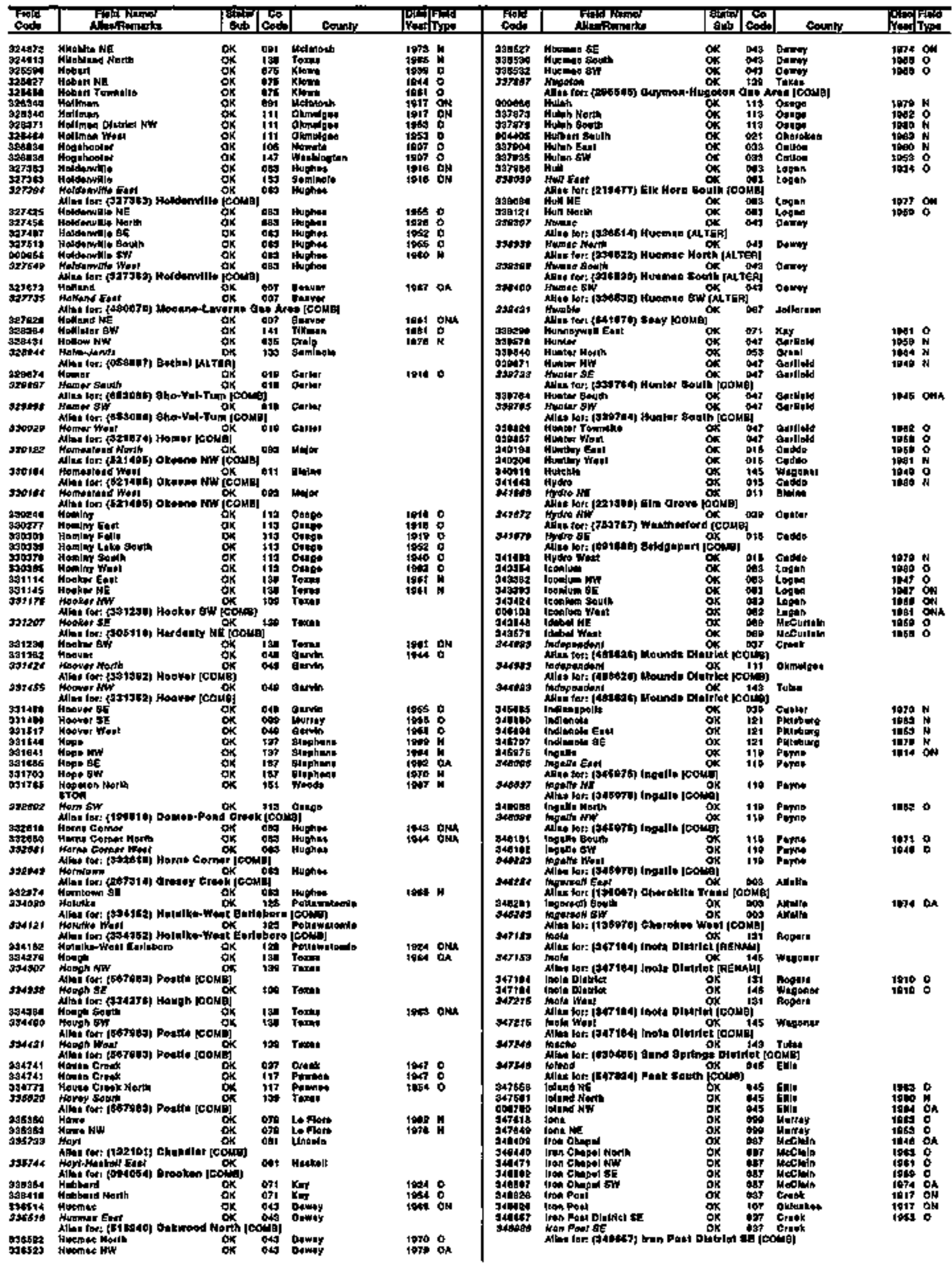


OKLAHOMA

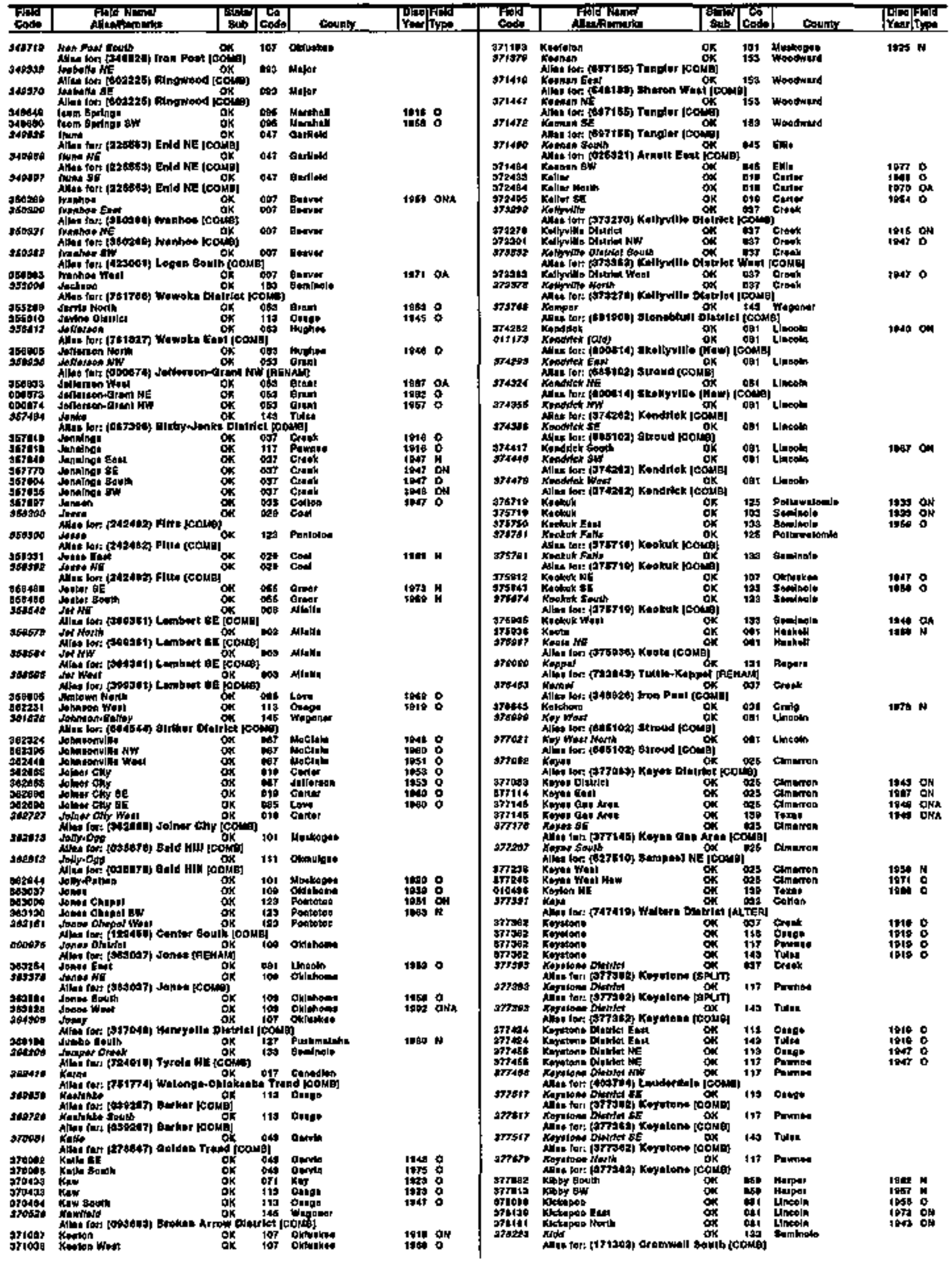


OKLAHOMA

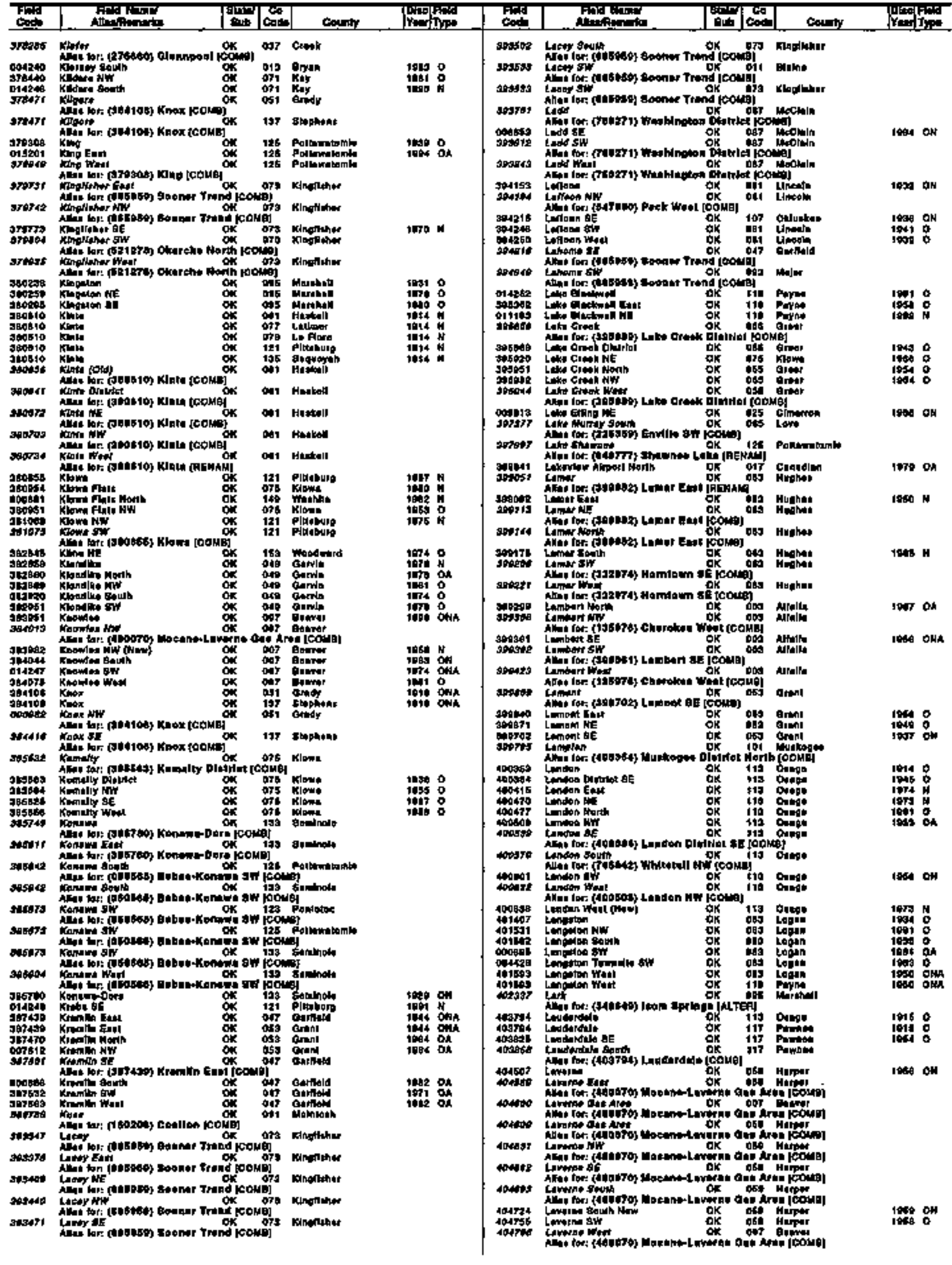


okLAHOMA

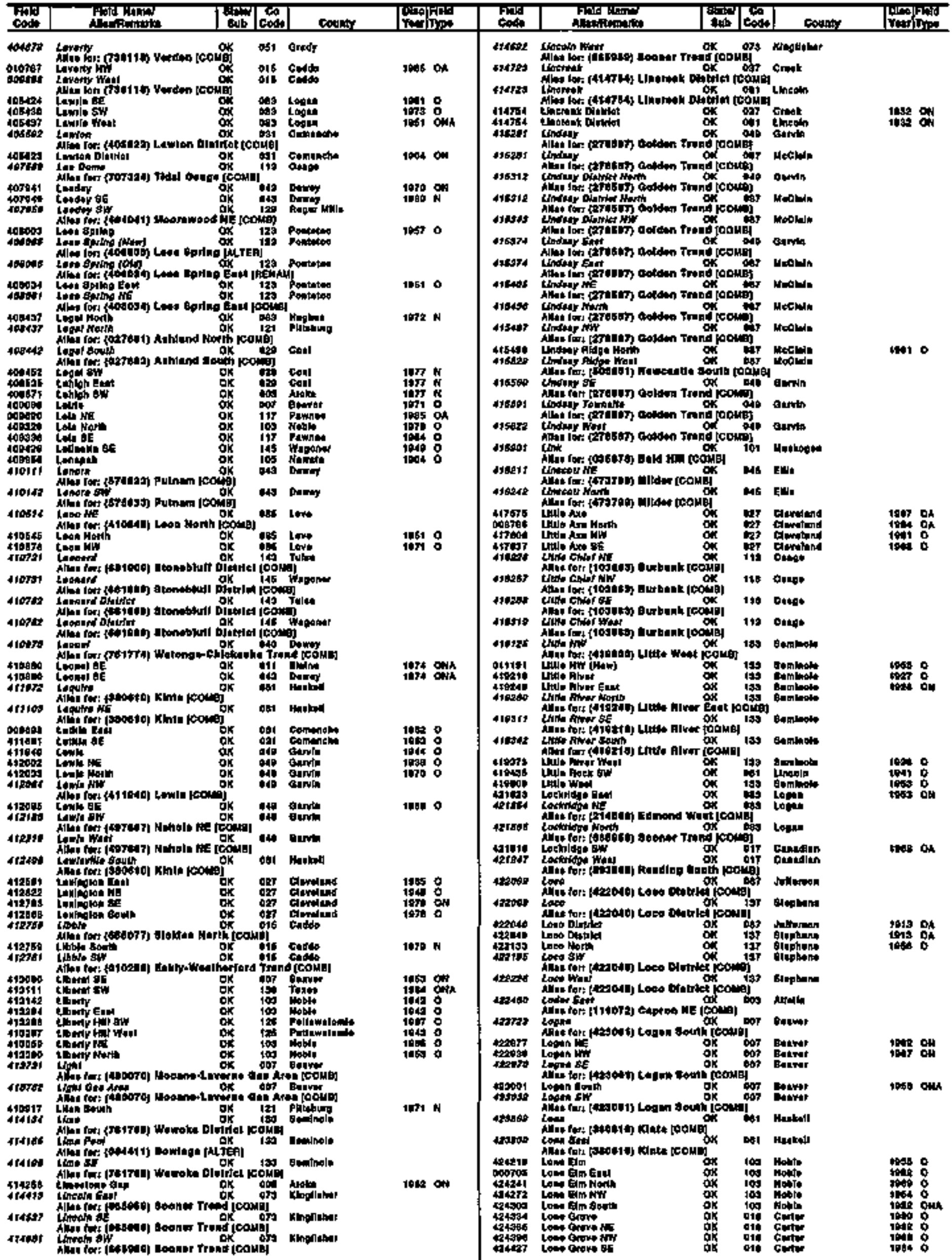




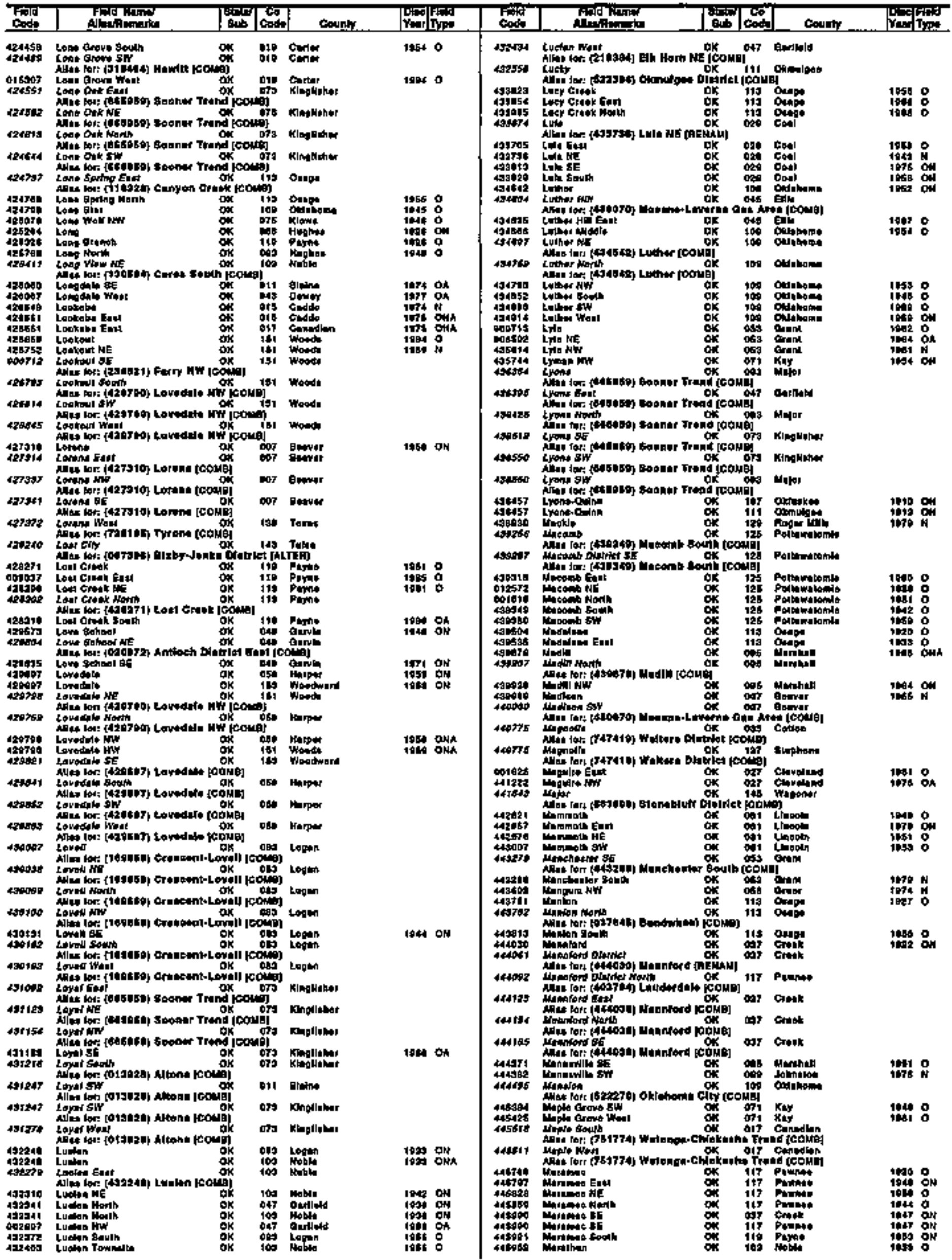


OKLAHOMA

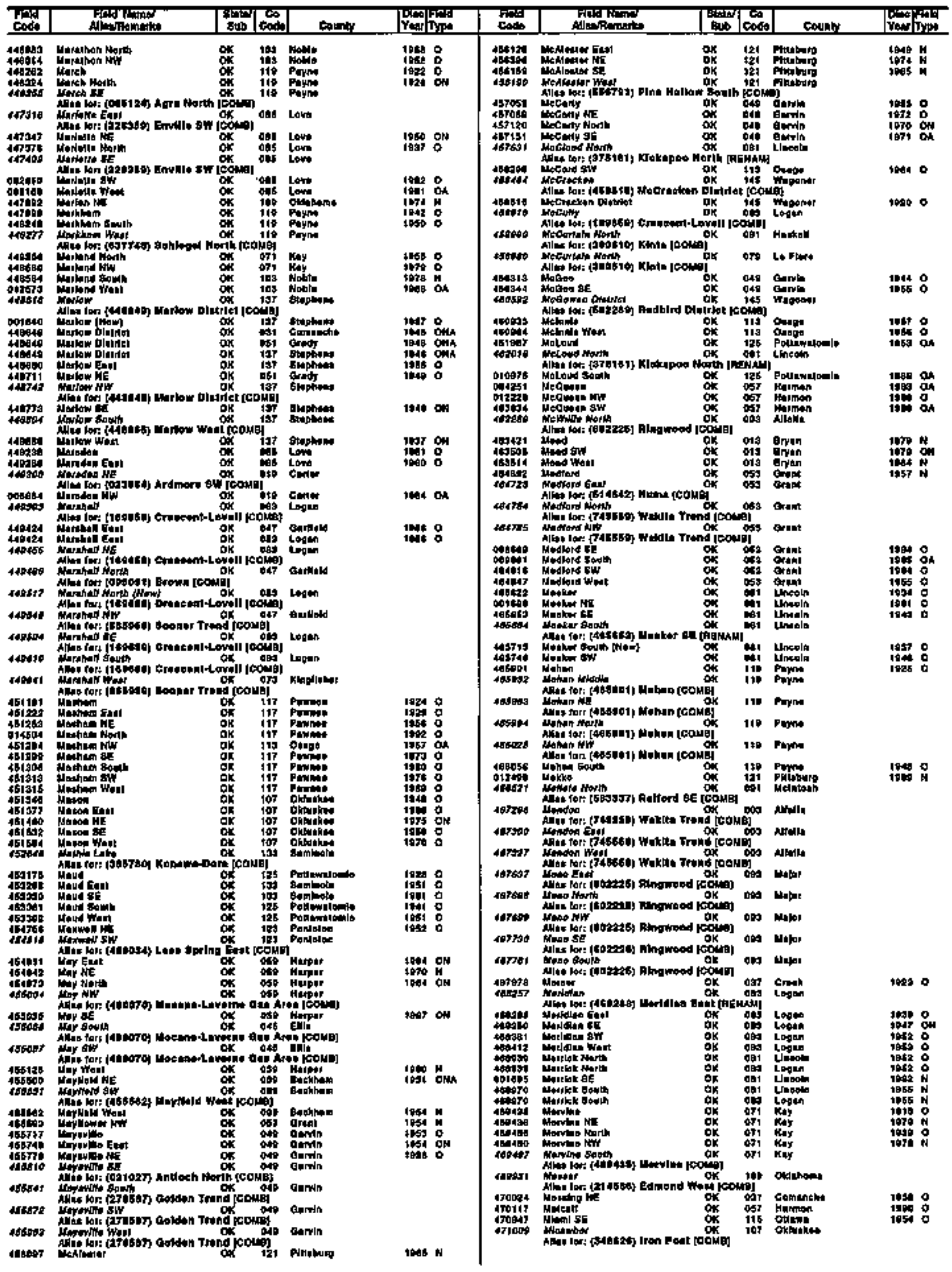


OKLAHOMA

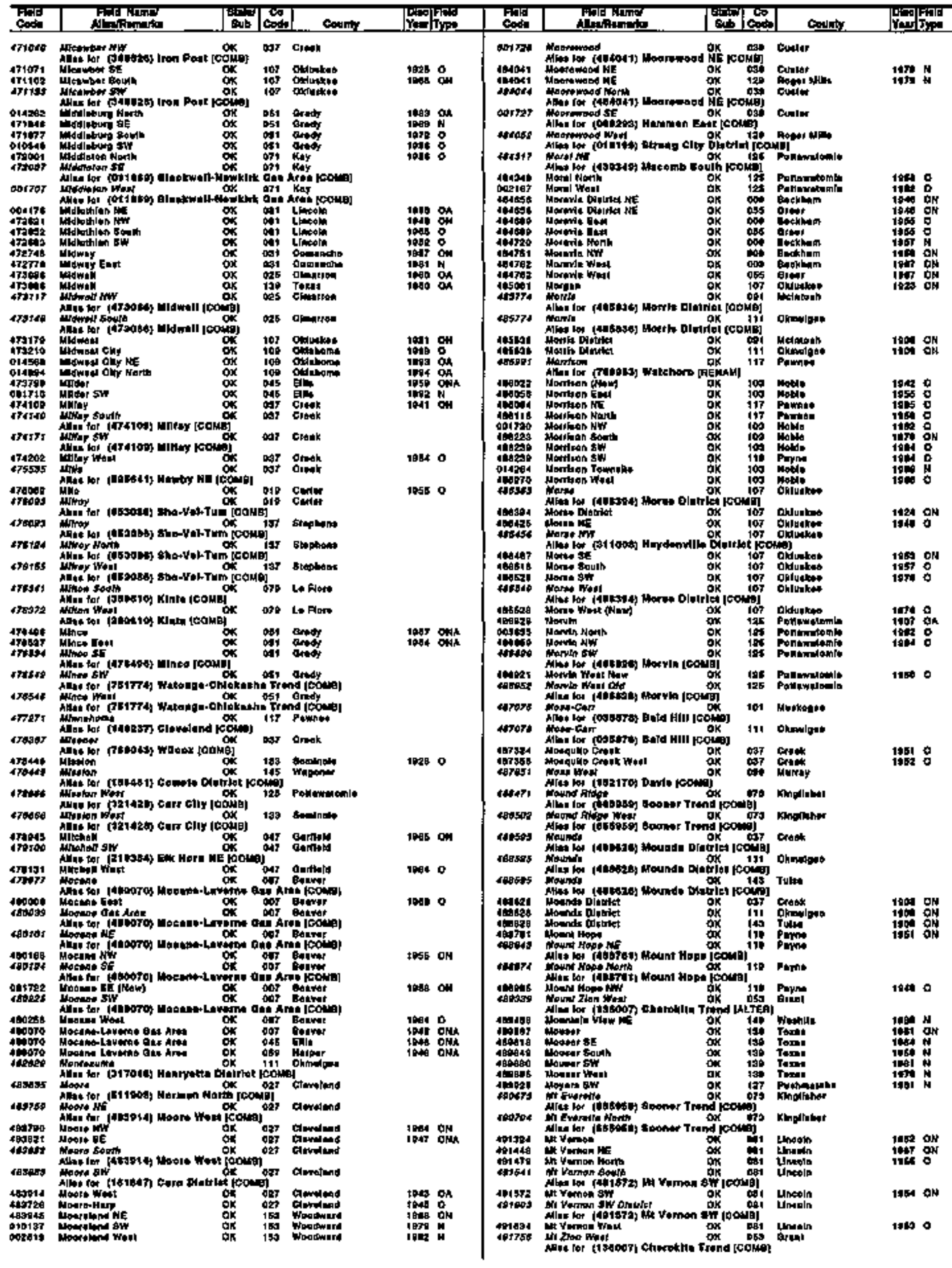


OKLAHOHA

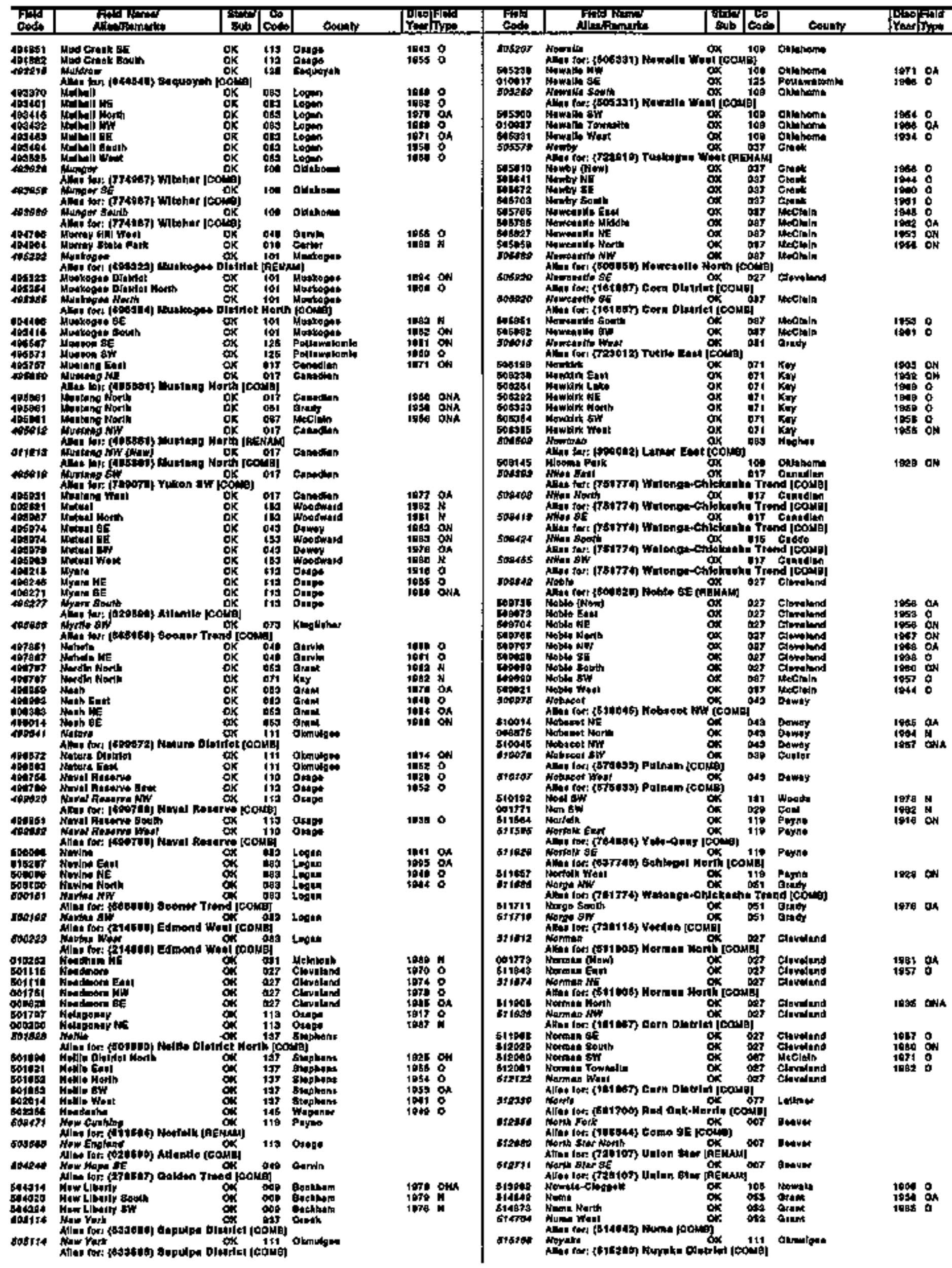




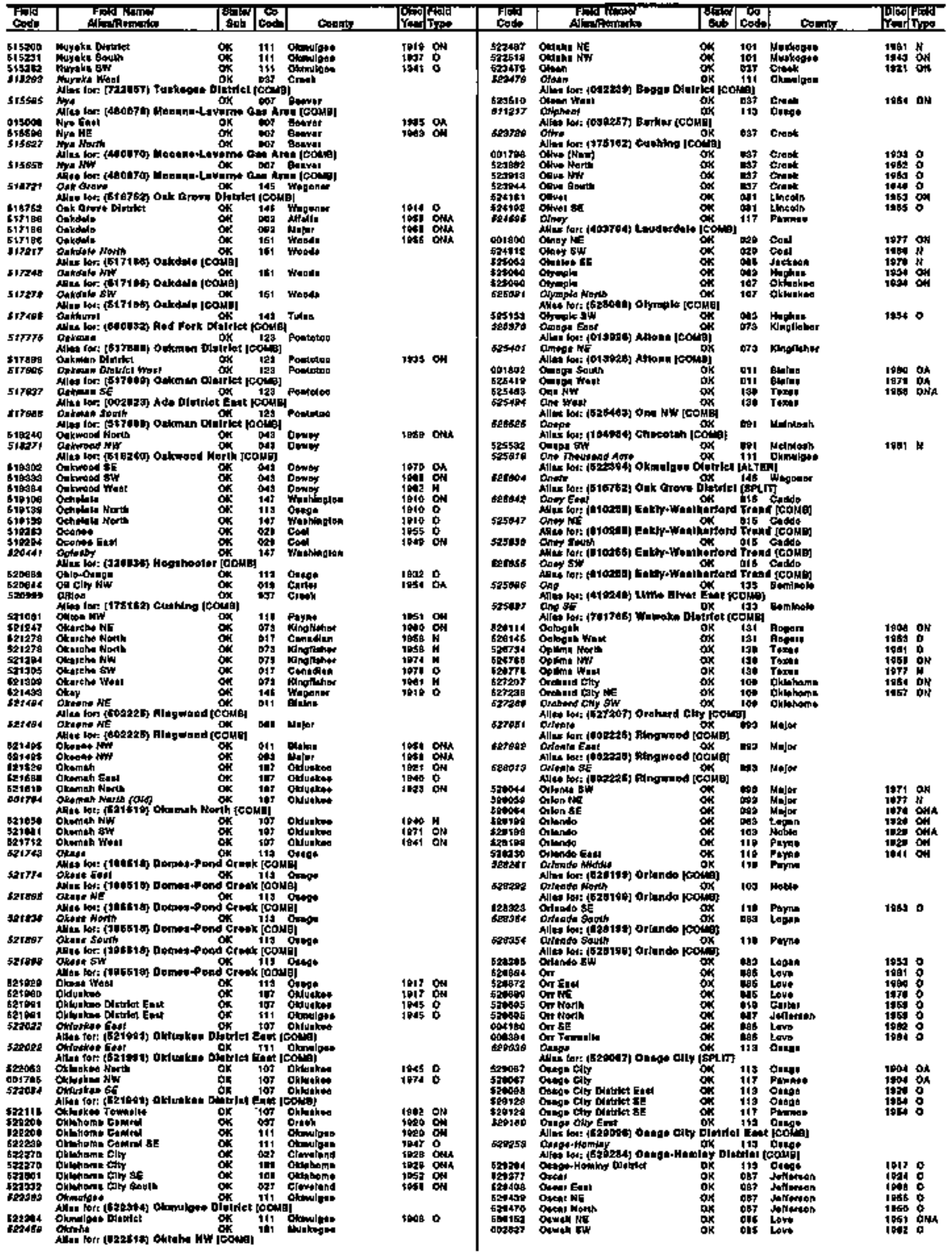


OKLAHOMA

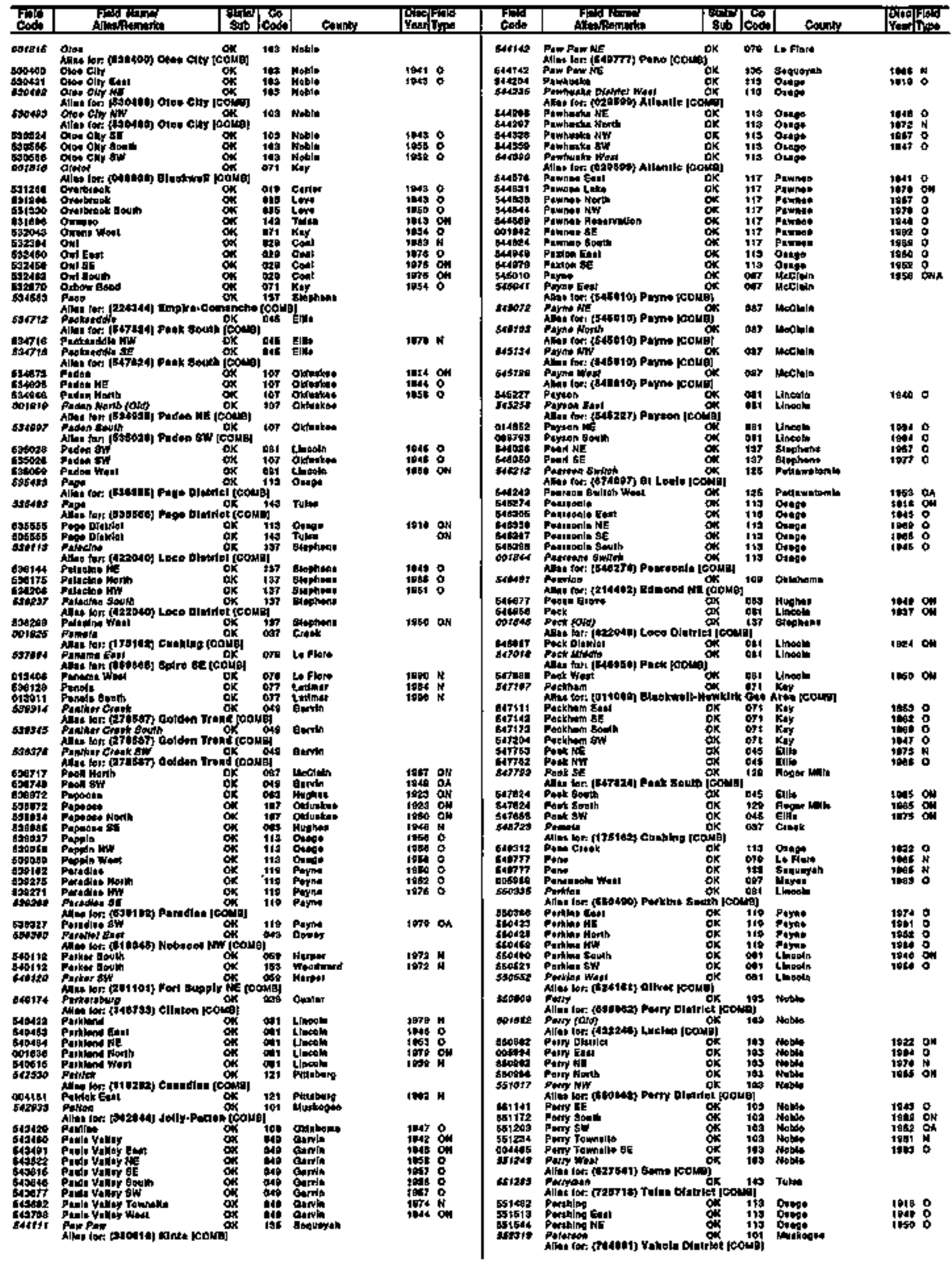




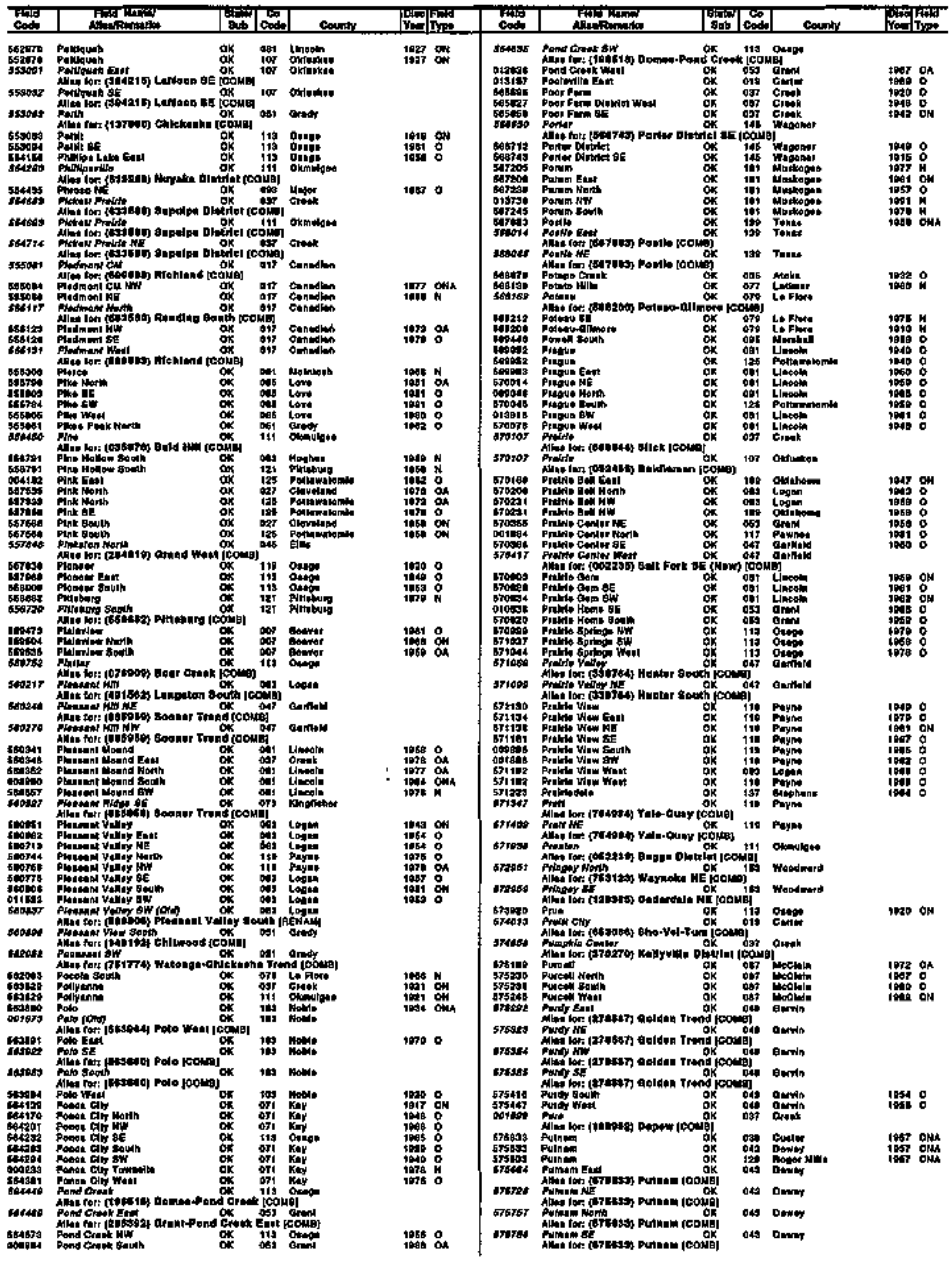


oklahoma

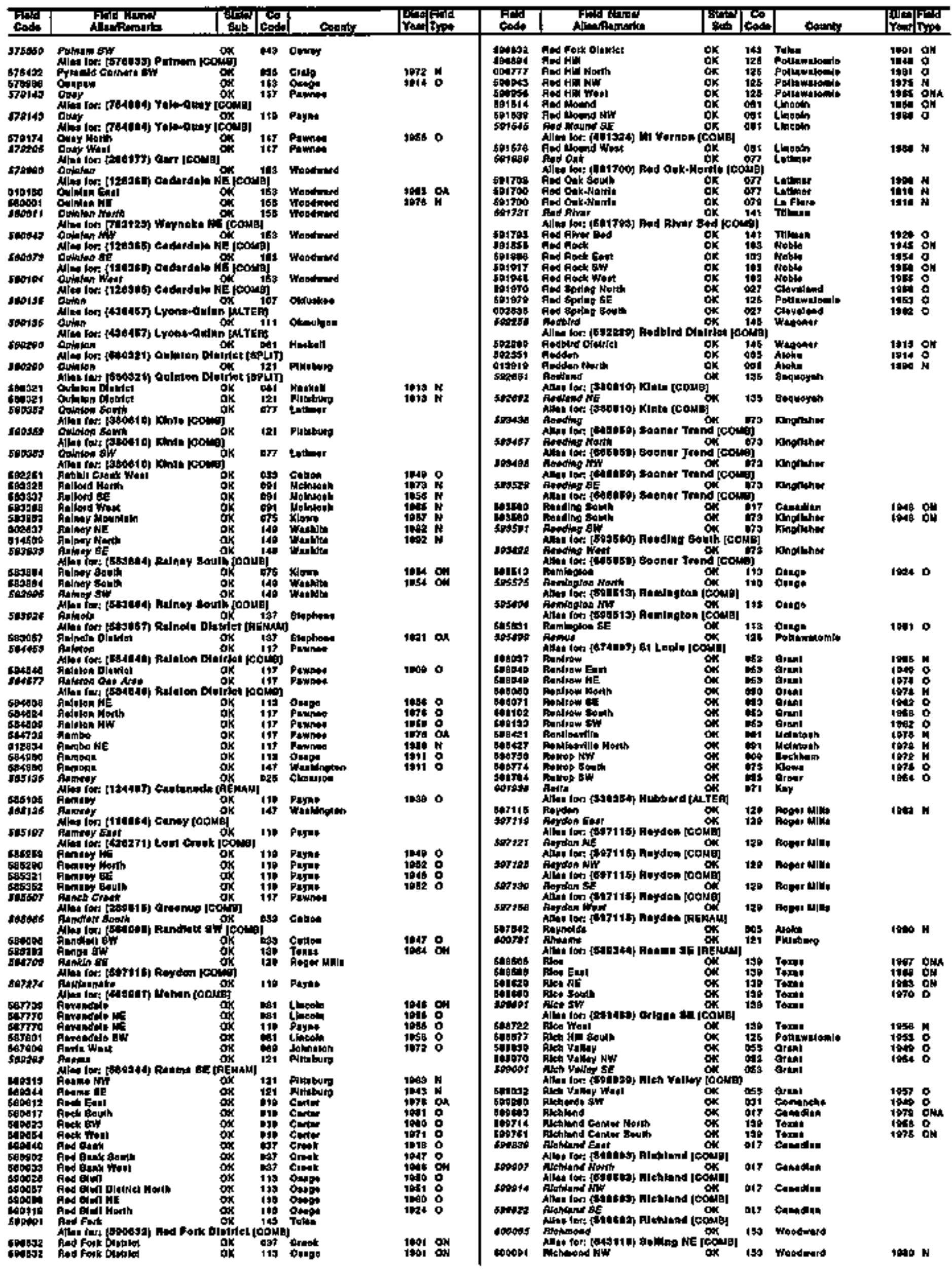




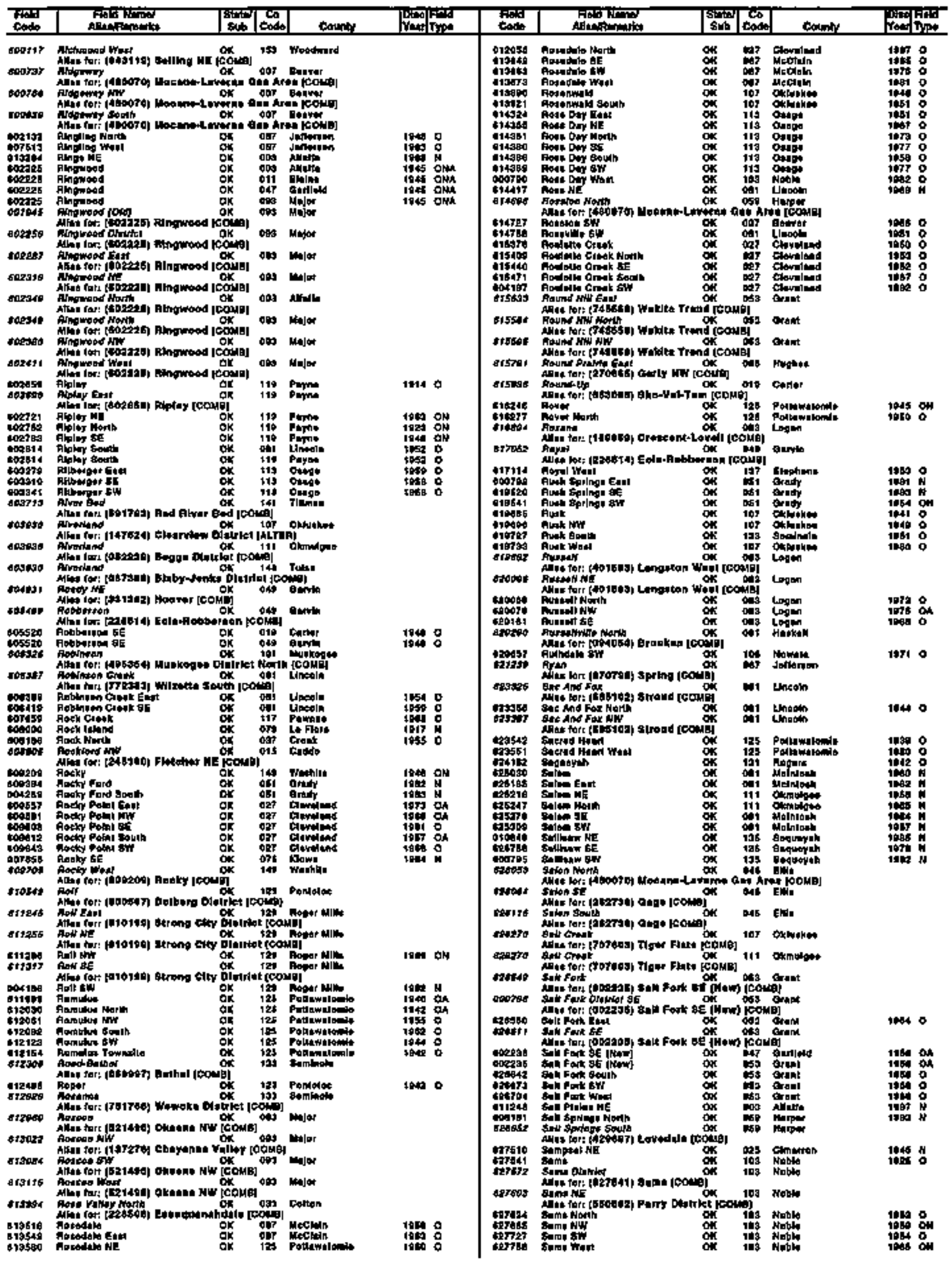


okLahoma

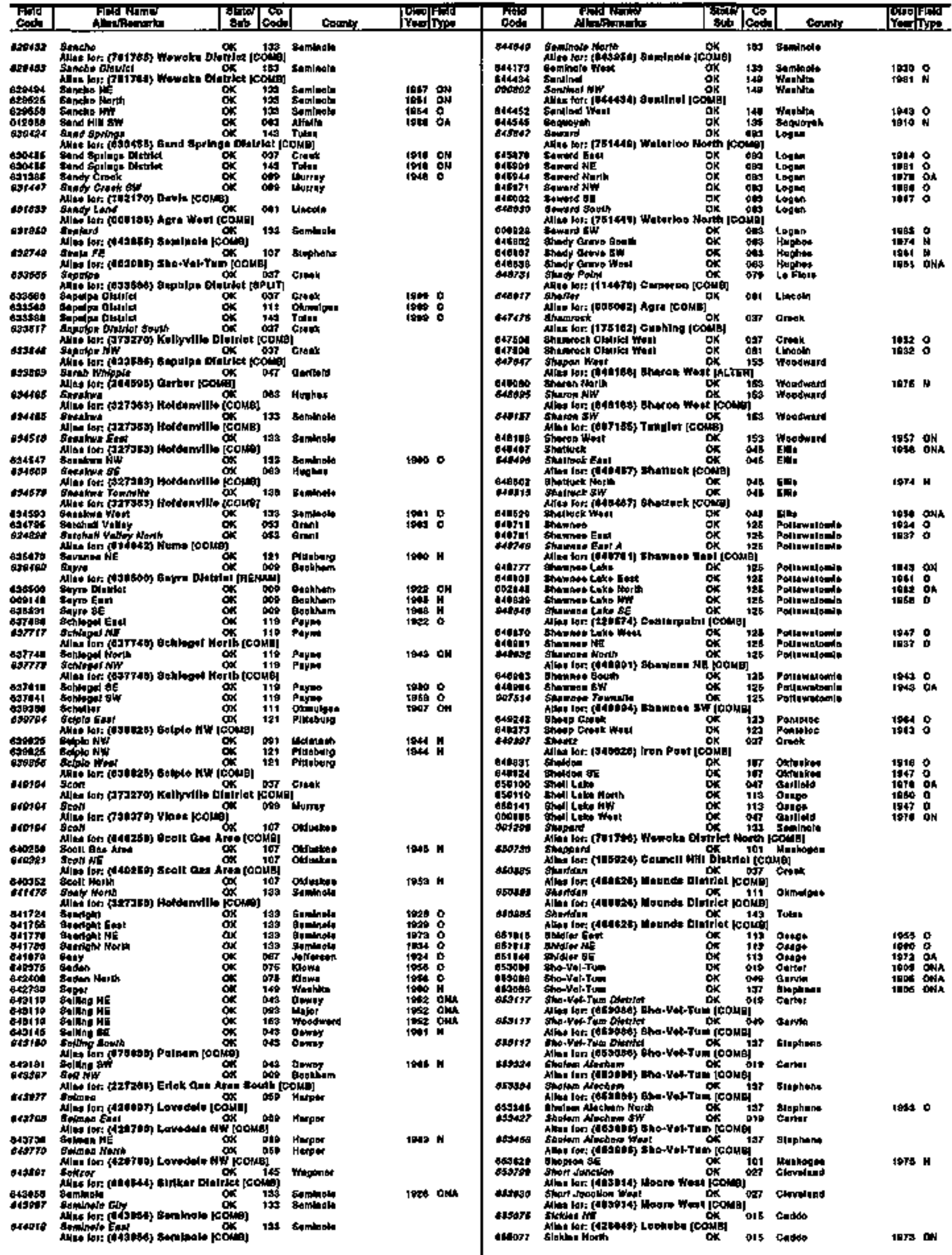




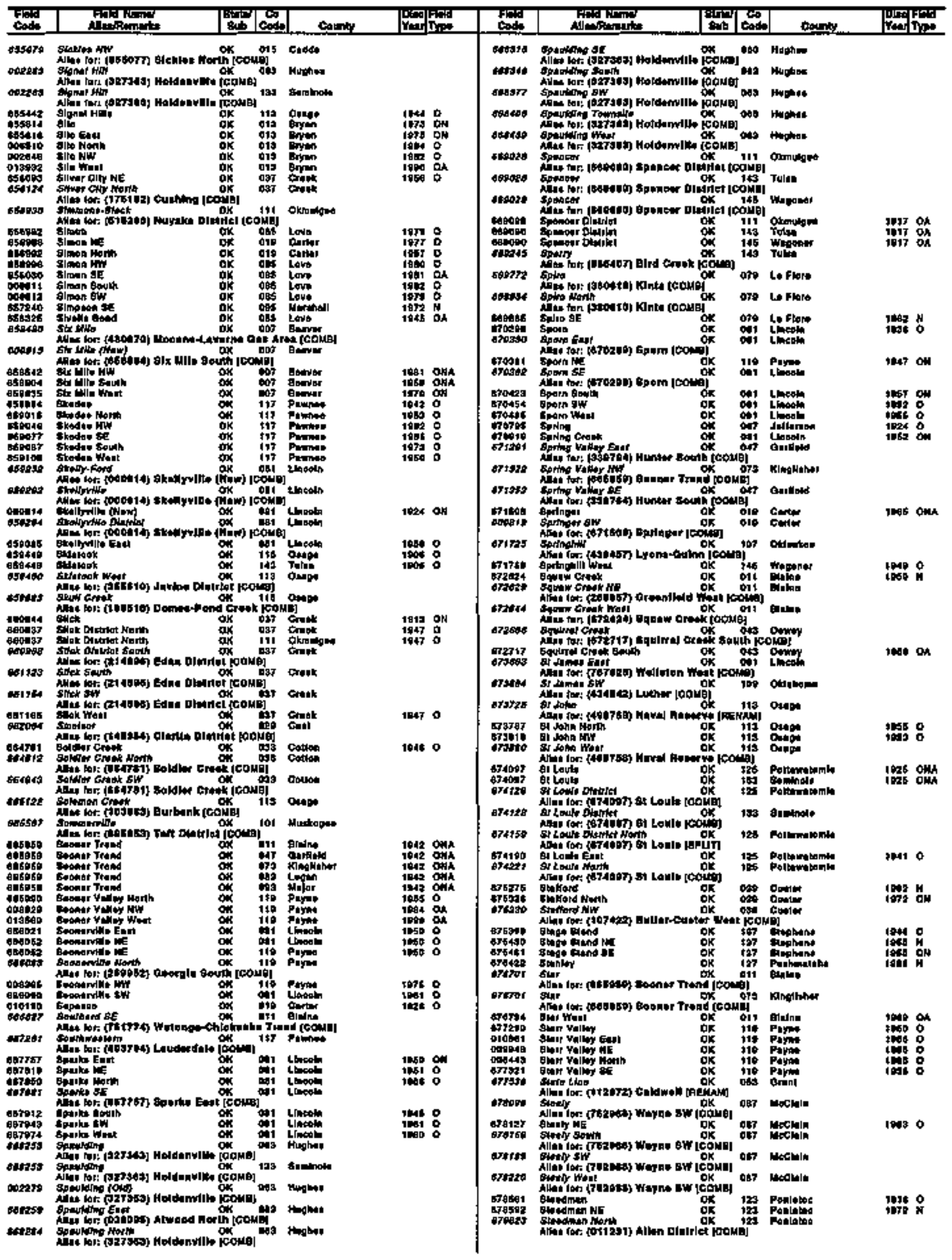




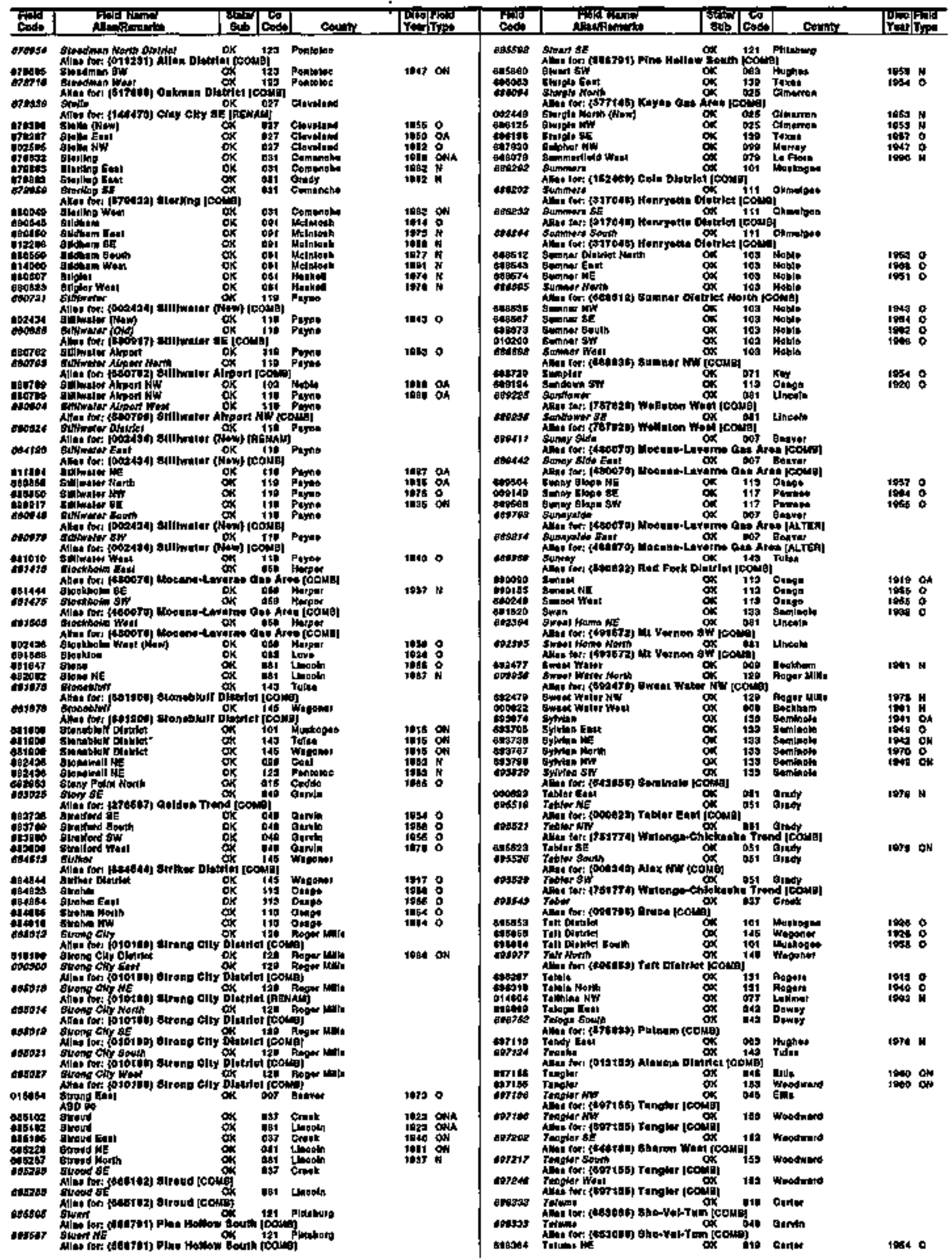


OKLAHOMA

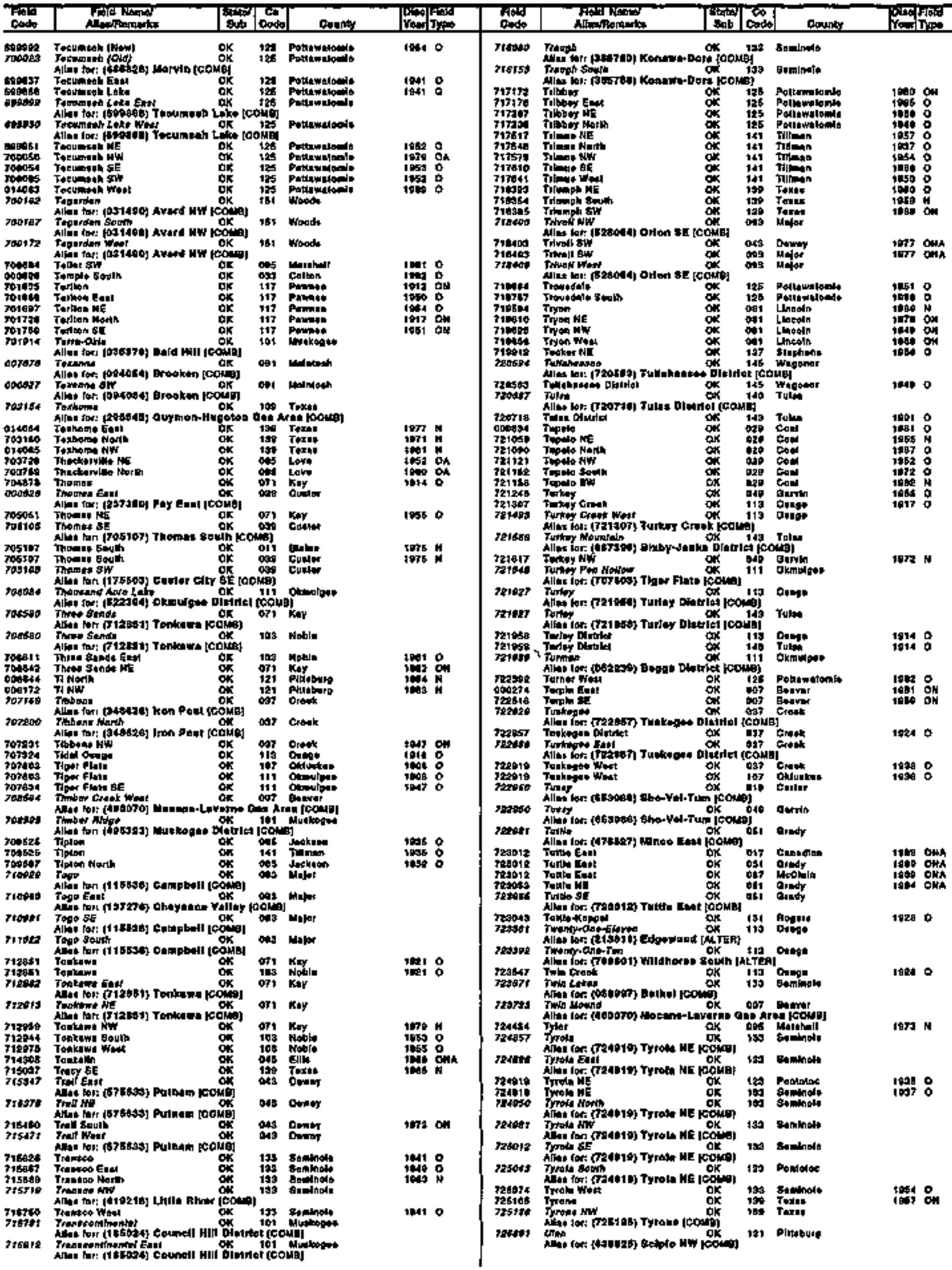


OKLAHOMA

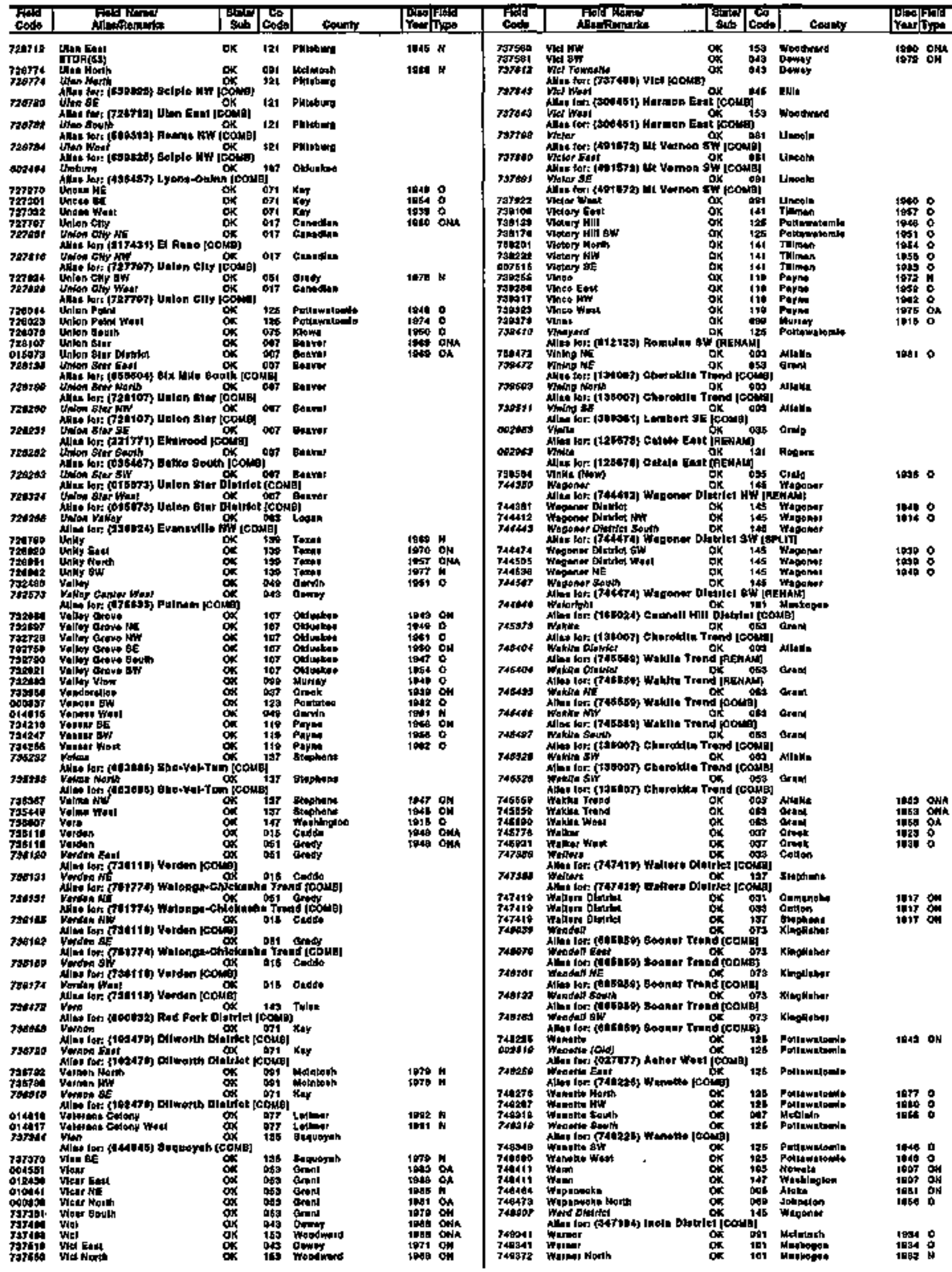


OKLAHOMA

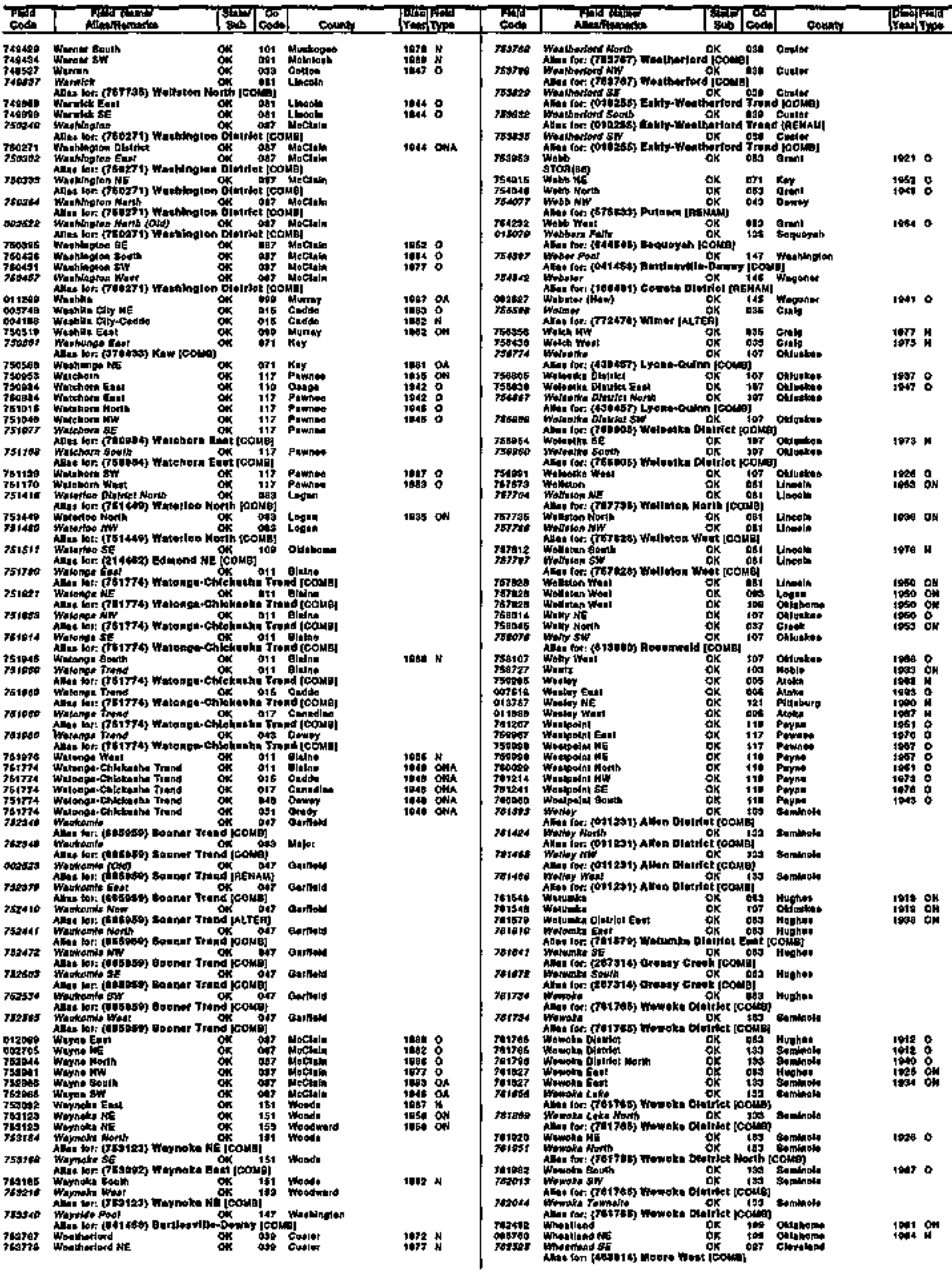


PENNSYLVANIA

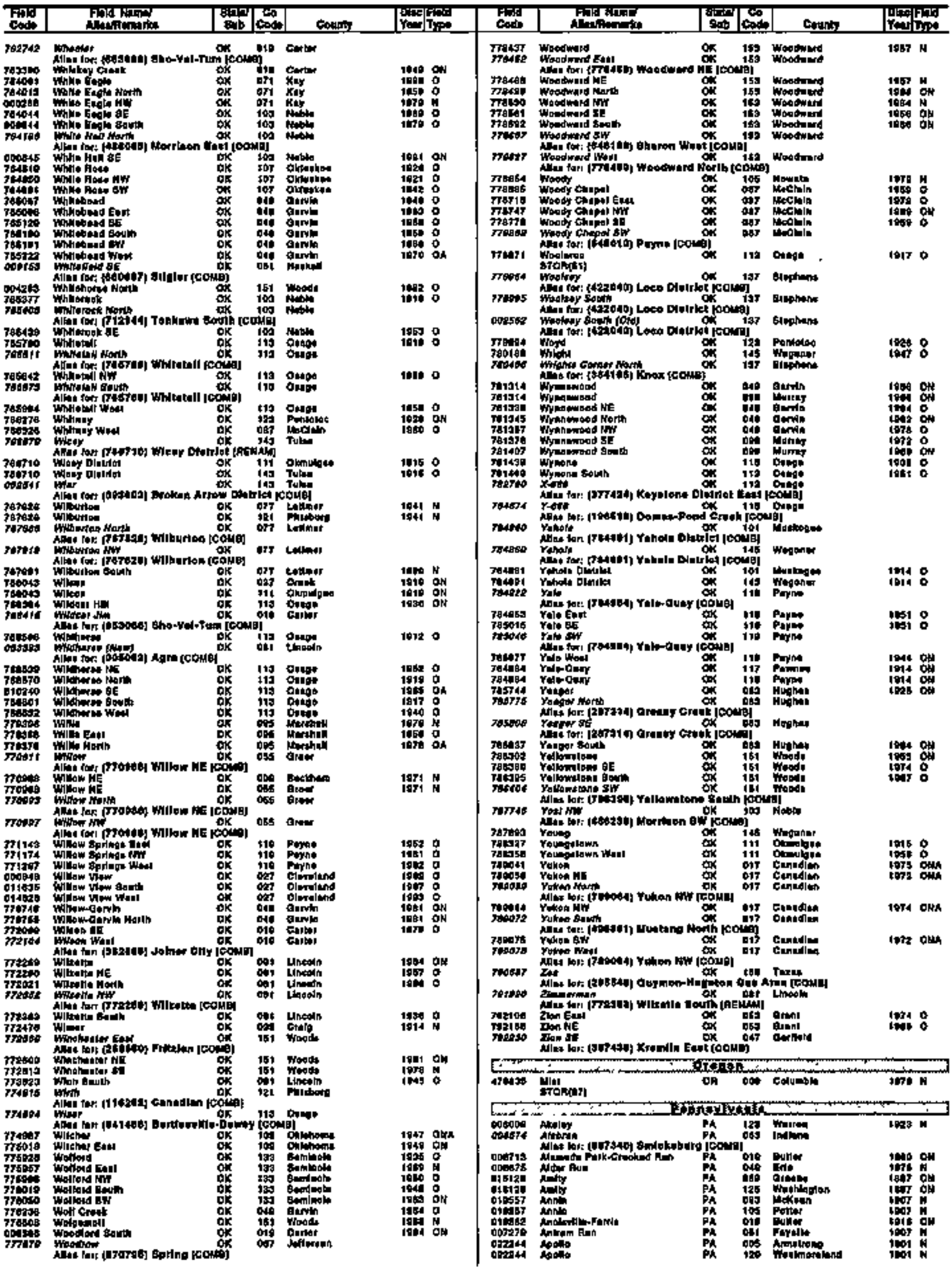


PENNSYLVANIA

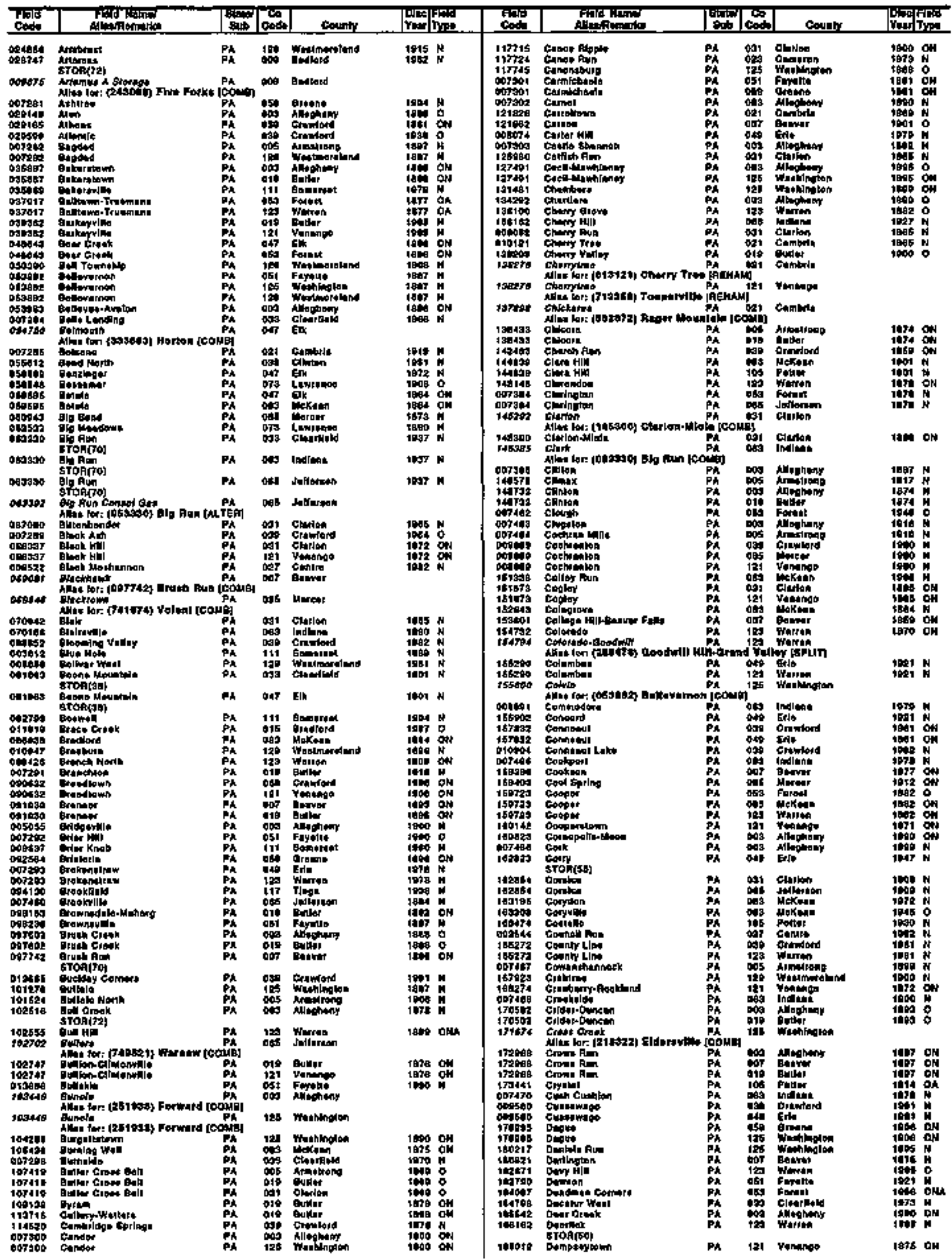


PENNSYLVANIA

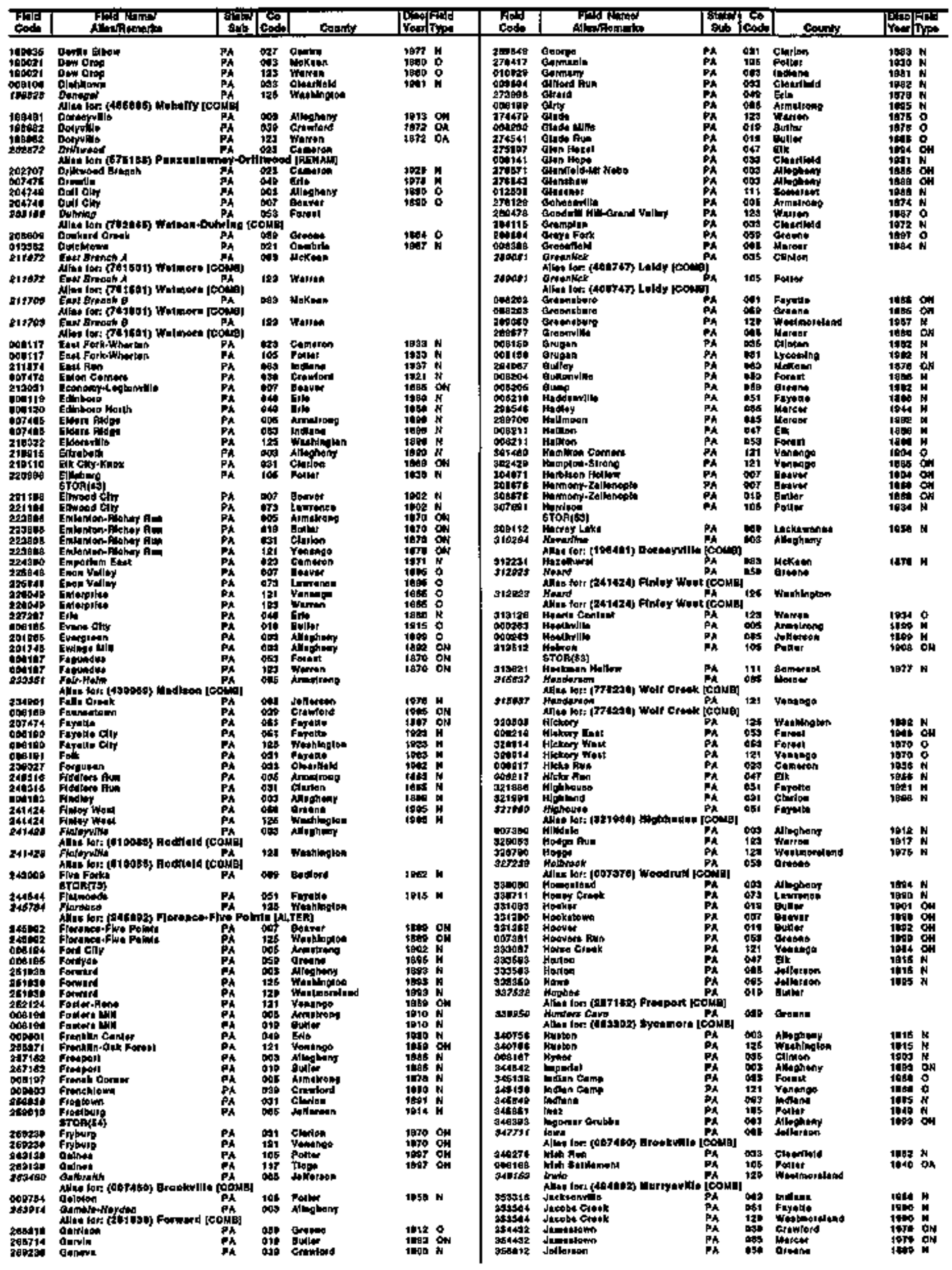


PENNSYLVANIA

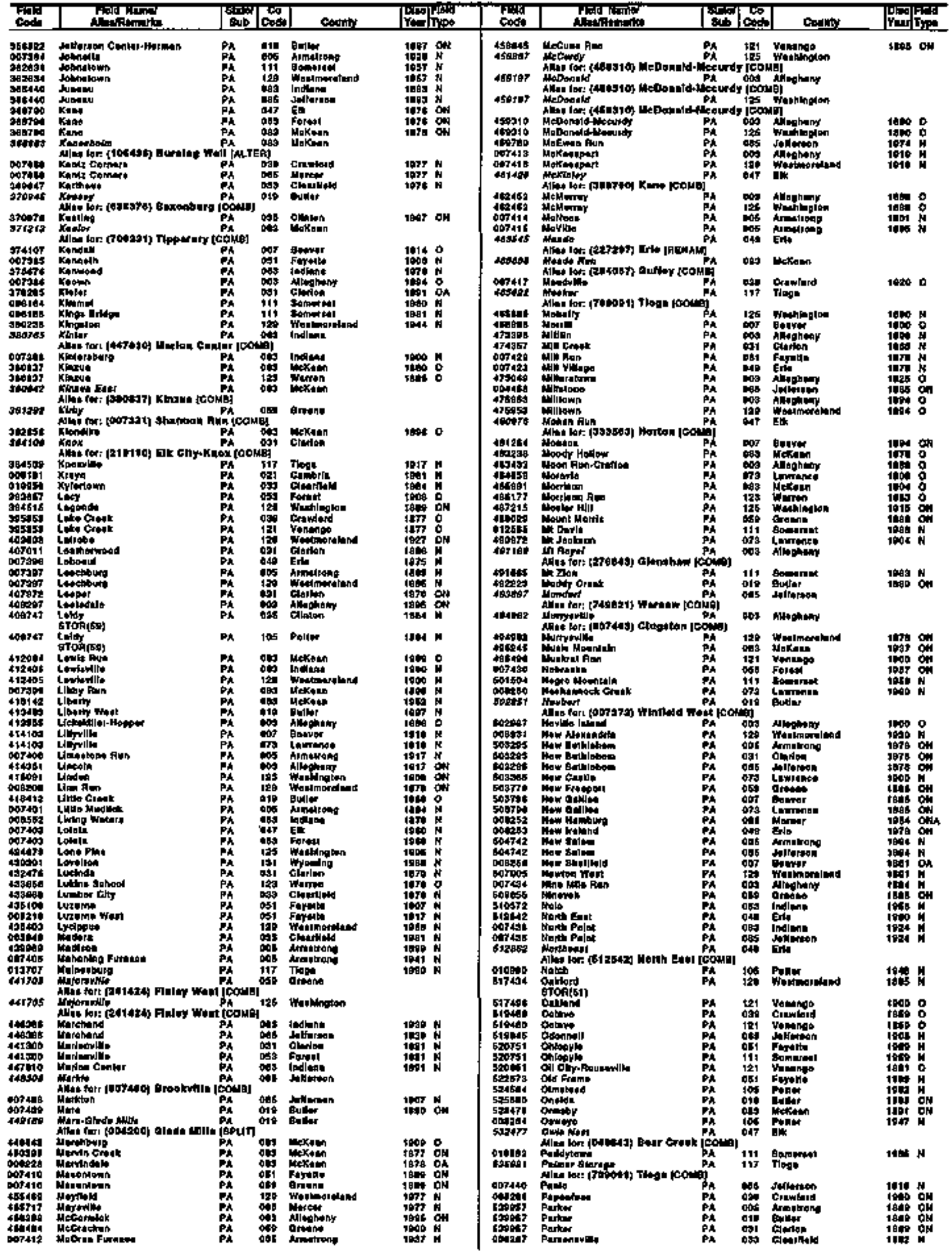


PENNSYLVANIA

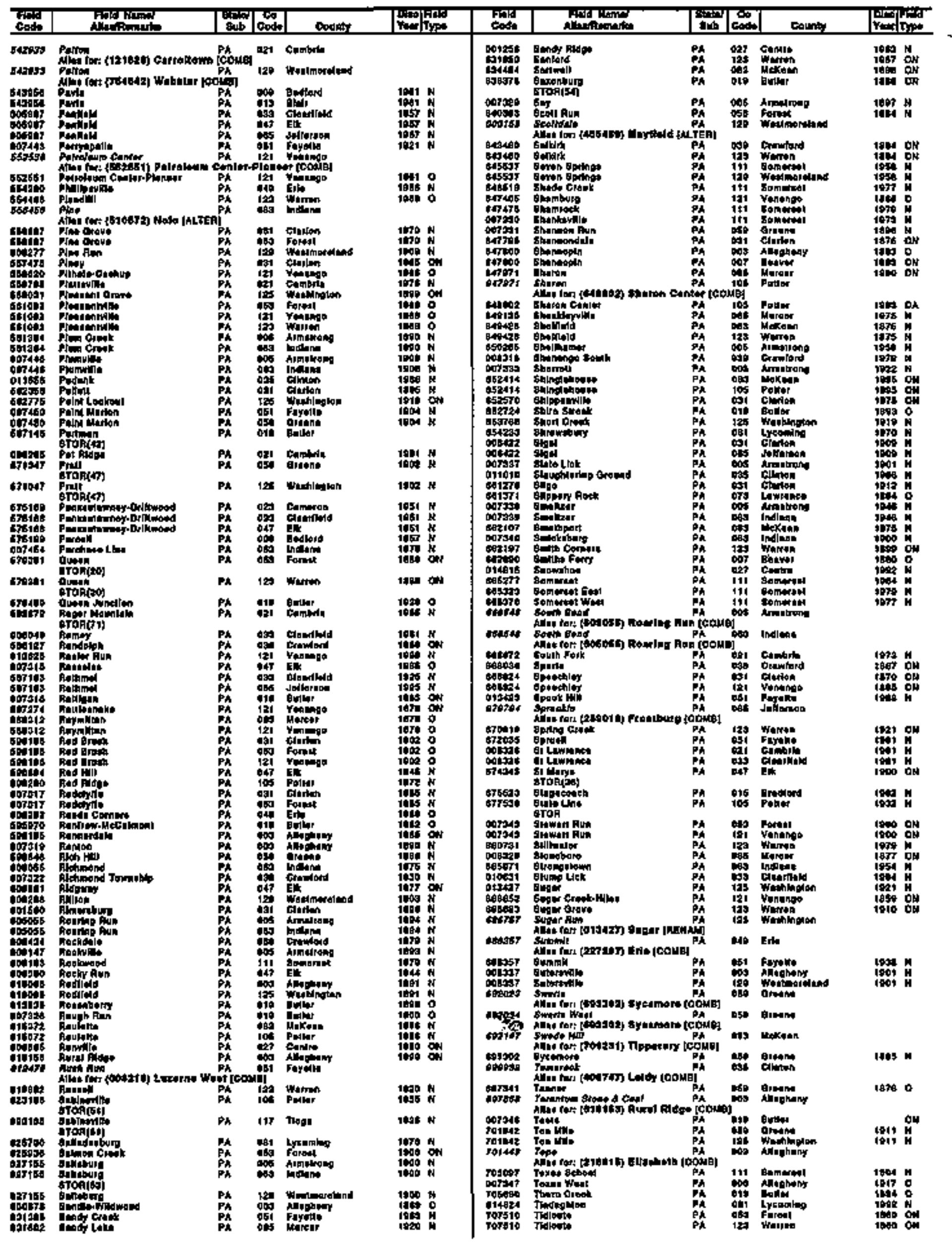




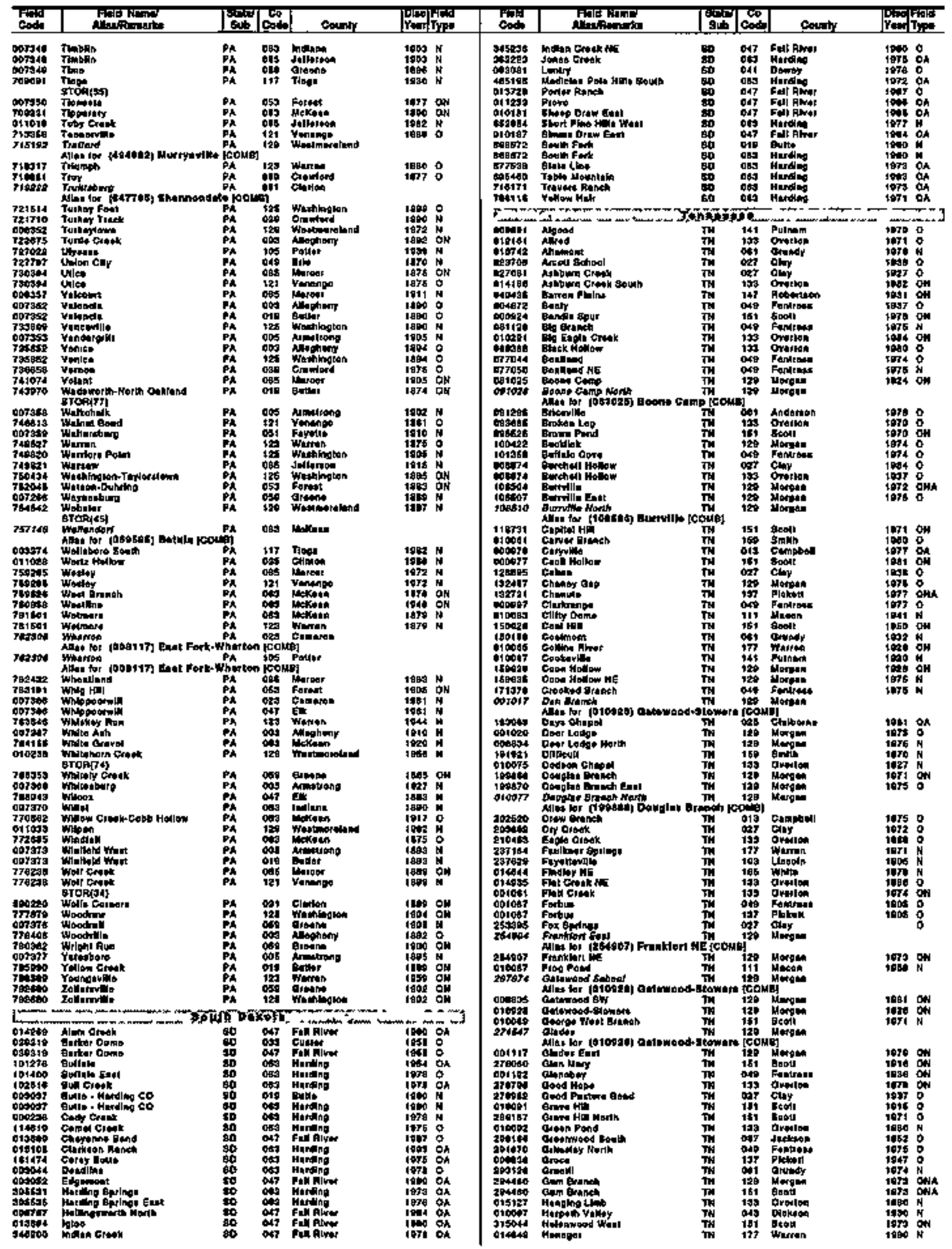


TEXAS

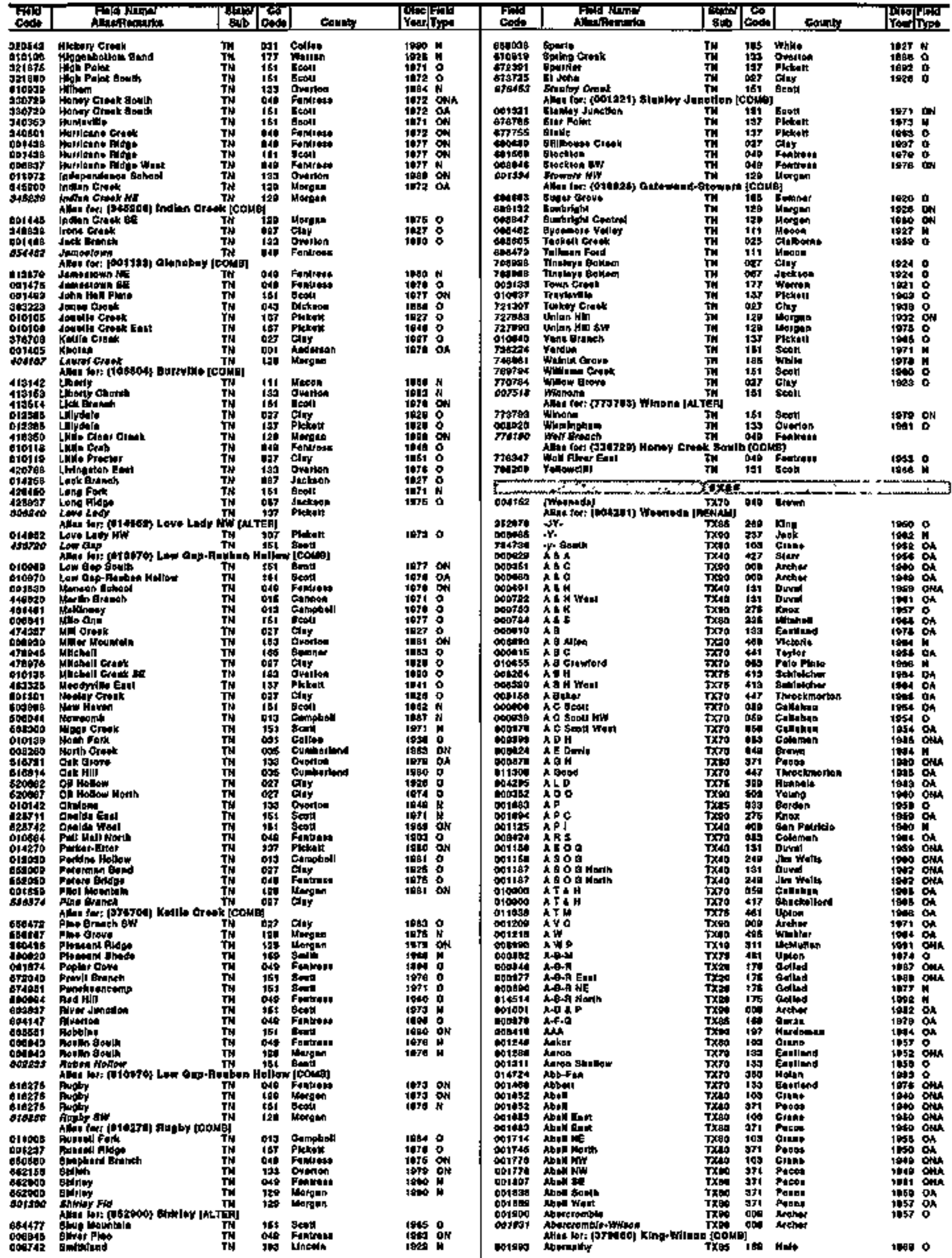


TEXAS

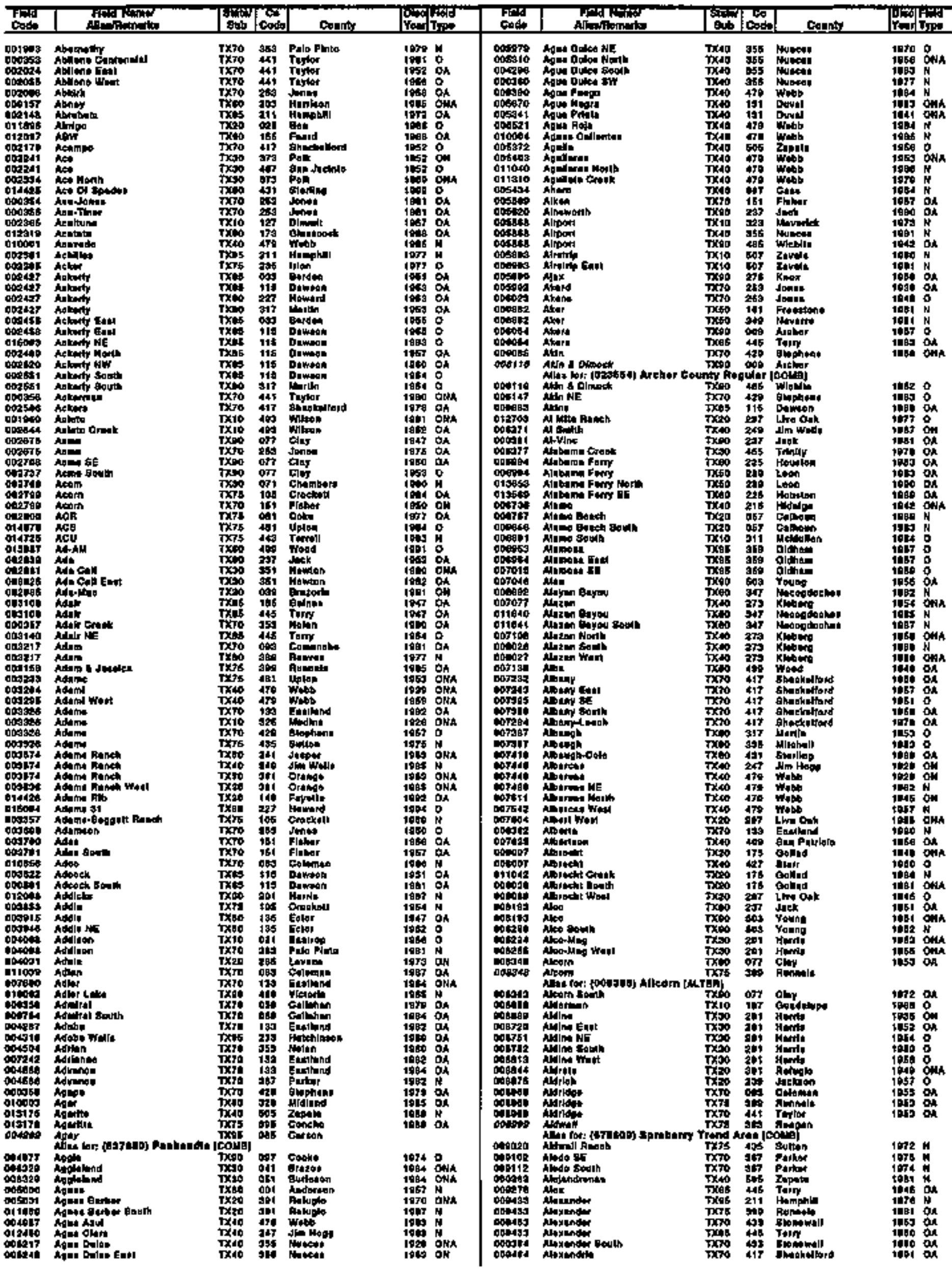


TEXA

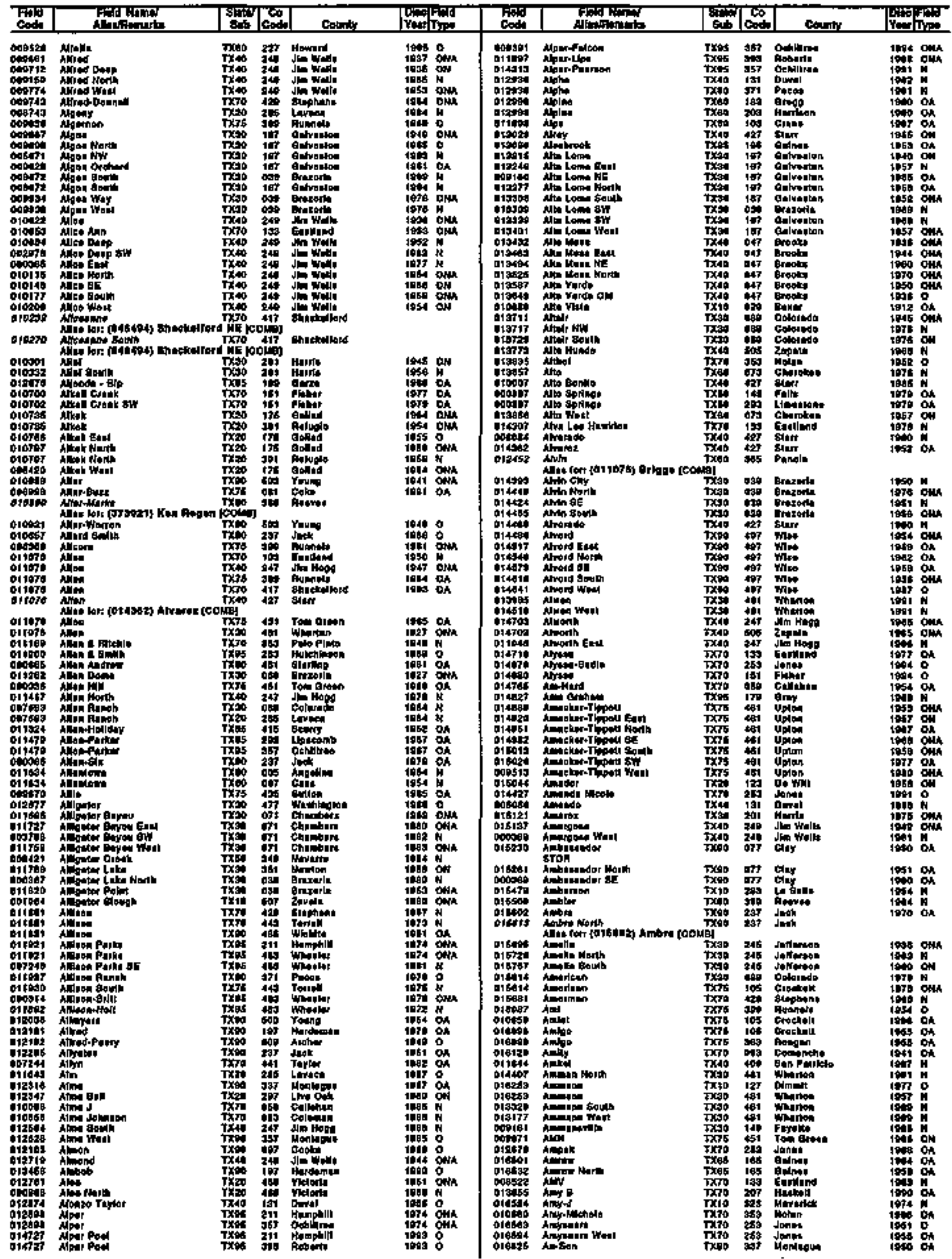


TEXAS

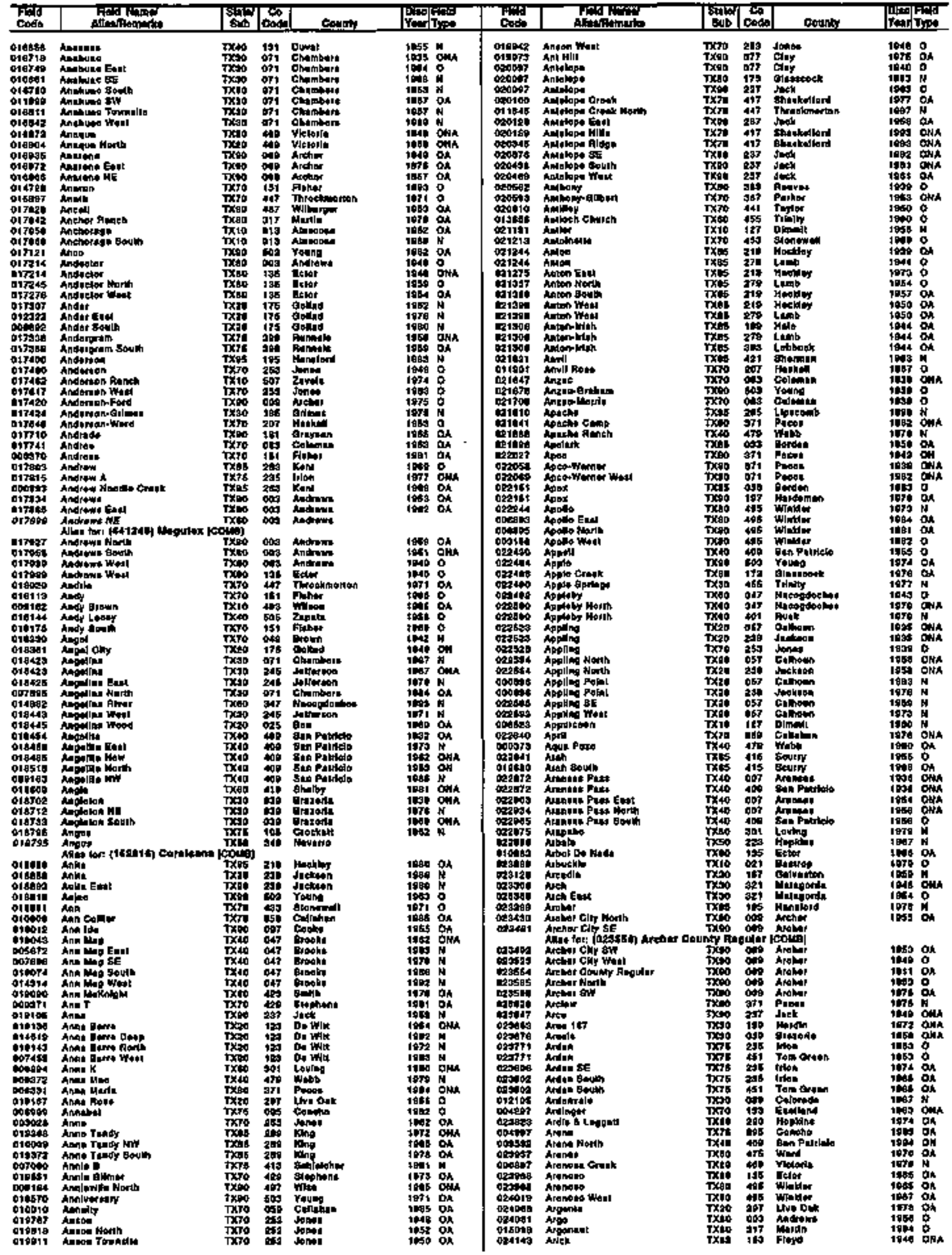


TEXAS

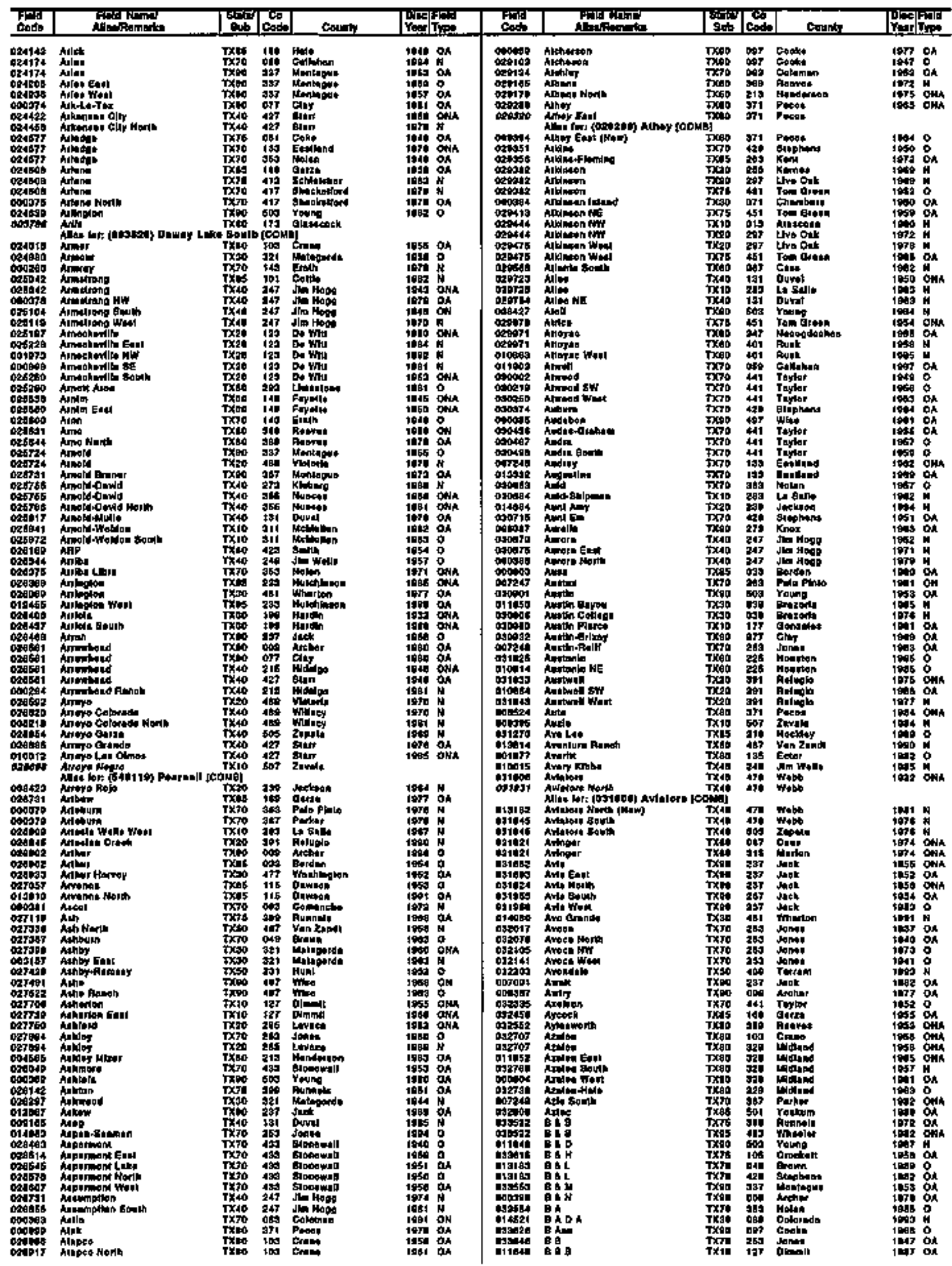


TEXA $\mathbf{S}$

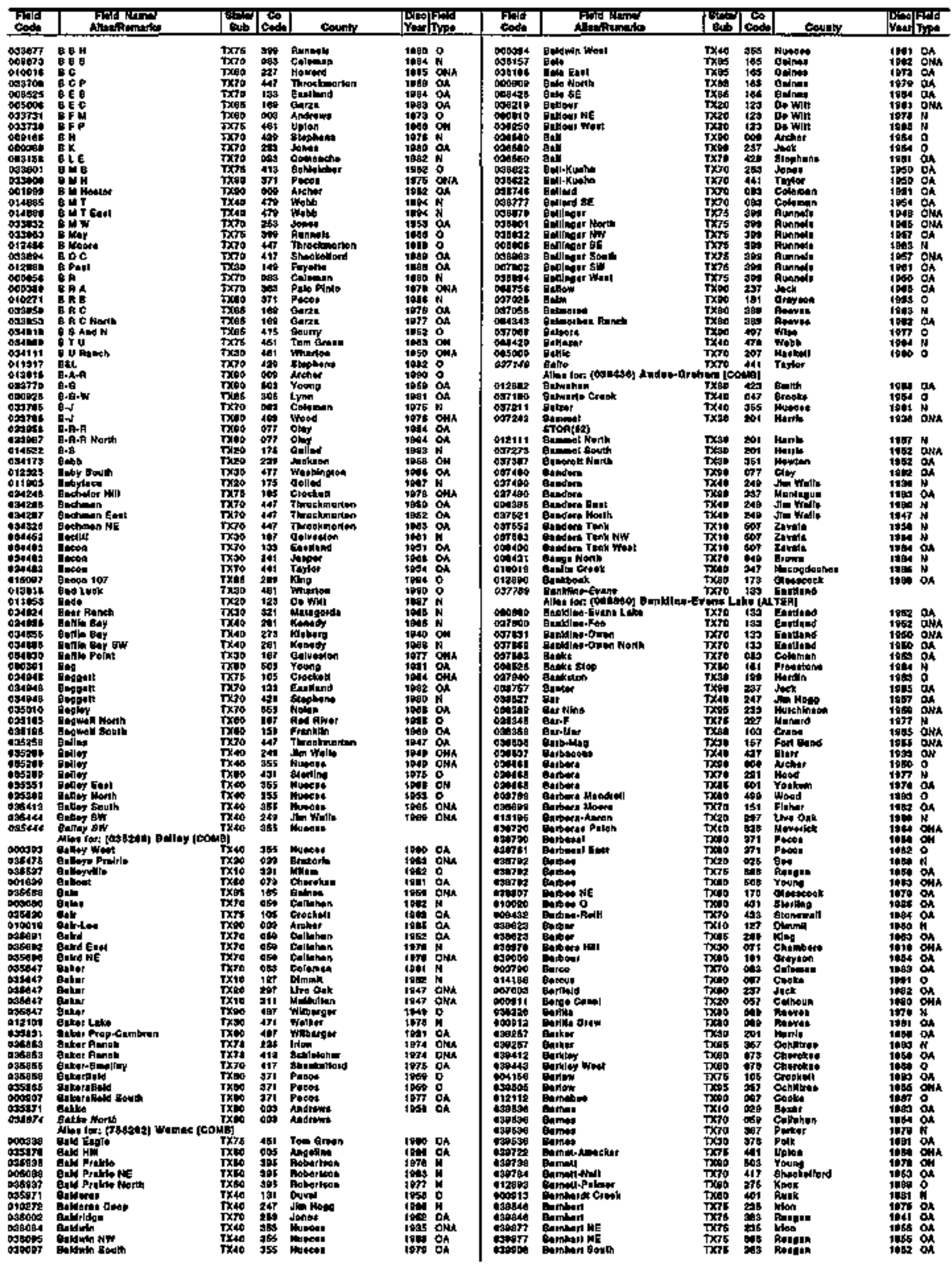


TEXAS

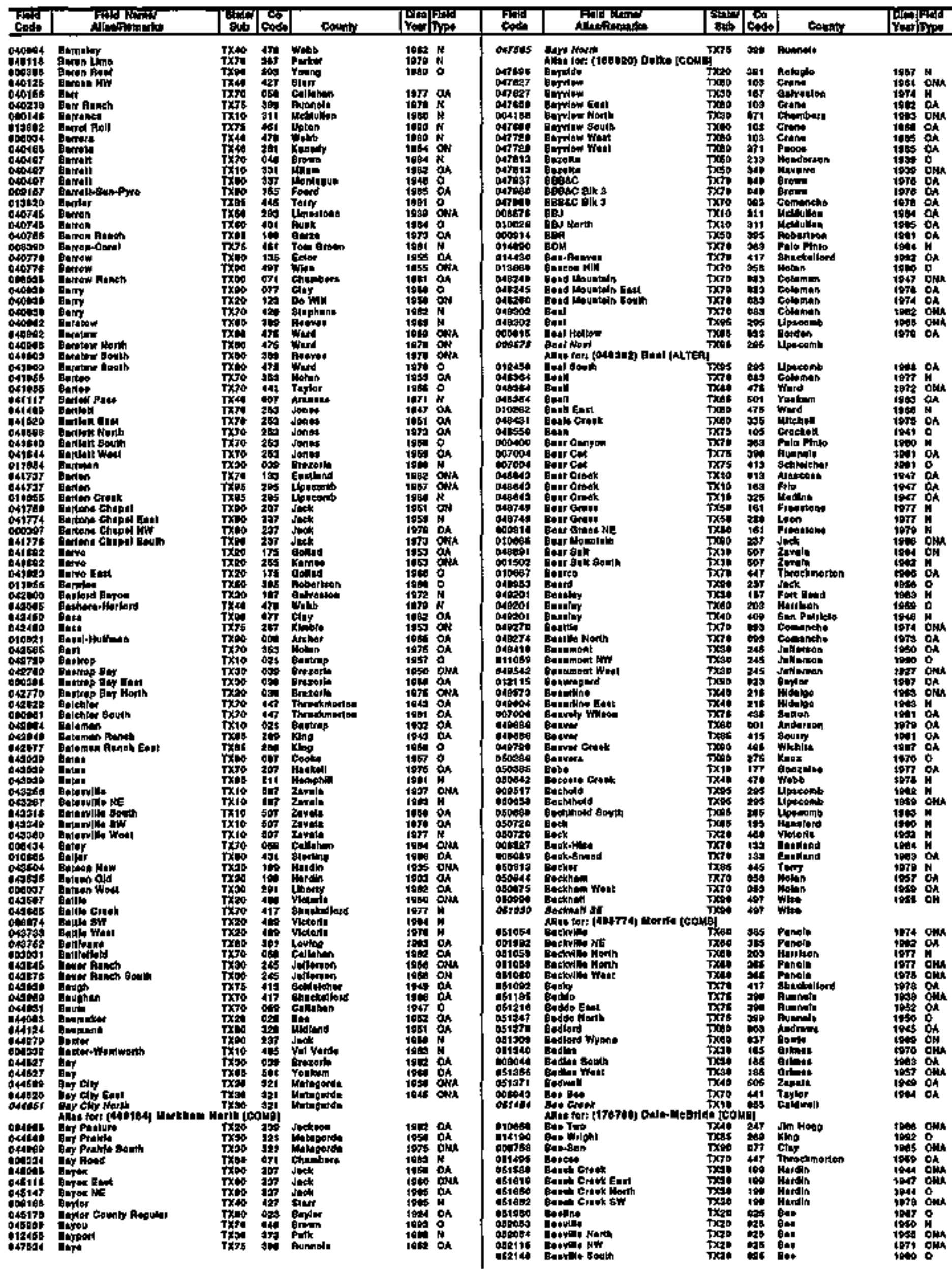


TEXAS

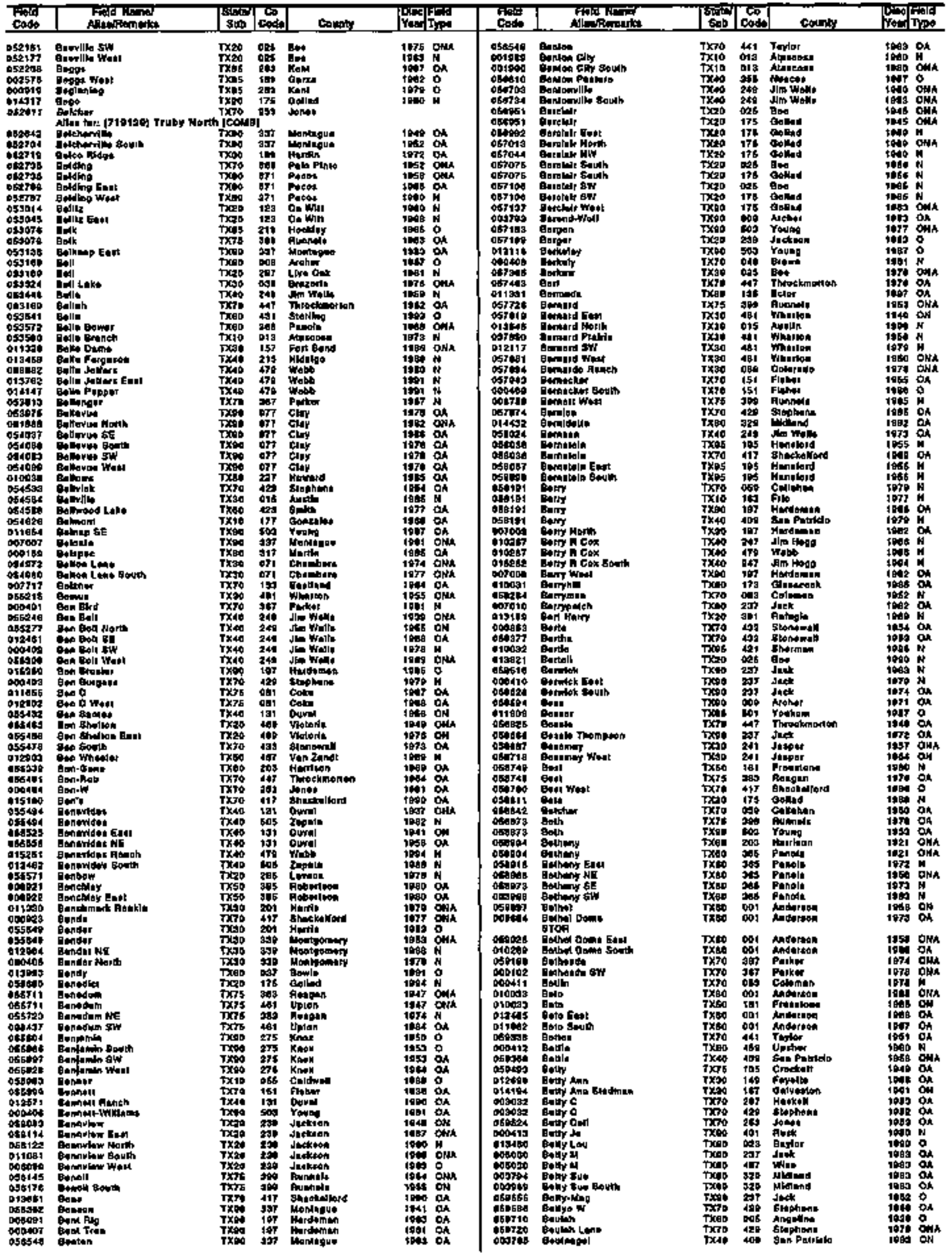


TEXAS

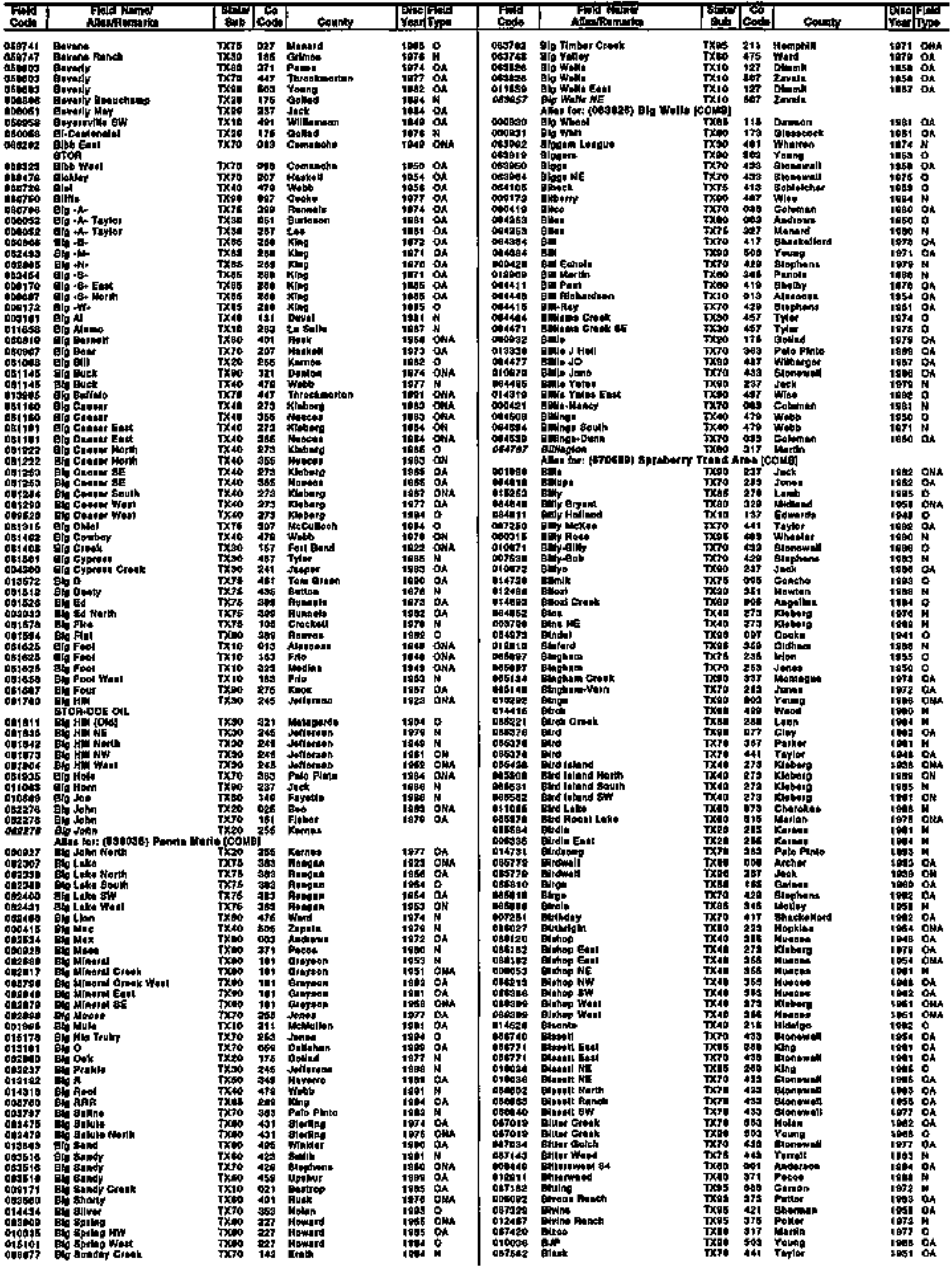


TEXAS

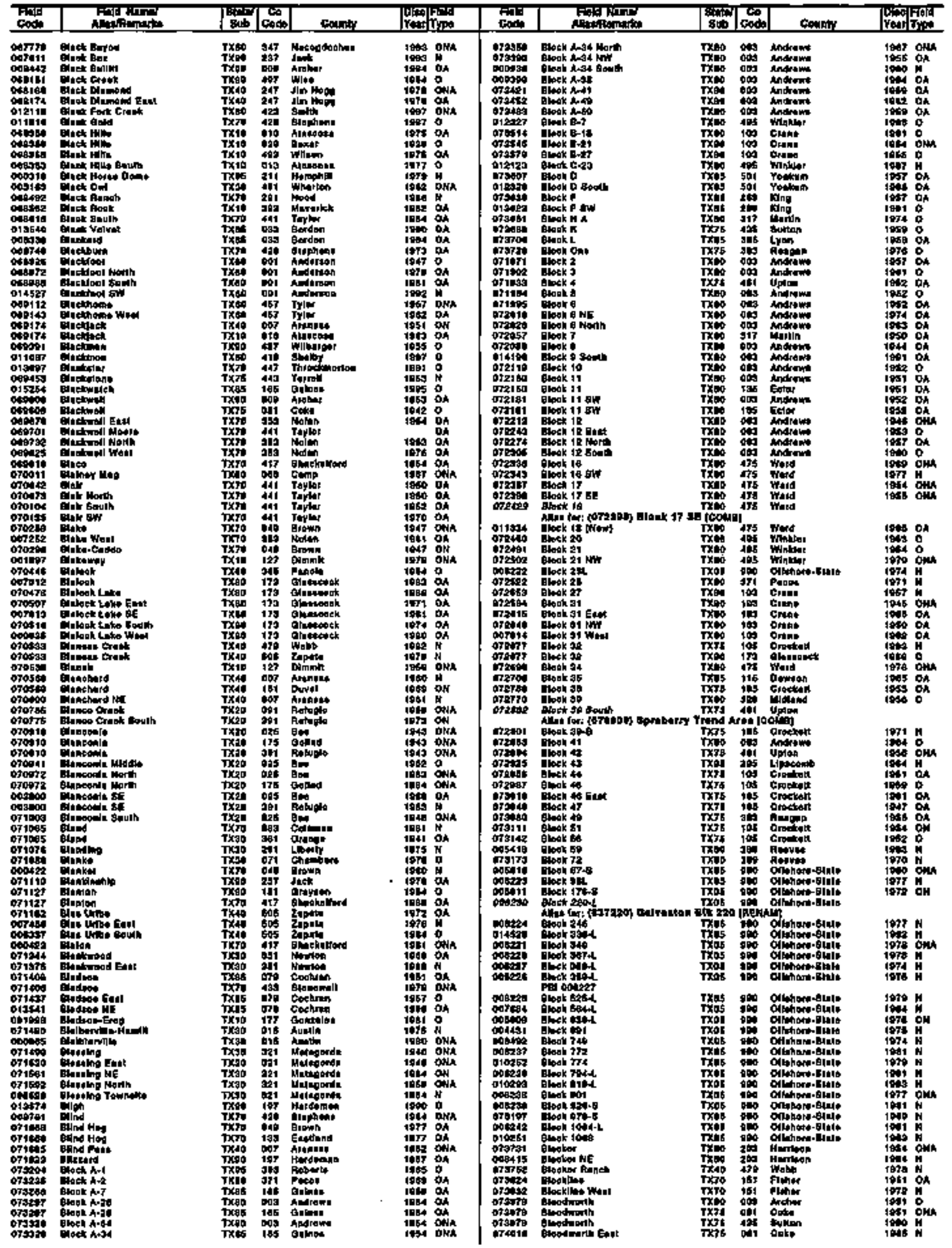


TEXA 3

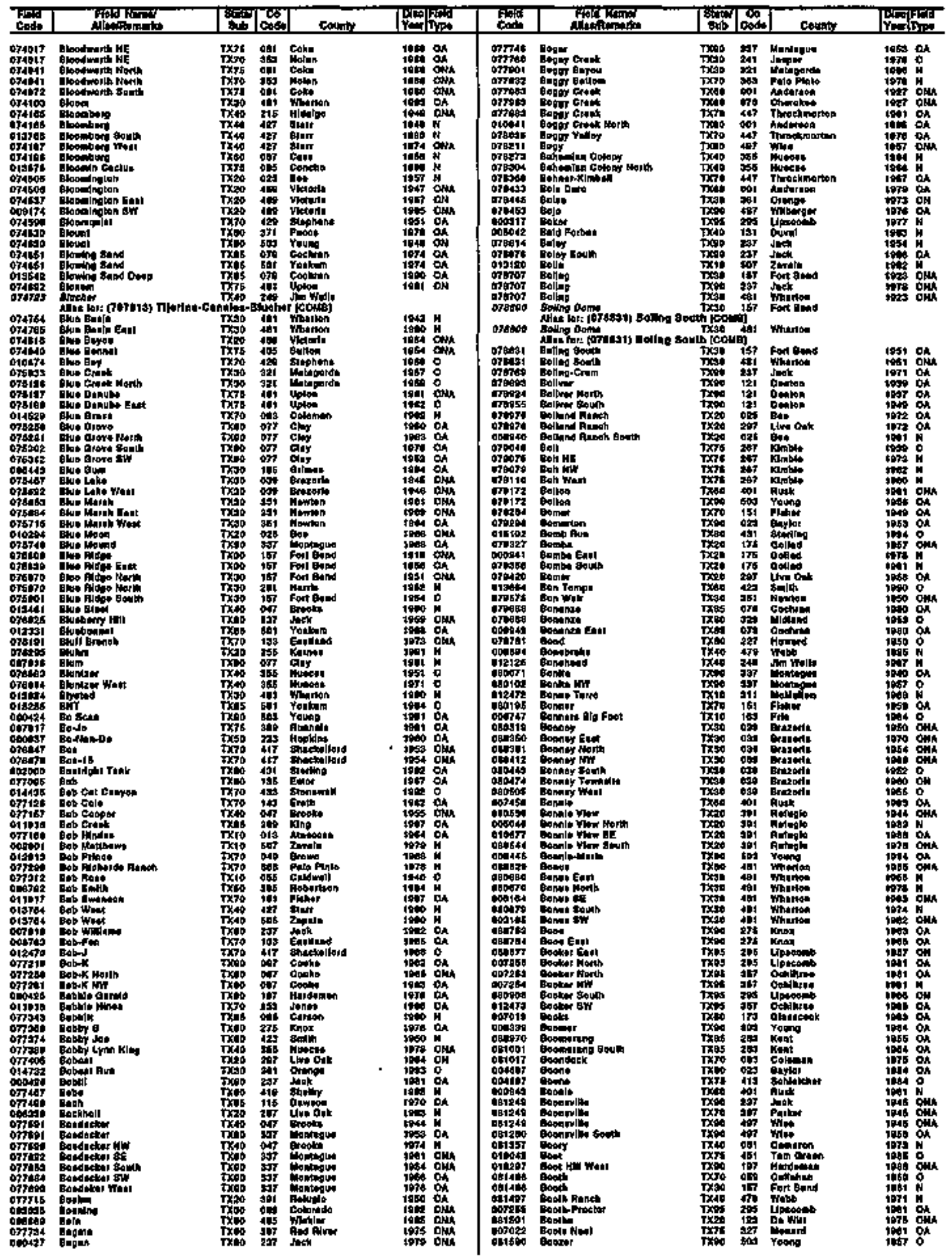


TEXAS

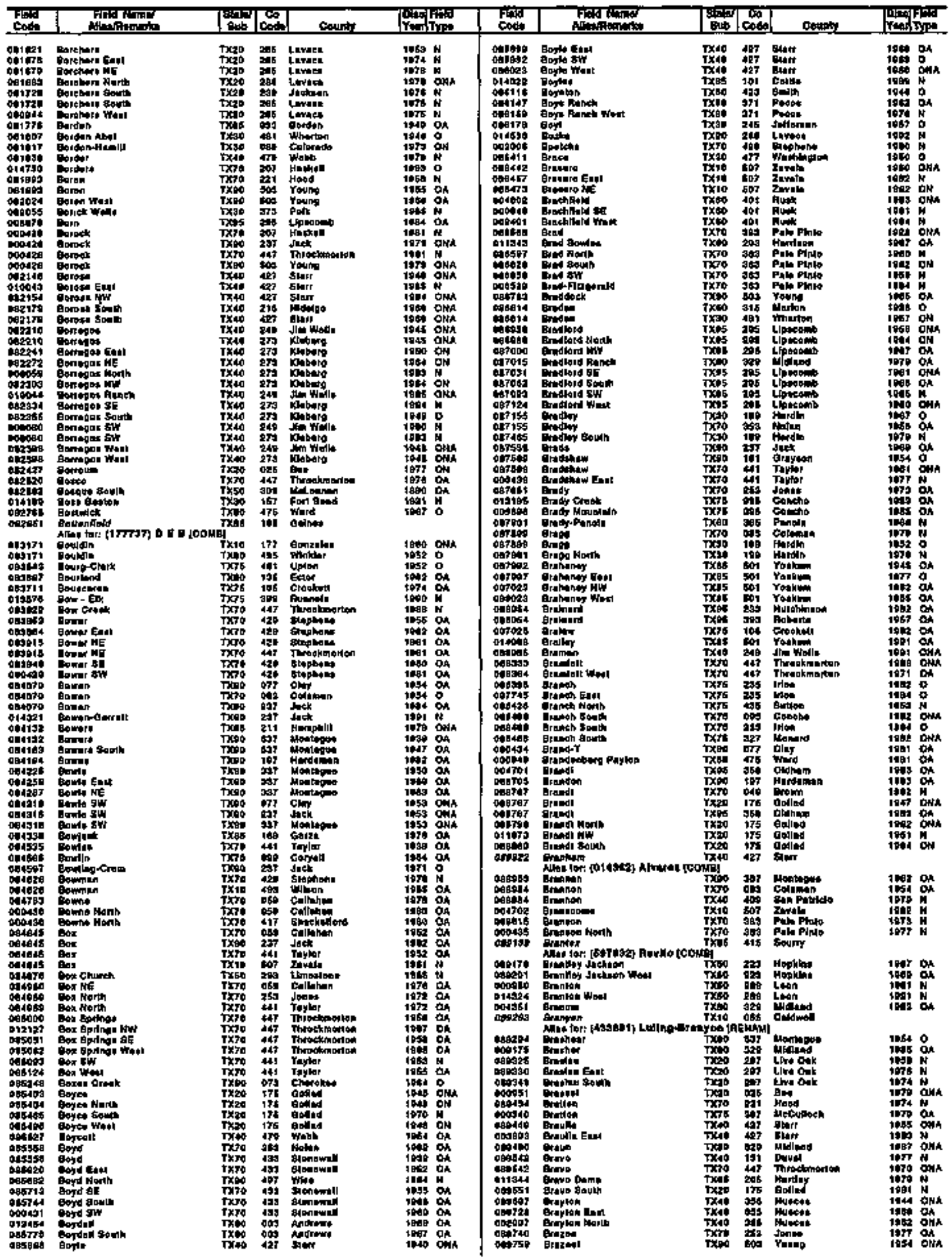


TEXAS

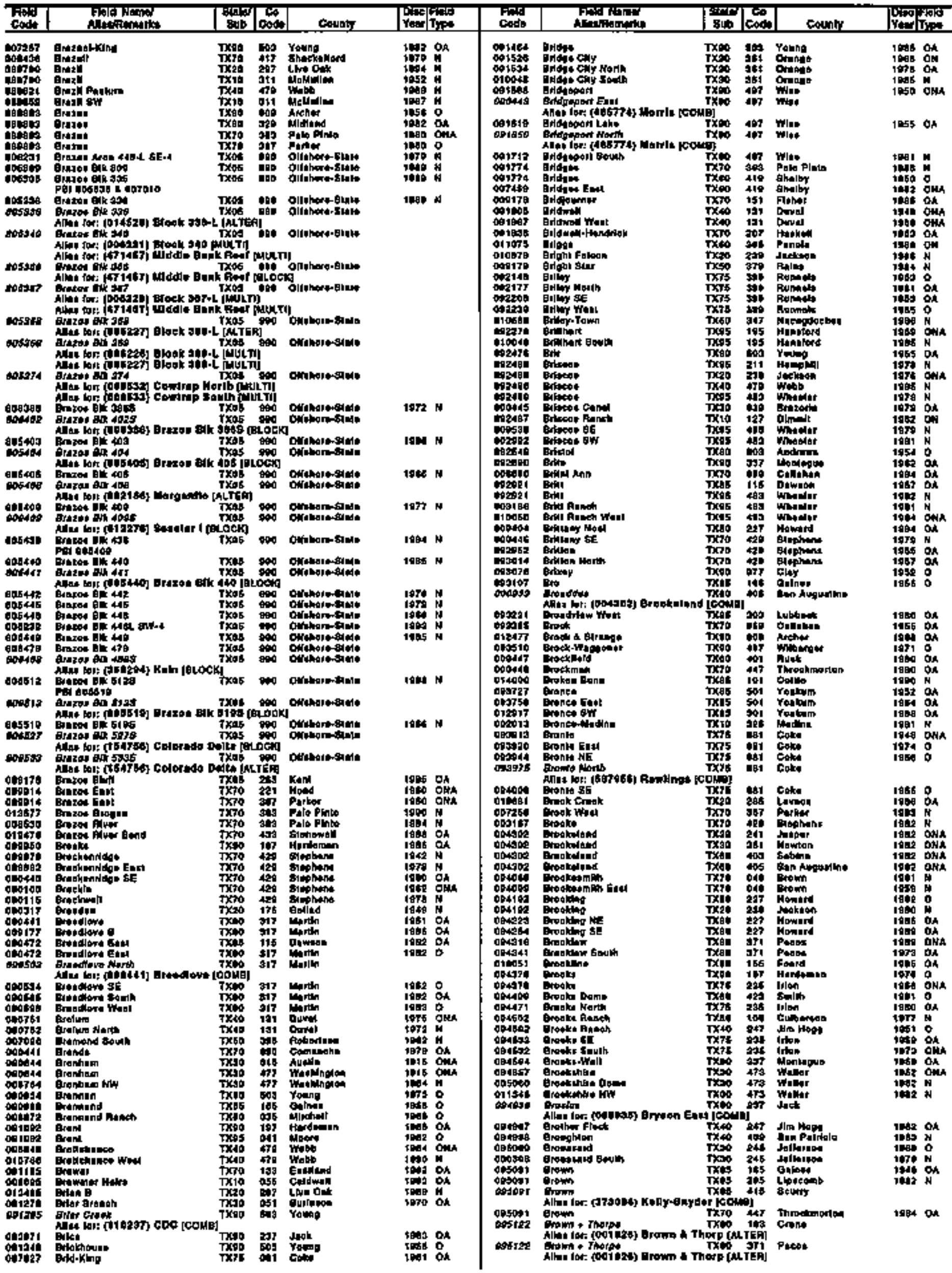


TEXAS

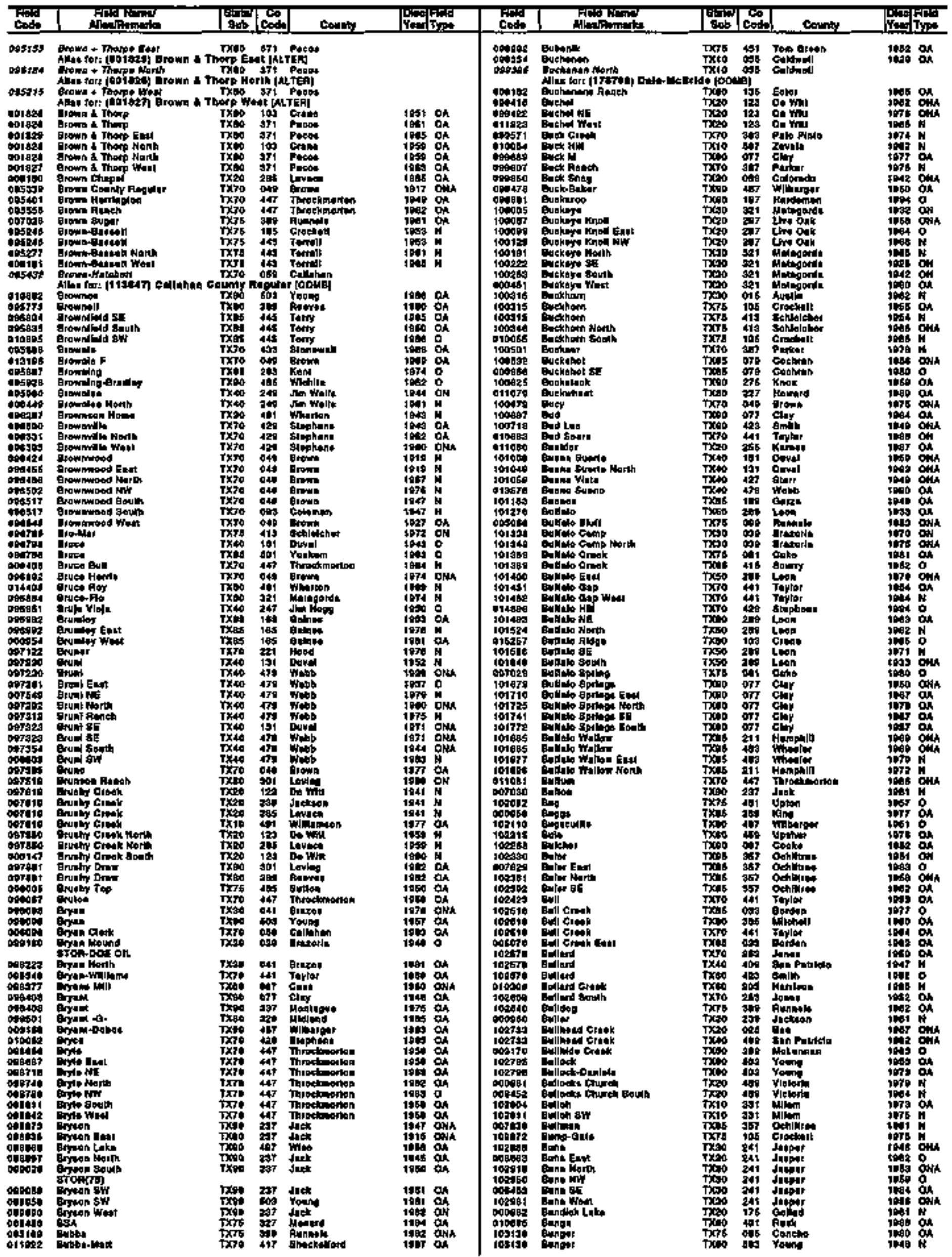


TEXAS

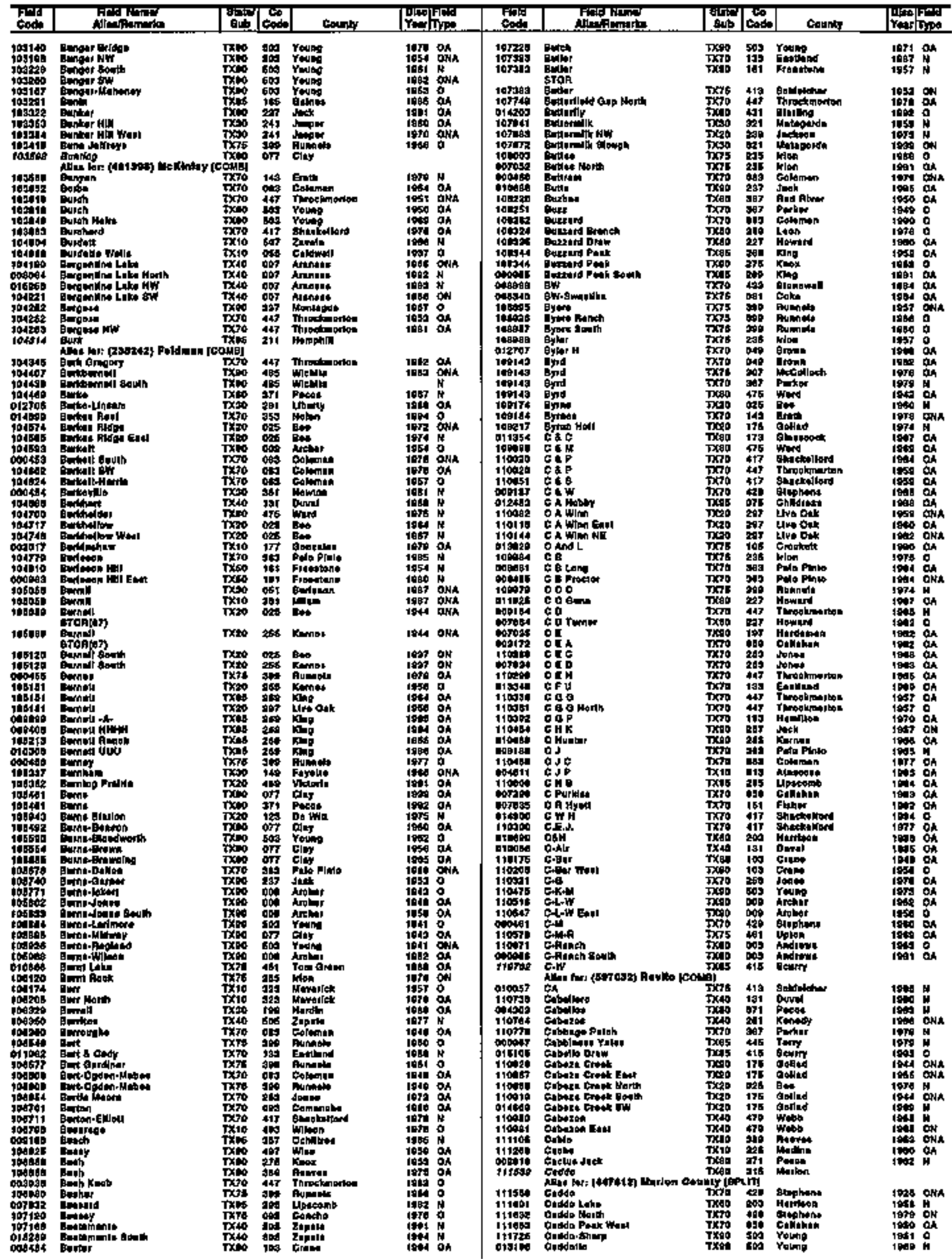


TEXAS

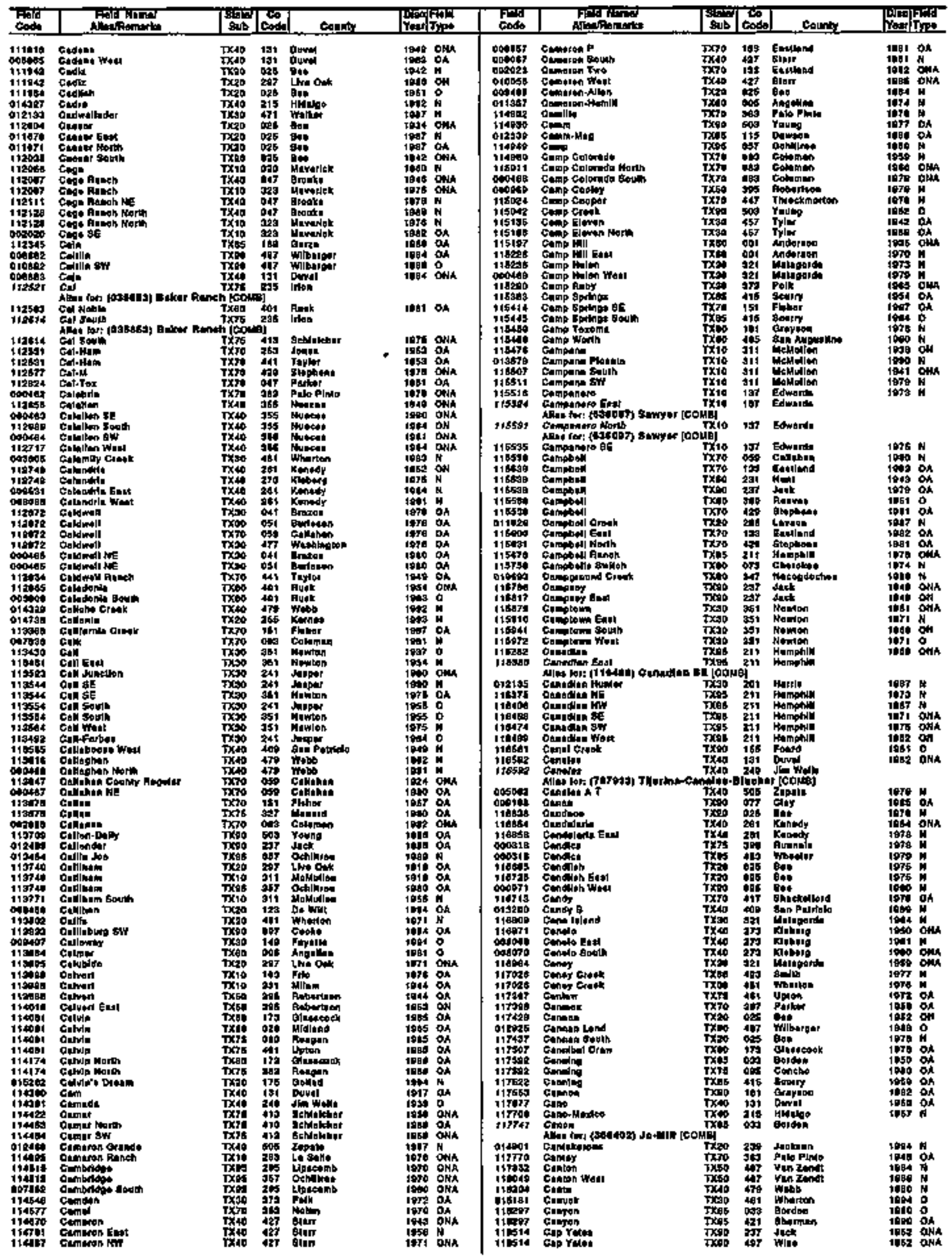


TEXA

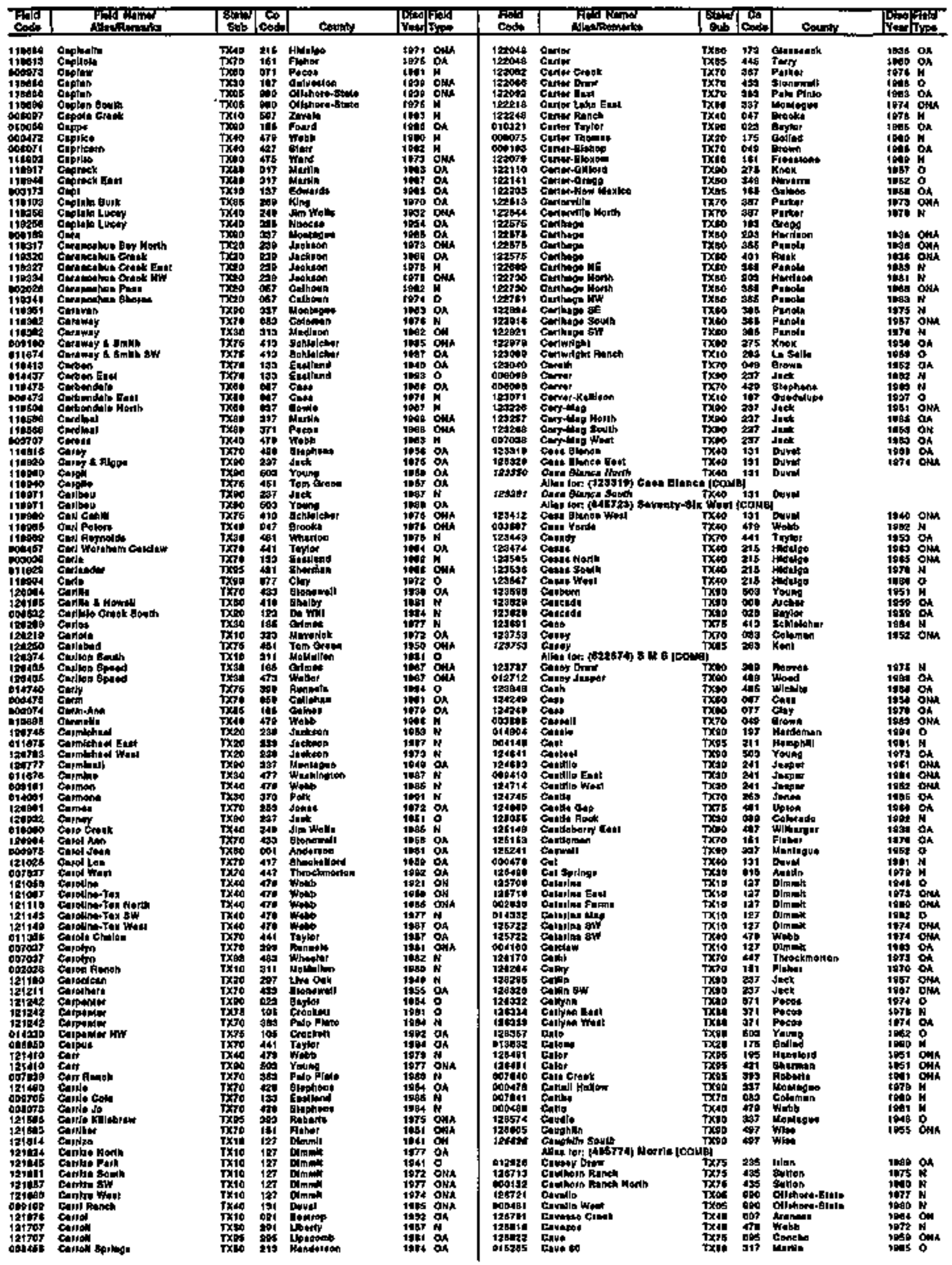


TEXAS

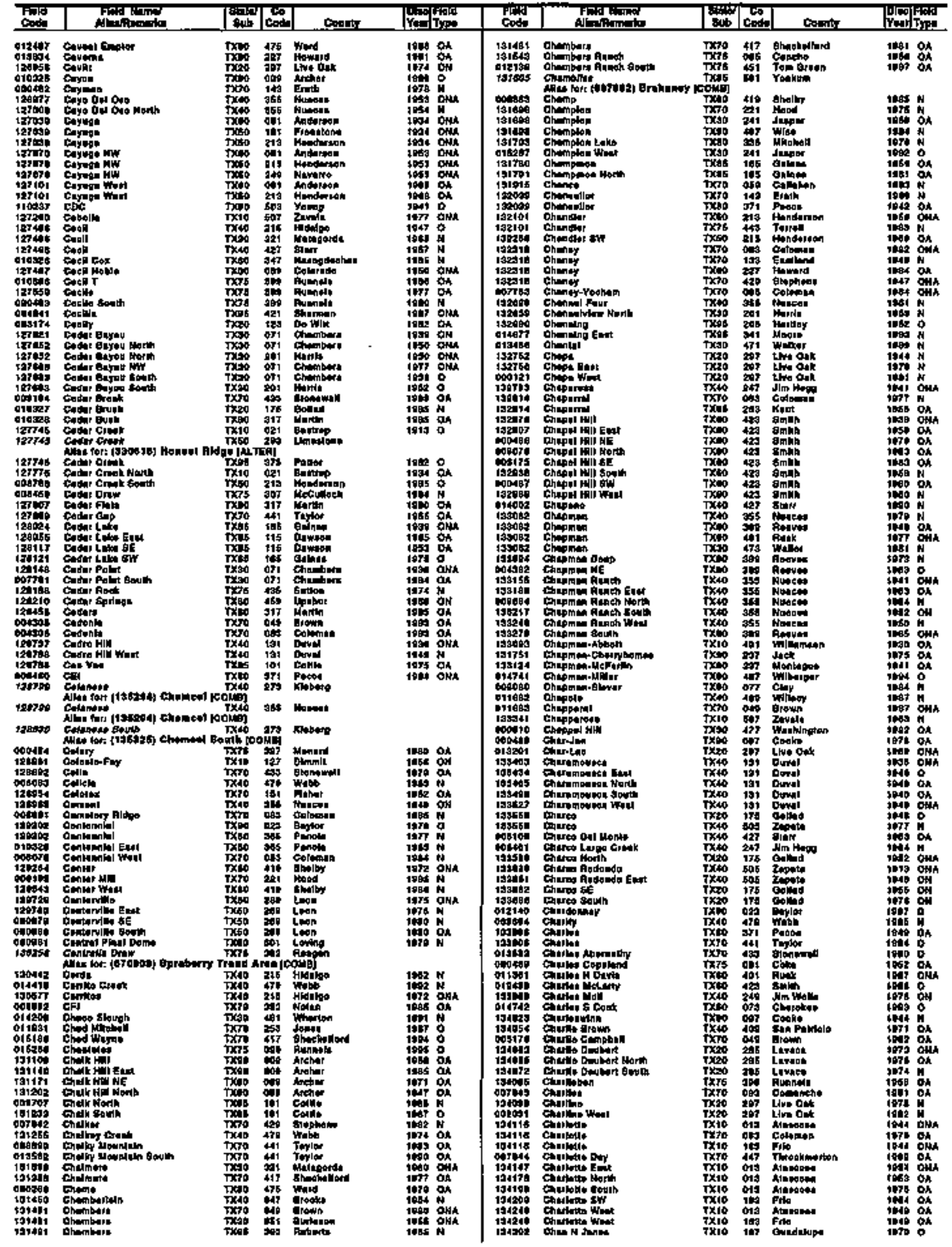


TEXAS

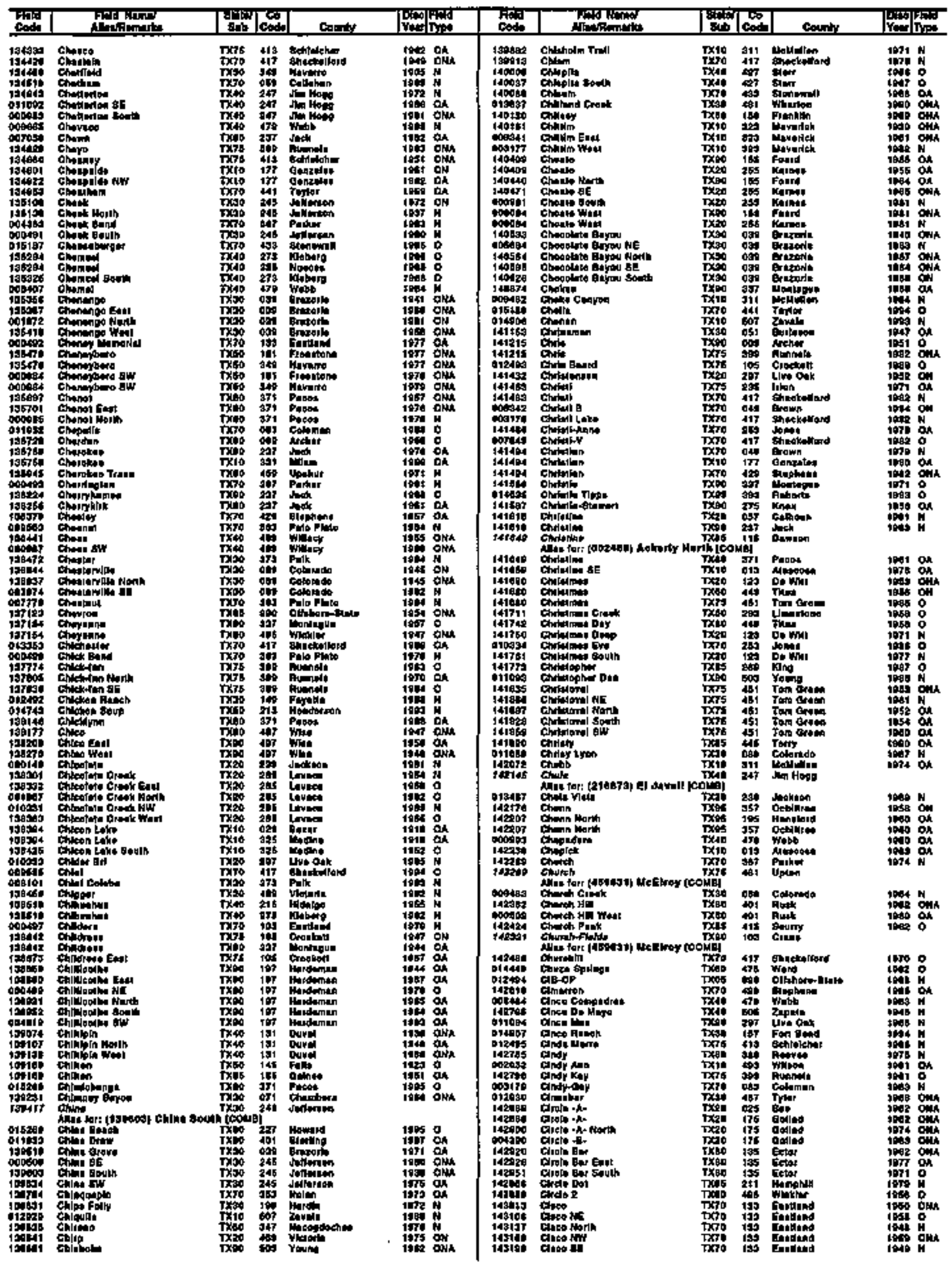


TEXAS

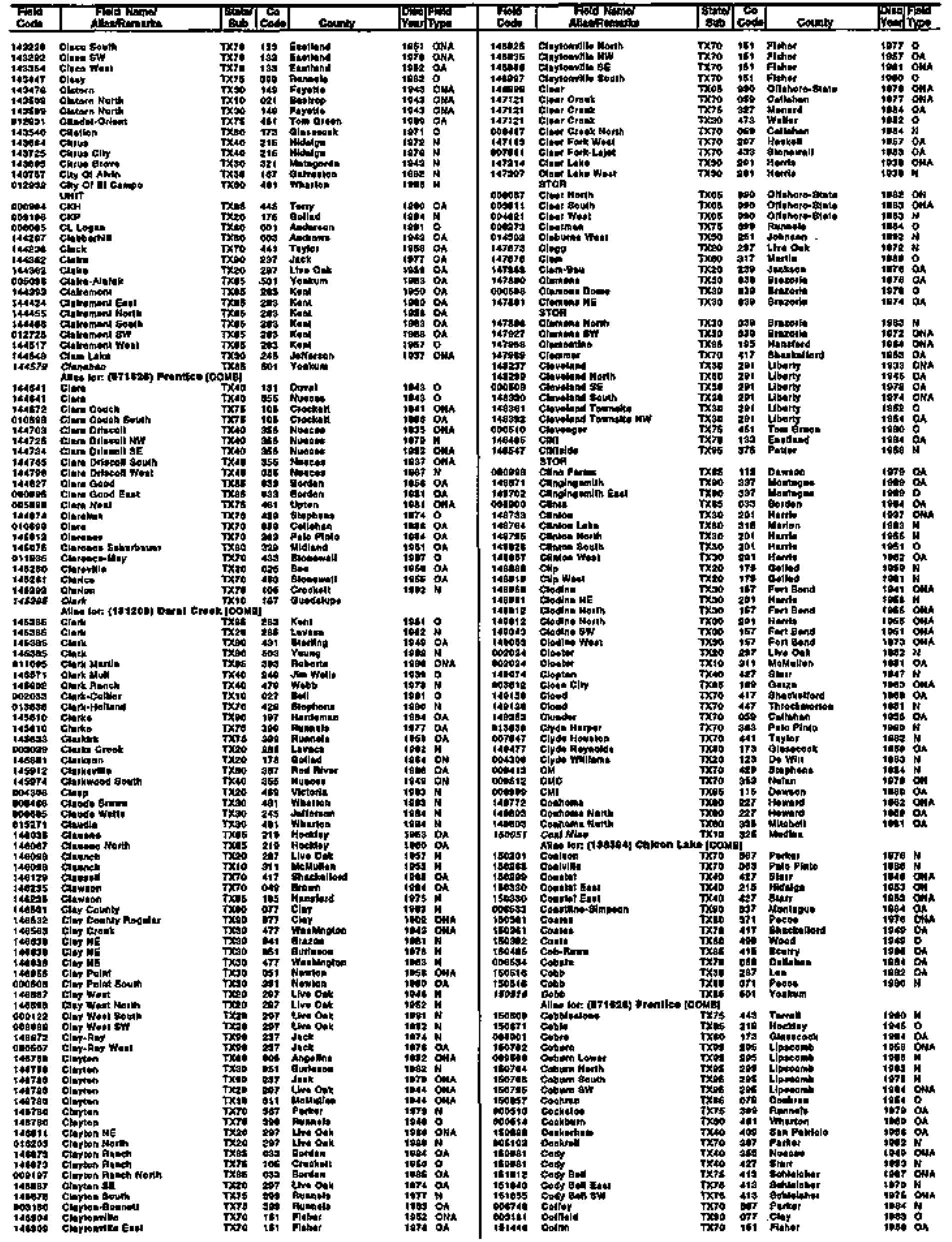


TEXAS

\begin{tabular}{|c|c|c|c|c|c|c|c|c|c|c|c|c|c|}
\hline $\begin{array}{l}\text { Fint: } \\
\text { Codo }\end{array}$ & 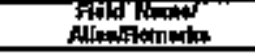 & Dי & tods & cons, & 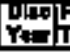 & $\begin{array}{l}\text { Alca } \\
\text { Iyre }\end{array}$ & Sadi & ADE & satin & Cods & Coptiot & |lons & 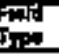 \\
\hline 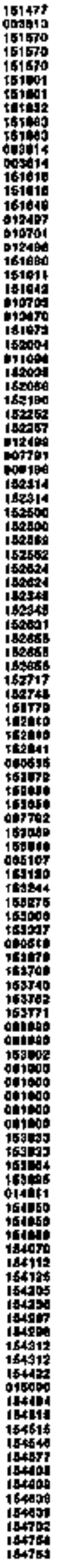 & 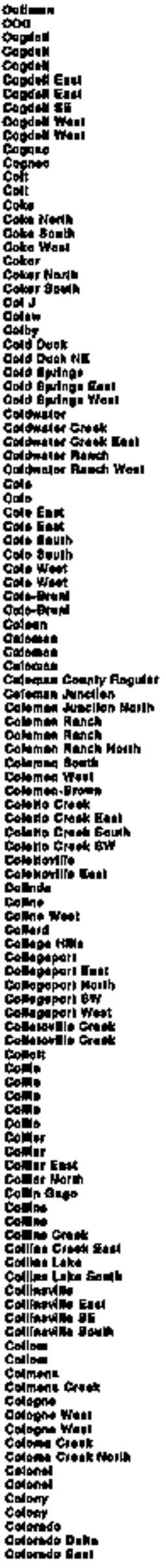 & 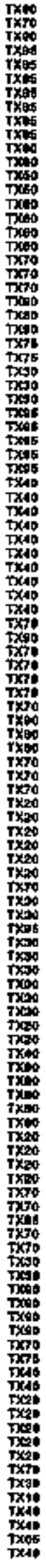 & 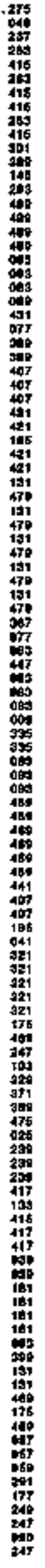 & 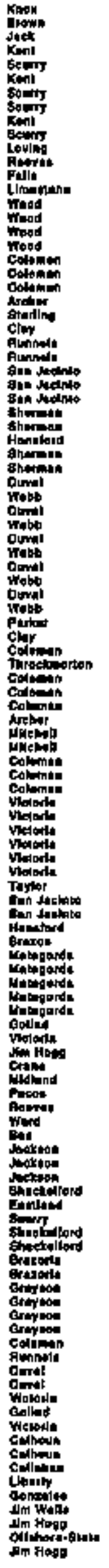 & 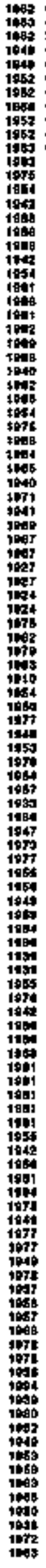 & 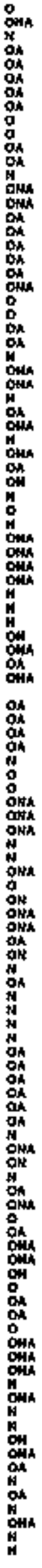 & 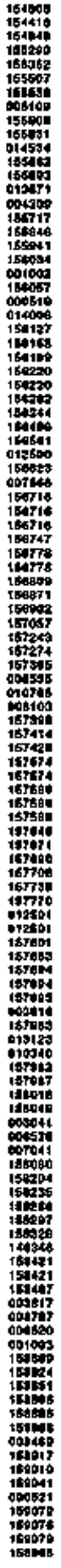 & 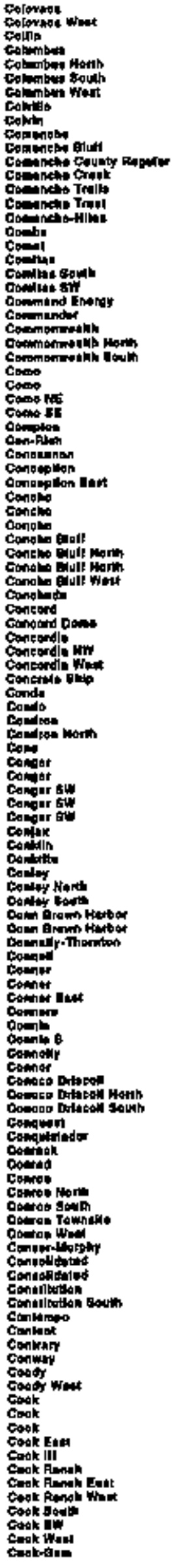 & 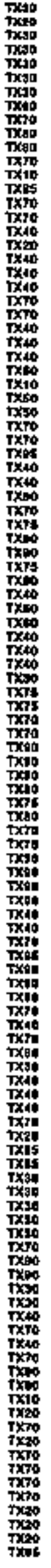 & 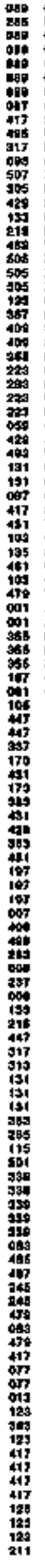 & 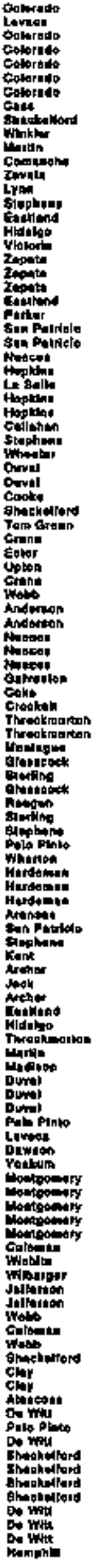 & 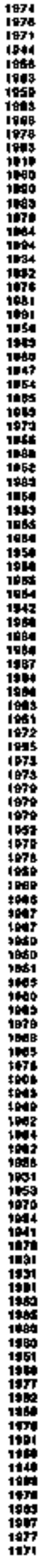 & 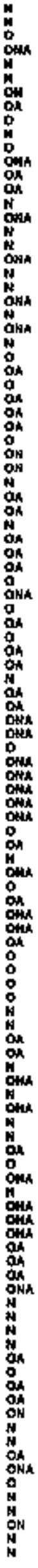 \\
\hline
\end{tabular}


TEXAS

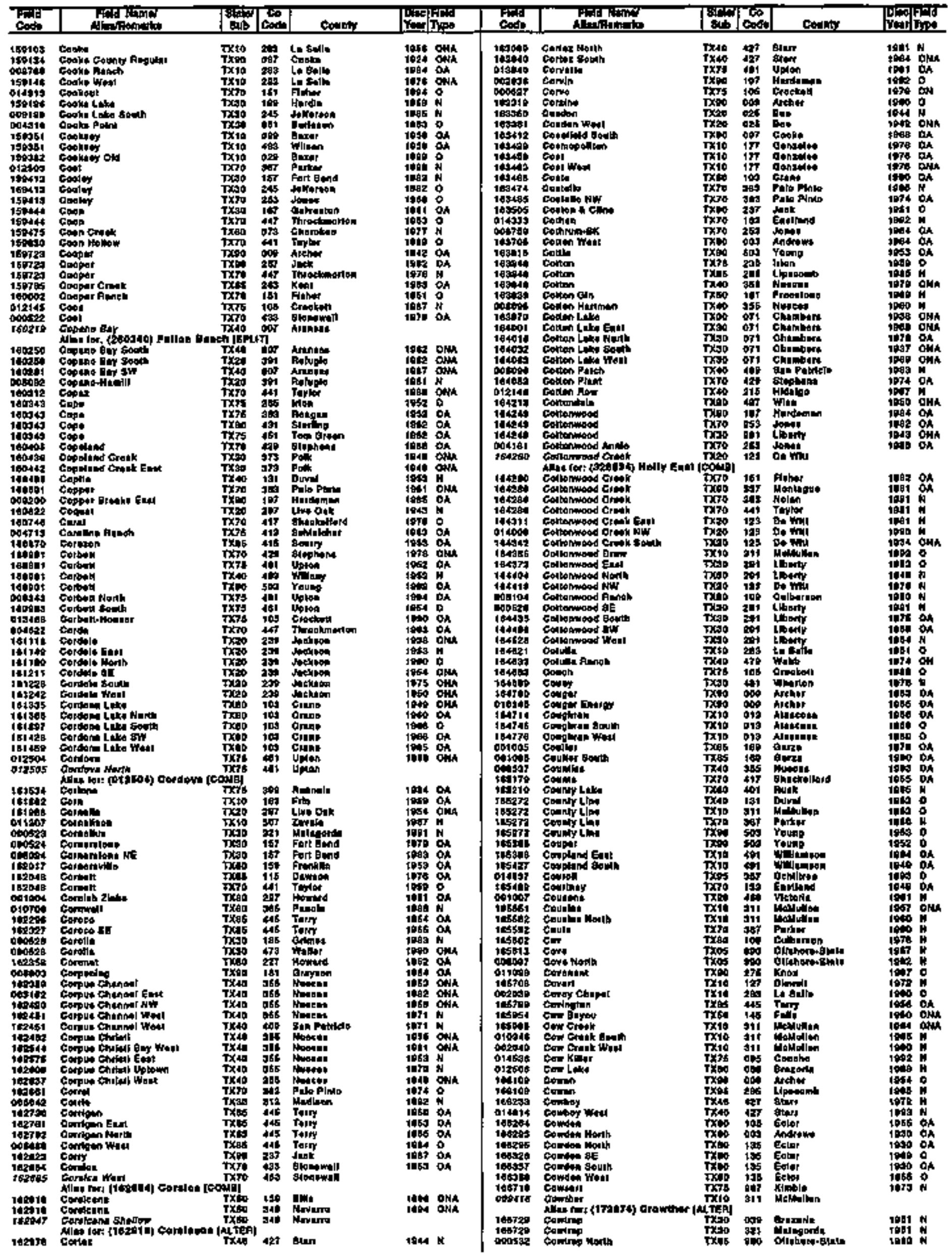


TEXAS

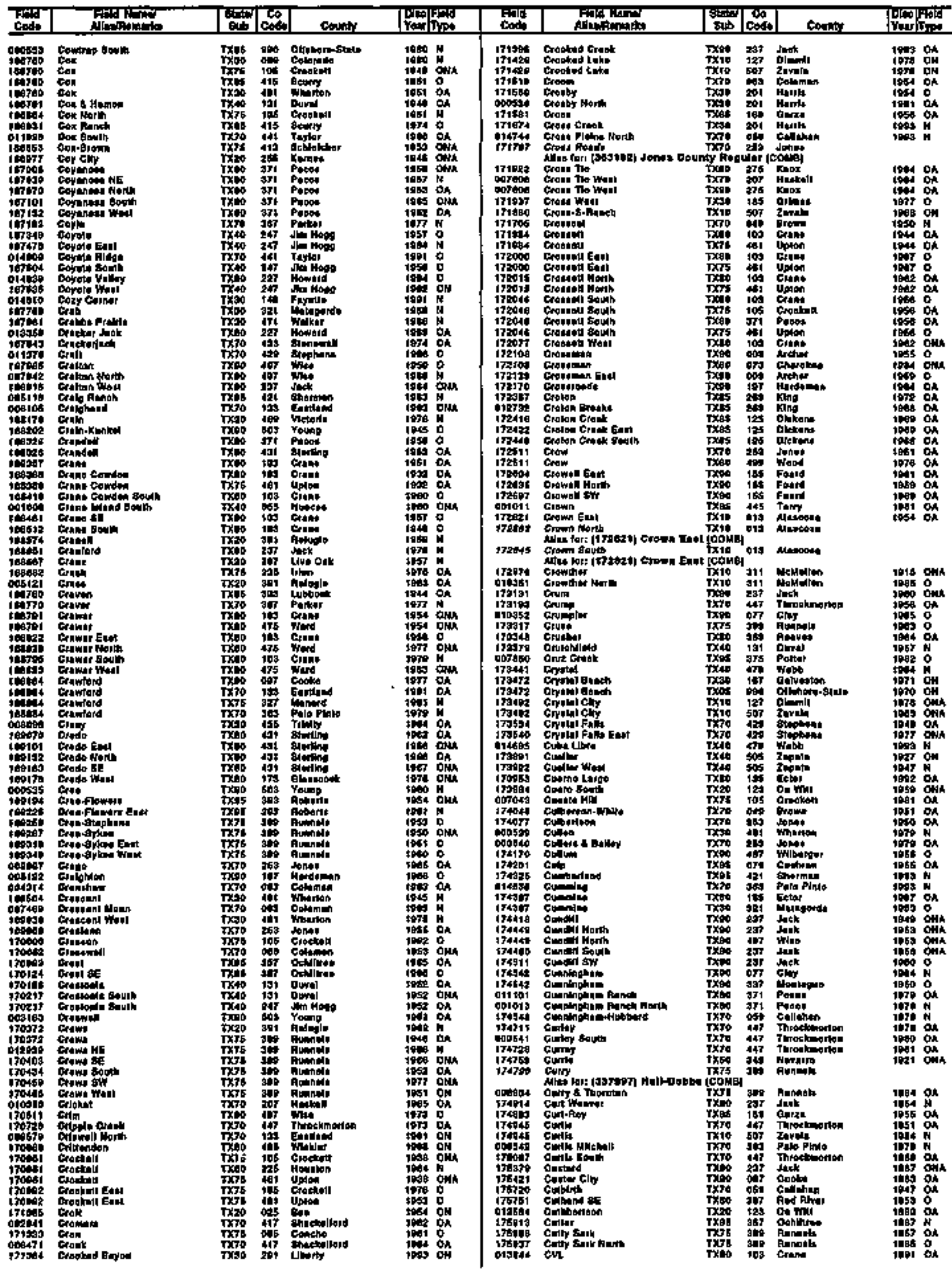


TEXAS

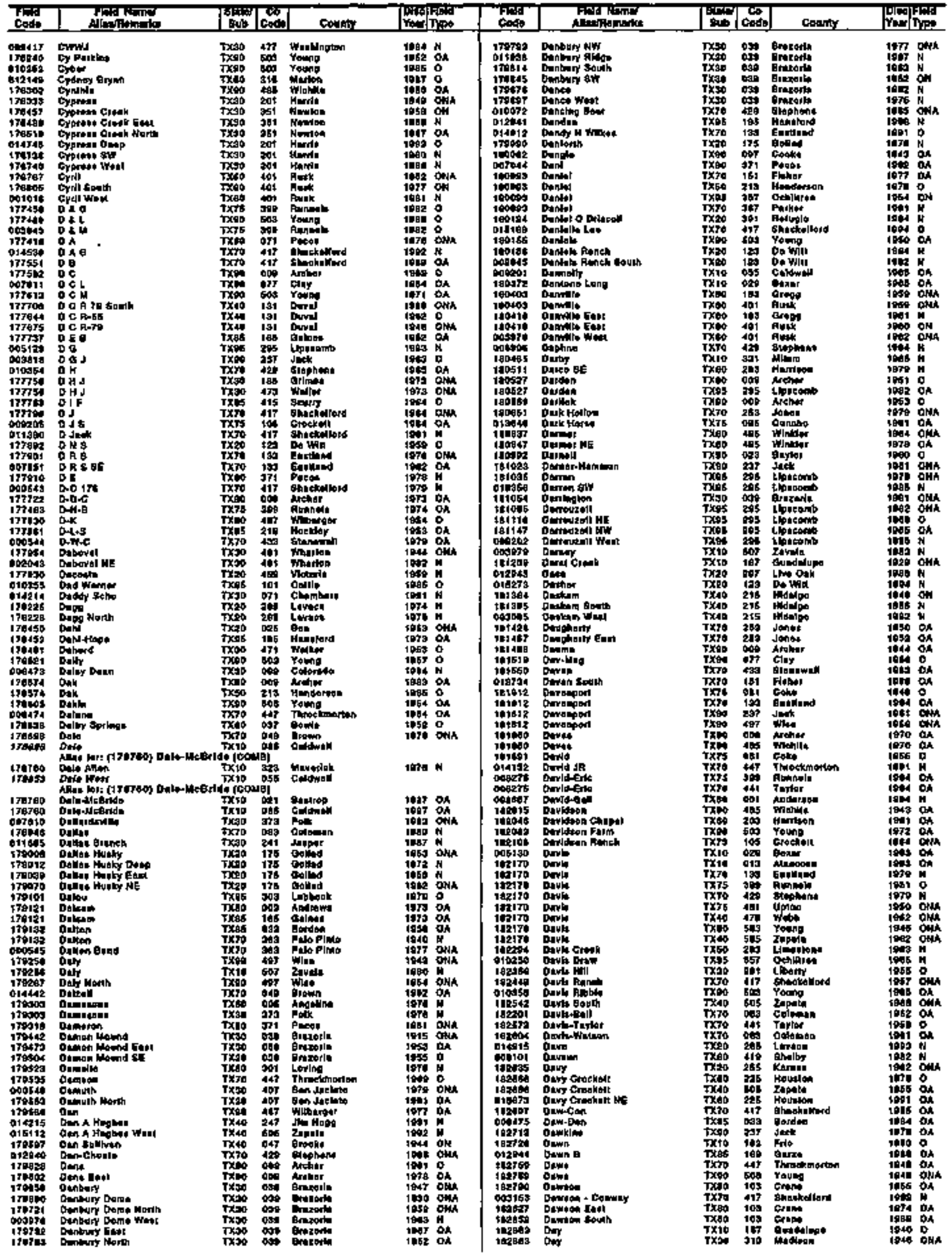


TEXAS

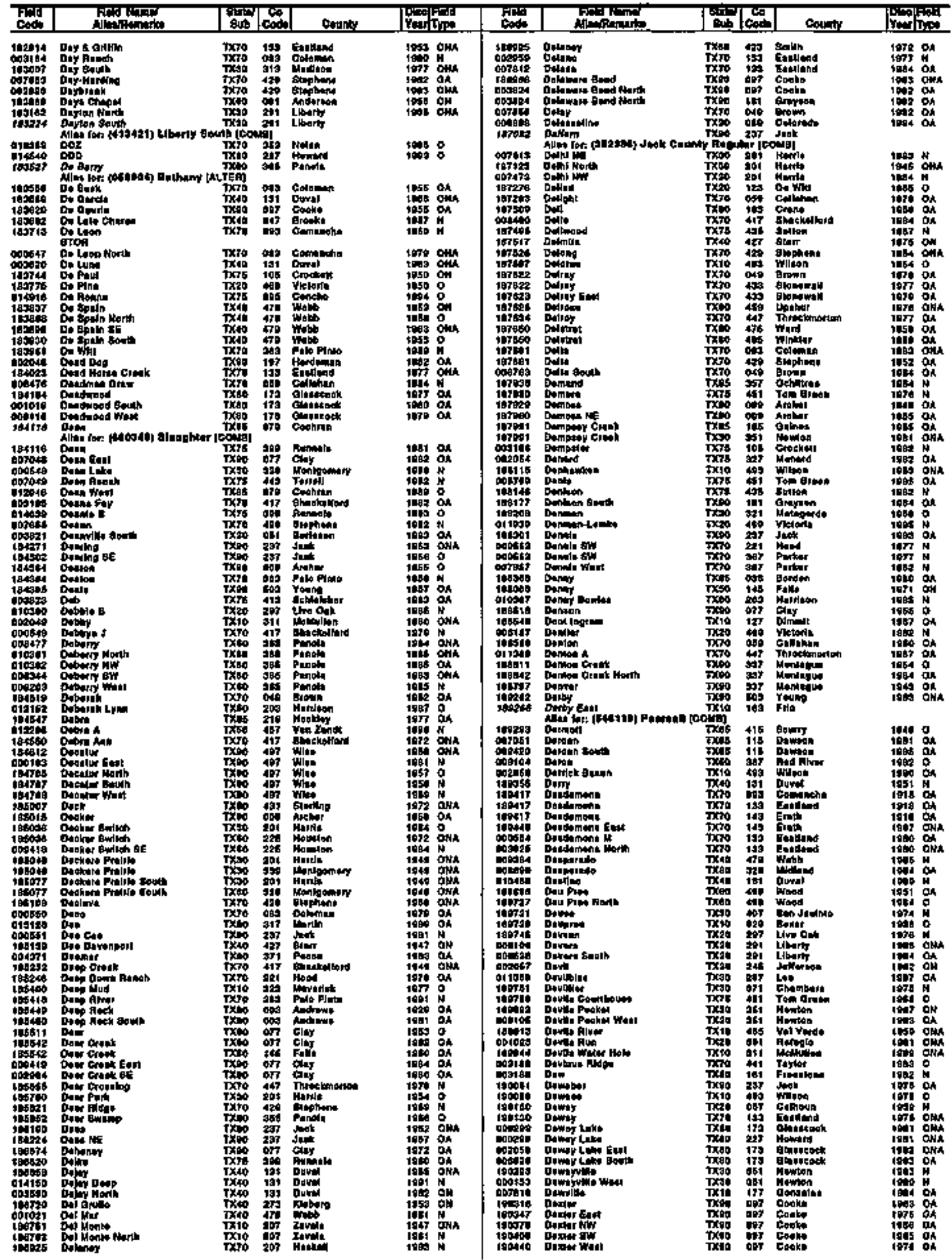


TEXA $\mathbf{S}$

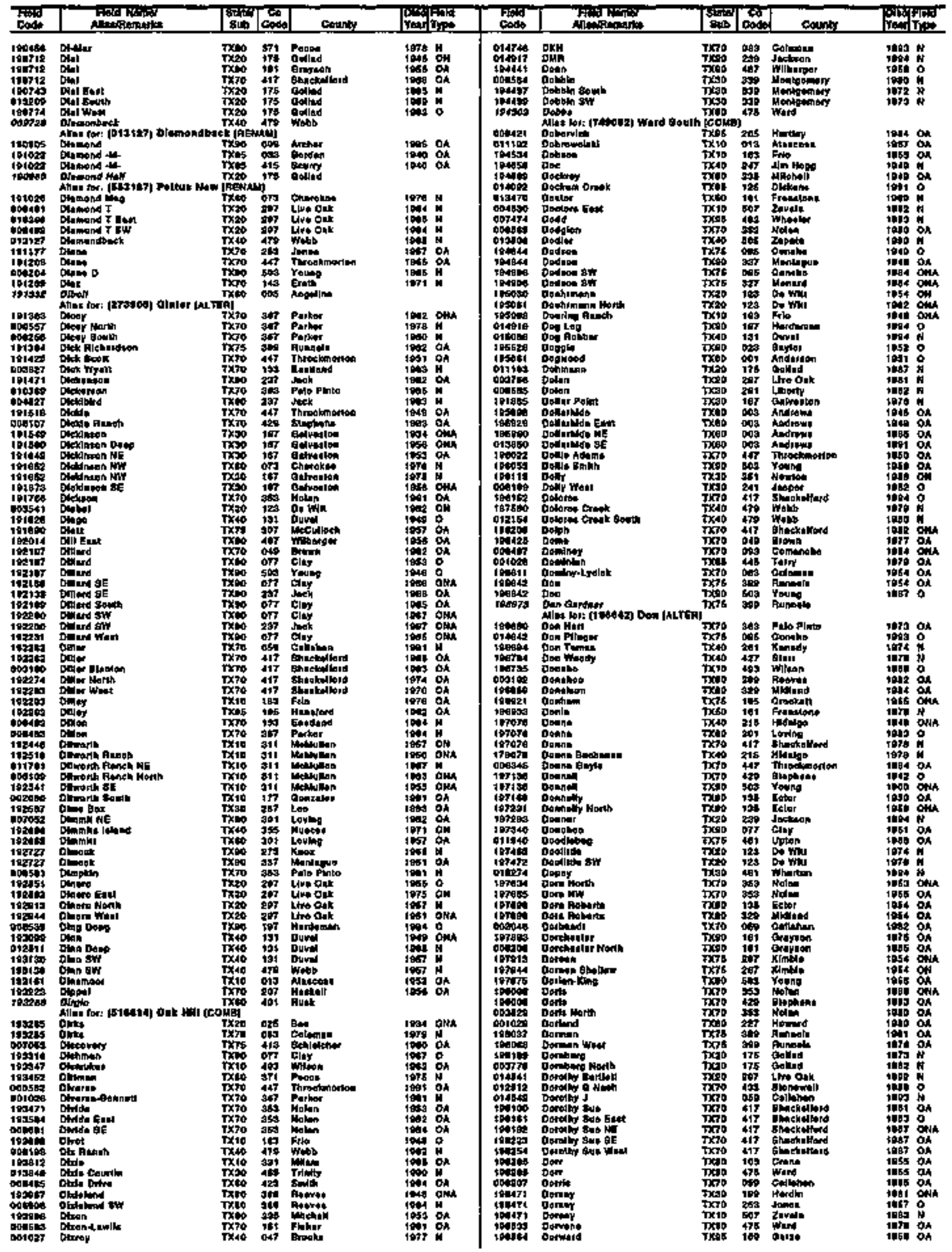


TEXAS

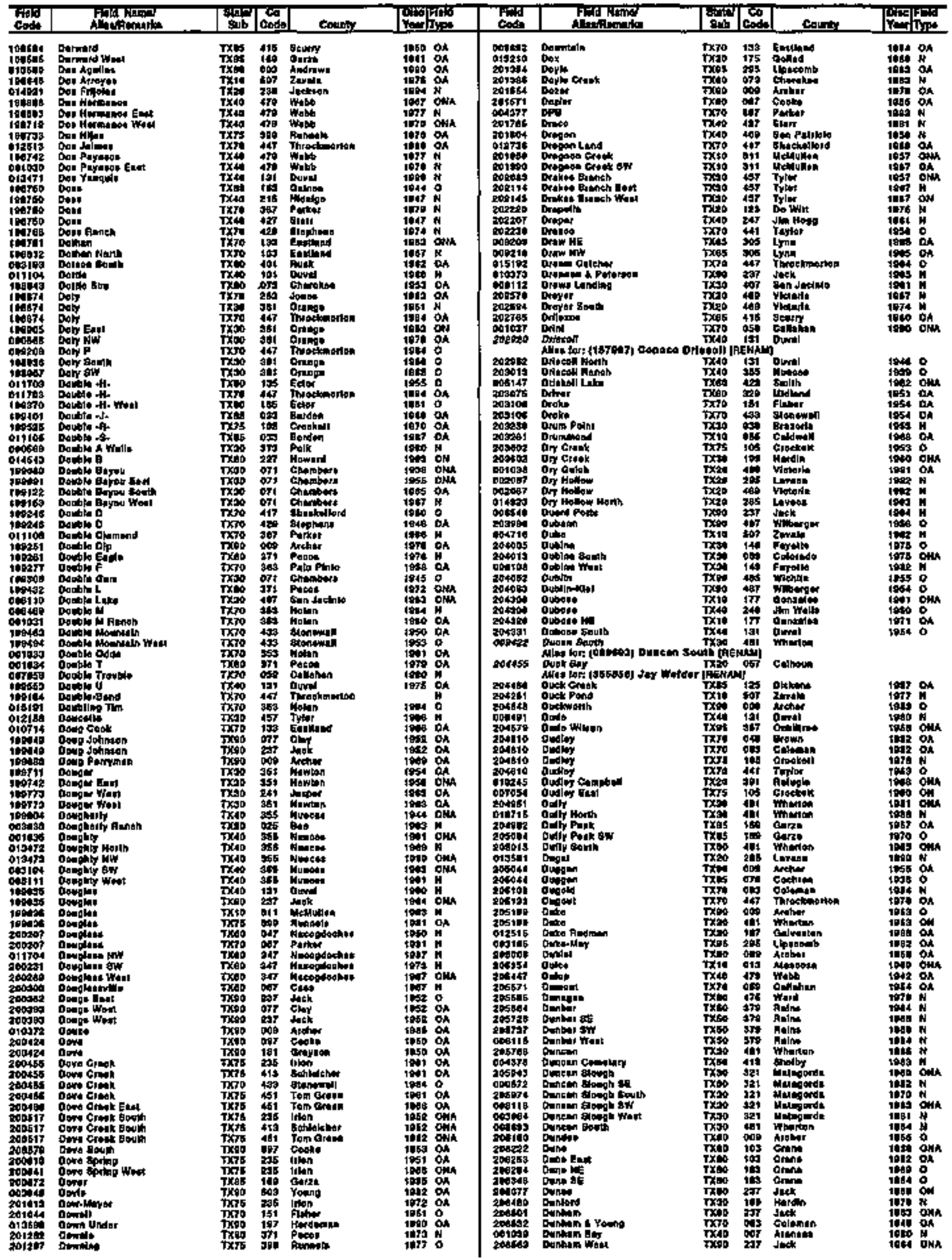


TEXAS

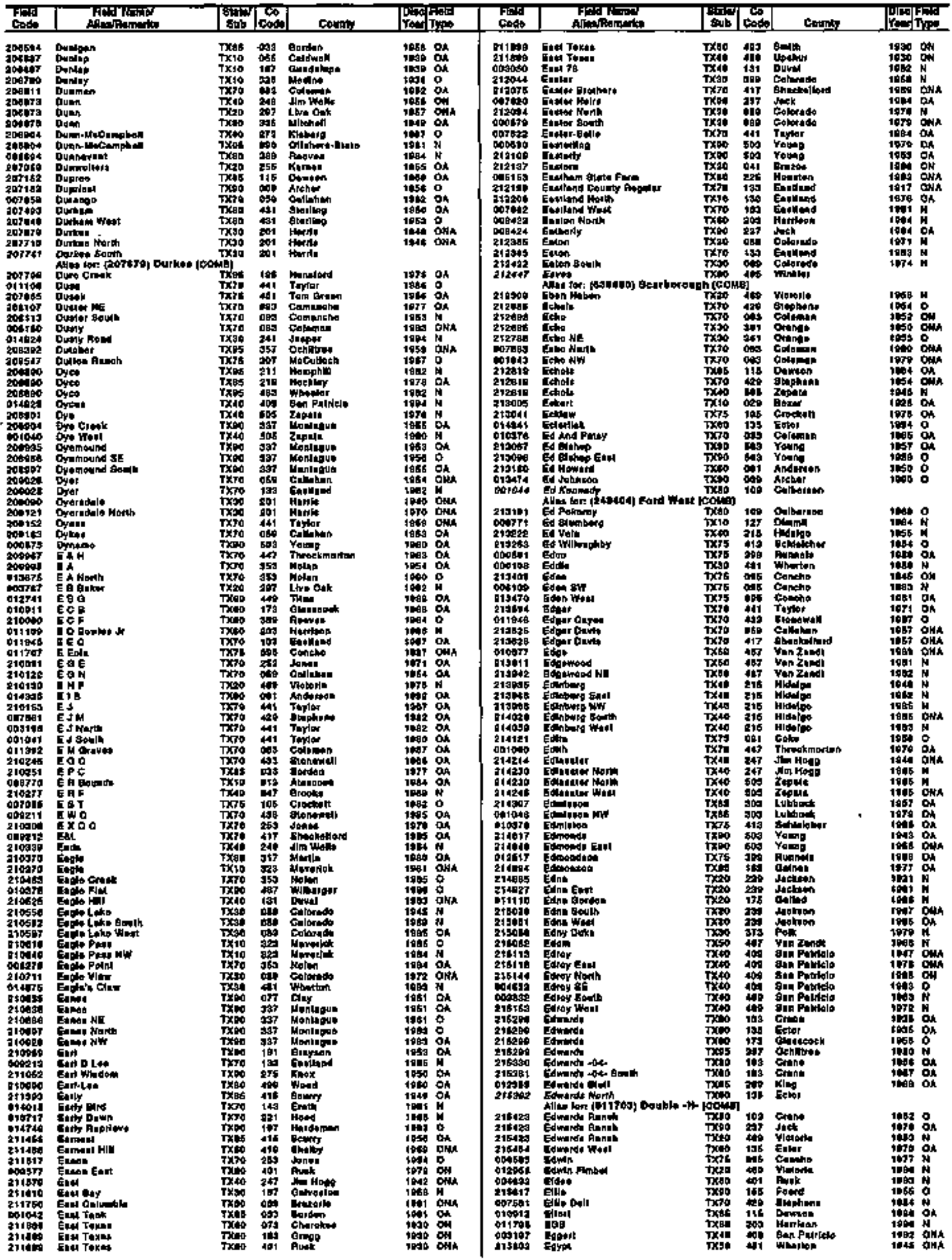


TEXAS

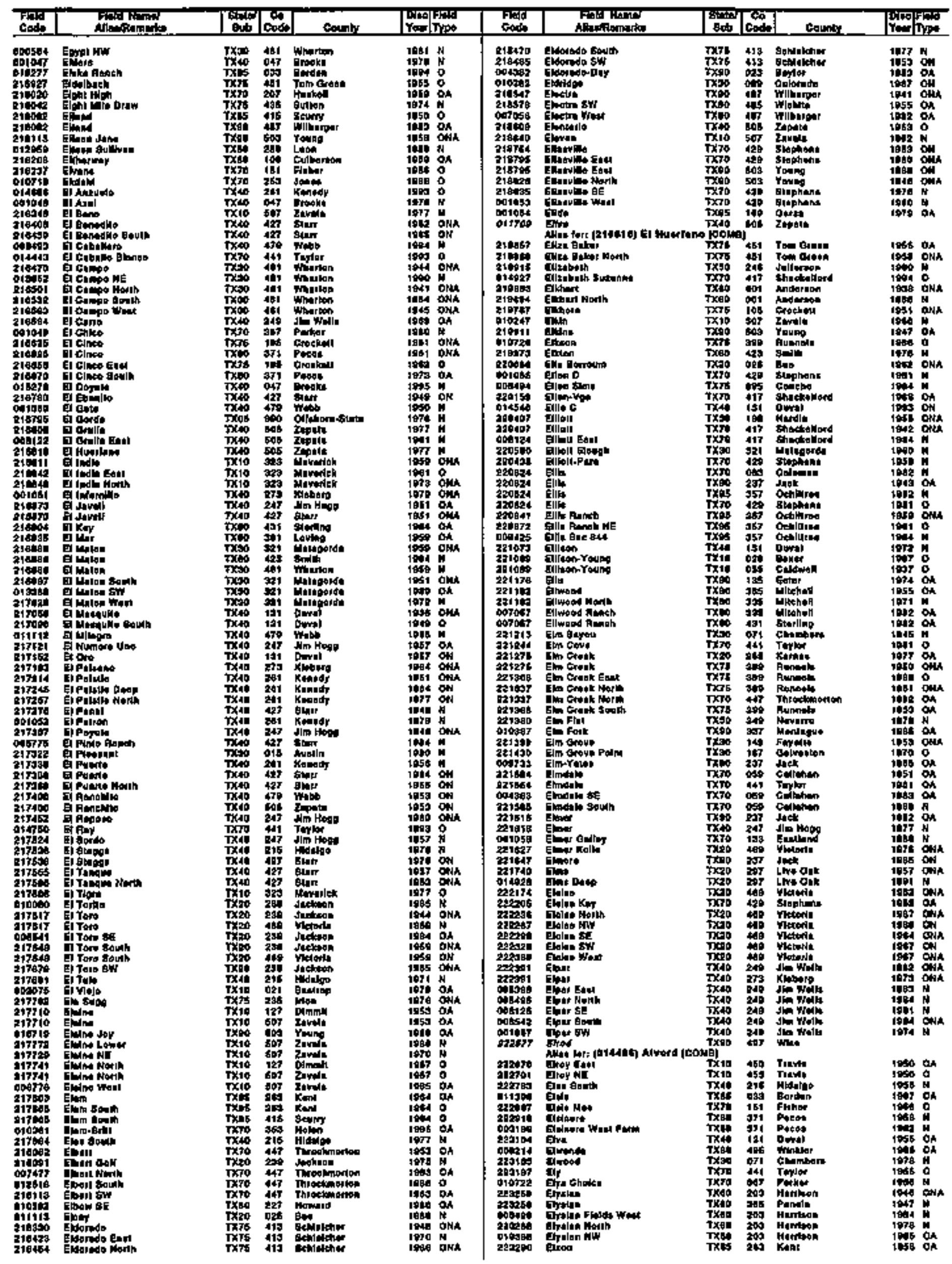

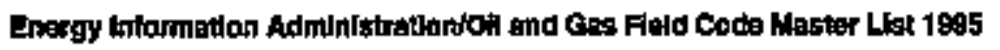


TEXAS

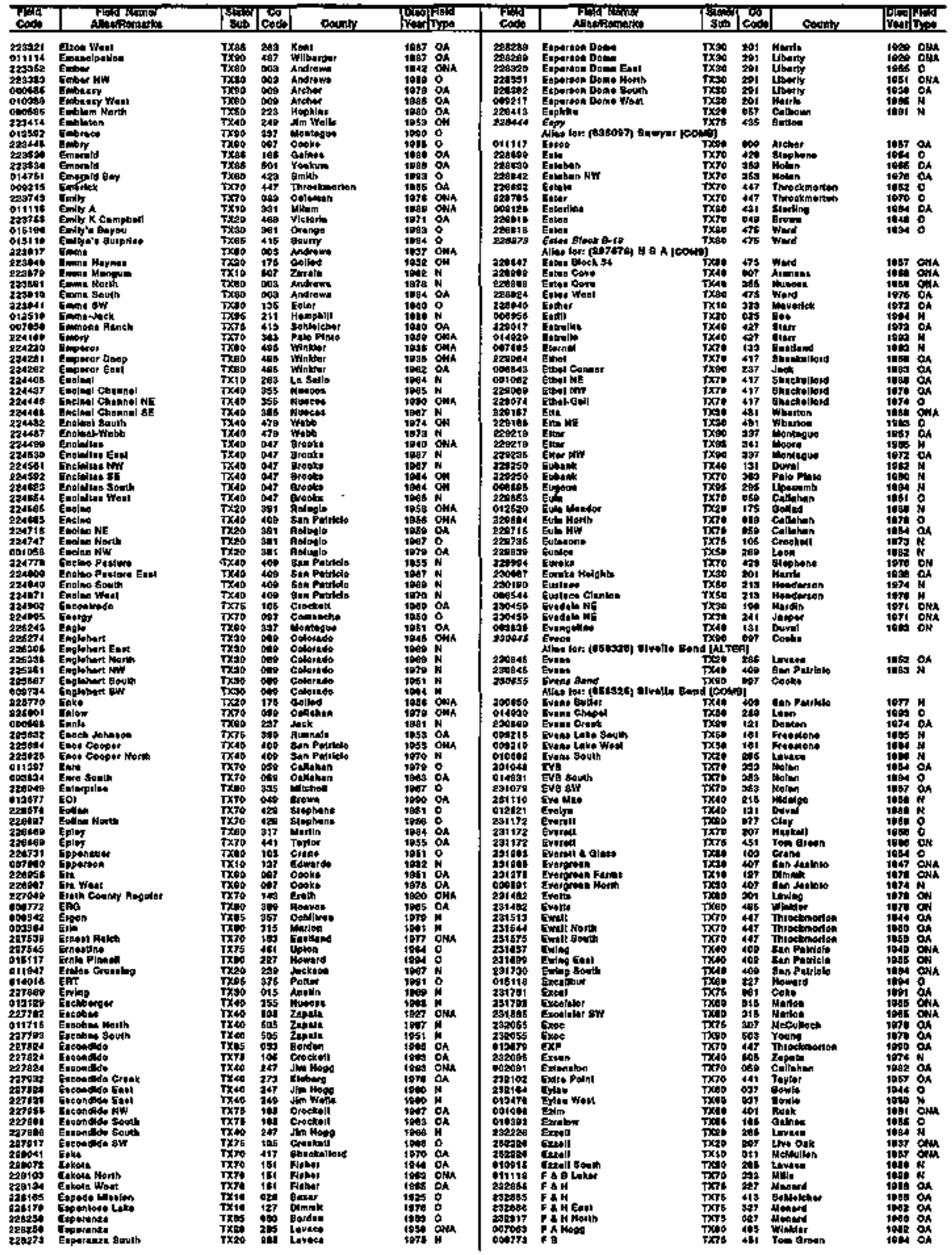


TEXAS

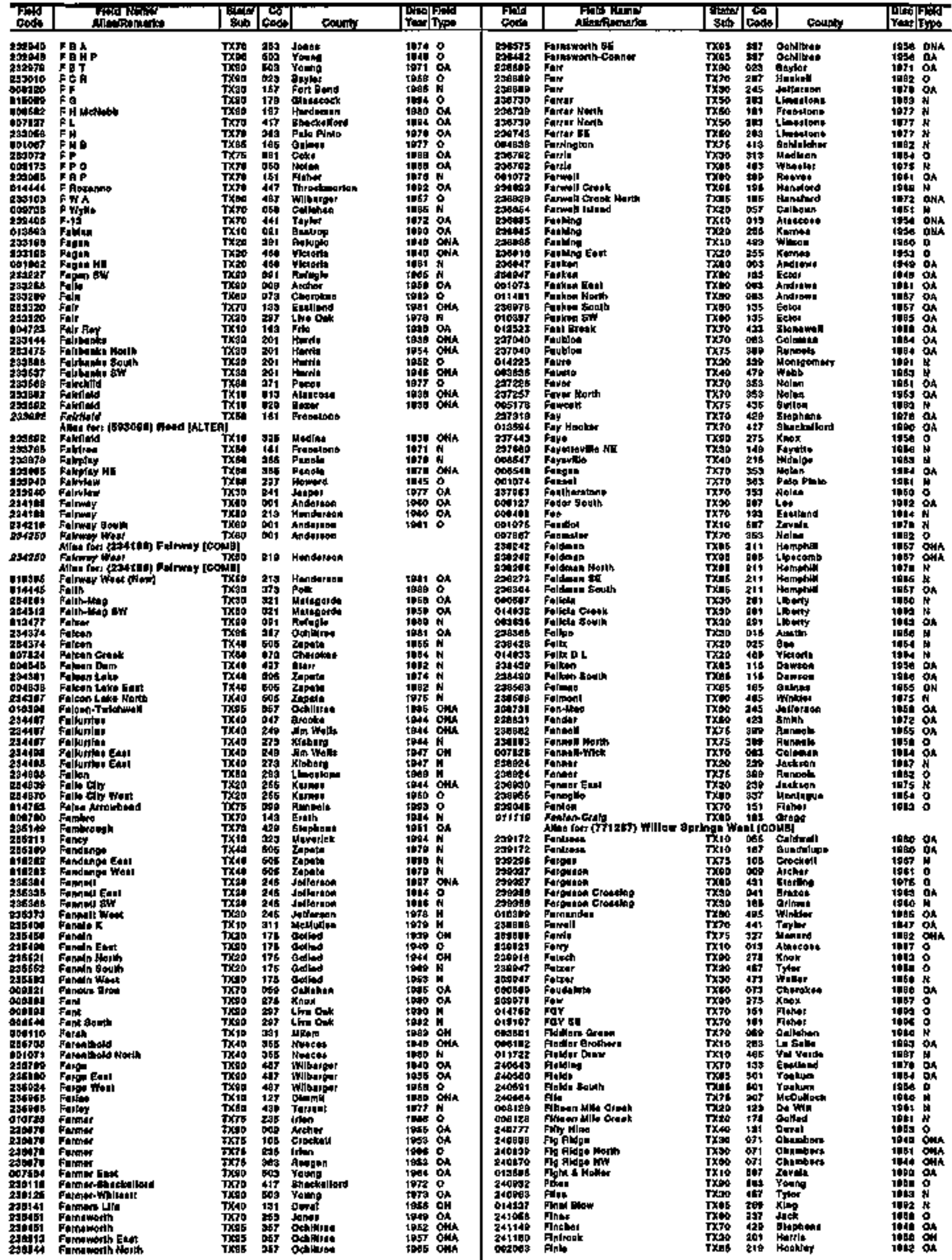


TEXAS

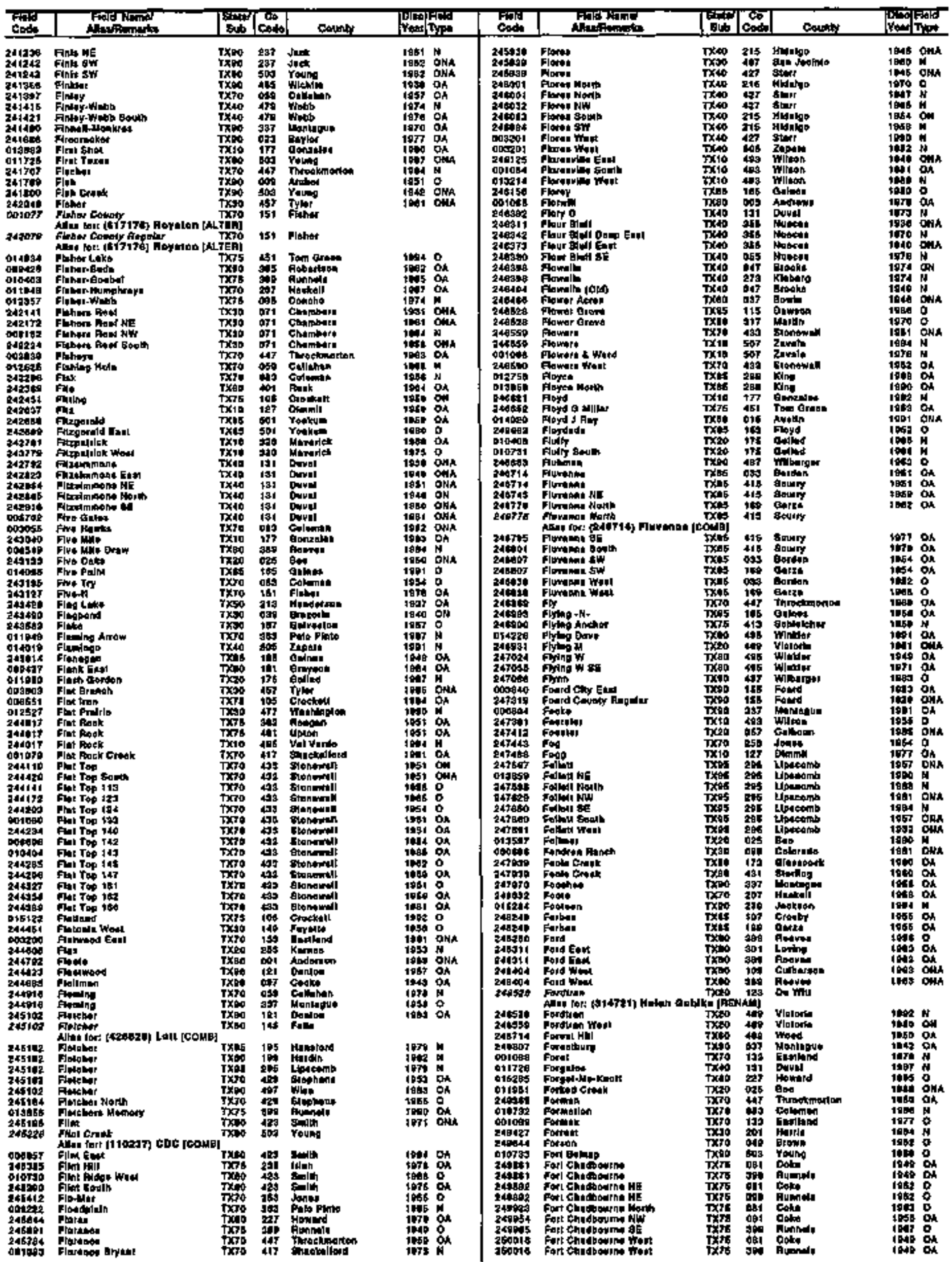


TEXAS

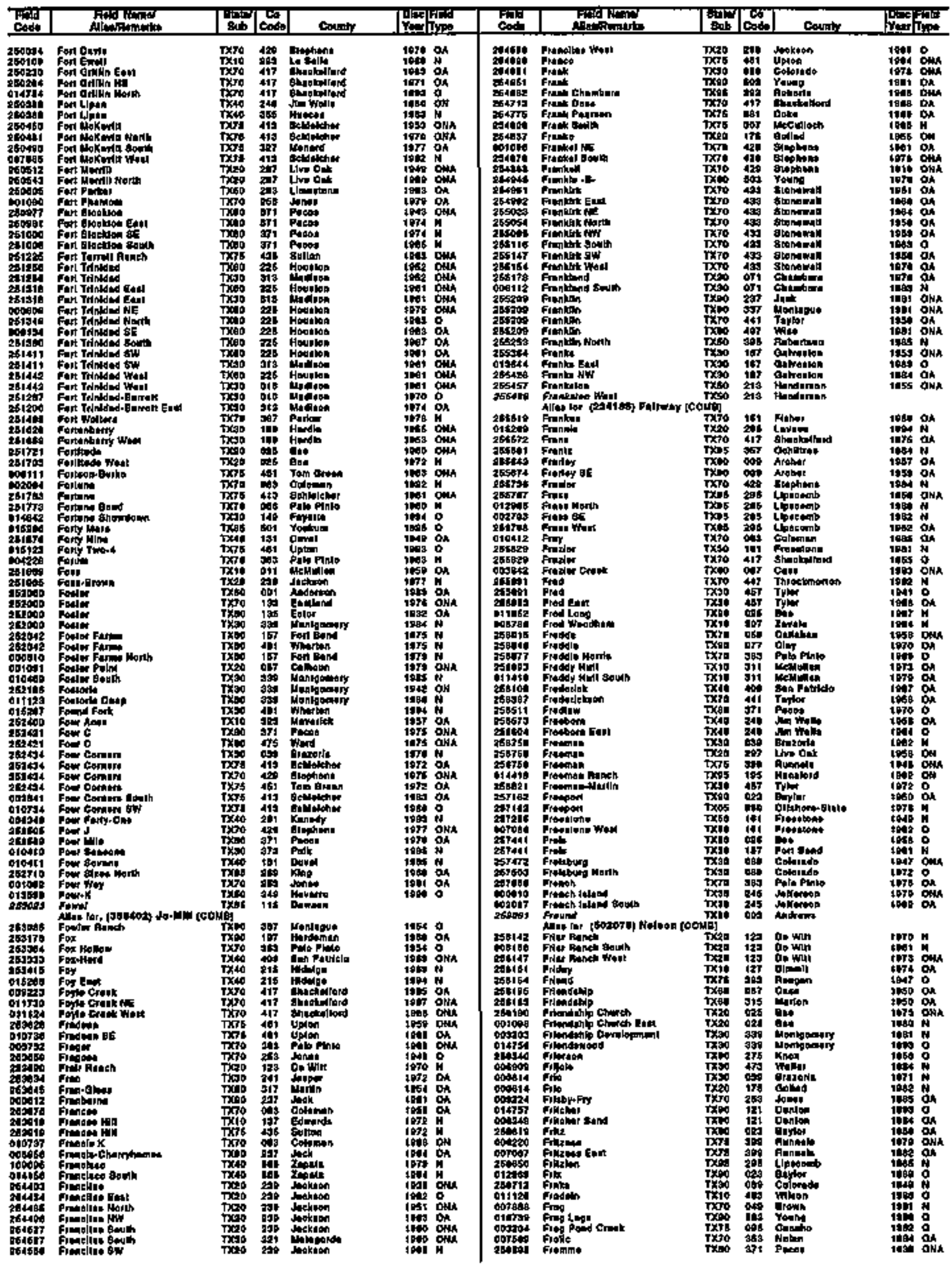




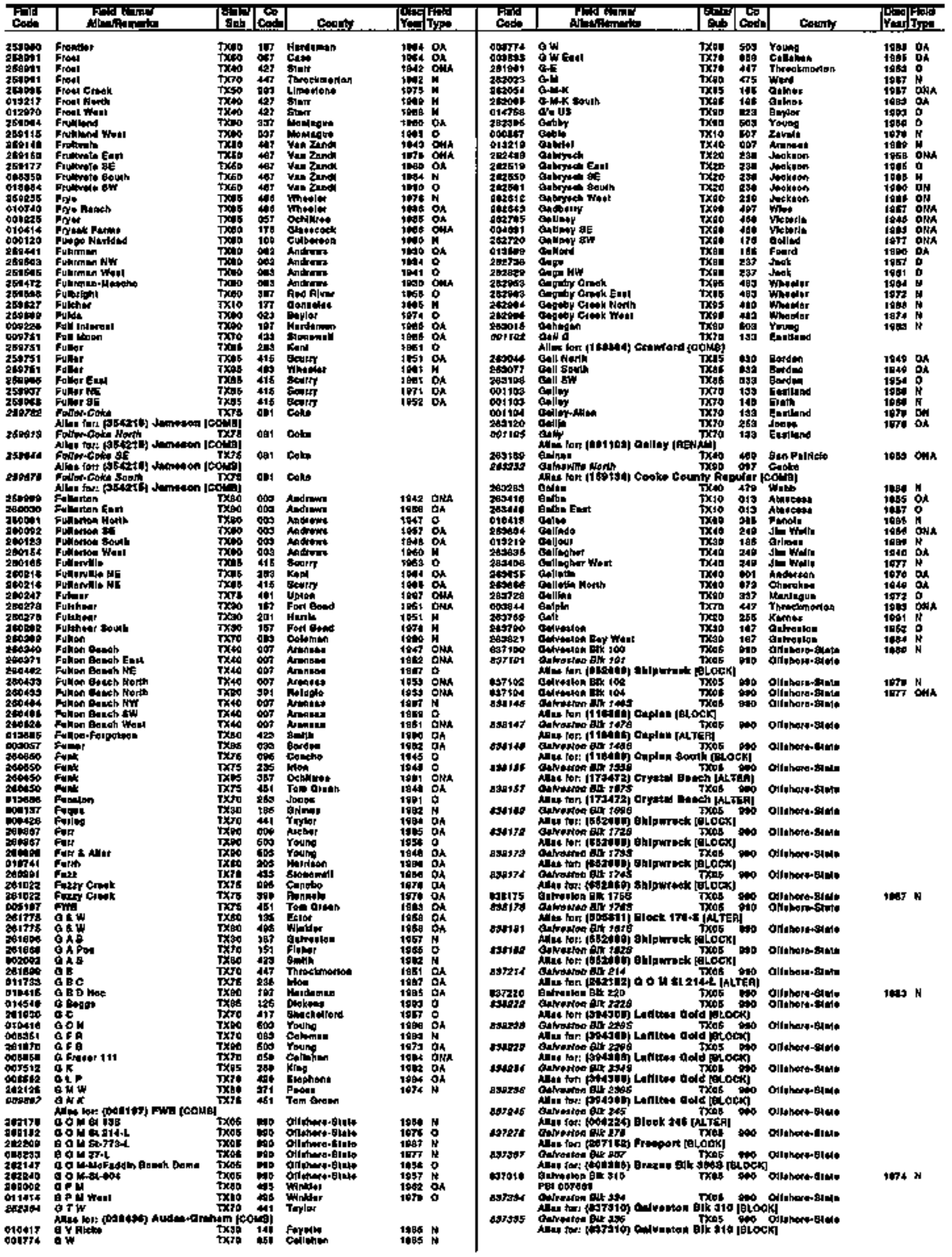


TEXAS

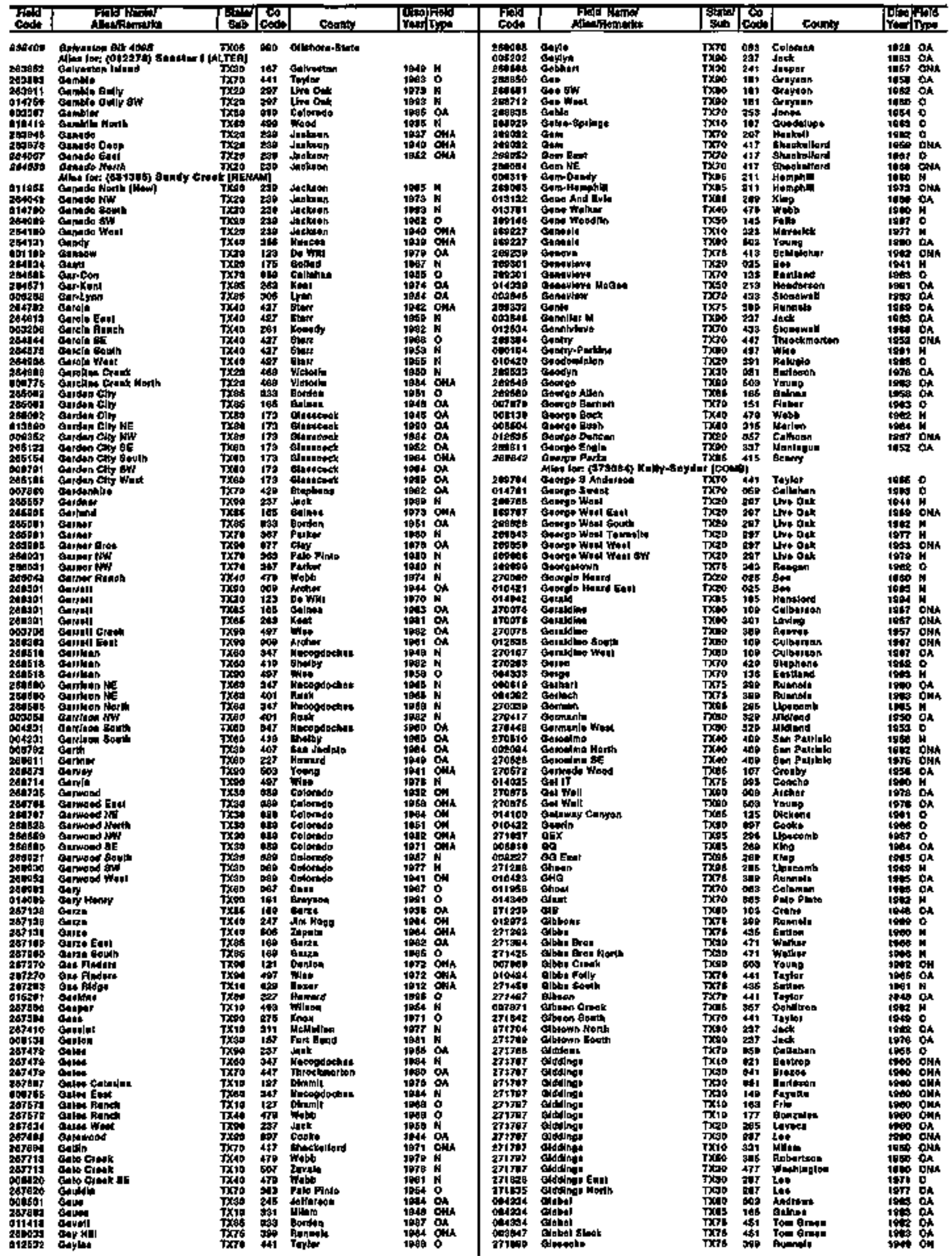




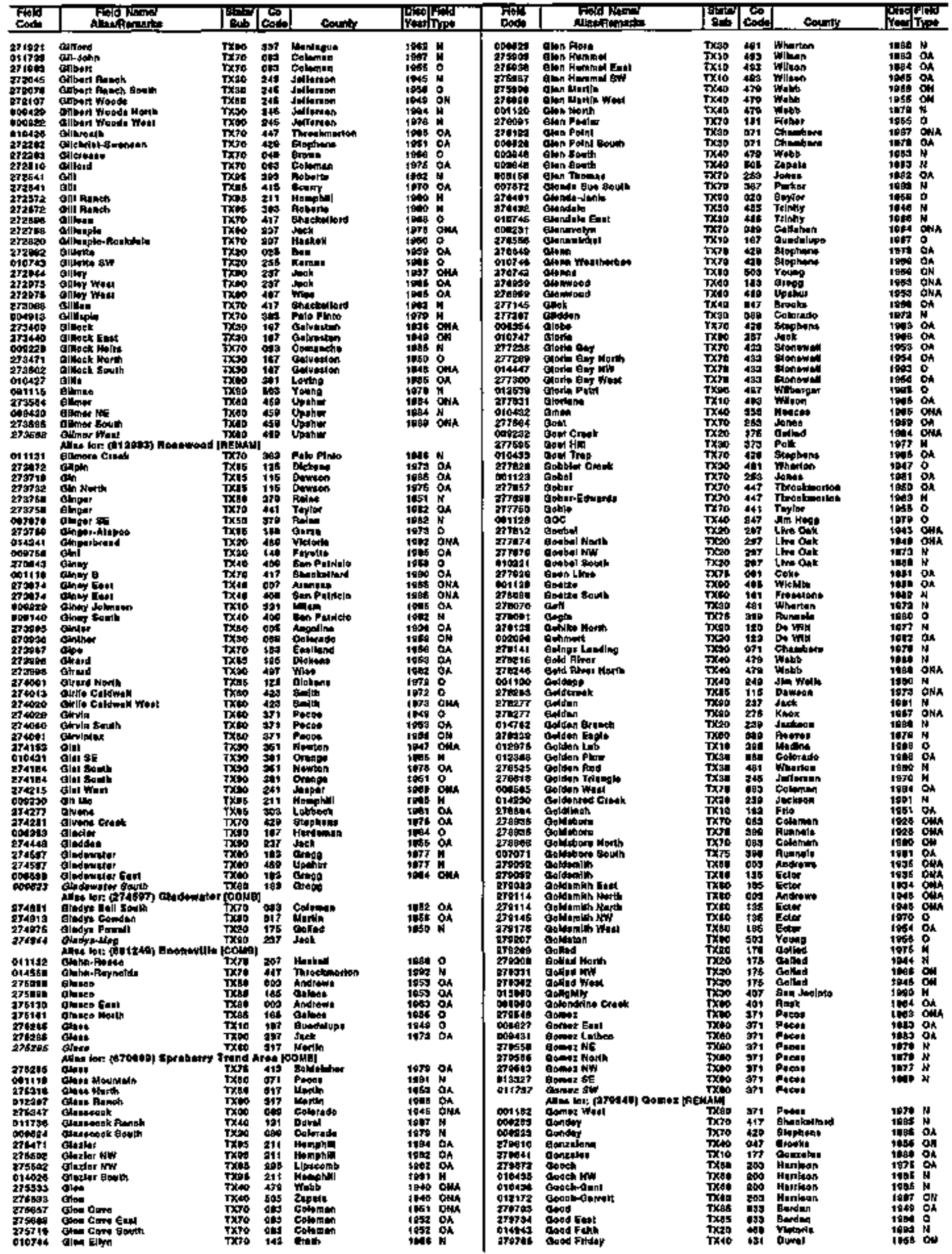


TEXAS

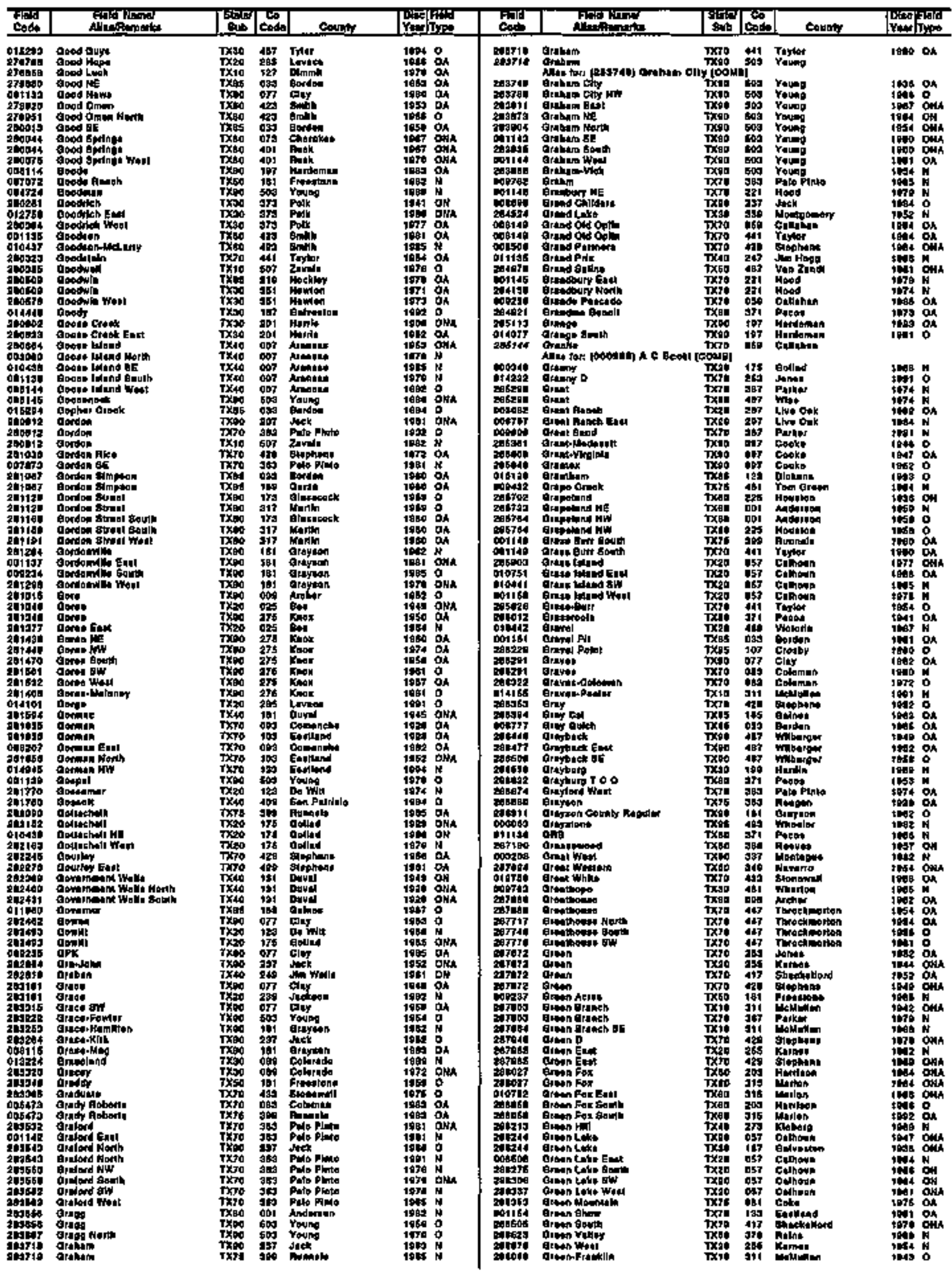


TEXAS

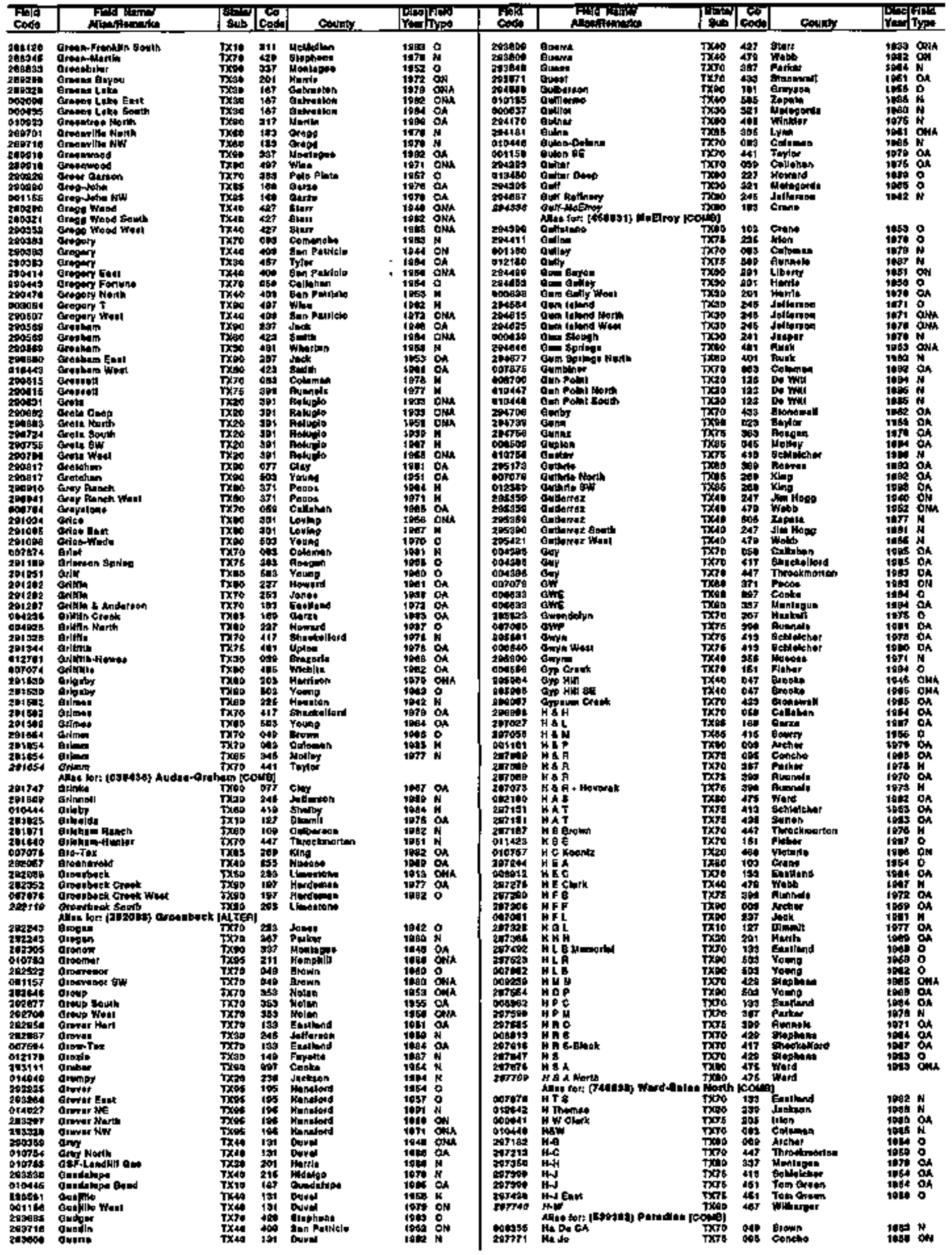


TEXas

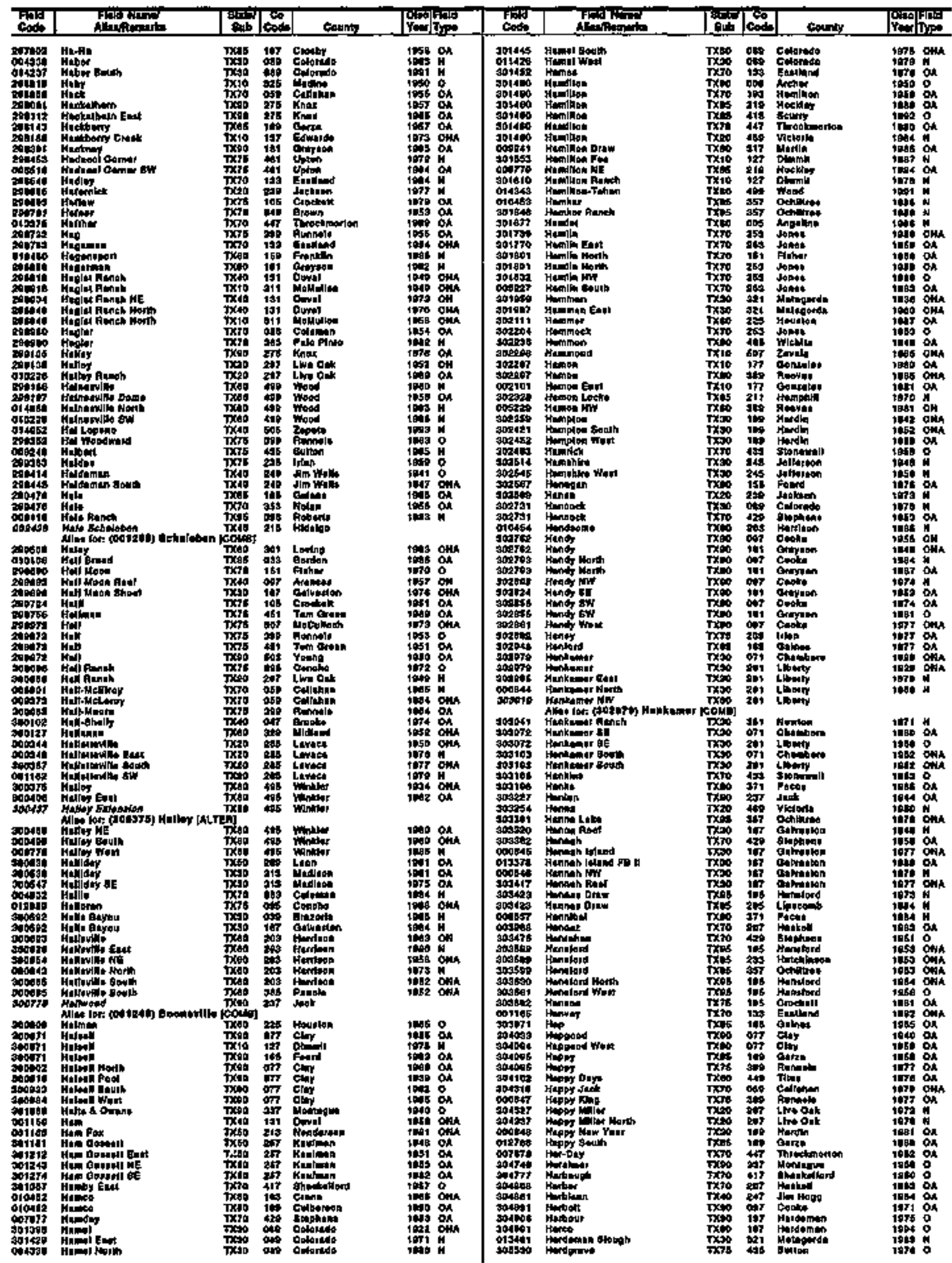


TEXAS

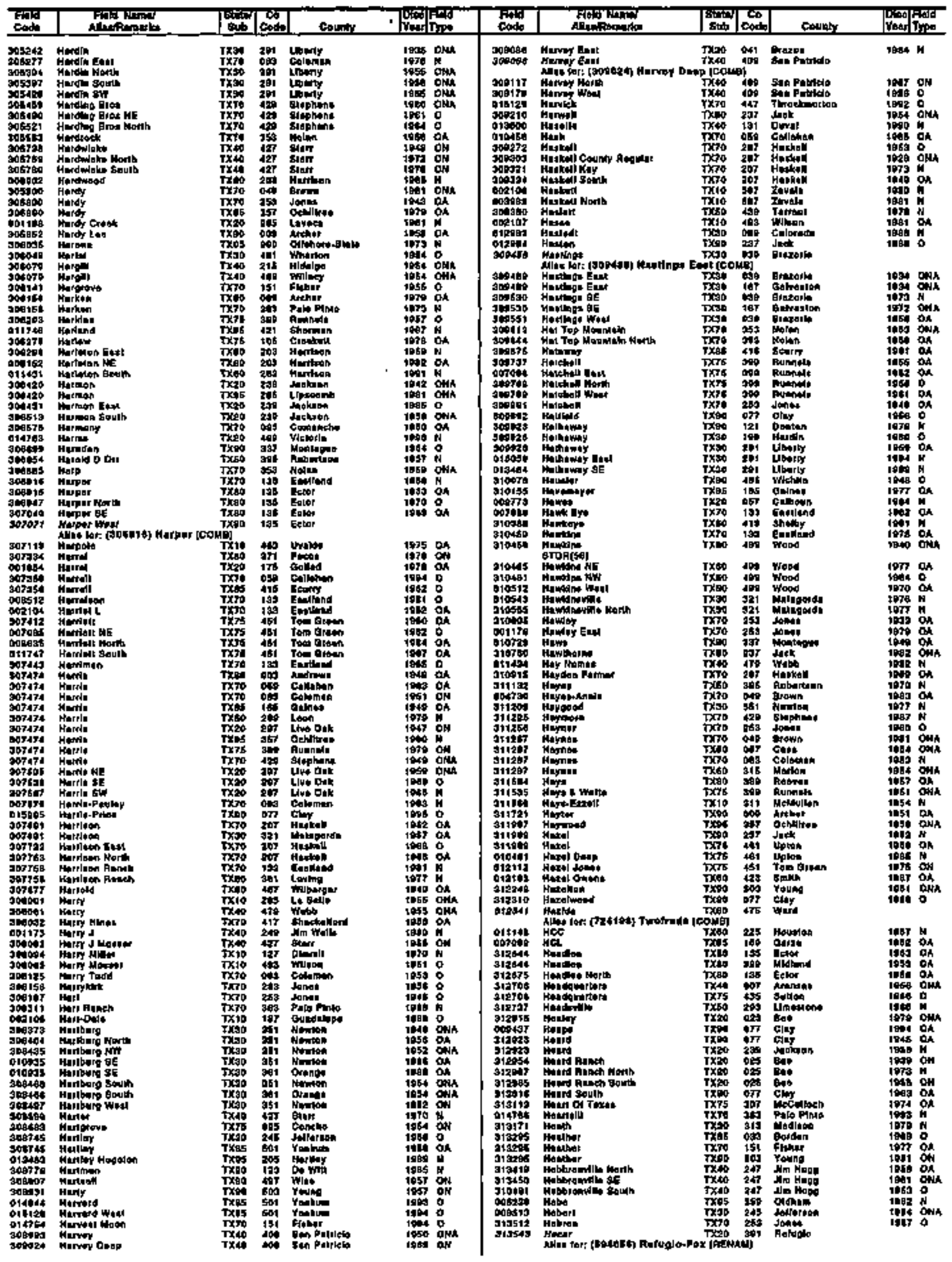


TEXAS

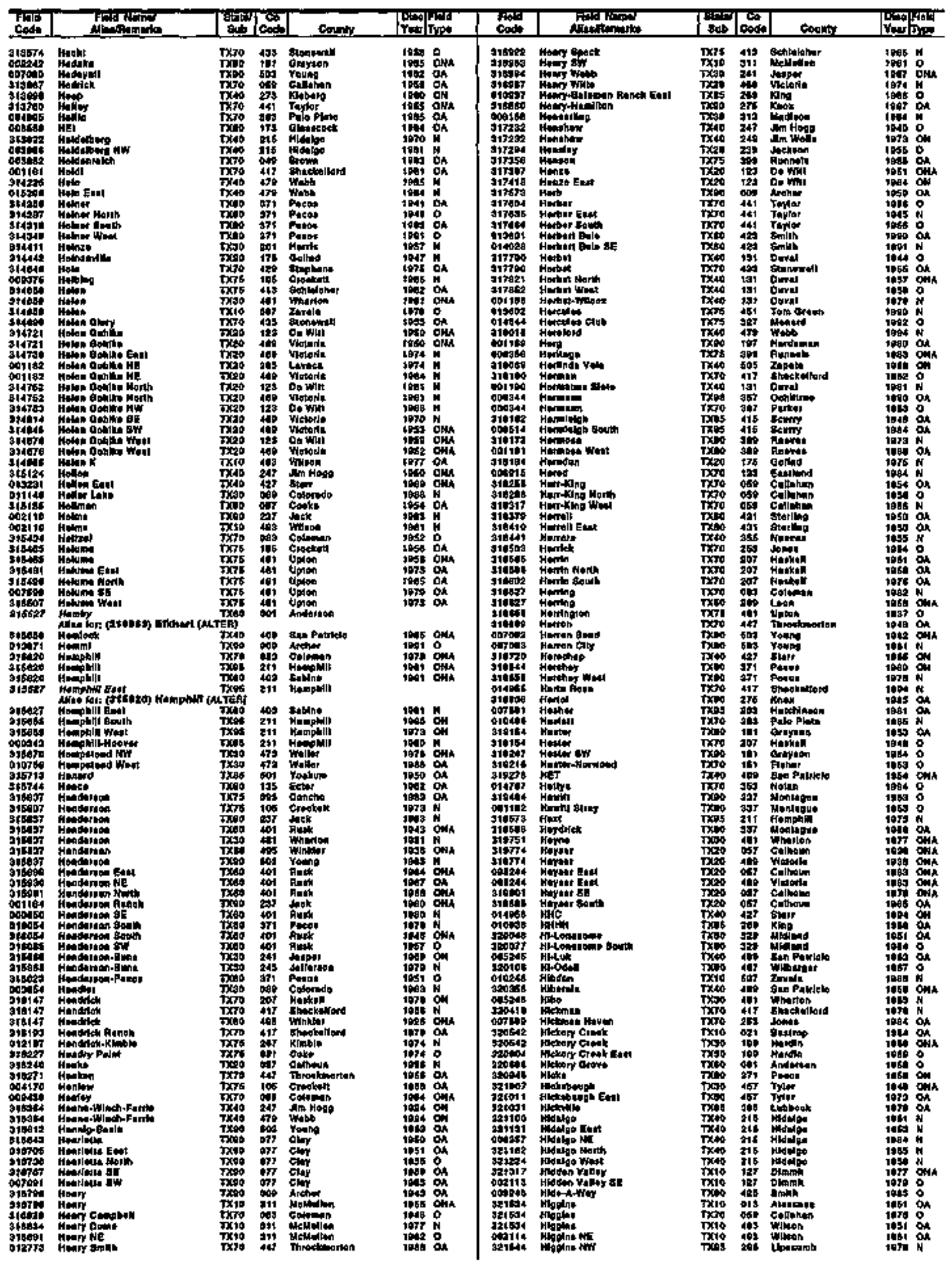


TEXAS

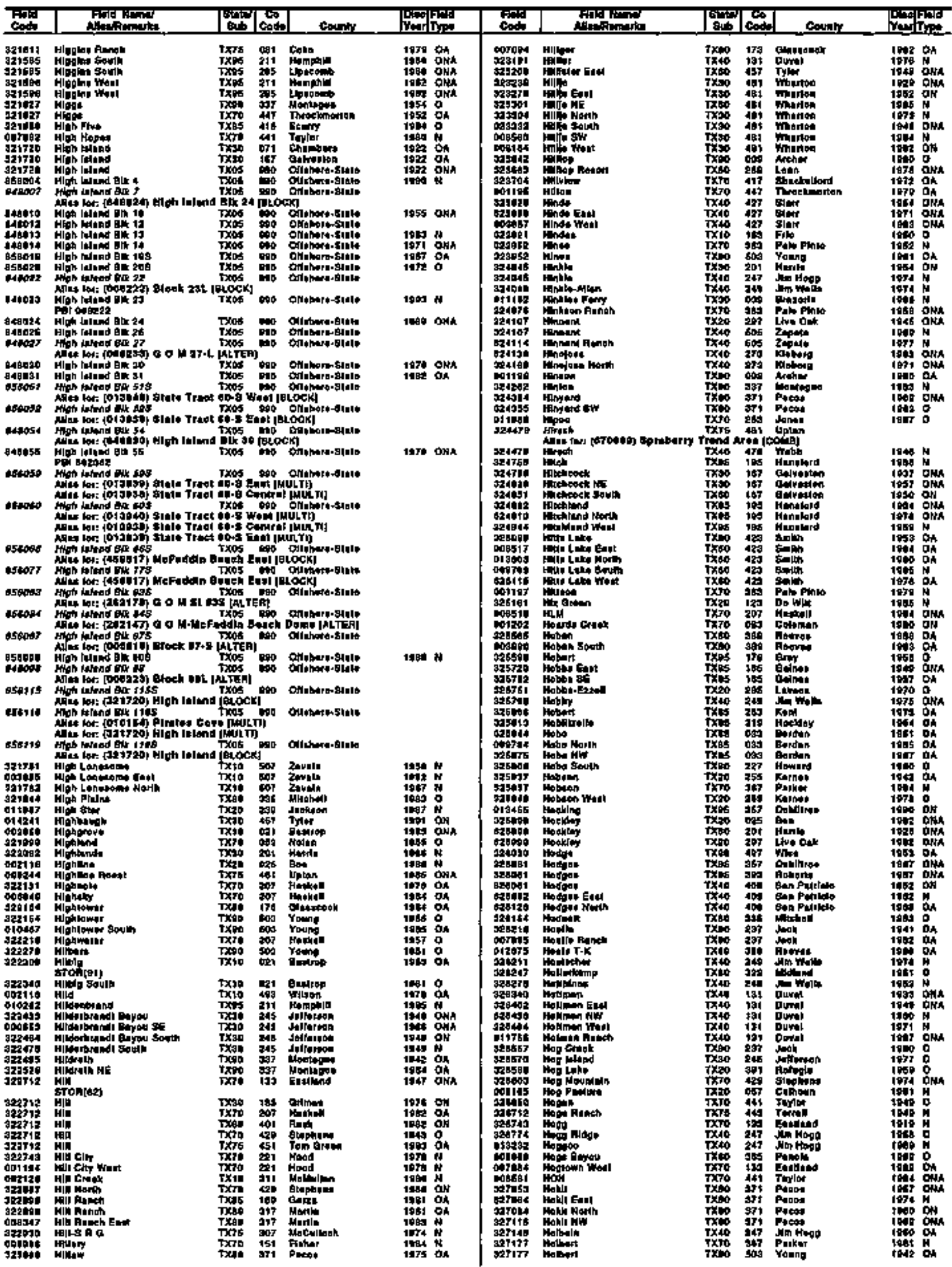


TEXAS

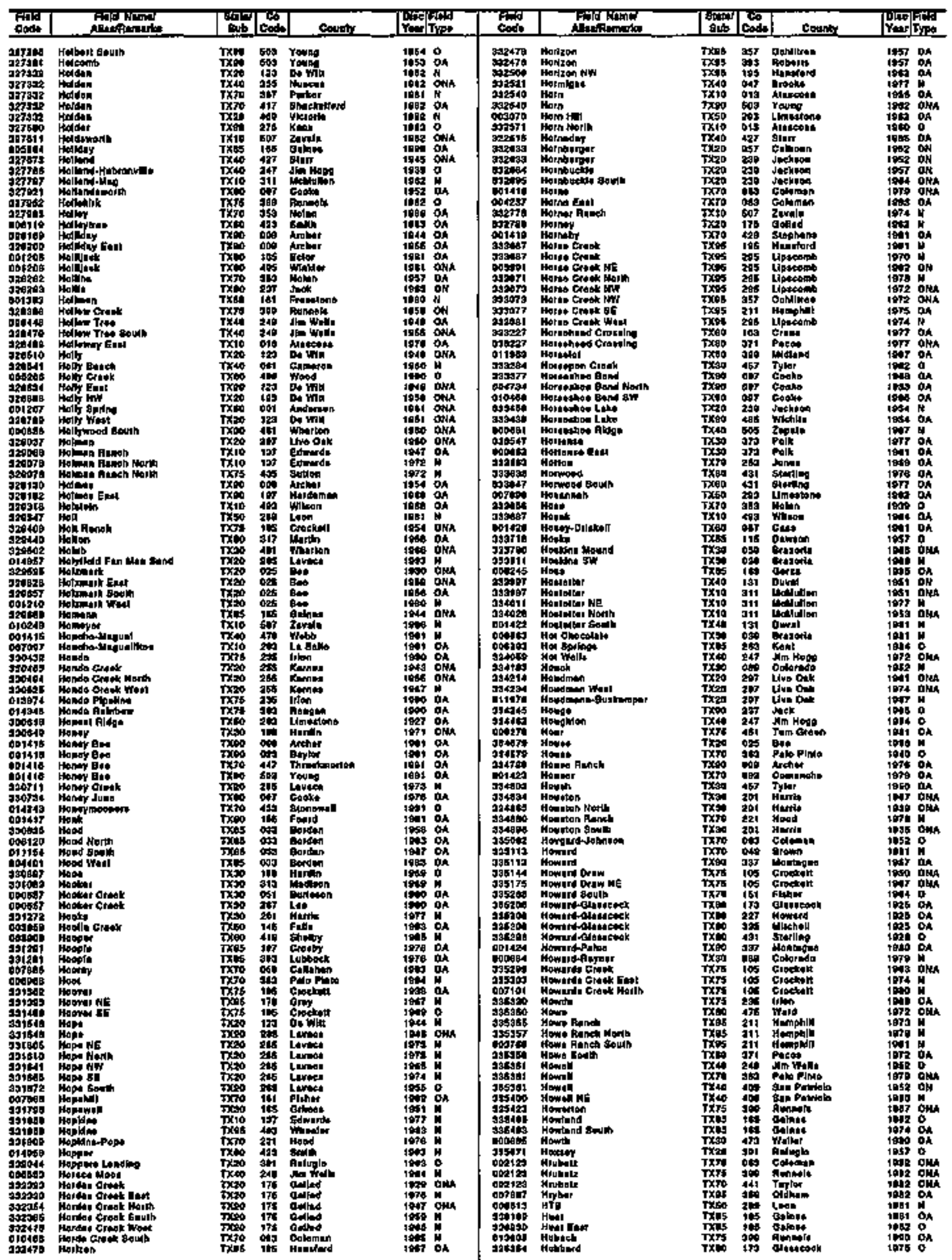


TEXAS

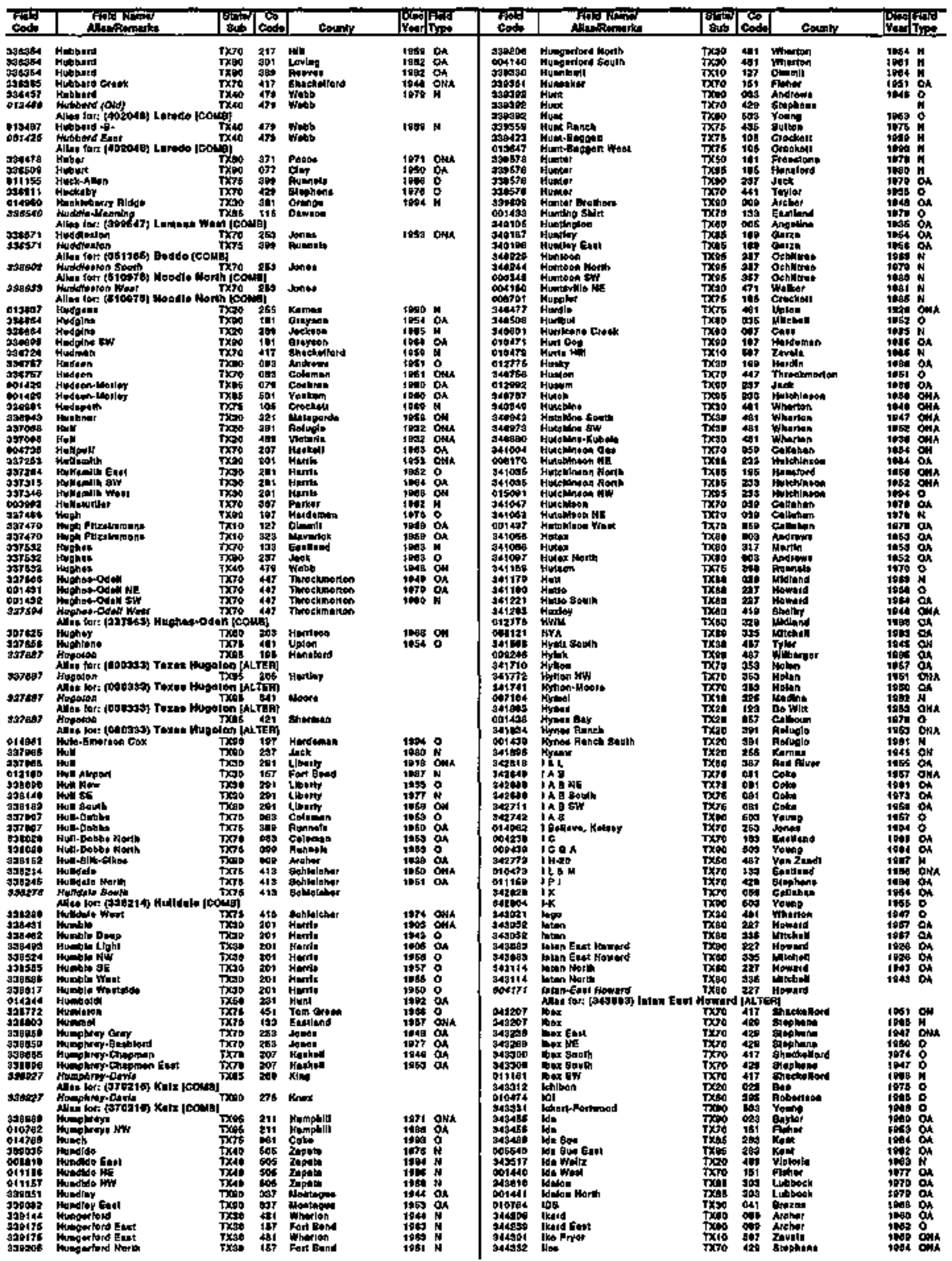


TEXAS

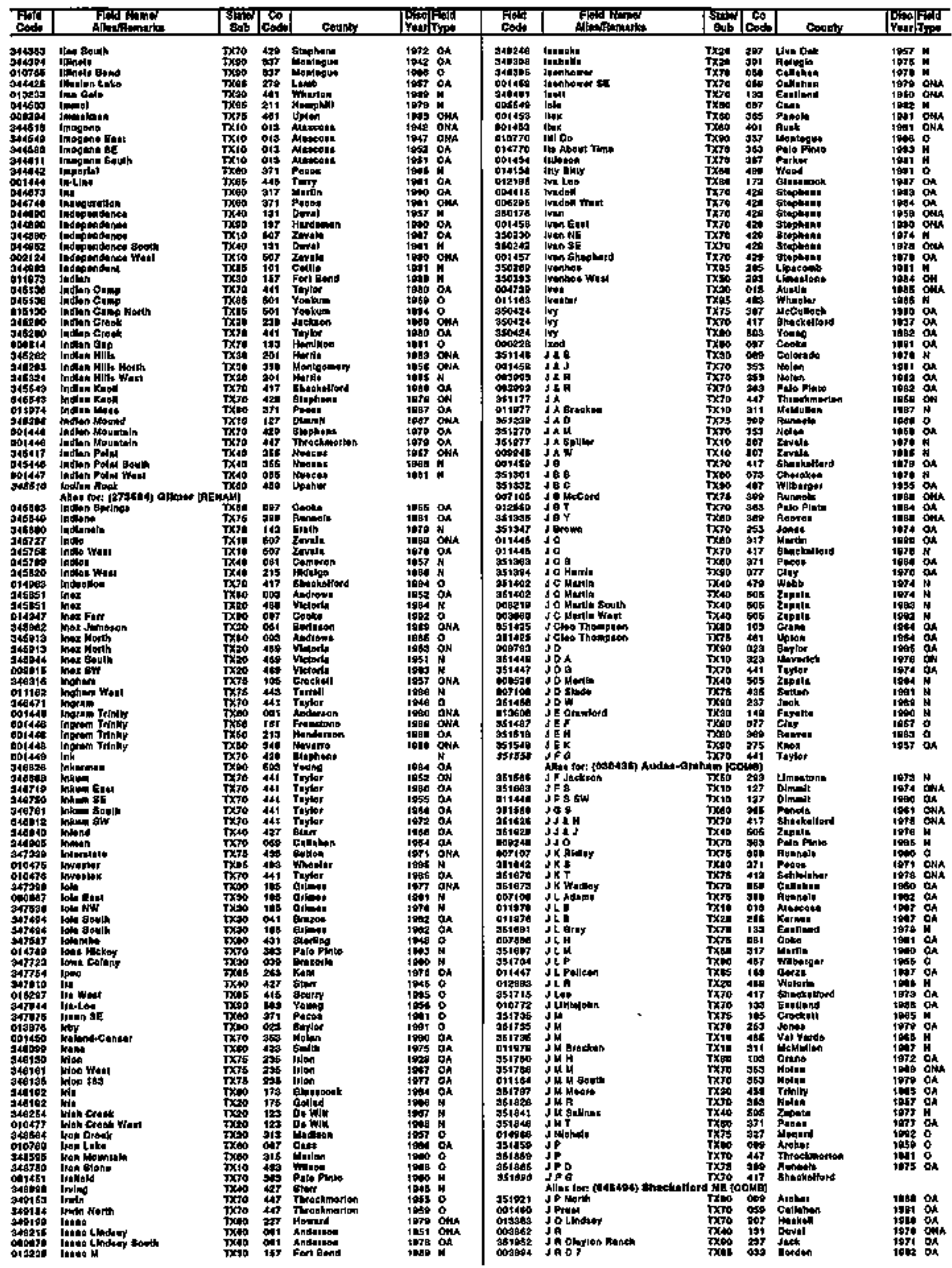




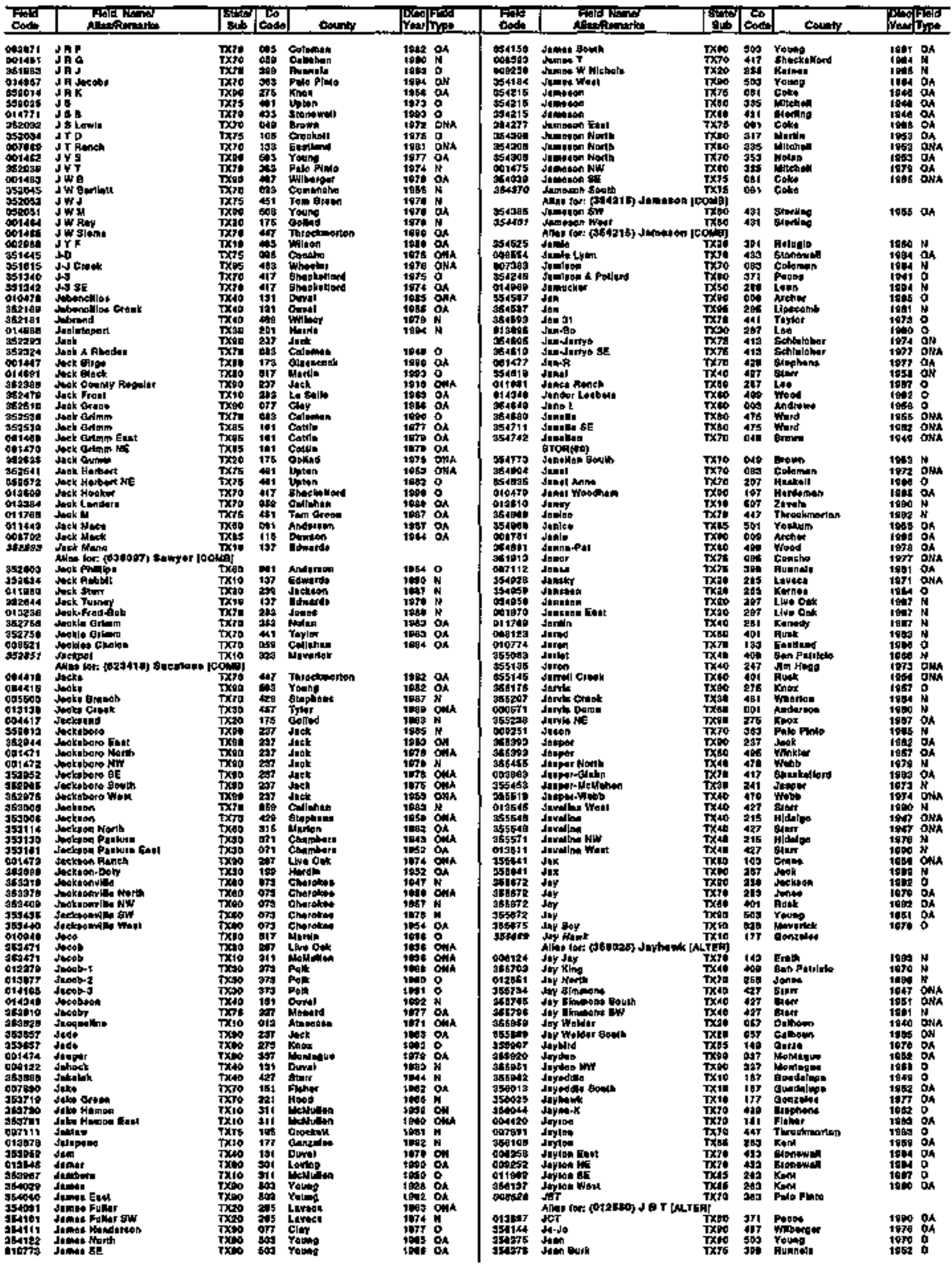


TEXAS

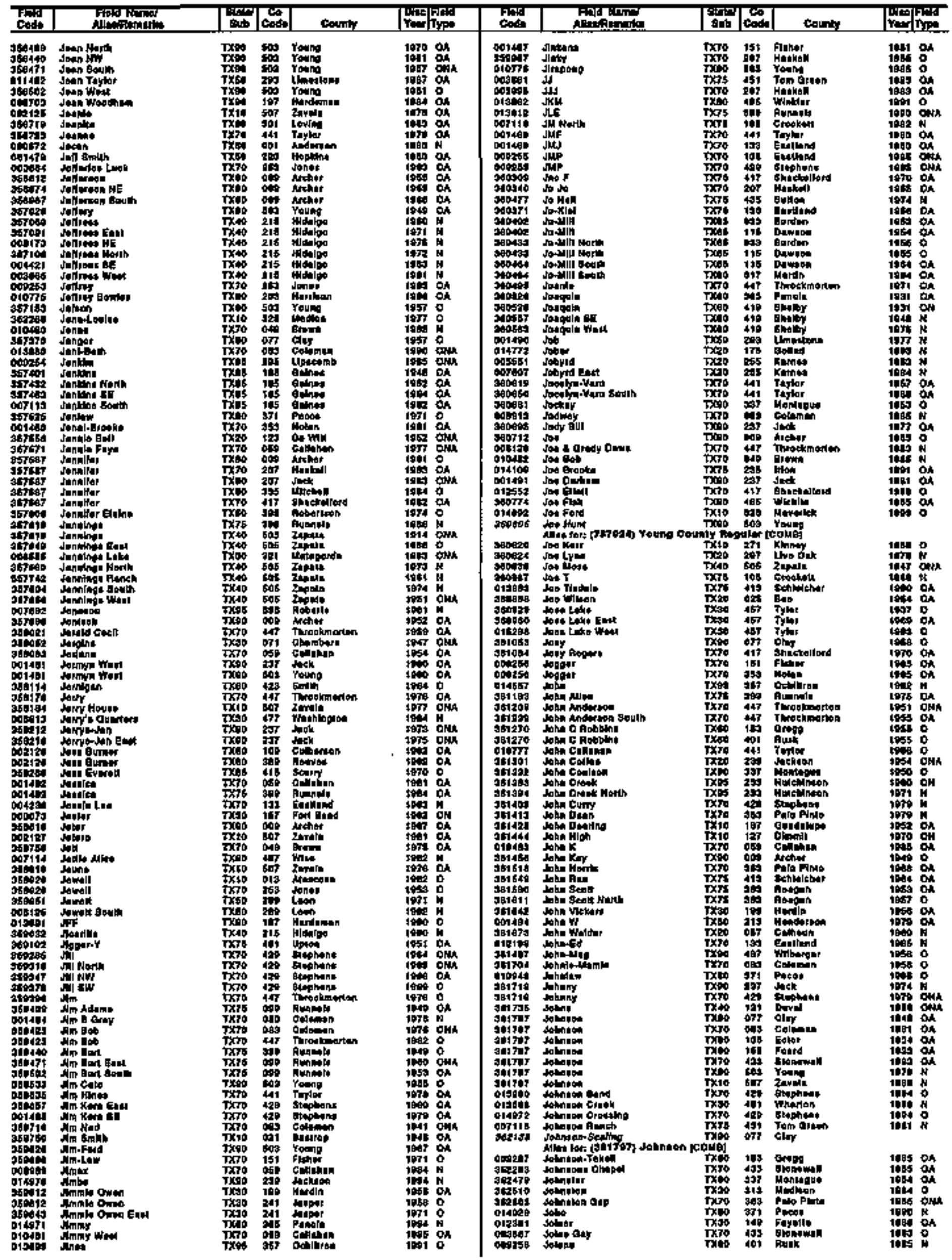




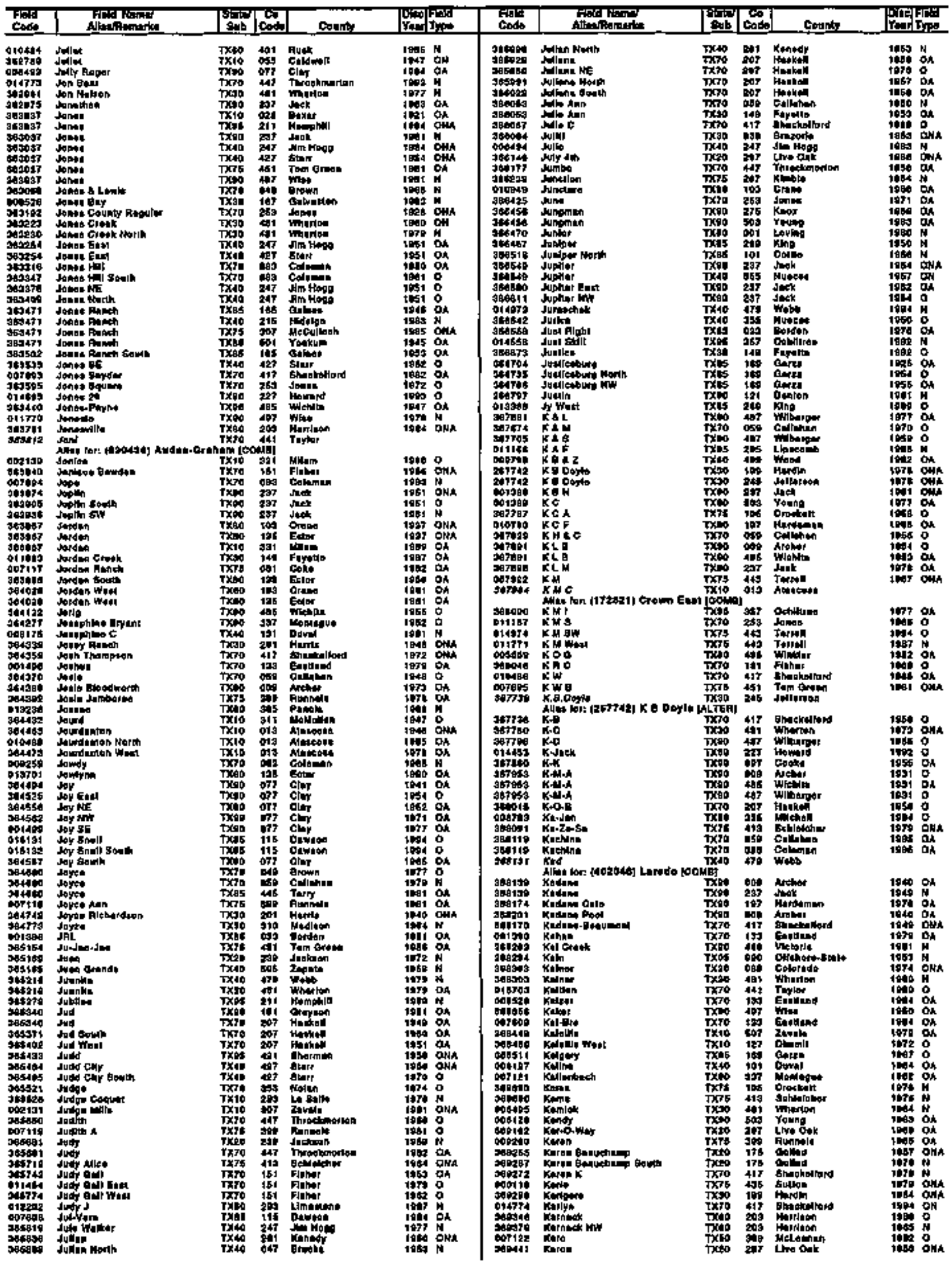


TEXAS

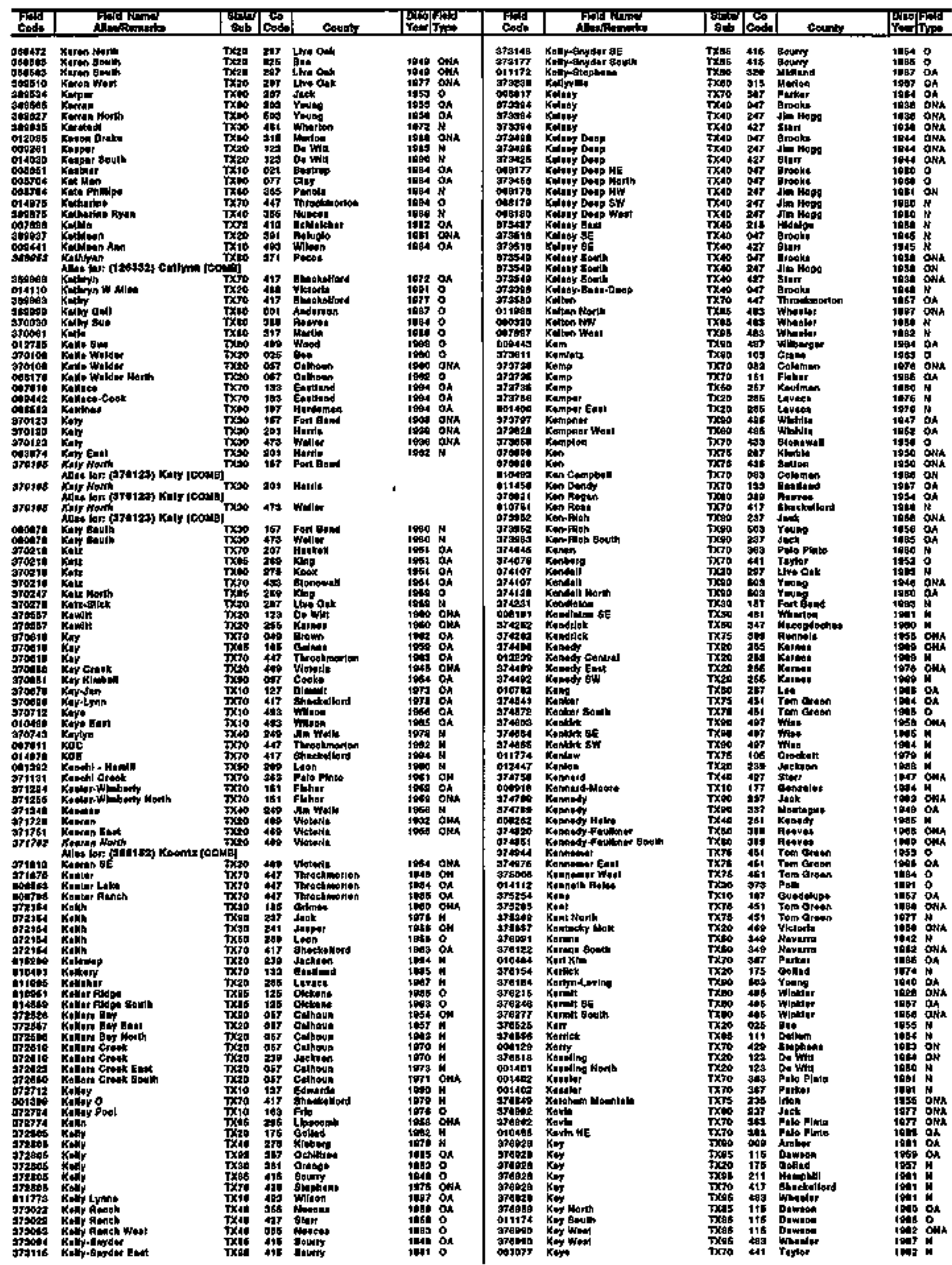




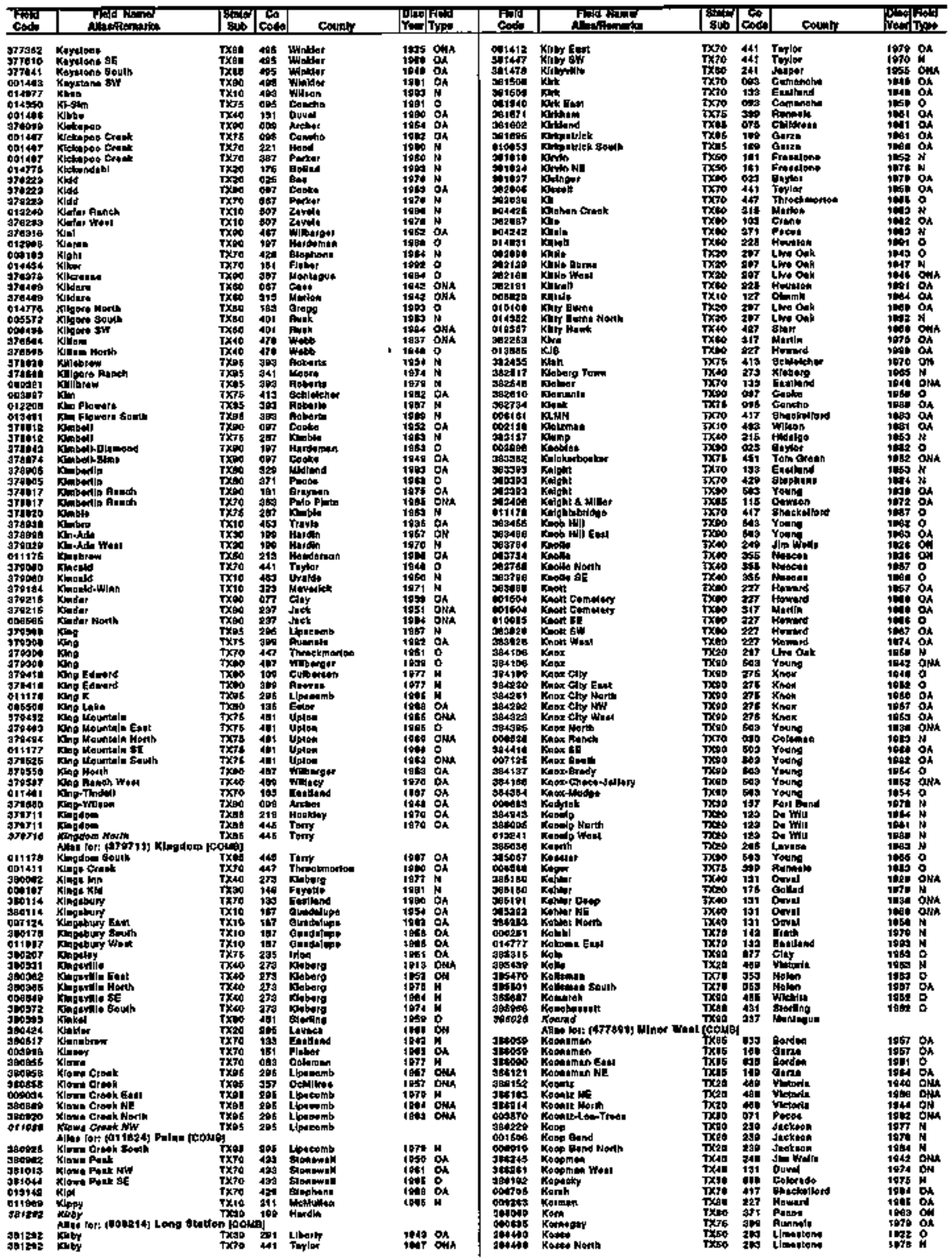


TEXAS

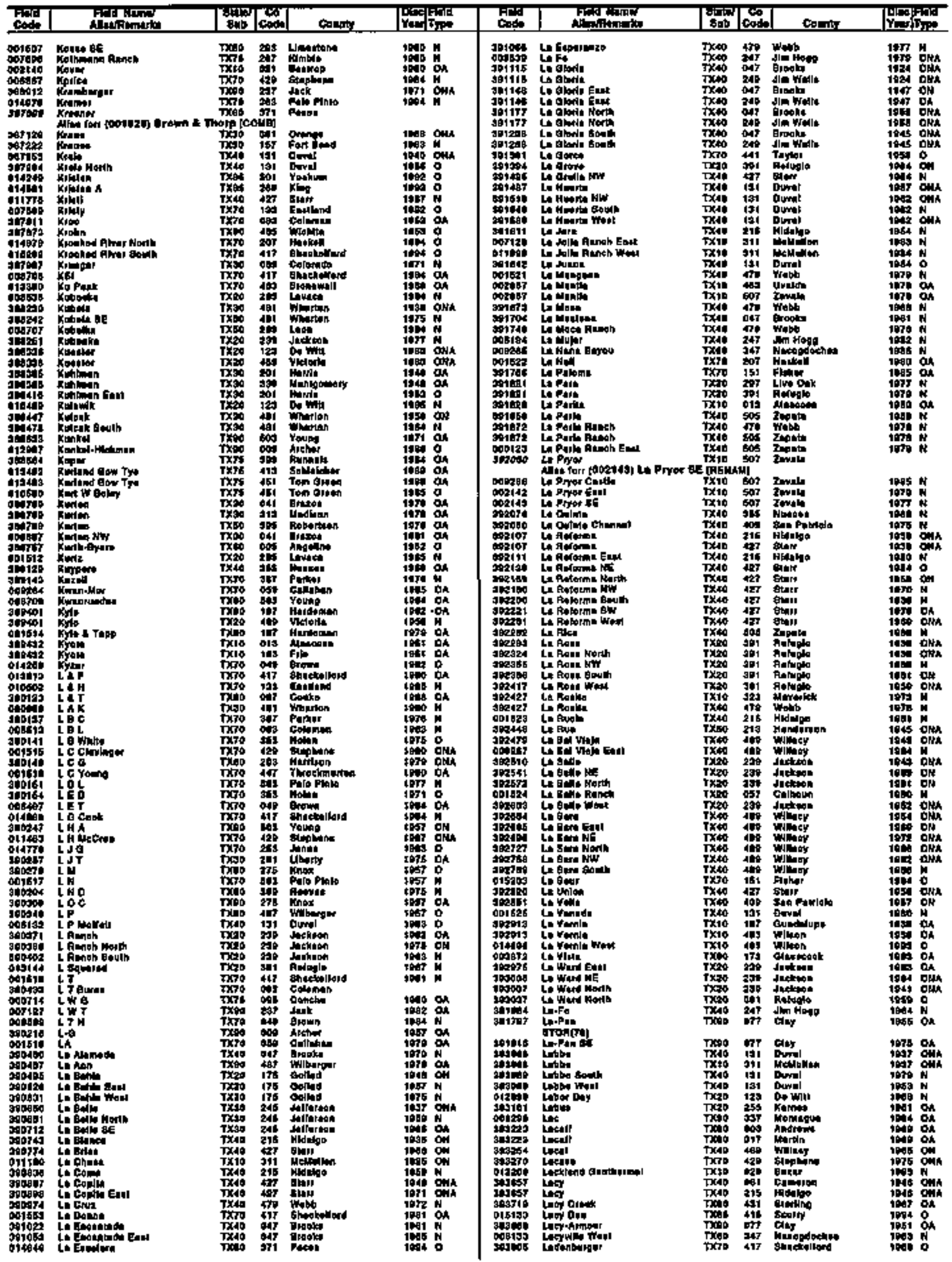


TEXAS

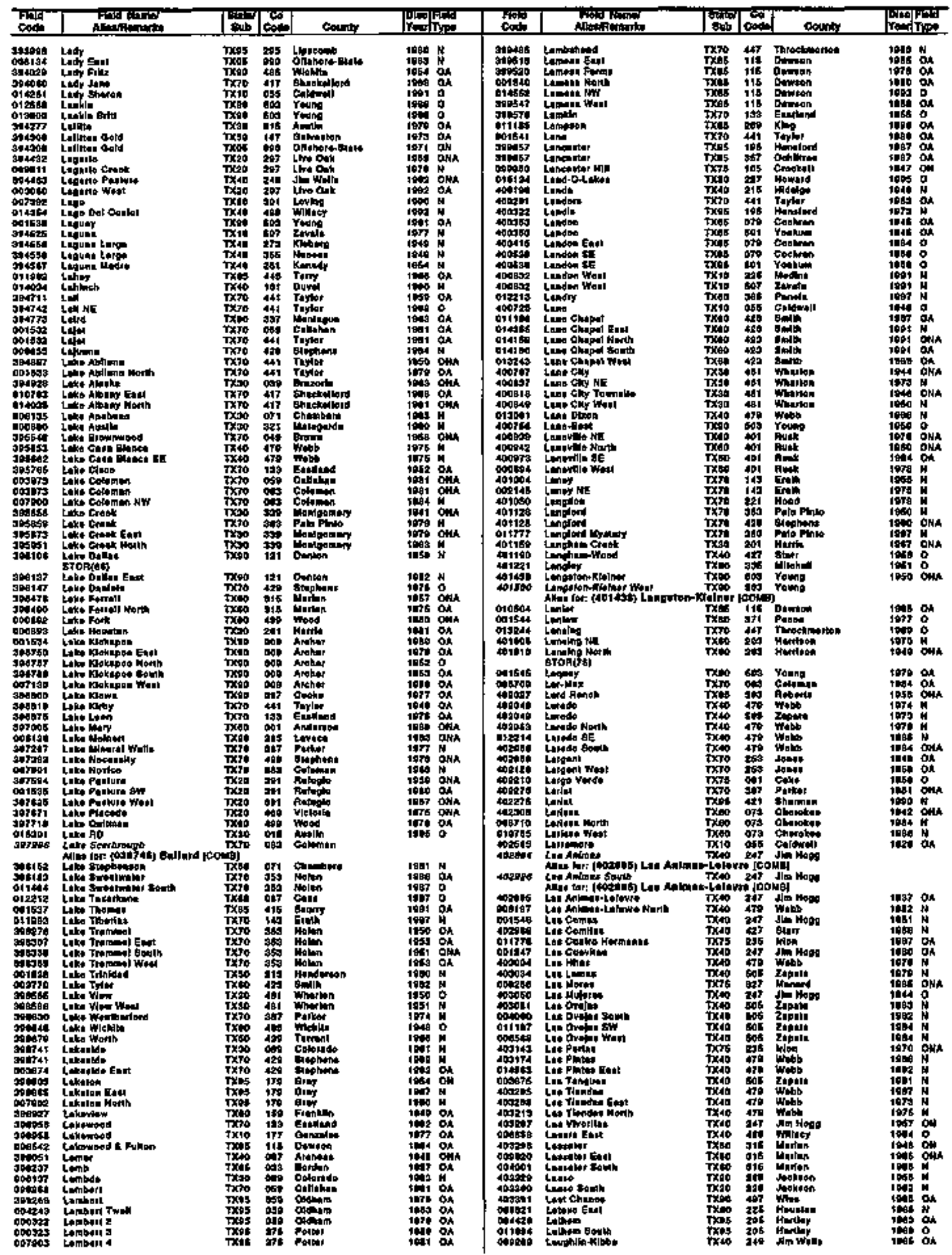


TEXAS

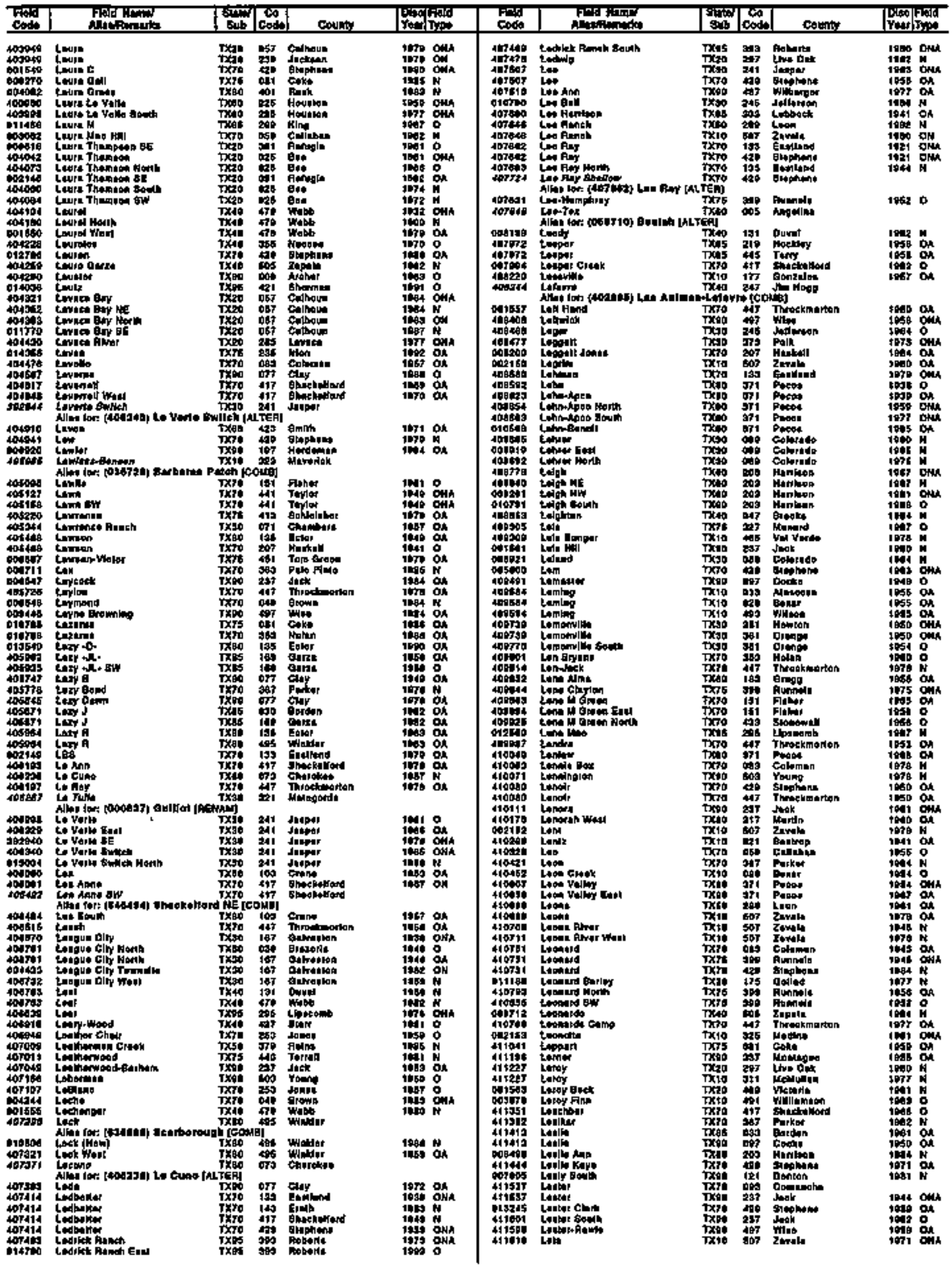


TEXAS

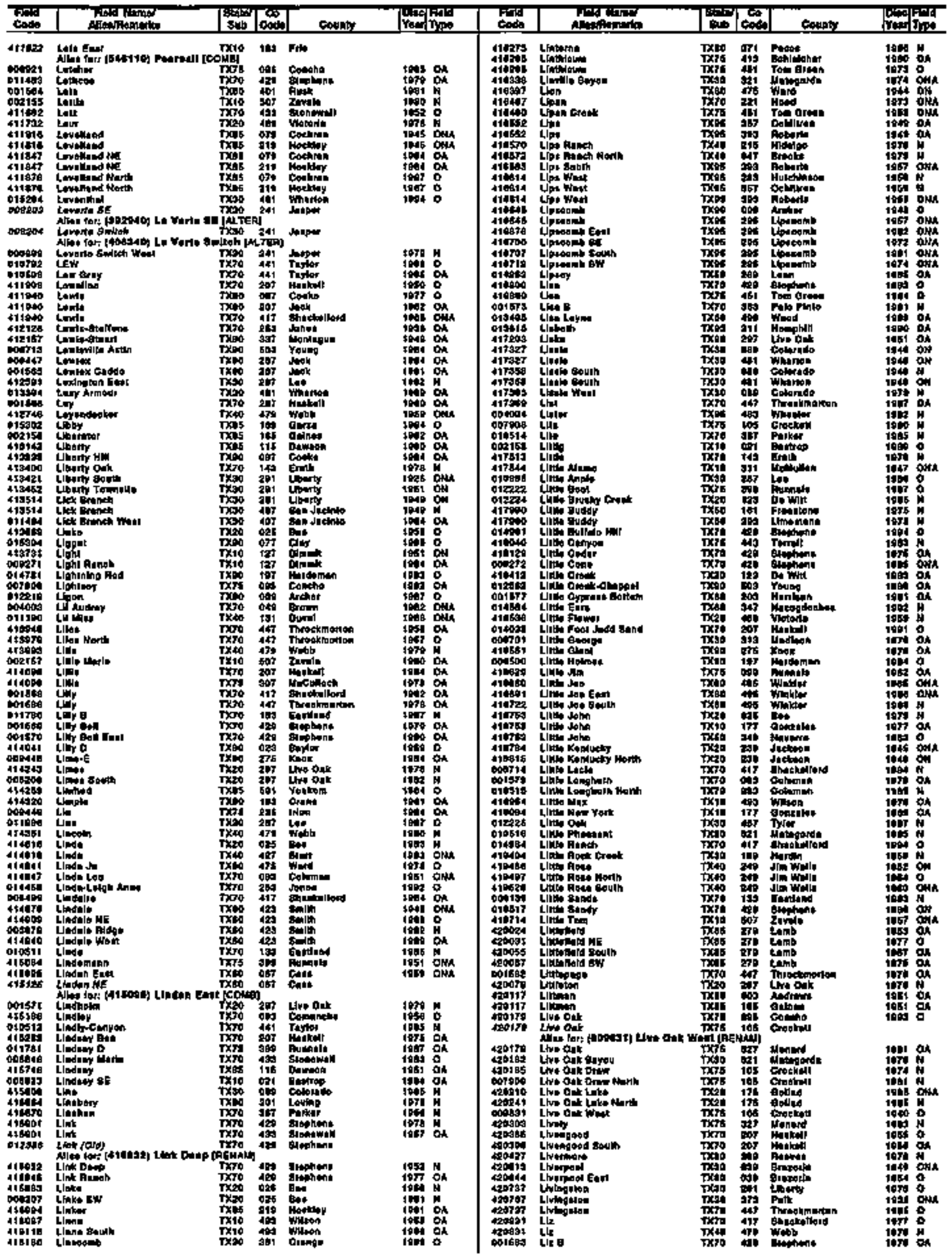


TEXAS

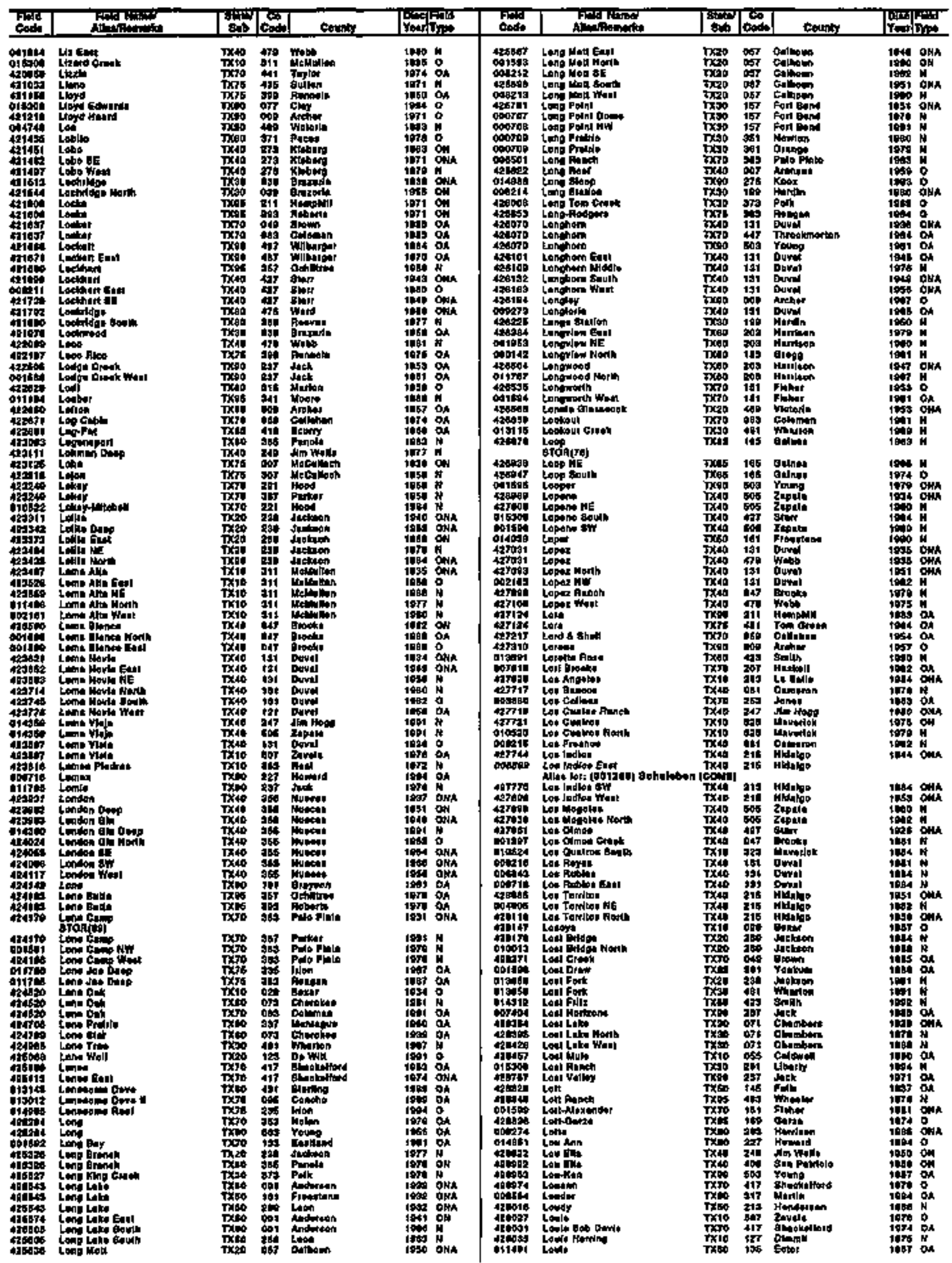




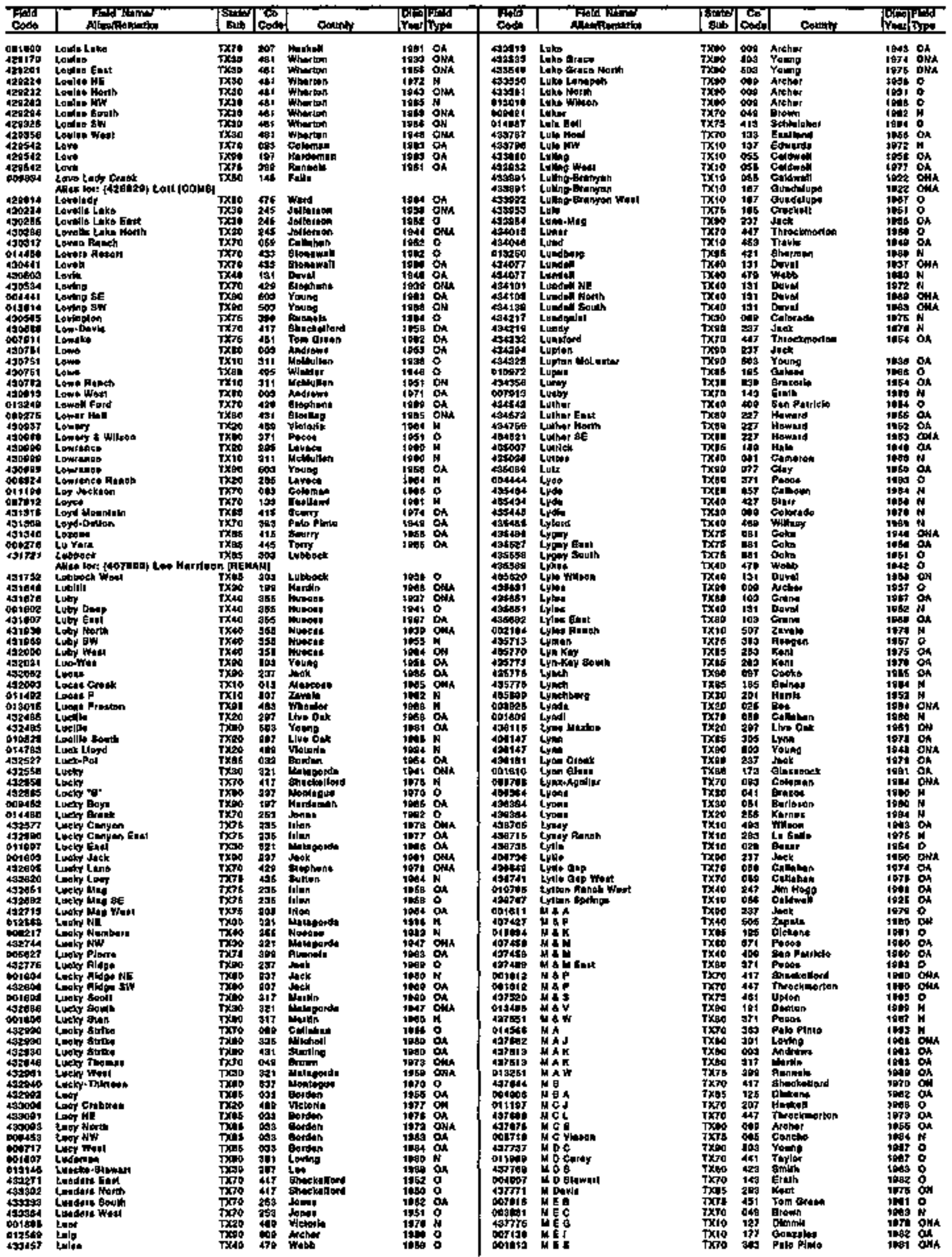


TEXA\&

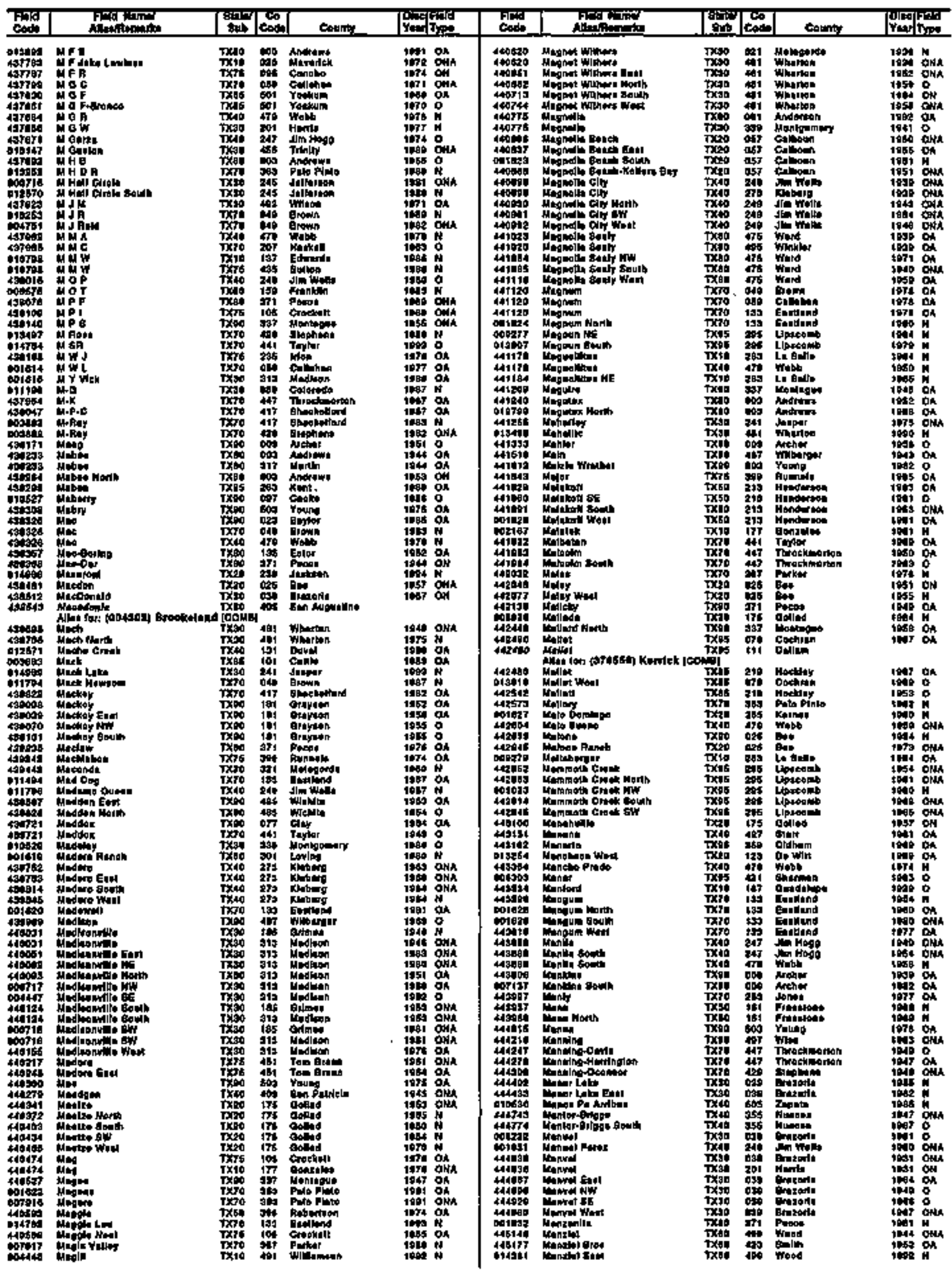




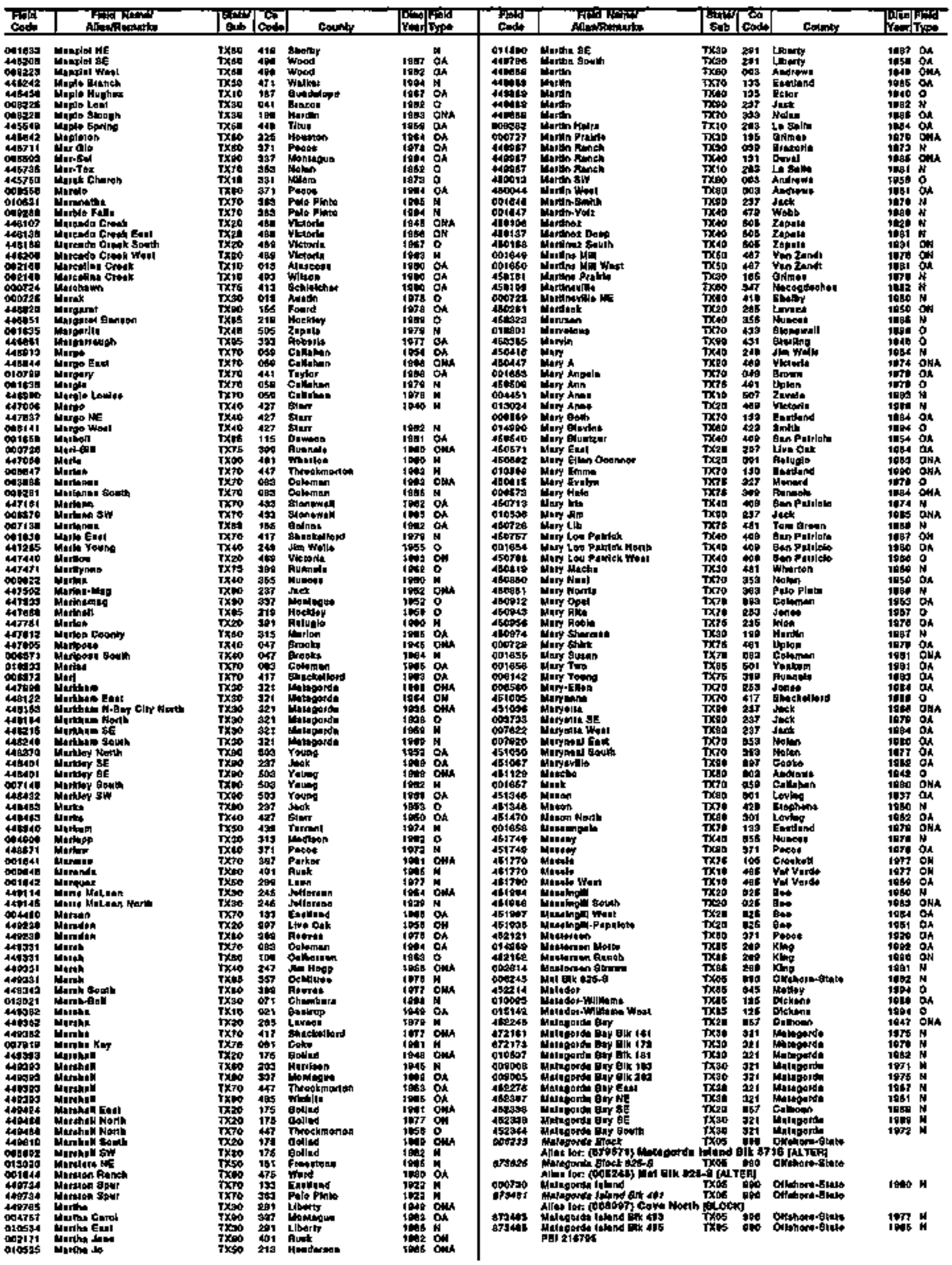


TEXAS

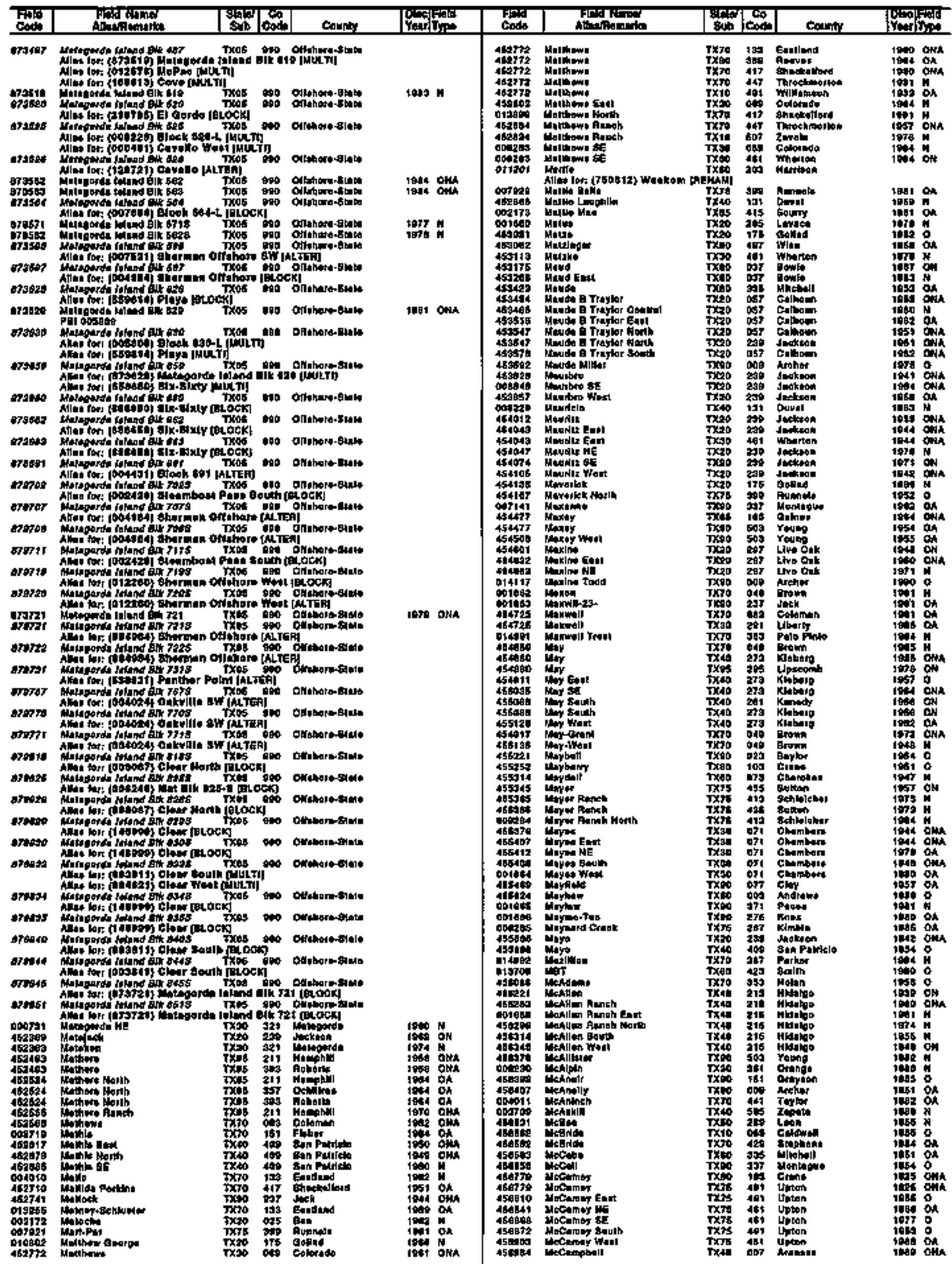




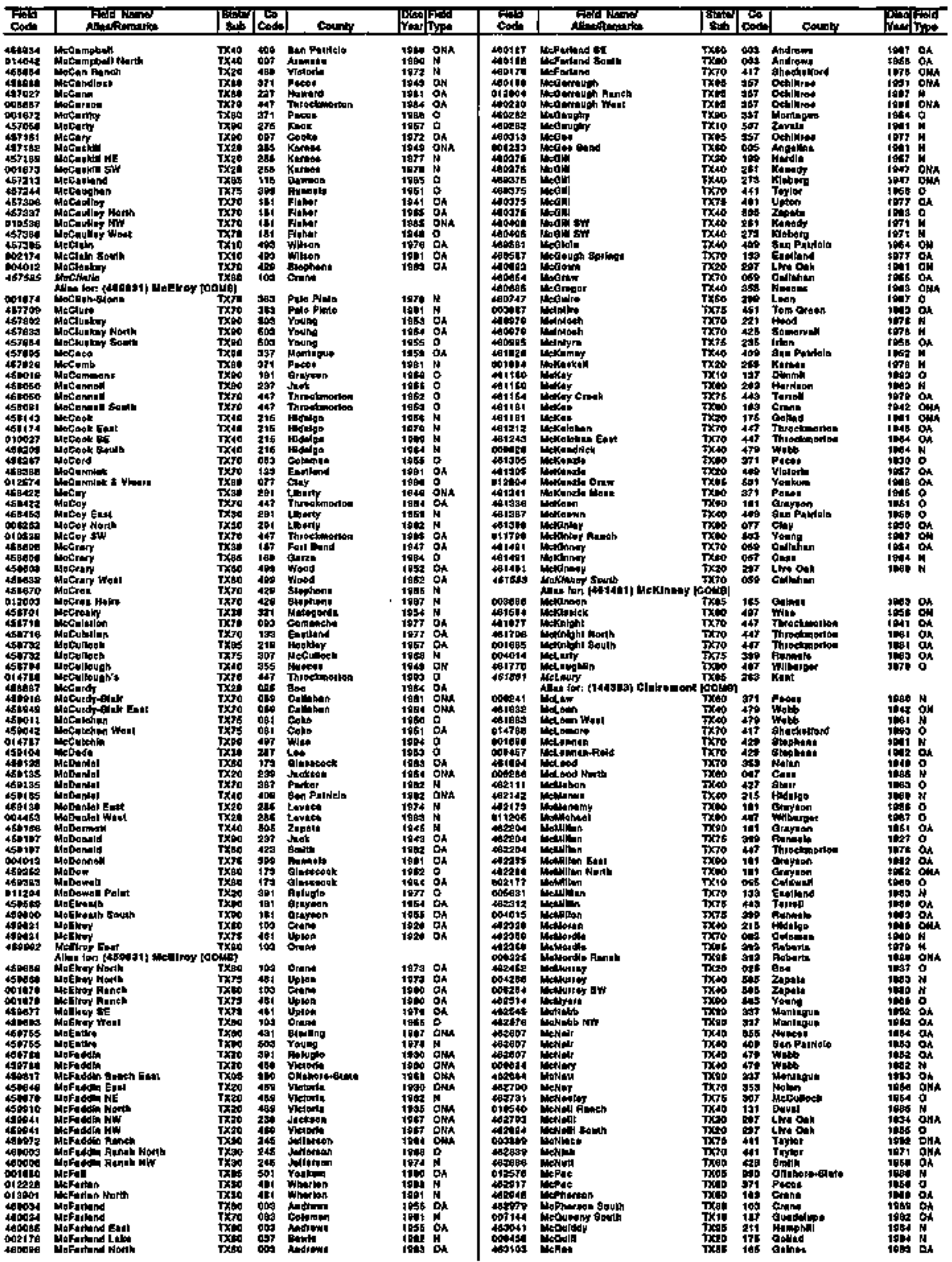


TEXAS

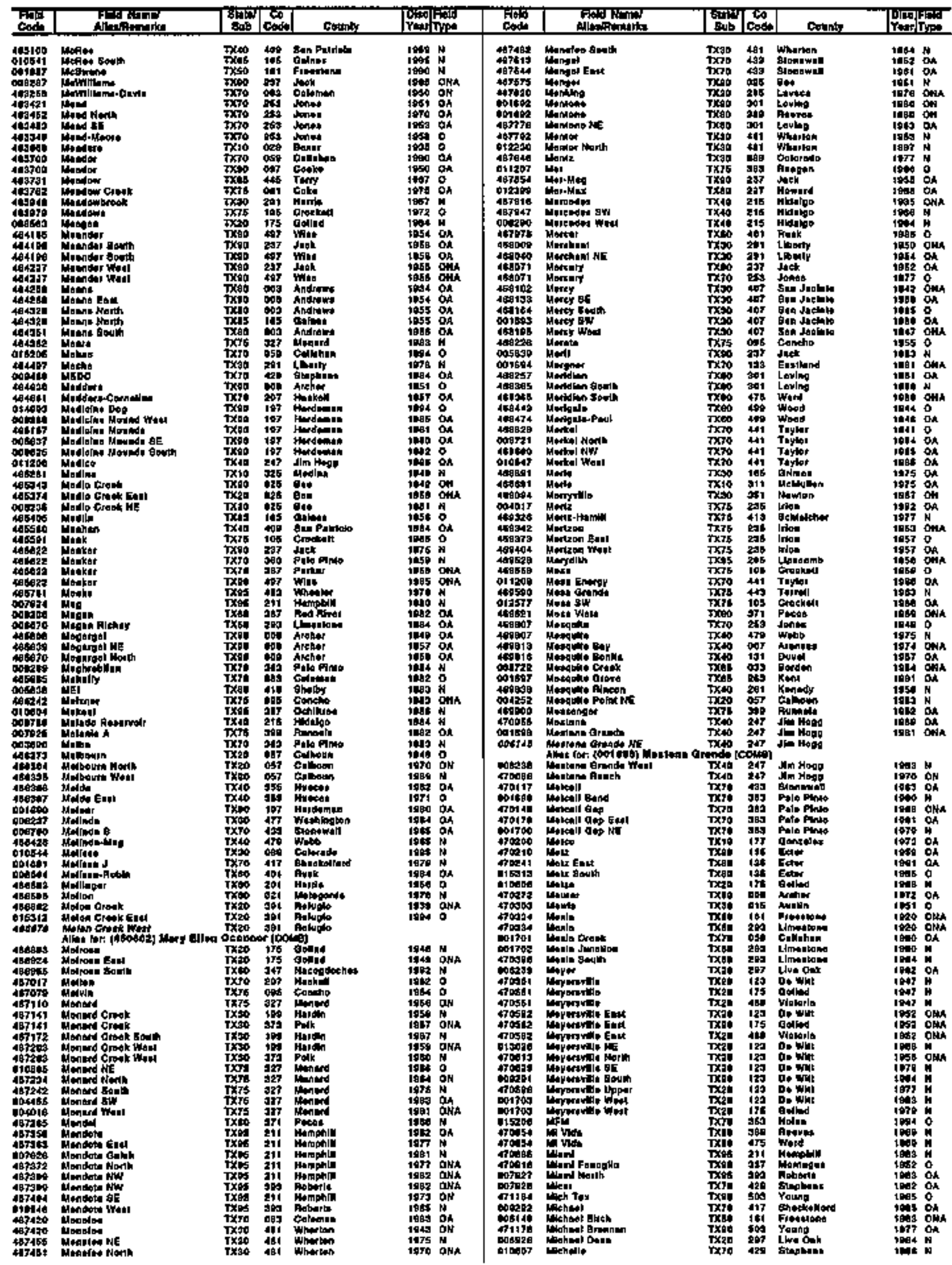


TEXA 8

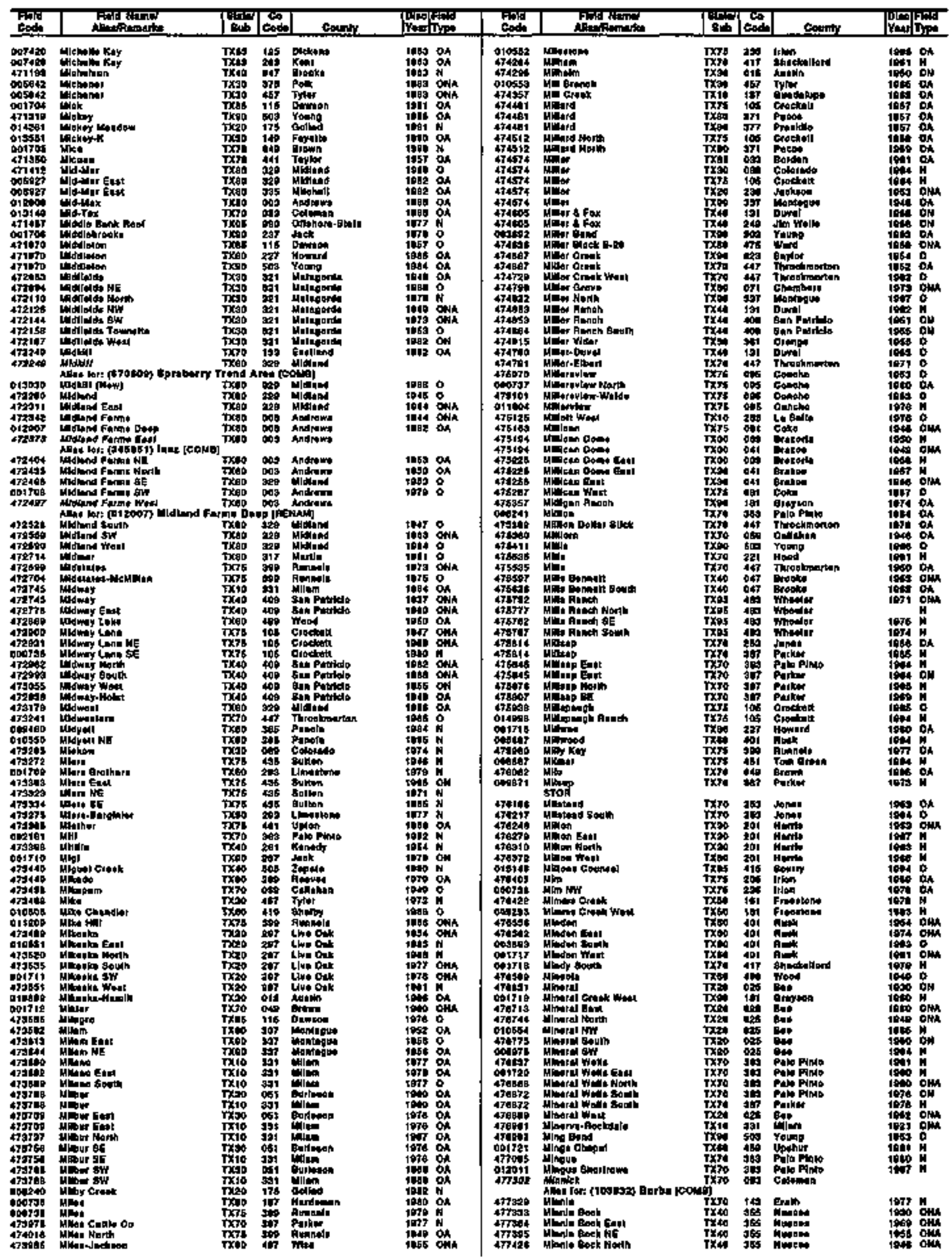


TEXAS

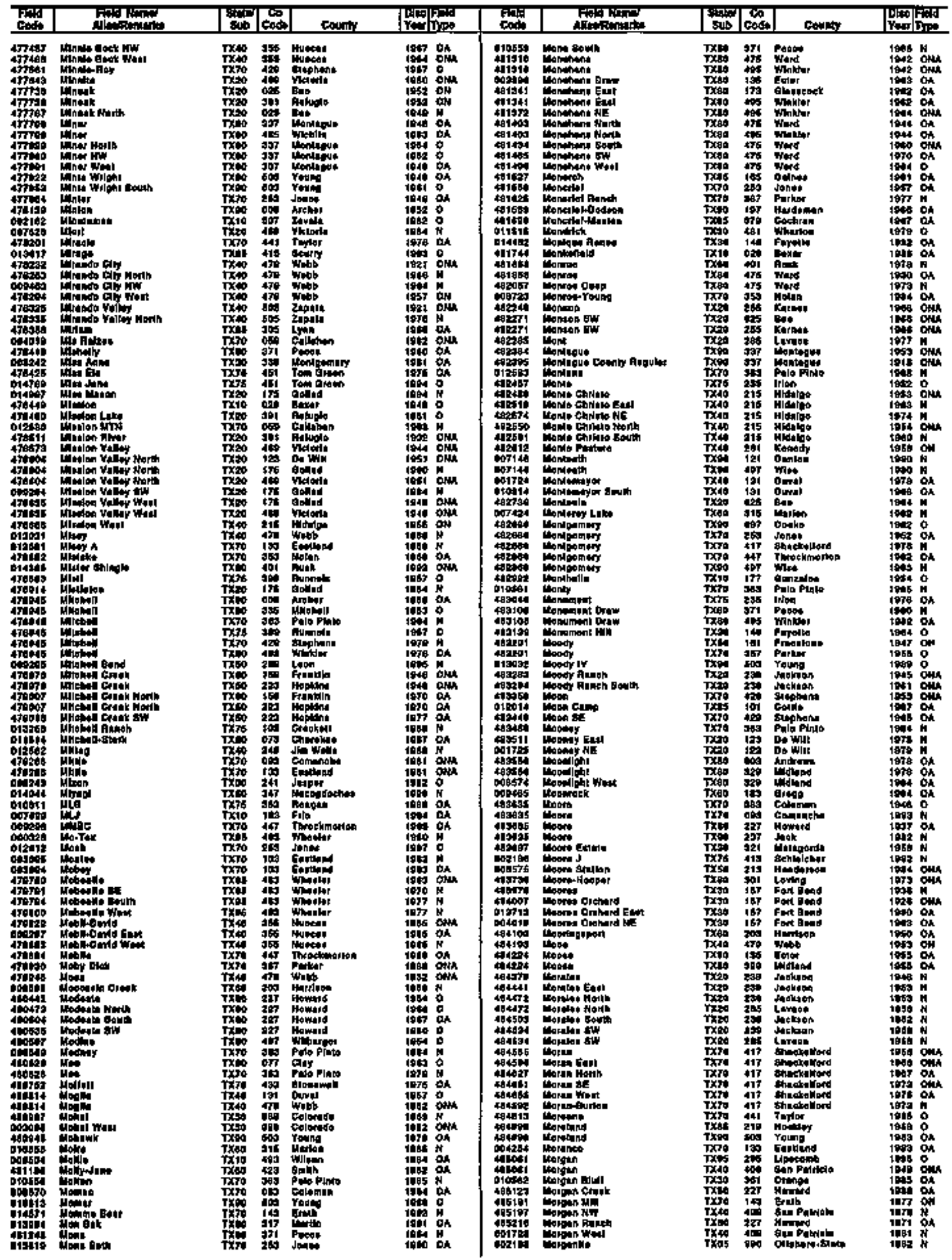


TEXAS

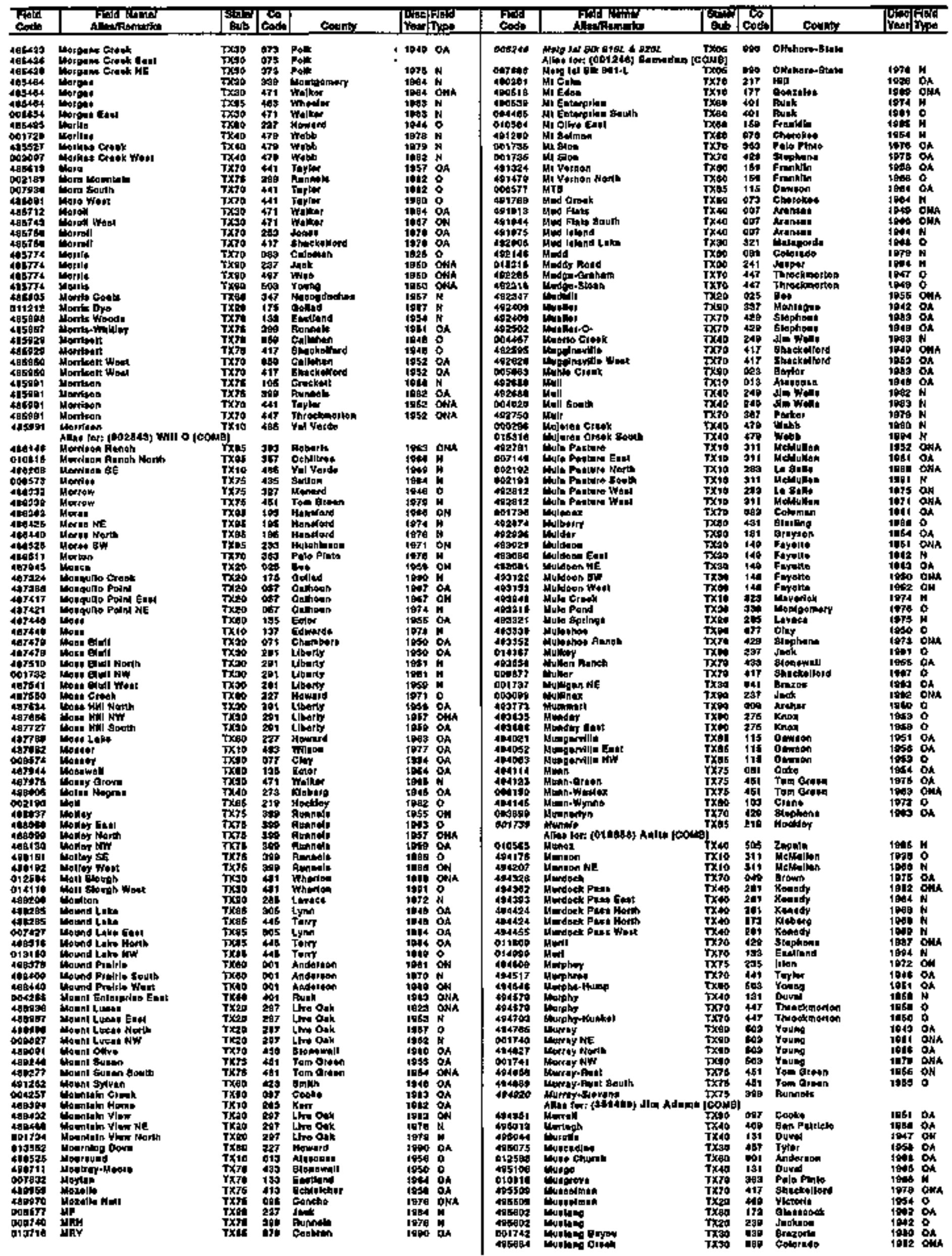


TEXA $\$$

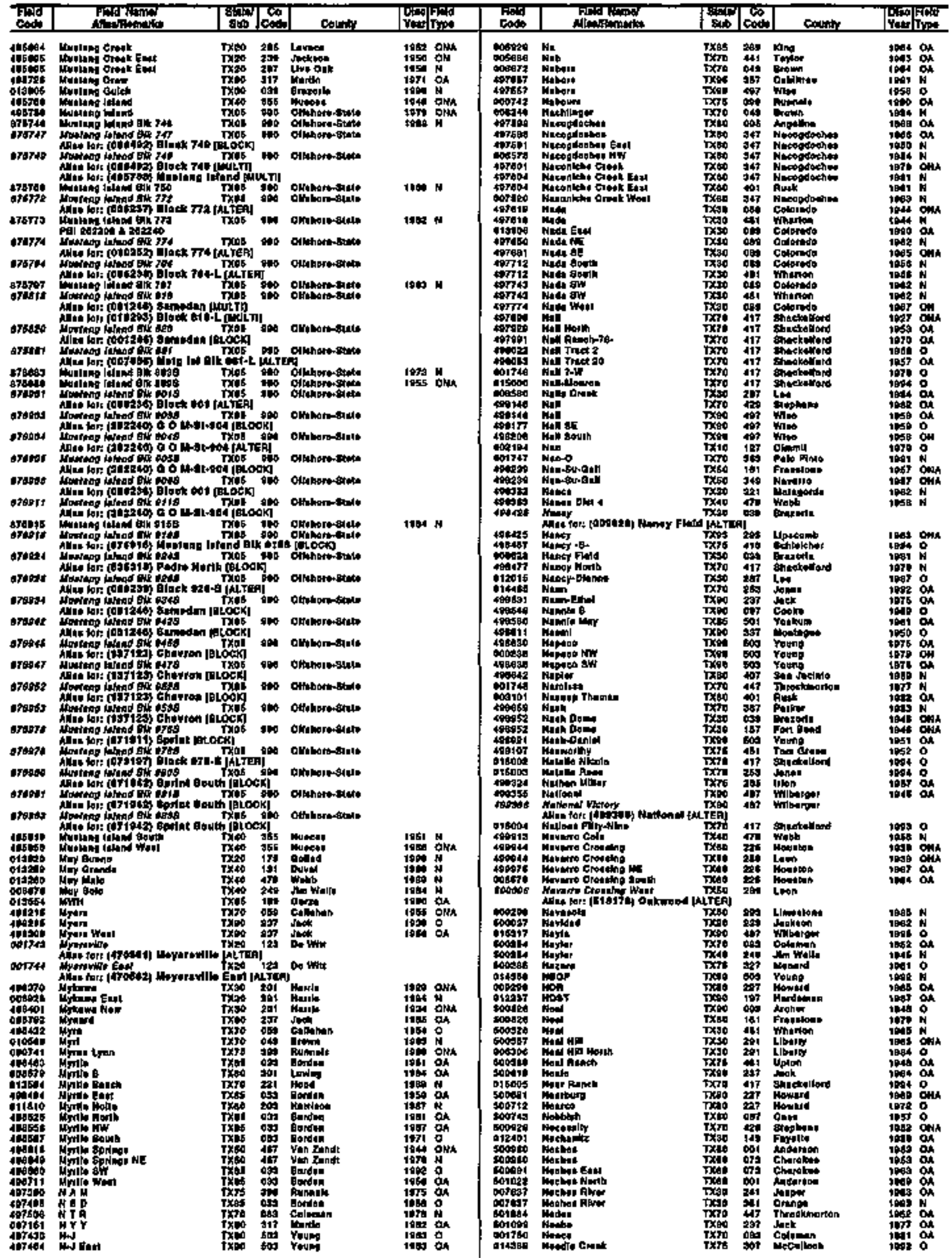


TEXAS

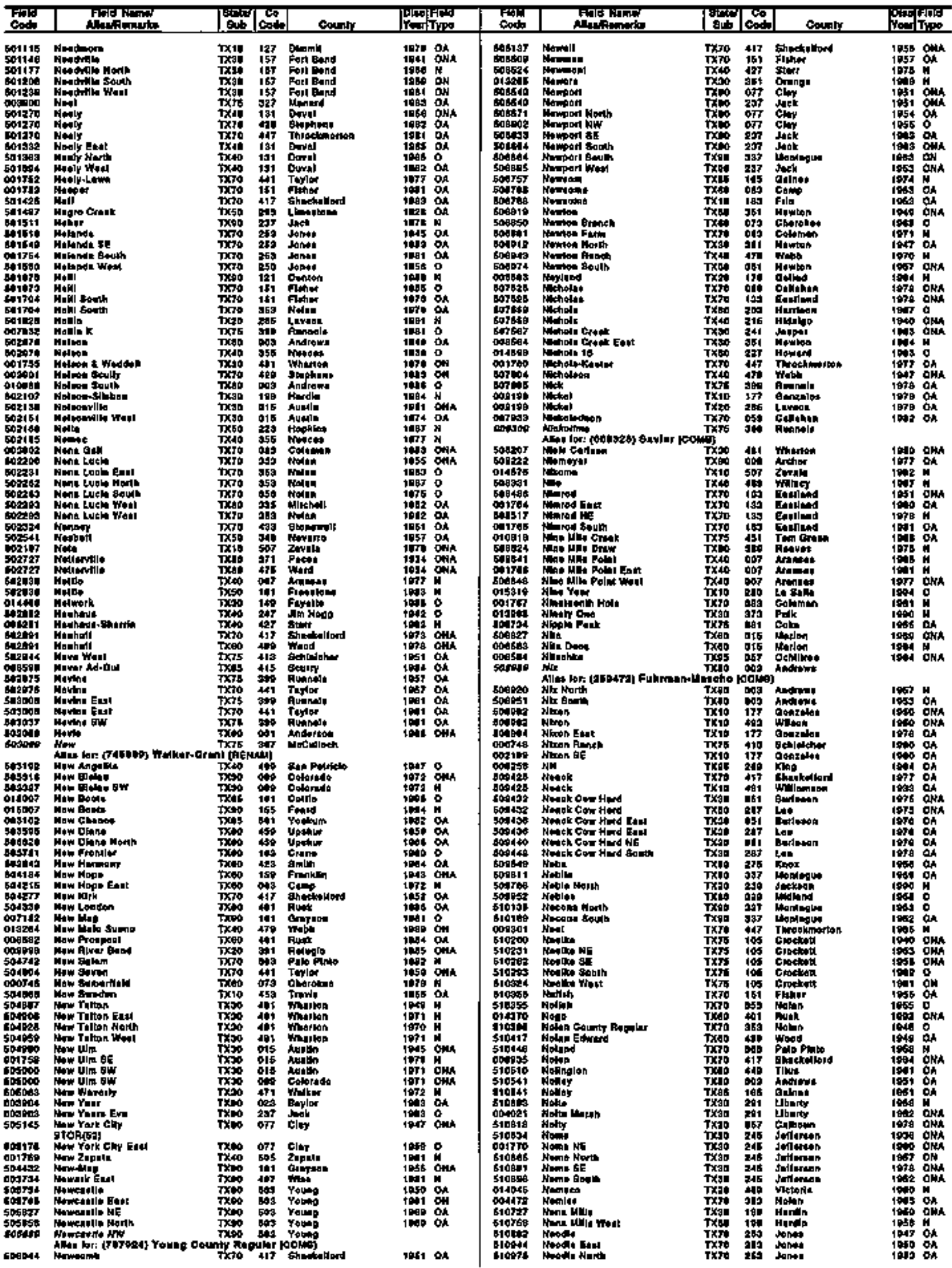


TEXAS

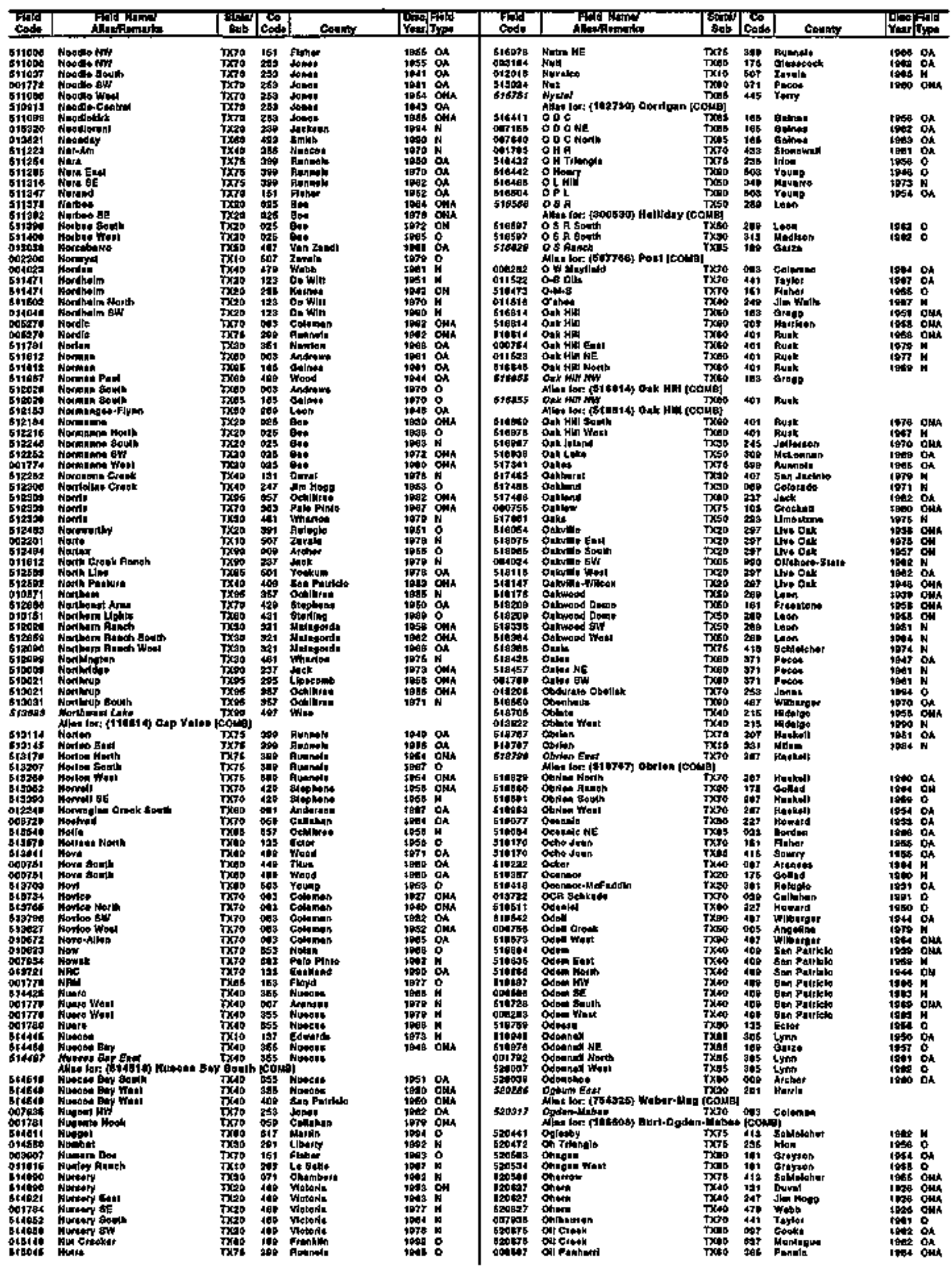




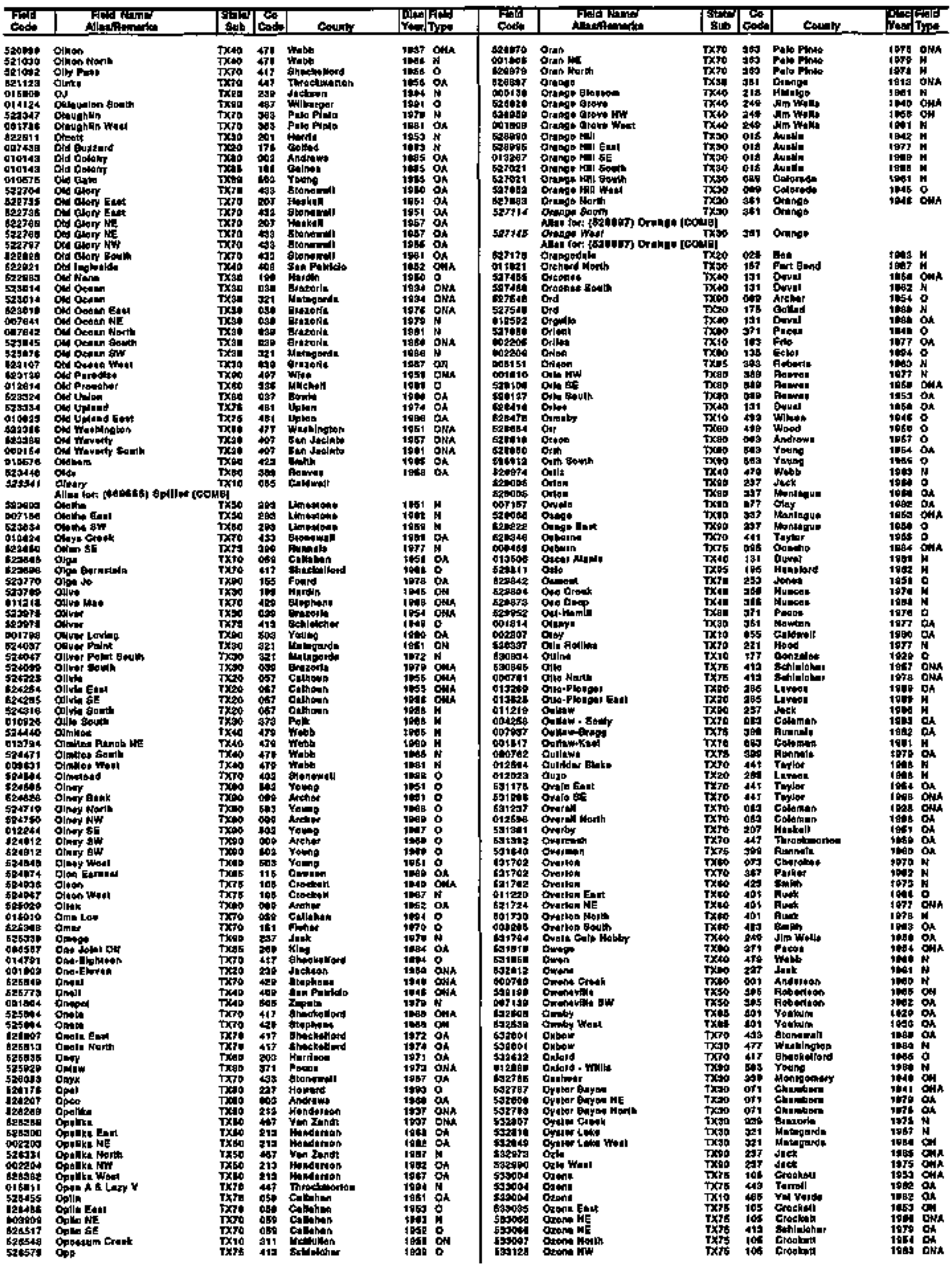


TEXAS

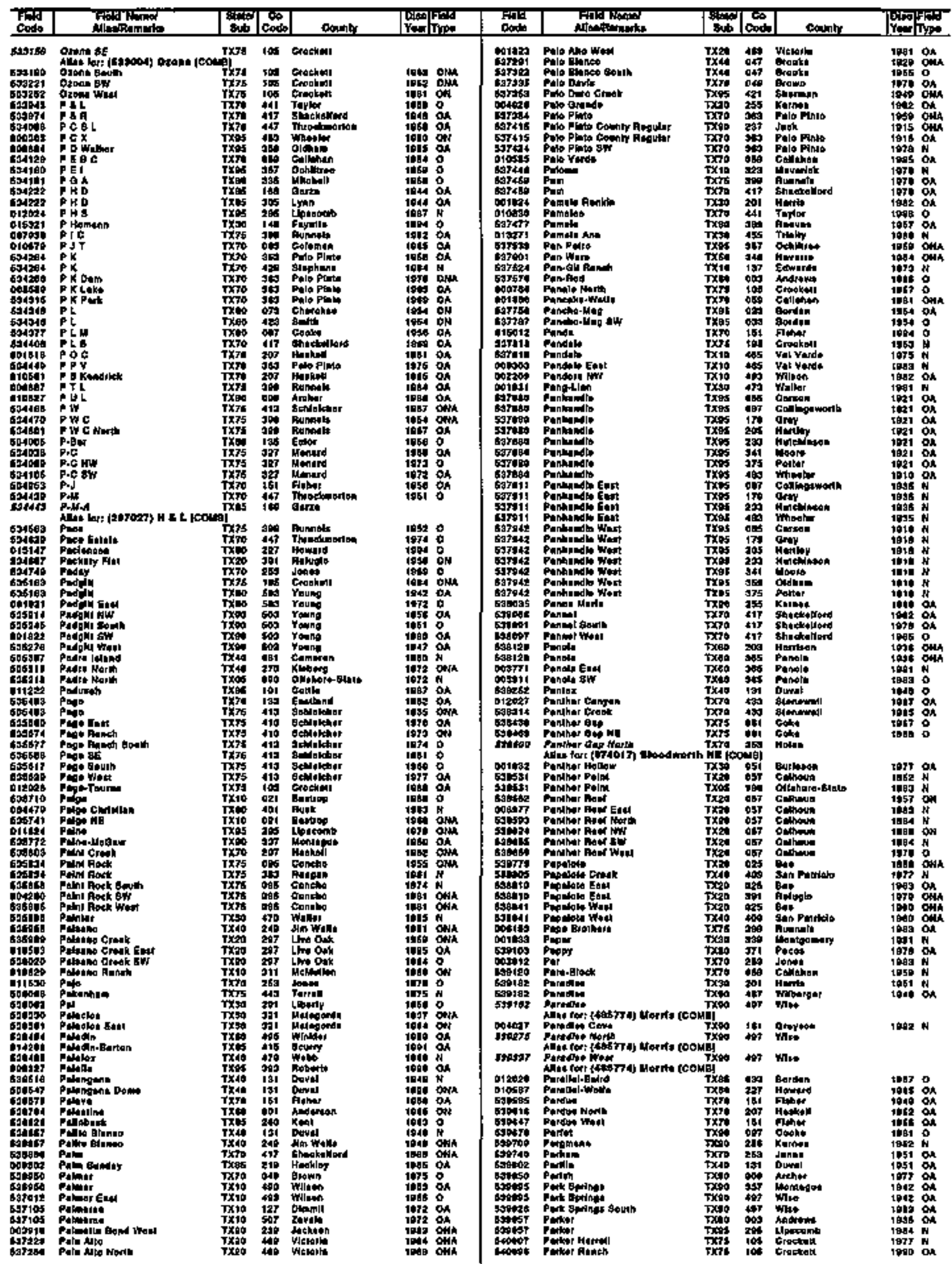


TEXAS

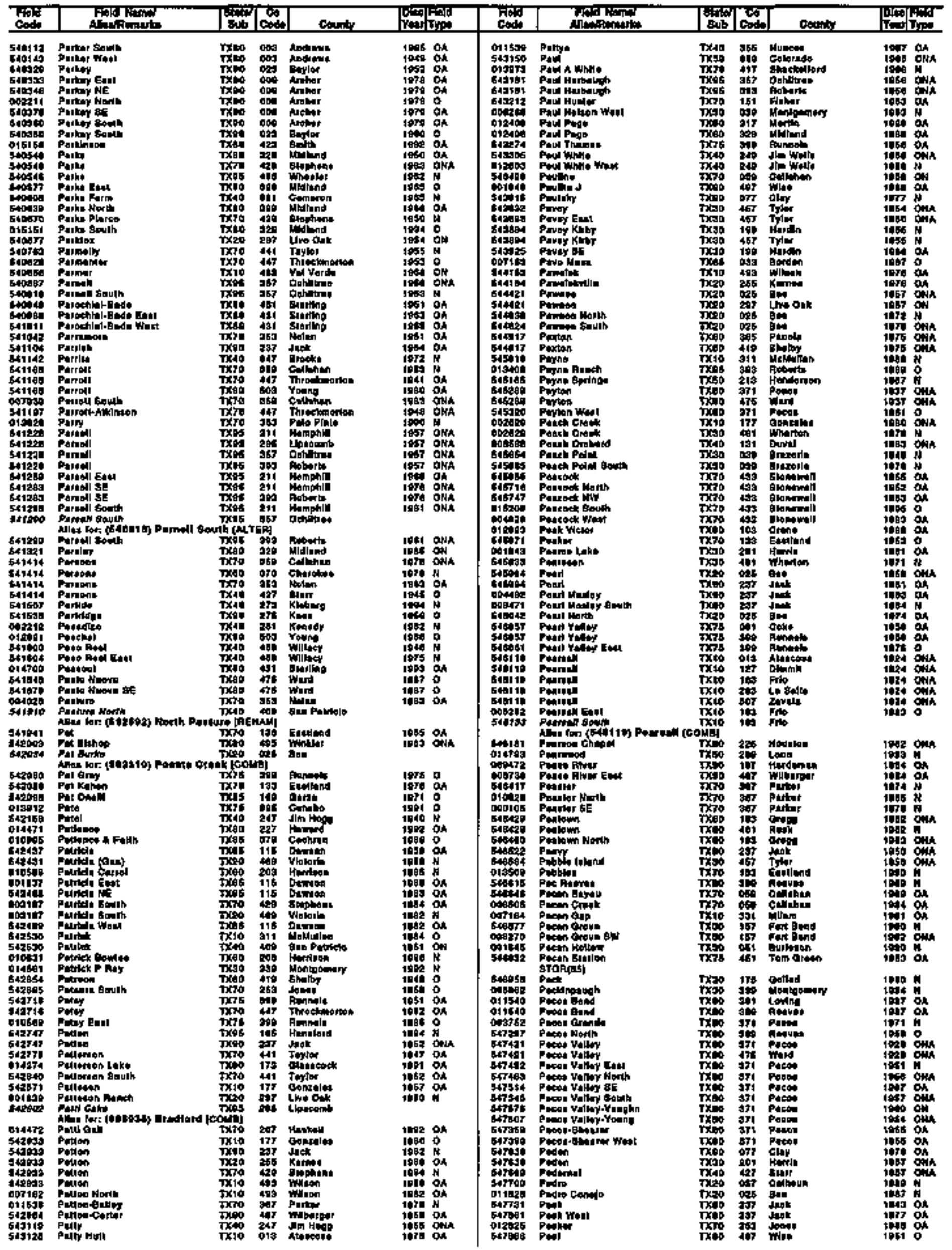


TEXAS

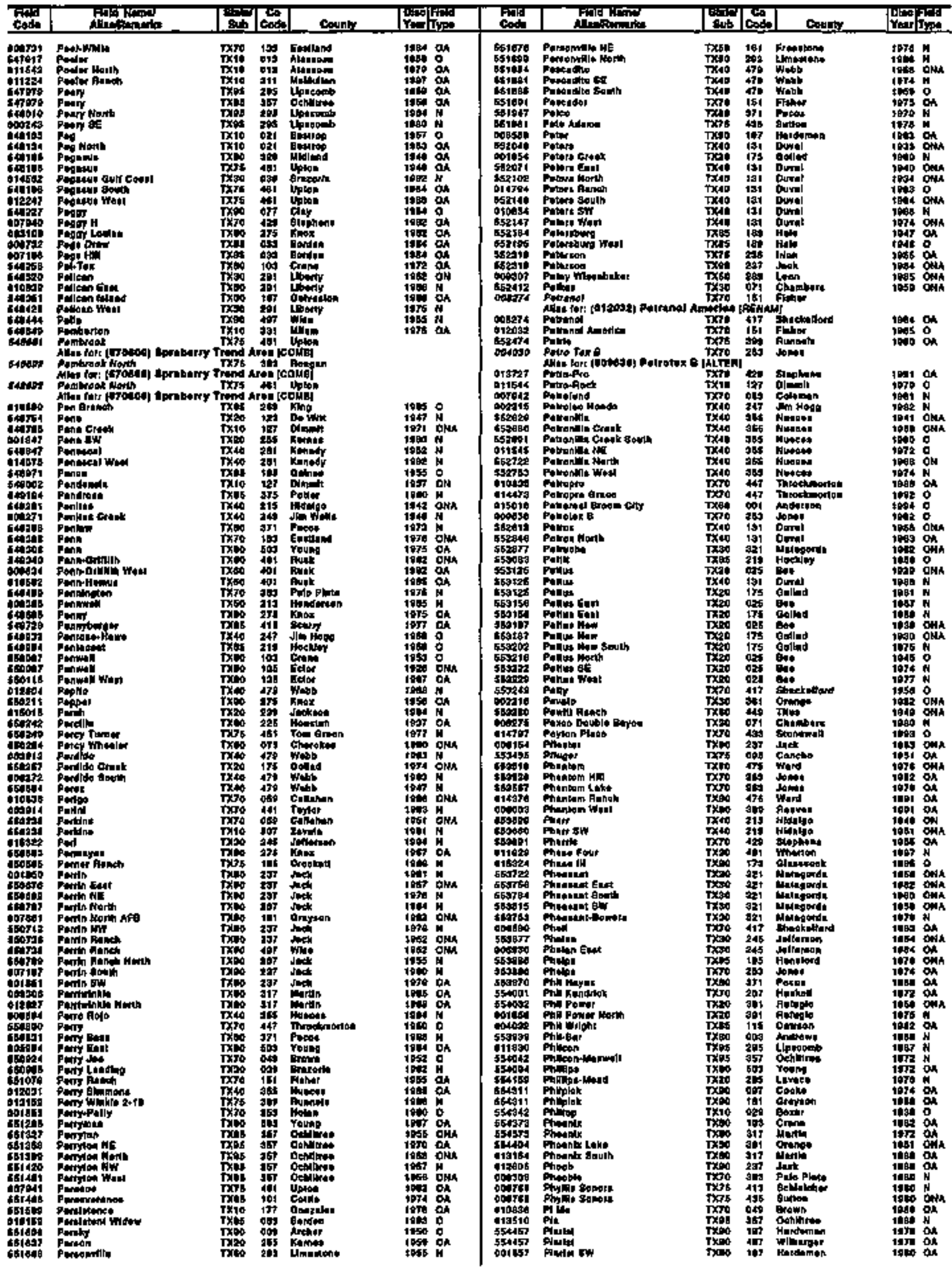


TEXAS

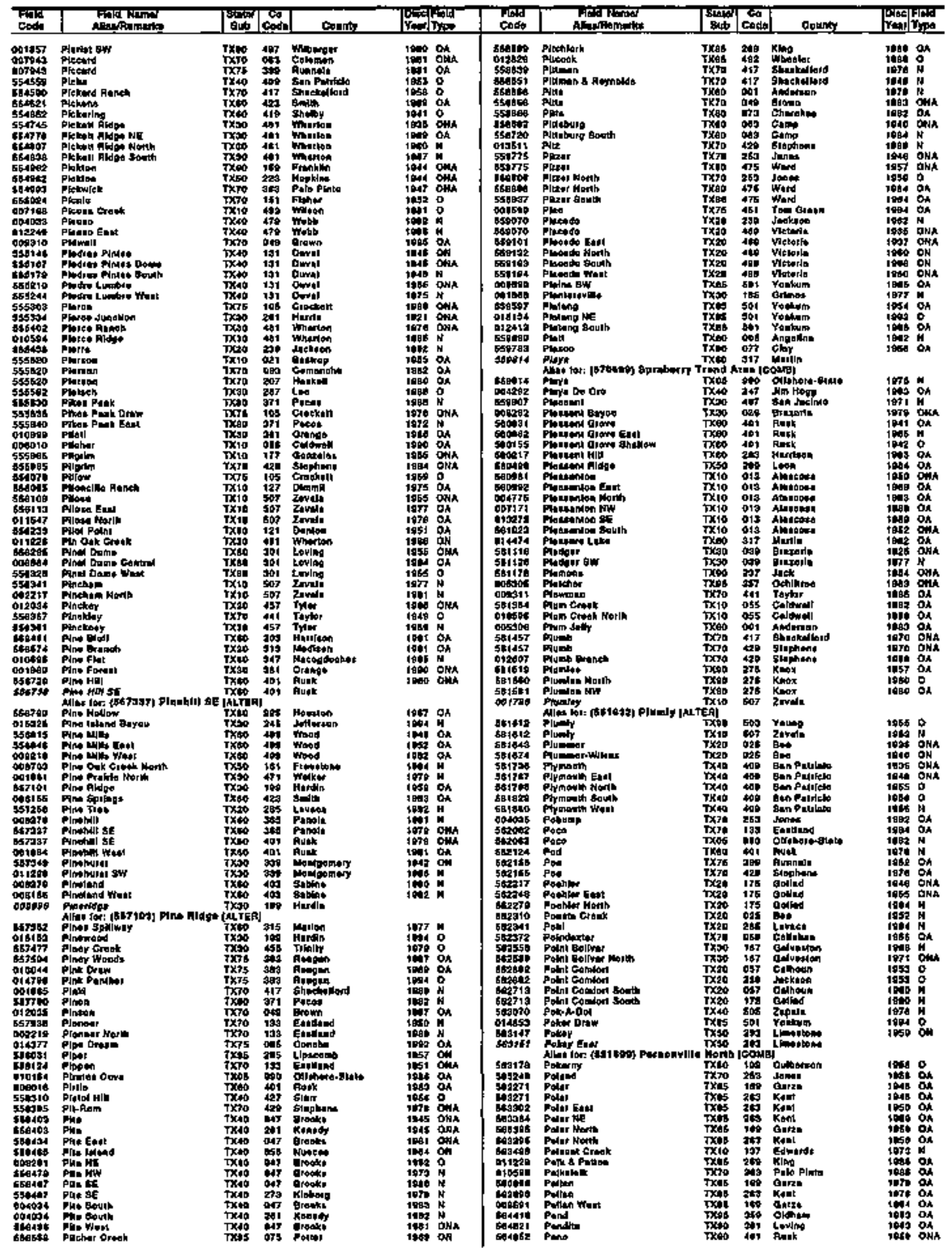


TEXAS

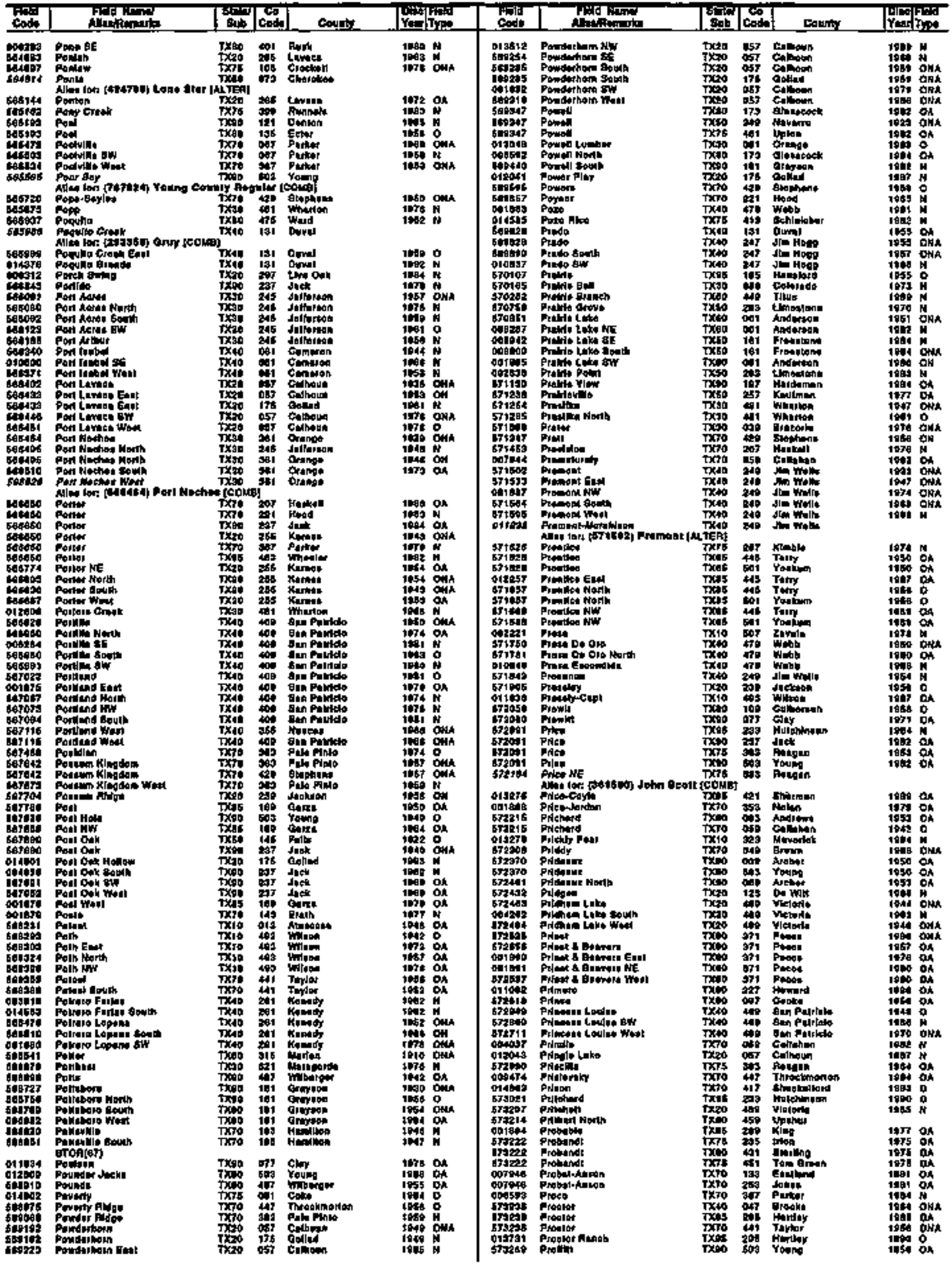


TEXAS

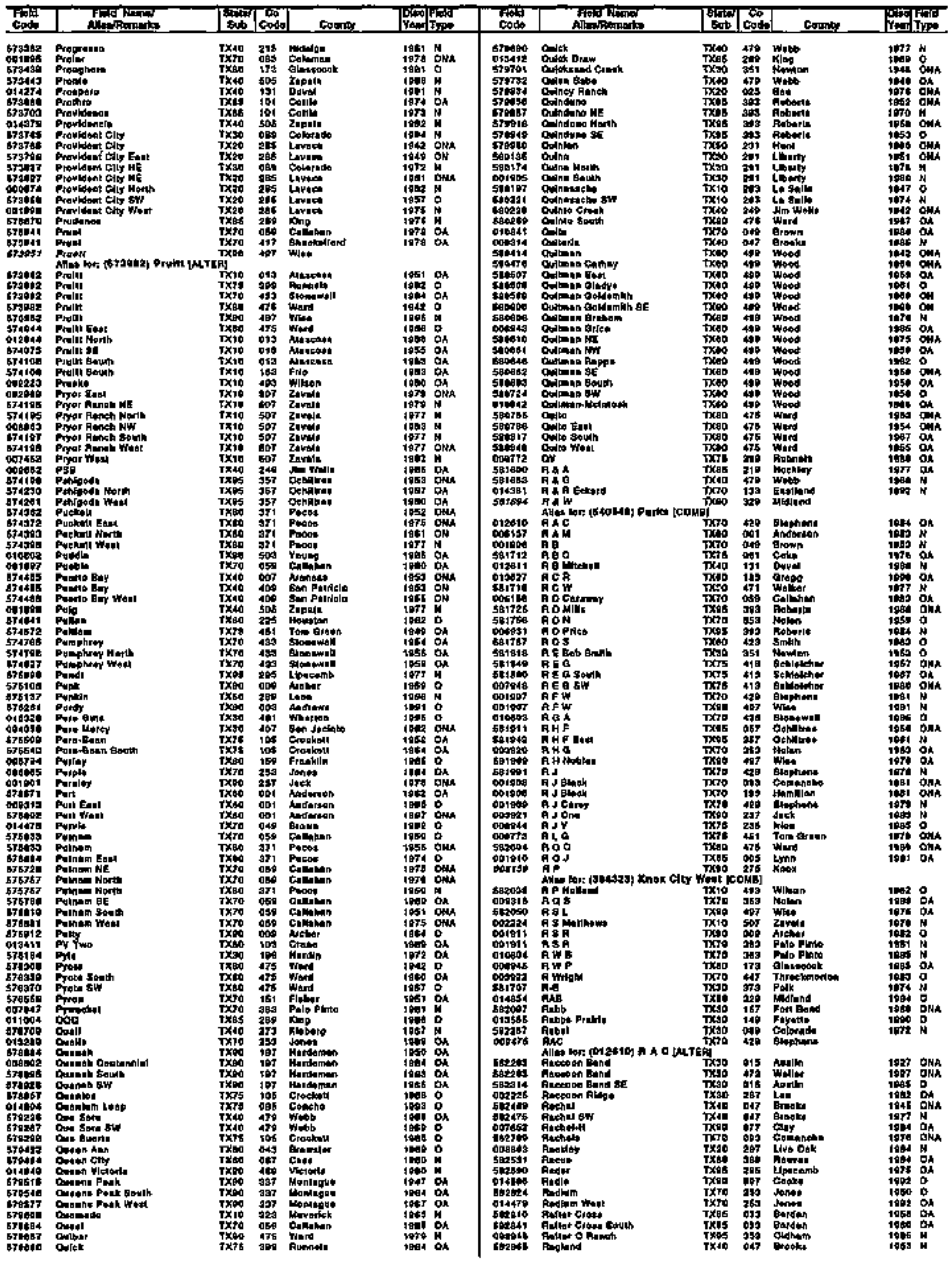


TEXAS

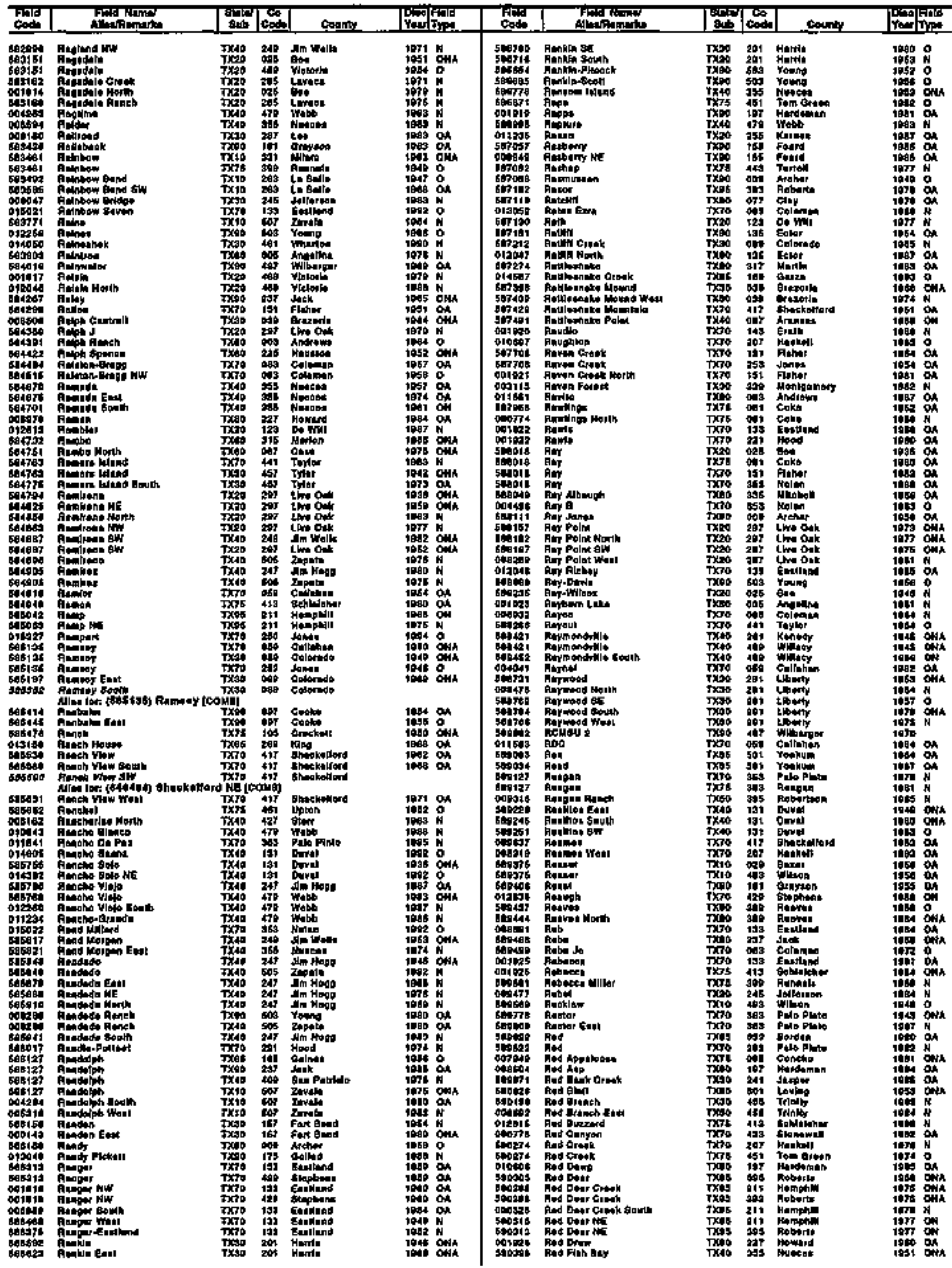


TEXAS

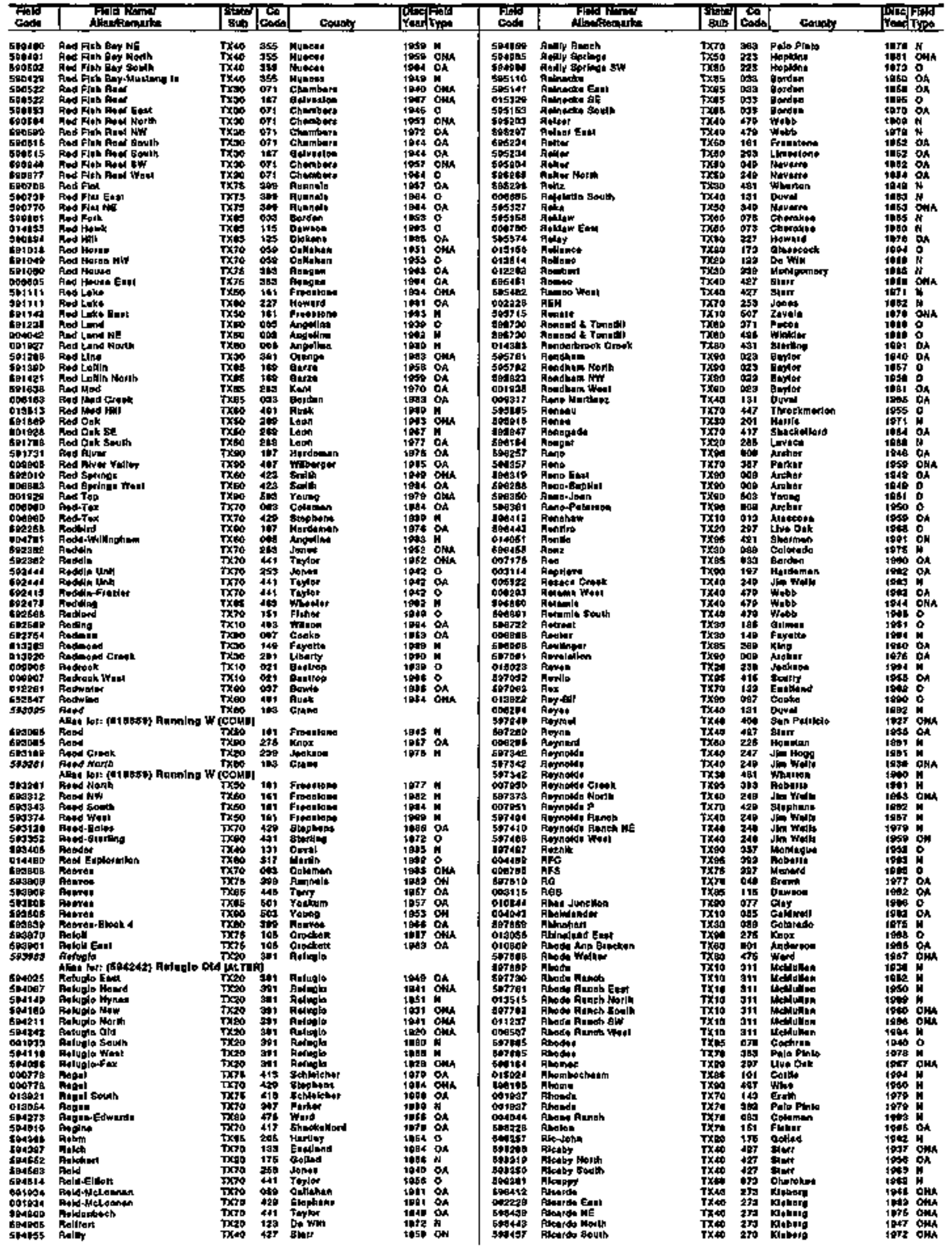


TEXAS

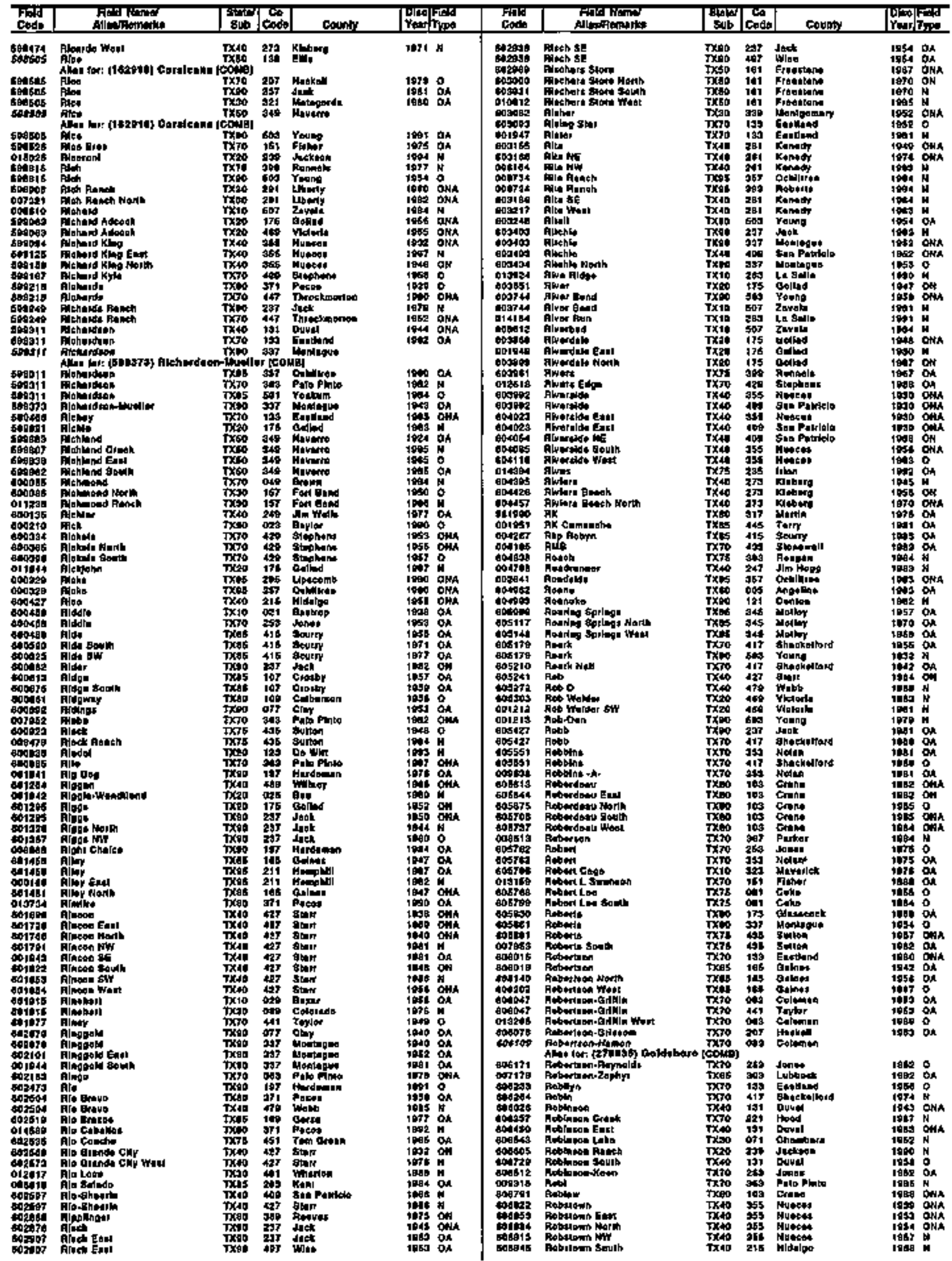




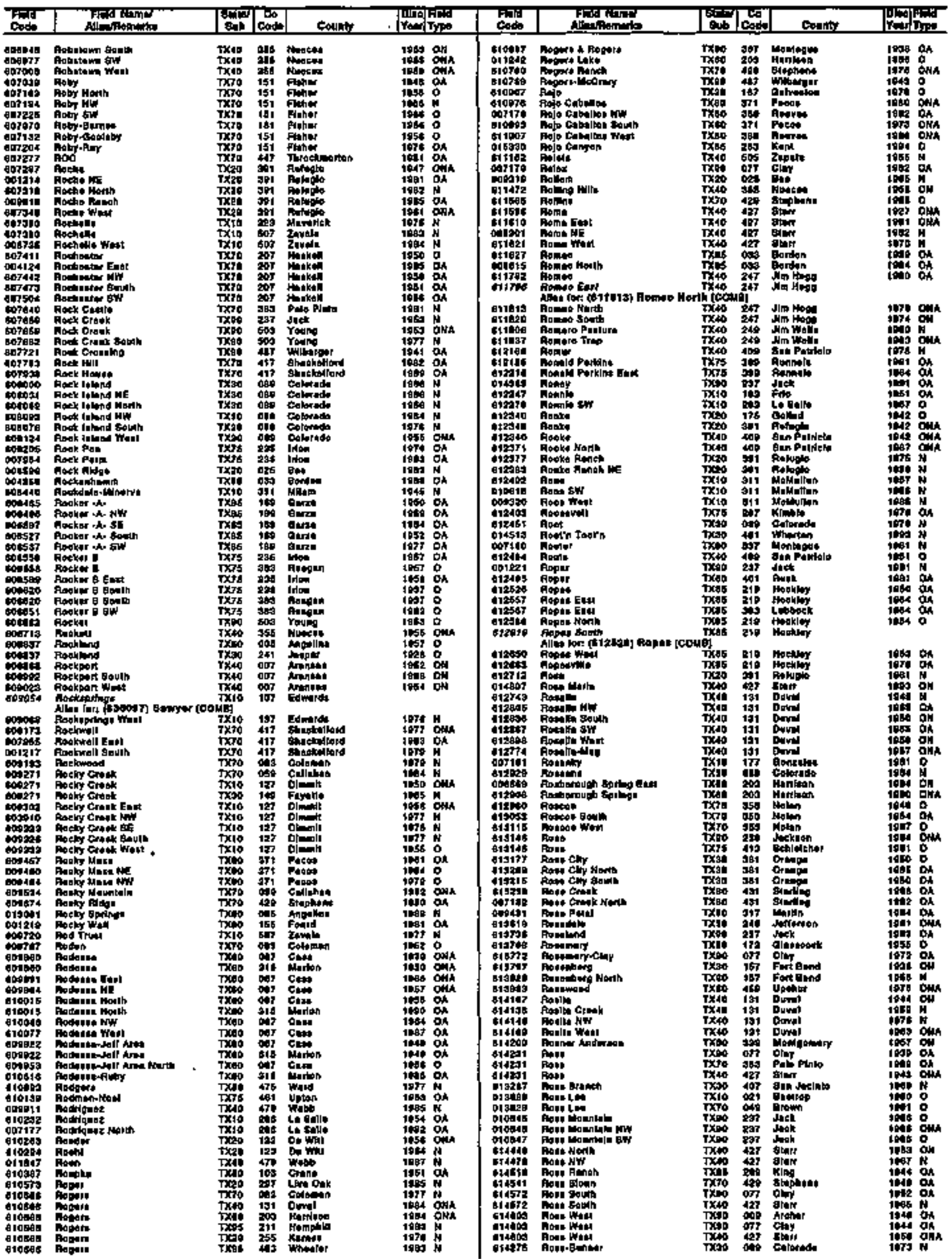


TEXAS

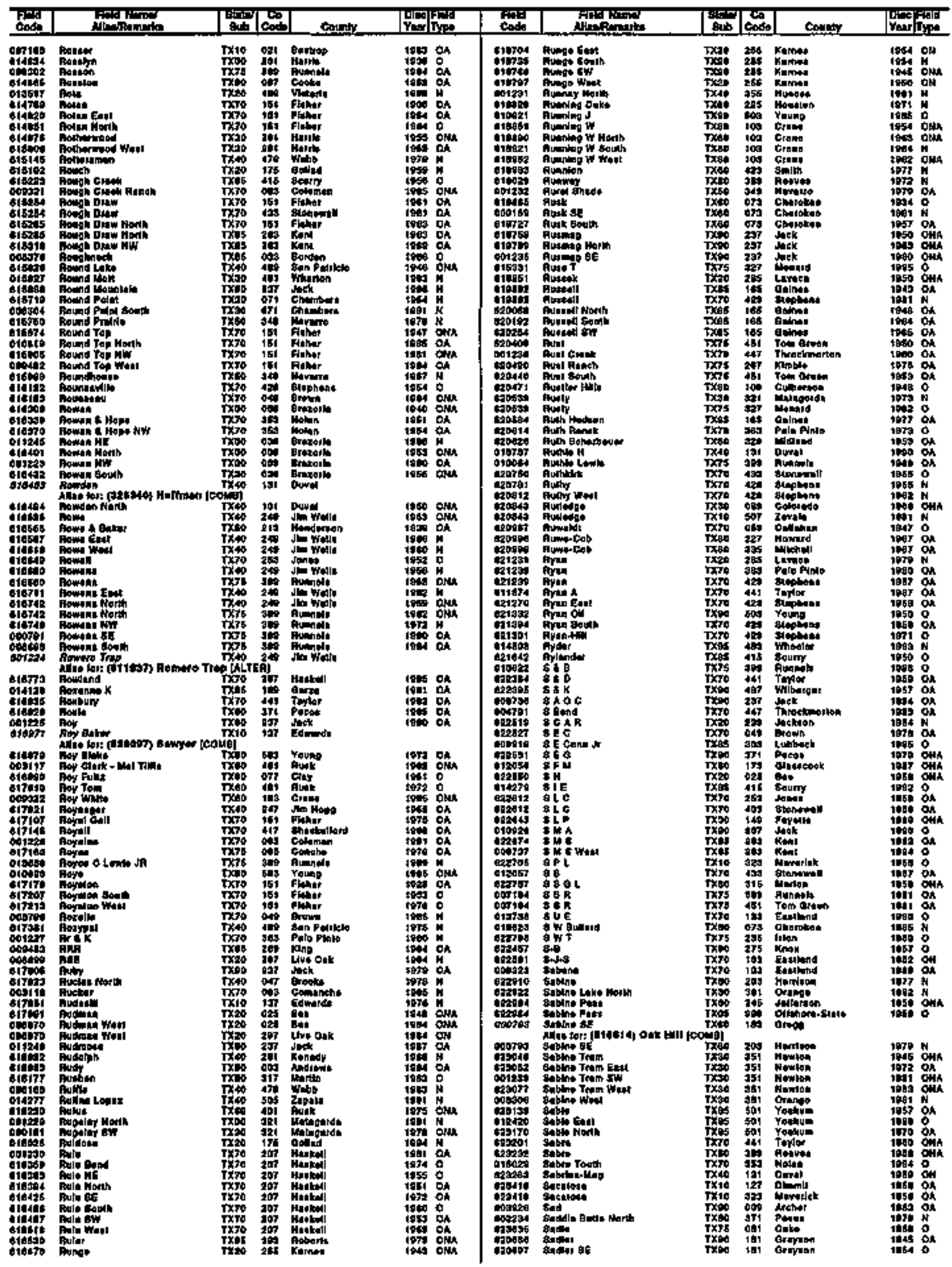


TEXAS

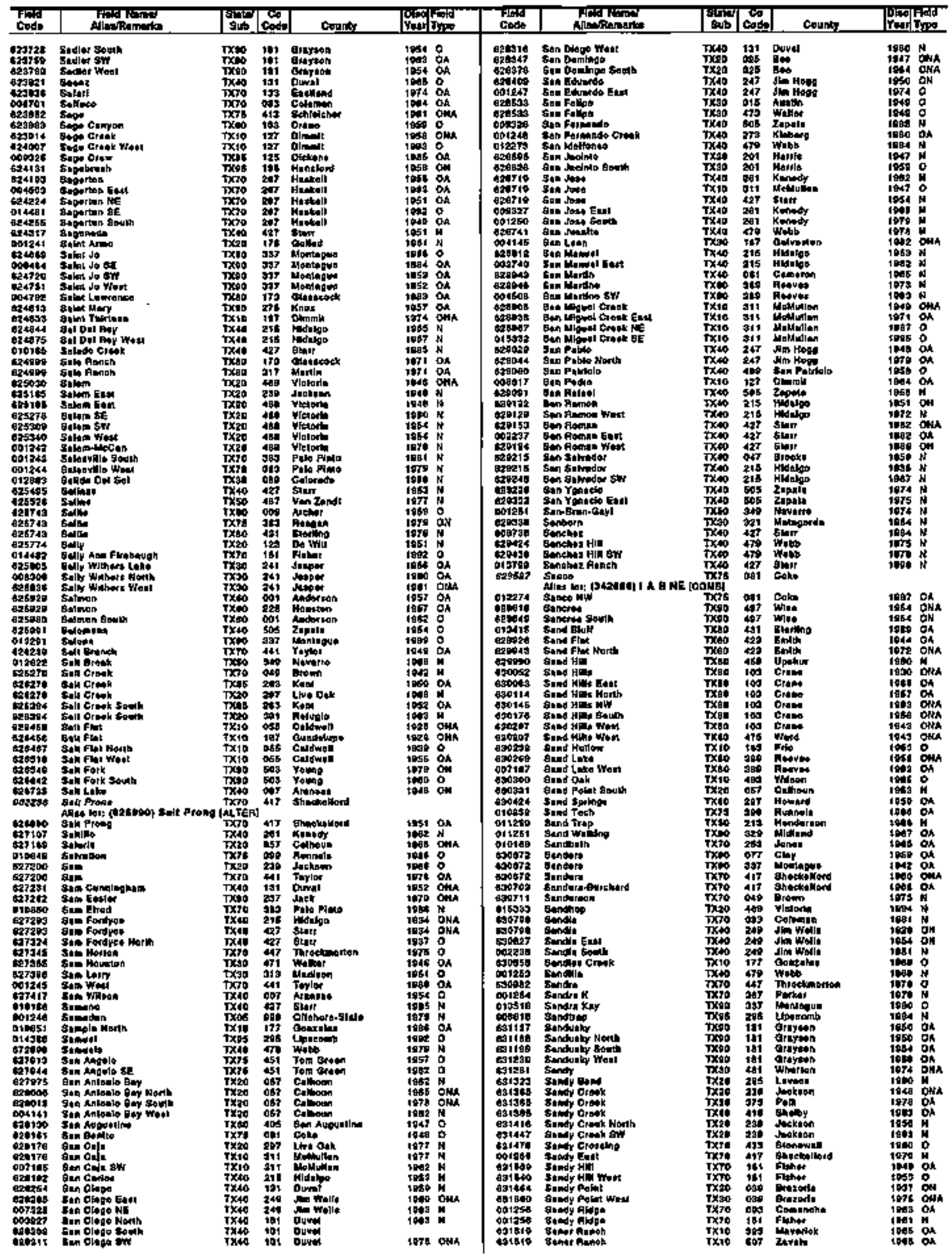


TEXAS

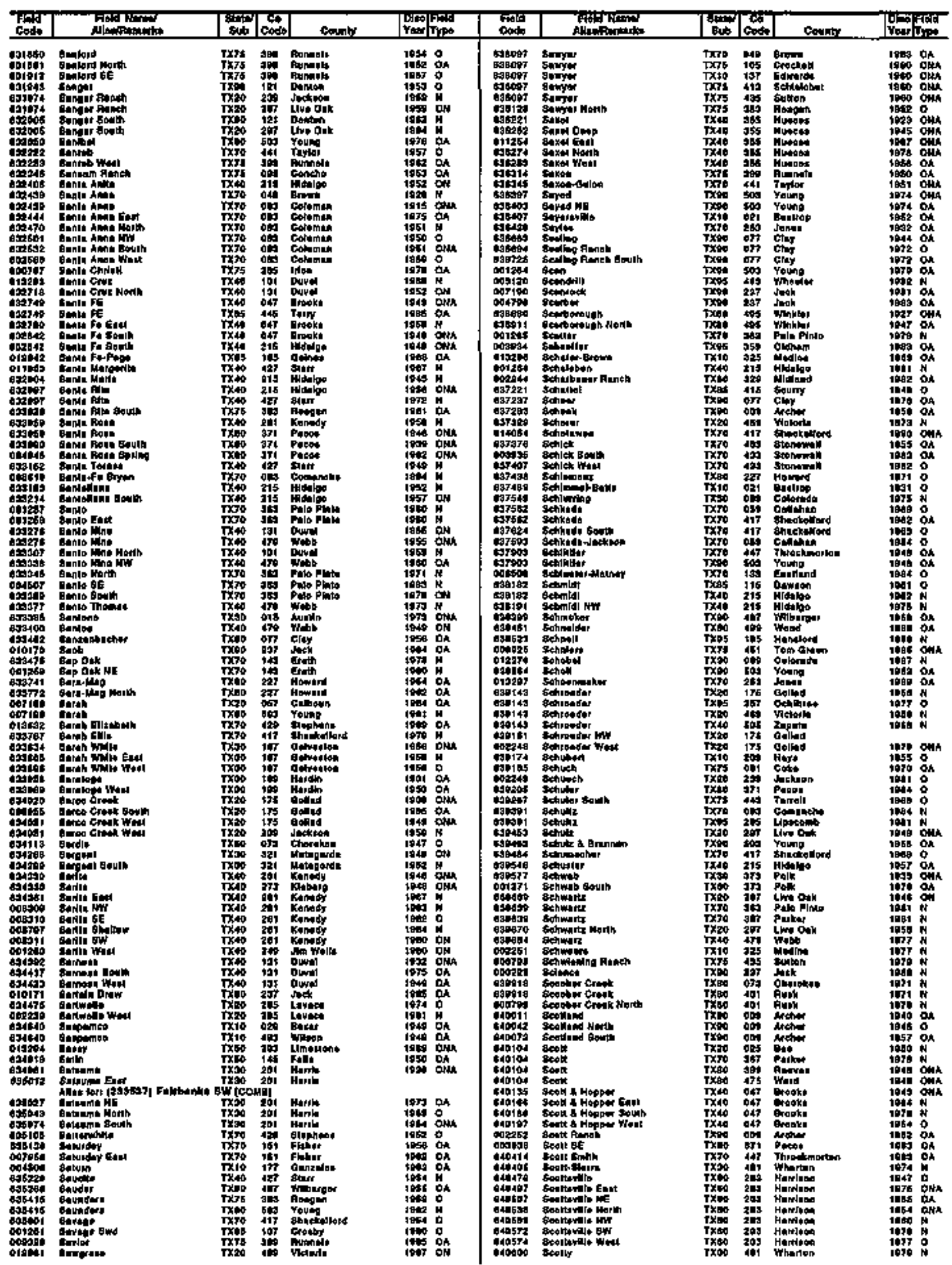




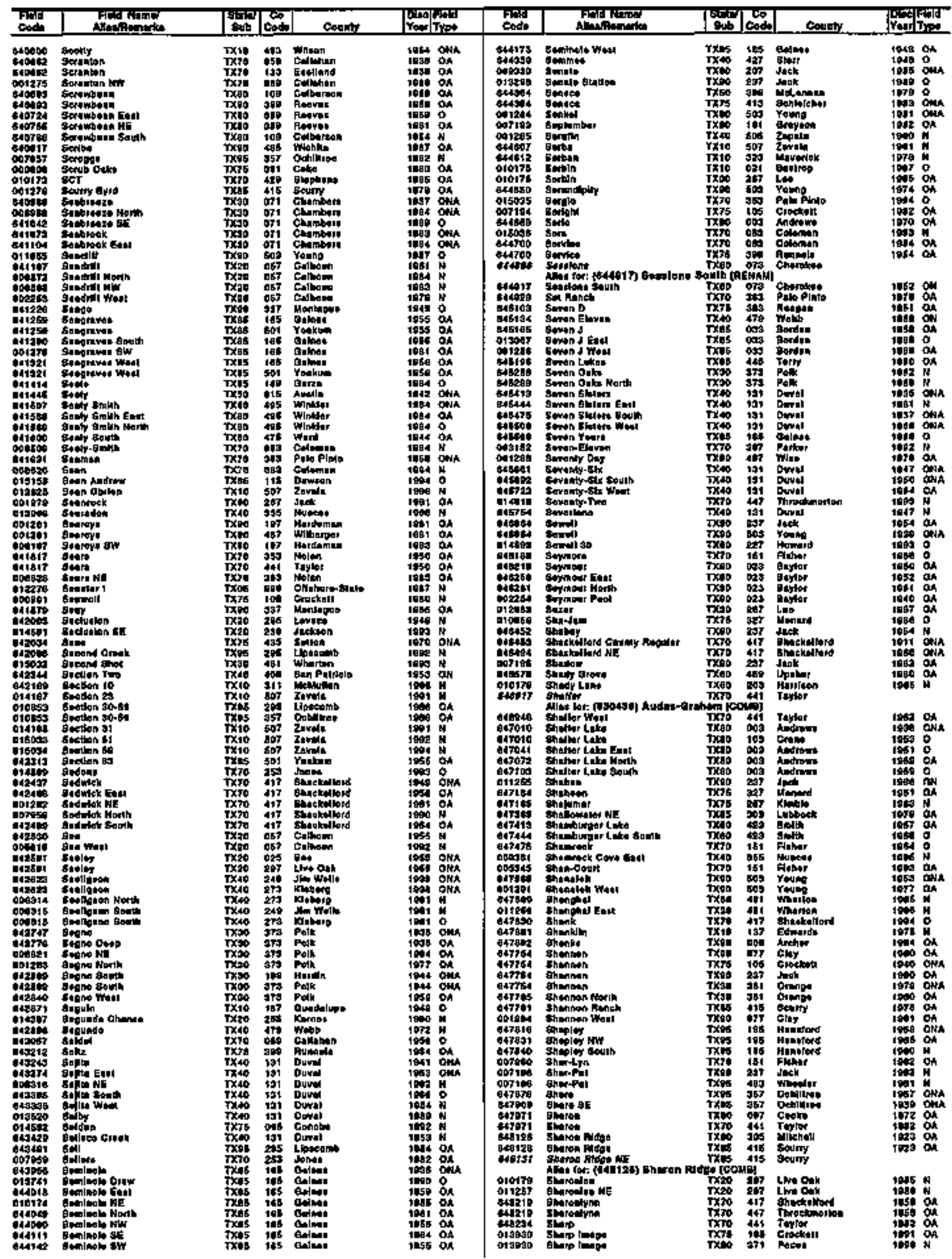


TEXAS

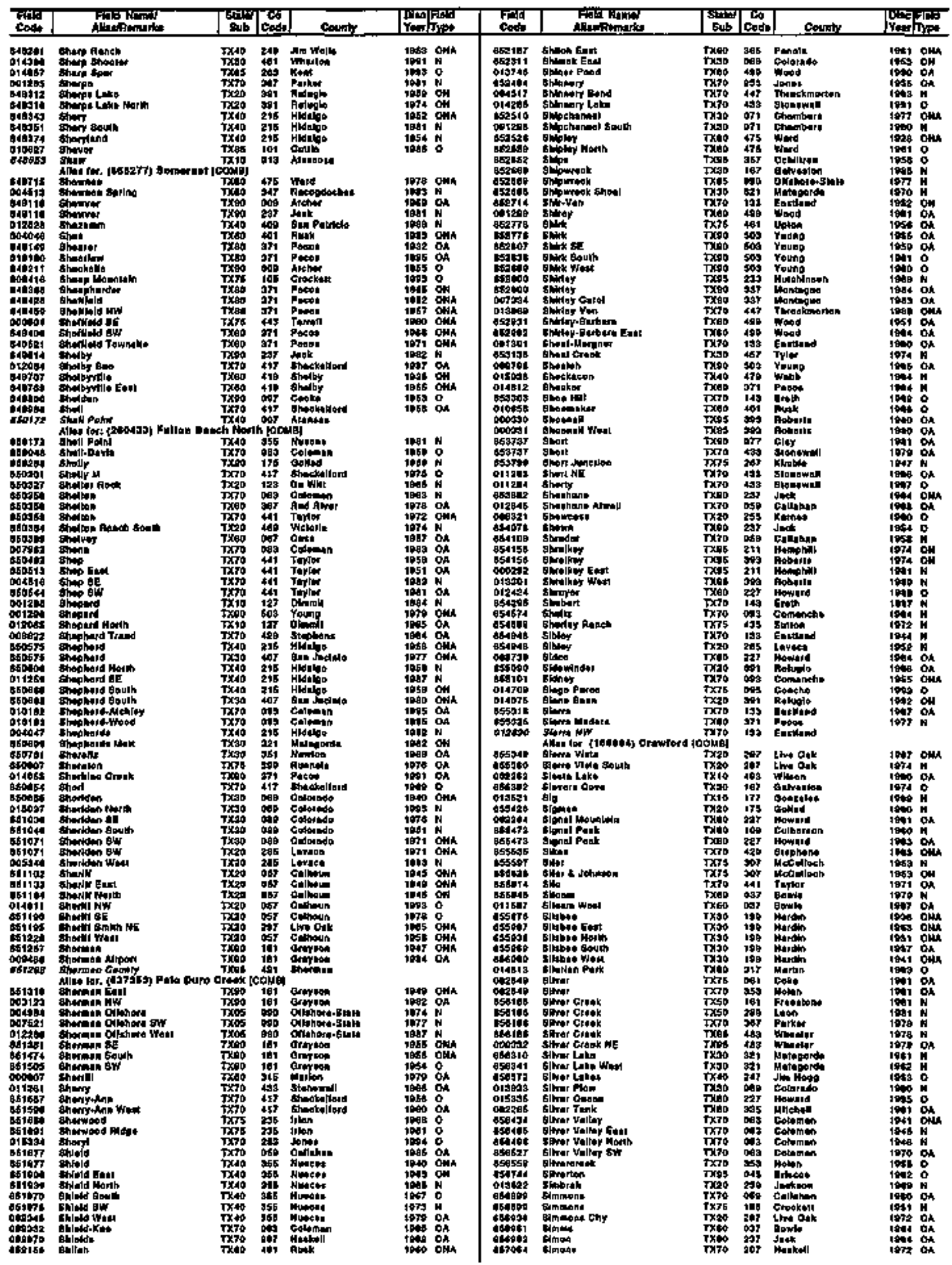


TEXAS

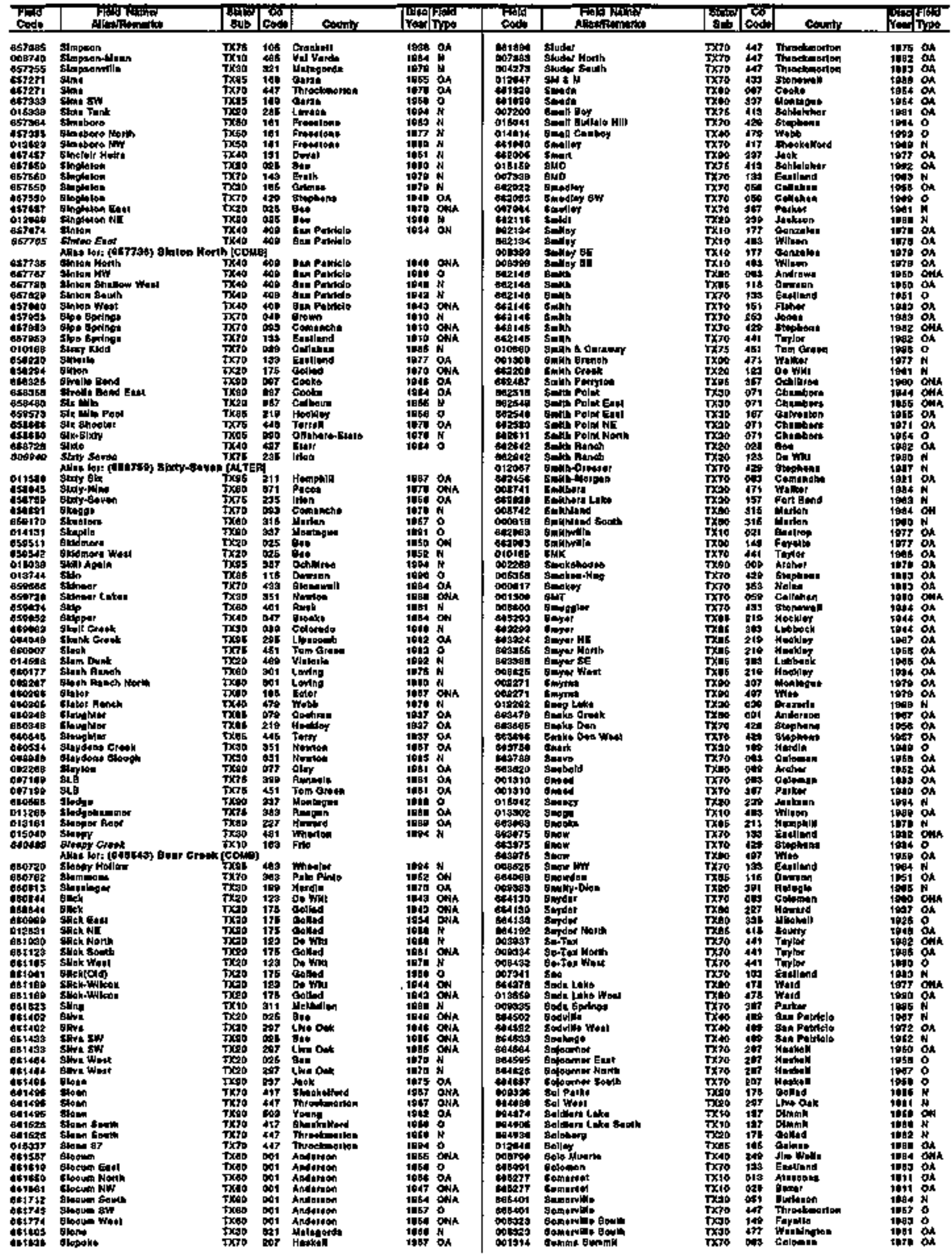


TEXAS

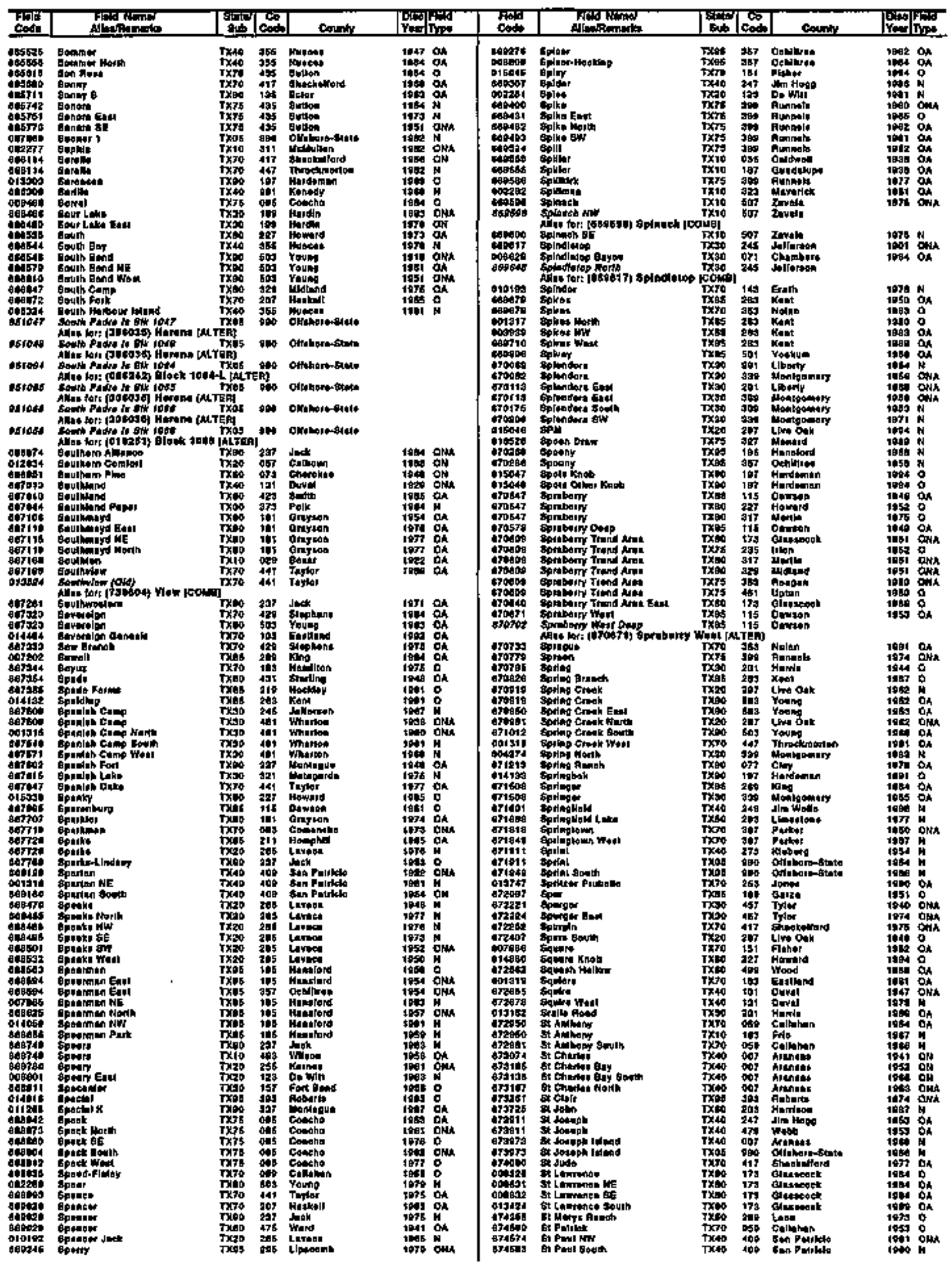


TEXAS

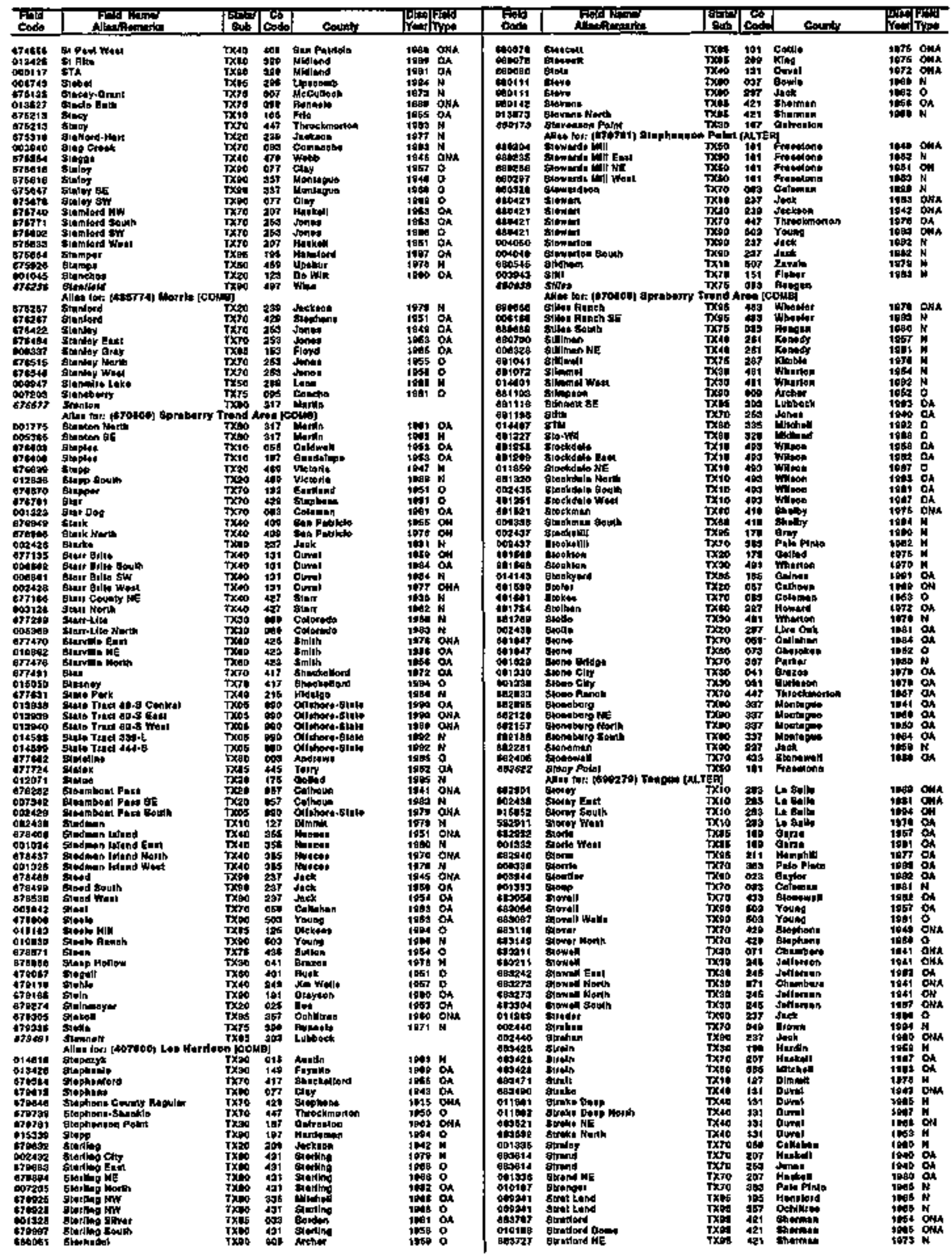


TEXAS

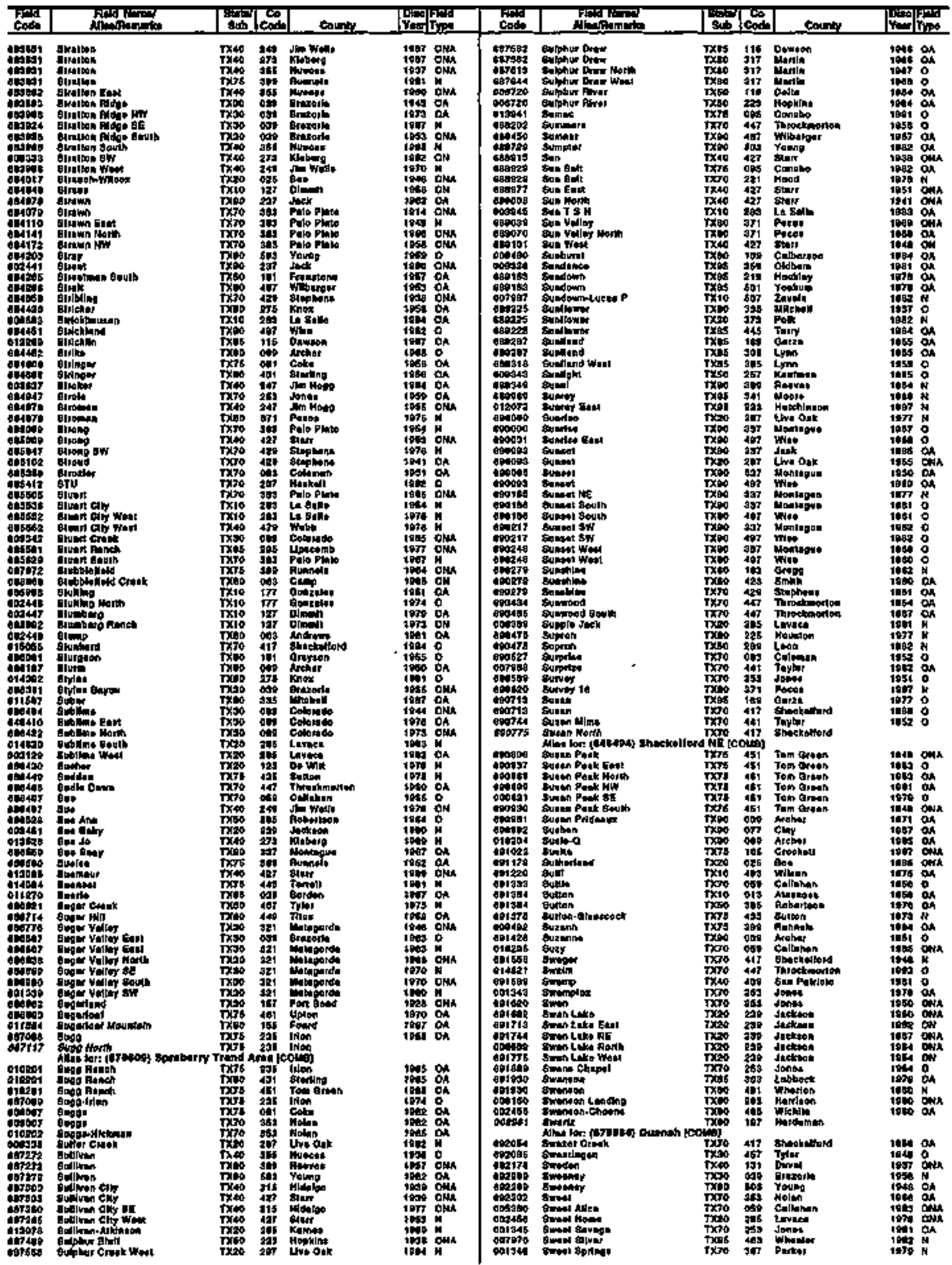


TEXAS

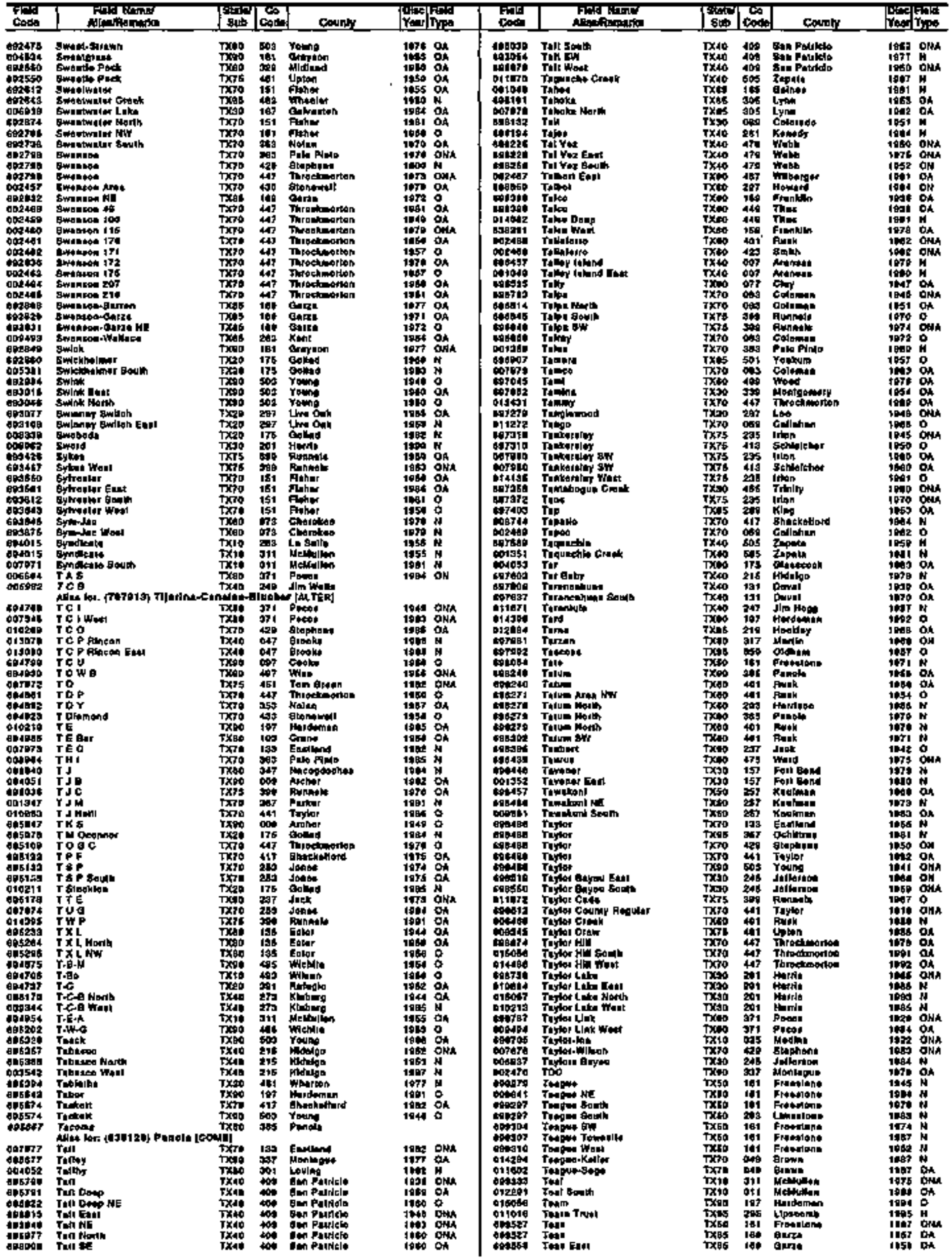


TEXAS

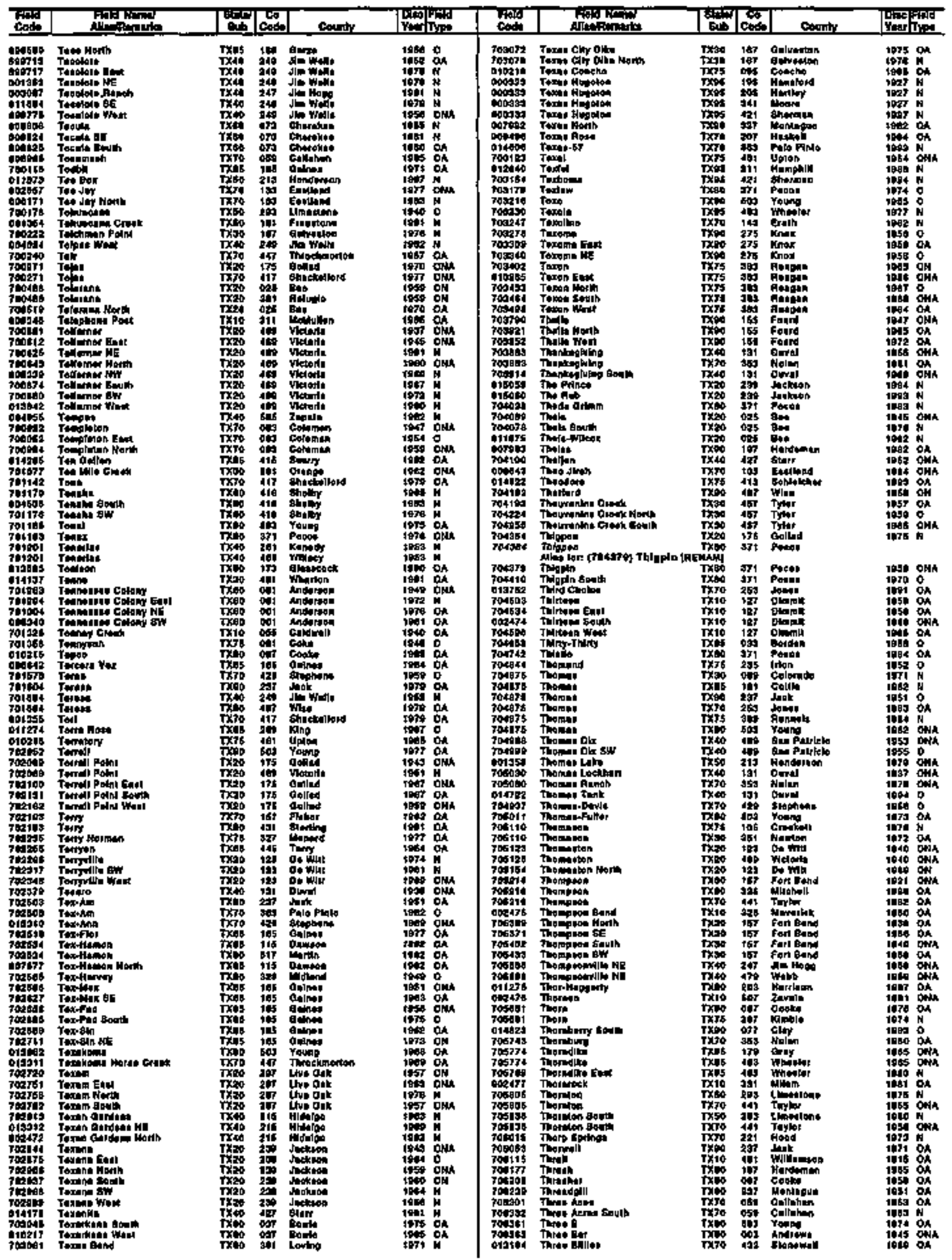


TEXAS

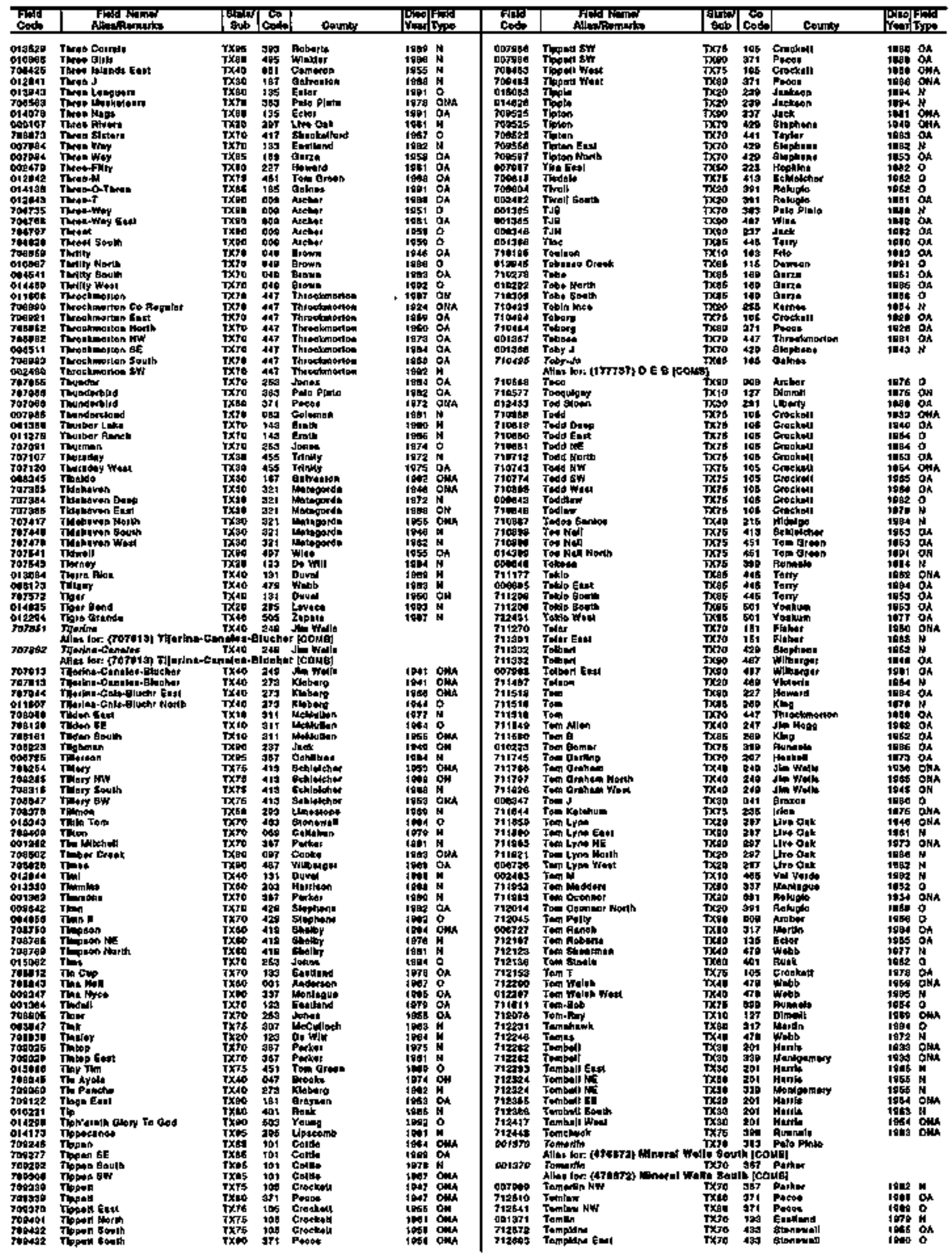


TEXAS

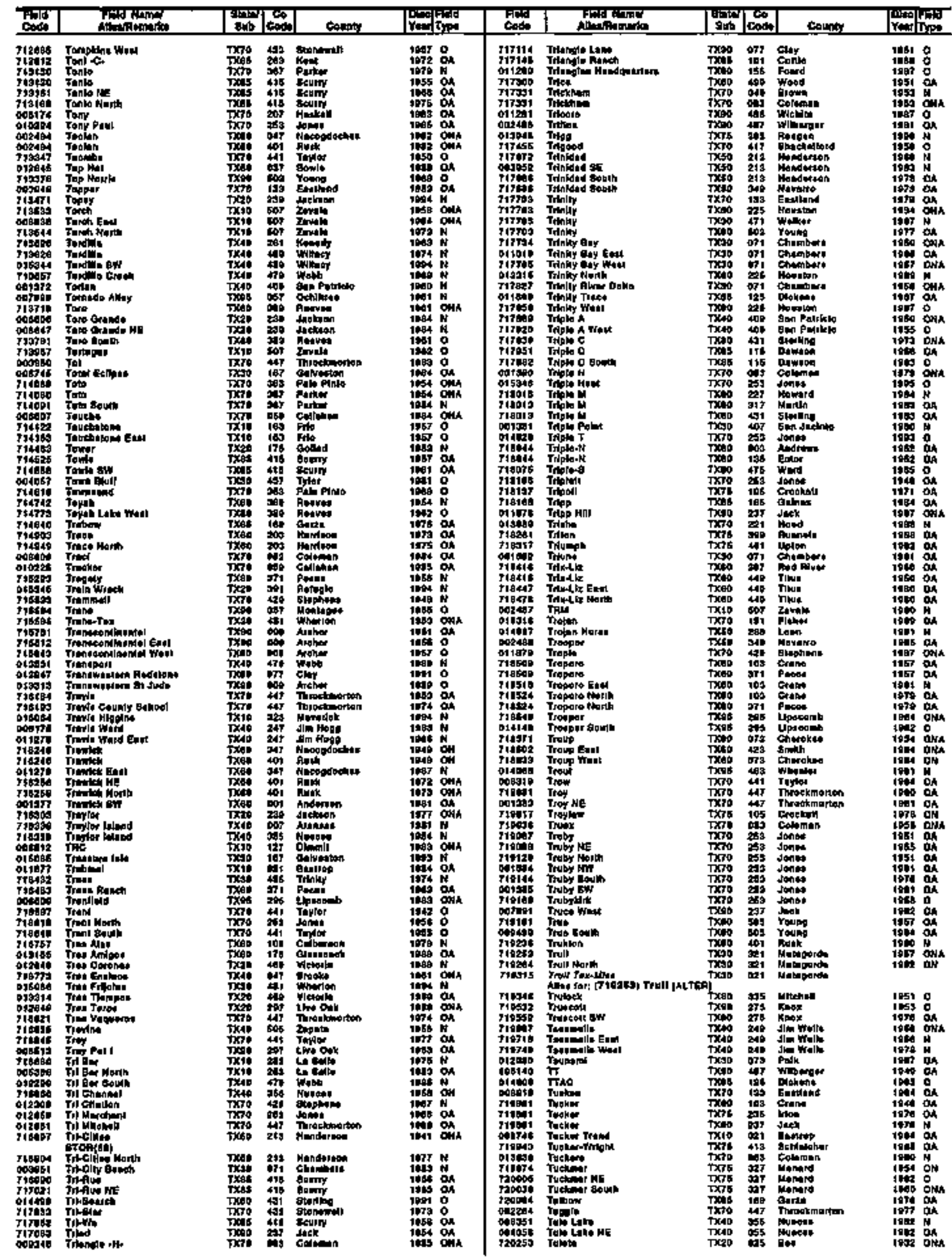


TEXAS

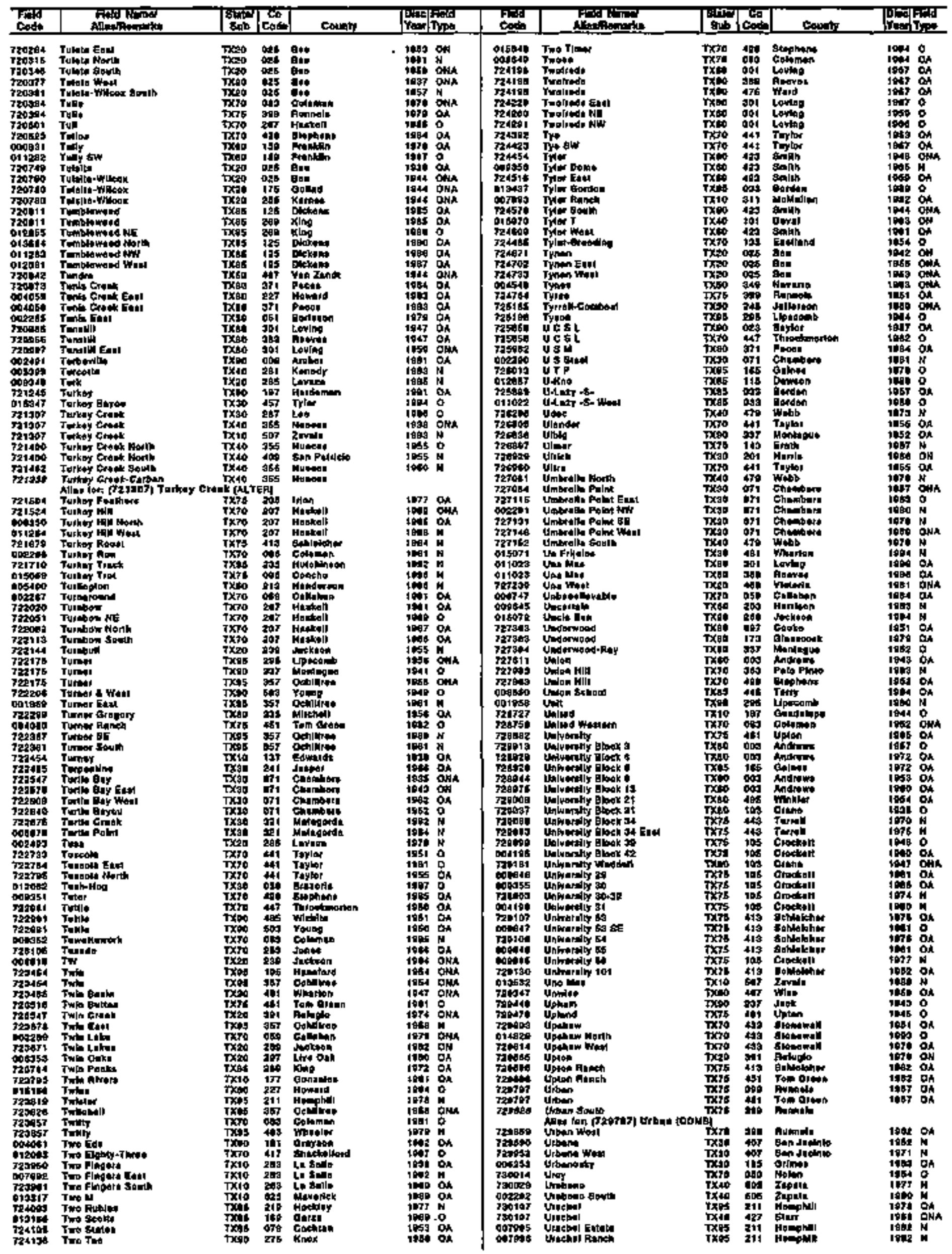


TEXAS

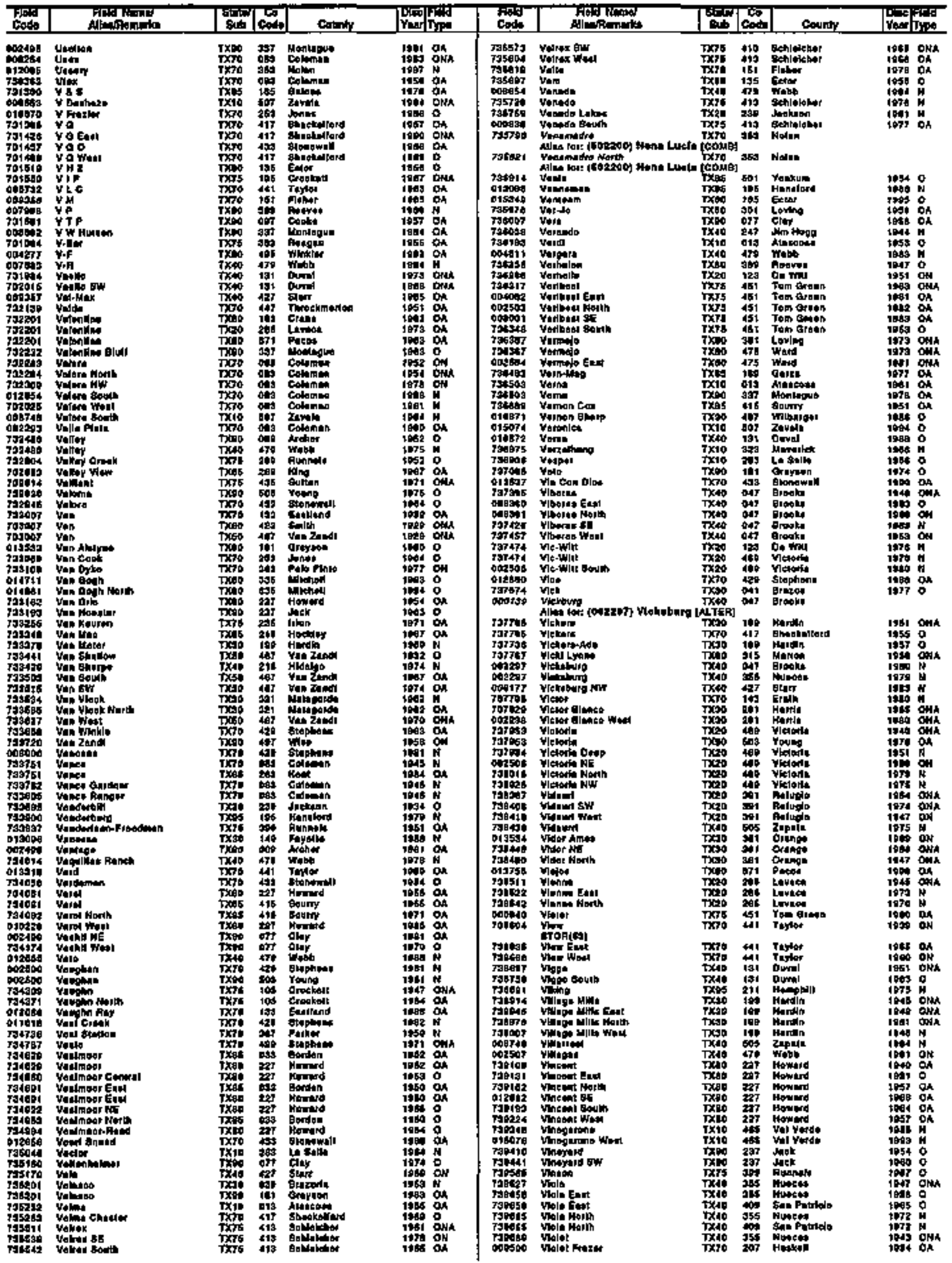


TEXAS

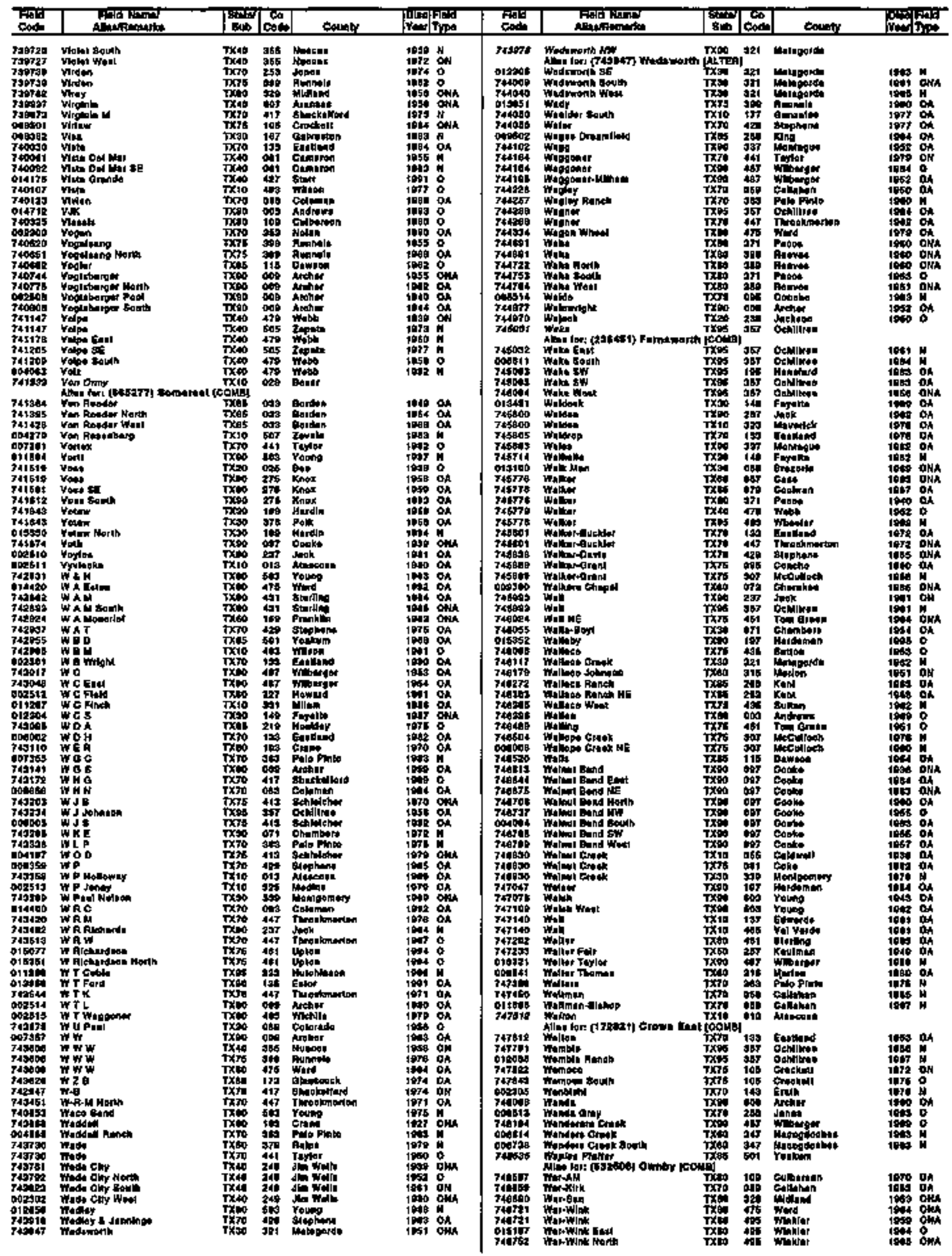


TEXAS

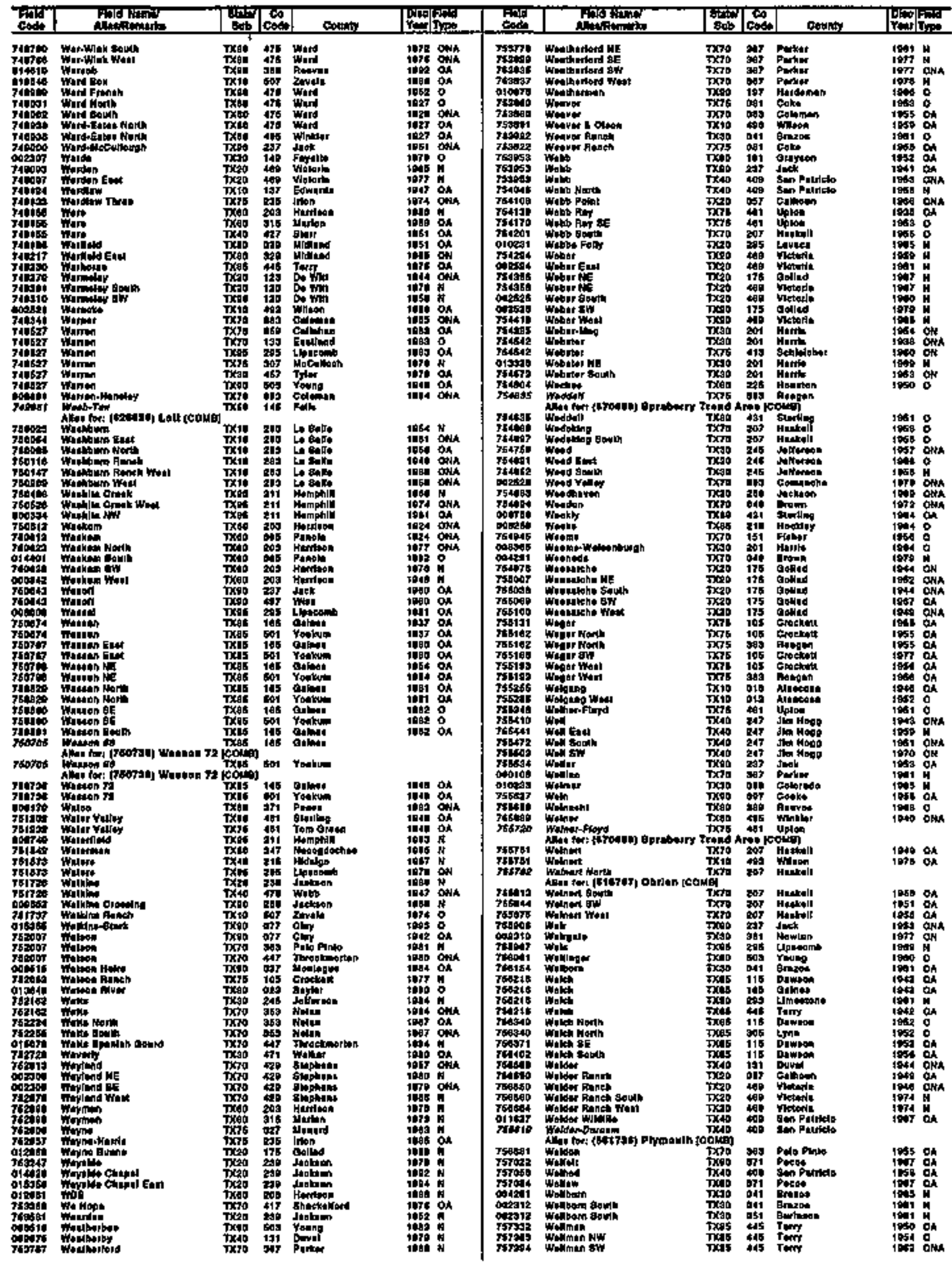


TEXAS

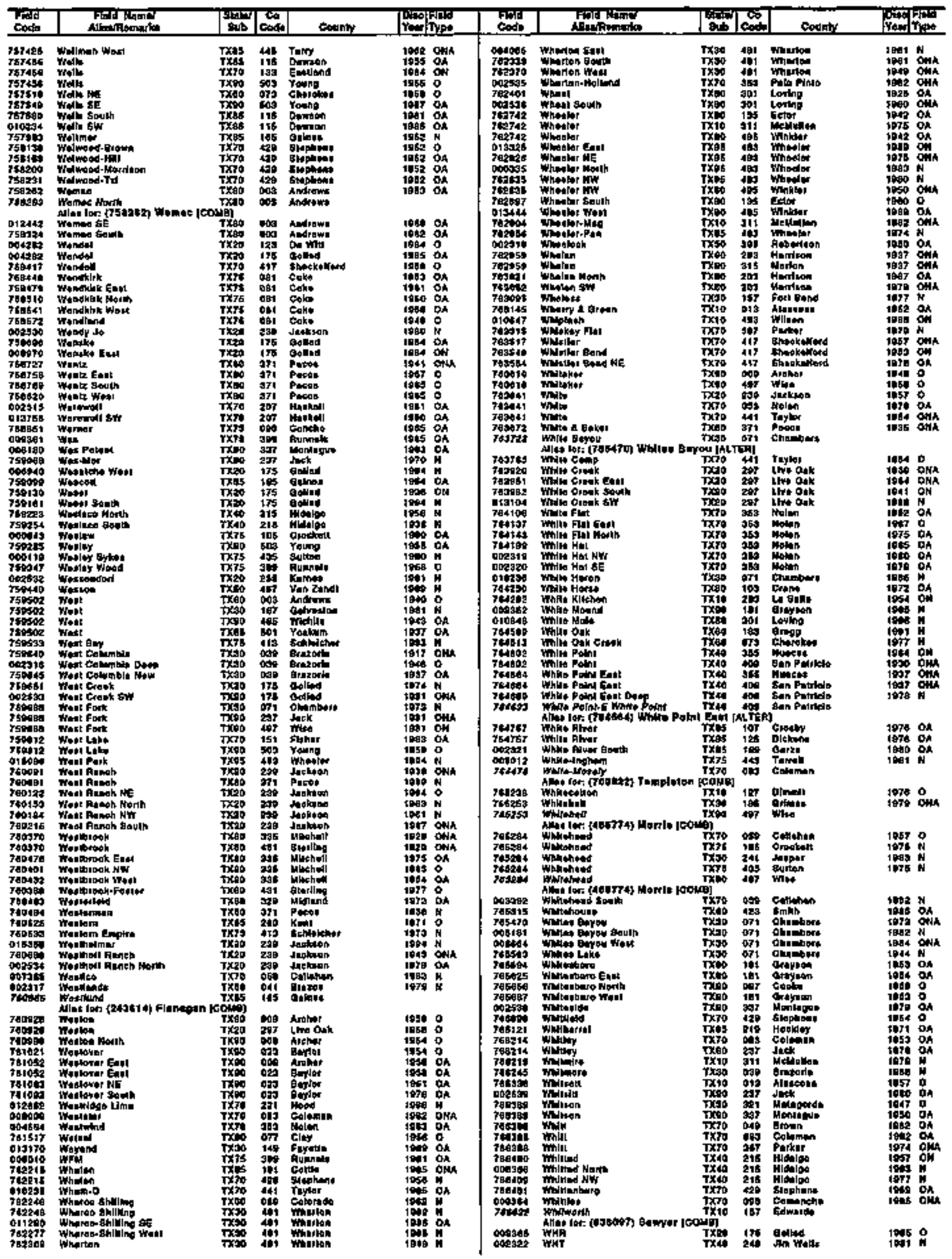


TEXAS

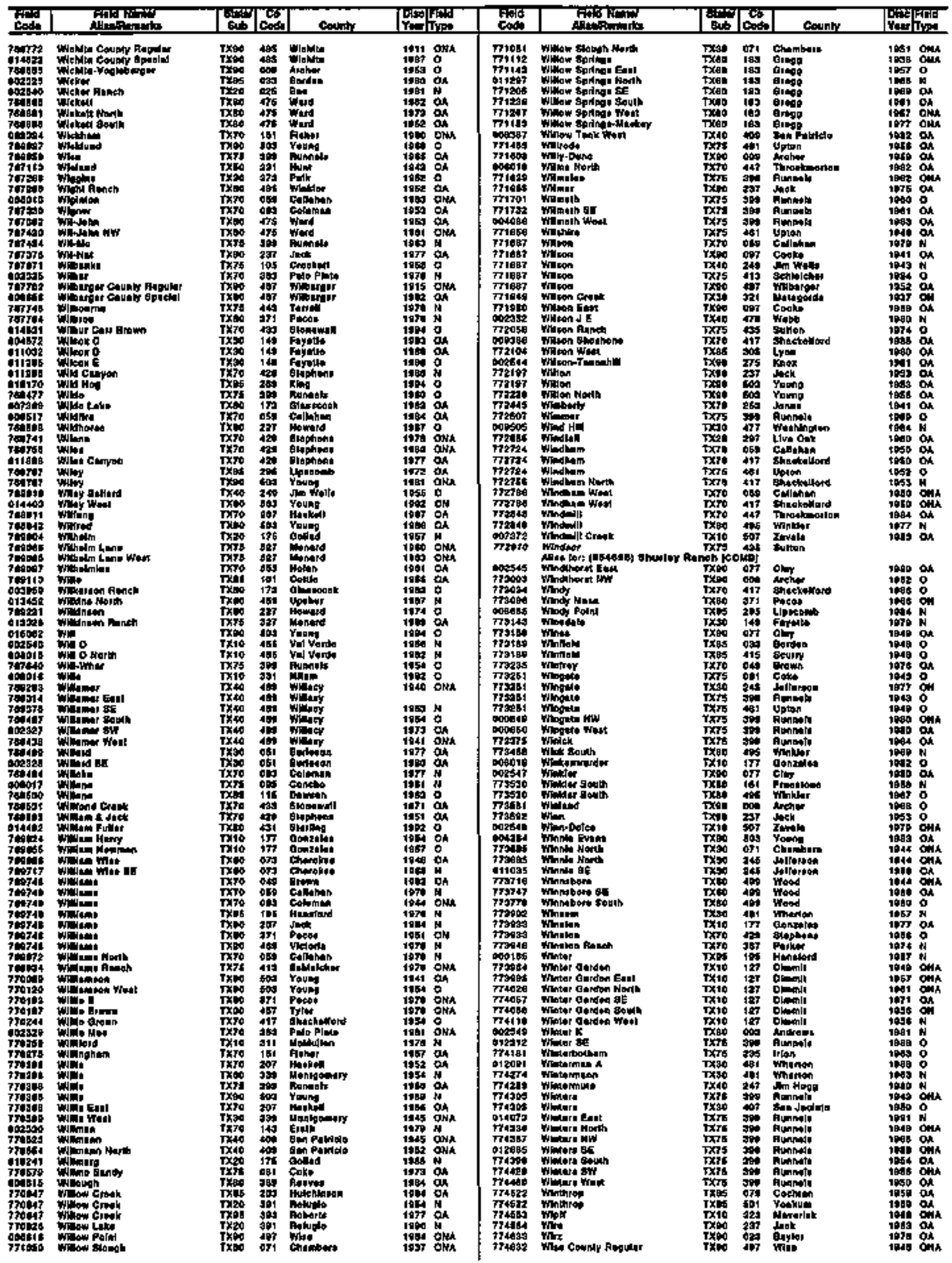


TEXAS

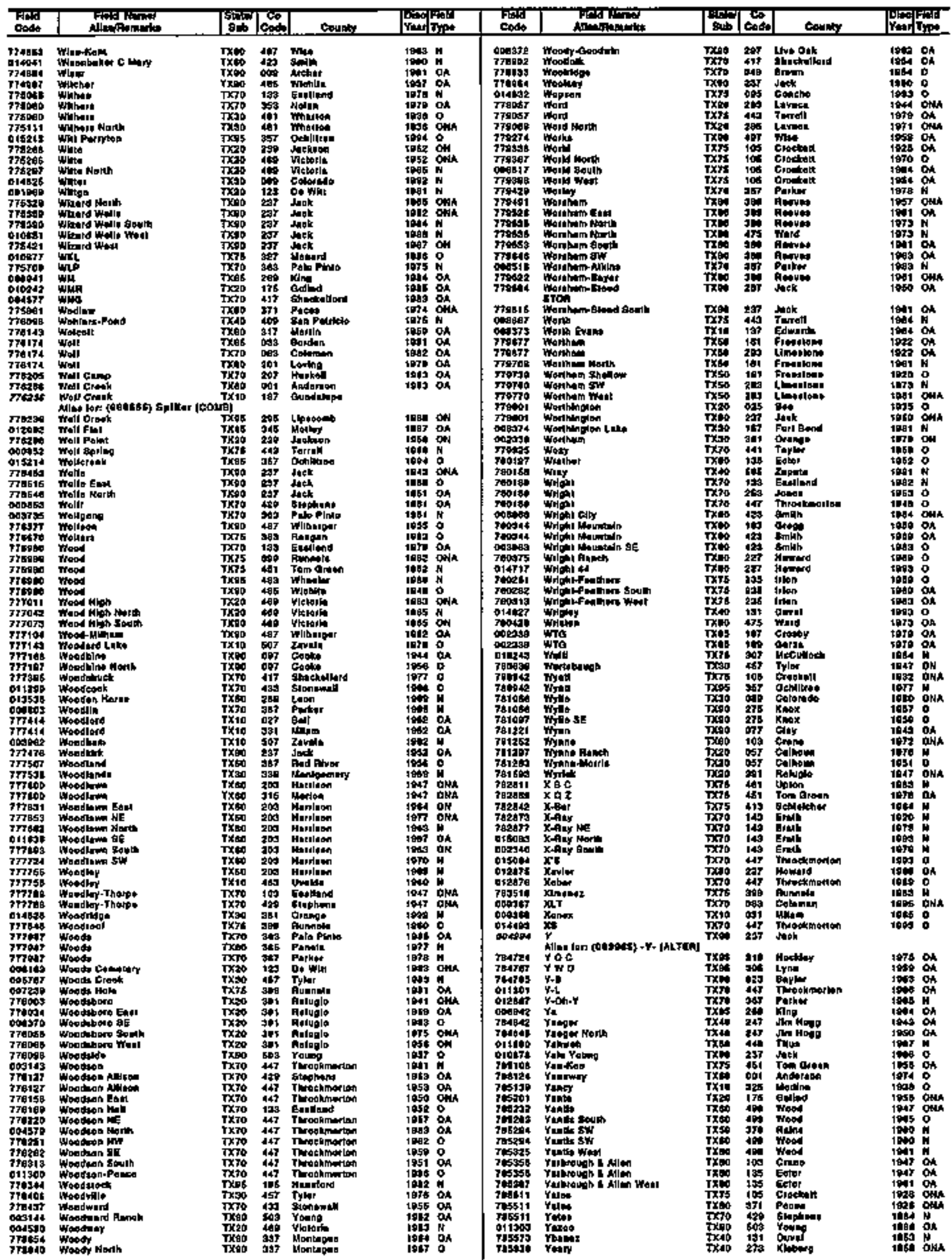


UT AH

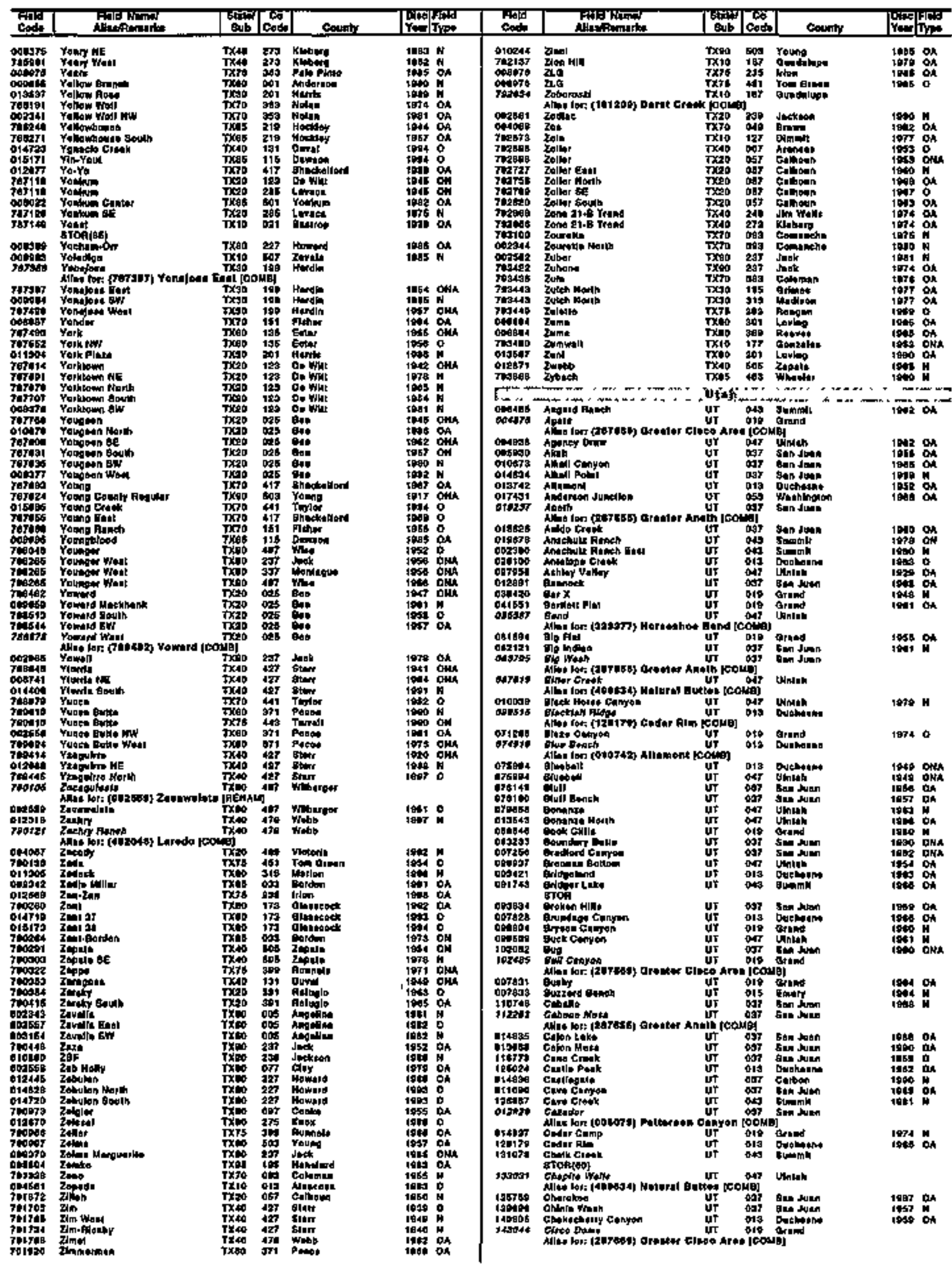




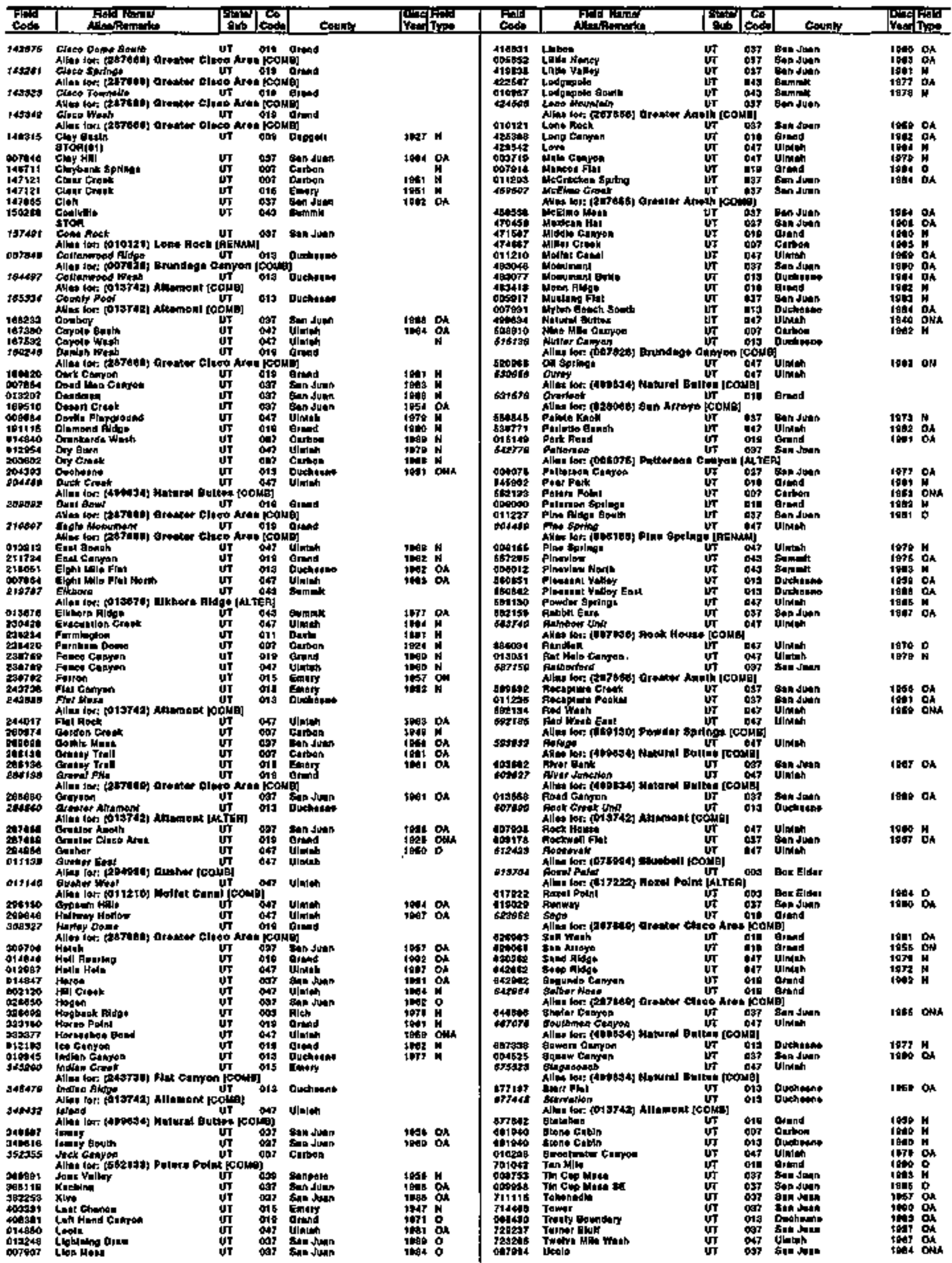


WEST VIRGINIA

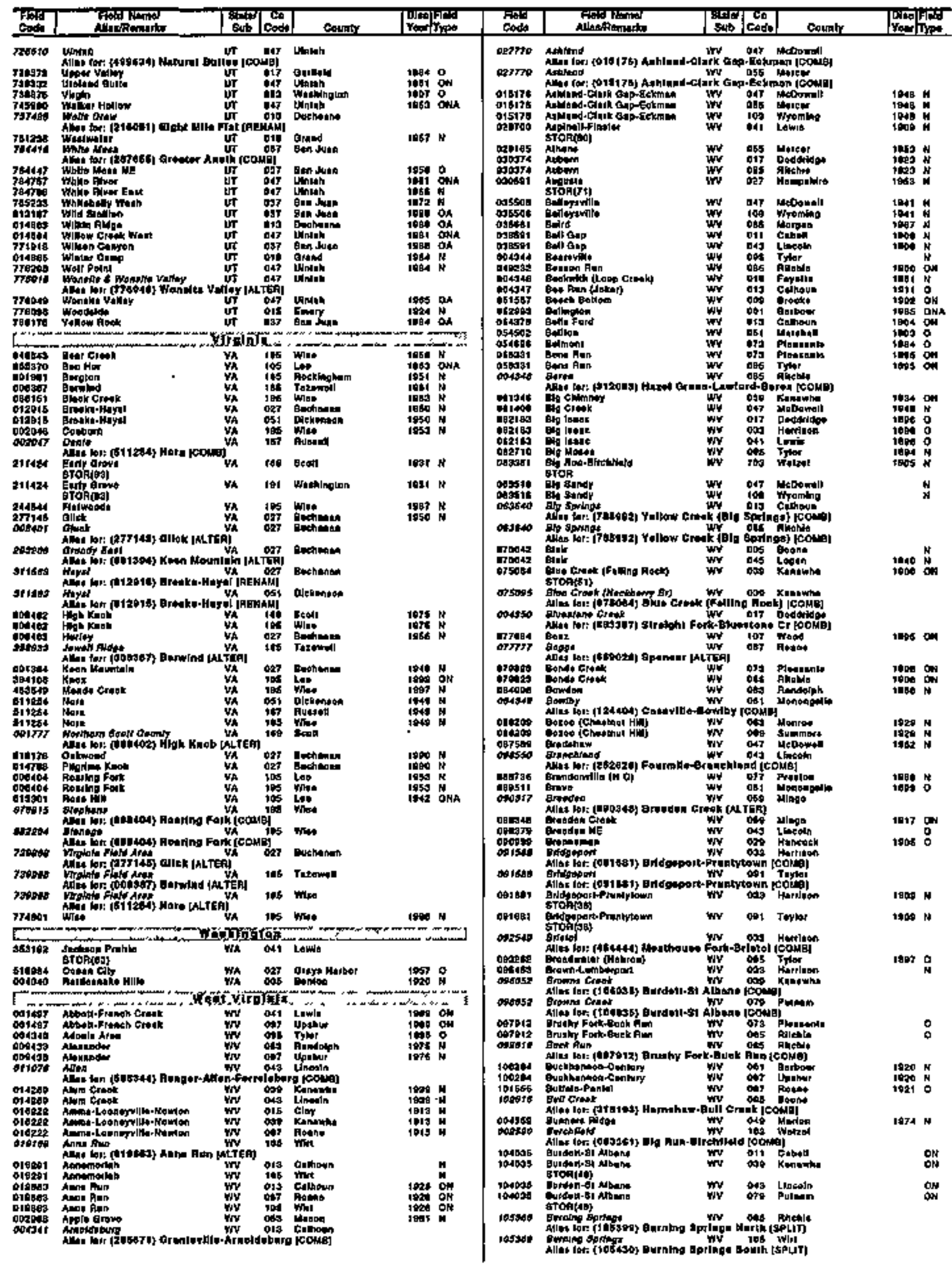




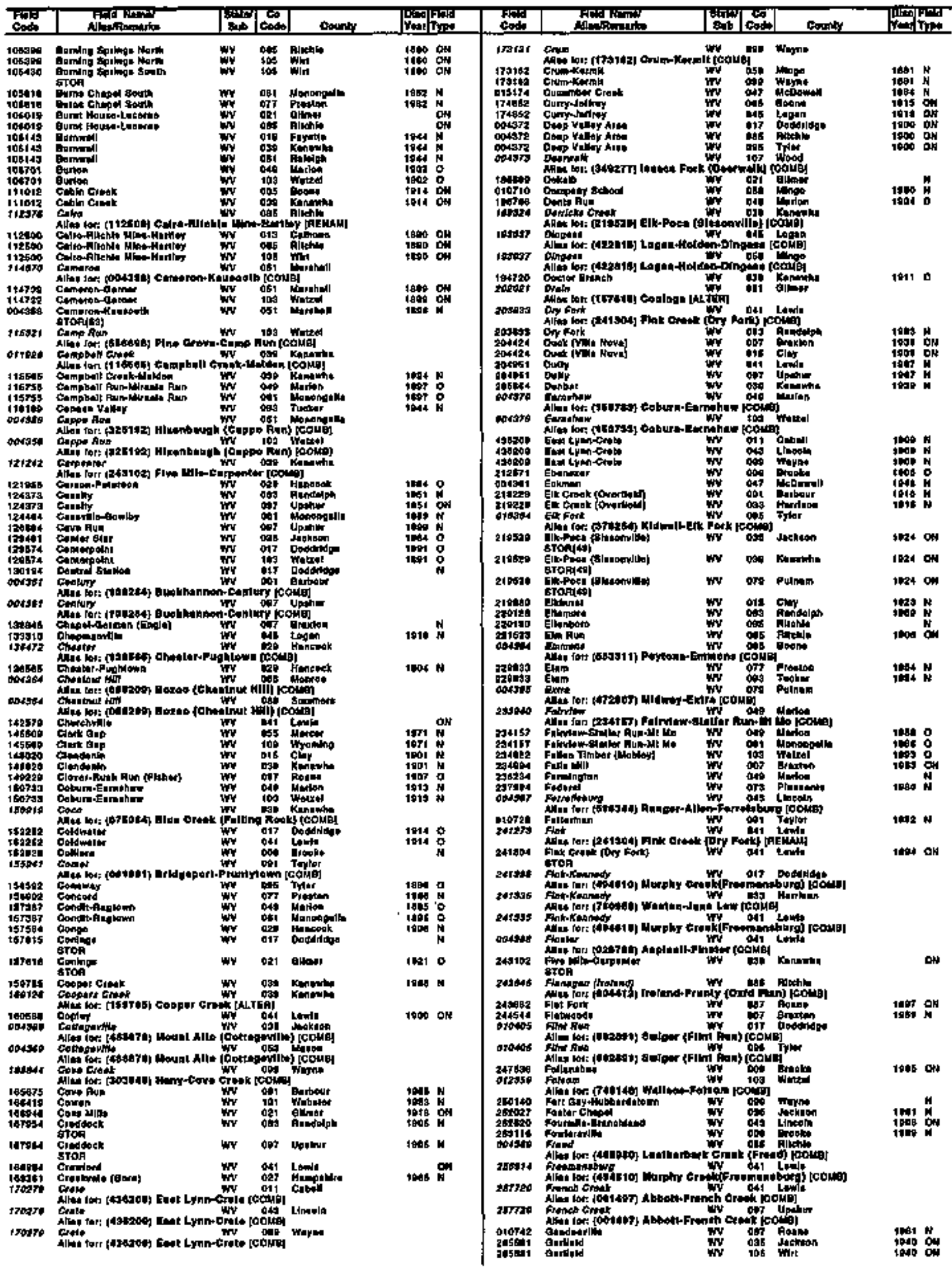


WEST VIRGINIA

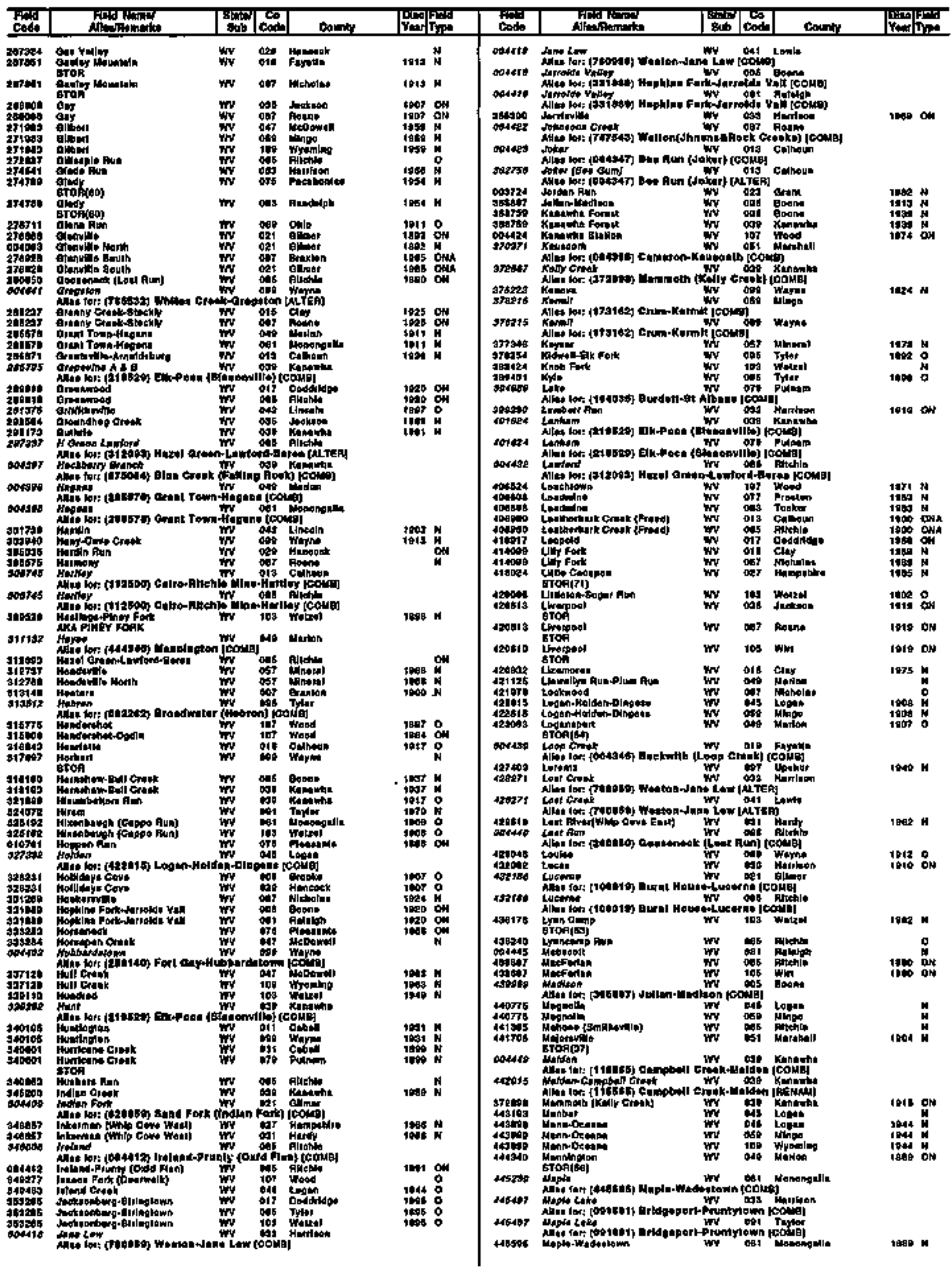




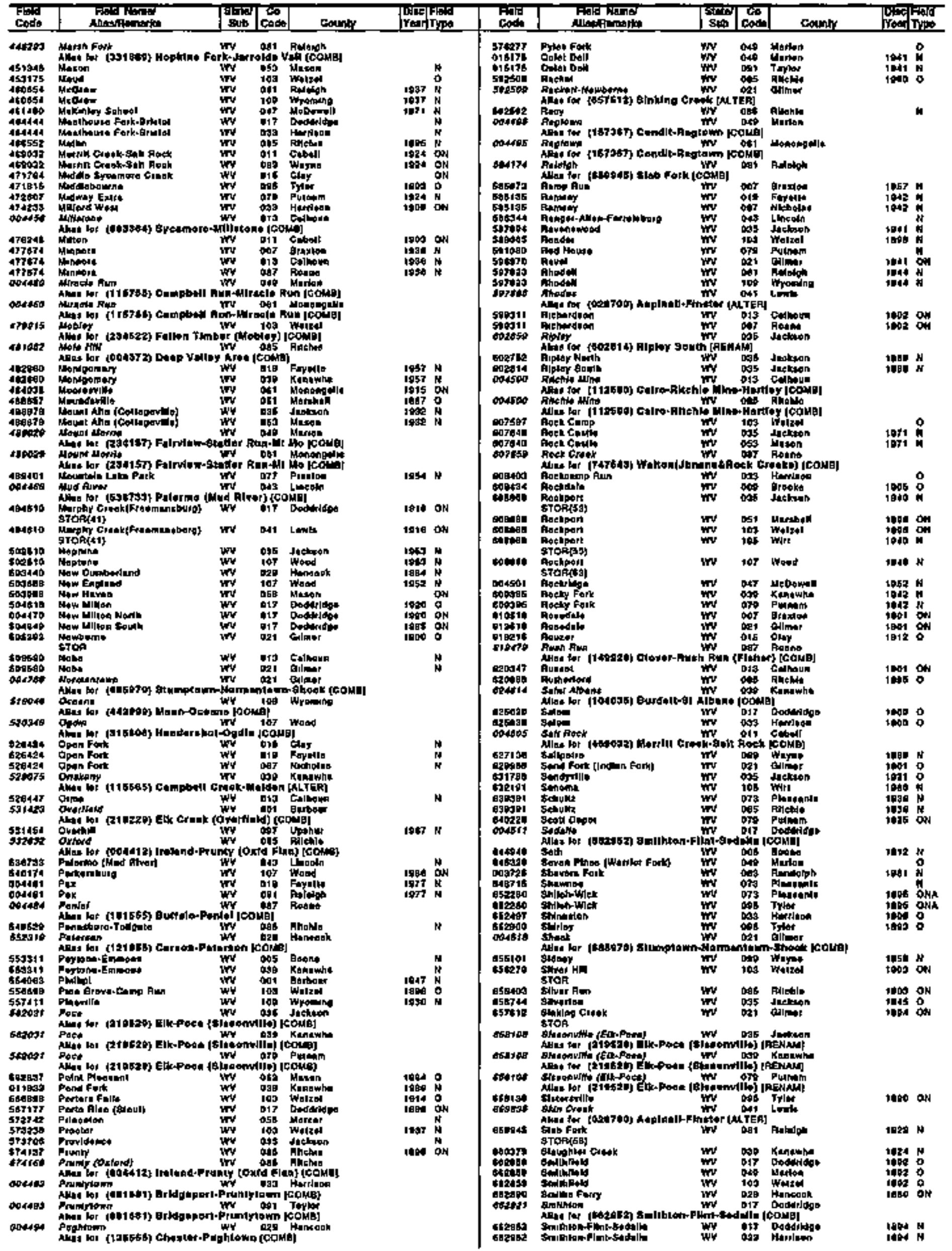


WYOMING

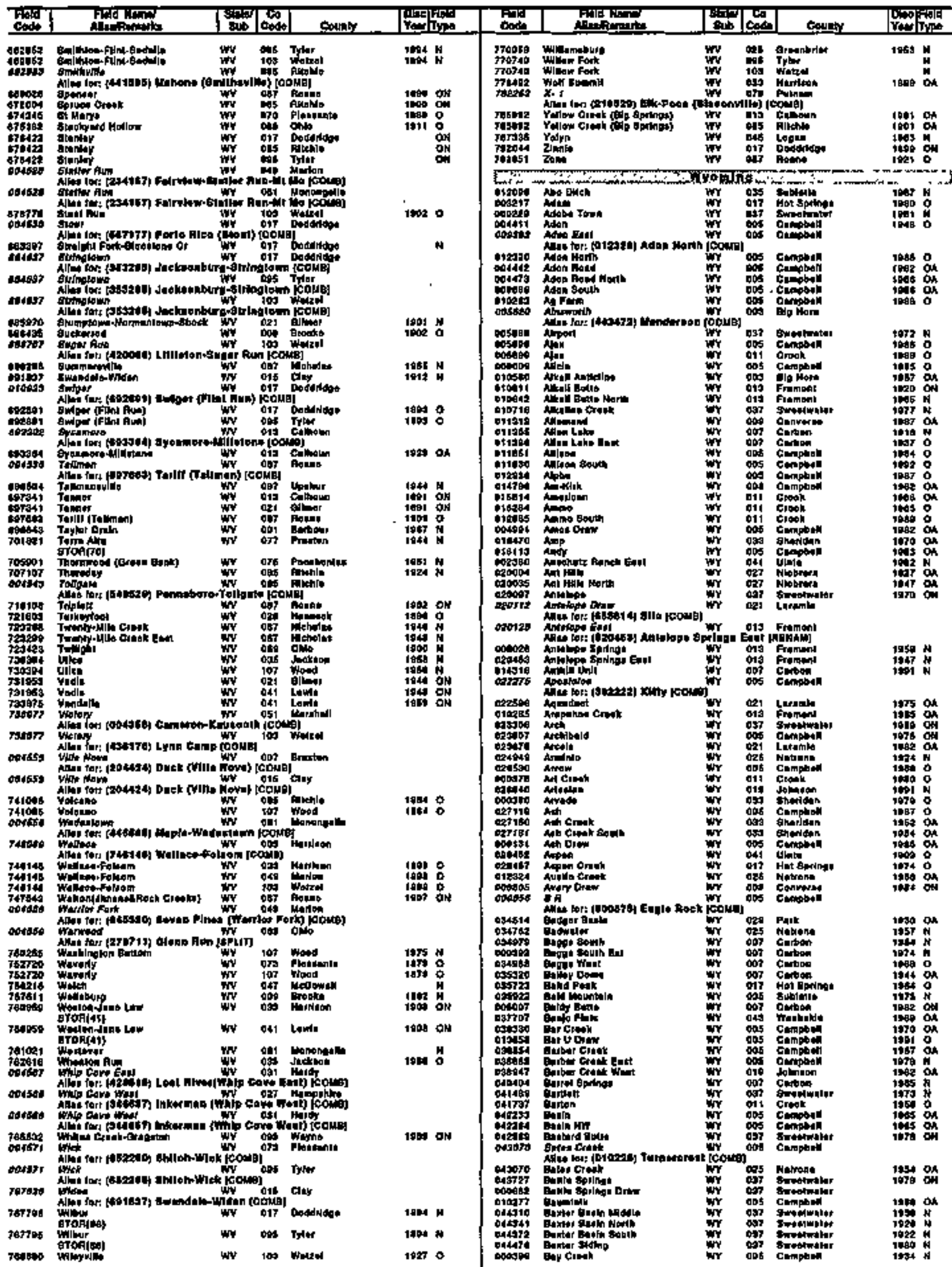




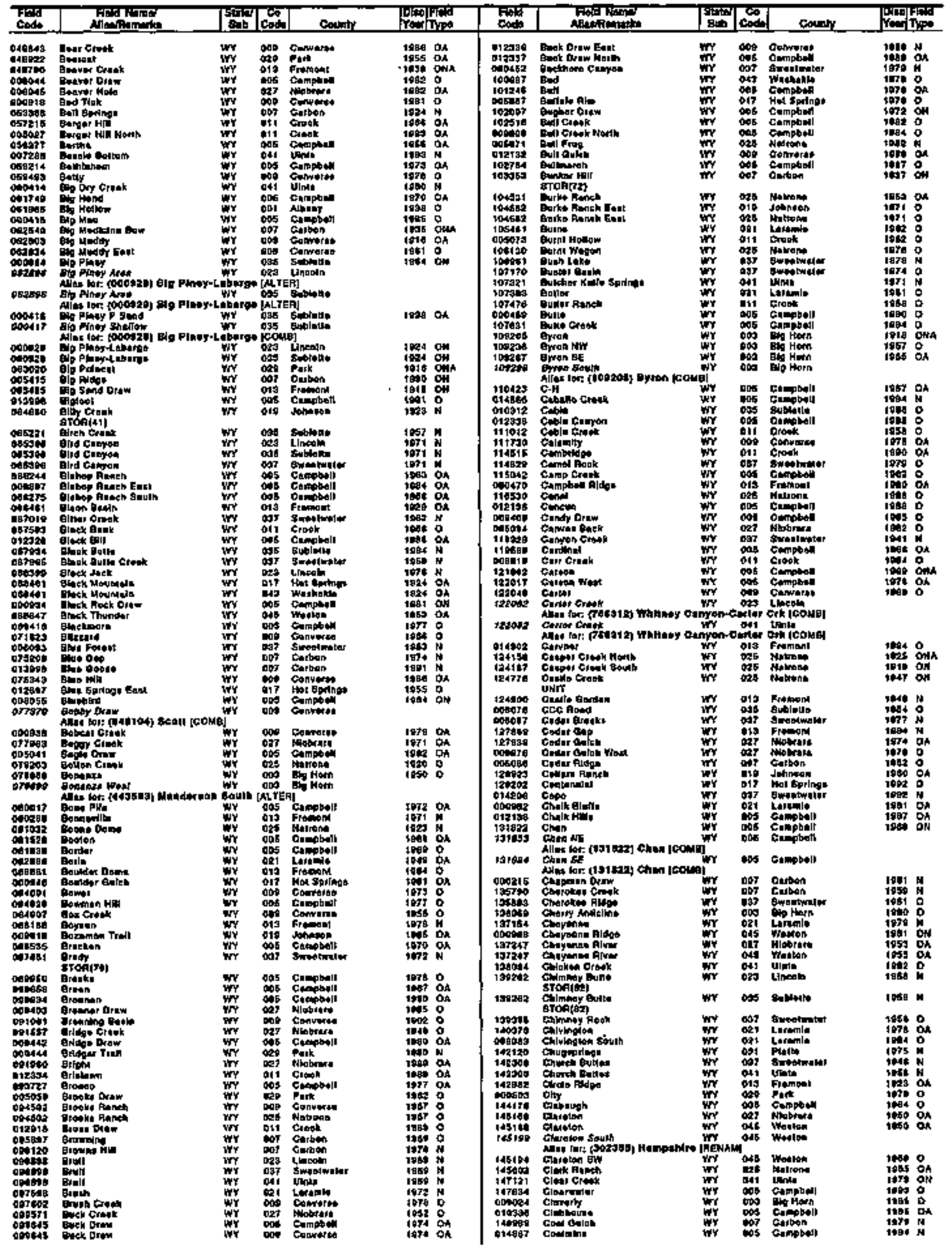


WYOWING

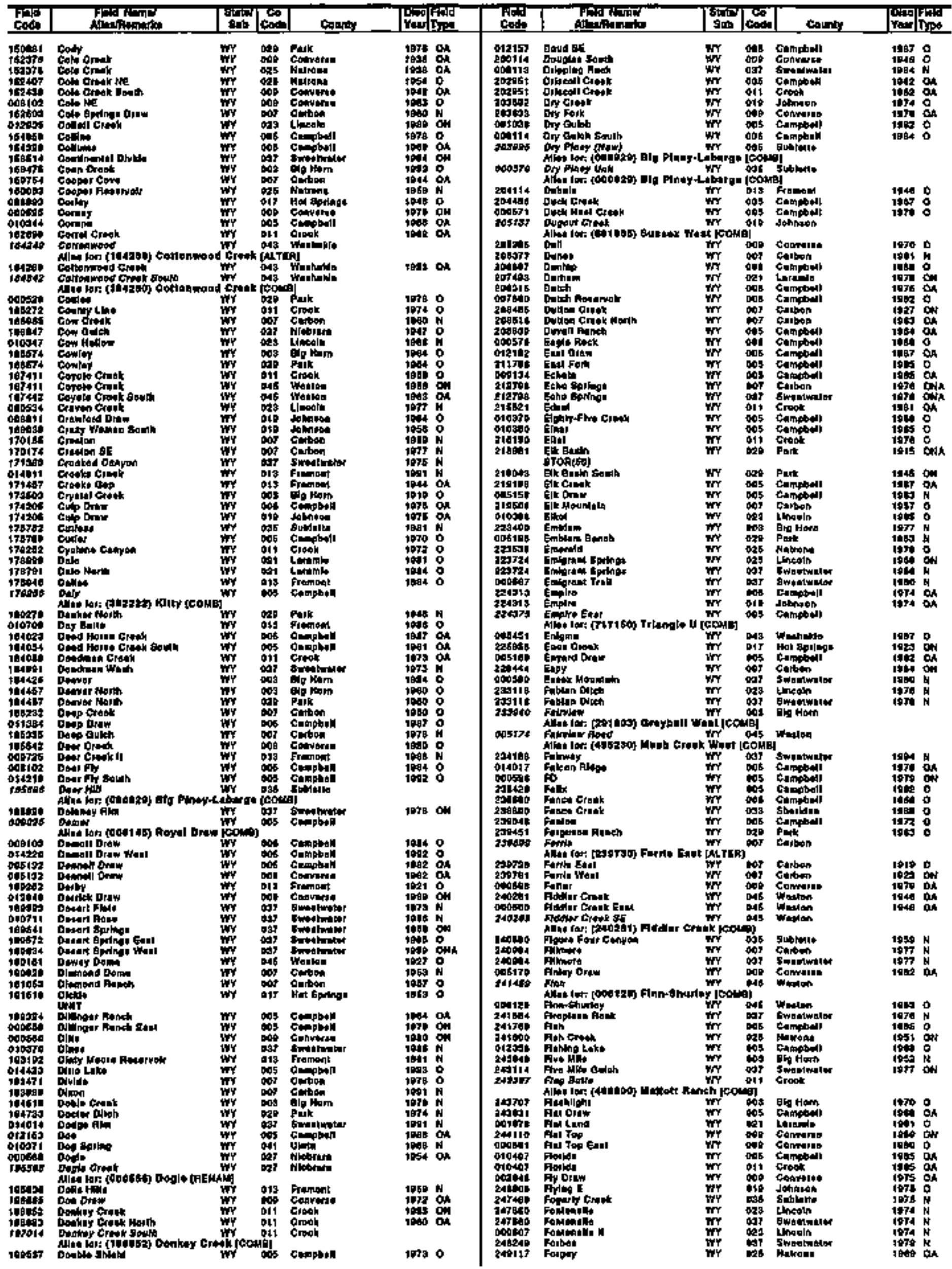




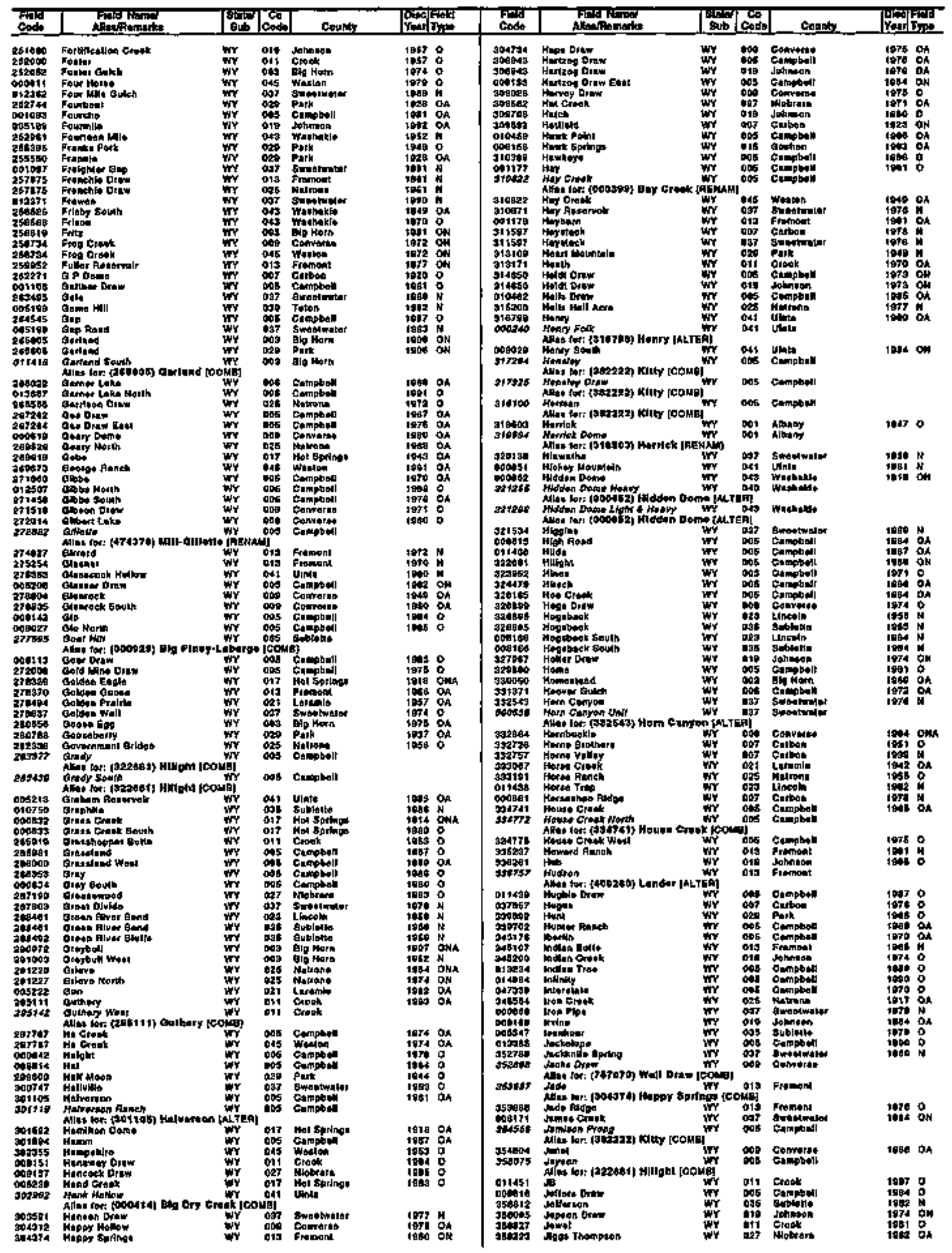


WYOMING

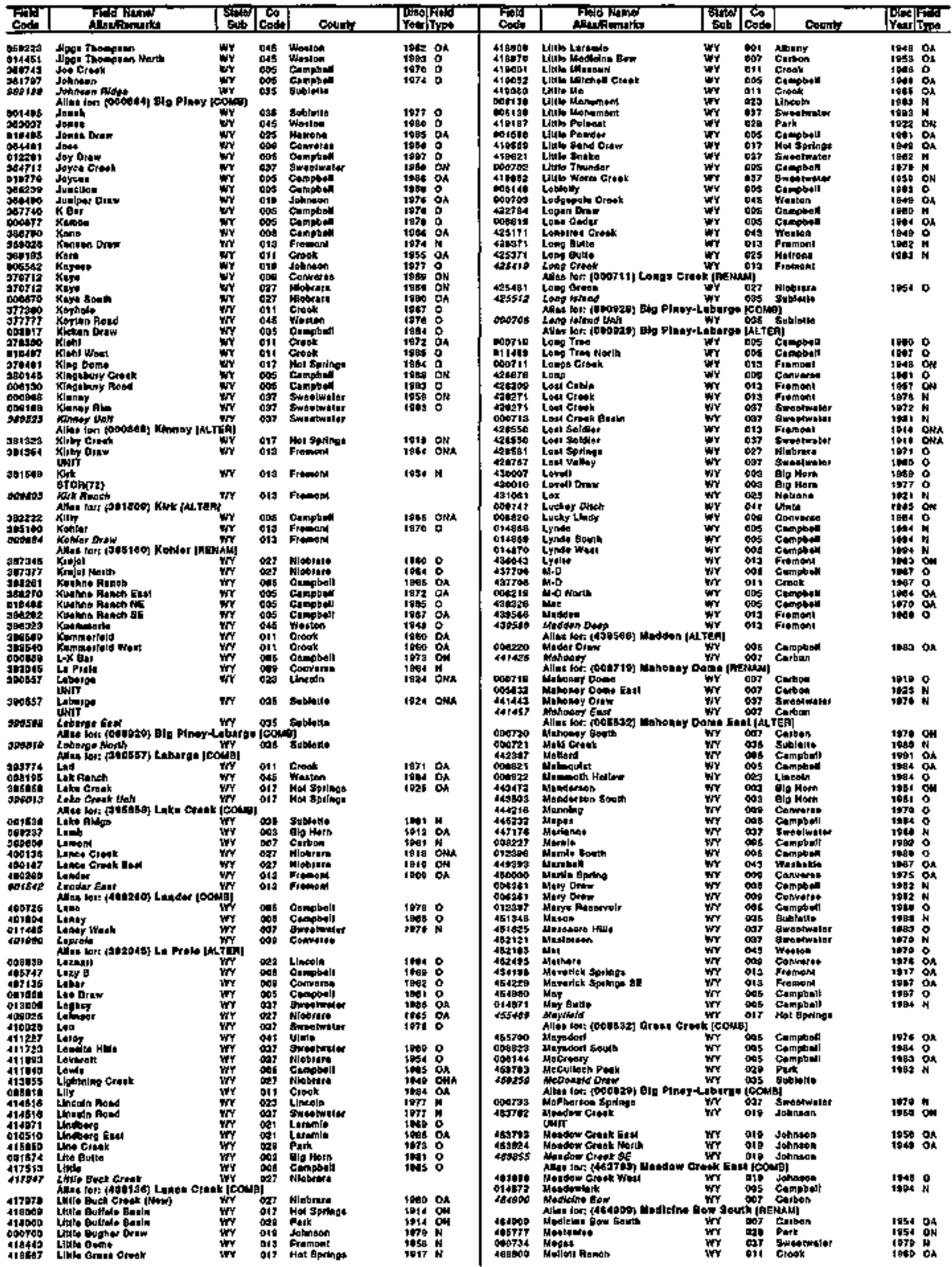




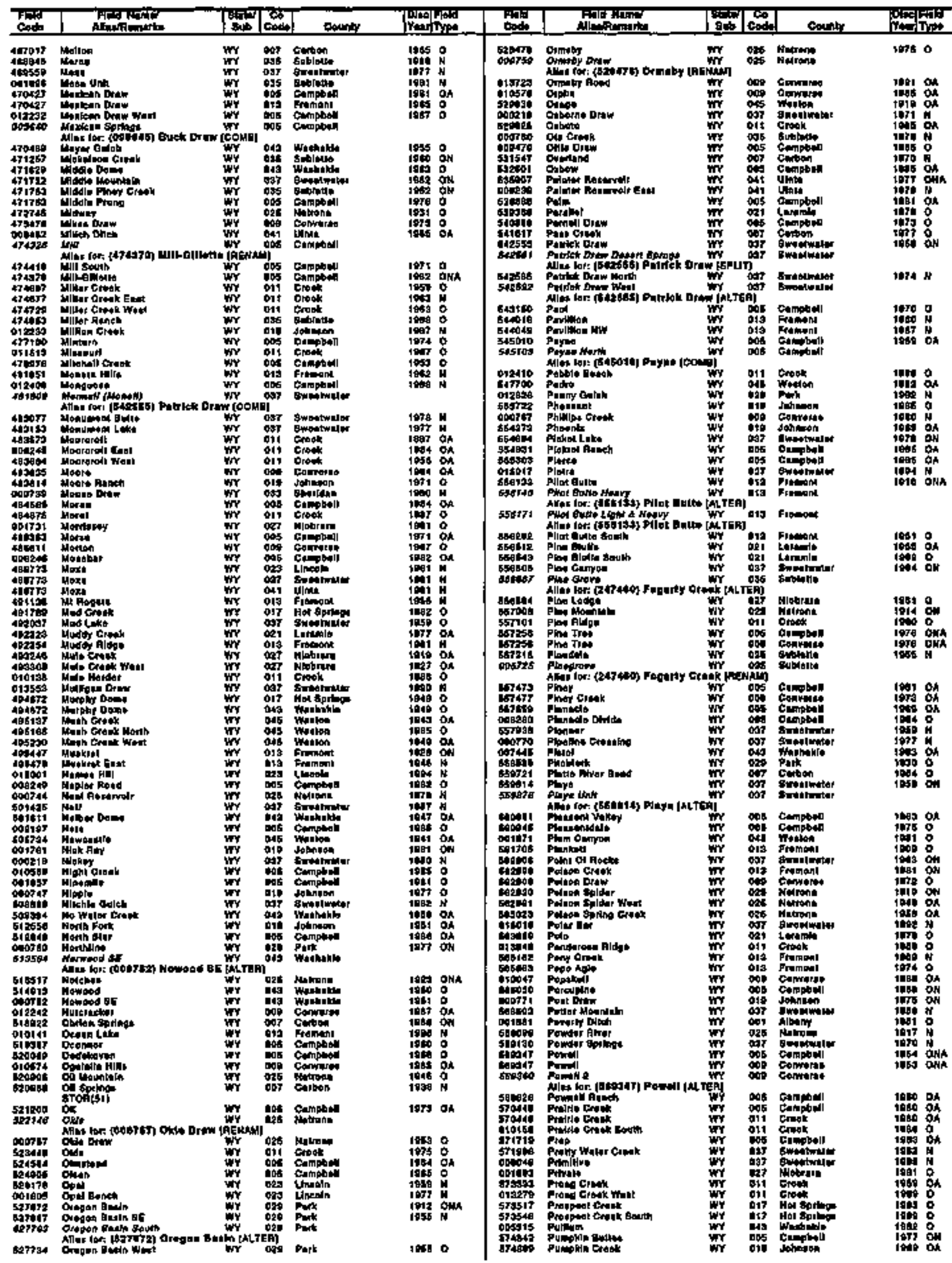


WYOMING

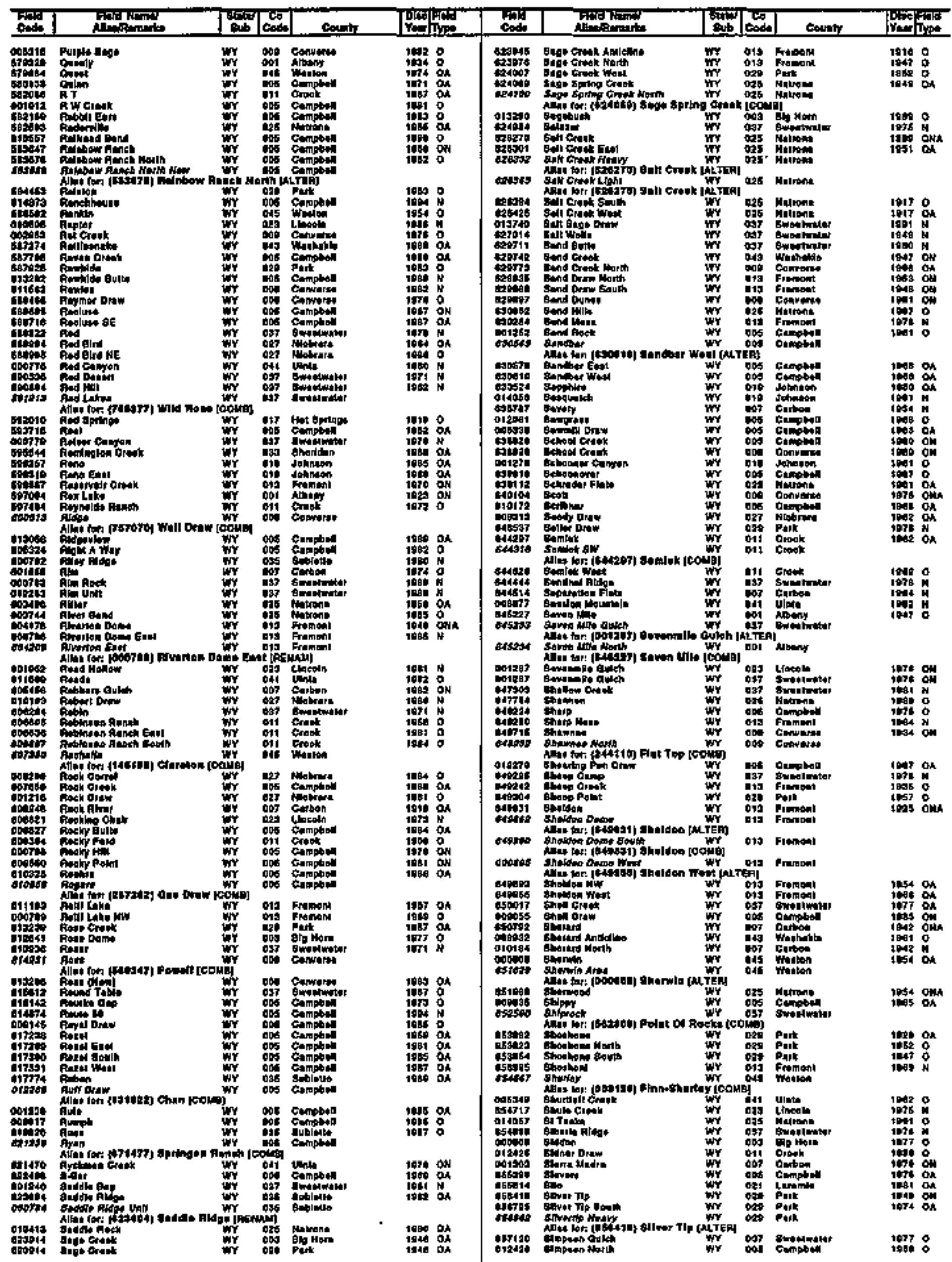




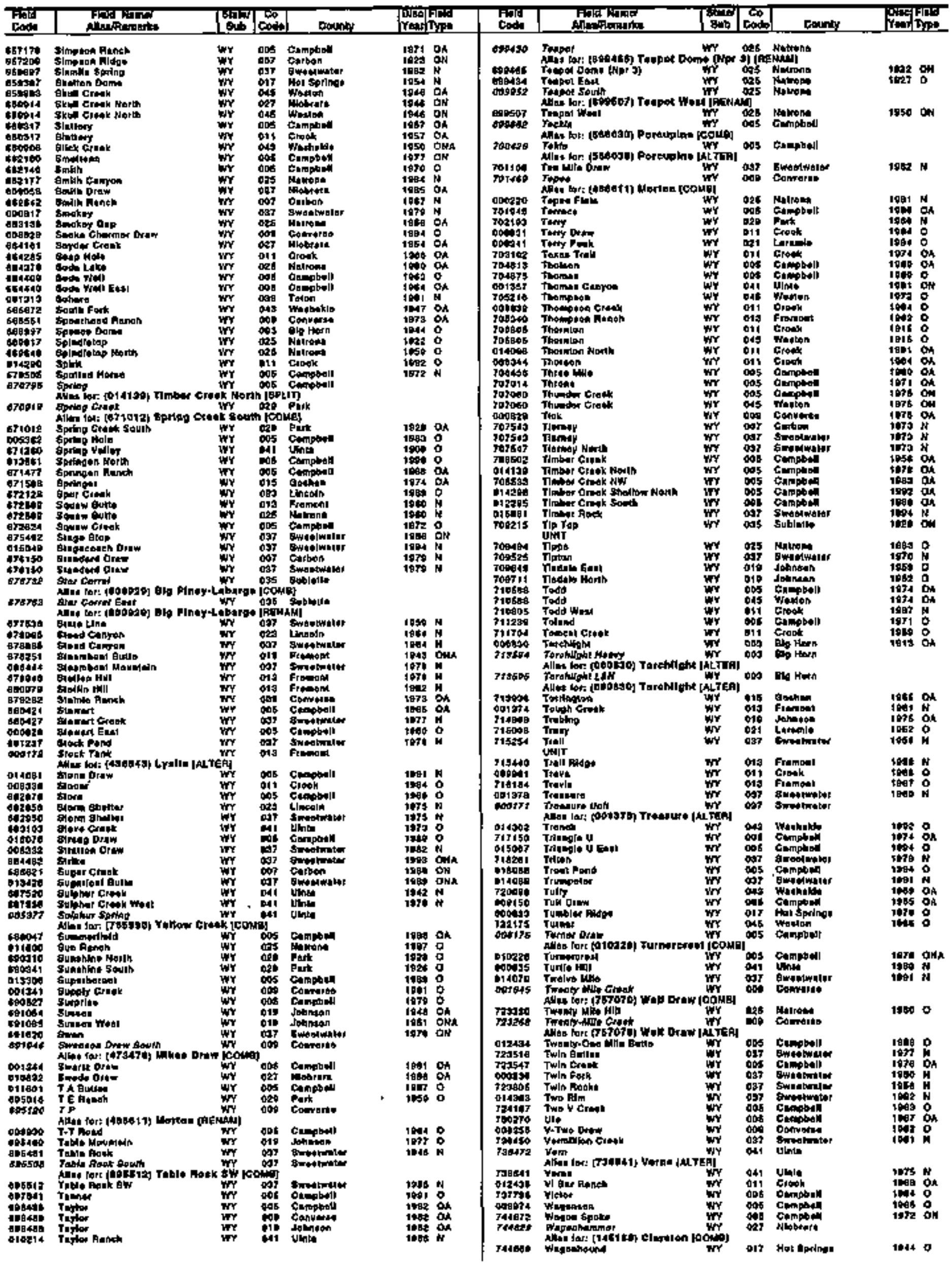




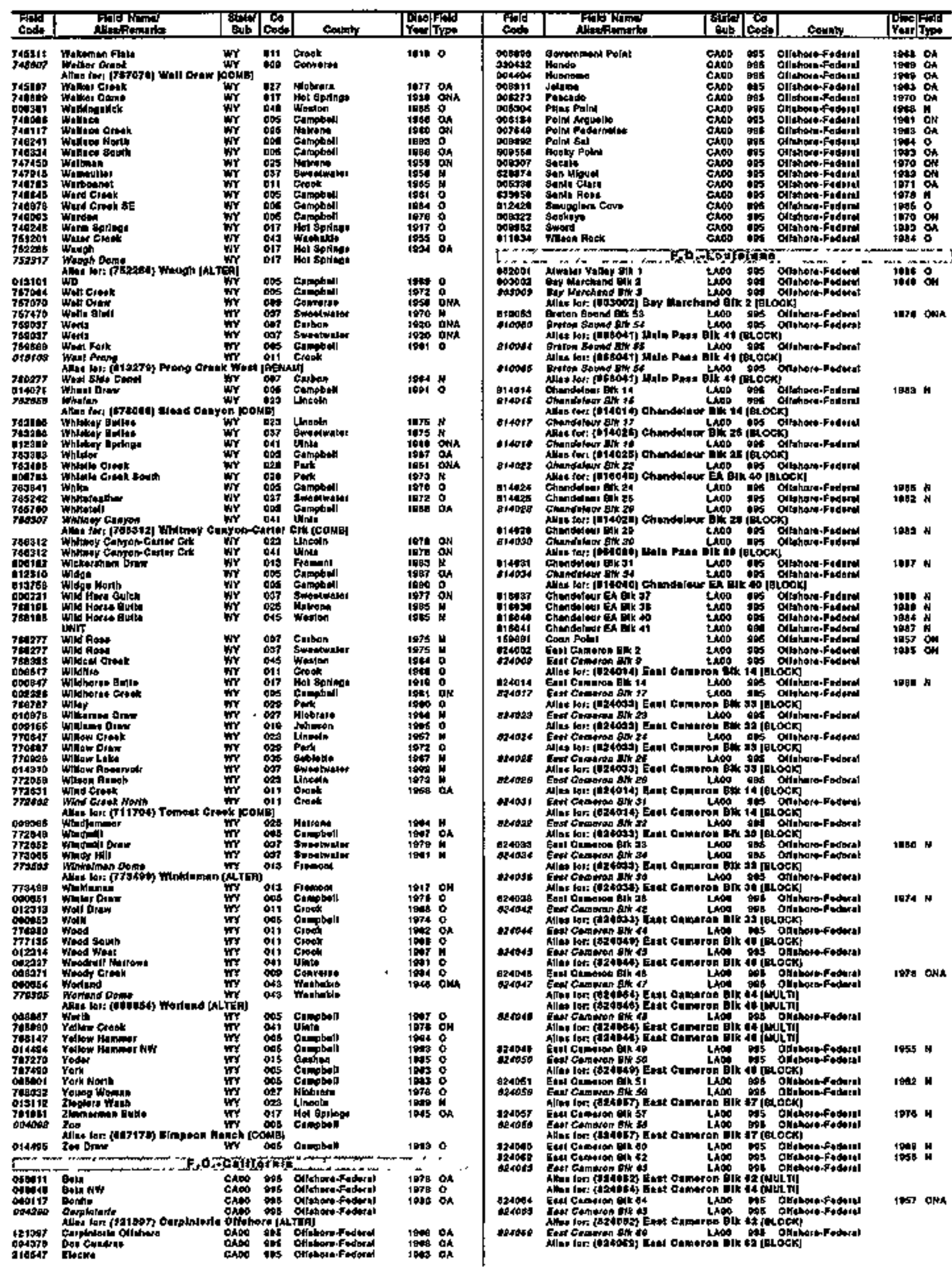




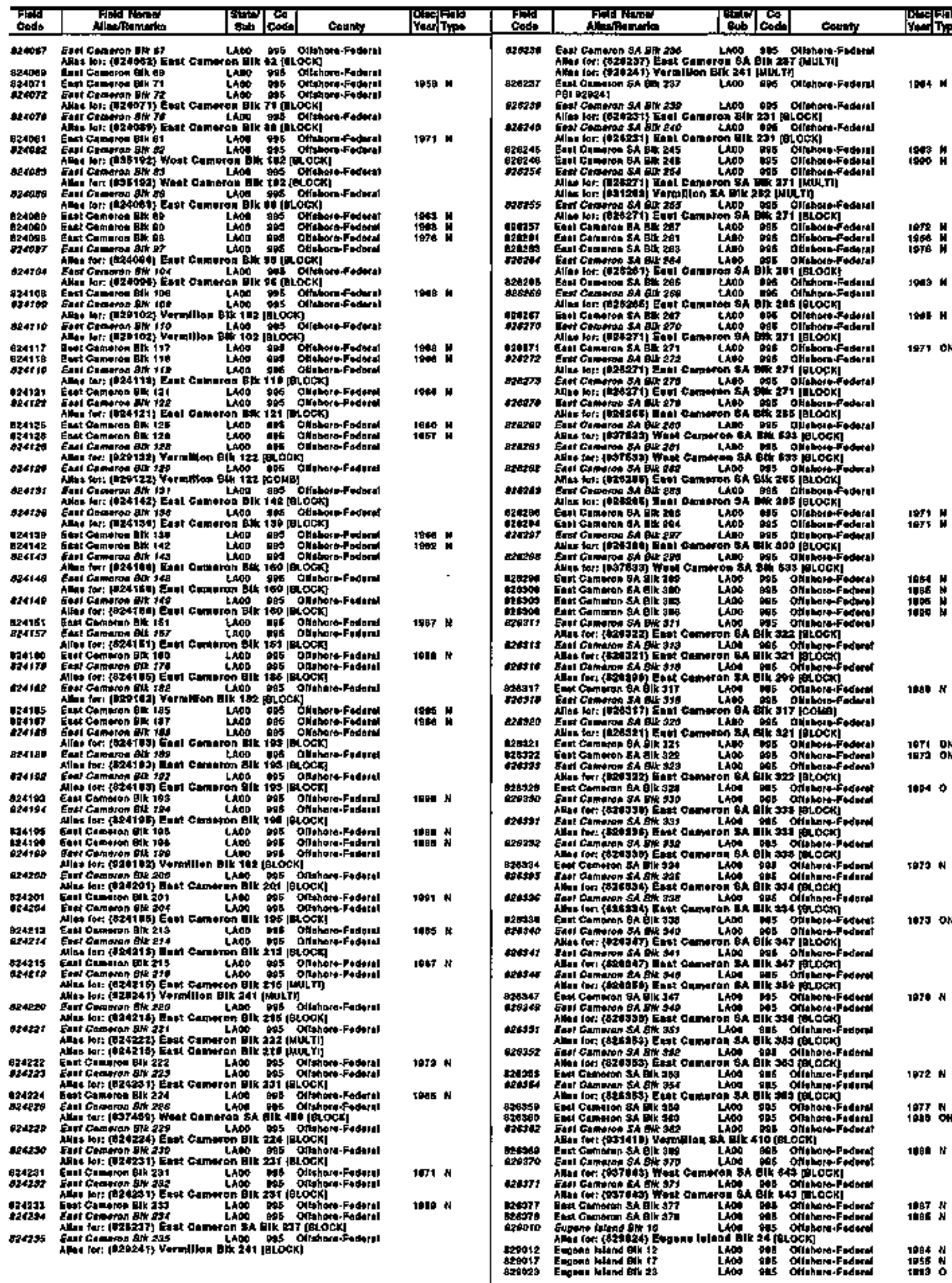




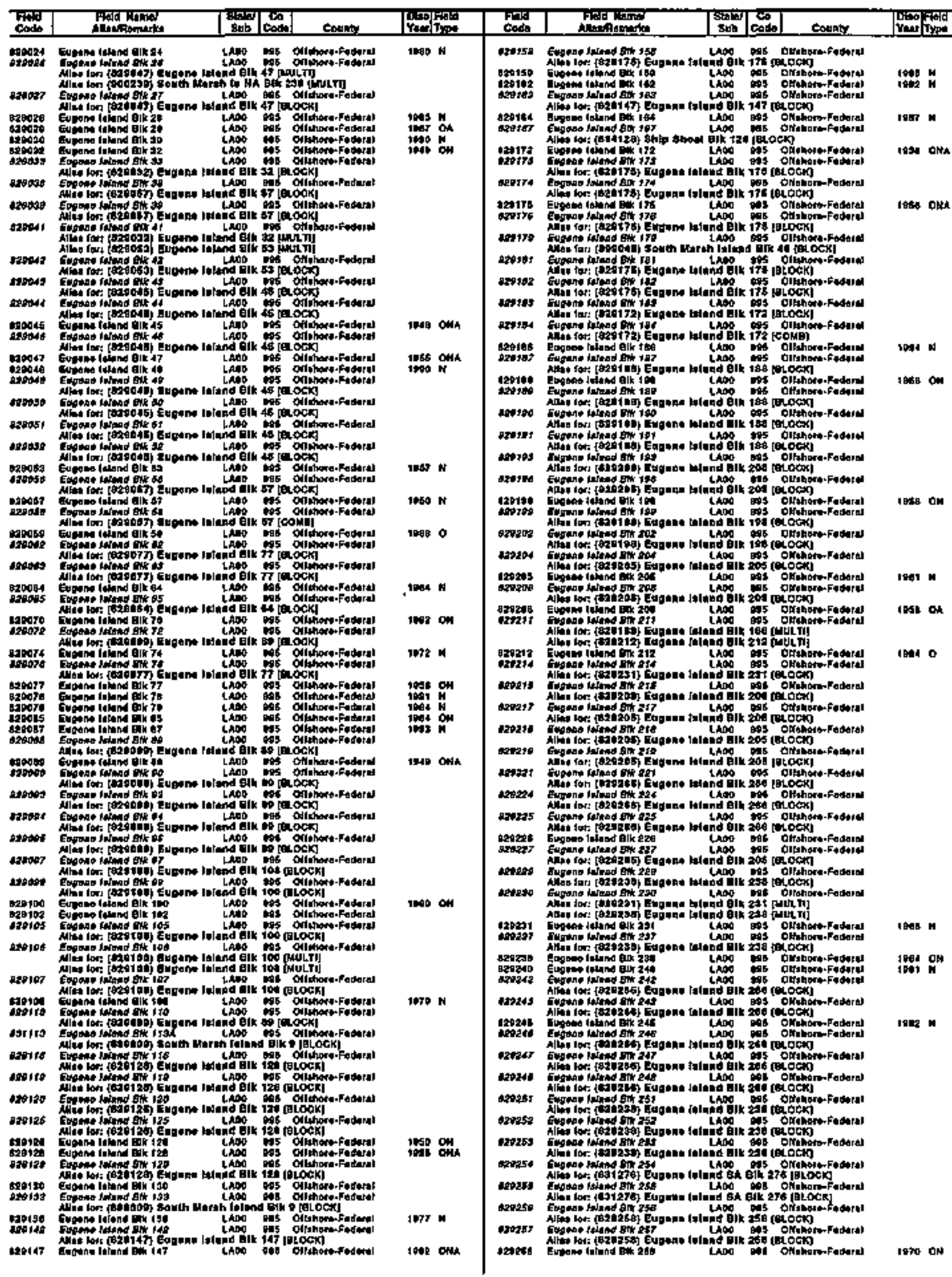




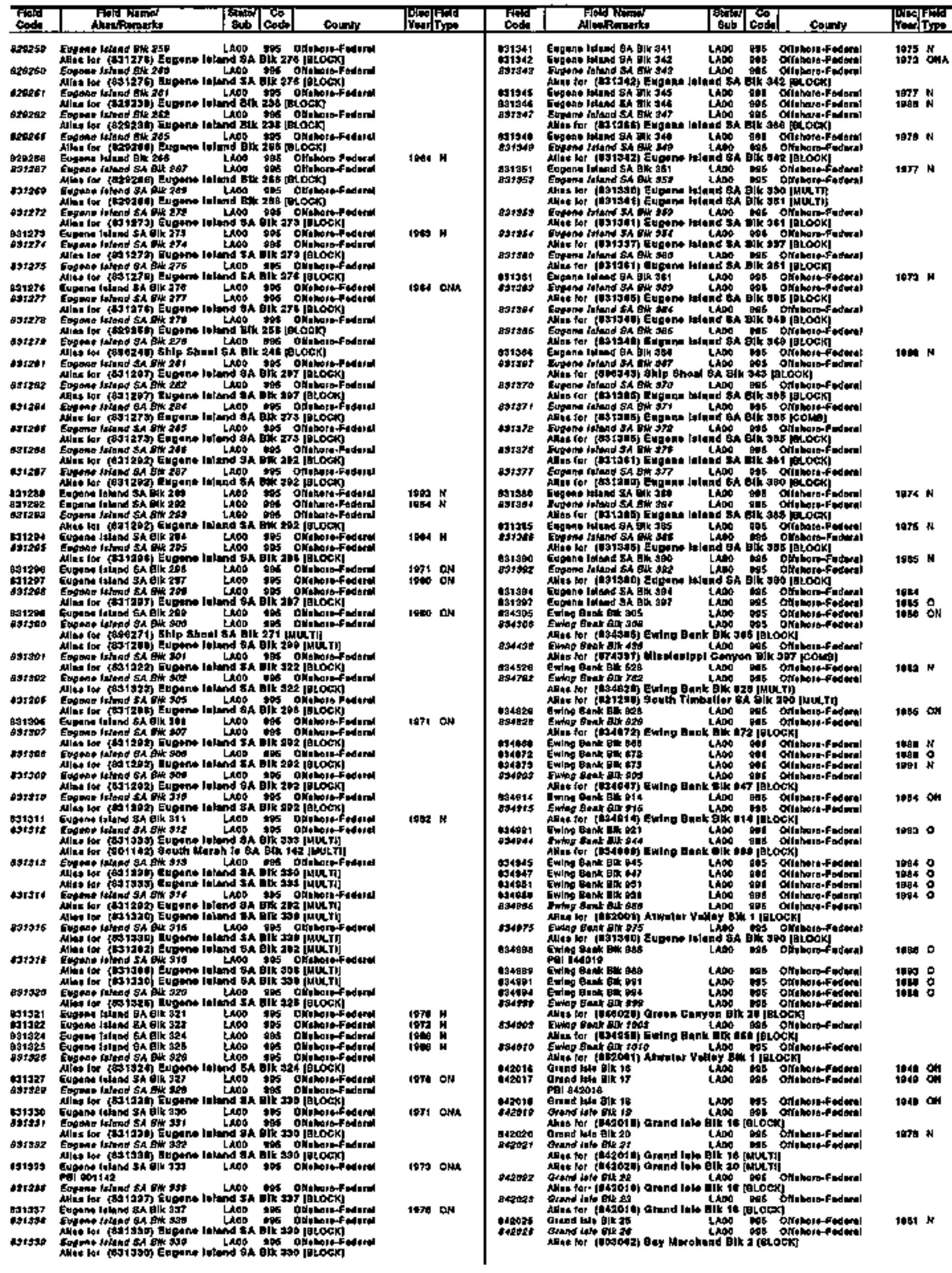




\begin{tabular}{|c|c|c|c|}
\hline Geds & 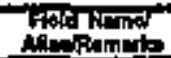 & $\mid \begin{array}{c}\infty \\
\text { Coded }\end{array}$ & Coustrt \\
\hline
\end{tabular}

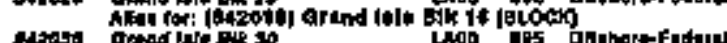

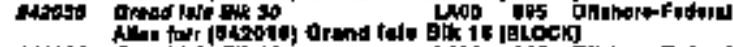

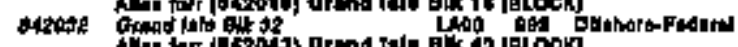

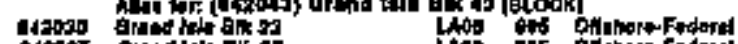

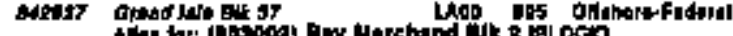

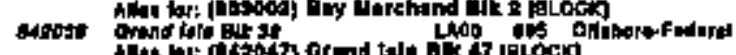

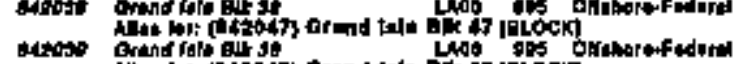

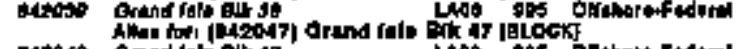

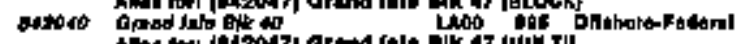

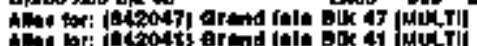

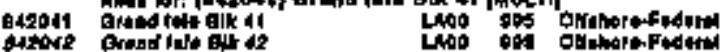

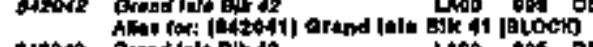

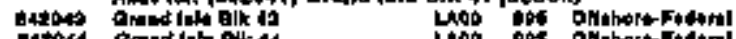

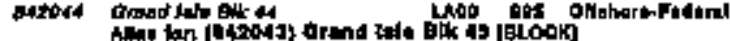

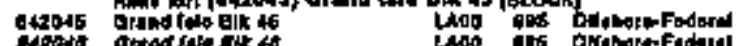

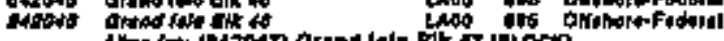

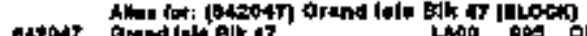

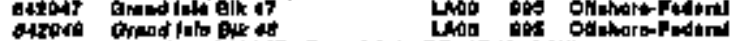

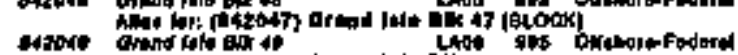

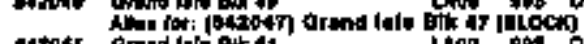

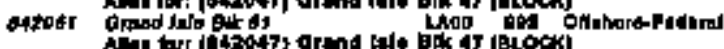

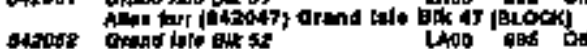

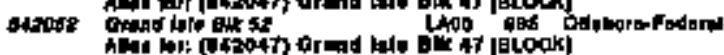

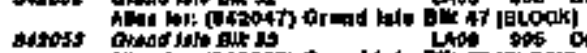

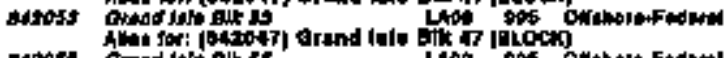

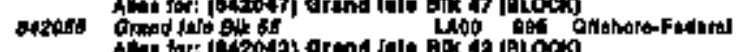

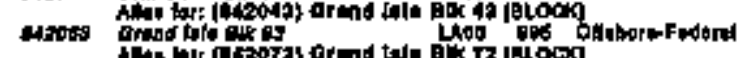

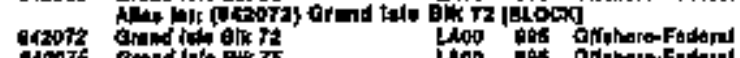

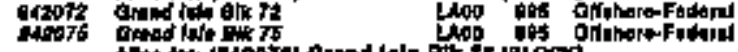

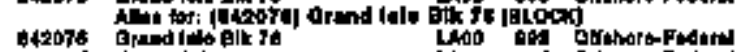

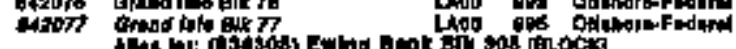

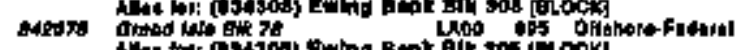

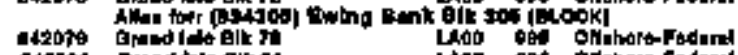

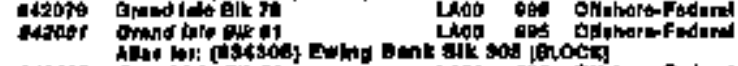

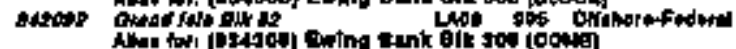

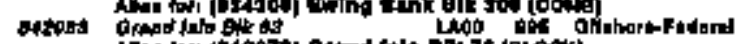

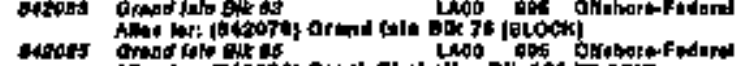

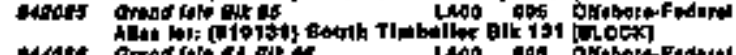

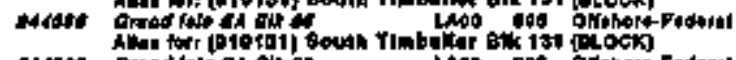

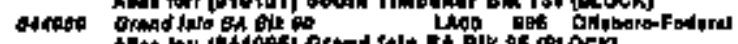

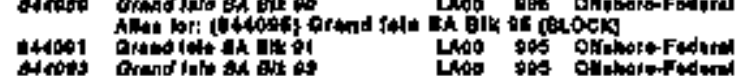

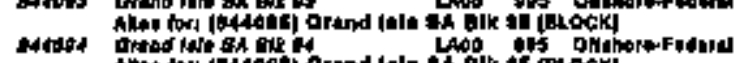

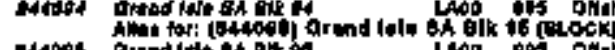

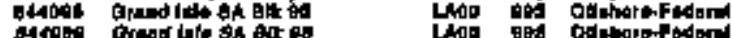

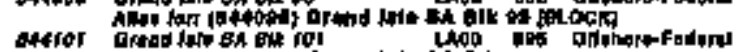

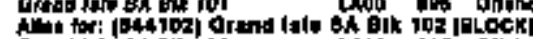

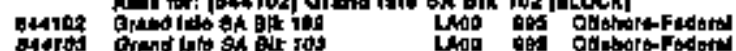

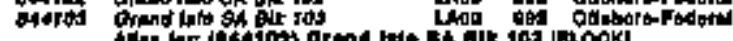

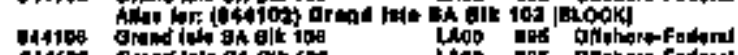

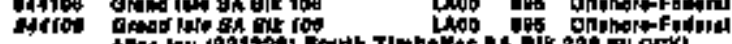

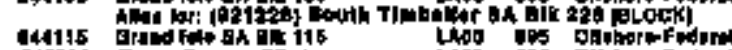

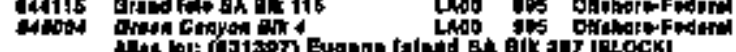

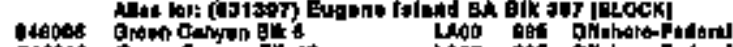

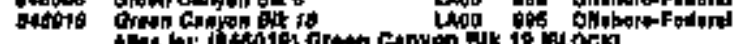

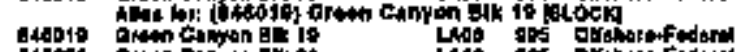

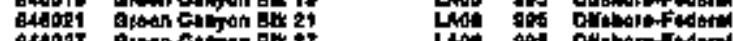

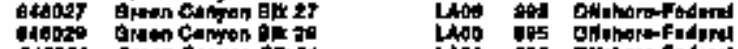

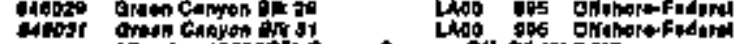

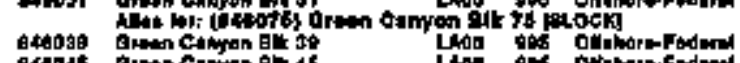

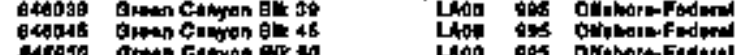

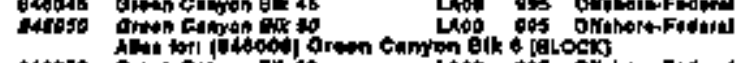

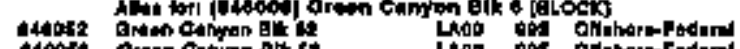

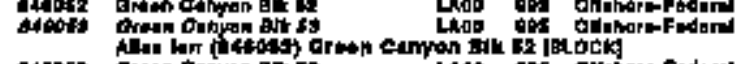

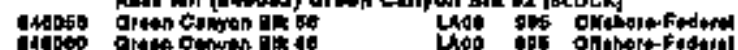

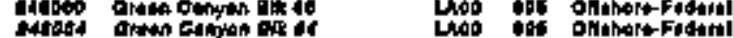

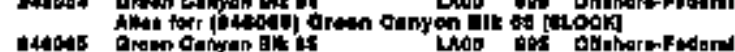

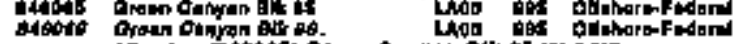

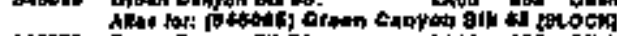

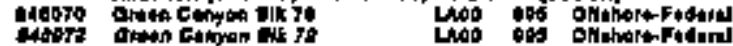

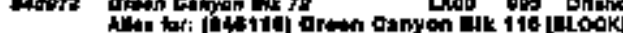

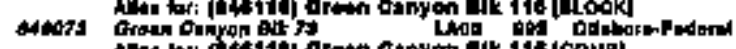

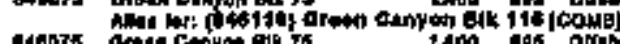

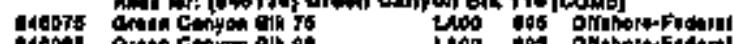

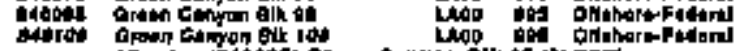

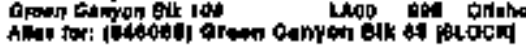

ting wat

Thats

cods

Hog hinet

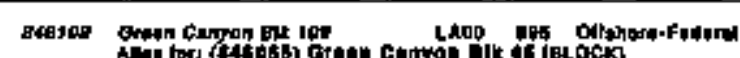

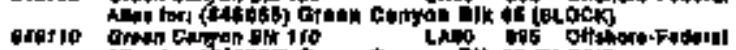

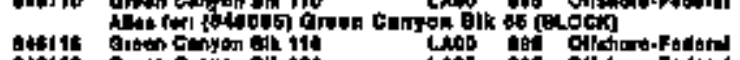

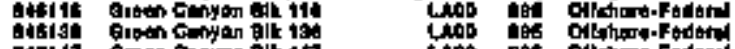

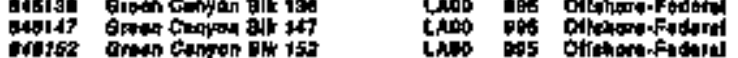

1900

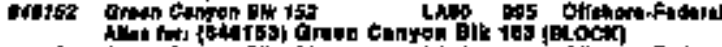

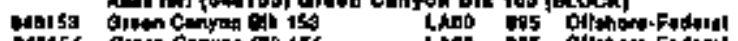

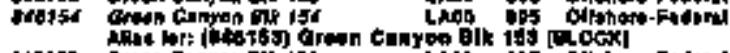

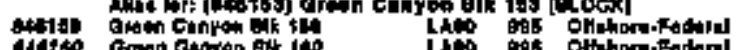

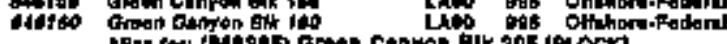

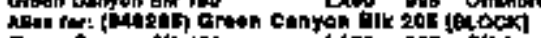

1080 on

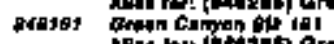

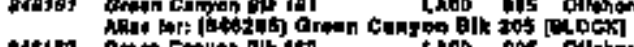

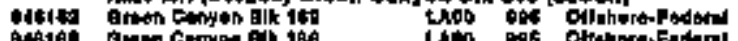

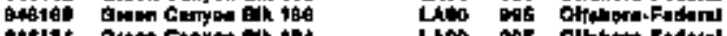

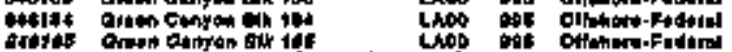

1940 ONA

Ibs $\mathrm{k}$

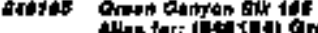

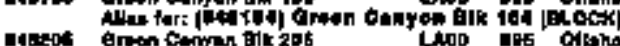

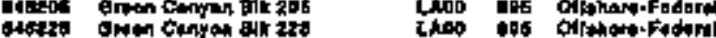

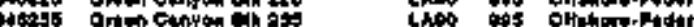

1958 on

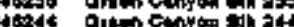

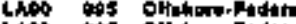

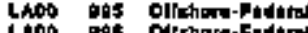

Lico of Odishare-Fudind

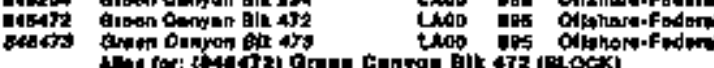

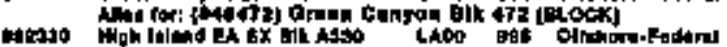

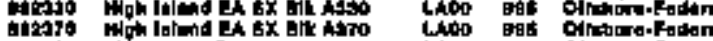

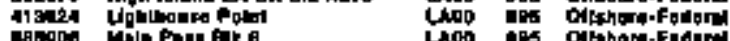

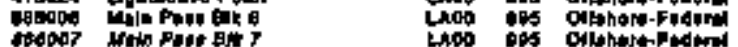

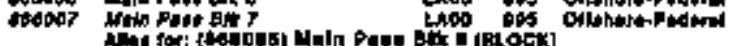

itcols Nhis pare git to

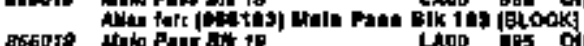

86605s itpa Panr

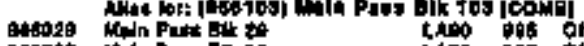

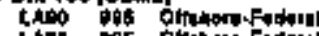

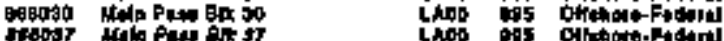

16할 $N$

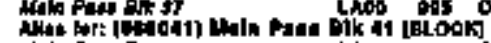

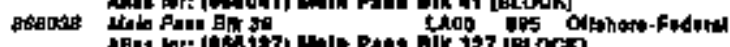

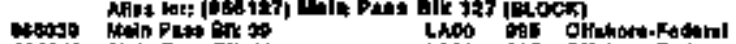

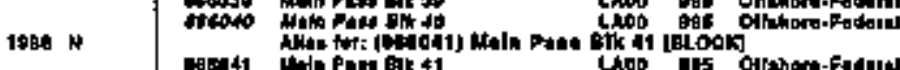

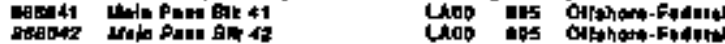

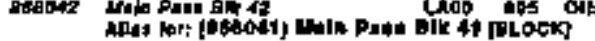

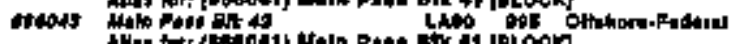

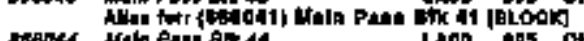

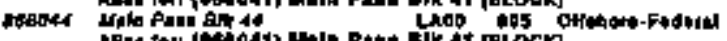

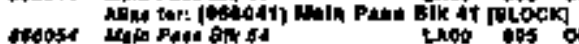

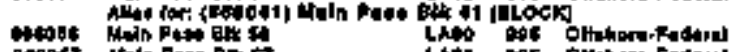

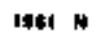

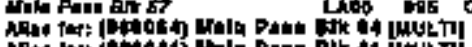

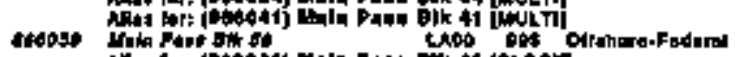

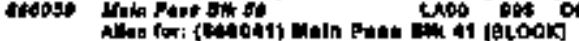

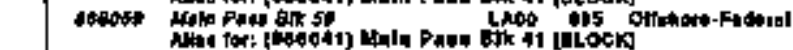

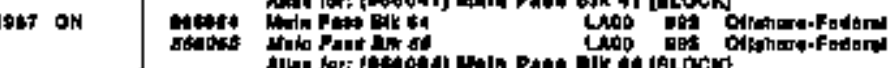

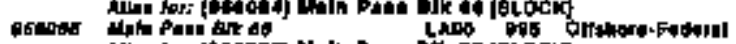

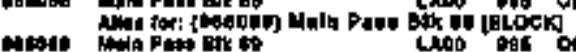

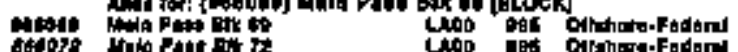

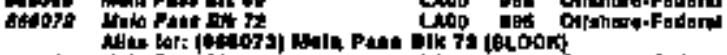

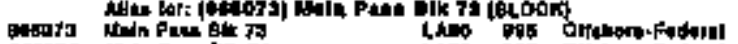

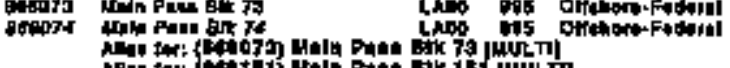

1074 $N$

10pa

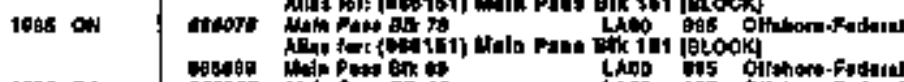

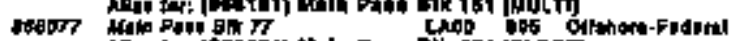

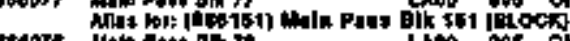

1910 on

19.04

10in

190

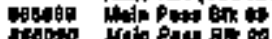

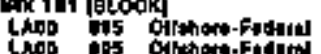

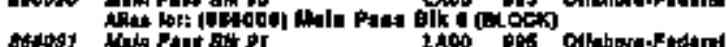

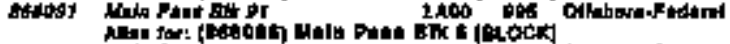

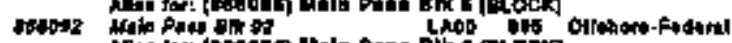

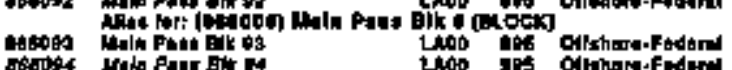

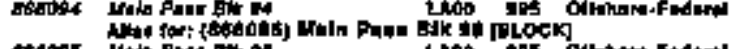

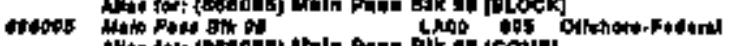

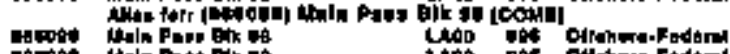

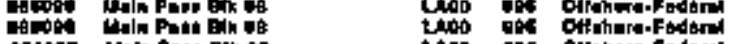

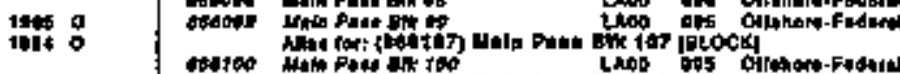

1014

10840

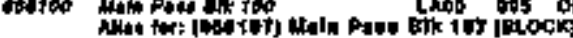

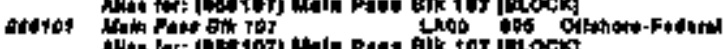

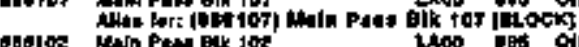

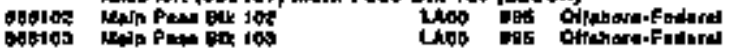

-

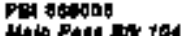

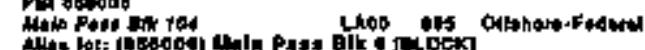

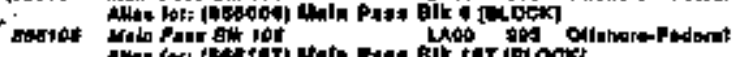

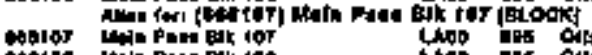

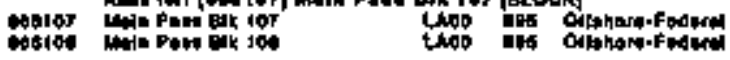

ins on

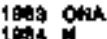

1004

1014 on

soto 0

10:

10101 othd

pors 0

tous

1044 \%

$2004 \%$

19ra $\mathbf{~}$

1056

gof 10

1904 $\mathrm{M}$

109t ons

104. $\mathrm{M}$

10020

198. On

IPrs OWA

104.

1045 $\boldsymbol{M}$

10ap on

rasp on

1016 os 


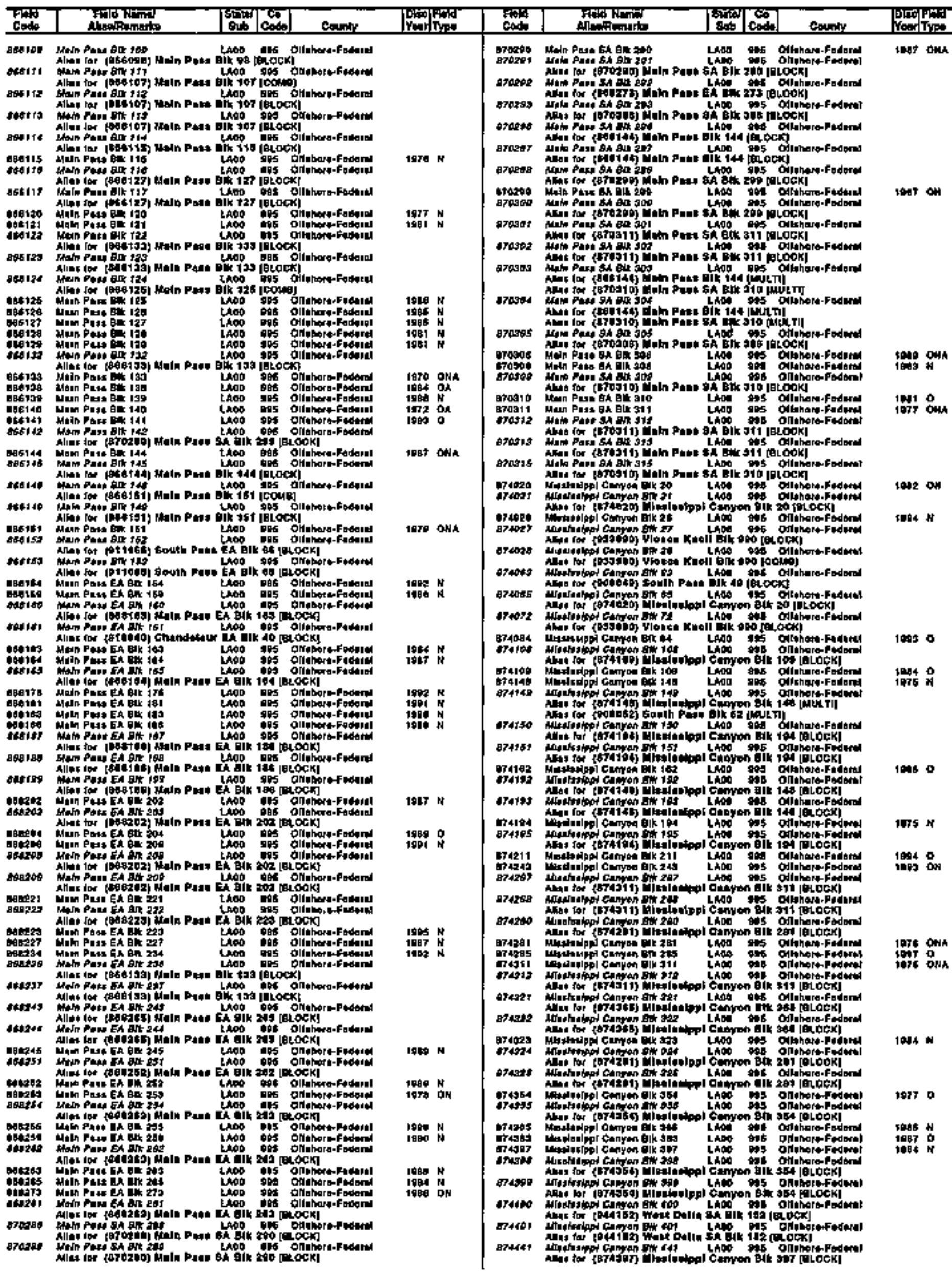




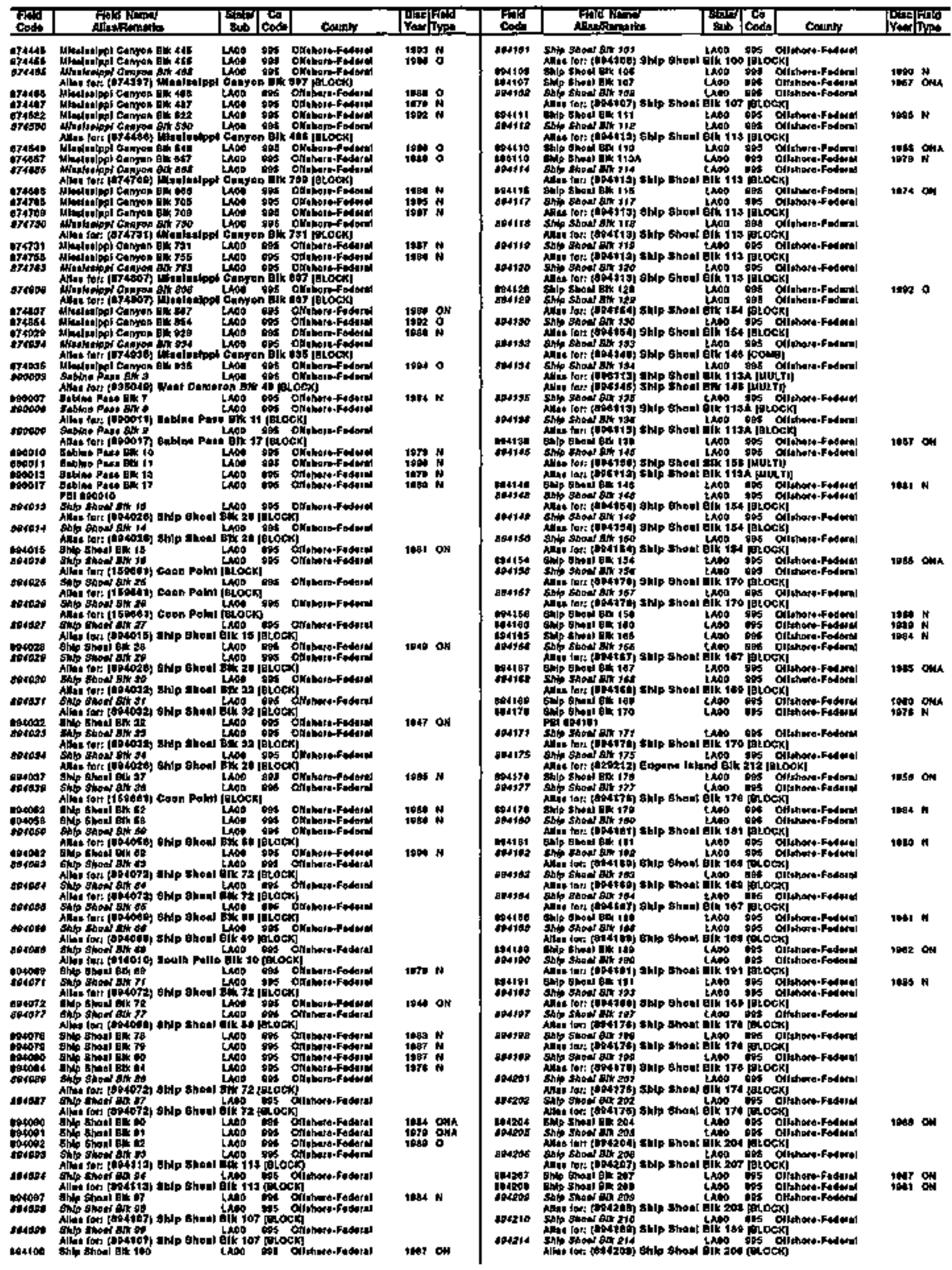




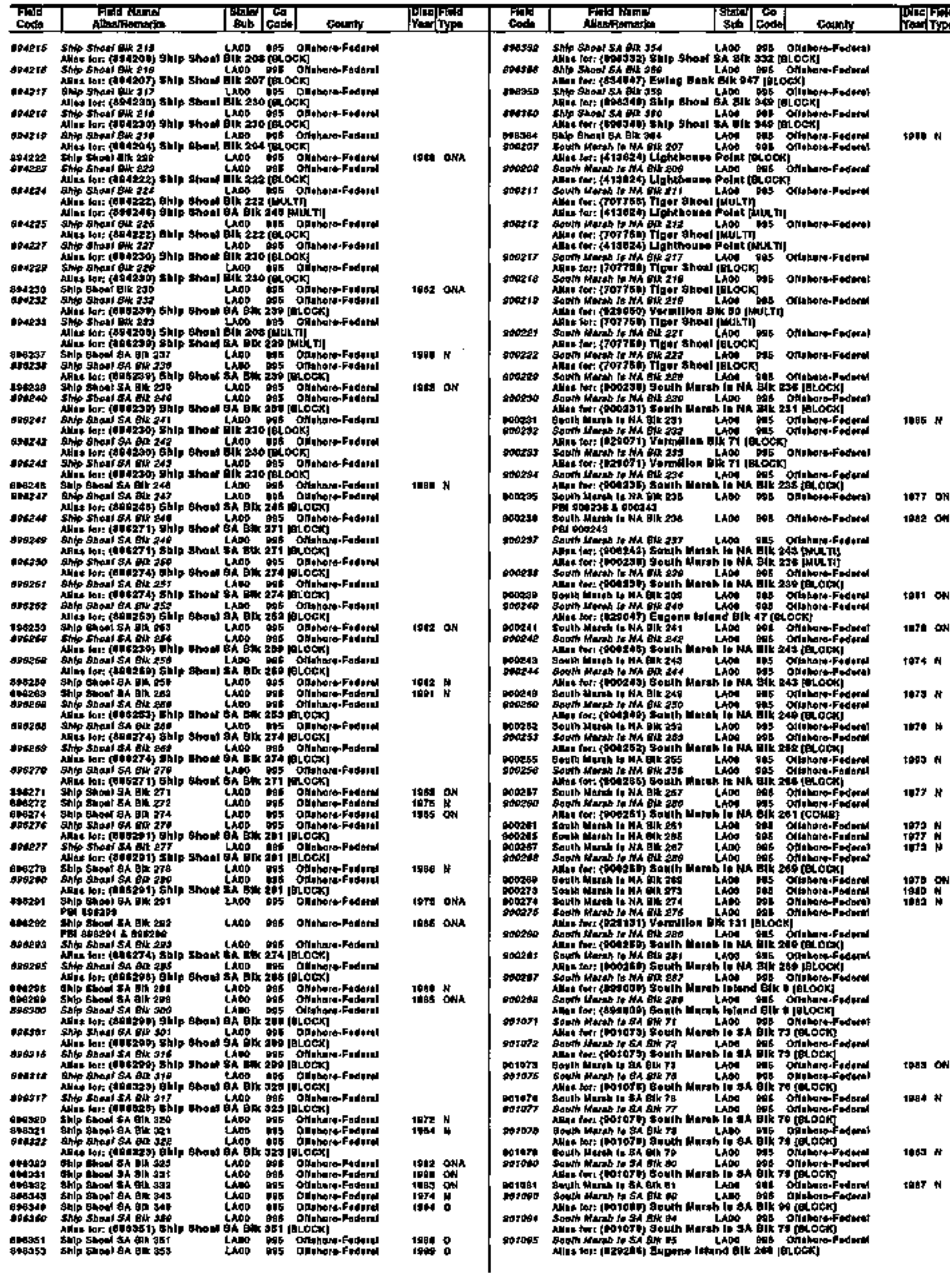




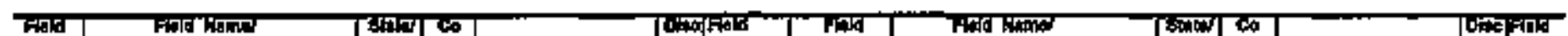

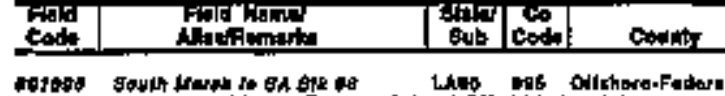

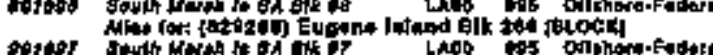

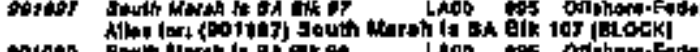

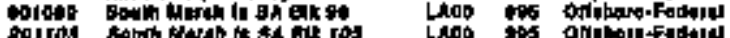

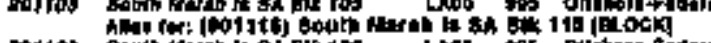

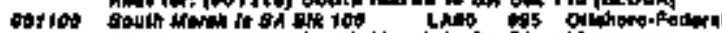

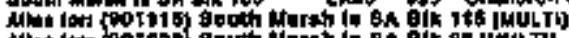

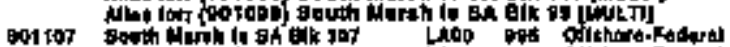

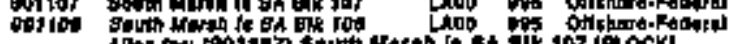

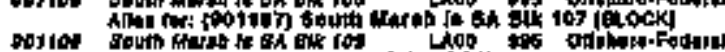

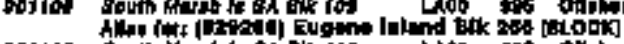

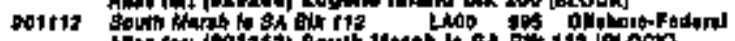

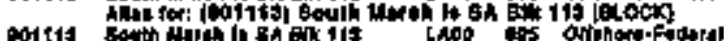

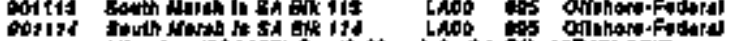

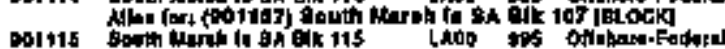

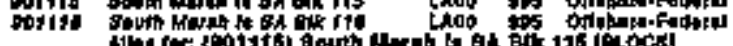

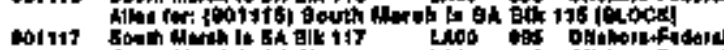

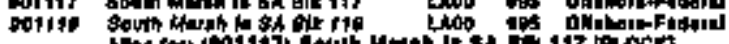

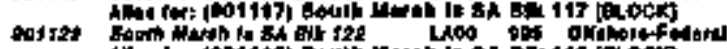

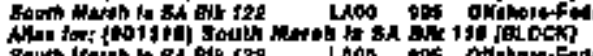

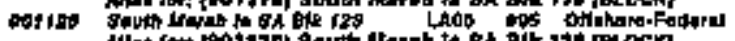

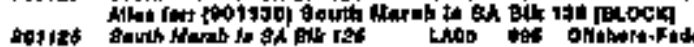

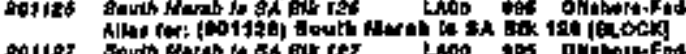

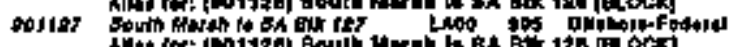

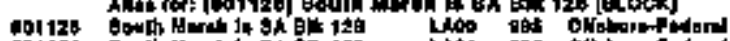

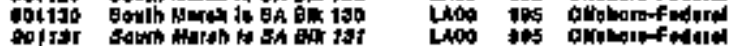

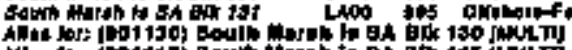

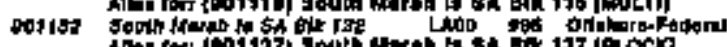

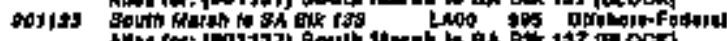

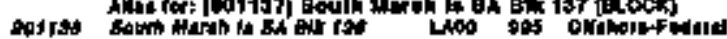

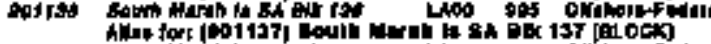

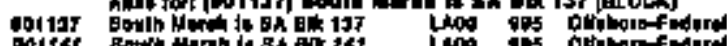

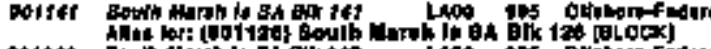

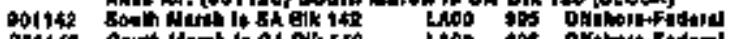

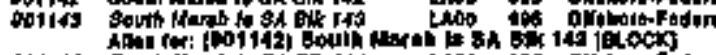

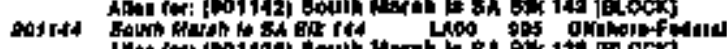

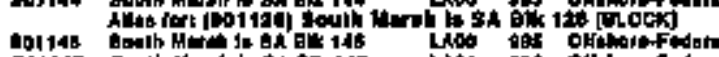

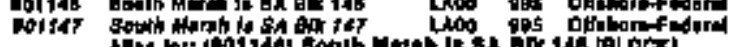

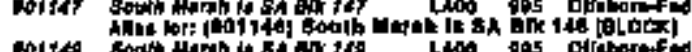

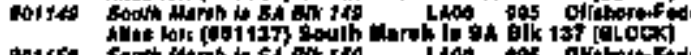

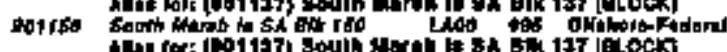

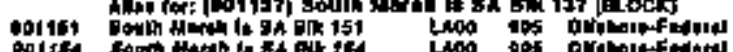

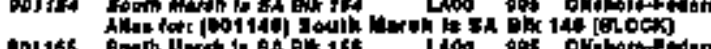

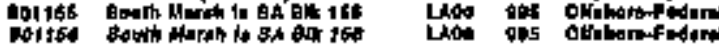

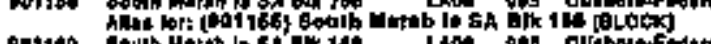

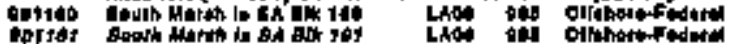

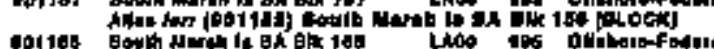

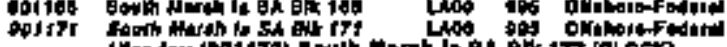

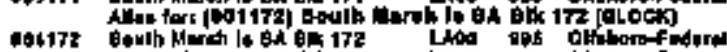

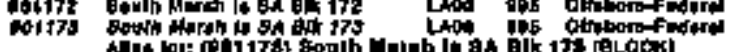

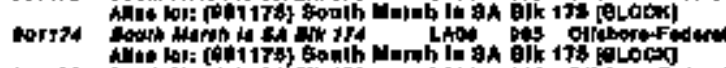

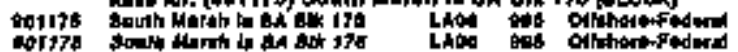

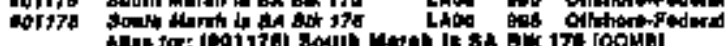

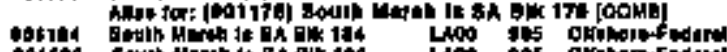

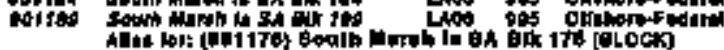

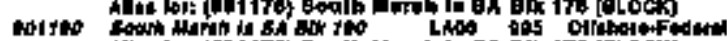

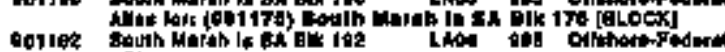

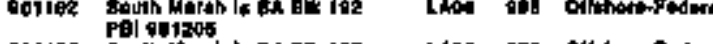

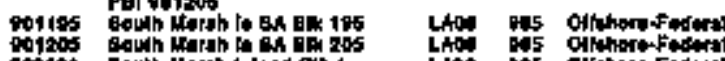

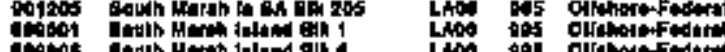

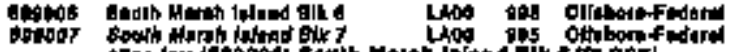

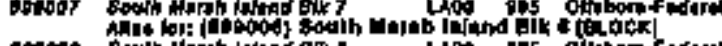

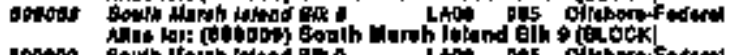

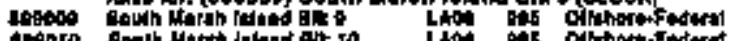

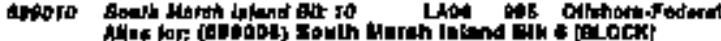

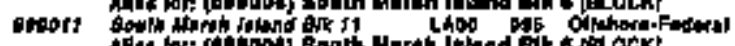

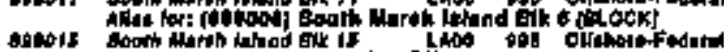

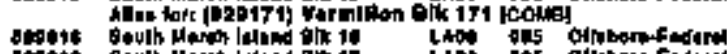

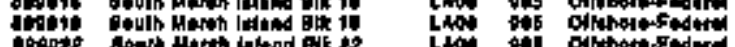

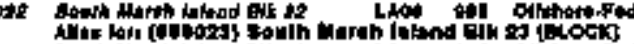

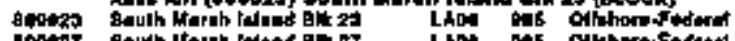

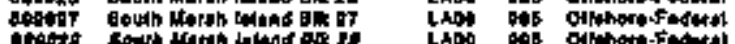

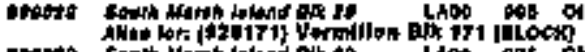

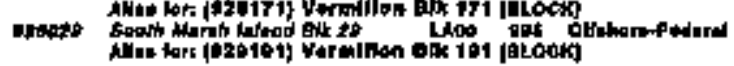

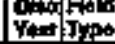

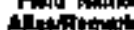

Bets

Conatr

Ving

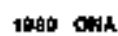

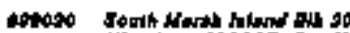

Lod Othore.Fodoral

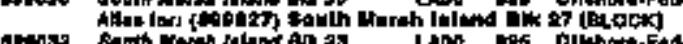

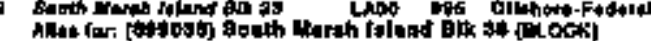

(2)

1804 ONA

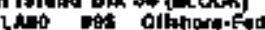

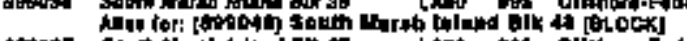

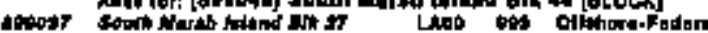

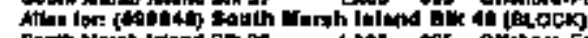

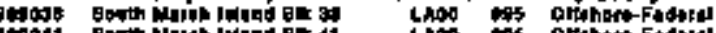

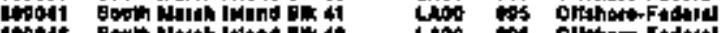

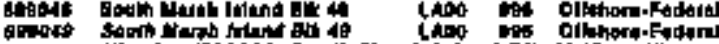

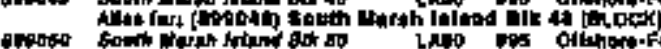

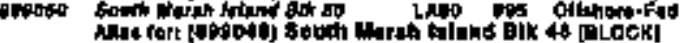

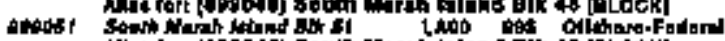

107.

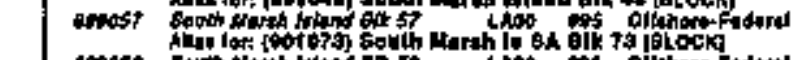

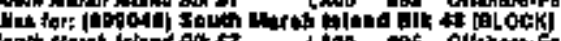

1 tot $\mathrm{O}$ 보

1016 H

arastr

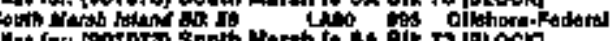

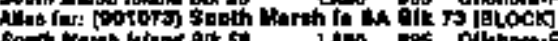

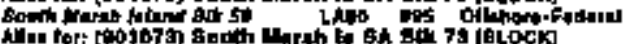

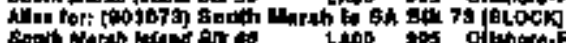

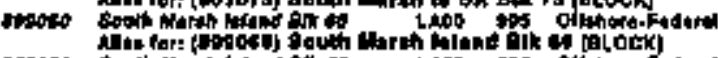

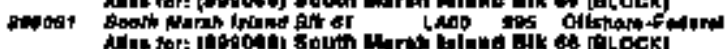

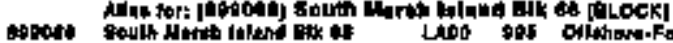

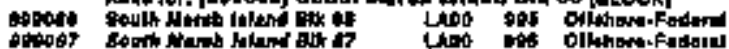

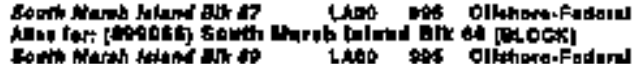

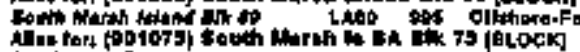

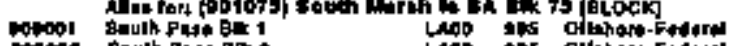

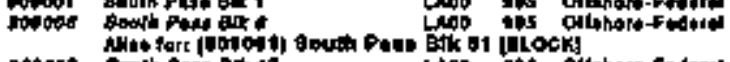

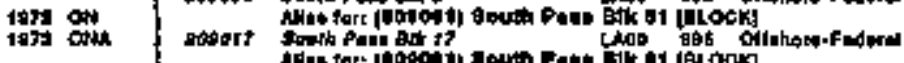

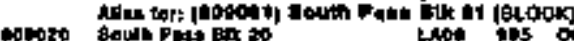

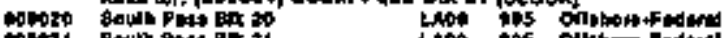

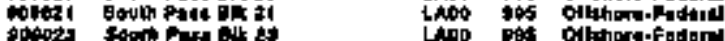

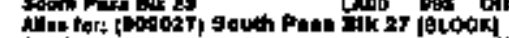

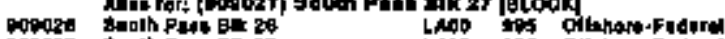

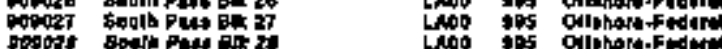

19 4 on

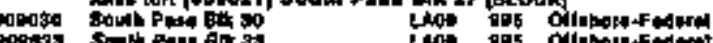

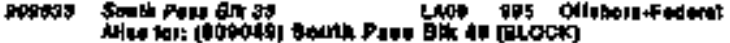

1084

popost sost

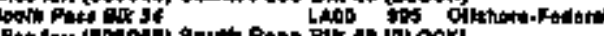

等

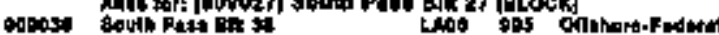

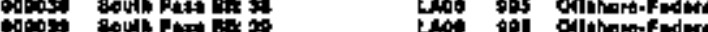

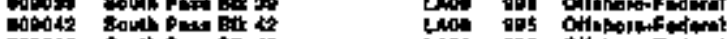

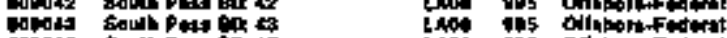

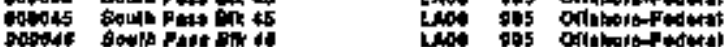

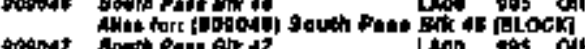

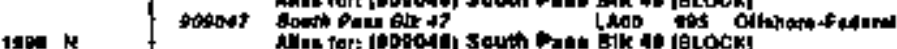

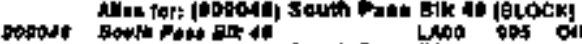

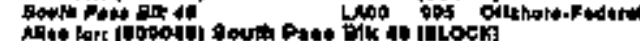

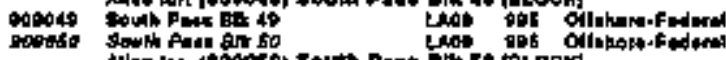

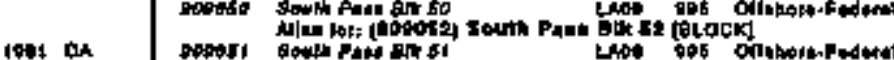

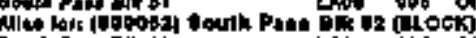

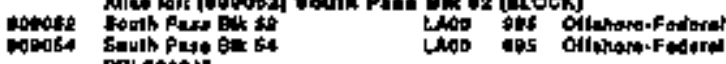

PEI $\$ 00045$

1 a) $\mathrm{k}$

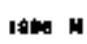

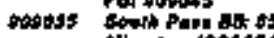

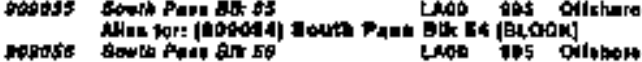

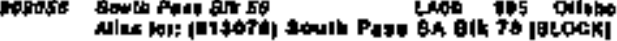

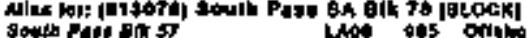

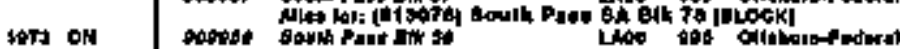

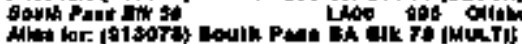

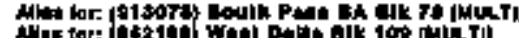

1974 $\mathrm{M}$

reapest sowit pros

nopst

5 . 5 .

opanta Dave

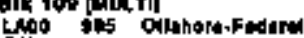

posedo sown

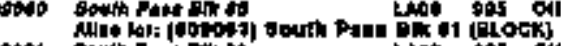

Iำ

Ipan

1902 oNh

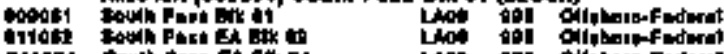

(Bock]

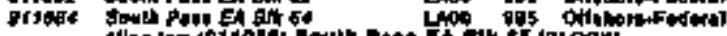

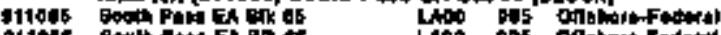

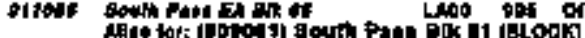

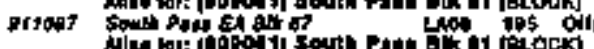

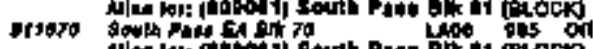

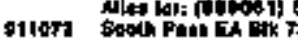

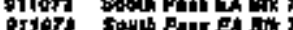

Lato bes on

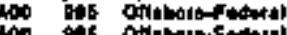

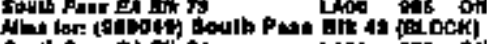

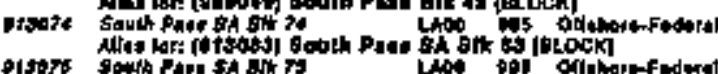

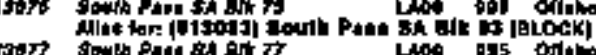

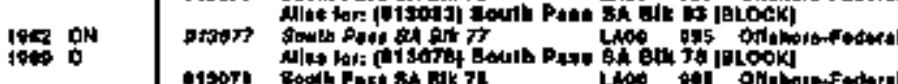

100 0

19.0\%

Fodith Part

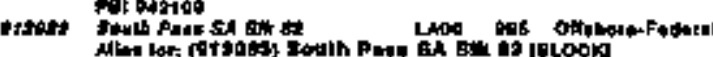

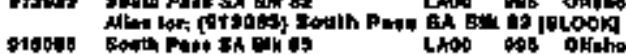

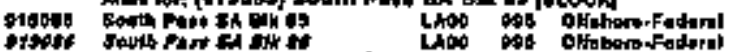

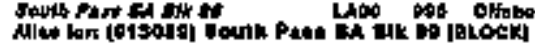

+ots

tod on

vatg ow

190 on

10H: N

1957 N

1055 N

10:2 oN

1094

1981 Oin

16) As

1094 DNA

10ine on

IDES DN

Iato on

1900 $\mathrm{A}$

1tos on

1904 ONA 
F.O.-LOUISIANA

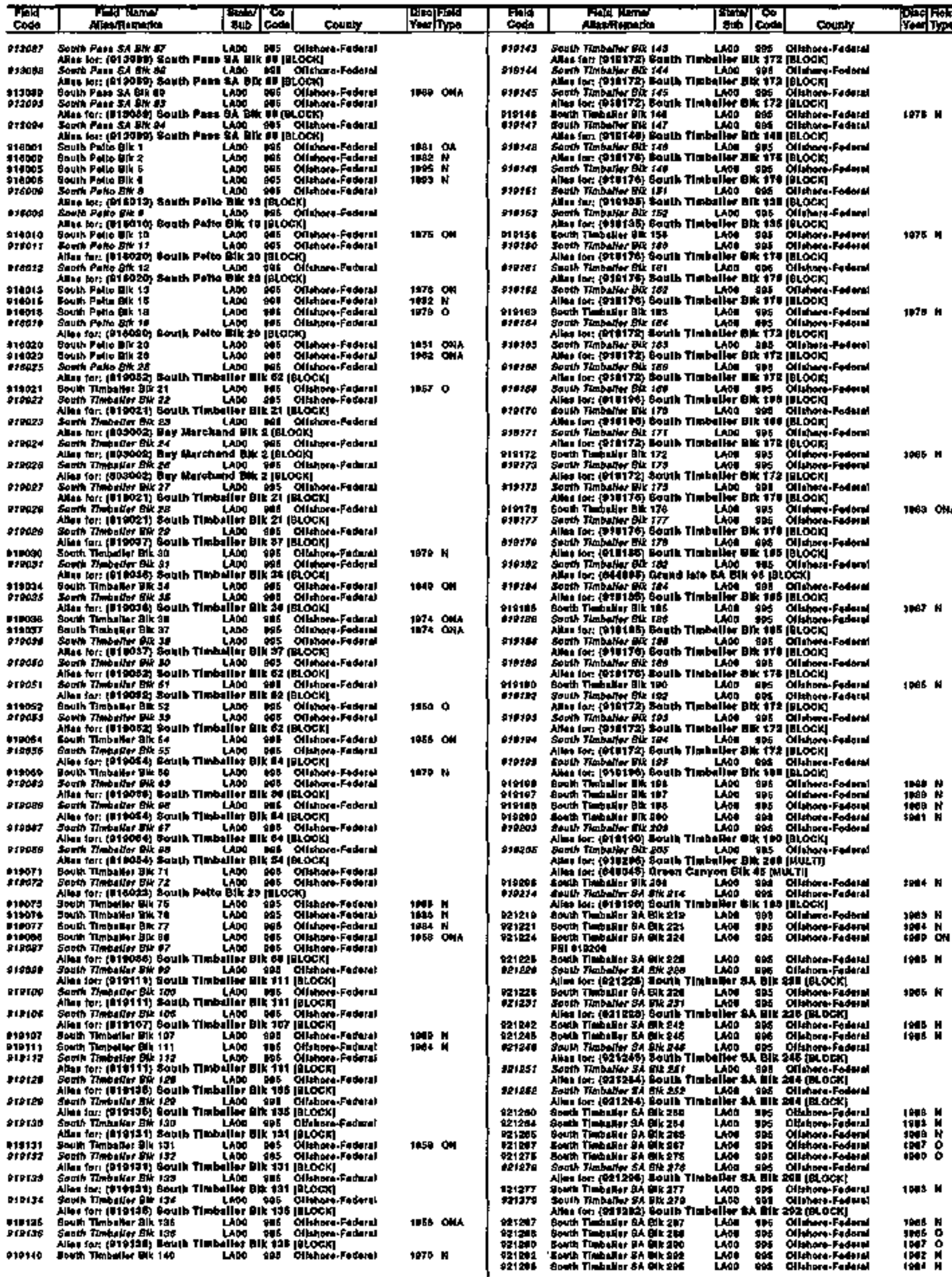


F.O.-LOUISIANA

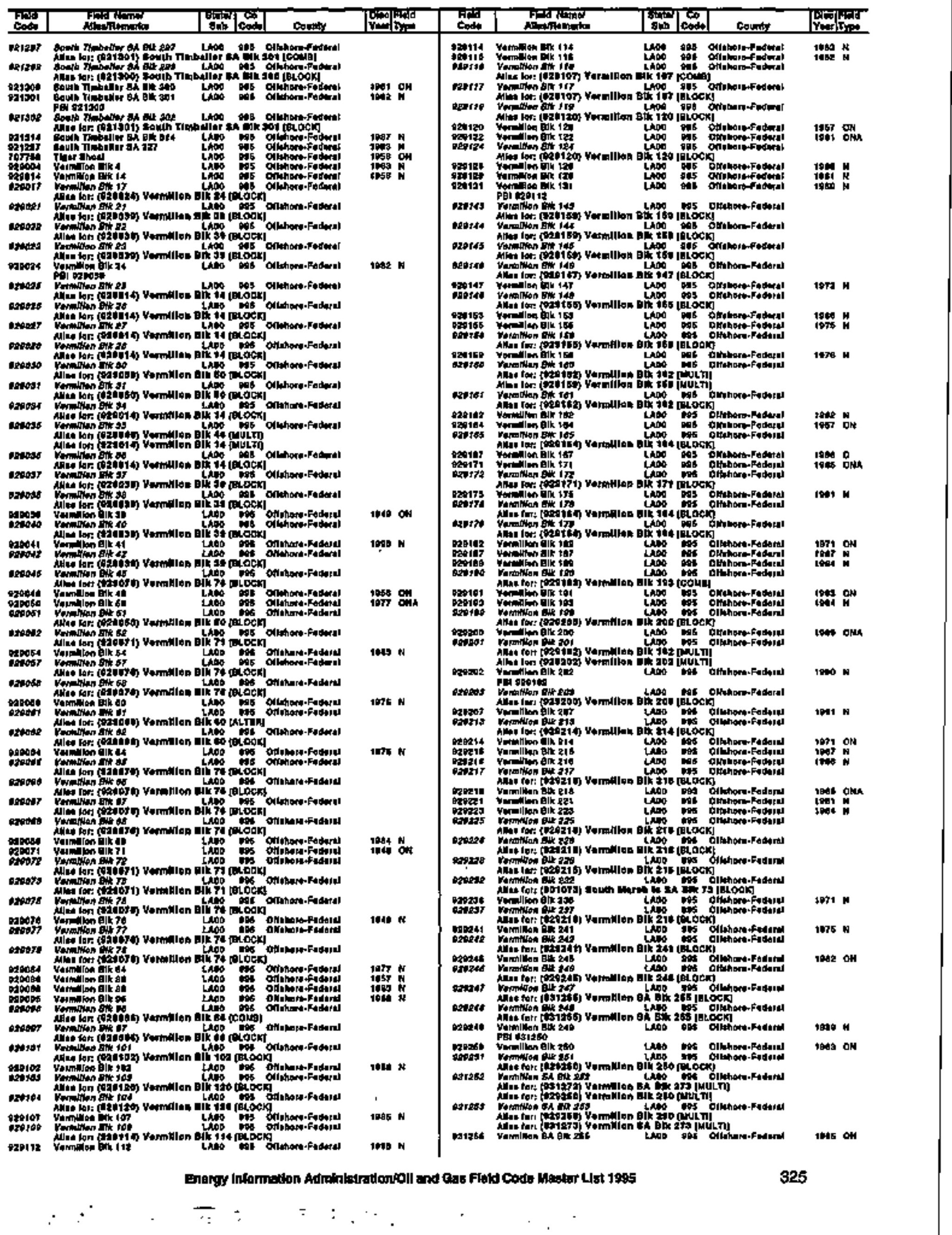




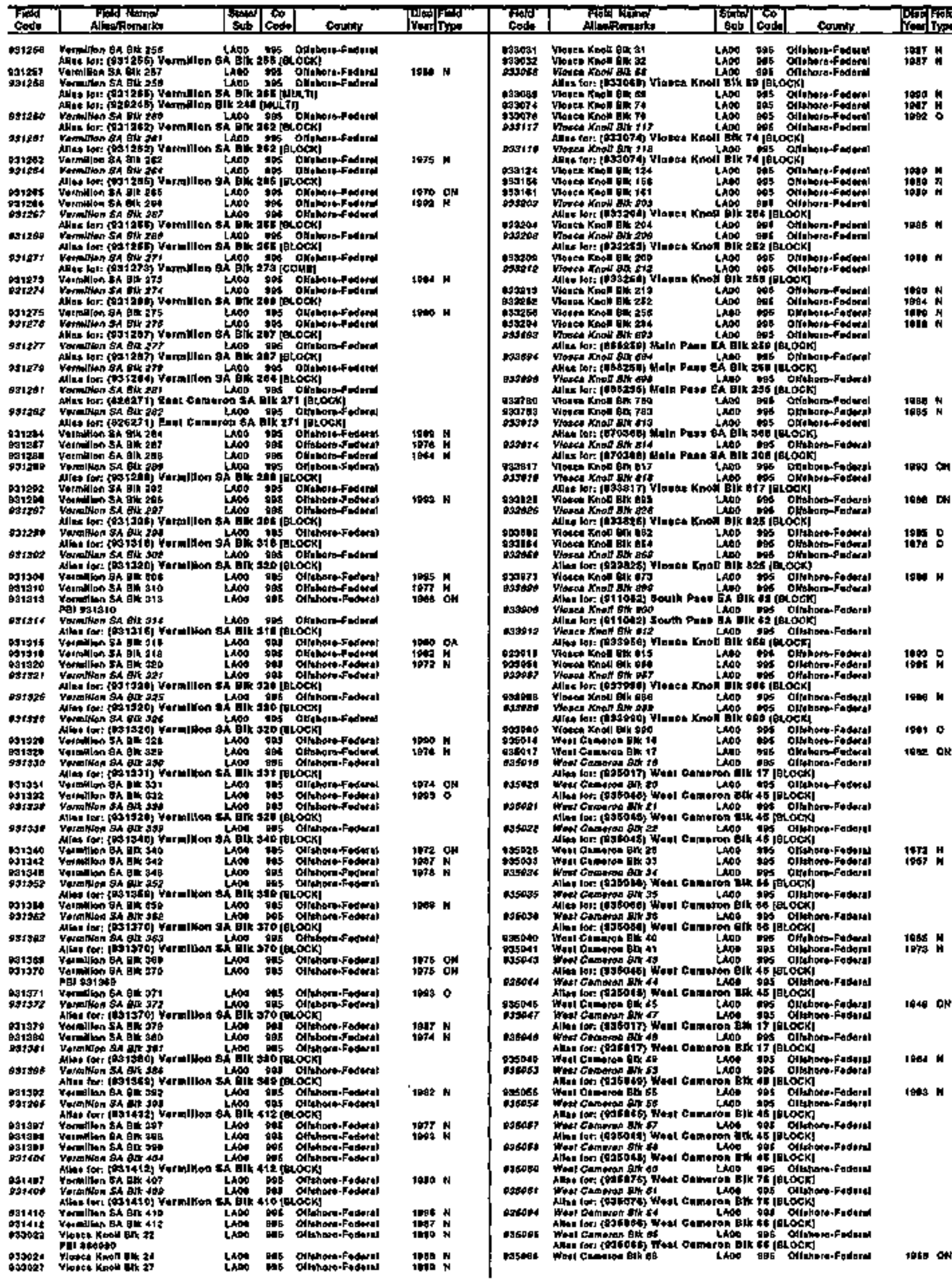


F.O.-LOUISIANA

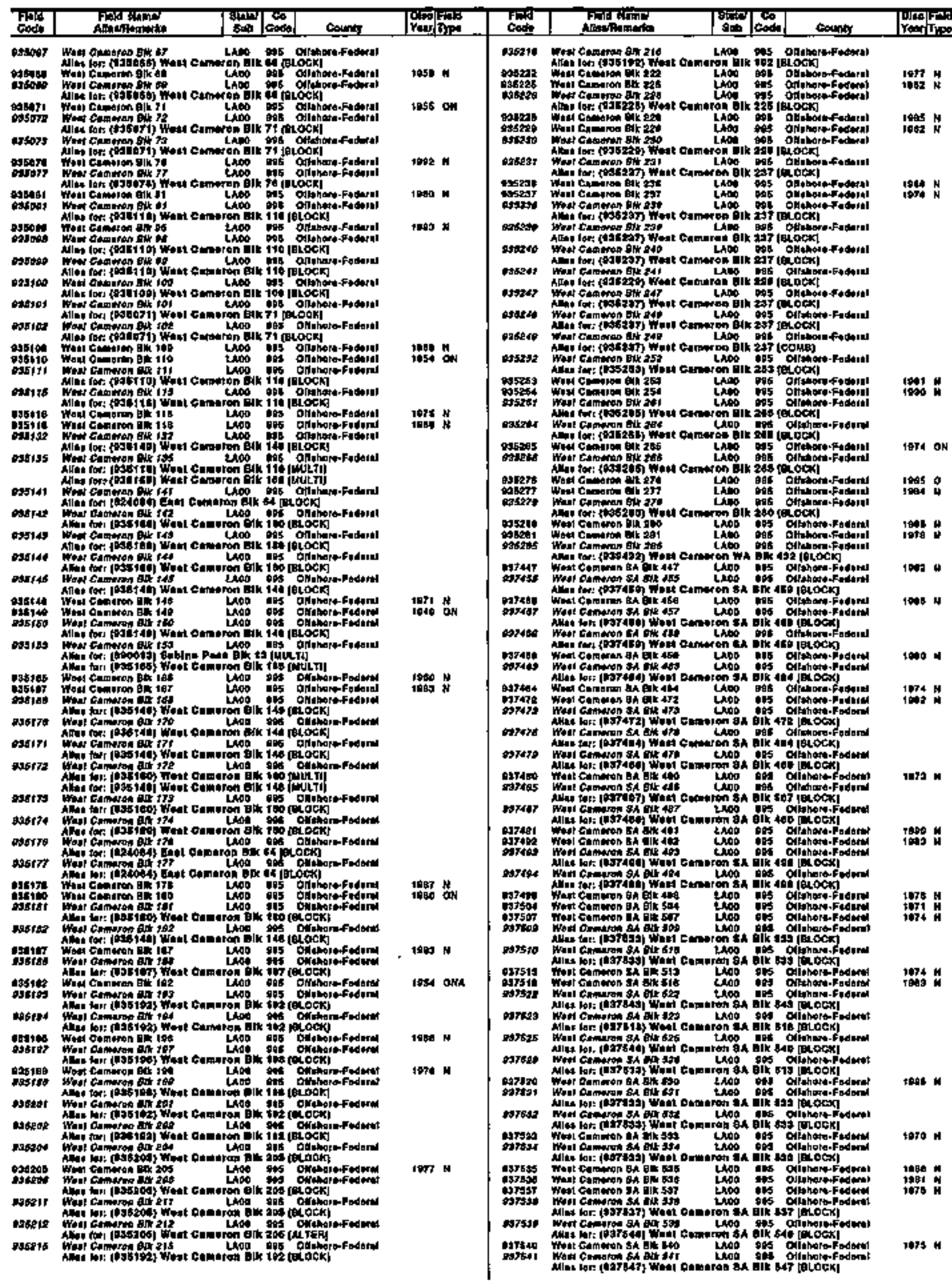




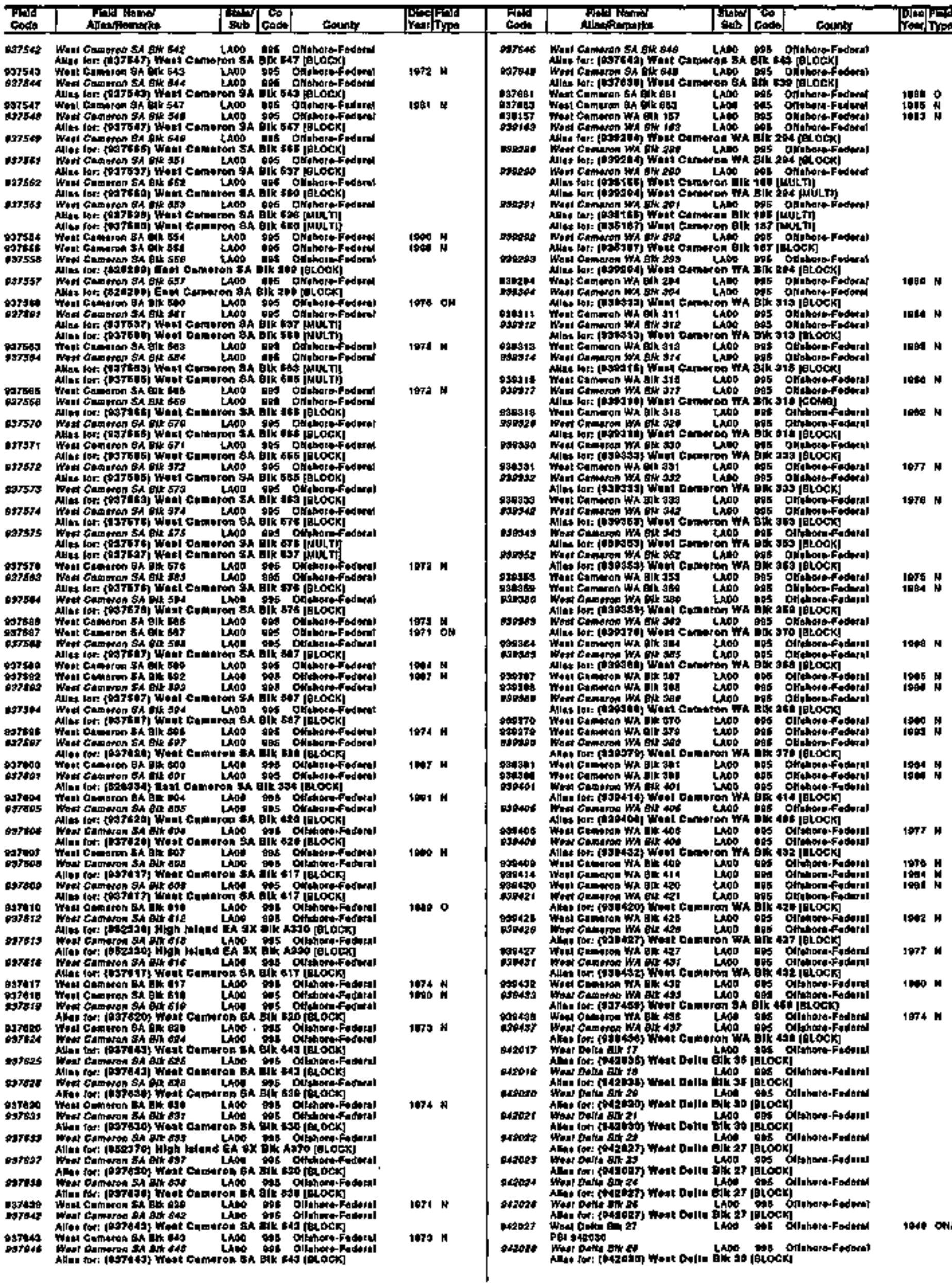




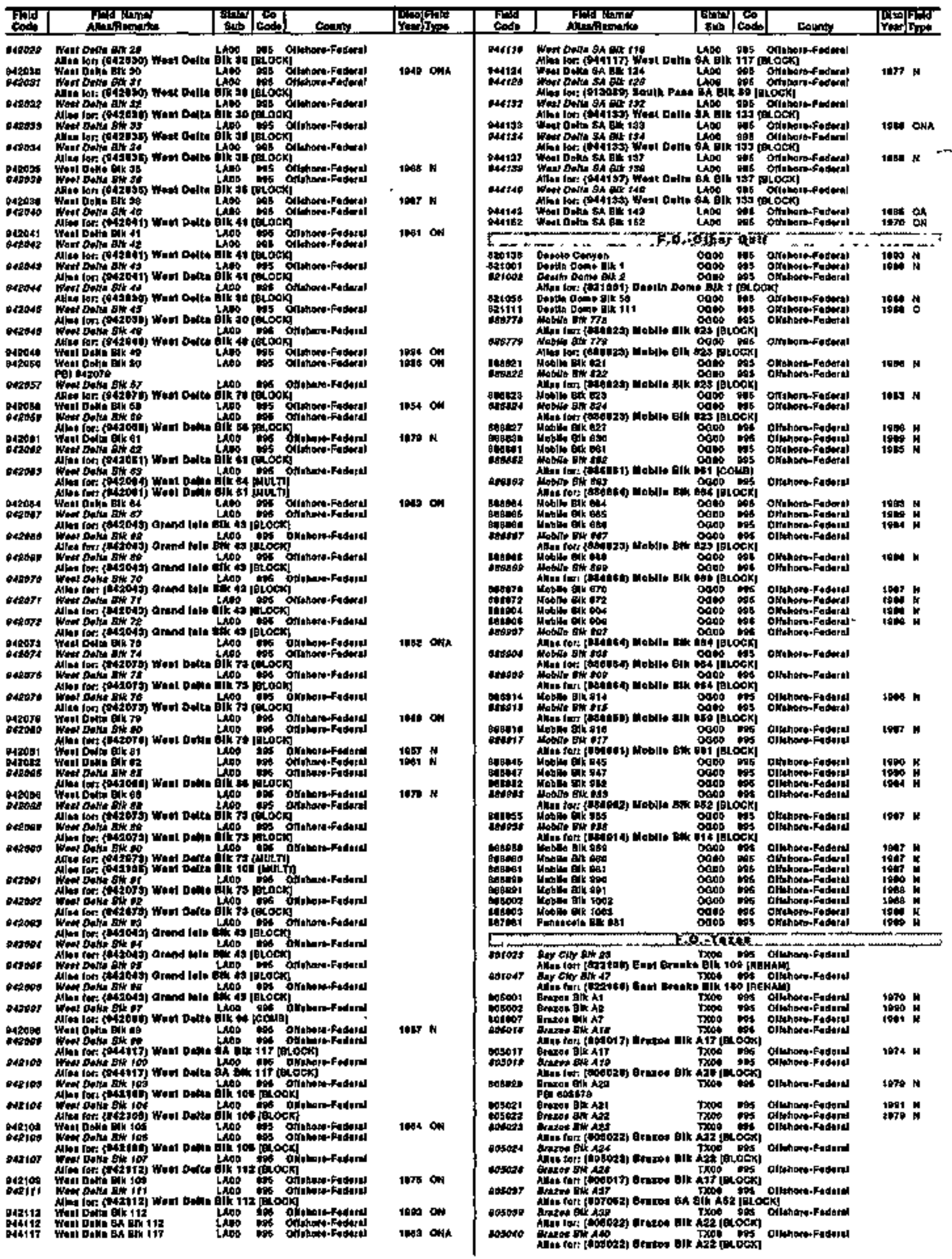




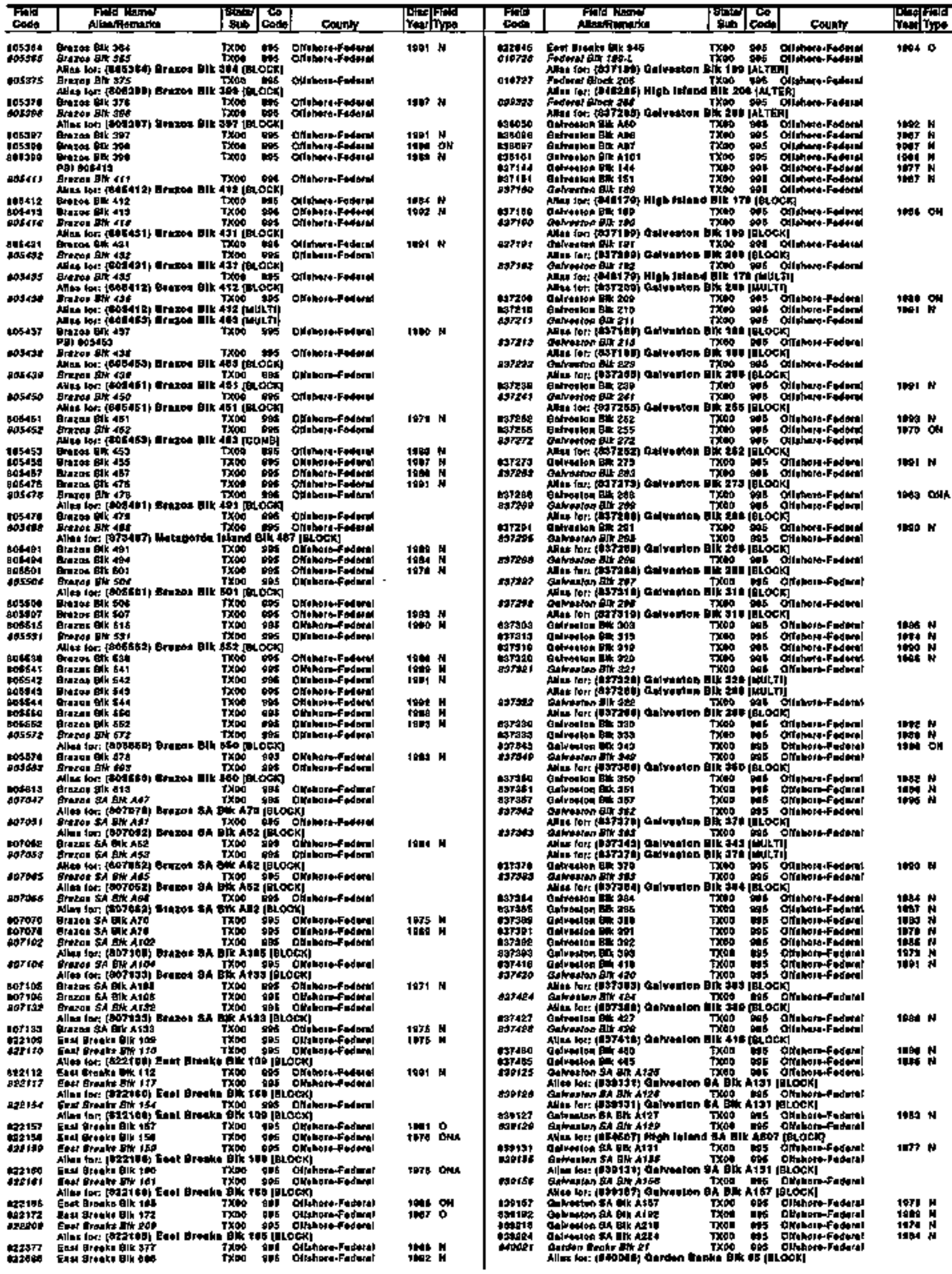




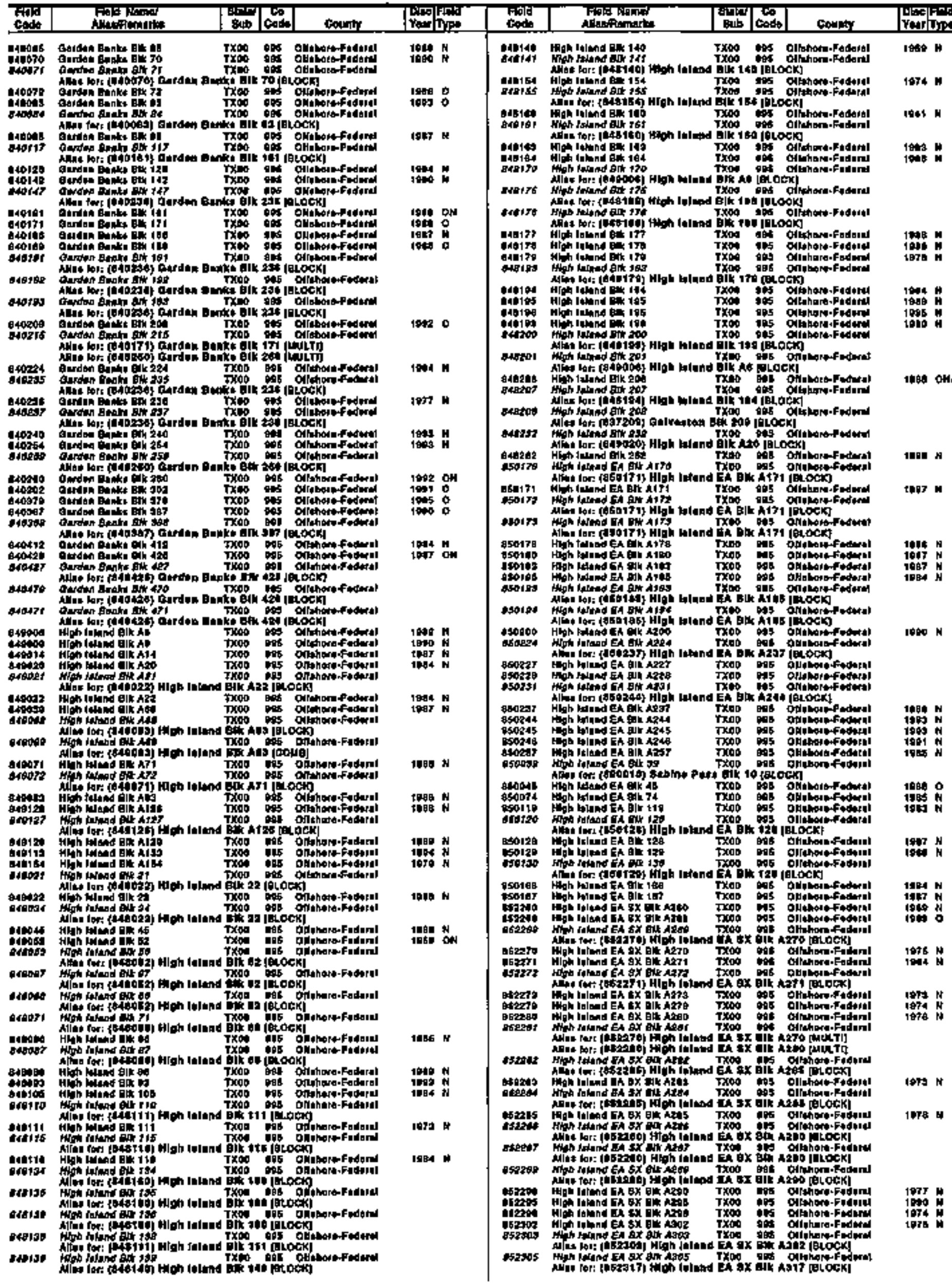




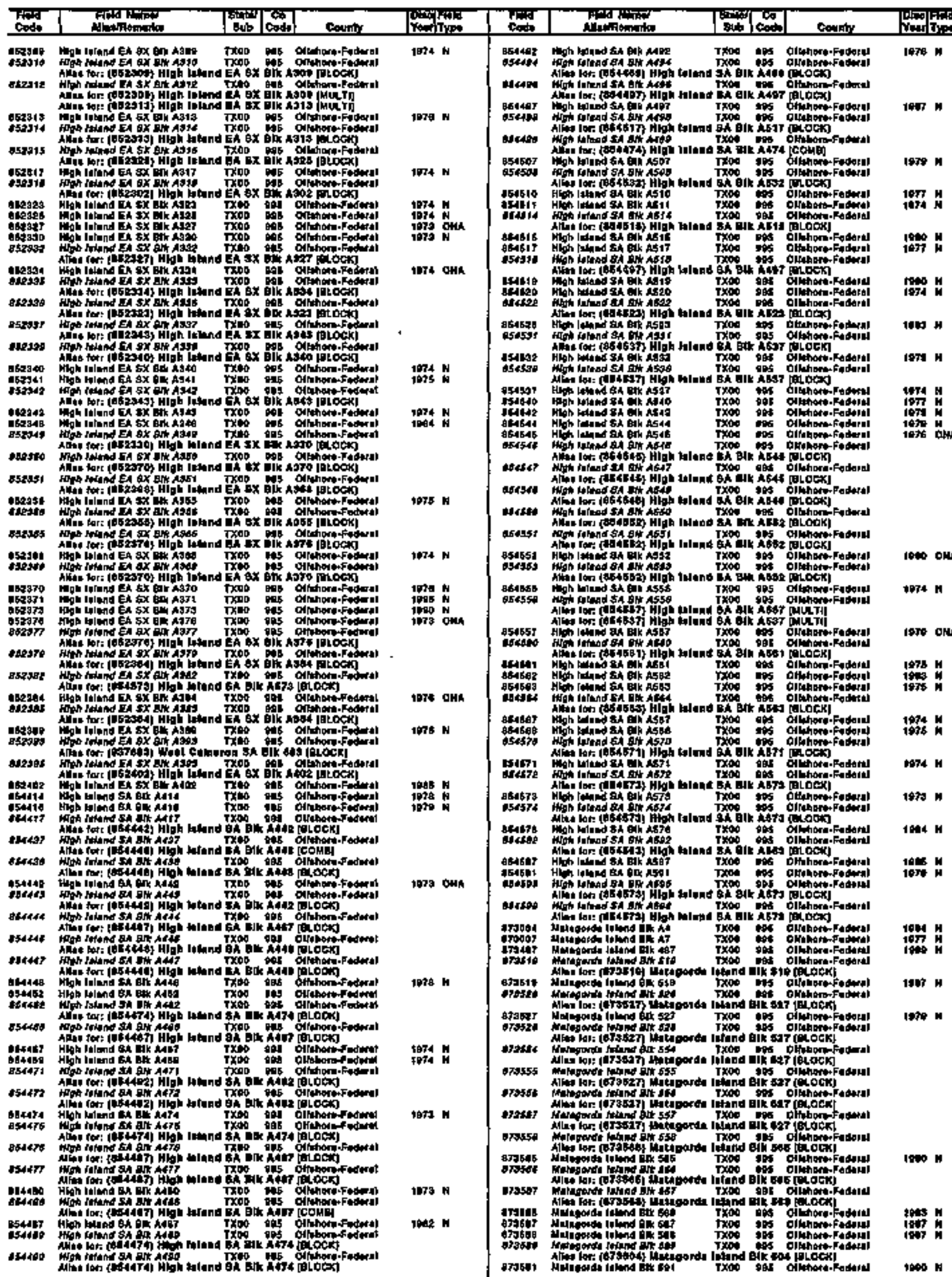




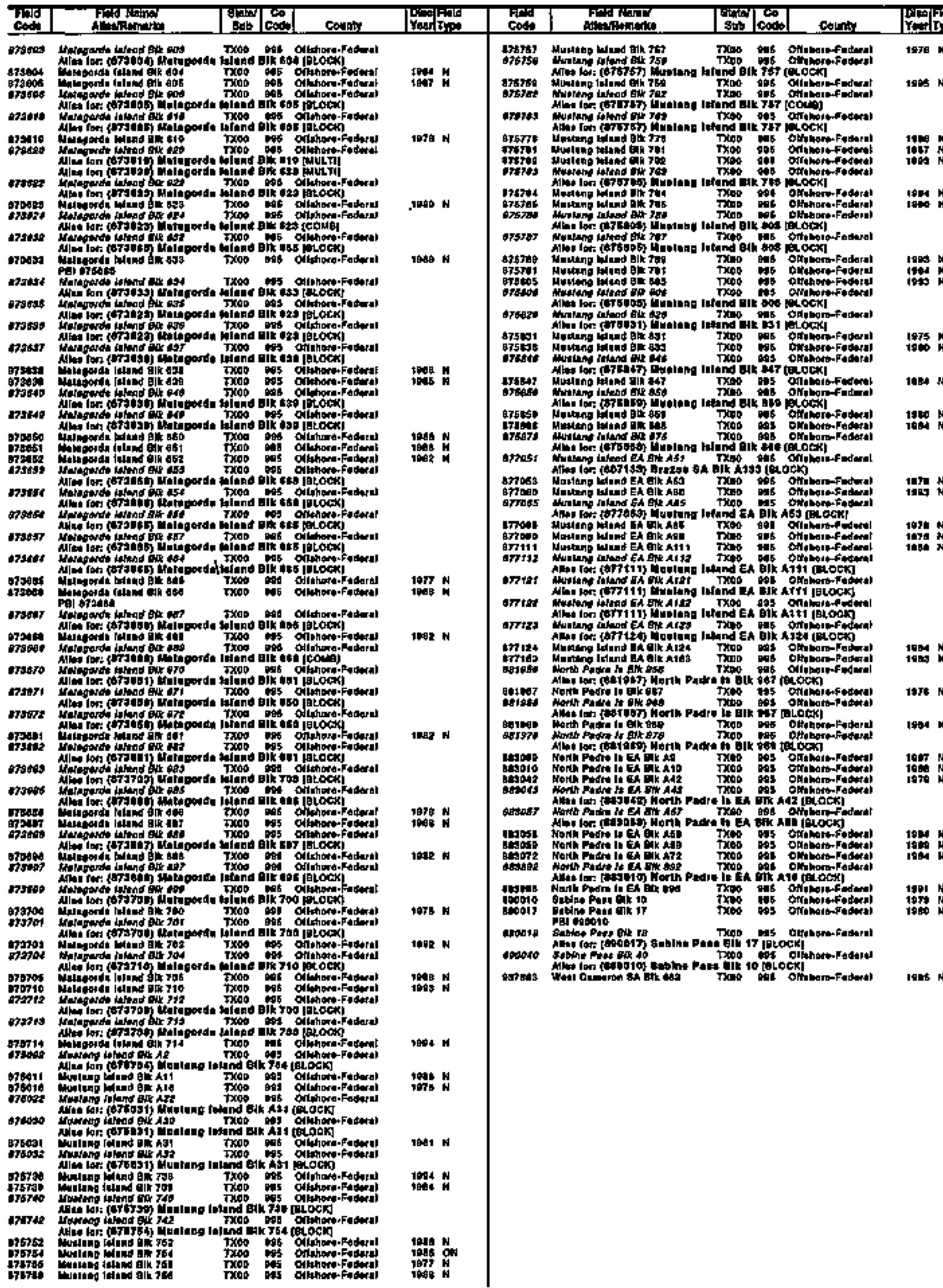




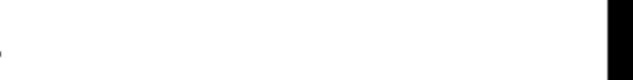




\section{Oil and Gas Invalid Field Record List}


KANSAS

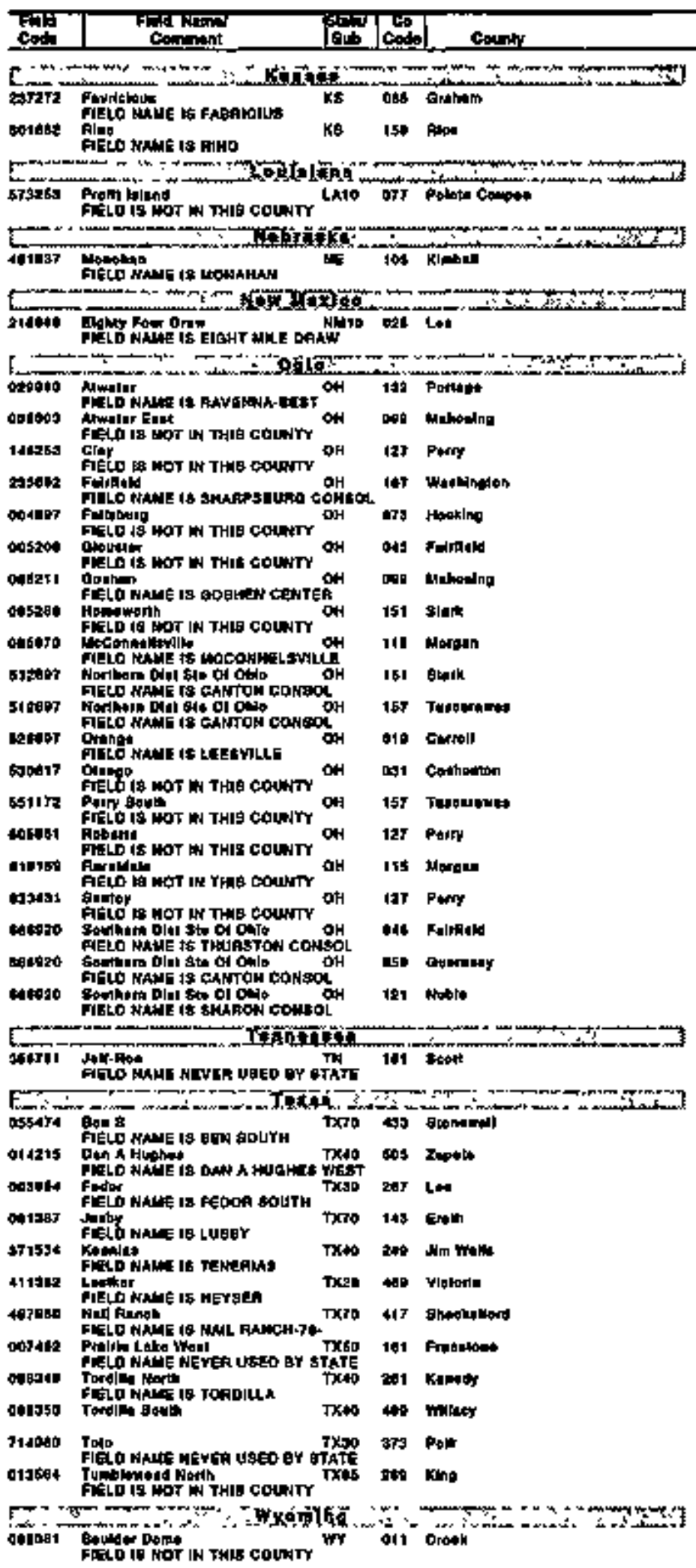




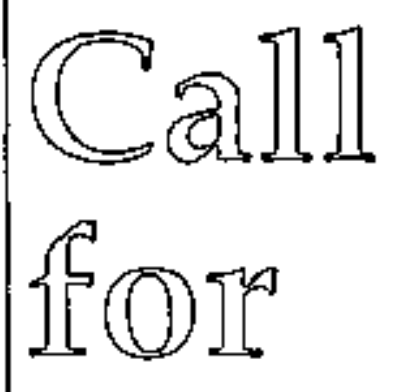

Yourfe
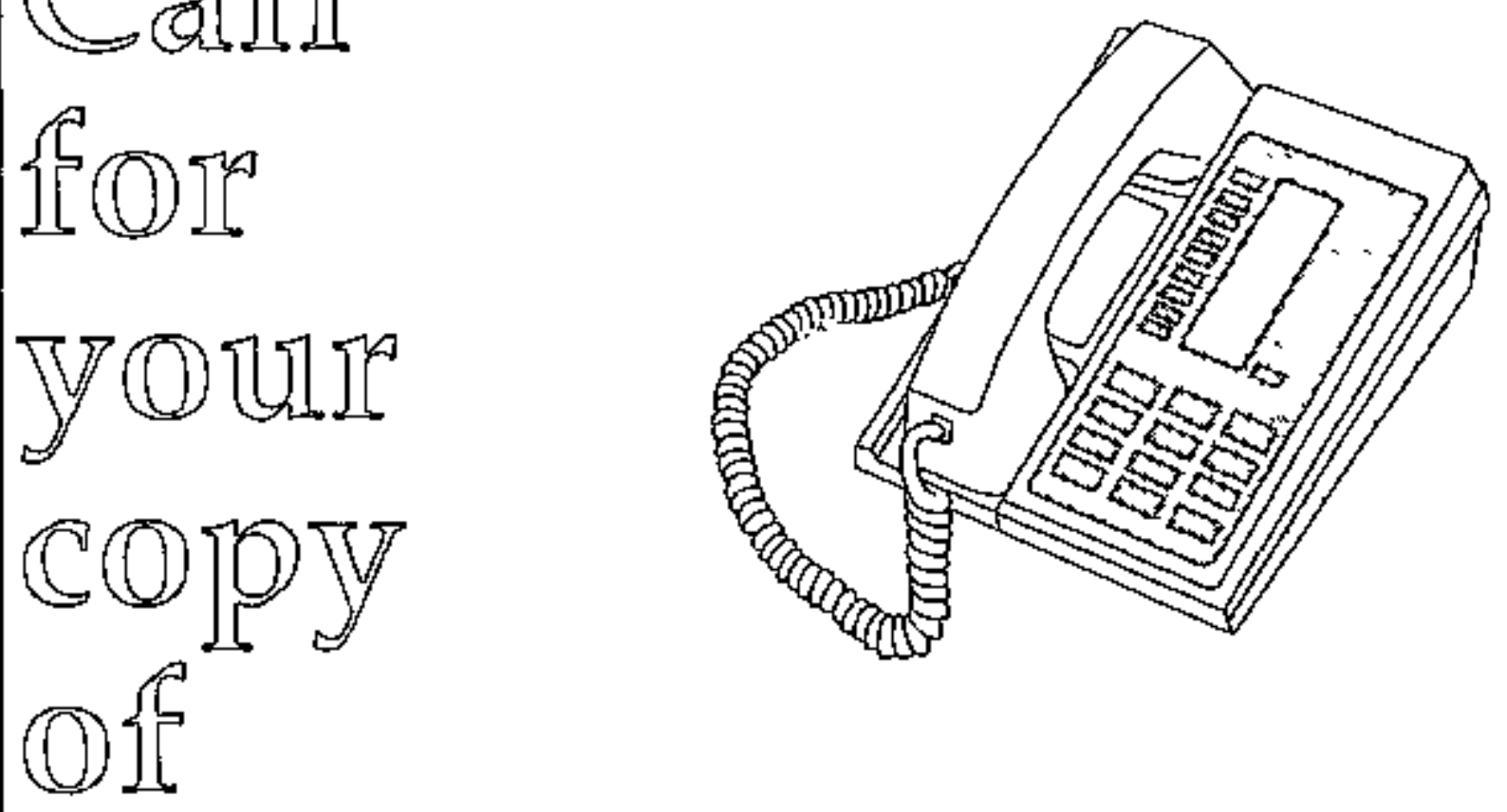

EIA Neru Releases

A concise and timely review of new statistical publicałions and energy news

Offered from the National Energy Information Center

For a complimentary copy of this bimonthly bulletin, or a complimentary subscription, call (202)586-8800. 
Energy Information Administration U.S. Department of Energy

Forrestal Bullding, El-231

Washington, DC 20585

OFFICIAL BUSINESS

PENALTY FOR PAIVATE USE. $\$ 300$

Do Not Forward, Do Not Return

Address Correction Requested

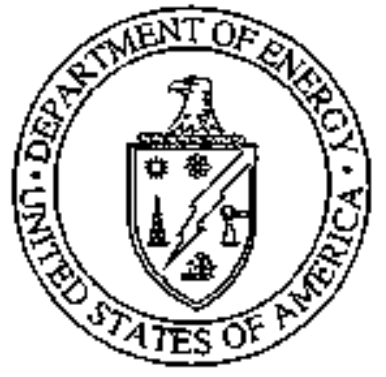

